\title{
Distribution of Recent Benthic Foraminifera off the North American Pacific Coast from Oregon to Alaska
}

\section{STEPHEN J. CULVER}

and MARTIN A. BUZAS
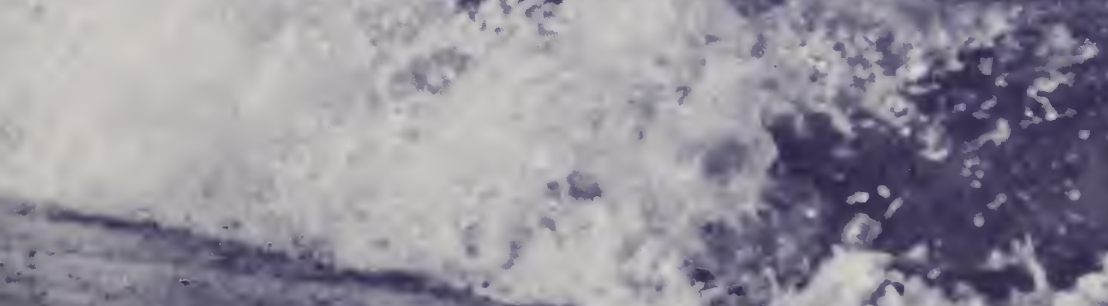

SMITHSONIAN CONTRIBUTIONS TO THE MARINE SCIENCES • NUMBER 26 


\title{
SERIES PUBLICATIONS OF THE SMITHSONIAN INSTITUTION
}

Emphasis upon publication as a means of "diffusing knowledge" was expressed by the first Secretary of the Smithsonian. In his formal plan for the Institution, Joseph Henry outlined a program that included the following statement: "It is proposed to publish a series of reports, giving an account of the new discoveries in science, and of the changes made from year to year in all branches of knowledge." This theme of basic research has been adhered to through the years by thousands of titles issued in series publications under the Smithsonian imprint, commencing with Smithsonian Contributions to Knowledge in 1848 and continuing with the following active series:

\author{
Smithsonian Contributions to Anthropology \\ Smithsonian Contributions to Astrophysics \\ Smithsonian Contributions to Botany \\ Smithsonian Contributions to the Earth Sciences \\ Smithsonian Contributions to the Marine Sciences \\ Smithsonian Contributions to Paleobiology \\ Smithsonian Contributions to Zoology \\ Smithsonian Folklife Studies \\ Smithsonian Studies in Air and Space \\ Smithsonian Studies in History and Technology
}

In these series, the Institution publishes small papers and full-scale monographs that report the research and collections of its various museums and bureaux or of professional colleagues in the world of science and scholarship. The publications are distributed by mailing lists to libraries, universities, and similar institutions throughout the world.

Papers or monographs submitted for series publication are received by the Smithsonian Institution Press, subject to its own review for format and style, only through departments of the various Smithsonian museums or bureaux, where the manuscripts are given substantive review. Press requirements for manuscript and art preparation are outlined on the inside back cover.

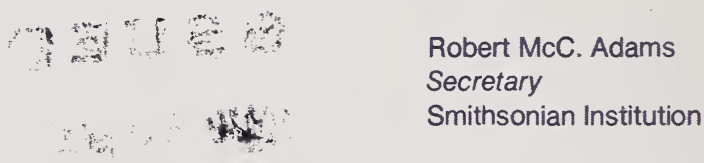

Robert McC. Adams

Smithsonian Institution 


\section{Distribution of Recent Benthic Foraminifera off the North American Pacific Coast from Oregon to Alaska}

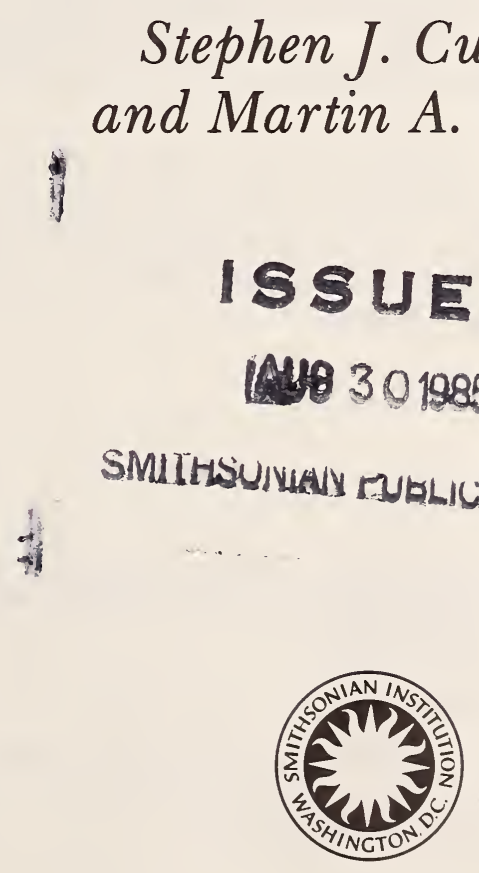

SMITHSONIAN INSTITUTION PRESS

City of Washington 1985 


\section{A B S T R A C T}

Culver, Stephen J., and Martin A. Buzas. Distribution of Recent Benthic Foraminifera off the North American Pacific Coast from Oregon to Alaska. Smithsonian Contributions to the Marine Sciences, number 26, pages 234, 139 figures, 2 tables, 1985.-A computer file of all published distributional data (presence or absence) on the living and dead recent benthic foraminifera off the North American Pacific Coast (Oregon to Alaska) was constructed from 31 papers published since 1886. Manipulation of this file produced 5 catalogs and 139 maps.

Catalog 1 lists alphabetically species names with publication and locality information as recorded in the literature (i.e., unsynonymized). Catalog 2 lists synonymized species names with publication and locality information. Catalogs 3 and 4 list alphabetically all unsynonymized and synonymized species names, respectively. Catalog 5 lists synonymized names by increasing latitude and longitude.

During the past 100 years, 523 names have been used to record benthic foraminifera in the study area. Through synonymization, this number was reduced to 404, of which 138 occur at 6 or more of the 157 sample localities. Computer-generated maps were drawn for the 138 most commonly recorded species.

Species were grouped by depth and geographic (latitudinal) distribution through visual examination of the maps. Eight species are coastal in their distribution, 63 occur mainly at depths of less than $200 \mathrm{~m}, 27$ at depths greater than $200 \mathrm{~m}$, and 40 are ubiquitous with depth. Many species appear to alter their depth distribution with latitude, but this may be due to a poor sampling framework.

Latitudinally, the species are grouped into three categories. Forty-two species occur mainly to the north of $52^{\circ}-55^{\circ} \mathrm{N}, 22$ species occur mainly to the south of $52^{\circ}-55^{\circ} \mathrm{N}$, and 74 species are latitudinally ubiquitous within the area of study. This preliminary analysis indicates a possible faunal break in the region of Queen Charlotte Island.

Official publication Date is handstamped in a limited number of initial copies and is recorded in the Institution's annual report, Smithsonian Year. SERIES Cover DESIGN: Seascape along the Atlantic coast of eastern North America.

Library of Congress Cataloging in Publication Data

Culver, Stephen J.

Distribution of recent benthic foraminifera off the North American Pacific coast from Oregon to Alaska.

(Smithsonian contributions to the marine sciences ; no. 26)

Bibliography: p.

Includes index.

Supt. of Docs. no.: SI 1.41:26

1. Foraminifera-Pacific Coast (U.S.)—Geographical distribution. 2. Foraminifera-Pacific Coast (B.C.)-Geographical distribution. 3. Protozoa-Geographical distribution. 4. Protozoa-Pacific Coast (U.S.) - Geographical distribution. 5. Protozoa-Pacific Coast (B.C.)-Geographical distribution. I. Buzas, Martin A. II. Title. III. Series.

QL368.F6C624 $1985 \quad 593.1$ '209795 85-600001 


\section{Contents}

Methods................................ 1

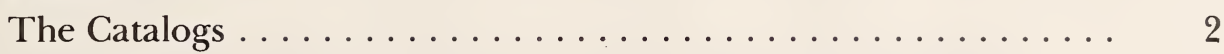

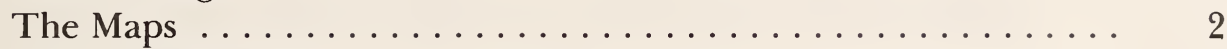

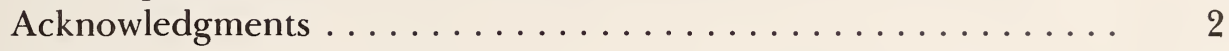

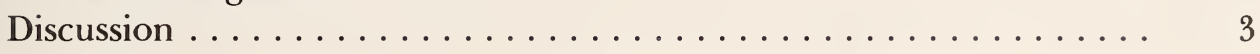

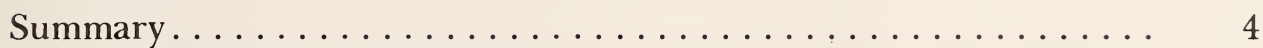

Key to the Species on the Maps................. 5

Index to Publications That Illustrate Our Concept of the 138 Most

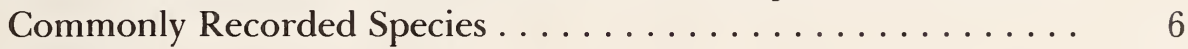

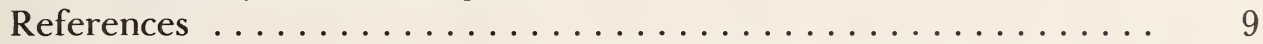

Catalog 1: Unsynonymized Species with Publication and Location . . . 12

Catalog 2: Synonymized Species with Publication and Location . . . . 38

Catalog 3: Unsynonymized Species . . . . . . . . . . . . . . . . . 64

Catalog 4: Synonymized Species . . . . . . . . . . . . . 67

Catalog 5: Latitude and Longitude of Synonymized Species . . . . . . 70

Maps: Figures $1-139 \ldots \ldots \ldots \ldots \ldots \ldots \ldots \ldots \ldots$ 



\title{
Distribution of Recent Benthic Foraminifera off the North American Pacific Coast from Oregon to Alaska
}

\author{
Stephen J. Culver and Martin A. Buzas
}

\section{Introduction}

Study of the recent benthic foraminifera off the North American Pacific Coast began in 1886 when Whiteaves published his work on the faunas off British Columbia. Since then, many other workers have contributed papers on the taxonomy, distribution, and ecology of northwestern Pacific foraminifera. One hundred and fifty-nine papers concerning recent benthic foraminifera of this region were published between 1886 and 1980. This paper represents the first attempt to synthesize these data and is the fourth of a series including and summarizing all published data on the distribution of recent benthic foraminifera around North America. All published distributional data for the east coast is presented in Culver and Buzas (1980) and the results of subsequent analysis are given in Buzas and Culver (1980), Culver and Buzas (1981a,b, 1982a). Gulf of Mexico data are given in Culver and Buzas (1981c,d, 1982a, 1983). Distribution data for the Caribbean region are presented in Culver and Buzas (1982b).

Stephen J. Culver, Department of Geological Sciences, Old Dominion University, Norfolk, VA 23508. Martin A. Buzas, Department of Paleobiology, National Museum of Natural History, Smithsonian Institution, Washington, D.C. 20560.
The work presented here can be divided into three main parts. First, there is a reference section consisting of (1) the papers used in compiling the presence-absence data utilized by this study and (2) additional references that are cited in this paper. Second, there are five computer-generated catalogs listing every species ever recorded in publications on the North American Pacific coast, north of California. Third, there are computer-generated maps of the 138 most commonly recorded species together with a map showing all sample localities in the study area. Explanatory notes for the catalogs and maps can be found later in this introduction.

Following the format of our studies of the benthic foraminifera off the east coast of North America, the Gulf of Mexico, and the Caribbean, we have included a key to our concept of each of the 138 most commonly recorded species.

Methods.-The computerization methods utilized in this work were the same as those described in detail in Culver and Buzas (1980). Of the 159 papers published on the benthic foraminifera of the North American Pacific continental margin, 31 contained data in a form enabling its incorporation in this study. The name of one or more species (living or dead) and the locality where they were found was all that 
was required. The information typed into the basic compilation file consisted of (1) author of publication, (2) date of publication, (3) generic name used in publication, (4) specific name used in publication, (5) locality, and (6) latitude and longitude (see Catalog 1).

When numerous sample sites of a particular study were in very close proximity they were summarized by a single or several representative localities. Thus, all species recorded in the sampled area were considered to have been found at the representative points. In a very few cases, where no detailed locality information was given, a point was arbitrarily chosen to be representative of the records in question. Modification and sorting of this basic file resulted in the production of 5 catalogs, which are described below. The 139 maps in this publication are also computergenerated and were drawn on a Calcomp Plotter.

The Catalogs.-Five catalogs are included in this section and are designed to enable the reader to have rapid access to all published distributional data on recent benthic foraminifera off the North American Pacific coast north of California. Catalog 1 lists (alphabetically by binomen) all the records of every species ever recorded in the study area and also includes publication and locality information. This catalog contains almost 2,500 entries and around 12,500 pieces of information. Catalog 2 is the synonymized version of number 1 . This list is identical to Catalog 1 except that generic and specific names have been changed where necessary. To aid referral between Catalogs 1 and 2, line numbers starting at 10001 are included at the lefthand margin of the files. Thus, for example, it can be seen that Trifarina angulosa from Ketchikan, Alaska (Catalog 2, line 10121) was originally recorded (Cushman and McCulloch, 1948) as Angulogerina angulosa (Catalog 1, line 10121). Due to an error, line 12047 in Catalogs 1 and 2 is blank.

Catalogs 3 and 4 aid rapid use of the first two catalogs. Catalog 3 is an alphabetized list of the 523 taxa that have been recorded on the North American Pacific continental margin north of
California. Catalog 4 lists alphabetically the 404 species recorded in this area after synonymization achieved by reference to published illustrations and specimens lodged in the USNM collections (National Museum of Natural History, Smithsonian Institution) and the British Museum (Natural History) collections. Catalogs 3 and 4, therefore, are derived directly from Catalogs 1 and 2 , respectively.

Catalog 5 is intended for the researcher interested in specific areas. This catalog contains alphabetized lists of synonymized species for each locality (localities are listed by increasing latitude and longitude). If, in the case of synonymized species, the investigator questions the generic or specific name used, he can refer to Catalogs 1 and 2 to find the original name used by the author of the publication in which that species was recorded.

THE MAPS. - The 139 computer-generated maps included in this section are, like the catalogs, provided as a reference tool for use in distributional studies of recent benthic foraminifera. Figure 1 illustrates the distribution of all the 157 sample localities included in this study. The $183 \mathrm{~m}$ and $1830 \mathrm{~m}$ submarine contours roughly approximate to the edge of the continental shelf and the bottom of the continental slope, respectively. The distribution of the sample localities must be taken into account when examining the maps illustrating the distributions of species.

Figures 2 to 139 are computer-generated maps of the 138 most commonly recorded species. These are species that have been recorded 6 times or more, or at approximately $4 \%$ or more of the 157 localities; the figure of $4 \%$ was chosen arbitrarily to conform with the studies of the Atlantic continental margin of North America, the Gulf of Mexico, and the Caribbean (Culver and Buzas, 1980, 1981c, 1982b). The maps are arranged alphabetically by genera and by species within each genus.

Acknowledgments. - We thank Dante Piacesi and Ken McCormick for their considerable efforts in the computer production of this work. 
D.A. Diddi, L. Collins, S. Richardson, and A. Lanham contributed invaluable library research and secretarial services. Dr. C.G. Adams kindly allowed access to British Museum (Natural History) collections.

\section{Discussion}

The main purpose of this publication is to present all the published distributional data of recent benthic foraminifera from the North American Pacific continental margin north of California. A total of 523 names have been recorded in this region from 157 localities. Synonymization reduced this number to 404 . Of these 404 species, 138 occurred at 6 or more of the 157 localities; that is, $29 \%$ of the species occur at $4 \%$ or more of the sample localities.

Distributional data have been summarized by tabulation. Table 1 is a synopsis of depth distribution and Table 2 summarizes latitudinal distribution. Depth distribution of the 138 most commonly recorded species was determined through visual inspection of the maps. Forty species are ubiquitous, 8 are coastal, 63 occur at depths of less than $200 \mathrm{~m}$ and 27 species at depths of greater than $200 \mathrm{~m}$.

The sampling framework must be taken into account when considering these depth distributions. In this study, "ubiquitous" means that a species occurs both on the continental shelf and slope. As there are few abyssal samples, ">200 $\mathrm{m}$ " indicates that a species is found mainly on the slope. Similarly, " $<200 \mathrm{~m}$ " indicates that a species occurs mainly on the shelf. The category "coastal" includes lagoons, marshes, bays, and beaches.

Many of the species listed under "ubiquitous" occur on the shelf in the south of the study area, but with increasing latitude they extend their bathymetric ranges down onto the slope. This could be interpreted as species changing their habitats with latitude as indicated by depth distributions (e.g., Hazel, 1970). However, there are so few bathyal samples in the south of the study area that this interpretation cannot be accepted without further study.

The poor sampling framework, together with taxonomic inconsistencies, can also be suggested as the reason for some unexpected distributions. For example, Trochammina inflata, a well-known littoral marsh form, is listed in Table 1 as having a ubiquitous distribution. Examination of Figure 128 shows 12 records, of which 7 are coastal, 4 are bathyal and 1 is abyssal. Given these wide habitat differences it is reasonable to suggest that even with synonymization, two or more species are represented by these data.

One species, Cribrostomoides nitidum (Figure 29 ), is distinguishable from all others in the ">200 m" category. The six records are all from abyssal depths and so this species may be a useful deep water indicator in the study area. In comparison, in the Gulf of Mexico (Culver and Buzas, 1981c) C. nitidum occurs at bathyal and abyssal depths. This illustrates the danger of utilizing the isobathyal species concept (Bandy and Chierici, 1966) as a tool for bathymetric interpretations.

Latitudinal distribution of recent benthic foraminifera is the subject of Table 2 . Visual examination of Figures 2-139 resulted in three categories of distribution with a possible faunal boundary in the vicinity of Queen Charlotte Island $\left(52^{\circ}-55^{\circ} \mathrm{N}\right)$. Forty-two species occur mainly to the north of this region, 22 occur mainly to the south, whilst 74 species occur throughout most of the latitudinal spread of the study area from around $41^{\circ} \mathrm{N}$ to $61^{\circ} \mathrm{N}$.

Although a possible faunal break is noted above, we will reserve comment on foraminiferal provinces along the Pacific coast of North America until the final two volumes in this series covering California to Baja, California, and Baja to Panama (Culver and Buzas, in preparation) are published.

The summary distributions described above are based solely on the data presented in this study and do not take into account distributional data to the north or south of the area covered by this publication. 
TABLE 1.-Depth distribution.

Ubiquitous

Adercotryma glomerata

Astrononion gallowayi

Bolivina decussata

Bolivina pacifica

Buccella frigida

Bulimina ovata

Cassidulina barbara

Cibicides fletcheri

Cribrostomoides jeffreysii

Cyclogyra involvens

Epistominella exigua

Epistominella pacifica

Epistominella vitrea

Eponides leviculus

Fissurina lucida

Globobulimina auriculata

Globobulimina pacifica

Haplophragmoides planissimum

Islandiella californica

Islandiella norcrossi

Lagena elongata

Lagena striata

Miliolinella subrotunda

Nonionella auricula

Nonionella turgida digitata

Nonionellina labradorica

Oolina hexagona

Oolina melo

Oolina straitopunctata

Pullenia salisburyi

Pyrgo murrhina

Reophax scorpiurus

Saccammina atlantica

Spiroplectammina biformis

Thalmannammina parkerae

Trifarina fluens
Trochammina inflata

Trochammina nitida

Uvigerina juncea

Uvigerina peregrina

$$
>200 \mathrm{~m}
$$

Ammodiscus minutissimus

Bolivina alata

Bolivina spissa

Bulimina barbata

Bulimina inflata mexicana

Bulimina subacuminata

Bulimina tenuata

Cassidulina cushmani

Cassidulina subglobosa

Chilostomella oolina

Cibicides mckannai

Cribrostomoides nitidum

Cribrostomoides subglobosum

Eggerella bradyi

Eponides healdi

Eponides subtenera

Eponides umbonatus

Fursenkoina loeblichi

Gyroidina io

Haplophragmoides scitulum

Hyperammina elongata

Karreriella parkerae

Triloculina trihedra

Trochammina globigeriniformis

Uvigerina peregrina dirupta

Valvulineria araucana

Valvulineria glabra

$$
<200 \mathrm{~m}
$$

Ammodiscus gullmarensis
Ammonia beccarii

Buliminella elegantissima

Cibicides lobatulus

Cribrostomoides crassimargo

Dendrophyra arborescens

Dyocibicides biserialis

Eggerella advena

Elphidiella nitida

Elphidium articulatum

Elphidium bartletti

Elphidium crispum

Elphidium excavatum

Elphidium frigidum

Elphidium hughesi

Elphidium incertum

Elphidium lene

Elphidium subarcticum

Elphidium tumidum

Eponides repandus

Fissurina marginata

Gaudryina arenaria

Gaudryina subglabrata

Glabratella ornatissima

Haplophragmoides bradyi

Haplophragmoides canariensis

Haplophragmoides columbiensis

Islandeilla helenae

Islandiella limbata

Islandiella tortuosa

Karreriella baccata

Lagena apiopleura

Lagena distoma

Lagena gracilis

Lagena laevis

Lagena pliocenica

Lagena semilineata

Lagena spicata

Miliammina fusca
Miliolinella circularis

Nonionella basispinata

Nonionella stella

Oolina borealis

Patellina corrugata

Polymorphina kincaidi

Pseudopolymorphina

charlottensis

Quinqueloculina seminula

Quinqueloculina stalkeri

Quinqueloculina sp.

Recurvoides turbinatus

Reophax curtus

Robertinoides charlottensis

Rosalina columbiensis

Sigmorphina gallowayi

Sigmomorphina trilocularis

Trifarina angulosa

Trifarina semitrigona

Trochammina charlottensis

Trochammina discorbis

Trochammina nana

Trochammina pacifica

Trochammina rotaliformis

Trochammina squamata

Coastal

Ammotium cassis

Buccella tenerrima

Elphidiella arctica

Elphideilla hannai

Elphidium microgranulosum

Eponides columbiensis

Quinqueloculina akneriana

Trochammina kellettae

\section{Summary}

1. All published data on the distribution of recent benthic foraminifera of the North American Pacific continental margin north of California is presented in the form of 5 catalogs and 139 maps.

2. 523 taxa have been recorded in the area of study.

3. The taxa have been reduced to 404 by synonymization.
4. Of the 404 synonymized species, 138 occur at 6 or more of the sample locations; that is, $29 \%$ of the species occur at $4 \%$ or more of the 157 sample localities.

5. Three groups of commonly recorded species characterize gross depth zones. Eight species are coastal in their distribution, 63 occur at depths of less than $200 \mathrm{~m}$ and 27 at depths of greater than $200 \mathrm{~m}$.

6. Another group, containing 40 species, is 
TABLE 2.-Latitudinal distribution.

North $\left(>50^{\circ}-55^{\circ} \mathrm{N}\right)$

Ammodiscus minutissimus

Ammotium cassis

Bolivina alata

Bolivina decussata

Bolivina spissa

Bulimina barbata

Bulimina inflata mexicana

Bulimina subacuminata

Cassidulina barbara

Cassidulina cushmani

Cassidulina subglobosa

Cibicides mckannai

Cribrostomoides crassimargo

Cribrostomoides jeffreysii

Elphidiella arctica

Elphidium bartletti

Epistominella vitrea

Eponides healdi

Eponides leviculus

Eponides subtenera

Fursenkoina loeblichi

Gyroidina io

Haplophragmoides scitulum

Hyperammina elongata

Islandiella helenae

Islandiella norcrossi

Karreriella baccata

Karreriella parkerae

Lagena laevis

Lagena semilineata

Oolina hexagona

Pyrgo murrhina

Quinqueloculina stalkeri

Quinqueloculina sp.

Reophax scorpiurus
Thalmannammina parkerae

Trifarina fluens

Triloculina trihedra

Uvigerina peregrina

Uvigerina peregrina dirupta

Valvulineria araucana

Valvulineria glabra

$$
\text { South }\left(<50^{\circ}-55^{\circ} \mathrm{N}\right)
$$

Ammonia beccarii

Buccella tenerrima

Bulimina ovata

Elphidium crispum

Elphidium incertum

Elphidium lene

Elphidium microgranulosum

Elphidium subarcticum

Eponides columbiensis

Gaudryina subglabrata

Haplophragmoides bradyi

Haplophragmoides canariensis

Miliammina fusca

Miliolinella circularis

Nonionella basispinata

Reophax curtus

Rosalina columbiensis

Trifarina semitrigona

Trochammina charlottensis

Trochammina discorbis

Trochammina kellettae

Trochammina nana

Ubiquitous

Adercotryma glomerata
Ammodiscus gullmarensis

Astrononion gallowayi

Bolivina pacifica

Buccella frigida

Bulimina tenuata

Buliminella elegantissima

Chilostomella oolina

Cibicides fletcheri

Cibicides lobatulus

Cribrostomoides nitidum

Cribrostomoides subglobosum

Cyclogyra involvens

Dendrophyra arborescens

Dyocibicides biserialis

Eggerella advena

Eggerella bradyi

Elphidiella hannai

Elphidiella nitida

Elphidium articulatum

Elphidium excavatum

Elphidium frigidum

Elphidium hughesi

Elphidium tumidum

Epistominella exigua

Epistominella pacifica

Eponides repandus

Eponides umbonatus

Fissurina lucida

Fissurina marginata

Gaudryina arenaria

Glabratella ornatissima

Globobulimina auriculata

Globobulimina pacifica

Haplophragmoides columbiensis

Haplophragmoides planissimum

Islandiella californica

Islandiella limbata
Islandiella tortuosa

Lagena apiopleura

Lagena distoma

Lagena elongata

Lagena gracilis

Lagena pliocenica

Lagena spicata

Lagena striata

Miliolinella subrotunda

Nonionella auricula

Nonionella stella

Nonionella turgida digitata

Nonionellina labradorica

Oolina borealis

Oolina melo

Oolina striatopunctata

Patellina corrugata

Polymorphina kincaidi

Pseudopolymorphina

charlottensis

Pullenia salisburyi

Quinqueloculina akneriana

Quinqueloculina seminula

Recurvoides turbinatus

Robertinoides charlottensis

Saccammina atlantica

Sigmomorphina gallowayi

Sigmomorphina trilocularis

Spiroplectammina biformis

Trifarina angulosa

Trochammina globigeriniformis

Trochammina inflata

Trochammina nitida

Trochammina pacifica

Trochammina rotaliformis

Trochammina squamata

Uvigerina juncea ubiquitous, occurring on the continental shelf and slope and sometimes at abyssal depths.

7. Commonly recorded genera and species vary in their usefulness for delimiting depth zones.

8. The depth distribution of many "ubiquitous" species increases towards the north. It may indicate habitat changes with increasing latitude, but it can just as easily be explained as an artifact of a poor sampling framework.

9. Three groups of commonly recorded spe- cies characterize biogeographic faunal categories. Forty-two species occur mainly to north of $52^{\circ}-55^{\circ} \mathrm{N}, 22$ occur mainly to the south of this region, and 74 species are latitudinally ubiquitous in the study area extending from around $41^{\circ} \mathrm{N}$ toward $61^{\circ} \mathrm{N}$.

\section{Key to the Species on the Maps}

Following Culver and Buzas (1980, 1981c, $1982 \mathrm{~b}$ ) we list below a key to our concept of the 
138 most commonly recorded species on the North American Pacific continental margin north of California. Genera are listed alphabetically as are the species within each genus. Under each species, the reference or references are given where the reader can find published illustrations that adequately convey our concept of each of the commonly recorded species in this study. Many of the 138 species were also included in the keys to commonly recorded species for the east coast of North America, the Gulf of Mexico, and the Caribbean (Culver and Buzas, 1980, 1981c, 1982b); the references given here for these species are generally the same.

\section{Index to Publications That Illustrate Our CONCEPT OF THE 138 MOST COMMONLY RECORDED SPECIES}

Adercotryma glomerata (Brady): Parker, 1954:486, pl. 1: fig. 18 [as Adercotryma glomeratum].

Ammodiscus gullmarensis Hoeglund: Hoeglund, 1947:123, pl. 8: figs. 2, 3, 8, pl. 20: figs. 17, 18 [as Ammodiscus planus].

Ammodiscus minutissimus Cushman and McCulloch: Cushman and McCulloch, 1939:70, pl. 5: figs. 3, 4.

Ammonia beccarii (Linné): Schnitker, 1974:217-223, pl. 1.

Ammotium cassis (Parker): Schafer and Cole, 1978:27, pl. 3: fig. 7 .

Astrononion gallowayi Loeblich and Tappan: Bergen and O'Neil, 1979:1289, pl. 2: figs. 1, 2.

Bolivina alata (Seguenza): Brady, 1884:422, pl. 53: figs. 24 [as Bolivina beyrichi var. alata].

Bolivina decussata Brady: Todd and Low, 1967:A27, pl. 4: fig. 11 .

Bolivina pacifica Cushman and McCulloch: Cushman and McCulloch, 1942:185, pl. 21: figs. 2, 3 [as Bolivina acerosa var. pacifica].

Bolivina spissa Cushman: Bergen and O’Neil, 1979:1289, pl. 3: fig. 25.

Buccella frigida (Cushman): Todd and Low, 1961:18, pl. 1: figs. 24,25 .

Buccella tenerrima (Bandy): Lankford and Phleger, 1973:116, pl. 4: fig. 19.

Bulimina barbata Cushman: Bergen and O'Neil, 1979:1289, pl. 4: fig. 14 .

Bulimina inflata Seguenza mexicana Cushman: Cushman, 1922:95, pl. 21 : fig. 2.

Bulimina ovata d'Orbigny: Cushman, 1922:100, pl. 21: fig. 3.

Bulimina subacuminata Cushman and Stewart: Bergen and O’Neil, 1979:1289, pl. 4: fig. 1.
Bulimina tenuata Cushman: Bergen and O'Neil, 1979:1289, pl. 5: fig. 8

Buliminella elegantissima (d'Orbigny): Schnitker, 1971:194, pl. 4: fig. 17.

Cassidulina barbara Buzas: Buzas, 1965:25, pl. 5: figs. 2, 3. Bergen and O'Neil, 1979:1289, pl. 4: fig. 25 [as Cassidulina islandica].

Cassidulina cushmani Stewart and Stewart: Bergen and O'Neil, 1979:1289, pl. 4: fig. 34.

Cassidulina subglobosa Brady: Parker, 1948:237, pl. 6: figs. 3a,b.

Chilostomella oolina Schwager: Parker, 1954:537, pl. 11: fig. 15.

Cibicides fletcheri Galloway and Wissler: Uchio, 1960, pl. 10: figs. 1-3. Bergen and O'Neil, 1979:1289, pl. 1: fig. 17.

Cibicides lobatulus (Walker and Jacob): Sen Gupta, 1971:89, pl. 2: figs. 34-36.

Cibicides mckannai Galloway and Wissler: Bergen and O'Neil, 1979:1289, pl. 3: figs. 8-10 [as Cibicides aff. mckannai].

Cribrostomoides crassimargo (Norman): Schafer and Cole, 1978:27, pl. 4: figs. 2a, b.

Cribrostomoides jeffreysii (Williamson): Todd and Low, 1967:A15, pl. 1: fig. 21.

Cribrostomoides nitidum (Goës): Parker, 1954:486, pl. 1: fig. 17 [as Alveolophragmium nitidum].

Cribrostomoides subglobosum (Sars): Loeblich and Tappan, 1964:C225, fig. $136(1,2)$.

Cyclogyra involvens (Reuss): Schafer and Cole, 1978:27, pl. 7: fig. 6a,b.

Dendrophyra arborescens (Norman): Brady, 1884:262, pl. 28: figs. 12, 13 [as Hyperammina arborescens].

Dyocibicides biserialis Cushman and Valentine: Todd and Low, 1967:A35, pl. 5: fig. 3.

Eggerella advena (Cushman): Cushman, 1922:57, pl. 9: figs. 7-9 [as Verneuilina advena].

Eggerella bradyi Cushman: Cushman, 1922:59, pl. 11: fig. 1 [as Verneuilina bradyi].

Elphidiella arctica (Parker and Jones): Schafer and Cole, 1978:27, pl. 9: fig. 6.

Elphidiella hannai (Cushman and Grant): Cushman and McCulloch, 1940:177, pl. 20: fig. 11.

Elphidiella nitida Cushman: Bergen and O'Neil, 1979:1290, pl. 1 : figs. 3,4 .

Elphidium articulatum (d'Orbigny): Parker, 1952a:411, pl. 5: figs. 5, 7.

Elphidium bartletti Cushman: Sen Gupta, 1971:89, pl. 2: figs. 26, 27.

Elphidium crispum (Linné): Nicol, 1944:177, pl. 29: figs. 3, 11 [as Elphidium fax fax].

Elphidium excavatum (Terquem): Cushman, 1930:20, pl. 7: fig. 10a,b [as Elphidium incertum var. clavatum]. Buzas, 1966:585-594, pl. 71: figs. 1-8 [as Elphidium clavatum]. Schnitker, 1971:198, pl. 7: fig. 4 [as Elphidium incertum]. Elphidium frigidum Cushman: Cushman and McCulloch, 1940:171, pl. 19: figs. 6,8 . 
Elphidium hughesi Cushman and Grant: Bergen and O'Neil, 1979:1290, pl. 1: figs. 1, 2.

Elphidium incertum (Williamson): Buzas, 1966:592, pl. 72: figs. 1-6.

Elphidium lene Cushman and McCulloch: Cushman and McCulloch, 1940:170, pl. 19: fig. 2 [as Elphidium incertum var. lene].

Elphidium microgranulosum (Thalmann): Galloway and Wissler, 1927:83, pl. 12: figs. 15, 16 [as Themeon decipiens].

Elphidium subarticum Cushman: Parker, 1952a:412, pl. 5: fig. 9

Elphidium tumidum Natland: Natland, 1938:144, pl. 5: figs. 5,6 .

Espistominella exigua (Brady): Phleger and Parker, 1951:28, pl. 15: figs. 6, 7, [as Pseudoparrella exigua].

Epistominella pacifica (Cushman): Todd and Low, 1967:A32, pl. 5: fig. 18.

Epistominella vitrea Parker: Parker, Phleger, and Peirson, 1953:9, pl. 4: figs. 34-36, 40, 41.

Eponides columbiensis (Cushman): Cushman, 1925:43, pl. 7: figs. la-c [as Pulvinulina columbiensis].

Eponides healdi Stewart and Stewart: Stewart and Stewart, 1930:70, pl. 8: figs. 8a-c.

Eponides leviculus (Resig): Bergen and O'Neil, 1979:1290, pl. 4: figs. 22-24.

Eponides repandus (Fichtel and Moll): Phleger and Parker, 1951:21, pl. 11: figs. 5, 6 .

Eponides subtenera (Galloway and Wissler): Bergen and O'Neil, 1979:1290, pl. 4: fig. 19-21 [as Eponides subtener].

Eponides umbonatus (Reuss): Cushman, 1931:52, pl. 11 : figs. 1-3 [as Eponides umbonata].

Fissurina lucida (Williamson): Todd and Low, 1967:A28, pl. 3: fig. 31.

Fissurina marginata (Montagu): Sen Gupta, 1971:82, pl. 7: figs. 4,5 .

Fursenkoina loeblichi (Feyling-Hanssen): Phleger and Parker, 1951:18, pl. 9: figs. 1-3 [as Virgulina complanata].

Gaudryina arenaria Galloway and Wissler: Cushman and McCulloch, 1939:91, pl. 8: figs. 2, 3.

Gaudryina subglabrata Cushman and McCulloch: Cushman and McCulloch, 1939:92, pl. 8: figs. 5-7.

Glabratella ornatissima (Cushman): Todd and Low, 1967:A30, pl. 4: fig. 23.

Globobulimina auriculata (Bailey): Schnitker, 1971:202, pl. 5: fig. 6.

Globobulimina pacifica Cushman: Bergen and O'Neil, 1979:1290, pl. 6: fig. 9.

Gyroidina io Resig: Uchio, 1960: pl. 8: figs. 16-18.

Haplophragmoides bradyi (Robertson): Hoeglund, 1947:134, pl. 10: fig. 1.

Haplophragmoides canariensis (d'Orbigny): Schnitker, 1971:204, pl. 1: fig. 6 .

Haplophragmoides columbiensis Cushman: Cushman and McCulloch, 1939:72, pl. 5: figs. 8-10 [as Haplophragmoides columbiense].
Haplophragmoides planissimum Cushman: Cushman, 1927:135, pl. 1: fig. 6 [as Haplophragmoides planissima].

Haplophragmoides scitulum (Brady): Parker, 1954:487, pl. 1: figs. 20, 21 [as Alveolophragmium scitulum].

Hyperammina elongata Brady: Parker, 1952a:395, pl. 1: fig. 10.

Islandiella californica (Cushman and Hughes): Bergen and O’Neil, 1979:1289, pl. 2: figs. 23, 24 [as Cassidulina californica].

Islandiella helenae Feyling-Hanssen and Buzas: Feyling-Hanssen and Buzas, 1976:155, figs. 1-4. Todd and Low, 1967:A38, pl. 5: fig. 10 [as Cassidulina teretis].

Islandiella limbata (Cushman and Hughes): Bergen and O'Neil, 1979:1289, pl. 2: figs. 3-5 [as Cassidulina limbata].

Islandiella norcrossi (Cushman): Parker, 1948, pl. 6: figs. 2a,b [as Cassidulina norcrossi].

Islandiella tortuosa (Cushman and Hughes): Todd and Low, 1967:A38, pl. 5: fig. 12 [as Cassidulina tortuosa].

Karreriella baccata (Schwager): Bergen and O'Neil, 1979:1291, pl. 3: figs. 13, 14.

Karreriella parkerae Uchio: Uchio, 1960:56, pl. 2: figs. $21-$ 23, Bergen and O'Neil, 1979:1291, pl. 5: figs. 3, 4, 22.

Lagena apiopleura Loeblich and Tappan: Bergen and O'Neil, 1979:1291, pl. 2: fig. 19.

Lagena distoma Parker and Jones: Todd and Low, 1967:A24, pl. 3: fig. 18.

Lagena elongata (Ehrenberg): Todd and Low, 1967:A24, pl. 3: fig. 22.

Lagena gracilis Williamson: Bergen and O'Neil, 1979:1291, pl. 4: fig. 4.

Lagena laevis (Montagu): Schafer and Cole, 1978:28, pl. 6: fig. 10.

Lagena pliocenica Cushman and Gray: Todd and Low, 1967:A25, pl. 3: fig. 19.

Lagena semilineata Wright: Cushman and McCulloch, 1950:345, pl. 46: fig. 11.

Lagena spicata Cushman and McCulloch: Cushman and McCulloch, 1950:360, pl. 48: figs. 3-7 [as Lagena sulcata var. spicata].

Lagena striata (d'Orbigny): Todd and Low, 1967:A25, pl. 3: fig. 20.

Miliammina fusa (Brady): Parker, 1952b:452, pl. 2: figs. 6a,b. Miliolinella circularis (Bornemann): Cushman and Todd, 1947:6, pl. 1: fig. 33 [as Triloculina circularis].

Miliolinella subrotunda (Montagu): Brooks, 1973, pl. 8: figs. 6, 9. Brady, 1884:169, pl. 4: fig. 3, pl. 5: figs. 13, 14 [as Miliolina circularis].

Nonionella auricula Heron-Allen and Earland: Cushman and McCulloch, 1940:159, pl. 17: figs. 6, 7 .

Nonionella basispinata Cushman and Moyer: Cushman and McCulloch, 1940:158, pl. 17: figs. 8, 9, pl. 18: figs. 4, 5 [as Nonion pizarrense var. basispinatum].

Nonionella stella Cushman and Moyer: Cushman and McCulloch, 1940:162, pl. 18: fig. 2 [as Nonionella miocenica 
var. stella].

Nonionella turgida digitata Norvang: Todd and Low, 1967:A36, pl. 5: fig. 8 .

Nonionellina labradorica (Dawson): Cushman, 1930:11, pl. 4: figs. 6-12 [as Nonion labradoricum].

Oolina borealis Loeblich and Tappan: Todd and Low, 1967:A28, pl. 3: fig. 34 .

Oolina hexagona (Williamson): Todd and Low, 1967:A29, pl. 3: fig. 28.

Oolina melo d'Orbigny: Schafer and Cole, 1978:28, pl. 6: fig. 5.

Oolina striatopunctata (Parker and Jones): Todd and Low, 1967:A29, pl. 3: fig. 25.

Patellina corrugata Williamson: Schnitker, 1971:206, pl. 6: fig. 12.

Polymorphina kincaidi Cushman and Todd: Cushman and Todd, 1947:12, pl. 2: figs. 9, 10.

Pseudopolymorphina charlottensis (Cushman): Cushman and Todd, 1947:12, pl. 2: fig. 11 [as Polymorphina charlottensis].

Pullenia salisburyi Stewart and Stewart: Todd and Low, 1967:A38, fig. 20.

Pyrgo murrhina (Schwager): Cushman, 1929:71, pl. 19: figs. $6,7$.

Quinqueloculina akneriana d'Orbigny: D'Orbigny, 1846:290, pl. 18: figs. 16-21.

Quinqueloculina seminula (Linné): Sen Gupta, 1971:85, pl. 1: figs. 25-27.

Quinqueloculina stalkeri Loeblich and Tappan: Todd and Low, 1967:A 19, pl. 2: fig. 17.

Quinqueloculina sp.: Bergen and O'Neil, 1979:1292, pl. 2: fig. 22 [as Quinqueloculina akneriana].

Recurvoides turbinatus (Brady): Sen Gupta, 1971:84, pl. 1: figs. 10-12.

Reophax curtus Cushman: Sen Gupta, 1971:84, pl. 1: figs. 24.

Reophax scorpiurus Montfort: Cushman, 1920:6, pl. 1: figs. 5-7.

Robertinoides charlottensis (Cushman): Cushman and Todd, 1947:18, pl. 3: fig. 2 [as Robertina charlottensis].

Rosalina columbiensis (Cushman): Lankford and Phleger, 1973:127, pl. 5: figs. 10-12.

Saccammina atlantica (Cushman): Sen Gupta, 1971:83, pl. 1: fig. 1 .

Sigmomorphina gallowayi Cushman and Ozawa: Cushman and
Todd, 1947:12, pl. 2: fig. 8 .

Sigmomorphina trilocularis (Bagg): Cushman and Todd, 1947:11, pl. 2: fig. 7.

Spiroplectammina biformis (Parker and Jones): Schafer and Cole, 1978:29, pl. 3: fig. 2.

Thalmannammina parkerae (Uchio): Bergen and O'Neil, 1979:1291, pl. 5: fig. 28 [as Haplophragmoides (Recurvoidella) parkerae].

Trifarina angulosa (Williamson): Sen Gupta, 1971:89, pl. 2: figs. $16-17$.

Trifarina fluens (Todd): Todd and Low, 1967:A30, pl. 4: fig. 5 [as Angulogerina fluens].

Trifarina semitrigona (Galloway and Wissler): Cushman and Todd, 1947:19, pl. 3: fig. 7 [as Angulogerina semitrigona].

Trilocuiina trihedra Loeblich and Tappan: Bergen and O'Neil, 1979:1292, pl. 5: figs. 1, 2.

Trochammina charlottensis Cushman: Lankford and Phleger, 1973:130, pl. 3: figs. 3, 4.

Trochammina discorbis Earland: Cushman and McCulloch, 1939:106, pl. 11: fig. 10.

Trochammina globigeriniformis Brady: Bergen and O'Neil, 1979:1292, pl. 5: figs. 20, 21.

Trochammina inflata (Montagu): Parker, 1952b:459, pl. 3: figs. la,b.

Trochammina kellettae Thalmann: Cushman and McCulloch, 1939:101, pl. 11: fig. 1.

Trochammina nana Brady: Brady, 1884:311, pl. 35: figs. 68 [as Haplophragmium nanum].

Trochammina nitida Brady: Cushman and McCulloch, 1939:105, pl. 11: figs. 7-9.

Trochammina rotaliformis Wright: Cushman and McCulloch, 1939:107, pl. 12: fig. 2.

Trochammina squamata Jones and Parker: Schafer and Cole, 1978:29, pl. 5: fig. 1.

Uvigerina juncea Cushman and Todd: Bergen and O'Neil, 1979:1292, pl. 3: fig. 4.

Uvigerina peregrina Cushman: Cushman, 1923:166, pl. 42: figs. 7-10. Pflum and Frerichs, 1976:108, pl. 8: figs. 2, 3 [as Uvigerina peregrina peregrina].

Uvigerina peregrina Cushman dirupta Todd: Pflum and Frerichs, 1976:108, pl. 8: figs. 4, 5 .

Valvulineria araucana d'Orbigny: Uchio, 1960, pl. 8: figs. 3-5.

Valvulineria glabra Cushman: Bergen and O'Neil, 1979:1292, pl. 5: figs. 14, 15. 


\section{References}

(An asterisk marks each of the 31 publications from which distribution data were drawn for construction of the catalogs)

Bandy, O.L., and M. Chierici

1966. Depth-Temperature Evaluation of Selected California and Mediterranean Bathyal Foraminifera. Marine Geology, 4:254-271.

Bergen, F.W., and P. O’Neil

* 1979. Distribution of Holocene Foraminifera in the Gulf of Alaska. Journal of Paleontology, 53(6):12671292.

Bradshaw, J.S.

* 1961. Laboratory Experiments on the Ecology of Foraminifera. Contributions from the Cushman Foundation for Foraminiferal Research, 12(3):87-106.

Brady, H.B.

1884. Report on the Foraminifera Collected by H.M.S. Challenger during the Years 1873-1876. In Report... Challenger, Zoology, 9(22):xxi +814 pages, 115 plates. London.

Brooks, W.A.

1973. Distribution of Recent Foraminifera from the Southern Coast of Puerto Rico. Micropaleontology, 19(4):385-416.

Buzas, M.A.

1965. Foraminifera from Late Pleistocene Clay near Waterville, Maine. Smithsonian Miscellaneous Collections, 145(8):1-30.

1966. The Discrimination of Morphological Groups of Elphidium (Foraminifer) in Long Island Sound through Canonical Analysis and Invariant Characters. Journal of Paleontology, 40(3):585-594.

Buzas, M.A., and S. J. Culver

1980. Foraminifera: Distribution of Provinces in the Western North Atlantic. Science, 209:687-689.

Cockbain, A.E.

* 1963. Distribution of Foraminifera in Juan de Fuca and Georgia Straits, British Columbia, Canada. Contributions from the Cushman Foundation for Foraminiferal Research, 14(2):37-57.

Cooper, W.C.

* 1961. Intertidal Foraminifera of the California and Oregon Coast. Contributions from the Cushman Foundation for Foraminiferal Research, 12(2):47-63.

Culver, S.J., and M.A. Buzas

1980. Distribution of Recent Benthic Foraminifera off the North American Atlantic Coast. Smithsonian Contributions to the Marine Sciences, 6:1-512. 1981a. Recent Benthic Foraminiferal Provinces on the Atlantic Continental Margin of North America. Journal of Foraminiferal Research, 11(3):217-240.

1981b. Distribution of Selected Recent Benthic Foraminiferal Genera in the Western North Atlantic. In J.W. Neale and M.D. Brasier, editors, Microfossils from Recent and Fossil Shelf Seas, pages 336-349. Chichester, England: Ellis Horwood.

1981c. Distribution of Recent Benthic Foraminifera in the Gulf of Mexico. Smithsonian Contributions to the Marine Sciences, 8(1-2):1-898.

1981d. Foraminifera: Distribution of Provinces in the Gulf of Mexico. Nature, 290:328-329.

1982a. Recent Benthic Foraminiferal Provinces between Newfoundland and Yucatan. Bulletin of the Geological Society of America, 93:269-277.

1982b. Distribution of Recent Benthic Foraminifera in the Caribbean Region. Smithsonian Contributions to the Marine Sciences, 14:1-382.

1983. Recent Benthic Foraminiferal Provinces in the Gulf of Mexico. Journal of Foraminiferal Research, 13(1):21-31.

Cushman, J.A.

* 1910. A Monograph of the Foraminifera of the North Pacific Ocean, Part 1: Astrorhizidae and Lituolidae. Bulletin of the United States National Museum, $71: 1-134$.

* 1913. A Monograph of the Foraminifera of the North Pacific Ocean, Part 3: Lagenidae. Bulletin of the United States National Museum, 71(3):1-125.

* 1915. A Monograph of the Foraminifera of the North Pacific Ocean, Part 5: Rotalidiiae. Bulletin of the United States National Museum, 7 1(5):1-87.

* 1917. A Monograph of the Foraminifera of the North Pacific Ocean, Part 6: Miliolidae. Bulletin of the United States National Museum, 7 1(6):1-108.

1920. The Foraminifera of the Atlantic Ocean, Part 2: Lituolidae. Bulletin of the United States Museum, 104(2): 1-111.

1922. The Foraminifera of the Atlantic Ocean, Part 3: Texulariidae. Bulletin of the United States National Museum, 104(3): 1-228.

1923. The Foraminifera of the Atlantic Ocean, Part 4: Lagenidae. Bulletin of the United States National Museum, 104(4):1-228. 
* 1925. Recent Foraminifera from British Columbia. Contributions from the Cushman Laboratory for Foraminiferal Research, 1(2):38-47.

* 1927. Recent Foraminifera from off the West Coast of America. Bulletin of the Scripps Institution of Oceanography, Technical Series, 1:119-188.

1929. The Foraminifera of the Atlantic Ocean, Part 6: Miliolidae. Bulletin of the United States National Museum, 104(6):1-129.

1930. The Foraminifera of the Atlantic Ocean, Part 7: Nonionidae, Camerinidae, Peneroplidae, and Alveolinellidae. Bulletin of the United States National Museum, 104(7):1-79.

1931. The Foraminifera of the Atlantic Ocean, Part 8: Rotaliidae, Amphisteginidae, Calcarinidae, Cymbaloporettidae, Globorotaliidae, Anomalinidae, Planorbulinidae, Rupertiidae and Homotremidae. Bulletin of the United States National Museum, 104(8):1-179.

Cushman, J.A., and P.G. Edwards

1937. Astrononion, a New Genus of the Foraminifera and Its Species. Contributions from the Cushman Laboratory for Foraminiferal Research, 13(1):2936.

Cushman, J.A., and I. McCulloch

* 1939. A Report on Some Arenaceous Foraminifera. University of Southern California, Publications of the Allen Hancock Pacific Expeditions, 6(1):1-1 13.

* 1940. Some Nonionidae in the Collections of the Allen Hancock Foundation. University of Southern California, Publications of the Allen Hancock Pacific Expeditions, 6(3):145-178.

* 1942. Some Virgulininae in the Collections of the Allen Hancock Foundation. University of Southern California, Publications of the Allen Hancock Pacific Expeditions, 6(4):181-230.

* 1948. The Species of Bolivina and Related Genera in the Collection of the Allen Hancock Foundation. University of Southern California, Publications of the Allen Hancock Pacific Expeditions, 6(5):231-294.

* 1950. Some Lagenidae in the Collections of the Allen Hancock Foundation. University of Southern California, Publications of the Allen Hancock Pacific Expeditions, 6(6):295-364.

Cushman, J.A., and R. Todd

* 1943. The Genus Pullenia and its Species. Contributions from the Cushman Laboratory of Foraminiferal Research, 19(1):1-23.

* 1947. Foraminifera from the Coast of Washington. Cushman Laboratory of Foraminiferal Research, Special Publication, 21:1-23.

Detling, M.R

* 1958. Some Littoral Foraminifera from Sunset Bay, Coos County, Oregon. Contributions from the Cush- man Foundation of Foraminiferal Research, 9(2):25-31.

d'Orbigny. See Orbigny, A. de

Echols, R.J.

* 1969. Foraminiferal Trends within the Inner Sublittoral Zone of the Coast of Washington. In Research on the Continental Shelf off Washington and Western Alaska from August 1968 through June 1969. University of Washington, Department of Oceanography, Special Report, 41:114-131.

Feyling-Hanssen, R.W., and M.A. Buzas

1976. Emendation of Cassidulina and Islandiella helenae New Species. Journal of Foraminiferal Research, 6(2): 154-158.

Galloway, J.J., and S.G. Wissler

1927. Pleistocene Foraminifera from the Lomita Quarry, Palos Verdes Hills, California. Journal of Paleontology, 1(1):35-87.

Hamlin, W.H.

* 1960. Two New Species of Foraminifera from the West Coast of the United States. Contributions from the Cushman Foundation for Foraminiferal Research, $11(3): 87-88$.

Hazel, J.E.

1970. Atlantic Continental Shelf and Slope of the United States: Ostracod Zoogeography in the Southern Nova Scotian and Northern Virginian Faunal Provinces. United States Geological Survey Professional Paper, 529-E:El-E21.

Hoeglund, $\mathrm{H}$.

1947. Foraminifera in the Gullmar Fjord and the Skagerak. Zoologiska Bidrag Fran Uppsala, 26:1-328.

Jones, G.D., and C. A. Ross

* 1979. Seasonal Distribution of Foraminifera in Samish Bay, Washington. Journal of Paleontology, 53(2):245-257.

Lankford, R.R., and F.B Phleger

* 1973. Foraminifera from the Nearshore Turbulent Zone, Western North America. Journal of Foraminiferal Research, 3(3):101-132.

Loeblich, A.L., Jr., and H. Tappan

1964. Sarcodina Chiefly "Thecamoebans" and Foraminiferida. In Raymond C. Moore, editor, Treatise on Invertebrate Paleontology, C(1-2): 900 pages. Lawrence: University of Kansas Press for Geological Society of America.

Natland, M.L.

* 1933. The Temperature and Depth Distribution of Some Recent and Fossil Foraminifera in the Southern California Region. Bulletin of the Scripps Institution of Oceanography, Technical Series, $3(10): 225-230$.

* 1938. New Species of Foraminifera from off the West Coast of North America and from the Later Ter- 
tiary of the Los Angeles Basin. Bulletin of the Scripps Institution of Oceanography, Technical Series, 4(5):137-164.

Nicol, D.

* 1944. New West American Species of the Foraminiferal Genus Elphidium. Journal of Paleontology, 18(2):172-185.

Orbigny, A., d'

1846. Foraminifères fossiles du bassin tertiaire de Vienne (Autriche). Gide et Compe (Paris), 1-312.

Palmer, D.K.

* 1929. A Note on the Occurrence of Patellina corrugata Williamson in the San Juan Archipelago, Washington. Journal of Paleontology, 3(3):306-307.

Parker, F.L.

1948. Foraminifera of the Continental Shelf from the Gulf of Maine to Maryland. Bulletin of the Museum of Comparative Zoology, 100(2):213-241.

1952a. Foraminifera Species off Portsmouth, New Hampshire. Bulletin of the Museum of Comparative Zoology, 106(9):391-423.

1952b. Foraminiferal Distribution in the Long Island Sound-Buzzards Bay Area. Bulletin of the Museum of Comparative Zoology, 106(10):425-473.

1954. Distribution of the Foraminifera in the Northeastern Gulf of Mexico. Bulletin of the Museum of Comparative Zoology, $111(10): 451-588$.

Parker, F.L., F.B Phleger, and J.F. Peirson

1953. Ecology of Foraminifera from San Antonio Bay and Environs, Southwest Texas. Cushman Foundation for Foraminiferal Research, Special Publication, 2:1-75.

Pflum, C.E., and W.E. Frerichs

1976. Gulf of Mexico Deep-Water Foraminifers. Cushman Foundation for Foraminiferal Research, Special Publication, 14:1-125.

Phleger, F.B

* 1967. Marsh Foraminiferal Patterns, Pacific Coast of North America. Annales de Instituto de Biologiá, Universidad Nacional Autonoma de México, 38 Serie Ciencias del Mar y Limnology, 1:11-38.

Phleger, F.B, and F.L. Parker

1951. Ecology of Foraminifera, Northwest Gulf of Mexico, Part 2: Foraminifera Species. Geological Society of America, Memoir, 46:1-64.

Schafer, C.T., and F.E. Cole

1978. Distribution of Foraminifera in Chaleur Bay, Gulf of St. Lawrence. Geological Survey of Canada, 7730:1-55.

Schnitker, D

1971. Distribution of Foraminifera on the North Carolina Continental Shelf. Tulane Studies in Geology and Paleontology, 8(4):169-215.

1974. Ecotypic Variation in Ammonia beccarii (Linné). Journal of Foraminiferal Research, 4(4):216-223.

Scott, D.B.

* 1974. Recent Benthonic Foraminifera from Samish and Padilla Bays, Washington, Northwest Science, 4(4):211-218.

Sen Gupta, B.K.

1971. The Benthonic Foraminifera of the Tail of the Grand Banks. Micropaleontology, 17(1):69-98.

Smith, P.B.

* 1973. Foraminifera of the North Pacific Ocean. United States Geological Survey Professional Paper, 766:127.

Stewart, R.E., and K.C. Stewart

1930. Post-Miocene Foraminifera from the Ventura Quadrangle, Ventura County, California. Journal of Paleontology, 4(1):60-72.

Todd, R., and D. Low

1961. Near-Shore Foraminifera of Martha's Vineyard Island, Massachusetts. Contributions from the Cushman Foundation for Foraminiferal Research, 12(1):5-21.

* 1967. Recent Foraminifera from the Gulf of Alaska and Southeastern Alaska. United States Geological Survey Professional Paper, 573-A:A 1-A46.

Uchio, $\mathrm{T}$.

1960. Ecology of Living Benthonic Foraminifera from the San Diego, California, Area. Cushman Foundation for Foraminiferal Research, Special Publication, 5:1-72.

Whiteaves, J.F.

* 1886. On Some Marine Invertebrata Dredged or Otherwise Collected by D.G.M. Dawson, in 1885, in the Northern Part of the Strait of Georgia, in Discovery Passage, Johnstone Strait, and Queen Charlotte and Quatsino Sounds, British Columbia: With a Supplementary List of a Few Land and Freshwater Shells, Fishes, Birds, etc., from the Same Region. Transactions of the Royal Society of Canada, 4(4):111-137. 


\section{Unsynonymized Species with Publication and Location}

\section{Publication}

10001 COCKBAIN 1963

10003 COCKBAIN 1963

10004 COCKBAIN 1963

10005 COCKBAIN 1963

10006 COC KBAIN 1963

10007 COCKBAIN 1963

10008 COC KBA IN 1963

10009 BERGEN O'NEIL 1979

10010 BERGEN O'NEIL 1979

10011 BERGEN O'NEIL 1979

10012 BERGEN O'NEIL 1979

10013 BERGEN O'NEIL 1979

10014 BERGEN O'NEIL 1979

10015 SMITH 1973

10016 SMI TH 1973

10017 SMITH 1973

10018 SMI TH 1973

10019 SMI TH 1973

10020 COOPER 1961

10022 LANKFORD PHLEGER 1973

$\begin{array}{lll}10022 & \text { LANKFORD PHLEGER } & 1973 \\ 10023 & \text { LANKFORD PHLEGER } & 1973\end{array}$

10023 LANKF ORD PHLE
10024 COCKBAIN 1963

10025 COCKBAIN 1963

10026 COC KBAIN 1963

10027 COCKBAIN 1963

10028 COC KBAIN 1963

10029 COCKBAIN 1963

10030 COCKBAIN 1963

10031 COCKBAIN 1963

10032 SMITH 1973

10033 SMI TH 1973

10034 SMITH 1973

10035 SMITH 1973

10036 SMITH 1973

10037 SMI TH 1973

10038 SMITH 1973

10039 SMI TH 1973

10040 COC KBAIN 1963

10041 COCKBAIN 1963

10042 COCKBAIN 1963

10043 COCKBAIN 1963

10044 COCKBAIN 1963

10045 SMITH 1973

10046 SMITH 1973

10047 SMITH 1973

10048 SMITH 1973

10049 SMI TH 1973

10050 SMI TH 1973

10051 SMI TH 1973

10052 SMITH 1973

10053 SMITH 1973

10054 SMITH 1973

10055 SMITH 1973
10056 SMITH 1973

10057 BERGEN O'NEIL 1979

10058 SMITH 1973

10059 SMITH 1973

10060 SMI TH 1973

10061 SMITH 1973

10062 TODD LOW 1967

10063 CUSHMAN MCCULLOCH 1939

10064 CUSHMAN MCCULLOCH 1939

10065 CUSHMAN MCCULLOCH 1939

10066 CUSHMAN MCCULLOCH 1939

10067 JONES ROSS 1979

10068 SCOTT 1974

10069 PHLEGER 1967

10070 PHLEGER 1967

10071 SMITH 1973

10072 SMITH 1973

10073 TODD LOW 1967

10074 TODD LOW 1967

10075 TODD LOW 1967

10076 TODD LOW 1967

10077 TODD LOW 1967

10079 BERGEN O'NEIL 1979

10079
10080
BERGEN O'NEIL 1979

10081 BERGEN O'NEIL 1979

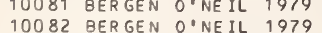

10082 BERGEN O'NEIL 1979
10083 SERGEN O'NEIL 1979

$\begin{array}{ll}10083 & \text { SERGEN O'NEIL } 1979 \\ 10084 & \text { BERGEN O'NEIL } 1979\end{array}$

10085 BERGEN O'NEIL 1979

\section{Generic Name}

ADERCOTRYMA

ADERCOTRYMA

ADERCOTRYMA

ADERCOTRYMA

ADERCOTRYMA

ADERCOTRYMA

ADERCOTRYMA

ADERCOTRYMA

ADERCOTRYMA

ADERCOTRYMA

ADERCOTRYMA

ADERCOTRYMA

ADERCOTRYMA

A DERCOTRYMA

ADERCOTRYMA

ADERCOTRYMA

ADERCOTRYMA

ALVEOLOPHRAGMIUM

ALVEOLOPHRAGMIUM

ALVEOLOPHRAGMIUM

ALVEOLOPHRAGMIUM

ALVEOLOPHRAGMIUM

ALVEOLOPHRA GMIUM

AL VEOLOP HRA GMIUM

ALVEOL OPHRAGMIUM

ALVEOLOPHRAGMIUM

ALVEOLOPHRAGMIUM

ALVEOLOPHRAGMIUM

ALVEOLOPHRAGMIUM

ALVEOLOPHRAGMIUM

ALVEOLOPHRAGMIUM

ALVEOLOPHRAGMIUM

ALVEOLOPHRAGMIUM

AL VEOLOPHRAGMIUM

ALVEOLOPHRAGMIUM

ALVEOL OPHRAGMIUM

ALVEOL OP HRAGMIUM

ALVEOLOPHRAGMIUM

ALVEOLOPHRAGMIUM

ALVEOLOPHRAGMIUM

ALVEOLOPHRA GMIUM

AL VEOLOPHRAGMIUM

ALVEOLOPHRA GMIUM

AL VEOL OPHRA GMIUM

AL VEOL OPHRA GMIUM

ALVEOL OPHRA GMIUM

ALVEOLOPHRAGMIUM

AL VEOL OPHRAGM IUM

ALVEOLOPHRAGMIUM

ALVEOLOPHRAGMIUM

AL VEOLOPHRAGMIUM

AMMOBACULITES

AMMOBACULITES

AMMOBACULITES

AMMOBACULITES

AMMOBACULITES

AMMOBACULITES

AMMOBACULITES

AMMOBA CUL ITE

AMMOBACULITES

AMMOBACULITES

AMMOBACULITES

AMMOBACULITES

AMMOBACULITES

AMMOBACULITES

AMMOBACULITES

AMMOBACUL ITES

AMMOBAC

AMMOBACULITES

AMMOBACULITES

AMMODISCUS

AMMODISCUS

AMMODISCUS

AMMODISCUS

AMMODISCUS

AMMODISCUS

AMMODISCUS

AMMODISCUS

AMMOD IS

AMMODISCUS

AMMODISCUS

AMMODISCUS

AMMODISCUS

\section{Specific Name}

GLOMERATA

GLOMERATA

GLOMERATA

GLOMERATA

GLOMERATA

GLOMERATA

GLOMERATA

GLOMERATA

GLOMERATA

GLOMERATA

GLOMERATA

GLOMERATA

GLOMERATA

GLOMERATUM

GLOMERATUM

GLOMERATUM

GLOMERATUM

GLOMERATUM

C OL UMB IENSE

COL UMBIENSE

COLUMB IENSIS

COL UMBIENSIS

COL UMBIENSIS

COL UMB I ENSIS

COL UMBIENSIS

COLUMBIENSIS

COL UMBIENS IS

COL UMBIENSIS

COL UMBIENSIS

NIT I DUM

NIT IDUM

NIT I DUM

NIT I DUM

NITI DUM

NIT IDUM

RIN GENS

RIN GENS

ROB USTUM

ROBUSTUM

ROBUSTUM

ROBUSTUM

ROB USTUM

SCITULUM

SUB GLOB OSUM

SUBGLOBOSUM

SUBGLOBOSUM

SUBGLOBOSUM

SUBGLOBOSUM

SUB GLOBOSUM

SUB GLOBOSUM

WEI SNER I

WEI SNER I

AGGLUTINANS

AGG LUTINANS

A GGLUT INANS

AGGLUTINANS FILAFORMIS

AGGLUTINANS FILAFORMIS

AME RI CANUS

AMERI CANUS

ARE NAR I US

CASSIS

CASSIS

CASSIS

CASSIS

DILATATUS

DXI GUUS

EXI GUUS

EXI GUUS

FILAFORMIS

FILAFORMIS

ARENACEUS

ARE NACEUS

GULLMARENSIS

GUL LMARENSIS

GULLMARENS IS

MINUTISSIMUS

MINUTISSIMUS

MINUTISSIMUS

MINUTISSIMUS

MINUTISSIMUS

MINUTISSIMUS

MINUTISSIMUS

MINUTISSIMUS

\section{Locality}

ERITISH COL UMBIA

BR ITISH COL UMBIA

BR ITISH COLUMBIA

BRITISH COLUMBIA

BRITISH COLUMBIA

BRIIISH COL UMBIA

BR ITISH COLUMBIA

BRITISH COLUMBI

GULF OF ALASKA

GULF OF ALASKA

GULF OF ALASKA

GULF OF ALASKA

GULF OF ALASKA

GULF OF ALASKA

OFF ALASKA PENINSULA

OFF ALASKA PENINSULA

NORTH PACIFIC

OFF ALASKA PENINSULA

OFF ALASKA PENINSULA

OREGON COAST

DEPOE BAY, OREGON

DEPOE BAY, OREGON
CRESCENT CITY, CALIF.

BRITISH COLUMBIA

BRITISH COLUMBIA

BR IT ISH COLUMBIA

BR ITISH COLUMBIA

RR IT ISH COL UNBBIA

BRITISH COLUMBIA

BRITISH COLUMBIA

BRITISH COL UMBIA

NORTH PACIFIC 


\section{Publication}

10086 BERGEN O NNEIL 1979 10087 BERGEN O'NEIL 1979

10088 SMITH 1973

10089 SMI TH 1973

10090 SMITH 1973

10091 BERGEN O'NEIL 1979

10092 BERGEN O'NEIL 1979

10093 BERGEN O'NEIL 1979

10094 BERGEN O०NEIL 1979

10095 JONES ROSS 1979

10096 SCOTT 1974

10097 BRADSHAW 1961

10098 BERGEN O 'NEIL 1979

10099 BERGEN O'NEIL 1979

10100 TODD LOW 1967

10101 TODD LOW 1967

10102 COCKBAIN 1963

10103 BERGEN O०NEIL 1979

10104 BERGEN O०NEIL 1979

10105 BERGEN O'NEIL 1979

10106 COCKBAIN 1963

10107 COCKBAIN 1963

10108 COCKBAIN 1963

10109 COCKBAIN 1963

10110 COCKBAIN 1963

10111 COCKBAIN 1963

10112 COCKBAIN 1963

10113 PHLEGER 1967

10114 PHLEGER 1967
10115 JONES ROSS 1979

10116 PHLEGER 1967

10117 SCOTT 1974

10118 LANKFORD PHLEGER 1973

10119 LANKFORD PHLEGER 1973

10120 CUSHMAN MCCULLOCH 1948

10121 CUSHMAN MCCULLOCH 1948

10122 COCKBAIN 1963

10123 COCKBAIN 1963

10124 COCKBAIN 1963
10125 COCKBAIN 1963

10126 COCKBAIN 1963

10127 SMITH 1973

10128 CUSHMAN MCCULLOCH 1948

10129 CUSHMAN MCCULLOCH 1948

10130 TODD LOW 1967

10131 TODD LOW 1967

10132 TODD LOW 1967

10133 TODD LOW 1967

10134 TODD LOW 1967

10135 DETLING 1958

10136 CUSHMAN TODD 1947

10137 COOPER 1961

10138 COCKBAIN 1963

10139 COCKBAIN 1963

10140 COCKBAIN 1963

10141 COCKBAIN 1963

10142 CUSHMAN TODD 1947

10143 CUSHMAN TODD 1947

10144 CUSHMAN TODD 1947

10145 CUS HMAN 1915

10146 NATLAND 1933

10147 BERGEN O'NEIL 1979

10148 BERGEN O NEIL 1979

10149 BERGEN O'NEIL 1979

10150 TODD LOW 1967

10151 TODD LOW 1967

10152 TODD LOW 1967

10153 TODD LOW 1967

10154 TODD LOW 1967

10155 BERGEN O'NEIL 1979

10156 BERGEN O'NEIL 1979

10157 BERGEN O'NEIL 1979

10158 BERGEN O'NEIL 1979

10159 BERGEN O'NEIL 1979

10160 BERGEN OPNEIL 1979

10161 BERGEN O'NEIL 1979

10162 BERGEN O'NEIL 1979

10163 DETLING 1958

10164 COCKBAIN 1963

10165 COCKBAIN 1963

10166 COCKBAIN 1963

10167 BERGEN O NEIL 1979

10168 BERGEN OONEIL 1979

10169 BERGEN O'NEIL 1979

10170 BERGEN O'NEIL 1979

10171 BERGEN O'NEIL 1979

10172 CUS HMAN TODD 1947

10173 CUS HMAN EDWARDS 1937

10174 CUSHMAN TODD 1947

10175 CUS HMAN 1927

10176 CUS HMAN 1927

10177 CUS HMAN 1927

10178 CUS HMAN 1917

10179 CUS HMAN 1927

10180 TODD LOW 1967
Generic Name Specific Name

PAC IFICUS

PACIFICUS

F OL I ACEA

FOL I ACEA

SANDIEGOENSIS

SAN DIEGOENSIS

SAN DIEGOENSIS

SAN DIEGOENSIS

BECCARII

BECCARII TEPIDA

PSE UDOSPIRALIS

PSEUDOSPIRAL IS

C AS SIS

PLANISSIMUM

PLANISSIMUM

PLANISSIMUM

PLANISSIMUM

PLANISSIMUM

PLANISSIMUM

PLANIS IMUM

PLANISSIMUM

PLANISSIMUM

PLANISSIMUM
PLANISSIMUM

SAL SUM

SAL SUM

SAL SUM

SAL SUM

SAL SUM

CHARL OTTENS IS

ANGULOSA

ANG UL OSA

ANGULOSA

ANGULOS

ANGULOSA

ANGULOSA

ANGULOSA

ANGULOSA

FLUENS

FLUENS

FLUENS

FLUENS

FLUENS

FLUENS

FLUENS

FLUENS

HUG HES I

HEGHESI

SEM I TR I GONA

SEMITRIGONA

SEM I TR I GONA

SEM I TR I GONA

SEM I TR I GONA

SEM I TRIGONA

ARI M I NE NS IS

SCHMITTI

HYALACRULUS

HYALACRULUS

PLANULATUS

PLANULATUS

GAL LOWAYI

GAL LOWAYI

GAL LOWAYI

GALL OWAYI

GALLOWAYI

GAL LOWAYI

GALLOWAYI

GALLOWAYI

GALLOWAYI

GALL OWAYI

GALL OWAYI

GAL LOWAYI

GALL LOWAYI

GALLOWAYI

GAL LOWAYI

GALL OWAYI

GALLOWAYI

GALLOWAYI

GALLOWAYI

GAL LOWAYI

GAL LOWAYI

STELLATUM

VIRAGOENSE

VIRAGOENSE

ARENACEA

DEPRESSA

DEPRESSA

LUCERNULA

SERRATA
Locality

Lat. Long.

GULF OF ALASKA

GULF OF ALASKA

OFF ALASKA PENINSULA

OFF ALASKA PENINSULA

OF F AL ASKA PENINSULA

GULF OF ALASKA

GULF OF ALASKA

GULF OF ALASKA

GULF OF ALASKA

SAMI SH BAY, WASH.

COOS BAY, OREGON

GULF OF ALASKA

GULF OF ALASKA

EXCURSION, ALASKA

BRITISH COL UMBIA

GULF OF ALASKA

GULF OF ALASKA

GULF OF ALASKA

BRITISH COLUMBIA

BRITISH COL UMBIA

BR IT ISH COL UMBIA

BRITISH COL UMBIA

BRITISH COL UMBIA

BRITISH COL UMBIA

BRITISH COL UMBIA

COOS, BAY, OREGON

FRASER R.. B.C.

SAMISH BAY, WASH.

GRAY'S HARBOUR, WASH.

SAMISH-PADILLA BAYS

DEPOE BAY, OREGON

CRESCENT CITY, CALIF.

OFF ALASKA

KETCHIKAN, ALASKA

BRITISH COLUMBIA

BRITISH COL UMBIA

BRITISH COL UMBIA

BRITISH COL UMBIA

BRITISH COL UMBIA

OFF ALASKA

KETCHIKAN, ALASKA

GAMBIER BAY, ALASKA

KASAAN BAY ALASKA

TAKU HARBOUR, ALASKA

EXCURSION, ALASK

GULF OF ALASKA
SUNSET BAY, OREGON

WASHINGTON COAST

OREGON COAST

BRITISH COL UMBIA

BRITISH COL UMBIA

BR ITISH COL UMBIA

BR IT ISH COL UMBIA

WASHINGTON COAST

WASHINGTON COAST

WASHINGTON COAST

OF F WASHINGTON

CRESCENT CITY, CALIF.

GULF OF ALASKA

GULF OF ALASKA

GULF OF ALASKA

GULF OF ALASKA

GULF OF ALASKA

EXCURSION, ALASKA

TAKU HARBOUR, ALASKA

GAMBIER BAY, ALASKA

GULF OF ALASKA

GULF OF ALASKA

GULF OF ALASKA

GULF OF ALASKA

GULF OF ALASKA

GULF OF ALASKA

GULF OF ALASKA

GULF OF ALASKA

SUNSET BAY, OREGON

BRITISH COLUMBIA

BR ITISH COL UMBIA

BRITISH COL UMBIA

GULF OF ALASKA

GULF OF ALASKA

GULF OF ALASKA

GULF OF ALASKA

GULF OF ALASKA

WASHINGTON COAST

VIRAGO SD.. B.C.

WASHINGTON COAST

OFF OREGON

OFF OREGON

OFF OREGON

OFF OREGON

OFF CALI FORNIA

GULF OF ALASKA

$5930 \mathrm{~N} 14520 \mathrm{~W}$

$\begin{array}{llll}59 & 00 N & 141 & 20 \mathrm{~W} \\ 48 & 61 \mathrm{~N} & 161 & 07 \mathrm{~W}\end{array}$

$5241 \mathrm{~N} 15536 \mathrm{~W}$

$5451 \mathrm{~N} 15524 \mathrm{~W}$

$5900 \mathrm{~N} 14120 \mathrm{~W}$

$5930 \mathrm{~N} 14255 \mathrm{~W}$

$5925 \mathrm{~N} 14525 \mathrm{~W}$

$5930 N 143$ OOW

$4835 \mathrm{~N} 12228 \mathrm{~W}$

$4836 \mathrm{~N} 12229 \mathrm{~W}$

$\begin{array}{llll}43 & 24 \mathrm{~N} & 124 & 14 \mathrm{~W} \\ 59 & 30 \mathrm{~N} & 142 & 55 \mathrm{~W}\end{array}$

$5925 \mathrm{~N} 14525 \mathrm{~W}$ 


\section{Publication}

10181 CUSHMAN MCCULLOCH 1942 10182 CUSHMAN MCCULLOCH 1942 10183 CUSHMAN TODD 1947 10184 CUSHMAN TODD 1947 10185 TODD LOW 1967 10186 BERGEN O'NEIL 1979 10187 BERGEN O NEIL 1979 10188 COCKBAIN 1963 10189 COCKBAIN 1963 10190 COCKBAIN 1963 10191 CUSHMAN TODD 1947 10192 LANKFORD PHLEGER 1973 10193 BERGEN O'NEIL 1979 10194 BERGEN O'NEIL 1979 10195 BERGEN O NE IL 1979 10196 BERGEN O.NEIL 1979 10197 BERGEN O.NEIL 1979 10198 BERGEN O'NEIL 1979 10199 BERGEN O'NEIL 1979 10200 BERGEN O'NEIL 1979 10201 BERGEN O'NEIL 1979 10202 BERGEN O'NEIL 1979 10203 BERGEN O'NEIL 1979 10204 BERGEN O'NEIL 1979 10205 BERGEN O०NEIL 1979 10206 BERGEN ONNEIL 1979 10207 SMITH 1973 10208 TODD LOW 1967 10209 TODD LOW 1967 10210 TODD LOW 1967 10211 TODD LOW 1967 10212 TODD LOW 1967 10213 TODD LOW 1967
10214 BERGEN O.NEIL 1979 10214 BERGEN O'NE IL
10215 TODD LOW 1967 10216 TODD LOW 1967 10217 TODD LOW 1967 10218 BERGEN O०NEIL 1979 10219 BERGEN O'NEIL 1979 10220 COCKBAIN 1963 10221 BERGEN O.NEIL 1979 10222 COCKBAIN 1963 10223 COCKBAIN 1963 10224 COCKBAIN 1963 10225 COCKBAIN 1963 10226 COCKBAIN 1963 10227 BERGEN O.NEIL 1979 10228 BERGEN O'NEIL 1979 10229 BERGEN O'NEIL 1979 10230 BERGEN O'NEIL 1979 10231 BERGEN O'NEIL 1979 10232 BERGEN O.NEIL 1979 10233 BERGEN O'NEIL 1979 10234 BERGEN O'NEIL 1979 10235 BERGEN O'NEIL 1979 10236 CUSHMAN MCCULLOCH 1942 10237 TODD LOW 1967

10238 COOPER 1961

10239 SMITH 1973

10240 BERGEN O.NEIL 1979

10241 CUSHMAN 1927

10242 CUSHMAN 1927

10243 COOPER 1961

10244 BERGEN O NNEIL 1979

10245 BERGEN O'NEIL 1979

10246 BERGEN O'NEIL 1979

10247 BERGEN O'NEIL 1979 10248 BERGEN O.NEIL 1979 10249 BERGEN O'NEIL 1979 10250 BERGEN O.NEIL 1979 10251 BERGEN O'NEIL 1979 10252 BERGEN O'NEIL 1979 10253 TODD LOW 1967 10254 TODD LOW 1967 10255 BERGEN O'NEIL 1979 10256 BERGEN O.NEIL 1979 10257 SMITH 1973 10257
10258

10258 SCOTT TOW 1967 10260 TODD LOW 1967 10261 TODD LOW 1967 10262 TODD LOW 1967 10263 TODD LOW 1967 10264 BERGEN O NEIL 1979 10265 BERGEN O.NEIL 1979 10266 PER GEN O NEIL 1979 10267 BERGEN O.NEIL 1979 10268 BERGEN ONE 1979 10269 BERGEN O'NEIL 1979 10270 BERGEN O०NEIL 1979 10271 BERGEN O'NEIL 1979 10272 BERGEN O'NEIL 1979 10273 BERGEN O'NEIL 1979 10274 BERGEN O'NEIL 1979 10275 BERGEN O'NEIL 1979

\section{Generic Name Specific Name}

ACEROSA PACIFICA

BOL IVINA

BOL IVINA

BOLIVINA

BOL IVINA

BOLIVINA

BOL IVINA

BOLIVINA

BOLIVINA

BOL IVINA

BOL IVINA

BOL IVINA

BOL IVINA

BOLIVINA

BOL IVINA

BOL IVINA

BOL IVINA

BOL IVINA

BOL IV INA

BOL I VINA

BOL IVINA

BOLIVINA

BOL IVINA

BOLIVINA

BOL IVINA

BOL IVINA

BOLIVINA

BOL IVINA

BOLIVINA

BOLIVINA

BOL IVINA

BOLIVINA

BOLIVINA

BOLIVINA

BOL IVINA

BOLIVINA

BOL IVINA

BOLIVINA

BOL IVINA

BOLIVINA

BOLIVINA

BOL IVINA

BOLIVINA

BOL IVINA

BOL IVINA

BOL IVINA

$B O L$ IVINA

BOL IVINA

BOL IVINA

BOL IVINA

BOL IVINA

BOLIVINA

BOL IVINA

BOLIVINA

BOLIVINA

BOL IVINA

BOLIVINA

BOL IVINA

BOL IVINA

BOL IV INA

BOLIVINA

BOLIVINA

BOL IVINA

BOL IVINA

BUCCELLA

BUCCELLA

BUCCELLA

BUCCELLA

BUC CELLA

BUCCELLA

BUCCELLA

BUCCELLA

BU C CELLA

BUCCELLA

BU C CELLA

BUCCELLA

BUCCELLA

BUCCELLA

BUCCELLA

BUCCELLA

BUCCELLA

BUCCELLA
Locality

Lat. Long.

OFF ALASKA

KETCHIKAN, ALASKA

WASHINGTON COAST

CLARENCE ST.. ALASKA

GULF OF ALASKA

GULF OF ALASKA

BRITISH COLUMBIA

BRITISH COLUMBIA

BRITISH COLUMBIA

WASHINGTON COAST

CRESCENT CITY, CALIF.

GULF OF ALASKA

GULF OF ALASKA

GULF OF ALASKA

GULF OF ALASKA

GULF OF ALASKA

GULF OF ALASKA

GULF OF ALASKA

GULF OF ALASKA

GULF OF ALASKA

GULF OF ALASKA

GULF OF ALASKA

GULF OF ALASKA

GULF OF ALASKA

OFF ALASKA PENINSULA

KASAAN BAY, ALASKA

EXCURSION, ALASKA

TAKU HARBOUR, ALASKA

GAMBIER BAY, ALASKA

CLARENCE ST.. ALASKA

GULF OF ALASKA

GULF OF ALASKA

GULF OF ALASKA

KASAAN BAY, ALASKA

GULF OF ALASKA

GULF OF ALASKA

GULF OF ALASKA

BRITISH COLUMBIA

GULF OF ALASKA

BRITISH COL UMBIA

BRITISH COL UMB IA

BR IT ISH COLUMBIA

BR IT ISH COL UMBIA

BR ITISH COL UMBIA

GULF OF ALASKA

GULF OF ALASKA

GULF OF ALASKA

GULF OF ALASKA

GULF OF ALASKA

GULF OF ALASKA

GULF OF ALASKA

GULF OF ALASKA

GULF OF ALASKA

KETCHIKAN, AL ASKA

GULF OF ALASKA

OREGON COAST

OFF ALASKA PENINSULA

GULF OF ALASKA

OFF OREGON

OFF OREGON

OREGON COAST

GULF OF ALASKA

GULF OF ALASKA

GULF OF ALASKA

GULF OF ALASKA

GULF OF ALASKA

GULF OF ALASKA

GULF OF ALASKA

GULF OF ALASKA

GULF OF ALASKA

CLARENCE ST.. ALASKA

CLARENCE ST.. ALASKA

GULF OF ALASKA

GULF OF ALASKA

KODIAK, ALASKA

SAMISH-PADILLA BAYS

GAMBIER BAY, ALASKA

EXCURSION, ALASKA

KASAAN BAY, ALASKA

LYNN CANAL

GULF OF ALASKA

GULF OF ALASKA

GULF OF ALASKA

GULF OF ALASKA

GULF OF ALASKA

GULF OF ALASKA

GULF OF ALASKA

GULF OF ALASKA

GULF OF ALASKA

GULF OF ALASKA

GULF OF ALASKA

$5628 \mathrm{~N} 13223 \mathrm{~W}$ $5521 \mathrm{~N} 13139 \mathrm{~W}$ $4833 \mathrm{~N} 12300 \mathrm{~W}$ $4832 \mathrm{~N} 12300 \mathrm{~W}$ $5521 \mathrm{~N} 13158 \mathrm{~W}$ $5930 \mathrm{~N} 14540 \mathrm{~W}$ 59 OON $14120 \mathrm{~W}$ $\begin{array}{llll}48 & 45 \mathrm{~N} & 123 & 00 \mathrm{~W} \\ 50 & 00 \mathrm{~N} & 125 & 07 \mathrm{~W}\end{array}$ $4900 \mathrm{~N} 12315 \mathrm{~W}$ $4833 \mathrm{~N} 12300 \mathrm{~W}$ $4145 \mathrm{~N} 12413 \mathrm{~W}$ $5850 \mathrm{~N} 14115 \mathrm{~W}$ $5930 \mathrm{~N} 14520 \mathrm{~W}$ $5925 \mathrm{~N} 14525 \mathrm{~W}$ $5930 \mathrm{~N} 14545 \mathrm{~W}$ $5930 \mathrm{~N} 14255 \mathrm{~W}$ 59 OON $14120 \mathrm{~W}$ 


\section{Publication}

10276 BERGEN O'NEIL 1979 10277 BERGEN O'NEIL 1979

10278 ECHOLS 1969

10279 ECHOLS 1969

10280 ECHOLS 1969

10281 JONES ROSS 1979

10282 BERGEN O NEIL 1979

10283 DETLING 1958

10284 SMITH 1973

10285 BERGEN O'NEIL 1979

10286 BERGEN O'NEIL 1979

10287 BERGEN O NEIL 1979

10288 COOPER 1961

10289 COOPER 1961

10290 COOPER 1961

10291 COOPER 1961

10292 COOPER 1961

10293 COOPER 1961

10294 ECHOLS 1969

10295 ECHOLS 1969

10296 ECHOLS 1969

10297 LANKFORD PHLEGER 1973

10298 LANKFORD PHLEGER 1973

10299 LANKFORD PHLEGER 1973

10300 CUSHMAN 1927

10301 SMI TH 1973

10302 CUSHMAN TODD 1947

10303 BERGEN O'NEIL 1979

10304 BERGEN O'NEIL 1979

10305 BERGEN O'NEIL 1979

10306 BERGEN O'NEIL 1979

10307 BERGEN O'NEIL 1979

10308 BERGEN O'NEIL 1979

10309 CUS HMAN $192 ?$

10310 SMITH 1973

10311 BERGEN O'NEIL 1979

10312 BERGEN O'NEIL 1979

10313 BERGEN O'NEIL 1979

10314 BERGEN O'NEIL 1979

10315 BERGEN O'NEIL 1979

10316 BERGEN O०NEIL 1979

10317 CUSHMAN 1927

10318 CUS HMAN 1927

10319 CUSHMAN 1927

10320 CUSHMAN 1927

10321 CUSHMAN 1927

10322 CUS HMAN 1927

10323 CUSHMAN 1927

10324 CUS HMAN 1927

10325 CUS HMAN 1927

10326 CUS HMAN 1927

10328 WHITEAVES 1886

10329 BERGEN O.NEIL 1979

10330 BERGEN O'NEIL 1979

10331 BERGEN O'NEIL 1979

10332 BERGEN O'NEIL 1979

10333 BERGEN O'NEIL 1979

10334 BERGEN O'NEIL 1979

10335 BERGEN O'NEIL 1979

10337 BERGEN O.NEIL 1979

10338 SMI TH 1973

10339 BERGEN O'NEIL 1979

10340 BERGEN O.NEIL 1979

10341 BERGEN O'NEIL 1979

10342 BERGEN OONEIL 1979

10343 BERGEN O'NEIL 1979

10344 BERGEN O'NEIL 1979

10345 COC KBAIN 1963

10346 COCKBAIN 1963

10347 COCKBAIN 1963

10348 COCKBAIN 1963

10349 COOPER 1961

10350 COOPER 1961

10351 COOPER 1961

10352 CUSHMAN 1925

10353 TODD LOW 1967

10354 TODD LOW 1967

10355 CUS HMAN MCCULLOCH 1948

10356 CUS HMAN MCCULLOCH 1948

10357 CUSHMAN MCCULLOCH 1948

10358 ECHOLS 1969

10359 ECHOLS 1969

10360 ECHOLS 1969

10361 DETLING 1958

10362 BERGEN O'NEIL 1979

10363 LANKFORD PHLEGER 1973

10364 LANKFORD PHLEGER 1973

10365 LANKFORD PHLEGER 1973

10366 PHLEGER 1967

10367 SMI TH 1973

10368 CUS HMAN TODD 1947

10369 CUSHMAN TODD 1947

10370 CUSHMAN MCCULLOCH 1948
Generic Name Specific Name

FRII IIDA

FRIGIDA

FRIGIDA

FRIGIDA

FRIGIDA

FRIGIDA

I NUS ITATA

I NUSITATA

INUSITATA

I NUSITATA

INUSITATA

TEN ERR I MA

TEN ERR IMA

TENERR IMA

TENERR IMA

TENERR IMA

TENERR IMA

TEN ERR IMA

TENERR IMA

TENERR IMA

TENERRIMA

A FFINIS

A UR I CULATA

A UR I CULATA
BARBATA

BARBATA
BAR BATA

BARBATA

BAR BATA

BARBATA

BAR BATA

INFLATA

MEXICANA

MEX I CANA

MEXI I ANA

MEXICANA

MEXI CANA

MEXI CANA

MEXICA

OVATA

OVATA

OVATA

OVATA

OVATA

OVATA

OVULA

OVULA

OVULA

OVULA

PYRULA

ROS TRATA

S UBACUMINATA

SUBACUMINATA

SUB ACUMINATA

SUBACUMINATA

S UB AC UM INATA

SUBACUMINATA

SUB ACUMINAT

BASI COSTATA

ELEGANT IS SIMA

ELEGANTISSIMA

ELEGANT IS SIMA

ELE GANTIS SIMA

ELE GANTIS SIMA

ELEGANT IS SIMA

ELEGANT ISSIMA

ELEGANTIS SIMA

ELEGANTISSIMA

ELEGANT IS SIMA

ELEGANTIS SIMA

ELEGANT ISSIMA

ELEGANT ISSIMA

ELEGANTISSIMA

ELEGANT IS SIMA

ELEGANT ISSIMA

ELEGANT ISSIMA

ELE GANTIS SIMA

ELEGANTISSIMA

ELEGANTISSIMA

ELEGANT IS SIMA

ELEGANTIS SIMA

ELEGANT ISSIMA

ELE GANTISSIMA

ELE GANTIS SIMA

ELEGANTIS SIMA

ELEGANT ISSIMA

ELEGANT IS IMA

ELE GANTIS SIMA

ELEGANT ISSIMA

ELEGANTISSIMA

ELEGANTISSIMA LIMBOSA
Locality

GULF OF ALASKA

GULF OF ALASKA

WASHINGT ON COAST

WASHINGTON COAST
WASHINGTON COAST

SAMISH BAY, WASH.

GULF OF ALASKA

SUNSET BAY, OREGON

KODIAK, ALASKA

GULF OF ALASKA

GULF OF ALASKA

GULF OF ALASKA

OREGON COAST

OREGON COAST

OREGON COAST

OREGON COAST

OREGON COAST

CALI FORNIA COAST

WASHINGTON COAST

WASHINGTON COAST

WASHINGTON COAST

CRESCENT CITY, CALIF.

LAPUSH, WASHINGTON

OFF OREGON

OFF ALASKA PENINSULA

WASHINGTON COAST

GULF OF ALASKA

GULF OF ALASKA

GULF OF ALASKA

GULF OF ALASKA

GULF OF ALASKA

GULF OF ALASKA

OFF OREGON

OFF ALASKA PENINSULA

GULF OF ALASKA

GULF OF ALASKA

GULF OF ALASKA

GULF OF ALASKA

GULF OF ALASKA

GULF OF ALASKA

OFF OREGON

OFF OREGON

OFF OREGON

OFF OREGON

OFF OREGON

OFF OREG ON

OFF OREGON

OFF OREGON

OFF OREGON

OFF OREGON

OFF OREGON

JOHNSTONE STRAIT, B.C.

GULF OF ALASKA

GULF OF ALASK

GULF OF ALASKA

GULF OF ALASKA

GULF OF ALASKA

GULF OF ALASKA

GULF OF ALASKA

GULF OF ALASKA

GULF OF ALASKA

OFF RLASKA PENINSULA

GULF OF ALASKA

GULF OF ALASKA

GULF OF ALASKA

GULF OF ALASKA

GULF OF ALASKA

GULF OF ALASKA

BRITISH COL UMBIA

BRITISH COLUMBIA

BRITISH COLUMBIA

BRITISH COLUMBIA

OREGON COAST

OREGON COAS

CALIFORNIA COAST

VIRAGO SD.. B.C.

EXCURSION, ALASKA

LYNN CANAL, ALASKA

OFF ALASKA

OFF ALASKA

KETCHIKAN, ALASKA

WASHINGTON COAST

WASHINGTON COAST

WASHINGTON COAST

SUNSET BAY, OREGON

GULF OF ALASKA

DEPOE BAY, OREGON

CRESCENT CITY, CALIF.

LAPUSH, WASHINGTON

GRAY'S HARBOUR WASH.

GRAY S HARBOUR,

WASHINGTON COAST

WASHINGTON COAST

OFF CALIFORNIA

Lat. Long.

$5630 \mathrm{~N} 15240 \mathrm{~W}$

$5835 \mathrm{~N} 13915 \mathrm{~W}$

$4730 \mathrm{~N} 12420 \mathrm{~W}$

47 OON $12410 \mathrm{~W}$

$4835 \mathrm{~N} 12228 \mathrm{~W}$

59 55N $14425 \mathrm{~W}$

$4321 \mathrm{~N} 12420 \mathrm{~W}$

$5749 N 15230$

$5635 \mathrm{~N} 15150 \mathrm{~W}$ 


\section{Publication}

10371 TODD LOW 1967

10372 TODD LOW 1967

10373 CUSHMAN 1927

10374 CUSHMAN 1927

10375 CUSHMAN 1927
10376 CUSHMAN 1927

10377 BERGEN O.NE IL 1979

10378 BERGEN O NEIL 1979

10379 BERGEN O.NEIL 1979

10380 BERGEN O'NEIL 1979

10381 BERGEN O.NEIL 1979

10382 BERGEN O NEIL 1979

10383 BERGEN O'NEIL 1979

10384 BERGEN O'NEIL 1979

10385 CUSHMAN TODD 1947

10386 CUSHMAN TODD 1947

10387 BERGEN O'NEIL 1979

10388 TODD LOW 1967

10389 BERGEN O'NEIL 1979

10390 BERGEN O'NEIL 1979

10391 TODD LOW 1967

10392 TODD LOW 1967

10393 TODD LOW 1967

10394 TODD LOW 1967

10395 BERGEN O'NEIL 1979

10396 BERGEN O'NEIL 1979

10397 BERGEN O'NEIL 1979

10398 BERGEN O'NEIL 1979

10399 BERGEN O.NEIL 1979

10400 BERGEN O.NE IL 1979

10401 BERGEN O.NEIL 1979

10402 BERGEN O'NE IL 1979

10403 BERGEN O'NEIL 1979

10404 BERGEN O'NEIL 1979

10405 BERGEN O'NEIL 1979

10406 BERGEN O'NEIL 1979

10407 BERGEN O.NEIL 1979

10408 BERGEN O'NEIL 1979

10409 COCKBAIN 1963

10410 COCKBAIN 1963

10411 COCKBAIN 1963

10412 CUSHMAN 1925

10413 CUSHMAN 1925

10414 SMITH 1973

10415 SMITH 1973

10416 BERGEN O.NEIL 1979

10417 BERGEN O'NEIL 1979

10418 BERGEN O.NEIL 1979

10419 BERGEN O'NEIL 1979

10420 BERGEN O.NEIL 1979

10421 BERGEN O'NEIL 1979

10422 BERGEN O'NEIL 1979

10423 BERGEN O'NE IL 1979

10424 BERGEN O'NEIL 1979

10425 BERGEN O.NEIL 1979

10426 BERGEN O.NEIL 1979

10427 BERGEN O०NEIL 1979

10428 CUSHMAN 1927

10429 COCKBAIN 1963

10430 COC KBAIN 1963

10431 TODD LOW 1967

10432 TODD LOW 1967

10432 TODD LOW 1967

10434 BERGEN O.NEIL 1979

10435 BERGEN O.NEIL 1979

10436 BERGEN O'NEIL 1979

10437 BERGEN O'NEIL 1979

10438 BERGEN O'NEIL 1979

10439 BERGEN O'NEIL 1979

10440 BERGEN O'NEIL 1979

10441 BERGEN O'NEIL 1979

10442 BERGEN O.NEIL 1979

10443 BERGEN O.NEII 1979

10444 BERGEN O NEEIL 1979

10445 BERGEN O'NEIL 1979

10446 BERGEN O'NEIL 1979

10447 COCKBAIN 1963

10448 COOPER 1961

10449 COOPER 1961

10450 COCKBAIN 1963

10451 COCKBAIN 1963

10451 COCKBAIN 1963
10452 COCKBAIN 1963

10453 COCKBAIN 1963

10454 COCKBAIN 1963

10455 COCKBAIN 1963

10456 CUSHMAN TODD 1947

10457 CUS HMAN TODD 1947

10458 CUS HMAN TODD 1947

10459 TODD LOW 1967

10460 TODD LOW 1967

10461 TODD LOW 1967

10467 TODD LOW 1967

10463 DETLING 1958

10464 BERGEN O'NEIL 1979

10465 BERGEN O.NEIL 1979
Generic Name

Specific Name

Locality

Lat. Long.

BUL IMINE LLA

BUL IMINE LLA

BUL IMINELLA

BUL IMINELLA

BUL IM I NE LLA

BULIMINELLA

BUL IM INE LLA

BUL IMINELLA

BUL IMINELLA

BUL IMINELLA

BUL IMINELLA

BULIMINELLA

BUL IMINE LLA

CASSIDULINA

CASSIDULINA

CASSIDULINA

CASSIDULINA

CASSIDULINA

CASSIDULINA

CASSIDULINA

CASSIDULINA

CASSIDULINA

CASSIDULINA

CASSIDULINA

CASSIDULINA

CASSIDULINA

CASSIDULINA

CASSIDULINA

CAS SIDULINA

CASSIDULINA

CASSIDUL INA

CASSIDULINA

CASSIDUL INA

CASSIDULINA

CASSIDULINA

CASSIDULINA

CAS SIDULINA

CASSIDULINA

CASSIDULINA

CASSIDULINA

CASSIDULINA

CASSIDULINA

CASSIDULINA

CASSIDULINA

CASSIDULINA

CASSIDULINA

CASSIDULINA

CASSIDULINA

CASSIDUL INA

CASSIDUL INA

CASSIDULINA

CASSIDULINA

CASSIDULINA

CASSIDULINA

CASSIDULINA

CASSIDULINA

CASSIDULINA

CASSIDULINA

CASSIDULINA

CASSIDUL INA

CASSIDULINA

CASSIDULINA

CASSIDULINA

CASSIDULINA

CASSI DUL INA

CASSIDULINA

CASSIDULINA

CASSIDULINA

CASSIDULINA

CASSIDULINA

CASSIDUL INA

CASSIDULINA

CASSI DULINA

CASSIDUL INA

CASSIDULINA

CASSI DUL INA

CASSI DULINA

CASSIDULINA

CASSIDULINA

CASSIDULINA

CASSIDULINA

CASSIDULINA

CASSIDULINA

CASSIDULINA

CASSIDULINA

CASSIDULINA

CASSIDULINA

CASSIDULINA

CASSIDULINA

CASSIDUL INA

CASSIDULINA

CASSI DUL INA

CASSIDUL INA
SUBFUSI FORMIS

SUBFUSIFORMIS

SUB FUSIFORMIS TENUATA

SUB FUS I FORMIS TENUATA

SUB FUSI FORMIS TENUATA

SUB FUSIFORMIS TENUATA

TENUATA

TENUATA

TENUATA

TENUATA

TENUATA

TENUATA

TENUATA

CALIFORNICA

C AL I FORNICA

CAL I FORNICA

CALIFORNICA

CAL IFORNICA

CAL IFORNICA

CALIFORNICA

CALI FORNICA

C AL IFORNICA

CAL I FORNICA

CAL I FORNICA

CAL I FORNICA

CAL I FORNICA

CAL I FORNICA

CAL I FORNICA

CAL I FORNICA

CAL IF ORNICA

C AL I FORNICA

CAL I FORNICA

CAL I FORNICA

CAL I FORNI CA

CAL I FORNICA

CALIFORNICA

CAL I FORNICA

CALIFORNI CA

CAL I FORNICA

CHARLOTTENSIS

CRASSA

CRASSA

C US HMAN I

CUSHMAN I

CUSHMANI

CUS HMANI

CUSHMAN I

CUS HMANI

CUS HMANI

CUS HMANI

C US HMANI

DEL I CATA

DEL I CATA

DEL I CATA

DEL I CATA

DEPRESSA

DEPRESSA

I SL ANDICA

I SL AND I CA

I SLANDICA

I SLANDICA

I SLANDICA

I SL ANDICA

I SLANDICA

I SL ANDI CA

I SL ANDI CA

I SL ANDICA

I SLANDICA

SLANDICA

I SLANDICA

I SLANDICA

I SL ANDICA

LIMBATA

LIMBATA

LIMBATA

LIMBATA

LIMBATA

LIMBATA

LIMBATA

LIMBATA

LIMBATA

LIMBATA

LIMBATA

LIMBATA

L IMBATA

LIMBATA

LIMBATA

LIMBATA

LIMBATA

LIMBATA

L IMBATA

LIMBATA
CAL IFORNICA

CLARENCE ST., ALASKA 


\section{Publication}

10466 BERGEN O'NEIL 1979 10467 BERGEN O'NEIL 1979 10468 BERGEN O'NEIL 1979 10469 BERGEN O'NEIL 1979 10470 BERGEN O'NEIL 1979 10471 BERGEN O'NEIL 1979 10472 BERGEN O'NEIL 1979 10473 BERGEN O'NEIL 1979 10473 BERGEN O'NEIL 1979 10474 BERGEN O'NEIL 1979 10475 BERGEN O'NEIL 1979

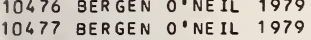
10477 BERGEN O'NEIL 1979 10479 CUS HMAN TODD 1947 10480 BERGEN O'NEIL 1979 10481 BERGEN O'NEIL 1979 10482 BERGEN O'NEIL 1979 10483 ECHOLS 1969

10484 ECHOLS 1969

10485 TODD LOW 1967

10486 BERGEN O'NEIL 1979

10487 BERGEN OPNEIL 1979

10488 COC KBAIN 1963

10489 TODD LOW 1967

10490 BERGEN O'NEIL 1979

10491 BERGEN O'NEIL 1979

10492 BERGEN O'NEIL 1979 10493 BERGEN O'NEIL 1979 10494 BERGEN O'NEIL 1979 10495 BERGEN O'NEIL 1979 10496 BERGEN O'NEIL 1979 10497 BERGEN O'NEIL 1979 10498 BERGEN O'NEIL 1979 10499 BERGEN O'NEIL 1979 10500 BERGEN O'NEIL 1979 10501 BERGEN O'NEIL 1979 10502 BERGEN O'NEIL 1979 10502 BERGEN O'NEIL 1979 10504 CUSHMAN 1925

10505 CUS HMAN 1925

10506 BERGEN O'NEIL 1979 10507 BERGEN O'NEIL 1979 10508 BERGEN OPNEIL 1979 10509 BERGEN O'NEIL 1979 10510 BERGEN O'NEIL 1979 10511 BERGEN O'NEIL 1979 10512 BERGEN O'NEIL 1979 10512 BERGEN OTNEIL 1979

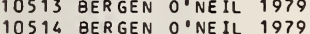
$\begin{array}{lll}10514 & \text { BERGEN O'NEIL } 1979 \\ 10515 & \text { BERGEN O'NEIL } 1979\end{array}$ 10516 BERGEN O.NE IL 1979 10517 SMI TH 1973

10518 TODD LOW 1967

10519 BERGEN O'NEIL 1979

10520 BERGEN O'NEIL 1979

10521 TODD LOW 1967

10522 TODD LOW 1967

10523 TODD LOW 1967

10524 BERGEN O'NEIL 1979 10525 BERGEN ODNEIL 1979 10526 BERGEN O'NEIL 1979 10527 BERGEN O'NEIL 1979 10528 BERGEN O'NEIL 1979 10529 SMITH 1973

10530 TODD LOW 1967

10531 LANKFORD PHLEGER 1973

10532 SMITH 1973

10533 TODD LOW 1967

10534 TODD LOW 1967

10535 BERGEN O'NEIL 1979

10536-BERGEN O'NEIL 1979

10537 BERGEN O'NEIL 1979

10538 BERGEN O'NEIL 1979

10539 COCKBAIN 1963

10540 BERGEN O'NEIL 1979

10541 BERGEN O'NEIL 1979

10542 BERGEN O'NEIL 1979

10543 CUSHMAN 1925

10544 COOPER 1961

10545 BERGEN O'NEIL 1979 10546 BERGEN O'NEIL 1979 10547 BERGEN O'NEIL 1979 10548 BERGEN O'NEIL 1979 10549 BERGEN O'NEIL 1979 10550 BERGEN O'NEIL 1979 10551 CUS HMAN 1927

10551 CUS HMAN 1927 1970 10552 BER GEN O NE IL 10553 CUS HMAN 1927
10554 BERGEN O.NEIL 1979 $\begin{array}{lll}10554 & \text { BERGEN O'NEIL } 1979 \\ 10555 & \text { BERGEN O'NEIL } 1979\end{array}$ 10556 BERGEN ONNEIL 1979 10557 CUS HMAN 1927

10558 CUS HMAN 1927

10559 BERGEN O'NEIL 1979 10560 BERGEN O'NEIL 1979
Generic Name Specific Name

LIMBATA

CASSIDULINA

CASSIDULINA

CASSIDULINA

CASSIDULINA

CASSIDULINA

CASSIDUL INA

CASSIDULINA

CASSIDUL INA

CASSIDULINA

CASSIDULLINA

CASSIDULINA

CASSIDULINA

CASSIDULINA

CASSIDUL INA

CASSIDULINA

CASSIDULINA

CASSIDULINA

CASSIDULINA

CASSIDULLINA

CASSIDUL INA

CASSIDULINA

CASSIDULINA

CASSIDULINA

CASSIDULINA

CASSIDUL INA

CASSIDUL INA

CASSIDULINA

CASSIDULINA

CASSIDULINA

CASSIDULINA

CASSIDULINA

CASSIDULINA

CASSIDULINA

CASSIDUL INA

CASSIDULINA

CASSIDULINA

CASSIDULINA

CASSIDULINA

CASSIDULINA

CASSIDULINA

CASSIDULINA

CASSIDULINA

CASSIDULINA

CASSIDULINA

CASSIDULINA

CASSIDULINA

CASSIDULINA

CASSIDUL INA

CASSIDULINA

CASSIDULINA

CASSIDULINA

CASSIDULINA

CASSIDUL INA

CASSIDULINA

CASSIDULINA

CASSIDULINA

CASSIDULINA

CASSIDULLINA

CASSIOULINA

CASSIDULINA

CASSIDULINA

CASSIDULINA

CASSIDULINA

CASSIDULINA

CASSIDULINA

CASSIDULINA

CASSIDULINA

CASSIDULINA

CASSIDULINA

CASSIDUL INA

CASSIDUL INA

CASSIDULINA

CASSIDULINA

CASSIDULINA

CASSIDULINA

CASSIDULINA

CASSIDULINA

CASSIDULINA

CASSIDULINA

CASS IDULINA

CASSIDULINOIDES

CHILOSTOMELLA

CHILOSTOMELLA

CHILOSTOMELLA

CHILOSTOMELLA

CHILOSTOMELLA

CHILOSTOMELLA

CHILOSTOMELLA

CHILOSTOMELLA

CHILOSTOMELLA

CHILOSTOMELLA

\section{Locality}

Lat. Long.

GULF OF ALASKA

GULF OF ALASKA

GULF OF ALASKA

GULF OF ALASKA

GULF OF ALASKA

GULF OF ALASKA

GULF OF ALASKA

GULF OF ALASKA

GULF OF ALASKA

GULF OF ALASKA

GULF OF ALASKA

WASHINGTON COAST

WASHINGTON COAST

GULF OF ALASKA

GULF OF ALASKA

WASHINGTON COAST

WASHINGTON COAST

KASAAN BAY, ALASKA

GULF OF ALASKA

GULF OF ALASKA

BR IT ISH COLUMBIA

GULF OF ALASKA

GULF OF ALASKA

GULF OF ALASKA

GULF OF ALASKA

GULF OF ALASKA

GULF OF ALASKA

GULF OF ALASKA

GULF OF ALASKA

GULF OF ALASKA

GULF OF ALASKA

GULF OF ALASKA

GULF OF ALASKA

GULF OF ALASKA

VIRAGO SD.. B.C.

Q. CHARLOTTE SD.., B.C.

GULF OF ALASKA

GULF OF ALASKA

GULF OF ALASKA

GULF OF ALASKA

GULF OF ALASKA

GULF OF ALASKA

GULF OF ALASKA

GULF OF ALASKA

GULF OF ALASKA

GULF OF ALASKA

GULF OF ALASKA

OFF ALASKA PENINSULA

GULF OF ALASKA

GULF OF ALASKA

GULF OF ALASKA

EXCURSION, ALASKA

GULF OF ALASKA

KASAAN BAY, ALASKA

GULF OF ALASKA

GULF OF ALASKA

GULF OF ALASKA

GULF OF ALASKA

GULF OF ALASKA

OFF ALASKA PENINSULA

LYNN CANAL, ALASKA

LAPUSH, WASHINGTON

OFF ALASKA PENINSULA

KASAAN BAY, ALASKA

GULF OF ALASKA

GULF OF ALASKA

GULF OF ALASKA

GULF OF ALASKA

GULF OF ALASKA

BRIT I SH COL UMBI A

GULF OF ALASKA

GULF OF ALASKA

GULF OF ALASKA

Q. CHARLOTTE SD.. B.C.

OREGON COAST

GULF OF ALASKA

GULF OF ALASKA

GULF OF ALASKA

GULF OF ALASKA

GULF OF ALASKA

GULF OF ALASKA

OFF OREGON

GULF OF ALASKA

OFF OREGON

GULF OF ALASKA

GULF OF ALASKA

GULF OF ALASKA

OFF OREGON

OFF OREGON

GULF OF ALASKA

GULF OF ALASKA

$5956 N 14425 \mathrm{~W}$

$5635 \mathrm{~N} 152$ 00W

$56 \angle 0 N 15155 \mathrm{~W}$

$\begin{array}{llll}58 & 35 N & 139 & 15 W\end{array}$

$5955 \mathrm{~N} 14540 \mathrm{~W}$

$5945 \mathrm{~N} 14600 \mathrm{~W}$ 


\section{Publication}

10561 BERGEN O'NEIL 1979 10562 CUSHMAN 1927

10563 CUS HMAN 1927

10564 BERGEN O'NEIL 1979

10565 SMITH 1973

10566 SMI TH 1973

10567 SMITH 1973

10568 BERGEN O'NEIL 1979

10569 BERGEN O'NEIL 1979

10570 BERGEN O'NEIL 1979

10571 BERGEN O'NEIL 1979

10572 BERGEN O'NEIL 1979

10573 BERGEN O'NEIL 1979

10574 BERGEN O'NEIL 1979

10575 BERGEN O'NEIL 1979

10576 BERGEN O'NEIL 1979

10577 BERGEN O'NEIL 1979

10578 BERGEN O'NEIL 1979

10579 BERGEN O'NEIL

10581 COCKBAIN 1963

10582 COCKBAIN 1963

10583 COCKBAIN 1963

10584 BERGEN O.NEIL 1979

10585 BERGEN O'NEIL 1979

10586 ECHOLS 1969

10587 BERGEN O'NEIL 1979

10588 BERGEN O'NEIL 1979

10589 BERGEN O'NEIL 1979

10590 BERGEN O'NEIL 1979

10591 BERGEN O'NEIL 1979

10592 BERGEN O'NEIL 1979

10593 COCKBAIN 1963

10594 COCKBAIN 1963

10595 COOPER 1961

10596 COOPER 1961

10597 COOPER 1961

10598 BERGEN O'NEIL 1979

10599 BERGEN O'NEIL 1979

10600 COCKBAIN 1963

10601 COC KBAIN 1963

10602 COCKBAIN 1963

10603 COCKBAIN 1963

10604 COCKBAIN 1963

10605 COCKBAIN 1963

10606 COCKBAIN 1963

10607 CUSHMAN TODD 1947

10608 CUSHMAN TODD 1947

10609 CUSHMAN TODD 1947

10610 CUSHMAN TODD 1947

10611 CUSHMAN TODD 1947

10612 CUSHMAN TODD 1947

10613 TODD LOW 1967

10614 TODD LOW 1967

10615 TODO LOW 1967

10616 TODD LOW 1967

10617 TODD LOW 1967

10618 TODD LOW 1967

10619 TOD D LOW 1967

10620 SMI TH 1973

10621 LANKFORD PHLEGER 1973

10622 BERGEN O'NEIL 1979

10623 BERGEN O'NEIL 1979

10624 BERGEN O'NEIL 1979

10625 BERGEN O'NEIL 1979

10626 BERGEN O'NEIL 1979

10627 BERGEN O०NEIL 1979

10628 BERGEN O.NEIL 1979

10629 ECHOLS 1969

10630 DETLING 1958

10631 BERGEN O'NEIL 1979

10632 BERGEN O'NEIL 1979

10633 BERGEN O'NEIL 1979

10634 BERGEN O'NEIL 1979

10635 BERGEN O'NEIL 1979

10636 BERGEN O'NEIL 1979

10637 BERGEN O'NEIL 1979

10638 COOPER 1961

10639 BERGEN O'NEIL 1979

10640 BERGEN O'NEIL 197

10641 BERGEN O'NEIL 1979

10642 BERGEN O'NEIL 1979

10643 BERGEN O'NEIL 1979

10644 BERGEN O'NEIL 1979

10645 BERGEN O'NEIL 1979

10646 BERGEN O'NEIL 1979

10647 BERGEN D.NEIL 1979

10648 SMITH 1973

10649 CUSHMAN TODD 1947

10650 COOPER 1961

10651 BERGEN O'NEIL 1979

10652 SMITH 1973

10653 SMITH 1973

10654 TODD LOW 1967

10655 TODD LOW 1967

\section{Generic Name Specific Name}

OOL INA

O CL INA

CHILOSTOMELLA

CHILOSTOMELLA

CHILOSTOMELLINA

CIBICIDES

CIBICIOES

CIBICIDES

CIBICIDES

CIBICIDES

CIBICIDES

CIBICIDES

CIBICIOES

CIBICIDES

CIBICIDES

CIBICIDES

CIBICIDES

CIBICIDES

CIBICIDES

CIBICIDES

CIBICIDES

CIBICIDES

CIBICIDES

CIBICIOES

CIBICIDES

CIBICIDES

CIBICIDES

CIBICIDES

CIBICIDES

CIBICIDES

CIBICIDES

CIBICIDES

CIBICIDES

CIBICIDES

CIBICIOES

CIBICIDES

CIBICIDES

CIBICIDES

CIBICIDES

CIBICIDES

CIBICIOES

CIBICIDES

CIBICIDES

CIBICIDES

CIBICIDES

CIBICIDES

CIBICIDES

CIBICIDES

CIBICIDES

CIBICIDES

CIBICIDES

CIBICIDES

CIBICIDES

CIBICIDES

CIBICIDES

CIBICIDES

CIBICIDES

CIBICIDES

CIBICIDES

CIBICIDES

CIBICIDES

CIBICIOES

CIBICIDES

CIBICIDES

CIBICIDES

CIBICIDES

CIBICIDES

CIBICIDES

CIBICIDES

CIBICIDES

CIBICIDES

CIBICIDES

CIBICIDES

CIBICIDES

CIBICIDES

CIBICIDES

CIBICIDES

CIBICIDES

CIBICIOES

CIBICIDES

CIBICIDES

CIBICIDES

CIBICIDES

CIBICIDES

CIBICIDES

CIBICIDES

CIBICIDES

CIBICIDES

CIBICIDES

CIBICIDES

CIBICIDOIDES

CORNUSPIRA

CORNUSPIRA

CORNUSPIRA

BRAOYI

BRADYI

FLETCHER I

FLETCHERI

FLETCHER

FLE TCHER I

FLETCHERI

FLETCHER

FLETCHERI

FLETCHERI

FLE TCHERI

FLETCHERI

FLE TCHER

FLETCHERI

F LE TCHERI

FLETCHER I

FLETCHER I

FLE TCHERI

FLETCHER I

FLETCHERI

FLETCHER I

FLETCHER

FLE TCHERI

FLETCHERI

FLETCHERI

FLE TCHERI

FLE T CHERI

FLE TCHER I

FLETCHERI

LOBATULUS

$\angle O B A T U L U S$

LOBATULUS

LOBATULUS
LOBATULUS

LOBATULUS

LOBATULUS

LOBATULUS

LOBATULUS

LOBATULUS

LBATULUS

LOBATULUS

LOBATULUS

LOBATULUS

$\angle O B A T U L U S$

LOBATULUS

$\angle O B A T U L U S$

LOBATULUS

LOBATULUS

LOBATULUS

LOBATULUS

LOBATULUS

LOBATULUS

LOBATULUS

LOBATULUS

LOBATULUS

LOBATULUS

LOBATULUS

LOBATULUS

LOBATULUS

LOBATULUS

LOBATULUS

LOB ATULUS

LOBATULUS

LOBATULUS

LOBATULUS

LOBATULUS

LOBATULUS

LOBATULUS

LOBATULUS

LOBATUS

MCKANNA I

MCK ANNA

MCK ANNA

MCK ANNA I

MCK ANNAI

MCK ANNA I

MCK ANNAI

MCKANNAI

MCK ANNA I

REFULGENS

REFULGENS

REF ULGENS

SPIRALIS

MUNDULUS

INCERT A

INVOLVENS

INVOLVENS

\section{Locality}

GULF OF ALASKA

OFF OREGON

GULF OF ALASKA

OFF ALASKA PENINSULA

NORTH PACIFIC

FLETCHER

Lat. Long.

$5930 \mathrm{~N} 14520 \mathrm{~W}$ $4308 \mathrm{~N} 12455 \mathrm{~W}$ 59 OON 14120 


\section{Publication}

10656 DETLING 1958

10657 CUS HMAN 1925

0658 CUSHMAN 1925

10659 BERGEN O.NEIL 1979

10660 CUSHMAN TODD 1947

10661 CUSHMAN TODD 1947

10662 LANKFORD PHLEGER 1973

10663 SCOTT 1974

10664 SCOTT 1974

10665 LANKFORD PHLEGER 1973

10666 LANKFORD PHLEGER 1973

10666 LANKF ORD PHLEGER 1973

10668 LANKFORD PHLEGER 1973

10669 LANKFORD PHLEGER 1973

10670 CUS HMAN 1910

10671 TODD LOW 1967

10672 TODD LOW 1967

10673 TODD LOW 1967

10674 BERGEN O.NEIL 1979

10675 TODD LOW 1967

10676 TODD LOW 1967

0677 TODD LOW 1967

0678 BERGEN O.NEIL 1979

10679 BERGEN O'NEIL 1979

0680 BERGEN O'NEIL 1979

10681 BERGEN O'NEIL 1979

0682 BERGEN O'NEIL 1979

10683 BERGEN O'NEIL 1979

10684 BERGEN O'NEIL 1979

10685 BERGEN O NE IL 1979

10686 ECHOLS 1969

10687 TODD LOW 1967

10688 TODD LOW 1967

10689 TODD LOW 1967

0690 TODD LOW 1967

10691 BERGEN O'NEIL 1979

10692 BERGEN O'NEIL 1979

10693 BERGEN O'NEIL 1979

10694 BERGEN O'NE IL 1979

10694 BERGEN O'NEIL 1979

10695 BERGEN O'NE IL
10696 TODD LOW 1967

10697 BERGEN O'NEIL 1979

0698 TODD LOW 1967

10699 BERGEN O'NEIL 1979

10700 BERGEN O'NEIL 1979

10701 BERGEN O०NEIL 1979

10702 BERGEN O'NEIL 1979

10703 BERGEN O'NEIL 1979

10704 BERGEN O'NEIL 1979

10705 TODD LOW 1967

0706 WHITEAVES 1886

10707 CUSHMAN 1913

10708 CUSHMAN 1910

10709 TODD LOW 1967

10710 SMITH 1973

10711 CUSHMAN 1910

10712 CUSHMAN 1910

10713 CUS HMAN 1910

10714 SMITH 1973

10715 SMITH 1973

0716 SMI TH 1973

10717 SMITH 1973

10718 SMI TH 1973

10720 BERGEN O'NEIL 1979

10721 BERGEN O'NEIL 1979

10722 BERGEN O.NEIL 1979

10723 TODD LOW 1967

10724 TODD LOW 1967

10725 CUSHMAN MCCULLOCH 1950

10726 TODD LOW 1967

10727 TODD LOW 1967

10728 BERGEN O.NEIL 1979

10729 TODD LOW 1967

10730 WHITEAVES 1886

10731 WHITEAVES 1886

10732 WHITEAVES 1886

10733 CUSHMAN 1925

10734 CUSHMAN 1925

10735 DETLING 1958

10736 CUS HMAN TODD 1947

10737 CUSHMAN TODD 1947

10738 CUSHMAN TODD 1947

10739 COOPER 1961

10740 COOPER 1961

10741 COOPER 1961

10742 COOPER 1961

10743 COOPER 1961

10744 CUSHMAN TODD 1947

10745 CUSHMAN TODD 1947

10746 CUSHMAN TODD 1947

10747 COCKBAIN 1963

10748 COCKBAIN 1963

10749 CUS HMAN 1925
10750 CUSHMAN 1925
Generic Name Specific Name

Locality

Lat. Long.

CORNUSPIRA

CORNUSPIRA

CORNUSPIRA

CORNUSPIRA

CORNUSPIRA

CORNUSPIRA

CRIBROELPHIDIUM

CRIBROELPHIDIUM

CRIBROELPHIDIUM

CRIBROELPHIDIUM

CRIBRONONION

CRIBRONONION

CRIBRONONION

CRIBRONONION

CRIBROST OMOIDES

CRIBROSTOMOIDES

CRIBROST OMOIDES

CRIBROST OMOIDES

CRIBROSTOMOIDES

CRIBROSTOMOIDES

CRIBROST OMOIDES

CRIBROSTOMOIDES

CRIBROSTOMOIDES

CRIBROSTOMOIDES

CRIBROST OMOIDES

CRIBROSTOMOIDES

CRIBROSTOMOIDES

CRI BROST OMOIDES

CRI BROST OMOIDES

CRIBROSTOMOIDES

CRIBROSTOMOIDES

CRIBROSTOMOIDES

CRIBROST OMOIDES

CRIBROSTOMOIDES

CRIBROST OMOIDES

CRIBROST OMOIDES

CRI BR OS T OMOIDES

CRIBROS TOMOIDES

CRIBROST OMOIDES

CRIBROST OMOIDES

CRIBROSTOMOIDES

CRIBROST OMOIDES

CRIBROSTOMOIDES

CRI IRR OSTOMOIDES

CRIBROSTOMOIDES

CRIBROST OMOIDES

CRIBROSTOMOIDES

CRIBROS TOMOIDES

CRIBROSTOMOIDES

CRIBROSTOMOIDES

CRISTELLARIA

CRISTELLARIA

CRITHIONINA

CRUCIL OCULINA

CYCLAMMINA

CYCLAMMINA

CYCLAMM INA

CY CLAMM INA

CYCLAMMINA

CYCLAMMINA

CYSTAMM INA

CYSTAMM I NA

CYSTAMMINA

DENTALINA

DENTALINA

DENTAL INA

DENTAL INA

DENTAL INA

DENTAL INA

DENTALINA

DENTAL INA

DENTALINA

DENTAL INA

DI SCORBINA

DIS CORBINA

DIS CORBINA

DISCORBIS

DI S CORB IS

DISCORBIS

DISCORBIS

DISCORBIS

DISCORBIS

DIS CORBIS

DISCORBIS

DISCORBIS

DISCORBIS

DISCORBIS

DISCORBIS

DIS CORBIS

DISCORBIS

DIS CORBIS

DISCORBIS

DISCORBIS
I NVOLVENS

I NVOL VENS

I NVOLVENS

PLANORBIS

PLANORBIS

FRIGIDUM

SEL SEYENSE

TUM I DUM

FRI GIDUM

LENE

LENE

LENE

CRASSIMARG

CRASS IMAR GO

C RA SS IMARGO

C RASS IMARGO

CRA SS IMARGO

CRA SS IMARGO

CRASS IMARGO

CRASSIMARGO

JEFFREYSI

JEF FREYSI

JEFFREYSI

JEFFREYSI

JEFFREYSI

JEF FEY I

JEFFREYSII

JEFFREYSII

JEFFREYSII

JEFFREYSII

JEFFREYSI I

SCI TULUS

SCITULUS

SCITULUS

SCITULUS

SCITULUS

SCITULUS

SCITULUS

SCITULUS

SCITULUS

SCITULUS

SCITULUS

SCITULUS

SCITULUS

VELERONIS

CUL TRATA

LATA

TRI ANGULARIS

CAN CELLATA

C ANCELLATA

PUS ILLA

TRULLISSATA

TRULLISSATA

GALEATA

GALEATA

GALEATA

BAG GI

BAG

BAGGI

BAGG

BAGG

CAL OMORPHA

DECEPTA

DECEPTA

DECEPTA

SUB SOLUTA

PAR ISIENSIS

PAR ISIENSIS

PARISIENSIS

CHARLOTTENSIS

COLUMBIENSIS

COL UMBIENSIS

COLUMB IENSIS

C OL UMBIENSIS

COLUMBIENSIS

MON I CANA

MONI CANA

MON I CANA

MON ICANA

MONICANA

OPERCULARIS

ORNATIS

ORNATISSIMA
PISUM HISPIDA

SUNSET BAY, OREGON

Q. CHARLOTTE SD.. B.C.

VIRAGO SD.. B.C.

GULF OF ALASKA

WASHINGTON COAST

WASHINGTON COAST

CRESCENT CITY, CALIF.

SAMISH-PADILLA BAYS

DEPOE BAY OREGON 


\section{Publication}

10751 CUSHMAN TODD 1947 10752 DETLING 1958

10753 COOPER 1961

10754 COOPER 1961

10755 COOPER 1961

10756 COOPER 196

10757 BERGEN O'NEIL 1979

10758 BERGEN O'NEIL 1979

10759 BERGEN O'NEIL 1979

10760 DETLING 1958

10761 CUS HMAN TODD 1947

10762 CUSHMAN TODD 1947

10763 CUSHMAN TODD 1947

10764 TODD LOW 1967

10765 BERGEN O'NEIL 1979

10766 BERGEN O'NEIL 1979

10767 SMITH 1973

10768 SMI TH 1973

10769 LANKFORD PHLEGER 1973

10770 BERGEN O'NEIL 1979

10771 BERGEN O'NEIL 1979

10772 BERGEN OINE 1979

10773 CUSHMAN TODD 1947

10774 CUSHMAN TODD 1947

10775 CUSHMAN TODD 1947

10776 TODD LOW 1967

10777 TODD LOW 1967

10778 TODD LOW 1967

10779 LANKFORD PHLEGER 1973

10780 LANKF ORD PHLEGER 1973

10781 LANKFORD PHLEGER 1973

10782 DETLING 1958

10783 SCOTT 1974

10784 ECHOLS 1969

10785 ECHOLS 1969

10786 COCKBAIN 1963

10787 COCKBAIN 1963

10788 COCKBAIN 1963

10789 COCKBAIN 1963

10790 COCKBAIN 1963

10791 BERGEN O.NEIL 1979

10792 BERGEN O.NEIL 1979

10793 BERGEN O'NEIL 1979

10794 BERGEN O'NEIL 1979

10795 BERGEN O'NEIL 1979

10796 BERGEN O'NEIL 1979

10797 BERGEN O'NEIL 1979

10798 COOPER 1961

10799 COOPER 1961

10800 COOPER 1961

10801 ECHOLS 1969

10802 COCKBAIN 1963

10803 COCKBAIN 1963

10804 COC KBAIN 1963

10805 TODD LOW 1967

10806 TODD LOW 1967

10807 TODD LOW 1967

10808 TODD LOW 1967

10809 CUSHMAN TODD 1947

10810 TODD LOW 1967

10811 CUS HMAN TODD 1947

10812 CUSHMAN TODD 1947

10813 CUSHMAN MCCULLOCH 1939

10814 CUS HMAN MCCULLOCH 1939

10815 CUS HMAN MCCULLOCH 1939

10816 CUSHMAN MCCULLOCH 1939

10817 SMITH 1973

10818 SMI TH 1973

10819 SMITH 1973

10820 SMITH 1973

10821 SMITH 1973

10822 BERGEN O'NEIL 1979

10823 BERGEN O'NEIL 1979

10824 BERGEN O'NE IL 1979

10825 BERGEN O'NEIL 1979

10826 BERGEN O NEIL 1979

10827 BERGEN O'NEIL 1979

10828 BERGEN O'NEIL 1979

10829 BERGEN O'NEIL 1979

10830 BERGEN O'NEIL 1979

10831 BERGEN O'NEIL 1979

10832 BERGEN O'NEIL 1979

10833 SMI TH 1973

10834 SMITH 1973

10835 SMI TH 1973

10836 SMITH 1973

10837 BERGEN O'NEIL 1979

10838 TODD LOW 1967

10839 SMITH 1973

10840 TODD LOW 1967

10841 TODD LOW 1967

10842 TODD LOW 1967

10843 TODD LOW 1967

10844 TOD D LOW 1967

10845 TODD LOW 1967

\section{Generic Name}

DISCORBIS

DISCORBIS

DISCORBIS

DISCORBIS

DISCORBIS

DISCORBIS

DIS CORBIS

DIS CORBIS

DIS CORBIS

DISCORBIS

DISCORBIS

DISCORBI

DOROTHIA

DOROTHIA

DOROTH IA

DOROTHIA

DOROTHIA

DYOCIBICIDES

DYOCIBICIDES

DYOCIBICIDES

DYOCIBICIDES

DYOCIBICIDES

DYOCIBICIDES

DYOCIBICIDES

DYOCIBICIDES

DYOCIBICIDES

DYOCIBICIDES

EGGERELLA

EGGERELLA

ERELLA

EGGERELLA

EGGERELLA

EGGERELLA

EGGERELLA

EGGERELLA

EGGERELLA

EGGERELLA

EGGERELLA

EGGERELLA

EGGERELLA

EGGERELLA

EGGERELLA

EGGERELLA

EGGERELLA

EGGERELLA

EGGERELLA

EGGERELLA

EGGERELLA

EGGERELLA

EGGERELLA

EGGERELLA

EGGERELLA

EGGERELLA

EGGERELLA

EGGERELLA

EGGERELLA

EGGERELLA

EGGERELLA

EGGERELLA

EGGERELLA

EGGERELLA

EGGERELLA

EGGERELLA

EGGERELLA

EGGERELLA

EGGERELLA

EGGERELLA

EGGERELLA

EGGERELLA

EGGERELLA

EGGERELLA

EGGERELLA

EGGERELLA

EGGERELLA

EGGERELLA

EGGERELLA

EGGERELLA

EGGERELLA

EGGERELLA

EHRENBER GINA

EHRENBER GINA

EHRENBER GINA

ELPHIDIELLA

ELPHIDIELLA

ELPHIDIELLA

ELPHIDIELLA

ELPHIDIELLA

ELPHIDIELLA
Specific Name

ORNATISSIMA

ORNATISSIMA

ORNATISSIMUS

ORNATISSIMUS

ORNATISSIMUS

PRAEGERI

PRAEGERI

PRAEGERI

SAN JUANENSIS

SANJUANENS IS

SANJUANEN S IS

SAN JUANENSIS

BRADYANA

BRADYANA

BRADYANA
EXILIS

EXILIS

B ISERIALIS

BISERIALIS

B ISERIALIS

BISERIALIS

BISERIALIS

BISERIALIS

BISERIALIS

B IS ERIALIS

BISERIALIS

BISER IALIS

A DVENA

A DVENA

ADVENA

A DVENA

A DVENA

A DVENA

ADVENA

A DVENA

ADVENA

ADVENA

A DVENA

A DVENA

A DVENA

A DVENA

ADVENA

ADVENA

ADVENA

ADVENA

A DVENA

A DVENA

ADVENA

ADVENA

A DVENA

A DVENA

ADV ENA

ADVENA

A DVENA

A DVENA

ADVENA

A DVENA

ADVENA

ADVENA

ADVENA

A DVENA

BRADYI

BRA DYI

BRADYI

BRADYI

BRADYI

BRADYI

BRADYI

BRADYI

BRADYI

HUMBOLDTI

HUMBOLDTI

PUS ILLA

PUS ILLA

PUS ILLA

PUS ILLA

SCABRA

SCABRA

SCABRA

C OMPRESS

COMPRESSA

HYSTRIX

ARC TICA

A RC T ICA

GROENLANDICA

GROENL AND I CA

GROENLANDICA

GROENLANDICA

Locality

Lat. Long.

WASHINGTON COAST

SUNSET BAY, OREGON

OREG ON COAST

OREGON COAST

OREGON COAST

CALIFORNIA COAST

GULF OF ALASKA

GULF OF ALASKA

GULF OF ALASKA

SUNSET BAY, OREGON 


\section{Publication}

10846 TOD D LOW 1967

10847 SMITH 1973

10848 LANKFORD PHLEGER 1973

10849 LANKFORD PHLEGER 1973

10850 LANKFORD PHLEGER 1973

10851 CUSHMAN TODD 1947

0852 CUSHMAN TODD 1947

10853 CUSHMAN TODD 1947

10854 CUSHMAN TODD 1947

10855 CUSHMAN TODD 1947

10856 CUSHMAN TODD 1947

10858 CUS HMAN MCCULLOCH 1940

10858 CUSHMAN MCCULLOCH 1940

10859 CUSHMAN MCCULLOCH 1940

10860 CUSHMAN MCCULLOCH 1940

10861 CUSHMAN MCCULLOCH 1940

10862 CUSHMAN MCCULLOCH 1940

10863 CUS HMAN MC

10864 SCOTT 1974

10866 COOPER 1961

10867 COOPER 1961

10868 COOPER 1961

10869 COOPER 1961

10870 COOPER 1961

10871 COOPER 1961

10872 COOPER 1961

10873 ECHOLS 1969

10874 ECHOLS 1969

10875 COCKBAIN 1963

10876 COCKBAIN 1963

10877 COCKBAIN 1963

$\begin{array}{lll}10878 & \text { BERGEN O'NEIL } 1979 \\ 10879 & \text { BERGEN O'NEIL } 1979\end{array}$

$\begin{array}{lll}10879 & \text { BERGEN O'NEIL } 1979 \\ 10880 & \text { BERGEN O'NEIL } 1979\end{array}$

$\begin{array}{ll}10880 & \text { BERGEN O'NEIL } 1979 \\ 10881 & \text { BERGEN O'NEIL } 1979\end{array}$

10882 COCKBAIN 1963

0883 COCKBAIN 1963

10884 COCKBAIN 1963

10885 BERGEN O'NE IL 1979

10886 BERGEN O'NEIL 1979

10887 BERGEN O NEEIL 1979

10888 DETLING 1958

10889 NAT LAN D 1933

10890 BERGEN O'NEIL 1979

10891 BERGEN O'NEIL 1979

10892 BERGEN O'NEIL 1979

10893 CUSHMAN MCCULLOCH 1940

10894 CUSHMAN MCCULLOCH 1940

10895 CUSHMAN MCCULLOCH 1940

10896 CUS HMAN TODD 1947

10897 CUSHMAN TODD 1947

10898 BERGEN O'NEIL 1979

10899 BERGEN O'NEIL 1979

10900 BERGEN O'NEIL 1979

0901 BERGEN O'NEIL 1979

10902 TODD LOW 1967

10903 TODD LOW 1967

10904 TODD LOW 1967

10905 SMITH 1973

10906 SMI TH 1973

10907 BERGEN O'NEIL 1979

10908 BERGEN O०NEIL 1979

10909 TODD LOW 1967

10910 BERGEN O'NEIL 1979

10911 BERGEN O'NEIL 1979

10912 BERGEN O'NEIL 1979

10913 BERGEN O'NEIL 1979

10914 COCKBAIN 1963

10915 COCKBAIN 1963

10916 COC KBAIN 1963

10917 COC KBAIN 1963

10918 COCKBAIN 1963

0919 COC KBAIN 196

10920 BERGEN O'NEIL 1979

10921 BERGEN O'NEIL 1979

10922 BERGEN O'NEIL 1979

10923 BERGEN O'NEIL 1979

10924 BERGEN O'NEIL 1979

10925 BERGEN O'NEIL 1979

10926 TODD LOW 1967

10927 TODD LOW 1967

10928 TOD D LOW 1967

10929 TODD LOW 1967

10930 BERGEN OONEIL 1979

10931 BERGEN O'NEIL 1979

10932 BERGEN O'NEIL 1979

10933 SMI TH 1973

10934 SMITH 1973

10935 COCKBAIN 1963

10936 CUSHMAN TODD 1947

10937 CUSHMAN TODD 1947

10938 CUSHMAN TODD 194 ?

10939 NICOL 1944

10940 COOPER 1961
Generic Name Specific Name

ELPHIDIELLA

ELPHIDIELLA

ELPHIDIELLA

ELPHIDIELLA

ELPHIDIELLA

ELPHIDIELLA

ELPHIDIELLA

ELPHIDIELLA

ELPHIOIELLA

ELPHIDIELLA

ELPHIDIELLA

ELPHIDIELLA

ELPHIDIELLA

ELPHIDIELLA

ELPHIDIELLA

ELPHIDIELLA

ELPHIDIELLA

ELPHIDIELLA

ELPHIDIELLA

ELPHIOIELLA

ELPHIDIELLA

ELPHIDIELLA

ELPHIDIELLA

ELPHIDIELLA

ELPHIDIELLA

ELPHIDIELLA

ELPHIDIELLA

ELPHIDIELLA

ELPHIDIELLA

ELPHIDIELLA

ELPHIDIELLA

ELPHIDIELLA

ELPHIDIELLA

ELPHIDIELLA

ELPHID IELLA

ELPHIDIELLA

ELPHIDIELLA

ELPHIDIELLA

ELPHIDIUM

ELPHIDIUM

ELPHIDIUM

ELPHIDIUM

ELPHIDIUM

ELPHIDIUM

ELPHIOIUM

ELPHIDIUM

ELPHIDIUM

ELPHIDIUM

ELPHIDIUM

ELPHIDIUM

ELPHIDIUM

ELPHIDIUM

ELPHIDIUM

ELPHIDIUM

ELPHIDIUM

ELPHIDIUM

ELPHIDIUM

ELPHIDIUM

ELPHIDIUM

ELPHIDIUM

ELPHIDIUM

ELPHIDIUM

ELPHIDIUM

ELPHIDIUM

ELPHIDIUM

ELPHIDIUM

ELPHIDIUM

ELPHIDIUM

ELPHIDIUM

ELPHIDIUM

ELPHIDIUM

ELPHIDIUM

ELPHIDIUM

ELPHIDIUM

ELPHIDIUM

ELPHIDIUM

ELPHIDIUM

ELPHIDIUM

ELPHIDIUM

ELPHIDIUM

ELPHIDIUM

ELPHIDIUM

ELPHIDIUM

ELPHIDIUM

ELPHIDIUM

ELPHIDIUM

ELPHIDIUM

ELPHIDIUM

ELPHIDIUM

ELPHIDIUM
GROENLANDICA

GROENLANDICA

HANNAI

HAN NAI

HANNAI

H ANNAI

HANNAI

HAN NAI

HANNAI

HANNAI

HANNAI

HANNAI
HANNAI

HANNAI

HANNAI

HANNAI

HAN N A I

HAN NAI

HANNAI

HAN NA

HAN NAI

HANNAI

H ANNAI

HANNAI

HAN NAI

HANNAI

HANNAI

HANNAI

$\begin{array}{llll}N & \text { IT I D A } \\ \text { N IT I D } & \text { DA } \\ \text { NIT I D }\end{array}$

NIT I DA

N IT I DA

N IT I DA

$N$ IT I DA

$N$ IT I DA

N IT I DA

NIT IDA

NIT I DA

N IT I DA

$N$ IT I DA

N IT I DA

ART I CULATUM

ART I CULATUM

ART I CULATUM

ART I CULATUM

ART I CULATUM

ART I CULATUM

ART I CULATUM

ART I CULATUM

ART I CULATUM

BAR TLETTI

BAR TLETTI

BAR TLET T

BAR TLETT

BAR TLETTI

BAR TLETTI

BAR TLETT I

BAR TLETT

BAR TLETTI

BAR TLETTI

BAR TLETT

CLAVATUM

CLAVATUM

CLAVATUM

CLAVATUM

CLAVATUM

CLAVATUM

CLAVATUM

CLAVATUM

CLAVATUM

CLAVATUM

CLAVATUM

CLAVATUM

CLAVATUM

CLAVATUM

CLAVATUM

CLAVATUM

CLAVATUM

CLAVATUM
CLAVATUM

CLAVATUM

CLAVATUM

CLAVATUM

CLAVATUM

CLAVATUM

CLAVATUM

CRI SPUM

CRISPUM

F AX BARBARENSE

FAX BARBARENSE

FAX BARBARENSE

FAX FAX

FAX FAX

Locality

Lat. Long.

GAMBIER BAY, ALASKA

OFF ALASKA PENINSULA

LAPUSH, WASHINGTON

CRESCENT CITY, CALIF.

DEPOE BAY, OREGO

WASHINGTION COAST

WASHINGTON COAST

WASHINGTON COAST

WASHINGTON COAST

WASHINGTON COAST

KETCHIKAN, ALASKA 


\section{Publication}

10941 PHLEGER 1967

10942 BERGEN O'NEIL 1979

10943 BERGEN O'NEIL 1979

10944 BERGEN O NEIL 1979

10945 BERGEN O.NEIL 1979

10946 BERGEN O'NEIL 1979

10947 BERGEN O.NEIL 1979

10948 TODD LOW 1967

10949 TODD LOW 1967

10950 TODD LOW 1967

10951 TODD LOW 1967

10952 TODD LOW 1967

10953 TOD D LOW 1967

10954 TODD LOW 1967

10955 CUSHMAN MCCULLOCH 1940

10956 CUSHMAN MCCULLOCH 1940

10957 CUSHMAN MCCULLOCH 1940

10958 CUS HMAN MCCULLOCH 1940

10959 CUSHMAN MCCULLOCH 1940

10960 CUS HMAN TODD 1947

10961 CUS HMAN TODD 1947

10962 BERGEN OPNE IL 1979

10963 BERGEN O'NEIL 1979

10964 BERGEN O'NEIL 1979

10965 BERGEN O'NEIL 1979

10966 JONES ROSS 1979

10967 NATLAND 1933

10968 NATLAND 1933

10969 BERGEN O'NEIL 1979

10970 BERGEN ODNEIL 1979

10971 BERGEN OONEIL 1979

10972 BERGEN O'NEIL 1979

10973 BERGEN O'NEIL 1979

10974 BERGEN O.NEIL 1979

10975 COOPER 1961

10976 COCKBAIN 1963

10977 COCKBAIN 1963

10978 COCKBAIN 1963

10979 COCKBAIN 1963

10980 DETLING 1958

10981 SMITH 1973

10982 CUSHMAN TODD 1947

10983 CUS HMAN TODD 1947

10984 CUS HMAN TODD 1947

10985 CUS HMAN TODD 1947

10987 CUSHMAN MCCULLOCH 1940

10987 CUSHMAN MCCULLOCH 1940
10988 CUSHMAN MCCULLOCH 1940

10989 PHLEGER 1967

10990 SMITH 1973

10991 ECHOLS 1969

10992 ECHOLS 1969

10993 ECHOLS 1969

10994 ECHOLS 1969

10995 ECHOLS 1969

10996 ECHOLS 1969

10997 COOPER 1961

10998 COOPER 1961

10999 COOPER 1961

11000 COOPER 1961

11001 COOPER 1961

11002 COOPER 1961

11003 COOPER 1961

11004 COOPER 1961

11005 SMITH 1973

11006 TODD LOW 1967

11007 JONES ROSS 1979

11008 COCKBAIN 1963

11009 COCKBAIN 1963

11010 COCKBAIN 1963

11011 COCKBAIN 1963

11012 COCKBAIN 1963

11013 PHLEGER 1967

11014 PHLEGER 1967

11015 DETLING 1958

11016 COOPER 1961

11017 COOPER 1961

11018 COOPER 1961

11019 CUSHMAN TODD 1947

11020 CUSHMAN TODD 1947

11021 CUS HMAN TODD 1947

11022 CUSHMAN MCCULLOCH 1940

11023 CUS HMAN MCCULLOCH 1940

11024 CUSHMAN MCCULLOCH 1940

11025 CUSHMAN MCCULLOCH 1940

11026 CUSHMAN TODD 1947

11027 CUS HMAN TODD 1947

11028 CUSHMAN TODD 1947

11029 CUS HMAN TODD 1947

11030 CUSHMAN TODD 1947

11031 CUSHMAN TODD 1947

11032 LANKFORD PHLEGER 1973

11033 SMITH 1973

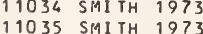

Generic Name Specific Name

FRI GIDUM

FRIGIDUM

FRIGIDUM

FRIGIDUM

FRI GIDUM

FRI GI DUM

FRI GIDUM

FRIGIDUM

FRIGIDUM

FRIGIDUM

FRI G I DUM

FRI GIDUM

FRI GIDUM

FRI GIDUM

FRIGIDUM

FRI GIDUM

FRI GIDUM

FRIGIDUM

FR I GIDUM

FRIGIDUM

FRI GIDUM

FRI GIDUM

HANNA

HUG HES I

HUGHES

HUG HES I

HUGHES I

HUG HES I

HUGHESI

HUGHES I

HUGHESI FORAMINOSUM

INCERT UM

INCERTUM

INC ERTUM

INCERTUM

I NC ERTUM

I NC ERTUM

INCERTUM LENE

INCERTUM LENE

INCERTUM LENE

INCERTUM LENE

INCERTUM LENE

INCERTUM LENE

INCERTUM LENE

LENE

MAGELLANI CUM

MAGELLANI CUM

MAGELLANI CUM

MAGELLANI CUM

MICROGRANULO SUM

MIC ROGRANULO SUM

MIC ROGRANULO SUM

MI C ROGR ANUL O SUM

MIC ROGR ANULO SUM

MIC ROGR ANULO SUM

MICROGRANULO SUM

MIC ROGRANULO SUM

MICROGRANULO SUM

MICROGRANULO SUM

MICROGRANULOSUM

ORB I CULARE

ORE GONENSE

SELSEYENSE

SUB ARC T I CUM

SUBARCT I CUM

SUB ARCTI I CUM

SUBARCT ICUM

SUBARCTICUM

SUB ARCTI CUM
SUBARCTI I UM

SUBARCTI CUM
SUBARCT I CUM

SUB ARCT I CUM
SUB ARCT I CUM

TRANSLUCENS

TRANSLUCENS

TRANSLUCENS

TUM I DUM

TUM I DUM

T UM I DUM

T UM I DUM

TUM IDUM

TUM I DUM

TUM I DUM

C AT ENULATA

CATENULATA

CATENULATA

COMPRESSA

COMPRESSA

LUC IDA

BRA DYANA

EXI GUA

EXIGUA

EXI GUA
Locality

Lat. Long.

GRAY'S HARBOUR, WASH.

GULF OF ALASKA

GULF OF ALASKA

GULF OF ALASKA

GULF OF ALASKA

GULF OF ALASKA

GULF OF ALASKA

GAMBIER BAY, ALASKA

LYNN CANAL, ALASKA

KASAAN BAY ALASKA

CLARENCE ST.. ALASKA

GULF OF ALASKA

TAKU HARBOUR, ALASKA

KETCHIKAN, ALASKA

OFF ALASKA

OFF BRIT - COLUMBIA

OFF WASHINGTON

OFF WASHING TON

WASHINGTON COAST

WASHINGTON COAST

GULF OF ALASKA

GULF OF ALASKA

GULF OF ALASKA

GULF OF ALASKA

SAMISH BAY, WASH.

CRESCENT CITY, CALIF.

CRESCENT CITY, CALIF.

GULF OF ALASKA

GULF OF ALASKA

GULF OF ALASKA

GULF OF ALASKA

GULF OF ALASKA

GULF OF ALASKA

CALIFORNIA COAST

BR ITISH COLUMBIA

BR IT ISH COLUMBIA

BRITISH COL

BRITISH COLUMBIA

SUNSET BAY, OREGON

OFF ALASKA PENINSULA

WASHINGTON COAST

WASHINGTON COAST

WASHINGTON COAST

WASH INGTON COAST

KETCHIKAN, ALASKA

OFF ALASKA

OFF BRIT. COLUMBIA

GRAY'S HARBOUR, WASH.

OFF ALASKA PENINSULA

WASHINGTON COAST

WASH INGTON COAST

WASHINGTON COAST

WASHINGTON COAST

WASHINGTON COAST

WASHINGTON COAST

OREGON COAST

OREGON COAST

CALIFORNIA COAST

OREGON COAST

OREGON COAST

CALIFORNIA COAST

OREGON COAST

OREGON COAST

KODIAK, ALASKA

KASAAN BAY, ALASKA

SAMISH BAY, WASH.

BRITISH COLUMBIA

BR IT ISH COLUMBIA

BR IT ISH COLUMBIA

BR ITISH COL UMBIA

BRITISH COLUMBIA

GRAY'S HARBOUR, WASH.

COOS, BAY, OREGON

SUNSET BAY, OREGON

CALIFORNIA COAST

OREGON COAST

OREGON COAST

WASHINGTON COAST

WASHINGTON COAST

WASHINGTON COAST

OFF BRIT. COLUMBIA

OFF ALASKA

OFF ALASKA

OFF ALASKA

WASH INGTON COAST

WASH INGTON COAST

WASHINGTON COAST

WASHINGTON COAST

WASHINGTON COAST

WA SHINGTON COAST

CRESCENT CITY, CALIF.

OFF ALASKA PENINSULA

OFF ALASKA PENINSULA

$4700 \mathrm{~N} 12408 \mathrm{~b}$

$5945 \mathrm{~N} 14455 \mathrm{~W}$

$5950 N 14547$

$5942 \mathrm{~N} 144 \quad 10$

$5925 N 14525 W$

$5930 \mathrm{~N} 14520 \mathrm{~W}$

$5720 \mathrm{~N} 13402 \mathrm{~W}$ 


\section{Publication}

11036 SMI TH 1973

11037 BERGEN O'NEIL 1979

11038 BERGEN O'NEIL 1979

11039 BERGEN O'NEIL 1979
11040 BERGEN O'NEIL 1979

11041 TODD LOW 1967

11042 TODD LOW 1967

11043 BERGEN O'NEIL 1979

11044 BERGEN O'NEIL 1979

11045 BERGEN O'NE IL 1979

1046 BERGEN O'NE IL 1979

1047 BERGEN O'NEIL 1979

1048 BERGEN O'NEIL 1979

11049 BERGEN O०NEIL 1979

11050 BERGEN O'NEIL 1979

11051 BERGEN O'NEIL 1979

11052 BERGEN O NEIL 1979

11053 COC KBAIN 1963

11054 COCKBAIN 1963

11055 COCKBAIN 1963

11056 BERGEN O'NEIL 1979

1057 BERGEN OPNEIL 1979

1058 BERGEN O'NEIL 1979

11059 SMI TH 1973

11060 TODD LOW 1967

11061 TODD LOW 1967

11062 TODD LOW 1967

11063 TODD LOW 1967

11064 TOD D LOW 1967

11065 BERGEN O.NEIL 1979

1066 BERGEN O'NEIL 1979

11067 BERGEN O'NEIL 1979

1068 BERGEN O'NEIL 1979

11069 BERGEN O-NEIL 1979

11070 BERGEN O'NEIL 1979

11071 BERGEN O'NEIL 1979

11072 BERGEN O'NEIL 1979

11073 BERGEN O'NEIL 1979

11074 BERGEN O'NEIL 1979

11075 BERGEN O'NEIL 1979

11076 BERGEN O'NEIL 1979

11077 BERGEN O'NEIL 1979

11078 BERGEN O'NEIL 1979

11079 BERGEN O'NEIL 1979

11080 BERGEN O'NEIL 1979

11081 COOPER 1961

11082 COOPER 1961

11083 COOPER 1961

11084 CUS HMAN TODD 1947

11085 DETLING 1958

11086 CUS HMAN TODD 1947

11087 CUSHMAN TODD 1947

11088 CUS HMAN TODD 1947

11089 CUSHMAN TODD 1947

11090 CUS HMAN TODD 1947

11091 CUS HMAN TODD 1947

11092 BERGEN O'NEIL 1979

11093 BERGEN O'NEIL 1979

11094 BERGEN O'NEIL 1979

11095 BERGEN O'NEIL 1979

11096 BERGEN O०NEIL 1979

11097 BERGEN O'NEIL 1979

11098 TODD LOW 1967

11099 BERGEN O'NEIL 1979

11100 BERGEN O'NEIL 1979

11101 BERGEN O'NEIL 1979

11102 BERGEN O'NEIL 1979

11103 BERGEN O'NEIL 1979

11104 BERGEN O.NEIL 1979

11105 BERGEN O'NEIL 1979

11106 BERGEN O'NEIL 1979

11107 BERGEN O'NEIL 1979

11108 BERGEN O'NEIL 1979

11109 BERGEN O'NEIL 1979

11110 BERGEN O'NEIL 1979

11111 BERGEN O'NEIL 1979

11112 BERGEN O'NEIL 1979

11113 BERGEN O'NEIL 1979

11114 NATLAND 1933

11115 BERGEN O'NEIL 1979

11116 BERGEN O'NEIL 1979

11117 BERGEN O'NEIL 1979

11118 BERGEN O'NEIL 1979

11119 CUSHMAN TODD 1947

11120 CUSHMAN TODD 1947

11121 BERGEN O'NEIL 1979

11122 BERGEN O'NEIL 1979

11123 BERGEN OONEIL 1979

11124 BERGEN OONEIL 1979

11125 BERGEN ODNEIL 1979

11126 BERGEN O.NEIL 1979

11127 BERGEN O'NEIL 1979

11128 BERGEN O'NEIL 1979

11129 BERGEN O'NEIL 1979
Generic Name Specific Name

EXIGUA

EXIGUA

EXIGUA

PACIFICA

PACIFICA

PACIFICA

PACIFICA

PACIFICA

PACIFICA

PAC IFICA

PACIFICA

PACIFICA

$P A C$ IFICA

PACIFICA

PACIFICA

PAC IFICA

PAC IFICA

PAC IFICA

PAC IFICA

PAC IFICA

UMB ONIFERA

VITREA

VITREA

VITREA

VITREA

VITREA

VITREA

VITREA

VITREA

VITREA

VITREA

VITREA

VITREA

VITREA

VITREA

VITREA

VITREA

VITREA

VITREA

COL UMBI IENSIS

COLUMBI IENSIS

COLUMBIENSIS

COL UMBIENSIS

COL UMBIENSIS

C OL UMB I ENS IS

C OL UMB I ENS IS

C OL UMB IENSIS

FRI GIDUS

FRIGIDUS

FRIGIDUS

HEALDI

HEALDI

HEALDI

HEALDI

HEALDI

HEALOI

ISABELLEANUS

LEV I CULUS

LEVICULUS

LEVICULUS

LEVICULUS

LEVICULUS

LEVICULUS

LEVICULUS

LEVICULUS

LEVICULUS

LEVICULUS

LEVICULUS

LEVICULUS

LEVICULUS

LEVICULUS

LEVICULUS

LEVICULUS

ORNATA

REPANDUS

REPANDUS

REPANDUS

REPANDUS

REPANDUS

REPANDUS

REP ANDUS

REPANDUS

REPANDU

SUB TENER

SUB TENER

SUB TENER

SUBTENER

SUBTENER

SUB TENER

SUB TENER
Locality

Lat. Long.

NORTH PACIFIC

GULF OF ALASKA

GULF OF ALASKA

GULF OF ALASKA

CLARENCE ST.. ALASKA

GULF OF ALASKA

GULF OF ALASKA

GULF OF ALASKA

GULF OF ALASKA

GULF OF ALASKA

GULF OF ALASKA

GULF OF ALASKA

GULF OF ALASKA

GULF. OF ALASKA

GULF OF ALASKA

BRITISH COLUMBIA

BR ITISH COLUMBIA

BRITISH COLUMBIA

GULF OF ALASKA

GULF OF ALASKA

GULF OF ALASKA

OFF ALASKA PENINSULA

TAKU HARBOUR, ALASKA

KASAAN BAY, ALASKA

EXCURSION, ALASKA

LYNN CANAL. ALASKA

GULF OF ALASKA

GULF OF ALASKA

GULF OF ALASKA

GULF OF ALASKA

GULF OF ALASKA

GULF OF ALASKA

GULF OF ALASKA

GULF OF ALASKA

GULF OF ALASKA

GULF OF ALASKA

GULF OF ALASKA

GULF OF ALASKA

GULF OF ALASKA

GULF OF ALASKA

GULF OF ALASKA

CALIFORNIA COAST

OREGON COAST

OREGON COAST

WASH INGTON COAST

SUNSET BAY, OREGON

WASHINGTON COAS

WASHINGTON COAS

WASHINGTON COAST

WASH INGTON COAS

WASH INGTON COAST

WASHINGTON COAST

GULF OF ALASKA

GULF OF ALASKA

GULF OF ALASKA

GULF OF ALASKA

GULF OF ALASKA

GULF OF ALASKA

GULF OF ALASKA

GULF OF ALASKA

GULF OF ALASKA

GULF OF ALASKA

GULF OF ALASKA

GULF OF ALASKA

GULF OF ALASKA

GULF OF ALASKA

GULF OF ALASKA

GULF OF ALASKA

GULF OF ALASKA

GULF OF ALASKA

GULF OF ALASKA

GULF OF ALA?

GULF OF ALA

GULF OF ALASKA

CRESCENT CITY, CALIF.

GULF OF ALASKA

GULF OF ALASKA

GULF OF ALASKA

GULF OF ALASKA

WASH INGTON COAST

WASHINGTON COAST

GULF OF ALASKA

GULF OF ALASKA

GULF OF ALASKA

GULF OF ALASKA

GULF OF ALASKA

GULF OF ALASKA

GULF OF ALASKA

GULF OF ALASKA

GULF OF ALASKA

GULF OF ALASKA

$4347 \mathrm{~N} 16036 \mathrm{~W}$

$5945 \mathrm{~N} 14600 \mathrm{~W}$

$5930 \mathrm{~N} 14540 \mathrm{~W}$

$\begin{array}{llll}59 & 35 \mathrm{~N} 146 & 30 \mathrm{~W} \\ 59 & 55 \mathrm{~N} 142 & 25 \mathrm{~W}\end{array}$

$5521 \mathrm{~N} 13158 \mathrm{~W}$

$5932 \mathrm{~N} 14236 \mathrm{~W}$

$5930 \mathrm{~N} 14520 \mathrm{~W}$

59 20N $14558 \mathrm{~W}$

$5945 \mathrm{~N} 14455 \mathrm{~W}$

$5850 \mathrm{~N} 14115 \mathrm{~W}$ 


\section{Publication}

11131 CUSHMAN 1927

11132 CUSHMAN 1927

11133 BERGEN O०NEIL 1979

11134 BERGEN O'NEIL 1979

11135 BERGEN O'NEIL 1979

11136 BERGEN O NE IL 1979

11137 BERGEN O'NEIL 1979

11138 BERGEN O'NEIL 1979

11139 TODD LOW 1967

11140 TODD LOW 1967

11141 BERGEN O'NEIL 1979

11142 BERGEN O'NEIL 1979

11143 BERGEN O'NEIL 1979

11144 DETLING 1958

11145 COOPER 1961

11146 COCKBAIN 1963

11147 COCKBAIN 1963

11148 BERGEN O'NEIL 1979

11149 BERGEN O'NEIL 1979

11150 BERGEN O'NEIL 1979

11151 BERGEN O'NEIL 1979

11152 BERGEN O'NEIL 1979

11153 BERGEN O'NEIL 1979

11155 BERGEN OPNEIL 1979

11156 BERGEN O'NEIL 1979

11157 BERGEN O'NE IL 1979

11158 BERGEN O'NEIL 1979

11159 BERGEN O'NEIL 1979

11160 BERGEN O'NEIL 1979

11161 BERGEN O'NEIL 1979

11162 BERGEN O'NEIL 1979

11163 TODD LOW 1967

11164 TODD LOW 1967

11165 TODD LOW 1967

11166 LANKFORD PHLEGER 1973

11167 LANKFORD PHLEGER 1973

11168 COOPER 1961

11169 BERGEN O'NEIL 1979

11170 COOPER 1961

11171 COOPER 1961

11172 BERGEN O'NEIL 1979

11173 BERGEN OONEIL 1979

11174 BERGEN O'NE IL 1979

11175 BERGEN O'NEIL 1979

11176 BERGEN O०NEIL 1979

11177 BERGEN O'NEIL 1979

11178 BERGEN O'NEIL 1979

11179 BERGEN O'NEIL 1979

11180 BERGEN O NEIL 1979

11181 BERGEN O.NEIL 1979

11182 BERGEN OONEIL 1979

11183 BERGEN O'NE IL 1979

11184 BERGEN O'NEIL 1979

11185 BERGEN O'NEIL 1979

11186 BERGEN O'NEIL 1979

11187 BERGEN O'NEIL 1979

11188 BERGEN O 'NEIL 1979

11189 BERGEN OONEIL 1979

11190 BERGEN O'NEIL 1979

11191 BERGEN O'NEIL 1979

11192 LANKFORD PHLEGER 1973

11193 ECHOLS 1909

11194 ECHOLS 1969

11195 ECHOLS 1969

11196 BERGEN O'NEIL 1979

11197 BERGEN O'NEIL 1979

11198 BERGEN O N NEIL 1979

11199 BERGEN O'NEIL 1979

11200 BERGEN O.NEIL 1979

11201 BERGEN O'NE IL 1979

11202 BERGEN O'NEIL 1979

11203 BERGEN O'NE IL 1979

11204 BERGEN $0^{\circ} \mathrm{NEIL} 1979$

11205 BERGEN O'NEIL 1979

11206 BERGEN O.NEIL 1979

11207 TODD LOW 1967

11208 TODD LOW 1967

11209 TODO LOW 1967

11210 TODD LOW 1967

11211 BERGEN O'NEIL 1979

11212 WHITEAVES 1886

11213 WHI TEAVES 1886

11214 TODD LOW 1967

11215 TODD LOW 1967

11216 TOD D LOW 1967

11217 TODD LOW 1967

11218 TOD D LOW 1967

11219 TODD LOW 1967

11220 CUSHMAN MCCULLOCH 1939

11221 COCKBAIN 1963

11222 COOPER 1961

11223 COCKBAIN 1963

11224 COCKBAIN 1963

11225 COCKBAIN 1963

\section{Generic Name Specific Name}

TENERA

UMB ONATUS

UMB ONATUS

UMB ONATUS

UMB ONATUS

UMB ONATUS

AGASSIZ I

A GASSI $Z$ I

C UC URB I TASEMA

CUCURBI TOSEMA

UUC I DA

LUC I DA

U UC I DA

LUC I DA

LUC IDA
LUC IDA

LUC I DA

LUC IDA

LUC IOA

LUCIDA

LUCIDA

LUC IDA

LUC IOA

LUC IOA

LUC IDA

LUC IOA

UC IDA

LUCIDA

LUC IDA

LUC IDA

UCIOA

$\angle U C I D A$

UC I DA

LUC IDA

LUC IDA

LUCIDA

MAR GINATA

MARGINATA

MAR GINATA

MAR GINATA

MARGINATA

MAR GINATA

MARGINATA

AUR I CULUS

AUR I CULUS

AUR I CULUS

A UR I CULUS

A UR I CULUS

AUR I CULUS

A UR I CULUS

A UR I CULUS

A UR I CULUS

A UR I CULUS

AUR ICULUS

A UR I CULUS

A UR I CULUS

A UR I CULUS

A UR I CULUS

A UR I CULUS

AUR I CULUS

B AS ISPINATUS

JAP ONICUS

JAP ONI CUS

JAP ONICUS

LABRADORI CUS

LABRADORICUS

LABRADORICUS

LABRADORI CUS

LABRADORICUS

LABRADORICUS

LABRADORICUS

LABRADORICUS

LABRADORICUS

LABRADORICUS

LABRADORI CUS

LABRADORICUS

LABRADORI CUS

LABRADORI CUS

LABRADORICUS

LABRADORI CUS

C AN AL I CULATA

CANALI CULATA

GIGAS

A RENAR I

ARE NAR I A

A RENAR I A

A RE NAR I A

ARENARIA

A RE NAR I A

A RE NAR I A

ARENAR I A

ARENAR IA

ARE NAR IA
Locality

OFF OREGON

OFF OREGON

GULF OF ALASKA

GULF OF ALASKA

GULF OF ALASK

GULF OF ALASKA

GULF OF ALASKA

GULF OF ALASKA

GULF OF ALASKA

GULF OF ALASKA

GULF OF ALASKA

SUNSET BAY, OREGON

CALIFORNIA COAST

BRITISH COLUMBIA

BRITISH COLUMBIA

GULF OF ALASKA

GULF OF ALASKA

GULF OF ALASKA

GULF OF ALASKA

GULF OF ALASKA

GULF OF ALASKA

GULF OF ALASKA

GULF OF ALASKA

GULF OF ALASKA

GULF OF ALASKA

GULF OF ALASKA

GULF OF ALASKA

GULF OF ALASKA

GULF OF ALASKA

GULF OF ALASKA

EXCURSION, ALASKA

CLARENCE ST.. ALASKA

GULF OF ALASKA

CRESCENT CITY, CALIF.

LAPUSH, WASHINGTON

CALIFORNIA COAST

GULF OF ALASKA

OREGON COAST

OREGON COAST

GULF OF ALASKA

GULF OF ALASKA

GULF OF ALASKA

GULF OF ALASKA

GULF OF ALASKA

GULF OF ALASKA

GULF OF ALASKA

GULF OF ALASKA

GULF OF ALASKA

GULF OF ALASKA

GULF OF ALASKA

GULF OF ALASKA

GULF OF ALASKA

GULF OF ALASKA

GULF OF ALASKA

GULF OF ALASKA

GULF OF ALASKA

GULF OF ALASKA

GULF OF ALASKA

GULF OF ALASKA

CRESCENT CITY, CALIF.

WASHINETON COAST

WASHINGTON COAST

WASHINGTON COAST

GULF OF ALASKA

GULF OF ALASKA

GULF OF ALASKA

GULF OF ALASKA

GULF OF ALASKA

GULF OF ALASKA

GULF OF ALASKA

GULF OF ALASKA

GULF OF ALASKA

GULF OF ALASKA

GULF OF ALASKA

CLARENCE ST.. ALASKA

KASAAN BAY, ALASKA

EXCURSION, ALASKA

GULF OF ALASKA

GULF OF ALASKA

STRAIT OF GEORGIA, B. C.

STRAIT OF GEORGIA, B.

GULF OF ALASKA

KASAAN BAY, ALASKA

EXCURSION, ALASKA

GULF OF ALASKA

KASAAN BAY, ALASKA

TAKU HARBOUR, ALASKA

OFF ALASKA

BRITISH COLUMBIA

OREGON COAST

BRITISH COLUMBIA

BRITISH COLUMBIA

BRITISH COLUMBIA

Lat. Long.

$4315 \mathrm{~N} 12453 \mathrm{~W}$ $4308 \mathrm{~N} 12459 \mathrm{~h}$ $5930 N 14520 \mathrm{~W}$ 


\section{Publication}

11226 BERGEN O'NEIL 1979 1227 BERGEN O'NEIL 1979 11228 BERGEN O'NEIL 1979 11229 BERGEN O NE IL 1979 11230 CUSHMAN TODO 1947 11231 CUSHMAN TODD 1947 11232 COOPER 1961

11233 COOPER 1961

11234 COCKBAIN 1963

11235 COOPER 1961

11236 COCKBAIN 1963
11237 COCKBAIN 1963

11237 COCKBAIN 1963
11238 COCKBAIN 1963

11239 CUS HMAN TODD 1947

11240 ECHOLS 1969

11241 COOPER 1961

11242 SCOTT 1974

11243 ECHOLS 1969

$\begin{array}{lll}11244 & \text { BERGEN O'NEIL } 1979 \\ 11245 & \text { BERGEN O'NEIL } 1979\end{array}$

$\begin{array}{lll}11245 & \text { BERGEN O'NEIL } 1979 \\ 11246 & \text { BERGEN O'NE IL } 1979\end{array}$

$\begin{array}{lll}11246 & \text { BERGEN O'NE IL } & 1979 \\ 11247 & \text { BERGEN O०NE IL } 1979\end{array}$

$\begin{array}{lll}11247 & \text { BERGEN } & \circ \\ 1 & \text { NEIL } & 1979 \\ 1248 & \text { BERGEN } & 0^{\circ} \text { NEIL } 1979\end{array}$

11249 BERGEN O NE IL 1979

11250 BERGEN O'NEIL 1979

11251 TODD LOW 1967

11252 COCKBAIN 1963

11253 COCKBAIN 1963

11254 COCKBAIN 1963

1255 COCKBAIN 1963

11256 BERGEN O NEIL 1979

11257 BERGEN O'NEIL 1979

11258 BERGEN O०NEIL 1979

11259 TODD LOW 1967

11260 TODD LOW 1967

11261 TODD LOW 1967

11262 TODD LOW 1967

11263 TODD LOW 1967

11264 SMITH 1973

11265 BERGEN O'NEIL 1979

11266 COCKBAIN 1963

11267 BERGEN O'NEIL 1979

11268 BERGEN O'NEIL 1979

11269 BERGEN O'NEIL 1979

11270 BERGEN O'NEIL 1979

11271 BERGEN O'NE IL 1979

11272 BERGEN O'NEIL 1979

1273 BERGEN O'NEIL 1979

11274 BERGEN O'NEIL 1979

11275 COCKBAIN 1963

11276 SMITH 1973

11277 SMITH 1973

11278 SMI TH 1973

11279 SMI TH 1973

11280 SMI TH 1973

11281 TODD LOW 1967
11282 CUS HMAN 1910

11282 CUS HMAN 1910
11283 CUS HMAN 1910

11283 CUSHMAN 1910

11285 BERGEN O'NEIL 1979

11286 BERGEN O'NEIL 1979

11287 BERGEN O'NEIL 1979

11288 BERGEN O०NEIL 1979

11289 BERGEN O'NEIL 1979

11290 BERGEN O'NEIL 1979

11291 BERGEN O'NEIL 1979

11292 BERGEN O'NEIL 1979

11293 BERGEN O.NEIL 1979

11294 BERGEN O०NEIL 1979

$\begin{array}{lll}11295 & \text { BERGEN O'NEIL } 1979 \\ 11296 & \text { BERGEN O'NEIL } 1979\end{array}$

$\begin{array}{lll}11296 & \text { BERGEN O'NE IL } 1979 \\ 11297 & \text { BERGEN O'NE IL } 1979\end{array}$

11298 BERGEN O'NEIL 1979

11299 BERGEN O'NEIL 1979

11300 BERGEN O०NEIL 1979

11301 BERGEN O.NEIL 1979

11302 BERGEN O NE IL 1979

11303 SMI TH 1973

11304 BERGEN O.NEIL 1979

11305 WHITEAVES 1886

11306 WHI TEAVES 1886

11307 WHI TEAVES 1886

11308 WHITEAVES 1886

11309 CUS HMAN 1925

11310 CUSHMAN 1925

11311 CUSHMAN MCCULLOCH 1939

11312 CUSHMAN TODD 1947

1313 CUSHMAN TODD 1947

11314 TODD LOW 1967

11315 TODD LOW 1967

11316 SMI TH 1973
11317 SMI TH 1973

11318 CUSHMAN 1910

11319 CUSHMAN MCCULLOCH 1939

11320 DETLING 1958
Generic Name Specific Name

Locality

Lat. Long.

GAUDRYINA

GAUDRYINA

GAUDRYINA

GAUDRY INA

GAUDRYINA

GAUDRYINA

GAUDRYINA

GAUDRYINA

GAUDRY INA

GAUDRY INA

GAUDRYINA

GAUDRYINA

GLABRAIELLA

GLABRATELLA

GLABRATELLA

GLABRATELLA

GLANDULINA

GLOBOBULIMINA

GLOBOBUL IM INA

GLOBOBUL IMINA

GLOBOBUL IMINA

GLOBOBUL IM INA

GLOBOBUL IMINA

GLOBOBUL IM INA

GLOBOBUL IMINA

GLOBOBUL IMINA

GLOBOBUL IMINA

GLOBOBULIMINA

GLOBOBUL IMINA

GLOBOBUL IM INA

GLOBOBUL IMINA

GLOBOBUL IMINA

GLOBOBUL IMINA

GLOBOBUL IMINA

GLOBOBUL IMINA

GLOBOBU IMINA

GLOBOBUL IMINA

GLOBOBUL IMINA

GLOBOBUL IMINA

GLOBOBUL IMINA

GLOBOBUL IMINA

GLOBOBUL IM INA

GLOBOBUL IMINA

GLOBOBUL IMINA

GLOBOBUL IMINA

GLOBOBUL IMINA

GLOBOBUL IMINA

GLOBOTEXTULARIA

GLOMOSPIRA

GLOMOSPIRA

GLOMOSPIRA

GLOMOSPIRA

GOE SELLA

GORDIAMM INA

GORDIAMMINA

GUT TUL INA

GUTTUL INA

GYROIDINA

GYROIDINA

GYROIDINA

GYROIDINA

GYROIOINA

GYROIDINA

GYROIDINA

GYROIDINA

GYROIDINA

GYROIDINA

GYROIDINA

GYROIDINA

GYROIDINA

GYROIDINA

GYROIDINA

GYROIDINA

GYROIDINA

GYROIDINA

HAPLOPHRAGMIUM

HAPLOPHRAGMIUM

HAPL OPHRAGM I UM

HAPLOPHRAGMIUM

HAPLOPHRAGMOIDES

HAPLOPHRAGMOIDES

HAPLOPHRAGMOIDES

HAPLOPHRAGMOIDES

HAPLOPHRAGMOIDES

HAPLOPHRAGMOIDES

HAPLOPHRAGMOIDES

HAPL OPHRAGMOIDES HAPLOPHRAGMOIDES HAPLOPHRAGMOIDES HAPLOPHRAGMOIDES HAPLOPHRAGMOIDES

\section{ARÉNAR I A}

ARENARIA

A RE NAR I A

ARE NAR I A

ARE NARIA

ATLANTI CA

A TLANTICA PACIFICA

SUBGLABRATA

SUBGLABRATA

SUBGLABRATA

SUB GLABRATA

SUBGLABRATA

SUBGLABRATA

CHARLOTTENSIS

LAURIEI

OPERCULARIS

ORNATISSIMA

LAE VIGATA

A UR I CULATA

A UR I CULATA

A UR I CULATA

A UR ICULATA

AUR I CULATA

A UR I CUL ATA

A UR I CULATA

A UR I CULATA

AUR I CULATA

A UR I CULATA

A UR I CULATA

A UR I CULATA

A UR I CULATA

A UR I CULATA

A UR I CULATA

A UR I CULATA

PAC IFICA

PACIFICA

PACIFICA

PACIFICA

PACIFICA

$P A C$ IF I CA

PAC IFICA

PACIFICA

PAC IF I C

ANCEPS

GOROIALIS

GOR DIALIS

GOR DIALIS

GORDIALIS

FLINTII

CHAROIDES

GOR DIALIS

ORI ENTALIS

PROBLEMA

ALTIFORMIS

ALTIFORMIS

ALTI FORMIS

ALT IFORMIS

GEMMA

GEMMA

GEMMA

GEMMA

I 0

10

10

10

10

10
10

LAM ARCKIANA

LAMARCKIANA

ORB I CULARIS

CANARIENSE

CANARIENSE

CANARIENSE

ADVENA

ADVENA

A DVENUM

ADVENUM

A DVENUM

BRA DYI

CANARIENSIS

CANARIENSIS

CANARIENSIS

COL UMB IENSE

COL UMB IENSE
GULF OF ALASKA

GULF OF ALASKA

GULF OF ALASKA

GULF OF ALASKA

WA SHINGTON COAST

OREGON COAST

OREGON COAST

BRITISH COL UMBIA

OREGON COAST

BRITISH COL UMBIA

BRITISH COL UMBIA 


\section{Publication}

11321 CUSHMAN TODD 1947

11322 CUSHMAN TODD 1947

11323 CUSHMAN MCCULLOCH 1939

11324 CUSHMAN MCCULLOCH 1939

11325 CUSHMAN MCCULLOCH 1939

11326 CUSHMAN 1925

11327 CUS HMAN TODD 1947

11328 CUSHMAN TODD 1947

11329 CUSHMAN MCCULLOCH 1939

11330 CUS HMAN MCCULLOCH 1939

11331 COCKBAIN 1963

11332 COCKBAIN 1963

11333 COCKBAIN 1963

11334 COCKBAIN 1963

11335 SMI TH 1973

11336 BERGEN O'NEIL 1979

11337 BERGEN O'NEIL 1979

11338 BERGEN O'NEIL 1979

11339 BERGEN O'NEIL 1979

11340 BERGEN O'NEIL 1979

11341 BERGEN O'NEIL 1979

11342 BERGEN O'NEIL 1979

11343 BERGEN O'NEIL 1979

11345 TODD LOW 1967

11346 TODD LOW 1967

11347 TODD LOW 1967

11348 BERGEN O'NEIL 1979

11349 TODD LOW 1967

11350 CUS HMAN MCCULLOCH 1939

11351 PHLEGER 1967

11352 PHLEGER 1967

11353 PHLEGER 1967

11354 CUS HMAN 1927

11355 CUSHMAN 1927

11356 BERGEN O N NEIL 1979

11357 SMI TH 1973

11358 SMITH 1973

11359 SMITH 1973

11360 SMITH 1973

11362 TODD LOW 1967

11363 BERGEN O'NEIL 1979

$\begin{array}{lll}1363 & \text { BERGEN O NEIL } 1979 \\ 11364 & \text { BERGEN O'NEIL } 1979\end{array}$

11365 BERGEN O.NE IL 1979

11366 BERGEN O'NEIL 1979

11367 BERGEN O०NEIL 1979

11368 SMITH 1973

11369 SMI TH 1973

11370 COCKBAIN 1963

11371 COCKBAIN 1963

11372 COCKBAIN 1963

11373 COCKBAIN 1963

11374 COCKBAIN 1963

11375 SMITH 1973

11376 SMITH 1973

11377 SMITH 1973

11378 SMITH 1973

11379 SMI TH 1973

11380 SMITH 1973

11381 PHLEGER 1967

11382 PHLEGER 1967

11383 PHLEGER 1967

11384 BERGEN O'NEIL 1979

11385 BERGEN O'NEIL 1979

11386 BERGEN O'NEIL 1979

11387 BERGEN O'NEIL 1979

11388 BERGEN O'NEIL 1979

11389 BERGEN O'NEIL 1979

11390 BERGEN O'NEIL 1979

11391 BERGEN O'NEIL 1979

11392 BERGEN O'NEIL 1979

11393 BERGEN O.NEIL 1979

11394 BERGEN O'NEIL 1979

11395 TODD LOW 1967

11396 TODD LOW 1967

11397 BERGEN O'NEIL 1979

11398 BERGEN O'NEIL 1979

11399 BERGEN O'NEIL 1979

11400 BERGEN O'NEIL 1979

11401 BERGEN O'NEIL 1979

11402 BERGEN O'NEIL 1979

11403 BERGEN O'NEIL 1979

11404 CUSHMAN MCCULLOCH 1950

11405 CUS HMAN MCCULLOCH 1950

11406 CUS HMAN TODD 1947

11407 TODD LOW 1967

11408 TODD LOW 1967

11408 TODD LOW 1967,1979

11410 BERGEN O'NE IL 1979

11411 BERGEN O.NEIL 1979

11412 BERGEN O'NEIL 1979

11413 BERGEN O'NEIL 1979

11414 BERGEN O'NEIL 1979

11415 BERGEN O'NEIL 1979
Generic Name

HAPLOPHRAGMOIDES HAPLOPHRAGMOIDES HAPLOPHRAGMOIDES HAPLOPHRAGMOIDES HAPLOPHRAGMOIDES HAPLOPHRAGMOIDES HAPLOPHRAGMOIDES HAPLOPHRAGMOIDES HAPLOPHRAGMOIDES HAPLOPHRAGMOIDES HAPLOPHRAGMOIDES HAPLOPHRAGMOIDES HAPLOPHRAGMOIDES HAPLOPHR AGMOIDES HAPLOPHRAGMOIDES HAPLOPHRAGMOIDES HAPLOPHRAGMOIDES HAPLOPHRAGMOIDES HAPLOPHRAGMOIDES HAPLOPHRAGMOIDES HAPLOPHRAGMOIDES HAPLOPHRAGMOIDES HAPLOPHRAGMOIDES HAPLOPHRAGMOIDES HAPLOPHRAGMOIDES HAPLOPHRAGMOIDES HAPLOPHRAGMOIDES HAPLOPHRAGMOIDES HAPLOPHRAGMOIDES HAPLOPHRAGMOIDES HAPLOPHRAGMOIDES HAPLOPHRAGMOIDES HAPLOPHRAGMOIDES HAPLOPHRAGMOIDES HAPLOPHRAGMOIDES

HOE GLUND INA

HOE GLUND INA

HOE GLUNDINA

HORMOS INA

HYPERAMM INA

HYPERAMM INA

HYPERAMM IN

HYPERAMM INA
HYPERAMM INA

HYPERAMM INA

HYPERAMMINA

HYPERAMMINA

HYPERAMMINA

HYPERAMM INA

INVOLUTINA

I NVOLUTINA

INVOLUTINA

INVOLUTINA

I NVOLUTINA

INVOLUTINA

INVOLUTINA

JACULELLA

JACULELLA

JA CULELLA

JACULELLA

JADAMMINA

J A D AMM I NA

JADAMMINA

KARRER IELLA

KARRER IELLA

KARRERI ELLA

KARRERIELLA

KARRERIELLA

KARRERIELLA

KARRERIELLA

KARRERIELLA

KARRERIELLA

KARRERIELLA

KARRERIELLA

KARRERIELLA

KARRER I ELLA

KARRER I ELLA

KARRERIELLA

KARRERIELLA

KARRERIELLA

KARRERIELLA

KARRERIELLA

KARRERIELLA

LAGENA

LAGENA

LAGENA

LAGENA

LAGENA

LAGENA

LAGENA

LAGENA

LAGENA

LAGENA

LAGENA

LAGENA

\section{Specific Name}

COL UMB IENSE

COLUMBIENSE

C OL UMB I ENSE

C OL UMBIENSE

COLUMBIENSE EVOLUTUM

GLOMERATUM

HAN COCKI

HANCOCKI

NEOBRADYI

NEOBRADYI

NEOBRADYI

NEOBRADYI

NIT I DA

PARKERAE

PARKERAE

PARKERAE

PARKERAE

PARKERAE

PARKERAE

PARKERAE

PARKERAE

PARKERAE

PLANISSIMUS

PLANISSIMUS

PLANISSIMUS
PLANISSIMUS

SPHAERI LOCULUS

SUBGLOB OSUM

SUB INVOLUTUM

SUB INVOLUTUM

SUB I NVOLUTUS

TENUIS

TENUIS

ELEGANS

ELE GANS

ELEGANS

GLOBUL IFERA

CYLINDRICA

CYL INDRICA

ELONGATA

ELONGATA

ELONGATA

ELONGATA

ELONGATA

ELONGATA

FRIABILIS

FRIABILIS

GULLMARENSIS

GULLMARENS IS

GULLMARENSIS

GULLMARENSIS

GULLMARENS IS

TENUIS

TENUIS

ACUTA

ACUTA

ACUTA

ACUTA

POLYSTOMA

POL YSTOMA

POLYSTOMA

BAC CATA

BAC CATA

BAC CATA

BAC CATA

BAC CATA

BAC CATA

BAC CATA

BAC CATA

BAC CATA

BAC CATA

BAC CATA

BAC CATA

BAC CATA

PARKERAE

PARKERAE

PARKERAE

PARKERAE

PARKERAE

PARKERAE

PARKERAE

ACUTICOSTA

A CUTICOSTA

A CUTICOSTA

AMP HORA

AMP HORA

API OPLEURA

API OPLEURA

API OPLEURA

API OPLEURA

API OPLEURA

API OPLEURA

Locality

WASHINGTON COAST

WASHINGTON COAST

KETCHIKAN, ALASKA

OFF BRIT. COLUMBIA

OFF ALASKA 


\section{Publication}

11416 CUSHMAN TODD 1947 11417 CUSHMAN TODD 1947 11418 CUS HMAN TODD 1947

11419 CUSHMAN MCCULLOCH 1950

11420 COCKBAIN 1963

11421 COCKBAIN 1963

11422 COCKBAIN 1963

11424 COCKBAIN 1963

11425 TODD LOW 1967

11426 TODD LOW 1967

11427 TODD LOW 1967

11428 CUSHMAN MCCULLOCH 1950

11429 CUSHMAN MCCULLOCH 1950

11430 TODD LOW 1967

11431 TODD LOW 1967

11432 BERGEN O'NEIL 1979

11433 BERGEN O'NEIL 1979

11434 COCKBAIN 1963

11435 CUSHMAN 1927

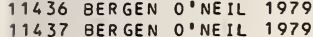

$\begin{array}{lll}11437 & \text { BER GEN } & \circ \\ 114 & \text { NEIL } & 1979 \\ 11438 & \text { BERGEN O'NEIL } 1979\end{array}$

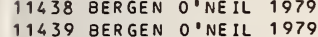

$\begin{array}{lll}11439 & \text { BERGEN O'NEIL } & 1979 \\ 11440 & \text { BERGEN } & \text { ' NEIL } 1979\end{array}$

11441 BERGEN O'NEIL 1979

11442 BERGEN O'NEIL 1979

$\begin{array}{lll}11443 & \text { BERGEN O'NEIL } & 1979 \\ 11444 & \text { BERGEN O'NEIL } 1979\end{array}$

11445 CUS HMAN TODD 1947

11446 CUSHMAN TODD 1947

11447 COCKBAIN 1963

11448 CUS HMAN 1927

11449 CUS HMAN 1927

11450 COCKBAIN 1963

11451 BERGEN O NEIL 1979

11452 BERGEN O'NEIL 1979

11453 BERGEN O'NEIL 1979

11454 BERGEN O'NEIL 1979

11455 BERGEN O'NEIL 1979

11456 CUSHMAN 1927

11457 CUS HMAN 1927

11458 TODD LOW 1967

11459 TODD LOW 1967

11460 BERGEN O.NEIL 1979

11461 BERGEN O'NEIL 1979

11462 BERGEN O'NEIL 1979

11463 BERGEN O०NEIL 1979

11464 BERGEN O N NEIL 1979

11465 CUSHMAN MCCULLOCH 1950

11466 CUS HMAN 1927

11467 BERGEN O'NEIL 1979

11468 BERGEN O'NEIL 1979

11469 BERGEN ONNEIL 1979

11470 TODD LOW 1967

11471 TODD LOW 1967

11472 BERGEN O'NEIL 1979

11473 BERGEN O'NEIL 1979

11474 CUSHMAN 1925

11475 BERGEN O'NEIL 1979

11476 BERGEN O'NEIL 1979

11477 BERGEN O'NEIL 1979
11478 CUSHMAN TODD 1947

11478 CUS HMAN TODD 1947
11479 CUSHMAN MCCULLOCH 1950

11480 CUSHMAN MCCULLOCH 1950

11481 CUS HMAN TODD 1947

11482 CUSHMAN TODD 1947

11483 CUS HMAN TODD 1947

11484 COCKBAIN 1963

11485 COCKBAIN 1963

11486 COCKBAIN 1963

11487 BERGEN O'NEIL 1979

11488 TODD LOW 1967

11489 CUSHMAN MCCULLOCH 1950

11490 BERGEN O NNEIL 1979

11491 BERGEN O.NEIL 1979

11492 BERGEN OONEIL 1979

11493 BERGEN O'NEIL 1979

11494 BERGEN O'NEIL 1979

11495 CUS HMAN MCCULLOCH 1950

11496 BERGEN O०NEIL 1979

11497 BERGEN O'NEIL 1979

11498 BERGEN O NEIL 1979

11499 BERGEN O०NEIL 1979

11500 BERGEN O०NEIL 1979

11501 BERGEN O'NEIL 1979

11502 BERGEN O'NEIL 1979

11503 BERGEN O'NEIL 1979

11504 BERGEN O.NEIL 1970

11505 LANKF ORD PHLEGER 1973

11506 TODD LOW 1967

11507 TODD LOW 1967

11508 CUSHMAN MCCULLOCH 1950

11509 BERGEN O'NEIL 1979
11510 BERGEN O.NEIL 1979
Generic Name Specific Name

CLAVATA

COSTATA

COSTATA

DENTALINI FORMIS

DIS TOMA

DIS TOMA

DIS TOMA

D IS TOMA

DIS TOMA

DIS TOMA

DIS TOMA

DIS TOMA

DIS TOMA

ELONGATA

ELONGATA

ELONGATA

ELONGAT

ELONGATA

ELONGATA

ELONGATA

ELONGATA

ELONGATA

ELONGATA

ELONGATA

ELONGATA

ELONGATA

ELONGATA

GRACILIS

GRACILIS

GRACILIS

GRACILIS

GRACILIS

GRACILIS

GRACILIS

GRACILIS

GRACILIS

GRACILIS

GRACIL IS

GRACILLIMA

GRA CILLIMA
HISPIDULA

LAE VIS

LAE VIS

LAE VIS

LAE VIS

LAE VIS

LAEVIS

LAEVIS BAGGI

LAMELLATA

MER IDIONALIS

MER IDIONALIS

MER IDIONALIS

MER I DIONALIS

MER I D IONALIS

MOLLIS

NEBULOSA

ORB I GNYANA

PARR

PAR R

PAR R I

PERLUCIDA

PERLUCIDA

PERLUCIDA

PERLUCIDA

PLIOCENICA

PLI OCENICA

PLI OCENICA

PLIOCENICA

PLIOCENICA

PLIOCENICA

PLIOCENICA

PLIOCENICA DISCREPANS

SEM I LINEATA

SEM ILINEATA

SEMILINEATA

SEM IL INEATA

SEM ILINEATA

SEM ILINEATA

SEM ILINEATA

SEM ILINEATA

STR IATA

STR I ATA

STR I ATA

STR IATA

STR IATA

STRIATA

STR IATA

STR IATA

STRIATA

STR IATA

STR IATA

STR IATA

STR IATA
Locality

Lat. Long.

WASHINGTON COAST WASHINGTON COAST WASHINGTON COAST OFF ALASKA

BRITISH COLUMBIA BRITISH COLUMBIA BRITISH COLUMBIA BRITISH COL UMBIA BR ITISH COLUMBIA

CLARENCE ST,. ALASKA

KASAAN BAY, ALASKA

GULF OF ALASKA

OFF ALASKA

OFF ALASKA

CLARENCE ST... ALASKA

GULF OF ALASKA

GULF OF ALASKA

BR IT ISH COL UMBIA

OFF OREGON

GULF OF ALASKA

GULF OF ALASKA

GULF OF ALASKA

GULF OF ALASKA

GULF OF ALASKA

GULF OF ALASKA

GULF OF ALASKA

GULF OF ALASKA

WASH INGTON COAST

WASHINGTON COAST

BR IT ISH COLUMBIA

OFF OREGON

OFF OREGON

BRITISH COL UMBIA

GULF OF ALASKA

GULF OF ALASKA

GULF OF ALASKA

GULF OF ALASKA

GULF OF ALA

OFF OREGON

GULF OF ALASKA

GULF OF ALASKA

GULF OF ALASKA

GULF OF ALASKA

GULF OF ALASKA

GULF OF ALASKA

GULF OF ALASKA

OFF ALASKA

OFF OREGON

GULF OF ALASKA

GULF OF ALASKA

GULF OF ALASKA

KASAAN BAY, ALASKA

GULF OF ALASKA

GULF OF ALASKA

GULF OF ALASKA

GULF OF ALASKA

GULF OF ALASKA

GULF OF ALASKA

WASHINGTON COAST

OFF ALASKA

OFF ALASKA

WASHINGTON COAST

WASHINGTON COAST

WASHINGTON COAST

BR IT ISH COL UMBIA

BRITISH COLUMBIA

BRITISH COLUMBIA

GULF OF ALASKA

GULF OF ALASKA

KETCHIKAN, ALASKA

GULF OF ALASKA

GULF OF ALASKA

GULF OF ALASKA

GULF OF ALASKA

GULF OF ALASKA

OFF ALASKA

GULF OF ALASKA

GULF OF ALASKA

GULF OF ALASKA

GULF OF ALASKA

GULF OF ALASKA

GULF OF ALASKA

GULF OF ALASKA

GULF OF ALASKA

CRESCENT CITY, CALIF.

KASAAN BAY, ALASKA

GULF OF ALASKA

OFF ALASKA

GULF CF ALASKA

GULF OF ALASKA

$4832 \mathrm{~N} 12310 \mathrm{~W}$ $4833 \mathrm{~N} 12300 \mathrm{~W}$ $5628 \mathrm{~N} 13223 \mathrm{~W}$ $4820 \mathrm{~N} 12300 \mathrm{~W}$ $4820 \mathrm{~N} 12300 \mathrm{~W}$ $4814 \mathrm{~N} 12330 \mathrm{~W}$ $4900 N 12315 \mathrm{~W}$ $4845 \mathrm{~N} 12300 \mathrm{~W}$ $5521 \mathrm{~N} 13158 \mathrm{~W}$ $5526 \mathrm{~N} 13214 \mathrm{~W}$ $5932 \mathrm{~N} 14236 \mathrm{~W}$ 


\section{Publication}

11511 BERGEN O'NEIL 1979

11512 BERGEN O'NEIL 1979

11513 CUSHMAN TODD 1947

11514 CUS HMAN MCCULLOCH 1950

11515 CUSHMAN MCCULLOCH 1950

11516 CUSHMAN 1927

11517 CUS HMAN 1927

11518 CUS HMAN 1927

11519 CUSHMAN 1927

11520 COCKBAIN 1963

11521 COCKBAIN 1963

11522 WHITEAVES 1886

11523 CUSHMAN TODD 1947

11524 CUSHMAN MCCULLOCH 1950

11525 BERGEN O'NEIL 1979

11526 BERGEN O'NEIL 1979

11527 BERGEN O.NEIL 1979

11528 BERGEN O'NEIL 1979

11529 CUSHMAN MCCULLOCH 1950

11530 CUS HMAN MCCULLOCH 1950

11531 LANKFORD PHLEGER 1973

11532 LANKF ORD PHLEGER 1973

11533 TODD LOW 1967

11534 BERGEN O'NEIL 1979

11535 TODD LOW 1967

11536 COCKBAIN 1963

11537 BERGEN O'NEIL 1979

11538 BERGEN O'NEIL 1979

11539 BERGEN O NEIL 1979

11540 BERGEN O'NEIL 1979

11541 BERGEN O'NEIL 1979

11542 BERGEN O'NEIL 1979

11543 BERGEN O'NEIL 1979

11544 BERGEN O'NEIL 1979

11545 BERGEN O'NEIL 1979

11546 SMI TH 1973

11547 CUSHMAN TODD 1947

11548 TODD LOW 1967

11549 BERGEN O'NEIL 1979

11550 BERGEN O.NEIL 1979

11551 HAMLIN 1960

11552 HAMLIN 1960

11553 LANKFORD PHLEGER 1973

11554 CUSHMAN TODD 1947

11555 CUSHMAN TODD 1947

11556 SMI TH 1973

11557 BERGEN O'NEIL 1979

11558 PHLEGER 1967

11559 JONES ROSS 1979

11560 SCOTT 1974

11561 PHLEGER 1967

11562 PHLEGER 1967

11563 PHLEGER 1967

11564 CUSHMAN TODD 1947

11565 CUSHMAN TODD 1947

11566 WHI TEAVES 1886

11567 WHI TEAVES 1886

11568 WHI TEAVES 1886

11569 WHITEAVES 1886

11570 LANKFORD PHLEGER 1973

11571 COOPER 1961

11572 COOPER 1961

11573 SMITH 1973

11574 SMI TH 1973

11575 SMITH 1973

11576 SMITH 1973

11576 SMITH 1973

11578 BERGEN O NEIL 1979

11579 WHITEAVES 1886

11580 WHITEAVES 1886

11581 CUS HMAN TODD 1947

11582 CUSHMAN TODD 1947

11583 CUSHMAN TODD 1947

11584 CUSHMAN MCCULLOCH 1950

11585 CUSHMAN MCCULLOCH 1940

11586 DETLING 1958

11587 CUSHMAN 1927

11588 CUS HMAN 1927

11589 CUSHMAN 1927

11590 CUSHMAN 1927

11591 CUSHMAN 1927

11592 CUS HMAN 1927

11593 CUSHMAN 1927

11594 SMITH 1973

11595 SMITH 1973

11596 CUS HMAN MCCULLOCH 1940

11597 CUSHMAN TODD 1947

11598 CUSHMAN TODD 1947

11599 CUSHMAN TODD 1947

11600 CUSHMAN 1927

11601 CUSHMAN 1927

11602 CUSHMAN 1927

11603 CUSHMAN 1927

11604 NATLAND 1933

11605 SMITH 1973

\section{Generic Name Specific Name}

STR IATA

STR IATO-PUNCTATA COMPLEXA

STR IATOPUNCT ATA

STRIATOPUNCTATA GEMMA

SUBSTRIATA

SUBSTRIATA

SUBSTRIATA

SUBSTRIATA

SULCATA

SUL CATA

SUL CATA APICULATA

SULCATA PECULIARIS

SULCATA SPICATA

SULCATA SPICATA

SULCATA SPICATA

SULCATA SPICATA

SULCATA SPICATA

SUL CATA SPI

A TL ANTI CA

SCALARIS

SCALARIS

SCALARIS

SCALARIS

SCALARIS

HYALASCIDIA

NIC OBARENS IS

PORRECTA

PSE UDOBEYRICHI

PSE UDOBEYRICHI

PSEUDOBEYRICHI

PSEUDOBEYRICHI

PSE UDOBEYRICH

AMY GDAL I F ORM IS

I NS TAB I LE

GLABRA

OCC I DENTALIS

OCC I DENTAL IS

CRE SCENTENSIS

CRE SCENTENSIS

PUL CHRA

PUL CHRA

PUL CHRA

AFF INE

POMPILIOIDES

FUSCA

FUS CA

FUSCA

FUS CA

FUS CA

FUS CA

FUS CA

F US CA

FERUSSACHII

SEM INULUM

SEM INUL UM

SEM I NUL UM

C AL I FORNI CA

CIR CULARIS

CIRCULARIS

SUBROTUNDA

SUBROTUNDA

SUBROTUNDA

SUBROTUNDA

MEMBRANACEUM

CAL OMORPHA

PAUPERATA

PAUPERATA

SCALARIS

SCALAR IS

SCALAR IS

SUBSCALARIS

GRATELOUPI

GRA TELOUPII

LABRADORICA

LABRADORICA

LABRADORICA

$\angle A B R A D O R I C A$

LABRADORICA

$\angle A B R A D O R I C A$

LABRADORICA

LABRADORI CUM

LABRADORI CUM

PIZ ARRENSE BASISPINATUM

PIZARRENSE BASISPINATUM

PIZARRENSE BASISPINATUM
PIZARRENSE BASISPINATUM

PIZ ARRENSE BASISPINATUM

PUNCTATA

PUNCTATA

PUNCTATA

SCAPHA

SCAPHA

SCAPHUM

\section{Locality}

GULF OF ALASKA

GULF OF ALASKA

WASHING TON COAST

OFF ALASKA

OFF ALASKA

OFF OREGON
OFF OREGON

OFF OREGON

OFF OREGON

BR ITISH COL UMBIA

BRITISH COLUMBIA

DISCOVERY PASSAGE, B. C.

WASHINGTON COAST

OFF ALASKA

GULF OF ALASKA

GULF OF ALASKA

GULF OF ALASKA

GULF OF ALASKA

KETCHIKAN, ALASKA

OFF ALASKA

DEPOE BAY, OREGON

CRESCENT CITY, CALIF.

TAKU HARBOUR, ALASKA

GULF OF ALASKA

GULF OF ALASKA

BRITISH COLUMBIA

GULF OF ALASKA

GULF OF ALASKA

GULF OF ALASKA

GULF OF ALASKA

GULF OF ALASKA

GULF OF ALASKA

GULF OF ALASKA

GULF OF ALASKA

GULF OF ALASKA

OFF ALASKA PENINSULA

WASHINGTON COAST

GULF OF ALASKA

GULF OF ALASKA

GULF OF ALASKA

CRESCENT CITY, CALIF.

PORT ORFORD, OREGON

CRESCENT CITY, CALIF.

WASHINGTON COAST

WASHINGTON COAST

OFF ALASKA PENINSULA

GULF OF ALASKA

GRAY'S HARBOUR, WASH.

SAMISH BAY, WASH.

SAMISH-PADILLA BAYS

COOS, BAY, OREGON

PHILLIPS ARM, B.C

FRASER R .. B.C.

WASHINGTON COAST

WASHINGTON COAST

QUEEN CHARLOTTE SD.. B.C.

DISCOVERY PASSAGE, B. C.

QUEEN CHARLOTTE SD.. B.C.

STRAIT OF GEORGIA, B. C

CRESCENT CITY, CALIF.

OREGON COAST

OREGON COAST

OFF ALASKA PENINSULA

OFF ALASKA PENINSULA

OFF ALASKA PENINSULA

NORTH PACIF IC

OFF ALASKA PENINSULA

GULF OF ALASKA

STRAIT OF GEORGIA, B. C.

STRAIT OF GEORGIA. B. C.

WASHINGTON COAST

WASHINGTON COAST

WASHINGTON COAST

OFF ALASKA

OFF ALASKA

SUNSET BAY, OREGON

OFF OREGON

OFF OREGON

OFF OREGON

OFF OREGON

OFF OREGON

OFF OREGON

OFF OREGON

KODIAK. ALASKA

OFF ALASKA PENINSULA

OFF ALASKA

WASHINGTON COAST

WASHINGTON COAST

WASHINGTON COAST

OFF OREGON

OFF OREGON

OFF OREGON

OFF OREGON

CRESCENT CITY, CALIF.

OFF ALASKA PENINSULA

Lat. Long.

$5955 N 14540 \mathrm{~W}$ $4955 N 14225 W$ 


\section{Publication}

11606 CUSHMAN TODD 1947

11607 SMITH 1973

11608 CUS HMAN TODD 1947

11609 COCKBAIN 1963

11610 COOPER 1961
11611 COCKBAIN 1963

11611 COCKBAIN 1963
11612 COCKBAIN 1963

11613 SMI TH 1973

11614 BERGEN O'NEIL 1979

11615 BERGEN O'NE IL 1979

11616 BERGEN O०NEIL 1979

11617 BERGEN O'NEIL 1979

11618 BERGEN O'NE IL 1979

11619 BERGEN O'NE IL 1979

11620 BERGEN O.NEIL 1979

$\begin{array}{lll}11621 & \text { BERGEN O'NE IL } & 1979 \\ 11622 & \text { BER GEN } & \text { O NEIL } 1979\end{array}$

$\begin{array}{lll}11622 & \text { BERGEN O०NEIL } 1979 \\ 11623 & \text { BERGEN } & 0^{\circ} \text { NEIL } 1979\end{array}$

11624 BERGEN O.NEIL 1979

11625 COCKBAIN 1963

11626 COCKBAIN 1963

11627 COCKBAIN 1963

11628 COCKBAIN 1963

11629 CUSHMAN 1927

11630 CUSHMAN MCCULLOCH 1940

11631 CUSHMAN MCCULLOCH 1940

11632 CUS HMAN MCCULLOCH 1940

11633 CUSHMAN MCCULLOCH 1940

11634 CUSHMAN TODD 1947

11635 COCKBAIN 1963

11636 COCKBAIN 1963

11637 COCKBAIN 1963

11638 COCKBAIN 1963

11639 ECHOLS 1969
11640 DETLING 1958

11640 DETLING 1958

11641 ECHOLS 1969
11642 ECHOLS 1969

11643 BERGEN O'NEIL 1979

11644 TOD D LOW 1967

11645 TODD LOW 1967

11646 TODD LOW 1967

11647 BERGEN O.NEIL 1979

11648 BERGEN O.NEIL 1979

11649 BERGEN O'NEIL 1979

11650 BERGEN O'NEIL 1979

11651 BERGEN O NEIL 1979

11652 BERGEN O'NEIL 1979

11653 BERGEN O०NEIL 1979

11654 BERGEN O.NEIL 1979

11655 BERGEN O.NE IL 1979

11656 BERGEN O'NEIL 1979

11657 BERGEN O 'NEIL 1979

11658 LANKFORD PHLEG
11659 TODD LOW 1967

11660 SMITH 1973

11661 TODD LOW 1967

11662 COCKBAIN 1963

11663 COCKBAIN 1963

11664 TOD D LOW 1967

11665 TODD LOW 1967

11666 CUSHMAN 1925
11667 CUSHMAN 1925

11668 WHITEAVES 1886

11669 WHI TEAVES 1886

11670 LANKFORD PHLEGER 1973

11671 CUSHMAN TODD 1947

11672 SMI TH 1973

11673 TODD LOW 1967

11674 TODD LOW 1967

11675 TODD LOW 1967

11676 TODD LOW 1967

11677 COCKBAIN 1963

11678 COCKBAIN 1963

11679 COCKBAIN 1963

11680 COCKBAIN 1963

11681 BERGEN O'NEIL 1979

11682 COCKBAIN 1963

11683 TODD LOW 1967

11684 BERGEN O'NEIL 1979

11685 BERGEN O'NEIL 1979

$\begin{array}{lll}11686 & \text { BERGEN O'NEIL } 1979 \\ 11687 & \text { BERGEN O'NEII } 1979\end{array}$

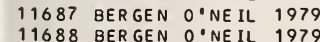

11688 BERGEN O'NEIL 197

11690 BERGEN O ONE IL 1979

11691 BERGEN OONEIL 1979

11692 BERGEN O'NEIL 1979

11693 BERGEN O'NEIL 1979

11694 BERGEN O'NEIL 1979

11695 BERGEN O'NEIL 1979

1696 BERGEN O'NE IL 197

11697 BERGEN O'NEIL 1979

11698 TODD LOW 1967

11699 BERGEN O'NE IL 1979

11700 BERGEN O.NEIL 1979
Generic Name Specific Name

A UR I CULA

AUR I CULA

AUR I CULA

BAS I SPINATA

AS I SP INATA

BAS ISPINATA

BRA DYI

DIGITATA

DIGITATA

DIG ITATA

DIG ITATA

D IG ITATA

DIG ITATA

DIG ITATA

DIG ITATA

DIG ITATA

DIG ITATA

DIG ITATA

LABRADORICA

$\angle A B R A D O R I C A$

$\angle A B R A D O R I C A$

$\angle A B R A D O R I C A$

MIO CEN I CA

MIOCEN I CA

MIOCENICA STELLA

MIOCENICA STELLA

MIOCENICA STELLA

MIOCENICA STELLA

MIOCENICA STELLA

MIOCENICA STELLA

MIOCENICA STELLA

MIOCENICA STELLA

MIOCENICA STELLA

MIOCENICA STELLA

MIOCENICA STELLA

MIOCENICA

PUL CHELLA

PULCHELLA

PUL CHELLA

PUL CHELLA

PUL CHELLA

PUL CHELLA

PUL CHELLA

PUL CHELLA

PULCHELLA

PULCHELLA

PULCHELLA

PULCHELLA

PUL CHELLA

S TE LLA

TUR GIDA

TUR GI DA

TUR GIDA

TUR GIDA DIGITATA

TURGIDA DIGITATA

TURGIDA DIGITATA

TURGIDA DIGITATA

AUR IS

A UR IS

SCAPHA

SCAPHA

POL YMORPH INO IDES

BRA DYI

LINEAR IS

API OPLEURA

APIOPLEURA

API OPLEURA

API OPLEURA

API OPLEURA

API OPLEURA

API OPLEURA

$B O R E A L I S$

BOREALIS

BOREALIS

BOREALIS

COSTATA

COSTATA

COSTATA

COSTATA

COS TATA

COSTATA

COSTATA

COS TATA

COSTATA

COS TATA

GLOBOSA

GLOBOSA

HEX AGONA

HEXAGONA

HEX AGONA

HEX AGONA

HEX AGONA
Locality

Lat. Long.

WASHINGTON COAST

OFF ALASKA PENINSULA

WASHINGTON COAST

BRITISH COL UMBIA

OREGON COAST

BR ITISH COLUMBIA

BR IT ISH COLUMBIA

OF F ALASKA PENINSULA

GULF OF ALASKA

GULF OF ALASKA

GULF OF ALASKA

GULF OF ALASKA

GULF OF ALASKA

GULF. OF ALASKA

GULF OF ALASKA

GULF OF ALASKA

GULF OF ALASKA

BRITISH COLUMBIA

BR ITISH COLUMBIA

BRITISH COLU

BRITISH COL UMBIA

OFF OREGON

OFF ALASKA

OFF ALASKA

KETCHIKAN, ALASKA

OFF ALASKA

WASHINGTON COAST

BRITISH COL UMBIA

BRITISH COL UMBIA

BR IT ISH COLUMBIA

BRITISH COLUMBIA

WASHINGTON COAST

SUNSET BAY, OREGON

WASHINGTON COAST

WA SH INGT ON COAST

CLARENCE ST.. ALASKA

GULF OF ALASKA

KASAAN BAY, ALASKA

GULF OF ALASKA

GULF OF ALASKA

GULF OF ALASKA

GULF OF ALASKA

GULF OF ALASKA

GULF OF ALASKA

GULF OF ALASKA

GULF OF ALASKA

GULF OF ALASKA

GULF OF ALASKA

CRESCENT CITY, CALIF.

GULF OF ALASKA

OFF ALASKA PENINSULA

BRITISH COL UMBIA

BRITISH COLUMBIA

GULF OF ALASKA

CLARENCE ST.. ALASKA

VIRAGO SD.. B.C.

Q. CHARL OTTE SD.. B.C.

JOHNSTONE STRAIT, B.C.

CRESCENT CITY, CALIF.

WASHINGTON COAST

OFF ALASKA PENINSULA

GULF OF ALASKA

KASAAN BAY, ALASKA

EXCURSION, ALASKA

TAKU HARBOUR, ALASKA

BR ITISH COLUMBIA

BR IT ISH COLUMB I A

BR ITISH COL UMBIA

BRITISH COLUMBIA

GULF OF ALASKA

BR ITISH COL UMBIA

EXCURSION, ALASKA

GULF OF ALASKA

GULF OF ALASKA

GULF OF ALASKA

GULF OF ALASKA

GULF OF ALASKA

GULF OF ALASKA

GULF OF ALASKA

GULF OF ALASKA

GULF OF ALASKA

GULF OF ALASKA

GULF OF ALASKA

GULF OF ALASKA

GULF OF ALASKA

GULF OF ALASKA

GULF OF ALASKA

GULF OF ALASKA

$4833 \mathrm{~N} 12300 \mathrm{~W}$

$5623 N 15427 W$

$4845 N 12300 W$

$4307 \mathrm{~N} 12426 \mathrm{~W}$

$4814 \mathrm{~N} 12330 \mathrm{~W}$

$4820 \mathrm{~N} 12300 \mathrm{~W}$

$5623 N 15427 W$

$5945 \mathrm{~N} 14455 \mathrm{~W}$

$5930 \mathrm{~N} 14255 \mathrm{~W}$

$5930 \mathrm{~N} 14520 \mathrm{~W}$

$5930 \mathrm{~N} 14300 \mathrm{~W}$

59 OON $14120 \mathrm{~W}$

$5920 \mathrm{~N} 14538 \mathrm{~W}$

$\begin{array}{llll}56 & 35 N & 151 & 50 W \\ 59 & 35 N & 143 & 00 W\end{array}$

$5942 \mathrm{~N} 14410 \mathrm{~W}$

$5930 \mathrm{~N} 14540 \mathrm{~W}$ 


\section{Publication}

11701 TODD LOW 1967

11702 BERGEN O'NEIL 1979

11703 TODD LOW 1967

11704 BERGEN O'NEIL 1979

11705 TODD LOW 1967

11706 LANKFORD PHLEGER 1973

11707 TODD LOW 1967

11708 DETLING 1958

11709 BERGEN O NEIL
11710 COCKBAIN 1963

11711 COCKBAIN 1963

11712 COCKBAIN 1963

11713 COCKBAIN 1963

11714 COCKBAIN 1963

11715 BERGEN O'NEIL 1979

11716 BERGEN O.NEIL 1979

11717 BERGEN O'NEIL 1979

11718 TODD LOW 1967

11719 BERGEN O.NEIL 1979

11720 BERGEN O'NEIL 1979

11721 BERGEN O'NEIL 1979

11722 TODD LOW 1967

11723 SMITH 1973

11724 BERGEN O'NEIL 1979

11725 LANKFORD PHLEGER 1973

11726 CUSHMAN TODD 1947

11727 COCKBAIN 1963

11728 COCKBAIN 1963

11729 BERGEN O NEIL 1979

11730 PALMER 1929

11731 BERGEN O'NEIL 1979

11732 DETLING 1958

11733 TODD LOW 1967

11734 TODD LOW 1967

11735 BERGEN OONEIL 1979

11736 BERGEN O'NEIL 1979

11737 SMI TH 1973

11738 SMITH 1973

11739 DETLING 1958

11740 COOPER 1961

11742 BERGEN O'NEIL 1979

11743 BERGEN O'NEIL 1979

11744 CUSHMAN 1925

11745 TODD LOW 1967

11746 TODD LOW 1967

11747 TODD LOW 1967

11748 BERGEN O'NEIL 1979

11749 CUS HMAN TODD 1947

11750 WHI TEAVES 1886

11751 WHITEAVES 1886

11752 WHITEAVES 1886

11753 WHITEAVES 1886

11754 BERGEN O.NEIL 1979

11755 BERGEN O'NEIL 1979

11756 COCKBAIN 1963

11757 COCKBAIN 1963

11758 BERGEN O'NEIL 1979

11759 CUSHMAN TODD 1947

11760 CUSHMAN TODD 1947

11761 TODD LOW 1967

11762 WHITEAVES 1886

11763 WHITEAVES 1886

11764 WHITEAVES 1886

11765 WHITEAVES 1886

19766 WHITEAVES 1886

11767 WHITEAVES 1886

11768 COCKBAIN 1963

11769 COOPER 1961

11770 COCKBAIN 1963

11771 COCKBAIN 1963

11772 DETLING 1958

11773 CUSHMAN MCCULLOCH 1939

11774 CUSHMAN TODD 1947

11775 CUSHMAN TODD 1947

11776 CUSHMAN 1927

11777 CUSHMAN 1927

11778 CUS HMAN 1927

11779 CUSHMAN 1927

11780 CUSHMAN TODD 1947

11781 CUSHMAN MCCULLOCH 1939

11782 PHLEGER 1967

11783 PHLEGER 1967

11784 PHLEGER 1967

11785 COCKBAIN 1963

11786 TODD LOW 1967

11787 CUS HMAN TODD 1947

11788 CUS HMAN TODD 1947

11789 TODD LOW 1967

11790 COCKBAIN 1963

11791 COCKBAIN 1963

11792 COCKBAIN 1963

11793 COCKBAIN 1963

11794 COCKBAIN 1963

11795 BERGEN O'NEIL 1979
Generic Name

OOL INA

OOL INA

OOL INA

OOL INA

OOL INA

OOL INA

OOL INA

OOLINA

OOL INA

OOL INA

OOL INA

OOLINA

OOL INA

OOL INA

OOL INA

OOL INA

OOL INA

OOL INA

OOLINA

OPHTHALMIDIUM

PARAFISSURINA

PATELLINA

PATELLINA

PATELLINA

PATELLINA

PATELLINA

PATELLINA

PATELLINA

PATEORIS

PELOSINA

PELOS INA

PELOSINELLA

PELOSINELLA

PLACOPSILINA

PLACOPSILINA

PLANORBULINA

PLANORBULINA

PLANULINA

PLANULINA

PLANULINA

POLYMORPHINA

POLYMORPHINA

POLYMORPHINA

POLYMORPHINA

POL YMORPHINA

POLYMORPHINA

POLYMORPHINA

POLYMORPHINA

POL YMORPHINA

POLYMORPHINA

POLYMORPHINA

POLYMORPHINA

POLYMORPHINA

POLYMORPHINA

POL YMORPHINA

POLYMORPHINA

POLYMORPHINA

POL YMORPHINA

POLYMORPHINA

POLYSTOMELLA

POLYSTOMELLA

POLYSTOMELLA

POLYSTOMELLA

POLYSTOMELLA

POROEPONIDES

POR OEPONIDES

POROEPONIDES

POROEPONIDES

POROEPONIDES

PROTEONINA

PROTEONINA

PROTEONINA

PROTEONINA

PROTEONINA

PROTEONINA

PROTEONINA

PROTEONINA

PROTEONINA

PROTOSCHISTA

PROTOSCH ISTA

PROTOSCHISTA

PSAMMATODENDRON

PSAMMA TODENDRON

PSAMMATODENDRON

PSAMMATODENDRON

PSAMMA TODENDRON

PSAMMATODENDRON

PSAMMATODENDRON

PSAMMAT ODENDRON

PSAMMATODENDRON

PS AMMATODENDRON

PSAMMATODENDRON
Specific Name

LAE VIGATA

LAE VIGATA

LINEATA

INEATOPUNCTATA

MELO

MELO

MELO

MELO

MELO

MELO

MELO

MELO

MELO

STR IATOPUNCTATA

STR IATOPUNCTATA

STR IATOPUNC TATA

STR IATOPUNCTATA

STR IATOPUNCTATA

WILLIAMSON

FUS ILI FORMIS

CORRUGATA

C CRRUGATA

C ORRUGATA

C ORRUGATA

CORRUGATA

CORRUGATA

C CRRUGATA

HAUERINOIDES

VAR I ABILIS

VAR IAB ILIS

DIDERA

DIDERA

BRADYI

BRADYI

A CERVALIS

MED I TERRANEN SIS

ORNATA

WUELLERS TORF I

WUELLERSTORFI

CHARLOTTENSIS

CHARLOTTENSIS

CHARLOTTENSIS

CHARLOTTENSIS

CHARLOTTENSIS

CHARLOTTENSIS

COMPRESSA

C CMPRESSA

COMPRESSA

COMPRESSA

KINCAIDI

$K$ INCAIDI

KINCAID

KINCAIDI

KINCAIDI

KINCAIDI

$K I N C A I D I$

KINCA I DI

LAC TEA

CRISPA

CRISPA

CRISPA

STR IATOPUNCTATA

STR IA TOPUNCTATA

CRI BROREPANDUS

CRI BROREPANDUS

CRI BROREPANDUS

LATERALIS

COMPRESSA

DIF FLUGIFORM IS

DIF FLUG I FORM IS

DIF FLUG IFORM IS

DIF DUGIFORMIS

DIFFLUGIFORMIS

DIF FLUGIFORM IS LAGENARI UM

DIF FLUGIFORMIS LAGENARIUM

FINDENS

FINDENS

FIN DENS

ARB ORE S CENS

A RB ORE S CENS

ARBORESCENS

ARBORESCENS

ARBORESCENS

ARB ORES CENS

ARBORE S CENS

A RB ORES CENS

ARBORESCENS

ARB ORES CENS

ARBORESCENS

Locality

Lat. Long.

GAMBIER BAY, ALASKA

GULF OF ALASKA

GULF OF ALASKA

GULF OF ALASKA

CRESCENT CITY, CALIF.

GULF OF ALASKA 


\section{Publication}

11796 BERGEN O'NEIL 1979

11797 TODD LOW 1967

11799 TODD LOW 1967

11799 TODD LOW 1967

11801 TODD LOW 1967

11802 TODD LOW 1967

11803 TODD LOW 1967

11804 COCKBAIN 1963

11805 COCKBAIN 1963

11806 TODD LOW 1967

11807 TODD LOW 1967

11808 COCKBAIN 1963

11809 COCKBAIN 1963

11810 COCKBAIN 1963

11811 COCKBAIN 1963

11812 SMITH 1973

11813 BERGEN O.NEIL 1979

11814 BERGEN O'NEIL 1979

11815 BERGEN O'NE IL 1979

11816 BERGEN O'NEIL 1979

11818 CUSHMAN AND TODD 1943

11819 CUSHMAN 1927

11820 COCKBAIN 1963

11821 COCKBAIN 1963

11822 COCKBAIN 1963

11823 COCKBAIN 1963

11824 COCKBAIN 1963

11825 COCKBAIN 1963

11826 CUSHMAN TODD 1947

11827 CUSHMAN TODD

11829 BERGEN O.NEIL 1979

11829 BERGEN O NEIL 1979

11830 BERGEN O'NEIL 1979

$\begin{array}{lll}11831 & \text { BERGEN O NE IL } 1979 \\ 11832 & \text { BERGEN O०NEIL } 1979\end{array}$

11832 BERGEN O०NEIL 1979

11833 BERGEN O N NEIL 1979

11834 BERGEN O'NEIL 1979

11836 BERGEN O'NEIL 1979

11837 BERGEN O'NE IL 1979

11838 BERGEN O'NEIL 1979

11839 BERGEN O'NEIL 1979

11840 BERGEN O'NEIL 1979

11841 BERGEN O'NEIL 1979

11842 BERGEN O'NEIL 1979

11843 BERGEN O NEIL 1979

11844 BERGEN O'NEIL 1979

11845 SMITH 1973

11846 SMITH 1973

11847 SMITH 1973

11848 CUS HMAN 1925

11849 CUSHMAN 1925

11850 WHI TEAVES 1886

11851 WHITEAVES 1886

11852 CUS HMAN 1925

11853 CUSHMAN 1925

11854 CUSHMAN 1915

11855 CUSHMAN 1927

11856 CUSHMAN 1927

11857 CUSHMAN 1927

11858 CUS HMAN 1927

11859 CUSHMAN 1927

11860 TODD LOW 196

11861 TODD LOW 1967

11862 TODD LOW 1967

11863 TODD LOW 1967

11864 TODD LOW 1967

11865 TODD LOW 1967

11866 BERGEN O.NEIL 1979

11867 BERGEN O'NEIL 1979

11868 BERGEN O'NEIL 1979

11869 BERGEN O NEIL 1979

11870 BERGEN O'NEIL 1979

11871 BERGEN O'NEIL 1979

11872 BERGEN O'NEIL 1979

11873 TODD LOW 1967

11874 TODD LOW 1967

11875 COCKBAIN 1963

11876 COCKBAIN 1963

11877 TODD LOW 1967

11878 TODD LOW 1967

11879 TODD LOW 1967

11880 BERGEN O'NEIL 1979

11881 BERGEN O'NEIL 1979

11882 BERGEN O'NEIL 1979

11883 BERGEN O'NEIL 1979

11884 BERGEN O'NEIL 1979

11885 BERGEN O'NEIL 1979

11886 BERGEN O'NEIL 1979

11887 BERGEN O'NEIL 1979

11888 TODD LOW 1967

11889 TODD LOW 1967

11890 TODD LOW 1967
Generic Name Specific Name

PSAMMATODENDRON

PSAMMOSPHAERA

PSEUDONODOSARIA

PSEUDONONION

PSEUDONONION

PSEUDCNONION

PSEUDONONION

PSEUDONONION

SEUDONONION

PSEUDONONION

PSEUDONONION

PSEUDOPOLYMORPH INA

PSEUDOPOLYMORPHINA

PSEUDOPOLYMORPHINA

PSEUDOPOLYMORPHINA

PULLENIA

PULLENIA

PULLENIA

PULLENIA

PULLENIA

PULLENIA

PULLENIA

PULLENIA

PULLENIA

PULLENIA

PULLENIA

PULLENIA

PULLENIA

PULLENIA

PULLENIA

PULLENIA

PULLENIA

PULLENIA

PULLENIA

PULLEN I A

PULLENIA

PULLENIA

PULLEN I A

PULLENIA

PULLENIA

PULLENIA

PULLENIA

PULLENIA

PULLENIA

PULLENIA

PULLEN I A

PULLENIA

PULVINUL INA

PULVINUL INA

PULVINULINA

PULVINUL INA

PULVINUL INA

PULVINULINA

PULVINUL INA

PULVINUL INELLA

PULVINUL INELLA

PULVINUL INELLA

PULVINUE INELLA

PULVINUL INELLA

PYRGO

PYRGO
PYRGO

PYRGO

PYRGO

PYRGO

PYRGO

PYRGO

PYRGO

PYRGO

PYRGO
PYRGO

PYRGO

PYRGO

PYRGO

PYRGO

PYRGO

PYRGO

PYRGOELLA

QU INQUELOCULINA

QUINQUELOCULINA

QUINQUELOCULINA

QUINQUELOCULINA

QU INQUELOCUL INA

QU I NQUEL OCULINA

QUINQUEL OCUL INA

QU INQUEL OCUL INA

QU INQUEL OCUL INA

QUINQUELOCULINA

QUINQUELOCULINA

QUINQUELOCULINA

QUINQUEL OCUL INA
ARB ORESCENS

FUSCA

A TL ANTI I CA

RADICULA

A UR I CULA

A UR I CULA

A UR I CULA

A UR I CULA

A UR I CUL A

A UR I CULA

AUR I CULA

A UR I CULA

AUR I CULA A

CHARLOTTENSIS

CHARLOTTENSI

CHARLOTTENSIS

LINGUA

BUL LOIDES

BULLOIDES

BULLOIDES

BUL LOIDES

MUL TI LOBA TA

QUINQUELOBA

SAL I SBURY I

SAL ISBURYI

SAL I SBURY I

SAL I SBURYI

SAL I SBURYI

SAL I SBURYI

SAL I SBURYI

ALISBURYI

SALI ISBURYI

SAL I SBURYI

SAL ISBURYI

SAL ISBURYI

SAL I SBURYI

SAL I SBURYI

SAL I SBURYI

SAL ISBURYI

SAL ISBURYI

SAL ISBURYI

SAL ISBURYI

SAL I SBURYI

SAL I SBURYI

SAL I SBURYI

SAL I SBURYI

SAL ISBURYI

SUBCARINATA

SUBCARINATA

SUBCARINATA

COLUMBIENSIS

COL UMB I ENS I

KARSTENI

KAR STEN I

REPANDA

REPANDA

UMB ONATA

PACIFICA

PAC IFICA

PACIFICA

PACIFICA

PAC IFICA

ABYSSORUM

DEPRESSA

DEPRESSA

LUCERNULA

LUCERNULA

LUCERNULA

MURRH INA

MURRHINA

MUR RHINA

MURRHINA

MURRH INA

MURRH INA

MURRHINA

ROT ALARIA

VES PER TILIO

WILLIAMSON

SPHAERA

AGGLUTINATA

A GGLUTINATA

AGGLUTINATA

A GG LUT INATA

AKNERIANA

AKNER I ANA

AKN ERI I ANA

AKNERIANA

AKNERIANA

AKNERIANA

AKNERIANA

AKNERIANA

Locality

Lat. Long.

GULF OF ALASKA

OFF ALASKA PENINSULA

GULF OF ALASKA

GAMBIER BAY, ALASKA

LYNN CANAL, ALASKA

KASAAN BAY, ALASKA

TAKU HARBOUR, ALASKA

BRIIISH COL UMBIA

BRITISH COLUMBI

EXCURSION, ALASKA

BRITISH COLUMBIA

BRIT ISH COL UMB IA

BRITISH COL UMBIA 


\section{Publication}

11891 TODD LOW 1967 11892 TODD LOW 1967

11893 TODD LOW 1967

11894 COOPER 1961

11895 BERGEN O'NEIL 1979

11896 BERGEN O'NEIL 1979

11897 COOPER 1961

11898 COOPER 1961

11899 LANKFORD PHLEGER 1973

11900 LANKFORD PHLEGER 1973

11901 TODD LOW 1967

11902 TOD D LOW 1967

11903 BERGEN O.NEIL 1979

11904 BERGEN O'NEIL 1979

11905 CUSHMAN TODD 1947

11906 CUSHMAN TODD 1947

11907 CUS HMAN TODD 1947

11908 CUSHMAN TODD 1947

11909 CUS HMAN TODD 1947

11909 CUSHMAN TODD 1947

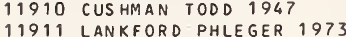

11912 TODD LOW 1967

11913 CUSHMAN TODD 1947

11914 CUSHMAN 1917

11915 LANKFORD PHLEGER 1973

11916 NATLAND 1933

11917 BERGEN O'NEIL 1979

11918 TODD LOW 1967

11919 TODD LOW 1967

11920 TODD LOW 1967

11921 TODD LOW 1967

11922 TODD LOW 1967

11923 TODD LOW 1967

11924 TODD LOW 1967

11925 TODD LOW 1967

11926 BERGEN O'NEIL 1979

11927 BERGEN O'NEIL 1979

11928 BERGEN OONEIL 1979

11929 BERGEN O'NEIL 1979

11930 BERGEN O'NEIL 1979

11931 BERGEN OONEIL 1979

11932 BERGEN O'NEIL 1979

11933 TODD LOW 1967

11934 BERGEN O NE IL 1979

11935 LANKFORD PHLEGER 1973

11936 LANKFORD PHLEGER 1973

11937 CUSHMAN TODD 1947

11938 CUSHMAN TODD 1947

11939 TODD LOW 1967

11940 TODD LOW 1967

11941 TODD LOW 1967

11942 TODD LOW 1967

11943 COCKBAIN 1963

11944 COCKBAIN 1963

11945 COCKBAIN 1963

11946 COCKBAIN 1963

11947 COCKBAIN 1963

11948 ECHOL S 1969

11949 COC KBAIN 1963

11950 COCKBAIN 1963

11951 ECHOLS 1969

11952 ECHOLS 1969

11953 BERGEN O.NEIL 1979

11954 COCKBAIN 1963

11955 COCKBAIN 1963

11956 COCKBAIN 1963

11957 COCKBAIN 1963

11958 COCKBAIN 1963

11959 COCKBAIN 1963

11960 COCKBAIN 1963

11961 SMI TH 1973

11961 SMI TH 1973

11962 SMITH 1973

11964 SMI TH 1973

11965 SMITH 1973

11966 SMI TH 1973

11967 SMITH 1973

11968 SMI TH 1973

11969 SMI TH 1973

11970 CUSHMAN 1927

11971 SMI TH 1973

11972 SMITH 1973

11973 CUSHMAN MCCULLOCH 1939

11974 BERGEN O'NEIL 1979

11975 BERGEN O'NEIL 1979

11976 BERGEN O'NEIL 1979

11977 BERGEN O NEIL 1979

11978 BERGEN O'NEIL 1979

11979 TODD LOW 1967

11980 SMI TH 1973

11981 SMI TH 1973

11982 BERGEN O NEIL 1979

11983 BERGEN O.NEIL 1979

11984 COC KBAIN 1963

11985 COC KBAIN 1963
Generic Name

QUINQUEL OCUL INA QUINQUEL OC ULINA QUINQUEL OCULINA QUINQUEL OCULINA QUINQUELOCULINA QUINQUEL OCULINA QUINQUEL OCULINA QUINQUEL OCUL INA QUINQUEL OCULINA QUINQUEL OCUL INA QUINQUEL OCULINA QUINOUELOCULINA QUINQUEL OCUL INA QUINQUEL OCULINA QUINQUEL OCULINA QUINQUEL OCULINA QUINQUEL OCULINA QUINQUEL OCULINA QUINQUELOCULINA QUINQUEL OCUL INA QUINQUEL OCULINA QUINQUEL OCULINA QUINQUELOCULINA QUINQUEL OCULINA QUINQUEL OCULINA QU INQUEL OCUL INA QUINQUELOCULINA QUINQUEL OCULINA QUINQUEL OCULINA QUINQUEL OCULINA QUINQUEL OCULINA QUINQUEL OCULINA QUINQUEL OCULINA QUINQUEL OCUL INA QUINQUEL OCULINA QUINQUEL OCUL INA QUINQUE LOCUL INA QUINQUEL OCULINA QUINQUEL OCUL INA QUINQUEL OCULINA QUINQUEL OCUL INA QUINQUEL OCULINA QUINQUEL OCUL INA QUINQUEL OCUL INA QUI NQUEL OCUL INA QUINQUEL OCUL INA QUINQUEL OCULINA QUINQUEL OCULINA RECURVOIDES RECURVOI DES RECURVOIDES RECURVOIDES RECURVOIDES RECURVOIDES RECURVOIDES RECURVOIDES RECURVOIDES RECURVOIDES RECURVOIDES

REC URVOIDES

RECURVOIDES

RECURVOIDES

RECURVOIDES

RECURVOIDES

REOPHAX

REOPHAX

REOPHAX

REOPHAX

REOPHAX

REOPHAX

REOPHAX

REOPHAX
REOPHAX

RE OPHAX

REOPHAX

RE OPHAX

REOPHAX

REOPHAX

RE OPHAX

REOPHAX

REOPHAX

REOPHAX

REOPHAX

REOPHAX

RE OPHAX

REOPHAX

REOPHAX

REOPHAX

REOPHAX

REOPHAX

REOPHAX

REOPHAX

REOPHAX

RE OPHAX
Specific Name

A KNER I ANA

AKNERIANA

AKNERIANA

AKNERIANA

AKNERIANA

AKNERIANA

AKNERIANA

AKNERIANA BELLATULA

AKNERIANA BELLATULA

ARC T I CA

RCTICA

ARCTICA

ARC TICA

CATALINENSIS

DISCIFORMIS

DIS CIFORMIS

DISCIFORMIS

E LONGATA

ELONGATA

ELONGATA

FRI GIDA

FUS CA

PROCERA

SABULOSA

SEM INULA

SEM INUL UM

SEM INULUM

SEM INULUM

SEM I NUL UM

SEM INULUM

STALKERI

STALKER I

STALKERI

STALKER

STALKER I

STALKER I

STALKER I

STALKER I

SUBROTUNDA

SUBROTUNDA

SUBROTUND

TENAGOS

VUL GARIS

VUL GARIS

VUL GAR IS

CON TORTUS

CONTORTUS

CON TORTUS

TURBINATUS

TURBINATUS

TURBINATUS

TURBINATUS

TURBINATUS

TURBINATUS

UR BINATUS

TURBINATUS

TURBINATUS

TURBINATUS

TURBINATUS

TURBINATUS

TURBINATUS

CURTUS

CURTUS

CURTUS

CURTUS

CURTUS

CURTUS

DENTALINAFORMIS

DEN TAL INAFORMIS

DIFFLUG IF ORM IS

DIFFLUG IFORM IS

DIF FLUG IF ORM IS

IFFLUG IF ORM IS

DIFFLUGIFORM IS

DI F FLUG IFORM IS

DISTANS

EXCENTRICUS

EXCENTRICUS

EXCENTRICUS

EXCENTRICUS

GUTTIFER

GUTTIFER

GUTTIFER

GUTTIFER

GUT TIFER

INSECTUS

NODULOSUS

PILULIFER

PILULIFER

PILULIFER

PILULIFERA

Locality

Lat. Long.

GAMBIER BAY, ALASKA

KASAAN BAY, ALASKA

TAKU HARBOUR, ALASKA

OREGON COAST 


\section{Publication}

11986 BERGEN O'NEIL 1979 11987 BERGEN O०NEIL 1979 11988 BERGEN O'NEIL 1979

11989 BERGEN O०NEIL 1979

11990 BERGEN O०NEIL 1979

11991 TODD LOW 1967

11992 SMITH 1973

11993 BERGEN O'NE IL 1979

11994 BERGEN O'NEIL 1979

11995 BERGEN O'NEIL 1979

11996 BERGEN O.NEIL 1979

11997 TODD LOW 1967

11998 TODD LOW 1967

11999 TODD LOW 1967

$\begin{array}{llll}12000 & \text { TOD D LOW } & 1967 \\ 12001 & \text { TOD D LOW } & 1967\end{array}$

12002 SMI TH 1973

12003 CUSHMAN MCCULLOCH 1939

12004 CUSHMAN MCCULLOCH 1939

12005 CUSHMAN MCCULLOCH 1939

12006 CUS HMAN MCCULLOCH 1939

12007 TODD LOW 1967

12008 WHITEAVES 1886

12009 WHITEAVES 1886

12010 WHI TEAVES 1886

12011 WHITEAVES 1886

12012 BERGEN O NEIL 1979

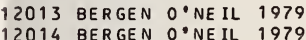

12015 TODD LOW 1967

12016 TODD LOW 1967

12017 CUS HMAN MCCUL

12018 COOPER 1961
12019 BERGEN O०NE IL 1979

12019 BERGEN O NE IL 1979
12020 CUSHMAN TODD 1947

12021 CUSHMAN TODD 1947

12022 BERGEN O'NEIL 1979

12023 BERGEN O'NEIL 1979

12024 BERGEN O NEIL 1979

12025 CUSHMAN MCCUL
12026 DETLING 1958

12027 CUSHMAN TODD 1947

12028 TODD LOW 1967

12029 TODD LOW 1967

12030 TODD LOW 1967

12031 CUSHMAN TODD 1947

12032 TODD LOW 1967

12033 COCKBAIN 1963

12034 ECHOL S 1969

12035 COCKBAIN 1963

12036 COCKBAIN 1963

12037 LANKF ORD PHLEGER 1973

12038 LANKFORD PHLEGER 1973

12039 LANKF ORD PHLEGER 1973

12040 TODD LOW 1967

12041 TODD LOW 1967

12042 TODD LOW 1967

12043 WHITEAVES 1886

12044 WHITEAVES 1886

12045 WHI TEAVES 1886

12046 TODO LOW 1967

12048 CUSHMAN 1915

12049 CUS HMAN 1915

12050 TODD LOW 1967

12051 BERGEN O'NEIL 1979

12052 BERGEN O०NEIL 1979

12053 COCKBAIN 1963

12054 COCKBAIN 1963

12055 COCKBAIN 1963

12056 T.ODO LOW 1967

12057 COCKBAIN 1963

12058 COCKBAIN 1963

12059 COCKBAIN 1963

12060 COCKBAIN 1963

12061 COCKBAIN 1963

12062 COCKBAIN 1963

12063 COCKBAIN 1963

12064 COCKBAIN 1963

12065 BERGEN O.NE IL 1979

12066 BERGEN O.NE IL 1979

12067 BERGEN O'NEIL 1979

12068 SMITH 1973

12069 BERGEN O.NEIL 1979

12070 BERGEN O'NEIL 197.9

12071 TODD LOW 1967

12072 BERGEN O NEIL 1979

12073 CUSHMAN TODD 1947

12074 BERGEN O.NEIL 1979

12075 TODD LOW 1967

12076 CUS HMAN 1917

12077 BERGEN O.NE IL 1979

12078 TODD LOW 1967

12079 CUSHMAN TODD
12080 CUSHMAN
Generic Name Specific Name

SCORPIURUS

SCORPIURUS

SCORPIURUS

SCORPIURUS

SCORPIURUS

SCORPIURUS

SCORPIURUS

SCORPIURUS

SCORPIURUS

SCORPIURUS

SCORPIURUS

SCORPIURUS

SCORPIURU

SCORPIURUS

SCORPIURUS

SCOTTI

SCOTIII

SUB FUSI FORMIS

SUB FUS I FORMIS

SUB FUS I FORMIS

ABYSSORUM

ABY S SORUM

ABY S SORUM

ABY SSORUM

ABY SSORUM

ALG AEF ORMIS

ALGAEFORMIS

I ND IV I SA

ARC TI CA

CHARLOTTENSI S

CHARL OTTENSIS

CHARLOTTENSI S

CHARLOTTENS I S

CHARLOTTENSI

CHARLOTTENSIS

CHARLOTTENSI S

CHARLOT TENS I S

CHA RLOTTENSIS

CHARLOTTENS I

D'ORBI GNYI

NIC OBARENSIS

NIC OBARENSIS

OCC I DENTAL IS

ORB I CULAR IS

STRONG I

CAMPANULATA

COL UMBIENSIS

COL UMBIENSIS

COL UMBIENSIS

COLUMBIENSIS

$C$ OL UMB IENS IS

COL UMBIENSIS

ORNAT IS SIMA

WRIGHTII

BEC CAR I

BEC CARII

BEC CARII

COL UMBIENSIS

ORB I CULARIS

SOLDANII

STABILIS

STABIL I S

STABILIS

DIFFLUG IFORMIS

DIF FLUG IF ORM IS

DIFFLUG IF ORM IS

DIF FLUGIFORM IS

DIF FLUG IF ORM IS

DIF FLUG IF ORM IS

DIF FLUG IF ORM IS

DIF FLUG IF ORM IS

DIF FLUG IF ORM IS

LONG I C OLL IS

LON G I COLL IS

LONGICOLL IS

SPHAERICA

SPH AERICA

SPHAERICA

SPHAERICA

RAM OSA

RAM OSA

RAM OSA

RAMOSA

RAM OSA

PRIMAEVA

PRIMAEVA
CELATA

CEL ATA
DISTORTA

DISTORTA

GAL LOWAYI
Locality

Lat. Long.

GULF OF ALASKA

GULF OF ALASKA

GULF OF ALASKA

GULF OF ALASKA

GULF OF ALASKA

GULF OF ALASKA

OFF ALASKA PENINSULA

GULF OF ALASKA

GULF OF ALASKA

GULF OF ALASKA

GULF OF ALASKA

GAMBIER BAY, ALASKA

TAKU HARBOUR, ALASKA

EXCURSION, ALASKA

KASAAN BAY, ALASKA

LYNN CANAL, ALASKA
OFF ALASKA PENINSULA

OFF ALASKA

OFF ALASKA

OFF ALSAKA

GULF OF ALASKA

QUEEN CHARLOTTE SD.. B.

QUATSINO SOUND, B.C.

STRAIT OF GEORGIA, B. C.

STRAIT OF GEORGIA, B.C.

GULF OF ALASKA

GULF OF ALASKA

GULF OF ALASKA

GAMBIER BAY, ALASKA

KETCHIKAN, ALASKA

OREGON COAST

GULF OF ALASKA

WASHINGTON COAST

WASHINGTON COAST

GULF OF ALASKA

GULF OF ALASKA

GULF OF ALASKA

OFF ALASKA

SUNSET BAY, OREGON

WASHINGTON COAST

GULF OF ALASKA

KASAAN BAY, ALASKA

GULF OF ALASKA

WASHINGTON COAST

KASAAN BAY, ALASKA

BRITISH COLUMBIA

WASHINGTON COAST

BR IT ISH COL UMBIA

BR IT ISH COL UMBIA

CRESCENT CITY, CALIF.

LAPUSH, WASHINGTON

DEPOE BAY, OREGON

KASAAN BAY, ALASKA

GULF OF ALASKA

DISCOVERY PASSAGE, B.C

QUATSINO SOUND. B.C. $\quad 5037 N 12801$

GAMBIER BAY, ALASKA B. C. $5025 \mathrm{~N} 12657 \mathrm{~W}$

\section{OFF OREGON}

OFF OREGON

GULF OF ALASKA

GULF OF ALASKA

BRITISH COL UMBIA

BRITISH COLUMBIA

BRITISH COLUMBIA

GULF OF ALASKA

BRITISH COL UMBIA

BR ITISH COLUMBIA

BRITISH COL UMBIA

BRITISH COLUMBIA

BR IT ISH COLUMBIA

BR IT ISH COLUMBIA

BR IT ISH COL UMBIA

BR IT ISH COL UMB I A

GULF OF ALASKA

GULF OF ALASKA

GULF OF ALASKA

OFF ALASKA PENINSULA

GULF OF ALASKA

GULF OF ALASK

GULF OF ALASKA

WASH ING TON COAST

GULF OF ALASKA

GULF OF ALASKA

OFF OREGON

GULF OF ALASKA

GULF OF ALASKA

WASH INGTON COAST

WASHINGTON COAST

$5930 \mathrm{~N} 14520 \mathrm{~W}$

$5930 \mathrm{~N} 14255 \mathrm{~W}$

$5930 \mathrm{~N} 14300 \mathrm{~W}$

$5925 N 14525 \mathrm{~W}$

$5932 \mathrm{~N} 14236 \mathrm{~W}$

$4861 N 16107 \mathrm{~W}$

$55 N 14455 W$

$5942 \mathrm{~N} 14410 \mathrm{~W}$

$5640 N 15155 \mathrm{~W}$

$5720 \mathrm{~N} 13402 \mathrm{~W}$

$5802 \mathrm{~N} 13400 \mathrm{~W}$

$5825 \mathrm{~N} 13530 \mathrm{~W}$

$5526 N 13214 \mathrm{~W}$ 


\section{Publication}

12081 BERGEN O'NEIL 1979

12082 BERGEN O'NEIL 1979

12083 BERGEN O'NEIL 1979

12084 BERGEN O.NEIL 1979

12085 CUSHMAN TODD 1947

12086 COCKBAIN 1963

12087 COCKBAIN 1963

12088 LANKFORD PHLEGER 1973

12089 TODD LOW 1967

12090 CUSHMAN TODD 1947

12091 TODD LOW 1967

12092 COCKBAIN 1963

12093 BERGEN O NEIL 1979

12094 CUSHMAN 1925

12095 SCOTT 1974

12096 CUSHMAN TODD 1947

12097 CUSHMAN TODD 1947

12098 BERGEN O N NE IL 1979

12099 COCKBAIN 1963

12100 BERGEN O'NEIL 1979

12101 BERGEN O०NEIL 1979

12102 BERGEN O.NEIL 1979

12103 COCKBAIN 1963

12104 COCKBAIN 1963

12105 COCKBAIN 1963

12106 COCKBAIN 1963

12107 BERGEN O N NEIL 1979

12108 BERGEN O'NEIL 1979.

12109 ECHOLS 1969

12110 ECHOLS 1969

12111 ECHOLS 1969

12112 SMITH 1973

12113 SMITH 1973

12114 SMI TH 1973

12115 SMI TH 1973

12116 TODD LOW 1967

12117 COCKBAIN 1963

12118 COCKBAIN 1963
12119 COCKBAIN 1963

12120 BERGEN O.NEIL 1979

12121 LANKFORD PHLEGER 1973

12122 BERGEN O.NEIL 1979

12123 BERGEN O.NEIL 1979

12124 BERGEN O'NEIL 1979

12125 BERGEN O'NEIL 1979

12126 BERGEN O NEIL 1979

12127 ECHOLS 1969

12128 COC KBAIN 1963

12129 COCKBAIN 1963

12130 HAMLIN 1960

12131 LANKFORD PHLEGER 1973

12132 BERGEN O'NEIL 1979

12133 BERGEN O'NEIL 1979

12134 BERGEN O'NEIL 1979

12135 BERGEN O'NEIL 1979

12135 BERGEN O'NEIL 1979

12136 8ERGEN O'NEIL 1979

12138 BERGEN O.NE IL 1979

12139 BERGEN O'NEIL 1979

12140 BERGEN O'NEIL 1979

12141 BERGEN O'NEIL 1979

12142 BERGEN O' $\triangle E I L 1979$

12143 BERGEN O'NEIL 1979

12144 BERGEN O'NEIL 1979

12145 BERGEN O'NEIL 1979

12146 BERGEN O.NEIL 1979

12147 BERGEN O'NEIL 1979

12148 BERGEN O'NEIL 1979

12149 BERGEN O'NEIL 1979

12150 BERGEN O'NEIL 1979

12151 BERGEN O'NEIL 1979

12152 BERGEN O'NEIL 1979

12153 BERGEN O'NEIL 1979

12154 BERGEN O'NEIL 1979

12155 BERGEN O'NEIL 1979

12156 BERGEN O.NEIL 1979

12157 CUSHMAN TODD 1947

12158 CUSHMAN TODD 1947

12159 TODD LOW 1967

12160 TODD LOW 1967

12161 TODD LOW 1967

12162 CUSHMAN TODD 1947

12163 BERGEN O N NEIL 1979

12163 BERGEN O NEIL 1979

12165 BERGEN O'NEIL 1979

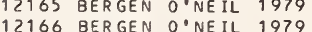

12166 BERGEN O'NEIL 1979

12167 BERGEN O'NEIL 1979

12168 BERGEN O'NEIL 1979

12169 BERGEN O'NEIL 1979

12170 BERGEN O'NEIL 1979

12171 TODD LOW 1967

12172 BERGEN O NEIL 1979

12173 TOOD LOW 1967

12174 COC KBAIN 1963

12175 COCKBAIN 1963
Generic Name Specific Name

GALLOWAYI

GALLOWAY

GALLOWAYI

TRILOCULARIS

TRI LOCULARIS

TRI LOCULARIS

TRILOCULARIS

TRI LOCULARIS

TRI LOCULARIS

TRI LOCULARIS

TRI LOCULAR I

BULLOIDES

SPINIGERA REDUCTA

$\checkmark$ IVIPARA

8 IF ORMI S

BIFORMIS

8 IF ORMIS

BIFORMIS

BIFORMIS

BIFORMIS

QIFORMIS

B IF ORM IS

B IF ORMIS

8 IF ORMIS

B IF ORMIS

BIF ORMIS

BIFORMIS

BIF ORMIS

BIF ORMIS

BIF ORMIS

BIF ORMIS

BIFORMIS

8 IF ORMIS

8 IF ORMIS

8 IF ORMIS

8 IF ORMIS

ECKIS I

EARLANDI

TOR QUATA

TOR QUATA

TOR QUA TA

TOR QUATA

TORQUATA

COL UMBIENSIS

COL UMBIENSIS

COL UMBIENSIS

ORF ORDENSIS

ORNATI SS IMA

ORNATISSIMUS

ORNATISSIMUS

ORNATISSIMUS

ORNATISSIMUS

ORNATISSIMUS

ORNATISSIMUS

FLUENS

FLUENS

FLUENS

FLUENS

FLUENS

FLUENS

FLUENS

FLUENS

FLUENS

FLUENS

FLUENS

FLUENS

FLUENS

F LUENS

FLUENS

FLUENS

FLUENS

FLUENS

FLUENS

CIRCULARIS

CIR CULARIS

ROTUNDA

ROTUNDA

TRI GONULA

TRI HED RA

TRIHEDRA

TRI HEDRA

TRI HEDRA

TRI HEDRA

TRI HEDRA

T RI HED RA

TRI HEDRA

ADVENA

A DVENA

ADVENA

CHARLOTTENSIS

TROCHAMM INA

TROCHAMMINA
Locality

Lat. Long.

GULF OF ALASKA

GULF OF ALASKA

GULF OF ALASKA

UASHINGTON

WA SHINGT

BRITISH

COLUMBIA

CRESCENT CITY, CALIF.

EXCURSION, ALASKA

WASHINGTON COAST

GULF OF ALASKA

BRITISH COLUMBI

GULF OF ALASKA

Q. CHARLOTTE SD.. 8.C.

SAMISH-PADILLA BAYS

WASHINGTON COAST

WASH INGTON COAS

GULF OF ALASKA

BRITISH COLUMBIA

GULF OF ALASKA

GULF OF ALASKA

GULF OF ALASKA

BRITISH COL UMBIA

BRITISH COLUMBIA

BRITISH COLUMBIA

BRITISH COLUMBIA

GULF OF ALASKA

GULF OF ALASKA

WASH INGTON COAST

WASHINGTON COAST

WASHINGT ON COAST

DFF ALASKA PENINSULA

OFF ALASKA PENINSULA

OFF ALASKA PENINSULA

NORTH PACIFIC

EXCURSION, ALASKA

BRITISH COLUMBIA

BRITISH COLUMBIA

BRITISH COLUMBIA

GULF OF ALASKA

CRESCENT CITY, CALIF.

GULF OF ALASKA

GULF OF ALASKA

GULF OF ALASKA

GULF OF ALASKA

GULF OF ALASKA

WASHINGTON COAST

BRITISH COLUMBIA

BRIT ISH COLUMBIA

PORT ORFORD, OREGON

DEPOE BAY, OREGON

GULF OF ALASKA

GULF OF ALASKA

GULF OF ALASKA

GULF OF

GULF OF ALASKA

GULF

GULF OF ALASKA

GULF OF ALASKA

GULF OF ALASKA

GULF OF ALASKA

GULF OF ALASKA

GULF OF ALASKA

GULF OF ALASKA

GULF

GULF OF ALASKA

GULF OF ALASKA

GULF OF ALASKA

GULF OF ALASKA

GULF OF ALASKA

GULF OF ALASKA

GULF OF ALASKA

GULF OF ALASKA

GULF OF ALASKA

GULF OF

WASHINGTON COAST

LYNN CANAL, ALASKA

GAMBIER BAY, ALAS

WASH

GULF OF ALASKA

GULF OF ALASKA

GULF OF ALASKA

GULF OF ALASKA

GULF OF ALASKA

GULF OF ALASKA

GULF OF ALASKA

GULF OF ALASKA

KASAAN BAY, ALASKA

GULF OF ALASKA

GULF OF ALASKA

BR IT ISH COLUMBIA

BRITISH COLUMBIA 


\section{Publication}

12176 COCKBAIN 1963

12177 COCKBAIN 1963

12178 CUS HMAN 1925

12179 CUS HMAN TODD 1947

12180 LANKFORD PHLEGER 1973

12181 LANKFORD PHLEGER 1973

12182 LANKFORD PHLEGER 1973

12183 CUSHMAN TODD 1947

12184 DETLING 1958

12185 ECHOLS 1969

12186 PHLEGER 1967

12187 COCKBAIN 1963

12188 COCKBAIN 1963

12189 COCKBAIN 1963

12191 CUS HMAN TODD 1947

$\begin{array}{lll}12191 & \text { CUS HMAN } \\ 12192 \text { CUS HMAN } & \text { TODD } & 1947\end{array}$

12193 DETLING 1958

12194 COCKBAIN 1963
12195 COCKBAIN 1963

12195 COCKBAIN 1963
12196 COCKBAIN 1963

12197 COCKBAIN 1963

12198 COCKBAIN 1963

12199 COCKBAIN 1963

12200 COCKBAIN 1963

12201 COCKBAIN 1963

12202 CUSHMAN MCCULLOCH 1939

12203 BERGEN O.NEIL 1979

$\begin{array}{lll}12204 & \text { BERGEN O'NEIL } 1979 \\ 12205 & \text { BERGEN } & 0 \\ \end{array}$

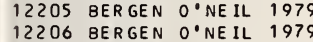

12207 BERGEN O'NEIL 1979

12208 BERGEN O'NEIL 1979

12209 SMI TH 1973

12210 SMI TH 1973

12211 SMITH 1973

12212 SMITH 1973

12213 SMI TH 1973

12214 SMITH 1973
12215 SMITH 1973

12216 SMITH 1973

12217 SMITH 1973

12218 SMITH 1973

12219 SMI TH 1973

12220 SMITH 1973

12221 PHLEGER 1967

12222 PHLEGER 196

$\begin{array}{lll}12223 & \text { PHLEGER } & 1967 \\ 12224 & \text { PHLEGER } & 1967\end{array}$

12225 PHLEGER 1967

12226 SCOTT 1974

12227 BERGEN O NE IL 1979

12228 BERGEN O'NEIL 1979

12229 BERGEN O NEIL 1979

12230 BERGEN O'NEIL 1979

12231 DETLING 1958

12232 LANKFORD PHLEGER 1973

$\begin{array}{lll}12233 & \text { COOPER } & 1961 \\ 12234 & \text { COOPER } & 1961\end{array}$

12235 COOPER 1961

12236 COOPER 1961

12237 COOPER 1961

12238 PHLEGER 1967

12239 PHLEGER 1967

12240 PHLEGER 196
12241 SMITH 1973

12241 SMITH 1973
12242 COCKBAIN 1963

12242 COCKBAIN 1963
12243 COC KBAIN 1963

12244 COCKBAIN 1963

12245 COCKBAIN 1963

12246 COCKBAIN 1963

12247 COCKBAIN 1963

12248 COCKBAIN 1963

12249 SMI TH 1973

12250 SMITH 1973

12251 SMITH 1973

12252 CUS HMAN MCCULLOCH 1939

12253 CUSHMAN MCCULLOCH 1939

12254 CUS HMAN MCCULLOCH 1939

12255 JONES ROSS 1979

12256 CUSHMAN 1925

12257 CUSHMAN 1925

12258 COOPER 1961

12259 COCKBAIN 1963

12260 COCKBAIN 1963

12261 COCKBAIN 1963

12262 COCKBAIN 1963

12263 COCKBAIN 1963
12264 COCKBAIN 1963

12264 COCKBAIN 1963
12265 COCKBAIN 1963

12266 COCKBAIN 1963

12267 COCKBAIN 1963

12268 LANKFORD PHLEGER 1973

12269 LANKFORD PHLEGER 1973

12270 SCOTT 1974
Generic Name Specific Name

CHARLOTTENSIS

CHARLOTTENSIS

CHARLOTTENSIS

CHARLOTTENS IS

CHARLOTTENSIS

CHARLOTTENSIS

CHARLOTTENSIS

CHARLOTTENSIS

CHARLOTTENSI

CHARLOTTENSI

CHARLOTTENSI

CHARLOTTENSI

CHARLOTTENSI

CHARLOTTENSIS

CHARLOTTENS IS

DIS CORBIS

DISCORBIS

DISCORBIS

DIS CORBIS

DIS CORBIS

D IS CORBIS

DIS CORBIS

DIS CORBIS

DIS CORBIS

DISCORBIS

DISCORBIS

DIS CORBIS

GLOBIGERINIF ORMIS

GLOBIGER IN I F ORM IS

GLOBI GER IN I F ORM I S

GLOBIGERINI F ORM IS

GLOBI GERIN I F ORM IS

GLOBIGERINIF ORM IS

GLOB IGER IN IF ORM IS

GLOBIGER IN IF ORMIS

GLOBGERINIFOF

GLOBIGERINIFORM I S

GLOBIGERINIF ORM IS

GLOB I GER IN I F ORM IS

GLOBI GER IN I F ORM I S

GRISEA

GRISEA

GRISEA

GRISEA

INFLATA

INF L ATA

I NF LATA

INFLATA

INFLATA

INF LATA

INFLATA

INFLATA

INF LATA

INF LATA

INF LATA

INF LATA

KELLET TAE

KELLET TAE

KELLETTAE

KELLET TAE

KELLETTAE

MACRESCENS

MACRESCENS

NAN A

N AN A

NAN A

NANA

NANA

NAN A

N AN A

NAN A

N IT I DA

N IT I DA

$N$ IT I DA

NITIDA

N IT I DA

N IT I DA

PAC IFICA

PACIFICA

PAC IFICA

PACIFICA

PAC IFICA

PAC IFICA

PAC IFICA

PAC IFICA

PAC IF ICA

PAC IFICA

PAC IFICA

PAC IFICA

PAC IFICA

PAC IFICA

\section{Locality}

Lat. Long.

BRITISH COLUMBIA

BR IT ISH COLUMBIA

Q. CHARLOTTE SD.. B.C.

WASHINGTON COAST

LAPUSH, WASHINGTON

DEPOE BAY, OREGON

CRESCENT CITY, CALIF.

WA SHINGTON COAST

SUNSET BAY, OREGON
WASHINGTON COAST

WASHINGTON COAST
GRAY'S HARBOUR. WASH.

BRITISH COLUMBIA

BRITISH COLUMBIA

BRITISH COLUMBIA

BRITISH COLUMBIA

WASHINGTON COAST

WASHINGTON COAST

SUNSET BAY, OREGON

BRITISH COLUMBIA

BR. ITISH COLUMBIA

BR ITISH COLUMBIA

BRITISH COLUMBIA

BRITISH COLUMBIA

BRITISH COL UMBIA

BRITISH COLUMBIA

BR ITISH COLUMBIA

KETCHIKAN, ALASKA

GULF OF ALASKA

GULF OF ALASKA

GULF OF ALASKA

GULF OF ALASKA

GULF OF ALASKA

GULF OF ALASKA

OFF ALASKA PENINSULA

OFF ALASKA PENINSULA

OFF ALASKA PENINSULA

OFF ALASKA PENINSULA

OFF ALASKA PENINSULA

OFF ALASKA PENINSULA

NORTH PACIFIC

OFF ALASKA PENINSULA

OFF ALASKA PENINSULA

NORTH PACIFIC

OFF ALASKA PENINSULA

GRAY'S HARBOUR, WASH

COPPER R.. ALASKA

FRASER R.. B.C.

COOS, BAY, OREGON

PHILLIPS ARM. B.C.

SAMISH-PADILLA BAYS

GULF OF ALASKA

GULF OF ALASKA

GULF OF ALASKA

GULF OF ALASKA

SUNSET BAY, OREGON

DEPOE BAY, OREGON

OREGON COAS

OREGON COAS

CALIFORNIA COAST

OREGON COAST

OREGON COAST

FRASER R.. B. C.

COOS, BAY, OREGON

GRAY'S HARBOUR, WASH

OFF ALASKA PENINSULA

BRITISH COLUMBIA

BRITISH COLUMBIA

BRITISH COLUMBIA

BR ITISH COLUMBIA

BR ITISH COLUMBIA

BR IT ISH COL UMBIA

BRITISH COLUMBIA

OFF ALASKA PENINSULA

NORTH PACIFIC

OFF ALASKA PENINSULA

OFF ALASKA

OFF ALASKA

OFF ALAS

SAMISH BAY, WASH.

Q. CHARLOTTE SD.. B.C.

VIRAGO SD.. B.C.

OREGON COAS

BRITISH COLUMBIA

BRITISH COLUMBIA

BRITISH COLUMBIA

BRITISH COLUMBIA

BR ITISH COLUMBIA

BRITISH COLUMBIA

BRITISH COLUMBIA

BR ITISH COL UMBIA

BRITISH COL UMBIA

CRESCENT CITY, CALIF.

CRESCENT CITY, CALI

DEPOE BAY, OREGON

$4835 \mathrm{~N} 12251 \mathrm{~W}$

$4900 \mathrm{~N} 12315 \mathrm{~W}$

$4832 \mathrm{~N} 12310 \mathrm{~N}$

$4755 \mathrm{~N} 12739 \mathrm{~W}$

$44 \quad 47 \mathrm{~N} 12405 \mathrm{~W}$

$4145 \mathrm{~N} 12413 \mathrm{~W}$

$4321 \mathrm{~N} 12420 \mathrm{~W}$

$4710 \mathrm{~N} 12414 \mathrm{~W}$

$4700 \mathrm{~N} 12408 \mathrm{~W}$

$4845 \mathrm{~N} 12300 \mathrm{~W}$

$4820 \mathrm{~N} 12300 \mathrm{~W}$

$4820 \mathrm{~N} 12410 \mathrm{~W}$ 


\section{Publication}

12271 CUSHMAN MCCULLOCH 1939 12272 CUSHMAN MCCULLOCH 1939 12273 CUSHMAN MCCULLOCH 1939 12274 CUSHMAN TODD 1947

12275 TODD LOW 1967

12276 TODD LOW 1967

12277 TODD LOW 1967

12278 TODD LOW 1967

12279 CUS HMAN TODD 1947

12280 BERGEN O'NEIL 1979

12281 BERGEN ONNEIL 1979

12282 DETLING 1958

12283 BERGEN O'NEIL 1979

2284 BERGEN O'NEIL 1979

$\begin{array}{lll}12285 & \text { BERGEN O'NEIL } & 1979 \\ 12286 & \text { BERGEN O'NEIL } 1979\end{array}$

12287 BERGEN O'NEIL 1979

12288 TODD LOW 1967

12289 CUSHMAN MCCULLOCH 1939

12290 COCKBAIN 1963

12291 COCKBAIN 1963

12292 COCKBAIN 1963

12293 COCKBAIN 1963
12294 WHITEAVES 1886

12294 WHITEAVES
122986
1229

12296 BERGEN O.NEIL 1979

12297 BERGEN O N NEIL 1979

12298 COCKBAIN 1963

12299 COCKBAIN 1963

12300 COCKBAIN 1963

12301 COCKBAIN 1963

12302 TODD LOW 1967

12303 COCKBAIN 1963

12304 COCKBAIN 1963

12305 COCKBAIN 1963

12306 COOPER 1961

12307 CUSHMAN 1910

12308 WHITEAVES 1886

12309 WHITEAVES 1886

12310 WHITEAVES 1886

12311 WHITEAVES 1886

12312 WHI TEAVES 1886

12313 WHITEAVES 1886

12314 WHITEAVES 1886

12315 WHITEAVES 1886

12316 WHITEAVES 1886

12317 WHITEAVES 1886

12318 WHITEAVES 1886

12319 WHITEAVES 1886

12320 WHITEAVES 1886

12321 WHI TEAVES 1886

12322 WHITEAVES 1886

12323 WHITEAVES 1886

12324 WHITEAVES 1886

12325 WHITEAVES 1886

12326 WHITEAVES 1886

12327 WHITEAVES 1886

12328 CUSHMAN 1915

12329 COOPER 1961

12330 BERGEN O'NEIL 1979

12331 BERGEN O'NEIL 1979

12332 BERGEN O'NEIL 1979

12333 BERGEN O'NEIL 1979

12334 BERGEN O०NEIL $1977^{\circ}$

12335 SMITH 1973

12336 BERGEN O'NEIL 1979

12337 BERGEN O.NEIL 1979

12338 BERGEN O'NEIL 1979

12339 BERGEN O'NEIL 1979

12340 BERGEN O'NEIL 1979

12341 BERGEN O'NEIL 1979

12342 BERGEN O NEIL 1979

12343 BERGEN O.NEIL 1979

12344 BERGEN O' $\mathrm{NEIL} 1979$

12345 BERGEN O'NEIL 1979

12346 BERGEN O'NEIL 1979

12347 BERGEN O'NEIL 1979

12348 BERGEN O'NEIL 1979

12349 CUSHMAN TODD 1947

12350 CUSHMAN TODD 1947

12351 COCKBAIN 1963

12352 COCKBAIN 1963

12353 COCKBAIN 1963

12354 COCKBAIN 1963

12355 BERGEN O'NEIL 1979

12356 BERGEN O'NEIL 1979

12357 BERGEN O'NEIL 1979

12358 BERGEN O'NEIL 1979

12359 BERGEN O'NEIL 1979

12360 COCKBAIN 1963

12361 COCKBAIN 1963

12362 BERGEN O'NEIL 1979

12363 BERGEN O'NEIL 1979

12363 BERGEN O'NEIL 1979

12365 BERGEN O'NEIL 1979

\section{Generic Name Specific Name}

TROCHAMM INA

TROCHAMM INA

TROCHAMM INA

TROCHAMM INA

TROCHAMM INA

TROCHAMM INA

TROCHAMM INA

TROCHAMM INA

TROCHAMMINA

TROCHAMMINA

TROCHAMMINA

TROCHAMM INA
TROCHAMM INA

TROCHAMMINA

TROC HAMM INA

TROCHAMM INA

TROCH AMM INA

TROCHAMM INA

TROCHAMMINA

TRO CHAMM INA

TROCHAMM INA

TROCHAMM INA

TROCHAMMINA

TROCHAMM INA

TROCHAMM INA

TROCHAMM INA

TROCHAMM INA

TROCHAMMINA

TROCHAMM INA

TROCHAMM INA

TROCHAMM INA

TROCHAMM INA

TROCHAMM INA

TROCHAMMINA

TROCHAMMINA

TRUNCATULINA

TRUNCATULINA

TRUNCATULINA

TRUNCATULINA

TRUNCATULINA

TRUNCATULINA

TRUNCATULINA

TRUNCATULINA

TRUNCATULINA

TRUNCATULINA

TRUNCATULINA

TRUNCATULINA

TRUNCATULINA

TRUNCATULINA

TRUNCATULINA

TRUNCATULINA

TRUNCATULINA

TRUNCATULINA

TRUNCATULINA

TRUNCATULINA

TRUNCATULINA
TRUNCATULINA

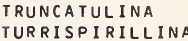

UVIGER INA

UVI GER INA

UVIGER INA

UVIGERINA

UVIGER INA

UV I GER INA

UVIGERINA

UVI GER INA

UVIGERINA

UVI GER INA

UV I GER INA

UVI GERINA

UVI GER INA

UV I GER INA

UV I GER INA

UV IGER INA

UVIGERINA

UVIGERINA

UV I GER INA

UVI GER INA

UV I GER I NA

UV I GER INA

UVI GER INA

UVIGER INA

UVI GER INA

UVI GER INA

UVI GER INA

UVIGERINA

UVIGERINA

UVI GER INA

UV I GER INA

UV I GER INA

UVI GERINA

UVI GER INA

UVI GER INA

UVI GER INA
PAC IFICA

PAC IF ICA

PACIFICA SIMPLEX

ROTALI FOR IS

ROTALIFORMIS

ROTALI FORMIS

ROTALI FORMI

ROTALIF ORMIS

ROTALIFORMIS

ROT AL IFORMIS

ROTALIFOR

ROTALIFORMIS

ROTALIFORMIS

ROT ALIFORMIS

ROTALI FORMI

ROT ALIFORMIS

SQU AMATA

SQU AMATA

SOUAMATA

SQU AMATA

SQUAMATA

SQU AMATA

SQU AMATA

SQU AMATA

SQUAMATA

SQU AMATA

SQUAMATA

SQU AMATA

SQUAMATA

SQUAMI FORMIS

SQU AMI F ORMI

SQUAMI FORMI

SQU AM I FORM I S

TURB INATA

LOBAT ULA

LOBATULA

LOBATULA

LOB ATUL A

LOBATULA

LOBATULA

LOB ATUL A

LOBATULA

LOBATULA

LOBATULA

LOBATULA

LOBATULA

LOBATULA

LOBATULA

LOBATULA

LOB ATULA

LOBATULA

LOBATULA

LOBATULA

LOB ATULA

WUELLERSTORF

WUELLER

A RC TICA

AUB ER I ANA

AUB ERI ANA

AUBER I ANA

AUBERIANA

AUBERIANA

CUSHMAN I

DIRUPTA

DIRUPTA

DIRUPTA

DIRUPTA

DIRUPTA

DIRUPTA

OIRUPTA

DIRUPTA

JUNCEA

JUNCEA

JUN CEA

JUNCEA

JUNCEA

JUNCEA

JUNCEA

JUNCEA

JUN CEA

JUNCEA

JUNCEA

JUN CEA

JUN CEA
JUN CEA

JUNCEA

JUNCEA

JUNCEA

JUNCEA

JUN CEA

JUNCEA

JUNCEA

JUNCEA

JUNCEA

\section{Locality}

Lat. Long.

KETCHIKANE ALASKA

OFF ALASKA

WASHINGTON COAST

EXCURSION. ALASKA 


\section{Publication}

12366 BERGEN O'NEIL 1979 12367 BERGEN O'NEIL 1979 12368 BERGEN O'NEIL 1979 12369 BERGEN O'NEIL 1979 12370 BERGEN O.NEIL 1979 $\begin{array}{lll}12370 & \text { BERGEN } \\ 12371 & \text { BERGEN O N NEIL } 1979 \\ 12372 & \text { BERGEN O.NE IL } 1979\end{array}$ 12372 BERGEN O'NEIL 1979 12373 BERGEN O'NEIL 1979 12374 BERGEN O'NEIL 1979 12376 BERGEN O.NE IL 1979 12377 SMITH 1973

12378 TODD LOW 1967

12379 TODD LOW 1967

12380 TODD LOW 1967

12381 TODD LOW 1967

12382 BERGEN O.NEIL 1979

12383 BERGEN O'NEIL 1979

12384 BERGEN O'NEIL 1979

12385 BERGEN O'NEIL 1979

12386 CUSHMAN MCCULLOCH

12387 BERGEN O'NE IL
12388 TODD LOW 1967

12388 TODD LOW 1967

$\begin{array}{lll}12389 & \text { BERGEN O'NEIL } 1979 \\ 12390 \text { BERGEN O'NEIL } 1979\end{array}$

12390 BERGEN O 'NEIL 1979

12391 BERGEN O'NEIL 1979

12392 BERGEN O'NEIL 1979

12393 BERGEN O'NEIL 1979

12394 BERGEN O'NEIL 1979

12395 BERGEN O'NEIL 1979

12396 BERGEN O'NEIL 1979

12396 BERGEN O NEIL 1979

12397 BERGEN O'NEIL 1979

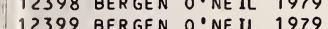

$\begin{array}{lll}12399 & \text { BERGEN O०NE IL } 1979 \\ 12400 & \text { BERGEN O'NE IL } 1979\end{array}$

12400 BERGEN O'NEIL 1979

12401 BERGEN O'NEIL 1979

12402 BERGEN O'NEIL 1979

12403 BERGEN O'NEIL 1979

12404 BERGEN O'NEIL 1979

12405 BERGEN O'NEIL 1979

12406 CUSHMAN 1927

12407 BERGEN O'NEIL 1979

12408 BERGEN OONEIL 1979

12409 BERGEN O.NEIL 1979

12410 BERGEN O'NEIL 1979

12411 BERGEN O'NEIL 1979

12412 BERGEN O'NEIL 1979

12413 BERGEN O'NE IL 1979

12414 BERGEN O'NEIL 1979

12415 BERGEN O.NEIL 1979

12416 BERGEN O NEIL 1979

12417 BERGEN ONNEIL 1979

12418 SMITH 1973

12419 SMITH 1973

12420 TODD LOW 1967

12421 TODD LOW 1967

12422 SMI TH 1973

12423 BERGEN O'NE IL 1979

12424 BERGEN O'NEIL 1979

12425 BERGEN O'NEIL 1979

12426 BERGEN O'NEIL 1979

12427 BERGEN O.NEIL 1979

12428 BERGEN O.NEIL 1979
Generic Name Specific Name

UVI GER INA

UVIGER INA

UVI GER INA

UVI GER INA

UVI GER I NA

UVI GER INA

UVI GER INA

UVI GERINA

UVI GERINA

UVI GER INA

UVI GER INA

UVI GER INA

UVI GER INA

UV I GER INA

UVI GER INA

UVIGER INA

UVI GER INA

UVI GER INA

UVIGER INA

UVI GER INA

UVI GER INA

VAG INUL INOPSIS

VAG INUL INOPS IS

VAL VUL INER IA

VAL VUL INER IA

VALVUL INERIA

VAL VUL INER IA

VAL VUL INER I A

VAL VUL INER IA

VAL VUL INER IA

VALVUL NER IA

VAL VUL INER IA

VALVUL INER IA

VAL VUL INER I A

VALVUL INER IA

VAL VUL INER I A

VAL VUL INER IA

VAL VUL INER IA

VAL VUL INER IA

VAL VUL INER IA

VERNEUIL INA

VIRGUL INA

VIRGUL INA

VIRGUL INA

VIRGUL INA

VIRGULINA

VIRGUL INA

VIRGUL INA

VIRGULINA

VIRGULINA

VIRGUL INA

VIR GUL INA

VIRGULINA

VIRGUL INA

VIRGUL INA

VIRGULINA

VIRGUL INA

VIRGULINA

VIRGULINA

VIRGUI INA

VIRGULINA

VIRGULINA

VIRGULINA

VIRGUL INA

VIRGULINA
Locality

GULF OF ALASKA

GULF OF ALASKA

GULF OF ALASKA

GULF OF ALASKA

GULF OF ALASKA

GULF OF ALASKA

GULF OF ALASKA

GULF OF ALASKA

GULF OF ALASKA

GULF OF ALASKA

GULF OF ALASKA

OFF ALASKA PENINSULA

CLARENCE ST.. ALASKA

GULF OF ALASKA

KASAAN BAY, ALASKA

TAKU HARBOUR, ALASKA

GULF OF ALASKA

GULF OF ALASKA

GULF OF ALASKA

GULF OF ALASKA

KETCHIKAN, ALASKA

GULF OF ALASKA

GULF OF ALASKA

GULF OF ALASKA

GULF OF ALASKA

GULF OF ALASKA

GULF OF ALASKA

GULF OF ALASKA

GULF OF ALASKA

GULF OF ALASKA

GULF OF ALASKA

GULF OF ALASKA

GULF OF ALASKA

GULF OF ALASKA

GULF OF ALASKA

GULF OF ALASKA

GULF OF ALASKA

GULF OF ALASKA

GULF OF ALASKA

OFF OREGON

GULF OF ALASKA

GULF OF ALASKA

GULF OF ALASKA

GULF OF ALASKA

GULF OF ALASKA

GULF OF ALASKA

GULF OF ALASKA

GULF OF ALASKA

GULF OF ALASKA

GULF OF ALASKA

GULF OF ALASKA

KODIAK. ALASKA

OFF ALASKA PENINSULA

CLARENCE ST.. ALASKA

EXCURSION, ALASKA

OF $F$ ALASKA PENINSULA

GULF OF ALASKA

GULF OF ALASKA

GULF OF ALASKA

GULF OF ALASKA

GULF OF ALASKA

GULF Of ALASKA
Lat. Long.

$5955 \mathrm{~N} 14225 \mathrm{~W}$

$5945 N 14455 \mathrm{~W}$

$5925 \mathrm{~N} 14525 \mathrm{~W}$

$5930 N 14520 \mathrm{~W}$

$5930 \mathrm{~N} 14300 \mathrm{~W}$

$5930 N 14545 \mathrm{~W}$

$5635 N 15150 \mathrm{~W}$

$5930 \mathrm{~N} 14540 \mathrm{~W}$

$5900 \mathrm{~N} 14120 \mathrm{~W}$

$5850 \mathrm{~N} 14115 \mathrm{~W}$

$5935 \mathrm{~N} 14300 \mathrm{~W}$

$5353 \mathrm{~N} 16140 \mathrm{~W}$

$5521 \mathrm{~N} 13158 \mathrm{~W}$

$5932 \mathrm{~N} 14236 \mathrm{~W}$

$5526 \mathrm{~N} 13214 \mathrm{~W}$

$5802 \mathrm{~N} 13400 \mathrm{~W}$

$5930 \mathrm{~N} 14520 \mathrm{~W}$

59 OON $14120 \mathrm{~W}$

$5930 \mathrm{~N} 14300 \mathrm{~W}$

$5920 N \quad 14538 \mathrm{~W}$

$5925 \mathrm{~N} 145 \mathrm{~S}$

$5932 \mathrm{~N} 14236 \mathrm{~W}$

$5925 \mathrm{~N} 14525 \mathrm{~W}$

$5920 N 14538 \mathrm{~W}$

$5930 \mathrm{~N} 14520 \mathrm{~W}$

$5930 N 14545 \mathrm{~W}$

$5930 \mathrm{~N} 14255 \mathrm{~W}$

$5930 \mathrm{~N} 14540 \mathrm{~W}$

$5930 \mathrm{~N} 14300 \mathrm{~W}$

59 OON $14120 \mathrm{~W}$

$5930 N 14300 \mathrm{~W}$

58 SON $14115 \mathrm{~W}$

$5930 \mathrm{~N} 14545 \mathrm{~W}$

$5930 N 14255 \mathrm{~W}$

59 OON $14120 \mathrm{~W}$

$5925 N 14525 \mathrm{~W}$

$5920 \mathrm{~N} 14538 \mathrm{~W}$

$5930 N 14520 \mathrm{~W}$

$5930 N 14540 \mathrm{~W}$

$4314 \mathrm{~N} 12443 \mathrm{~W}$

$5935 \mathrm{~N} 14300 \mathrm{~W}$

$5930 N 14540 \mathrm{~W}$

$5930 \mathrm{~N} 14300 \mathrm{~W}$

$5920 \mathrm{~N} 14538 \mathrm{w}$

$5930 \mathrm{~N} 14520 \mathrm{~W}$

59 OON $14120 \mathrm{~W}$

$5920 N 14538 \mathrm{~W}$

$5930 \mathrm{~N} 14540 \mathrm{~W}$

59 OON $14120 \mathrm{~W}$

$5930 \mathrm{~N} 14520 \mathrm{~W}$

$5925 \mathrm{~N} 14525 \mathrm{~W}$

$5749 \mathrm{~N} 15230 \mathrm{~W}$

$5623 N 15427 \mathrm{~W}$

$5521 \mathrm{~N} 13158 \mathrm{~W}$

$5825 \mathrm{~N} 13530 \mathrm{~W}$

$5353 \mathrm{~N} 16140 \mathrm{~W}$ 59 OON $14120 \mathrm{~W}$ $5930 N 14520 \mathrm{~W}$ $5930 N 14255 W$ 59 OON $14120 \mathrm{~W}$ $59.30 \mathrm{~N} 14300 \mathrm{~W}$ 
Catalog 2

\section{Synonymized Species with Publication and Location}

\section{Publication}

10001 COCKBAIN 1963

10002 COCKBAIN 1963

10003 COCKBAIN 1963

10004 COCKBAIN 1963

10005 COCKBAIN 1963

10006 COCKBAIN 1963

10007 COCKBAIN 1963

10008 COCKBAIN 1963

10009 BERGEN O'NEIL 1979

10010 BERGEN O'NEIL 1979

10011 BERGEN O'NEIL 1979

10012 BERGEN O'NEIL 1979

10013 BERGEN O'NEIL 1979

10014 BERGEN O'NEIL 1979

10015 SMITH 1973

10016 SMI TH 1973

10017 SMITH 1973

10018 SMI TH 1973

10019 SMITH 1973

10020 COOPER 1961

10021 COOPER 1961

10022 LANKFORD PHLEGER 1973

10023 LANKFORD PHLEGER 1973

10024 COC KBAIN 1963

10025 COC KBAIN 1963

10026 COCKBAIN 1963

10027 COCKBAIN 1963

10028 COCKBAIN 1963

10029 COCKBAIN 1963

10030 COCKBAIN 1963

10031 COCKBAIN 1963

10032 SMITH 1973

10033 SMITH 1973

10034 SMITH 1973

10035 SMITH 1973

10036 SMITH 1973

10037 SMITH 1973

10038 SMITH 1973

10039 SMITH 1973

10040 COCKBAIN 1963

10041 COCKBAIN 1963

10042 COCKBAIN 1963

10043 COCKBAIN 1963

10044 COCKBAIN 1963

10045 SMITH 1973

10046 SMITH 1973

10047 SMITH 1973

10048 SMITH 1973

10049 SMI TH 1973

10050 SMITH 1973

10051 SMITH 1973

10052 SMITH 1973

10053 SMITH 1973

10054 SMITH 1973

10055 SMITH 1973

10056 SMI TH 1973

10057 BERGEN O'NEIL 1979

10058 SMITH 1973

10059 SMITH 1973

10060 SMITH 1973

10061 SMITH 1973

10062 TODD LOW 1967

10063 CUS HMAN MCCULLOCH 1939

10064 CUSHMAN MCCULLOCH 1939

10065 CUSHMAN MCCULLOCH 1939

10066 CUSHMAN MCCULLOCH 1939

10067 JONES ROSS 1979

10068 SCOTT 1974

10069 PHLEGER 1967

10070 PHLEGER 1967

10071 SMITH 1973

10072 SMITH 1973

10073 TODD LOW 1967

10074 TODD LOW 1967

10075 TODD LOW 1967

10076 TODD LOW 1967

10077 TODD LOW 1967

10078 BERGEN O.NEIL 1979

10079 BERGEN O.NE IL 1979

10080 BERGEN O'NEIL 1979

10081 BERGEN O'NEIL 1979

10082 BERGEN O'NEIL 1979

10083 BERGEN O'NEIL 1979

10084 BERGEN O.NEIL 1979

10085 BERGEN O'NEIL 1979

\section{Generic Name Specific Name}

ADERCOTRYMA

ADERCOTRYMA

ADERCOTRYMA

ADERCOTRYMA

ADERCOTRYMA

ADERCOTRYMA

ADERC OTRYMA

ADERCOTRYMA

ADERCOTRYMA

ADERCOTRYMA

A DERCOTRYMA

ADERCOTRYMA

ADERCOTRYMA

ADERCOTRYMA

ADERCOTRYMA

ADERCOTRYMA

ADERCOTRYMA

HAPLOPHRAGMOIDES

HAP LOPHRAGMOIDE

HAPLOPHRAGMOIDES

HAPLOPHRAGMOIDES

HAPLOPHRAGMOIDES

HAPLOPHRAGMOIDES

HAPLOPHRAGMOIDES

HAPLOPHRAGMOIDES

HAPLOPHRAGMOIDES

HAPLOPHRAGMOIDE

HAPLOPHRAGMOIDES

HAPLOPHRAGMOIDES

CRIBROSTOMOIDES

CRIBROSTOMOIDES

CRIBROSTOMOIDES

CRI BROST OMOIDES

CRIBROSTOMOIDES

CRIBROSTOMOIDES

HAP LOPHRAGMOIDES

HAPLOPHRAGMOIDES

HAPLOPHR AGMOIDES

HAPLOPHRAGMOIDES

HAPLOPHRAGMOIDES

HAPLOPHRAGMOIDES

HAPLOPHRAGMOIDES

HAPLOPHRAGMOIDES

CRIBROSTOMOIDES

CRI BROST OMOIDES

CRIBROSTOMOIDES

CRIBROST OMOIDES

CRI BROST OMOIDES

CRIBROSTOMOIDES

CRIBROST OMOIDES

CRIBROSTOMOIDES

CRIBROSTOMOIDES

AMMOBA CUL I TES

AMMOBACULITE

AMM OBACULITES

AMMOBACULITES

AMMOBACULITES

AMM OBACULITES

AMMOBACULITES

AMMOBACULITES

AMMOTIUM

AMMOT I UM

AMMOTIUM

AMMOTIUM

AMMOBACULITES

AMM OBACULITES

AMMOBACULITES

AMMOBACULITES

AMM OBACULITES

AMMOBACULITES

AMMODISCUS

AMMODISCUS

AMMODISCUS

AMMODISCUS

AMMODISCUS

AMMODISCUS

AMMODISCUS

AMMODISCUS

AMMODISCUS

AMMODISCUS

AMMODISCUS

AMMODISCUS
GLOMERATA

GLOMERATA

GLOMERATA

GLOMERATA

GLOMERATA

GLOMERATA

GLOMERATA

GLOMERATA

GLOMERATA

GLOMERATA

GLOMERATA

GLOMERATA

GLOMERATA

GLOMERATA

GLOMERATA

GLOMERATA

GLOMERATA

C OL UMB IENS IS

COLUMBIENSIS

C OL UMB IENSIS

COL UMB IENSIS

COL UMB IENS IS

COL UMB IENSIS

COLUMBIENSIS

COL UMB I ENS IS

C OL UMB IENSIS

COLUMB I ENS I S

C OL UMB IENSIS

COLUMBIENSIS

NIT I DUM

NITI DUM

NIT I DUM

NITIDUM

NITI DUM

NIT I DUM

RINGENS

RINGENS

COL UMB I ENSIS

C OL UMB I ENS IS

C OL UMB I ENS IS

COL UMB I ENSIS

COLUMB IENS IS

SCI TULUM

SUB GLOB OSUM

SUBGLOB OSUM

SUBGLOBOSUM

SUBGLOBOSUM

SUBGLOBOSUM

SUBGLOBOSUM

SUBGLOB OSUM

WIE SNER I

W IE SNER I

AGGLUTINANS

A GG LUTINANS

AGGLUT INANS

FILIFORMIS

FIL I FORMIS

AME RICANUS

AMERICANUS

ARENARI US

CASSIS

CASSIS

CASSIS

CASSIS

EXIGUUS

EXIGUUS

EXIGUUS

EXIGUUS

FILIFORMIS

FILIFORMIS

ARENACEUS

ARENACEUS

GULLMARENSIS

GULLMARENS IS

GULLMARENSIS

GULLMARENSIS

MINUTISSIMUS

MINUTISSIMUS

MINUTISSIMUS

MINUTISSIMUS

MINUTISSIMUS

MINUT IS SIMUS

MINUTISSIMUS

MINUTISSIMUS

\section{Locality}

BRITISH COLUMBIA

BR ITISH COL UMBIA

BRITISH COLUMBIA

BRITISH COLUMBIA

BR ITISH COLUMBIA

BR IT ISH COL UMBIA

BRITISH COLUMBIA

BRITISH COLUMBIA

GULF OF ALASKA

GULF OF ALASKA

GULF OF ALASKA

GULF OF ALASKA

GULF OF ALASKA

OFF ALASKA PENINSULA

OFF ALASKA PENINSULA

NORTH PACIF IC

OFF ALASKA PENINSULA

OFF ALASKA PENINSULA

OREGON COAST

OREGON COAST

DEPOE BAY, OREGON

CRESCENT CITY, CALIF.

BRITISH COLUMBIA

BRITISH COLUMBIA

BRITISH COLUMBIA

BRIT ISH COLUMBIA

BRITISH COLUMBIA

BR ITISH COL UMBIA

BRITISH COLUMBIA

BRITISH COLUMB IA

OFF ALASKA PENINSULA 


\section{Publication}

10086 BERGEN O'NEIL 1979 10087 BERGEN O'NEIL 1979

10088 SMITH 1973

10089 SMITH 1973

10090 SMITH 1973

$\begin{array}{lll}10091 & \text { BERGEN O'NEIL } 1979 \\ 10092 & \text { BERGEN O'NE IL } 1979\end{array}$

$\begin{array}{lll}10092 & \text { BERGEN O'NEIL } 1979 \\ 10093 & \text { BERGEN O'NE IL } 1979\end{array}$

10093 BERGEN O'NEIL 1979

10095 JONES ROSS 1979

0096 SCOTT 1974

10097 BRADSHAW 1961

10098 BERGEN O'NEIL 1979

10099 BERGEN O'NEIL 1979

10100 TODD LOW 1967

10101 TODD LOW 1967

0102 COC KBAIN 1963

103 BERGEN O.NEIL 1979

10104 BERGEN O'NEIL 1979

10105 BERGEN O'NEIL 1979

10106 COCKBAIN 1963

10107 COCKBAIN 1963

10108 COCKBAIN 1963

10109 COCKBAIN 1963

10110 COCKBAIN 1963

10111 COCKBAIN 1963

0112 COCKBAIN 1963

10113 PHLEGER 1967

10114 PHLEGER 1967

10115 JONES ROSS 1979

10116 PHLEGER 1967

10118 LANKFORD PHLEGER 1973

10119 LANKFORD PHLEGER 1973

10120 CUS HMAN MCCULLOCH 1948

10121 CUSHMAN MCCULLOCH 1948

10122 COCKBAIN 1963

0123 COCKBAIN 1963

0124 COCKBAIN 1963

10125 COCKBAIN 1963

10126 COCKBAIN 1963

10127 SMITH 1973

10128 CUSHMAN MCCULLOCH 1948

10129 CUSHMAN MCCULLOCH 1948

10130 TCDD LOW 1967

10131 TODD LOW 1967

0132 TODD LOW 1967

10133 TOD D LOW 1967

10134 TODD LOW 1967

0135 DETLING 1958

10136 CUSHMAN TODD 1947

10137 COOPER 1961

10138 COCKBAIN 1963

10139 COCKBAIN 1963

10140 COCKBAIN 1963

10141 COCKBAIN 1963

10142 CUSHMAN TODD 1947

10143 CUSHMAN TODD 1947

10144 CUSHMAN TODD 1947

10145 CUSHMAN 1915

0146 NAT LAND 1933

10147 BERGEN O NEIL 1979

10148 BERGEN O०NEIL 1979

10149 BERGEN O'NEIL 1979

10150 TODD LOW 1967

10151 TODD LOW 1967

10152 TODD LOW 1967

10153 TODD LOW 1967

10154 TODD LOW 1967

10155 BERGEN O'NEIL 1979

10156 BERGEN O NEIL 1979

10157 BERGEN O NEIL 1979

10158 BERGEN O'NEIL 1979

10159 BERGEN O'NEIL 1979

10160 BERGEN O'NEIL 1979

10161 BERGEN O'NEIL 1979

10162 BERGEN O'NEIL 1979

10163 DETLING 1958

10164 COCKBAIN 1963

10165 COCKBAIN 1963

10166 COCKBAIN 1963

10167 BERGEN O'NEIL 1979

10168 BERGEN O'NE IL 1979

10169 BERGEN O'NEIL 1979

10170 BERGEN O'NEIL 1979

10171 BERGEN O'NEIL 1979

10172 CUSHMAN TODD 1947

10173 CUSHMAN EDWARDS 1937

10174 CUSHMAN TODD 1947

10175 CUSHMAN 1927

10176 CUSHMAN 1927

10177 CUSHMAN 1927

10178 CUS HMAN 1917

10179 CUSHMAN 1927

10180 TODD LOW 1967
Generic Name Specific Name

PAC-IFICUS

PACIFICUS

AMMODISCUS

AMM OMA RG INUL INA

AMM OMAR G INUL INA

AMM OMAR G I NUL I NA

AMMOMARG INUL INA

AMM OMA R G INUL INA

AMM OMARG INUL INA

AMM OMARG INULINA

AMMONIA

AMMONIA

AMMONI A

AMM OSCALARIA

AMMOSCALARIA

AMMOTIUM

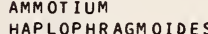

HAPLOPHRAGM OIDES

HAPLOPHRAGMOIDES

HAPLOPHRAGMOIDES
HAPLOPHRAGMOIDES

HAPLOPHRAGMOIDES

HAPLOPHRAGMOIOES

HAPLOPHRAGMOIDES

HAPLOPHRAGMOI DES

HAPLOPHRAGMOIDES

HAPLOPHRAGMOIDES

HAPLOPHRAGMOIDES

AMMOT IUM

AMMOTIUM

AMMOTIUM

AMMOTIUM

ANGULOD IS CORB IS

ANGULOD I SCORB IS

TR I FAR I NA

RI FARINA

TR I FAR INA
TRI FAR INA

TRI FAR INA

TRI FAR I NA

TRIFAR INA

TR I FAR INA

TRIFAR INA

TRIFARINA

IRI FARINA

TRI FAR INA

TRI FAR I NA

TRI FAR INA

TRIFAR INA

TRI FAR I NA

TR I FAR INA

TRIFAR INA

TRIFARINA

TRI FAR INA

TRIFARINA

TR I FAR INA

TR IFAR INA

PLANUL INA

ASTACOLUS

ASTACOLUS

ASTACOLUS

ASTACOLUS

ASTACOLUS

ASTRONONION

ASTRONONION

ASTRONONION

ASTRONONION

ASTRONONION

ASTRONONION

ASTRONONION

ASTRONONION

ASTRONONION

ASTRONONION

ASTRONONION

ASTRONONION

AST RONONION

ASTRONONION

ASTRONONION

ASTRONONION

ASTRONONION

ASTRONONION

ASTRONONION

ASTRONONION

ASTRONONION

ASTRONONION

ASTRONONION

BATHYSIPHON

PYRGO

PYRGO

PYRGO

BILOCUL INELLA

FOL I ACEA

F OL I ACEA

SANDIEGOENSIS

SANDIEGOENSIS

SANDIEGOENS IS

SANDIEGOENSIS

BEC CARI I

BECCARI

BECCARII

P SE UDOSPIRAL IS

CASSIS

CASSIS

PLANISSIMUM

PLANISS IMUM

PLANISSIMUM

PLANISS IMUM

PLANISSIMUM

PLANISSIMUM

PLANISSIMUM

PLANISSIMUM

PLANISS IMUM

PLANISSIMUM

SAL SUM

SAL SUM

SAL SUM

SAL SUM

SAL SUM

CHARLOTTENS IS

CHARLOTTENSIS

ANGULOSA

ANGULOSA

ANGULOSA

ANGULOSA

ANGULOSA

ANGULOSA

ANGULOSA

ANGULOSA

FLUENS

FLUENS

FLUENS

FLUENS

FLUENS

FLUENS

FLUENS

FLUENS

HUGHES I

SEM I TR I GONA

SEM ITRIGONA

SEM I TRI GONA

SEM ITR I GONA

SEM I TR I GONA

SEM ITRI GONA

SEM ITR I GONA

ARIMINENS IS

SCHMITTI

HYALACRULUS

HYALACRULUS

PLANULATUS

PLANULATUS

GAL LOWAYI

GAL LOWAYI

GALLOWAYI

GALLOWAYI

GALLOWAYI

GALLOWAYI

GALLOWAYI

GALLOWAYI

GALLOWAYI

GAL LOWAYI

GALLOWAYI

GALLOWAYI

GALLOWAYI

GALLOWAYI

GAL LOWAYI

GALLOWA

GALLOWA

GALLOWAYI

GALLOWAYI

GALLOWAYI

ARENACEA

DEPRESSA

LUCERNULA

LUCERNULA

GLOBULA
Locality

Lat. Long.

GULF OF ALASKA

GULF OF ALASKA

OFF ALASKA PENINSULA

PSE UDOSPIRAL IS

OFF ALASKA PENINSULA

OFF ALASKA PENINSULA

GULF OF ALASKA

GULF OF ALASKA

GULF OF ALASKA

GULF OF ALASKA

SAMISH BAY, WASH.

SAMISH-PADILLA BAYS

COOS BAY, OREGON

GULF OF ALASKA 


\section{Publication}

10181 CUSHMAN MCCULLOCH 1942 10182 CUSHMAN MCCULLOCH 1942

10183 CUSHMAN TODD 1947

10184 CUSHMAN TODD 1947

10185 TODD LOW 1967

10186 BERGEN O.NEIL 1979

10187 BERGEN O'NEIL 1979

10188 COCKBAIN 1963

10189 COCKBAIN 1963

10190 COCKBAIN 1963

10191 CUSHMAN TODO 1947

10192 LANKFORD PHLEGER 1973

10193 BERGEN O.NE IL 1979

10194 BERGEN O.NE IL 1979

10195 BERGEN O.NEIL 1979

10196 BERGEN O'NEIL 1979

10197 BERGEN O N NEIL 1979

10198 BERGEN O'NEIL 1979

10199 BERGEN O'NEIL 1979

10200 BERGEN O'NEIL 1979

10201 BERGEN O'NEIL 1979

10202 BERGEN O'NEIL 1979

10203 BERGEN O'NEIL 1979

10204 BERGEN O.NEIL 1979

10205 BERGEN O'NEIL 1979

10206 BERGEN O NEIL 1979

10207 SMITH 1973

10208 TODD LOW 1967

10209 TODD LOW 1967

10210 TODD LOW 1967

10211 TODD LOW 1967

10212 TODD LOW 1967

10213 TODD LOW 1967

10214 BERGEN O.NEIL 1979

10215 TODD LOW 1967

10216 TODD LOW 1967

10217 TODD LOW 1967

10218 BERGEN O.NEIL 1979

10219 BERGEN O'NE IL 1979

10220 COCKBAIN 1963

10221 BERGEN O.NEIL 1979

10222 COCKBAIN 1963

10223 COCKBAIN 1963

10224 COCKBAIN 1963

10225 COCKBAIN 1963

10226 COCKBAIN 1963

10227 BERGEN O'NEIL 1979

10228 BERGEN O'NEIL 1979

10229 BERGEN O'NEIL 1979

10230 BERGEN O.NEIL 1979

10231 BERGEN O.NEIL 1979

10232 BERGEN O'NEIL 1979

10233 BERGEN O'NEIL 1979

10234 BERGEN O'NEIL 1979

10235 BERGEN O'NEIL 1979

10236 CUSHMAN MCCULLOCH

10237 TODD LOW 1967

10238 COOPER 1961
10239 SMI TH 1973

10240 BERGEN O'NEIL 1979

10241 CUSHMAN 1927

10242 CUSHMAN 1927

10243 COOPER 1961

10244 BERGEN O'NEIL 1979

10245 BERGEN O'NEIL 1979

10246 BERGEN O'NEIL 1979

10247 BERGEN O N NEIL 1979

10248 BERGEN O N NEIL 1979

10249 BERGEN O०NE IL 1979

10250 BERGEN O'NE IL 1979

10251 BERGEN O'NEIL 1979

10252 BERGEN O'NEIL 1979

10253 TOOD LOW 1967

10254 TODD LOW 1967

10255 BERGEN O NEIL 1979

10256 BERGEN O.NEIL 1979

10257 SMI TH 1973

10258 SCOTT 1974

10259 TODD LOW 1967

10260 TODD LOW 1967

10261 TODD LOW 1967

10262 TODD LOW 1967

10263 TODD LOW 1967

10264 BERGEN O N NE IL 1979

10265 BERGEN O.NE IL 1979

10265 BER GEN O'NEIL 1979

10267 BERGEN O'NEIL 1979

10268 BERGEN O'NE IL 1979

10269 BERGEN O'NE IL 1979

10270 BERGEN O'NEIL 1979

10271 BERGEN O'NEIL 1979

10272 BERGEN O'NE IL 1979

10273 BERGEN O'NEIL 1979

10274 BERGEN O०NEIL 1979

10275 BERGEN O'NEIL 1979
Generic Name Specific Name

$P A C I F I C A$

BOL IVINA

BOL IVINA

BOL IVINA

BOL IVINA

BOL IVINA

BOL IVINA

BOL IVINA

BOLIVINA

BOL IVINA

BOLIVINA

BOLIVINA

BOL IVINA

BOL IVINA

BOL IVINA

BOL IVINA

BOLIVINA

BOLIVINA

BOLIVINA

BOLIVINA

BOL IVINA

BOLIVINA

BOL IVINA

BOL IVINA

BOLIVINA

BOLIVINA

BOL IVINA

BOLIVINA

BOL IVINA

BOL IVINA

BOLIVINA

BOLIVINA

BOL IVINA

BOL IVINA

BOLIVINA

BOL IVINA

BOLIVINA

BOLIVINA

BOL IVINA

BOL IVINA

BOL IVINA

BOL IVINA

BOLIVINA

BOLIVINA

BOL IVINA

BOLIVINA

BOLIVINA

BOL IVINA

BOL IVINA

BOL IVINA

RECTOBOL IVINA

BOL IVINA

BOL IVINA

BOLIVINA

BOL IVINA

BOL IVINA

BOL IVINA

BOL IVINA

BOLIVINA

BOL IVINA

BOL IVINA

BOL IVINA

BOLIVINA

BOL IVINA

BOLIVINA

BOL IVINA

BOL IVINA

BUCCELLA

BUCCELLA

BUCCELLA

BUCCELLA

BUCCELLA

BUCCELLA

BUCCELLA

BUCCELLA

BUCCELLA

BUCCELLA

BUCCELLA

BUCCELLA

BUCCELLA

BUCCELLA

BUCCELLA

BUCCELLA

BUCCELLA

BUCCELLA

BUC CELLA

BUCCELLA

BUCCELLA

\section{Locality}

OFF ALASKA

KETCHIKAN, ALASKA

WASHINGTON COAST

CLARENCE ST.. ALASKA

GULF OF ALASKA

GULF OF ALASKA

BRITISH COLUMBIA

BRITISH COLUMBIA

BRITISH COLUMBIA

WASHINGTON COAST

CRESCENT CITY, CALIF.

GULF OF ALASKA

GULF OF ALASKA

GULF OF ALASKA

GULF OF ALASKA

GULF OF ALASKA

GULF OF ALASKA

GULF OF ALASKA

GULF OF ALASKA

GULF OF ALASKA

GULF OF ALASKA

GULF OF $A L$

GULF OF AL

GULF OF ALASKA

GULF OF ALASKA

OFF ALASKA PENINSULA

KASAAN BAY, ALASKA

TAKU HARBOUR, ALASKA

GAMBIER BAY, ALASKA

CLARENCE ST.. ALASKA

GULF OF ALASKA

GULF OF ALASKA

GULF OF ALASKA

KASAAN BAY, ALASKA

GULF OF ALASKA

GULF OF ALASKA

GULF OF ALASK

BRIT ISH COL UMBIA

GULF OF ALASKA

BR ITISH COLUMB IA

BRITISH COL UMBIA

BRITISH COLUMBIA

BRITISH COL UMBIA

BRITISH COLUMBIA

GULF OF ALASKA

GULF OF ALASKA

GULF OF ALASKA

GULF OF ALASKA

GULF OF ALASKA

GULF OF ALASKA

GULF OF ALASKA

GULF OF ALASKA

GULF OF ALASKA

KETCHIKAN, ALASKA

GULF OF ALASKA

OREGON COAST

OFF ALASKA PENINSULA

GULF OF ALASKA

OFF OREGON

OFF OREGON

OREG ON COAST

GULF OF ALASKA

GULF OF ALASKA

GULF OF ALA

GULF OF

GULF OF ALASKA

GULF OF ALASKA

GULF OF ALASKA

GULF OF ALASKA

CLARENCE ST. ALASKA

CLARENCE ST.. ALASKA

GULF OF ALASKA

GULF OF ALASKA

KODIAK. ALASKA

SAMISH-PADILLA BAYS

GAMBIER BAY, ALASKA

EXCURSION, ALASKA

KASAAN BAY, ALASKA

LYNN CANAL, ALASKA

TAKU HARBOUR, ALASKA

GULF OF ALASKA

GULF OF ALASKA

GUiF OF ALASKA

GULF OF ALASKA

GULF OF ALASKA

GULF OF ALASKA

GULF OF ALASKA

GULF OF ALASKA

GULF OF ALASKA

GULF OF ALASKA

GULF OF ALASKA

GULF OF ALASKA

Lat. Long.

$5628 N 13223 \mathrm{~W}$ $\begin{array}{lllll}55 & 21 N & 13139 \mathrm{~W}\end{array}$ $4832 \mathrm{~N} 12300 \mathrm{~W}$ $5521 \mathrm{~N} 13158 \mathrm{~W}$ $5930 \mathrm{~N} 14540 \mathrm{~W}$ 59 OON $14120 \mathrm{~W}$ $48 \angle 5 N 12300 \mathrm{~W}$ $5000 \mathrm{~N} 12507 \mathrm{~W}$ 


\section{Publication}

10276 BERGEN O'NEIL 1979 10277 BERGEN O'NEIL 1979 10278 ECHOLS 1969 10279 ECHOLS 1969

10280 ECHOLS 1969

10281 JONES ROSS 1979

10282 BERGEN O.NEIL 1979

10283 DETLING 1958

10284 SMITH 1973

10285 BERGEN O'NEIL 1979

10286 BERGEN O'NEIL 1979

10287 BERGEN O'NEIL 1979

10288 COOPER 1961

10289 COOPER 1961

10290 COOPER 1961

10291 COOPER 1961

10292 COOPER 1961

10293 COOPER 1961

10294 ECHOLS 1969
10295 ECHOLS 1969

10295 ECHOLS 1969
10296 ECHOLS 1969

10297 LANKFORD PHLEGER 1973

10298 LANKFORD PHLEGER 1973

10299 LANKFORD PHLEGER 1973

10300 CUSHMAN 1927

10301 SMITH 1973

10302 CUS HMAN TODD 1947

10303 BERGEN O'NEIL 1979

10304 BERGEN O'NEIL 1979

10305 BERGEN O'NEIL 1979

10306 BERGEN O'NE IL 1979
10307 BERGEN O'NE IL 1979

10307 BERGEN O'NEIL 1979

10308 BERGEN O'NE IL
10309 CUSHMAN 1927

10310 SMITH 1973

10311 BERGEN O'NEIL 1979

10312 BERGEN O'NEIL 1979

10313 BERGEN O'NEIL 1979

10314 BERGEN O'NEIL 1979

10315 BERGEN O'NEIL 1979

10316 BERGEN O'NEIL 1979

10317 CUSHMAN 1927

10318 CUSHMAN 1927

10319 CUSHMAN 1927

10320 CUS HMAN 1927

10321 CUSHMAN 1927

10322 CUSHMAN 1927

10323 CUS HMAN 1927

10324 CUSHMAN 192

10325 CUSHMAN 1927

10326 CUSHMAN 1927

10328 WHITEAVES 1886

10329 BERGEN O'NEIL 1979

10330 BERGEN O'NEIL 1979

10331 BERGEN O'NEIL 1979

10332 BERGEN O'NEIL 1979

10333 BERGEN O'NEIL 1979

$\begin{array}{lll}10334 & \text { BERGEN O०NEIL } 1979 \\ 10335 & \text { BERGEN O०NEIL } 1979\end{array}$

10335 BERGEN O'NEIL 1979

10336 BERGEN O'NEIL 1979

10338 SMI TH 1973

10339 BERGEN O'NEIL 1979

10340 BERGEN O'NEIL 1979

10341 BERGEN O'NEIL 1979

10342 BERGEN OONEIL 1979

10343 BERGEN O'NE IL 1979

10344 BERGEN O'NE IL 1979

10345 COCKBAIN 1963

10346 COCKBAIN 1963

10347 COCKBAIN 1963

10348 COCKBAIN 1963

10349 COOPER 1961

10350 COOPER 1961

10351 COOPER 1961

10352 CUSHMAN 1925

10353 TODD LOW 1967
10354 TODD LOW 1967

10354 TODD LOW 1967

10355 CUSHMAN MCCULLOCH 1948

10356 CUS HMAN MCCULLOCH 1948
10357 CUSHMAN MCCULLOCH 1948

10358 ECHOLS 1969

10359 ECHOLS 1969

10360 ECHOLS 1969

10361 DETLING 1958

10362 BERGEN O'NEIL 197

10363 LANKFORD PHLEGER 1973

10364 LANKFORD PHLEGER 1973

10365 LANKFORD PHLEGER 1973

10366 PHLEGER 196

10367 SMITH 1973

10368 CUS HMAN TODD 1947

10369 CUSHMAN TODD 1947

10370 CUSHMAN MCCULLOCH 1948
Generic Name Specific Name Locality

GULF OF ALASKA

FRIGIDA

FRIGIDA

FRIGIOA

FRIGIDA

FRIGIDA

I NUS ITATA

INUSITATA

INU SITATA

INU SITATA

I NU SITATA

TEN ERR IMA

TENERRIMA

TENERR IMA

TENERR IMA

TENERR IMA

TENERR IMA

TENERR I MA

TENERR I MA

TENERR IMA

TENERR IMA

TENERR IMA

AFFINIS

AUR I CULATA

A UR I CULATA

BAR BATA

BAR BATA

BARBATA

BARBATA

BARBATA

BARBATA

INF LATA

INF LATA MEXI CANA

INFLATA MEXI CANA

INF LATA MEXI CANA

INF LATA MEXI CANA

INF LAT A MEXI CANA

INF LATA MEXI CANA

INF LATA MEXI CANA

OVATA

OVATA

OVATA

OVATA

OVATA

OVATA

OVATA

OVULA

OVULA

OVULA

OVULA

PYRULA

ROSTRATA

OS TRATA

SUBACUMINATA

SUB ACUMINATA

SUBACUMINATA

SUBACUMINATA

SUBACUMINATA

SUB ACUMINATA

SUBACUMINATA

BAS I COSTATA

ELEGANTIS SIMA

ELE GANT IS SIMA

ELEGANT ISSIMA

ELEGANT IS SIMA

ELEGANTISSIMA

ELEGANT ISS IMA

ELEGANTISSIMA

ELE GANT ISSIMA

ELE GANT IS SIMA

ELE GANT IS SIMA

ELEGANT ISSIMA

ELE GANT ISSIMA

ELEGANT ISSIMA

ELEGANTISSIMA

ELEGANT ISSIMA

ELE GANT IS SIMA

ELEGANT IS SIMA

ELEGANTI IS IMA

ELE GANT ISS IMA

ELEGAN IS SIMA

ELEGANTISSIMA

ELEGANTISSIMA

ELE GANTIS SIMA

ELEGANTISSIMA

ELEGANTISSIMA

ELEGANT ISSIMA

ELE GANTISSIMA

ELE GANT ISSIMA

ELEGANT IS SIMA

ELE GANT I SSIMA

ELE GANT ISSIMA

ELE GANT IS SIMA

ELE GANT IS SIMA
GULF OF ALASKA

WASHINGTON COAST

WASHINGTON COAST

WASHINGTON COAST

SAMISH BAY, WASH.

GULF OF ALASKA

SUNSET BAY, OREGON

KODIAK, ALASKA

GULF OF ALASKA

GULF OF ALASKA

GULF OF ALASKA

OREGON COAST

OREGON COAS

OREGON COAST

OREGON COAST

OREG ON COAST

CALIFORNIA COAST

WASHINGTON COAST

WASHINGTON COAST

WASHINGTON COAST

DEPOE BAY, OREGON

CRESCENT CITY, CALIF.

LAPUSH, WASHINGTON

OFF OREGON

OFF ALASKA PENINSULA

WASHINGTON COAST

GULF OF ALASKA

GULF OF ALASKA

GULF OF ALASKA

GULF OF ALASKA

GULF OF ALASKA

GULF OF ALASKA

OFF OREGON

OFF ALASKA PENINSULA

GULF OF ALASKA

GULF OF ALASKA

GULF OF ALASKA

GULF OF ALASKA

GULF OF ALASKA

GULF OF ALASKA

OFF OREGON

OFF OREGON

OFF OREGON

OFF OREGON

OFF OREGON

OFF OREGON

OFF OREGON

OFF OREGON

OFF OREGON

OFF OREGON

OFF OREGON

JOHNSTONE STRAIT, B.C.

GULF OF ALASKA

GULF OF ALASKA

GULF OF ALASKA

GULF OF ALASKA

GULF OF ALASKA

GULF OF ALASKA

GULF OF ALASKA

GULF OF ALASKA

GULF OF ALASKA

OFF ALASKA PENINSULA

GULF OF ALASKA

GULF OF ALASKA

GULF OF ALASKA

GULF OF ALASKA

GULF OF ALASKA

GULF OF ALASKA

BRITISH COLUMBIA

BRITISH COLUMBIA

BR ITISH COLUMB IA

BRITISH COLUMBIA

OREGON COAST

OREGON COAS T

CALIFORNIA COAST

VIRAGO SD.. B.C.

EXCURSION, ALASKA

LYNN CANAL, ALASKA

OFF ALASKA

OFF ALASKA

KETCHIKAN, ALASKA

WASH ING TON COAST

WASHINGION COAST

WASHINGTON COAS

WANHING

GULF OF ALASKA

DEPOE BAY, OREGON

CRESCENT CITY, CALIF.

LAPUSH. WASHINGTON

GRAY'S HARBOUR, WASH.

KODIAK, ALASKA

WASHING T ON COAST

WASHINGTON COAST

OFF CALIFORNIA

Lat. Long

$5630 \mathrm{~N} 15240 \mathrm{~W}$

$5835 \mathrm{~N} 13915 \mathrm{~W}$

$4730 \mathrm{~N} 12420 \mathrm{~W}$

47 OON $12410 \mathrm{~W}$

$4835 \mathrm{~N} 12228 \mathrm{~W}$

$5956 \mathrm{~N} 14425 \mathrm{~W}$

$4321 \mathrm{~N} 12420 \mathrm{~W}$ 


\section{Publication}

10371 TODD LOW 1967

10372 TODD LOW 1967

10373 CUSHMAN 19??

10374 CUSHMAN 1927

10375 CUSHMAN 1927

10376 CUSHMAN 1927

10377 BERGEN O'NEIL 1979

10378 BERGEN O'NEIL 1979

10379 BERGEN O.NE IL 1979

10380 BERGEN O'NEIL 1979

10381 BERGEN O'NEIL 1970

10382 BERGEN O.NEIL 1979

10383 BERGEN O'NEIL 1979

10384 BERGEN O'NEIL 1979

10385 CUSHMAN TODD 1947

10386 CUS HMAN TODD 1947

10387 BERGEN O'NEIL

10389 BERGEN O.NEIL 1979

10390 BERGEN O.NEIL 1979

10391 TODD LOW 1967

10392 TODD LOW 1967

10393 TODD LOW 1967

10394 TODD LOW 1967

10395 BERGEN O'NEIL 1979

10396 BERGEN O'NEIL 1979

10397 BERGEN O'NEIL 1979

10398 BERGEN O'NEIL 1979

10399 BERGEN O.NEIL 1979

10400 BERGEN O.NE IL 1979

10401 BERGEN O०NEIL 1979

10402 BERGEN O'NEIL 1979

10403 BERGEN O'NEIL 1979

10404 BERGEN O'NEIL 1979

10405 BERGEN O'NEIL 1979

10406 BERGEN O'NEIL 1979

10407 BERGEN O'NEIL 1979

10408 BERGEN O N NEIL 1979

10409 COCKBAIN 1963

10410 COCKBAIN 1963

10411 COCKBAIN 1963

10412 CUSHMAN 1925

10413 CUSHMAN 1925

10414 SMI TH 1973

10415 SMITH 1973

10416 BERGEN O'NEIL 1979

10417 BERGEN ODNEIL 1979

10418 BERGEN O'NEIL 1979

10419 BERGEN O.NEIL 1979

10420 BERGEN O.NE IL 1979

10421 BERGEN O'NEIL 1979

10422 BERGEN $0^{\circ}$ NEIL 1979

10423 BERGEN O.NEIL 1979

10424 BERGEN O'NEIL 1979

10425 BERGEN O'NEIL 1979

10426 BERGEN O.NEIL 1979

10427 BERGEN O.NEIL 1979

10428 CUSHMAN 1927

10429 COCKBAIN 1963

10430 COCKBAIN 1963

10431 TODD LOW 1967

10432 TODD LOW 1967

10433 TODD LOW 1967

10434 BERGEN O'NEIL 1979

10435 BERGEN O'NEIL 1979

10436 BERGEN O'NEIL 1979

10437 BERGEN O'NEIL 1979

10438 BERGEN O'NEIL 1979

10439 BERGEN O'NEIL 1979

10440 BERGEN O'NEIL 1979

$1 \cap 441$ BERGEN O'NEIL 1979

10442 BERGEN O'NEIL 1979

10443 BERGEN O'NEIL 1979

10444 BERGEN O NNEIL 1979

10445 BERGEN O'NEIL 1979

10446 BERGEN O'NEIL 1979

10447 COCKBAIN 1963

10448 COOPER 1961

10449 COOPER 1961

10450 COCKBAIN 1963

10451 COCKBAIN 1963

10452 COCKBAIN 1963

10453 COCKBAIN 1963

10454 COCKBAIN 1963

10455 COCKBAIN 1963

10456 CUSHMAN TODD 1947

10457 CUSHMAN TODD 1947

10458 CUSHMAN TODD 1947

10459 TODD LOW 1967

10460 TODD LOW 1967

10461 TOD D LOW 1967

10462 TODD LOW 1967

10462 TODD LOW 1967
10463 DETLING 1958

10464 BERGEN O'NEIL 1979

10465 BERGEN O'NEIL 1979
Generic Name Specific Name

BUL IMINA

BUL IMINA

BUL IMINA

BUL IMINA

BUL IM INA

BUL IMINA

BUL IMINA

BUL IM INA

BUL IMINA

BUL IMINA

BUL IMINA

BUL IM INA

BUL IMINA

ISL ANDIELLA

I SL AND IELLA

ISLANDIELLA

ISLANDIELLA

ISLAND IELLA

ISLANDIELLA

ISLANDIELLA

I SLANDIELLA

ISLAND IELLA

I SLANDIELLA

ISLANDIELLA

ISLAND IELLA

ISLAND IELLA

ISLANDIELLA

I SLANDIELLA

ISL AND IELLA

ISLANDIELLA

ISLANDIELLA

ISLAND IELLA

I SL AN D IELLA

ISLANDIELLA

ISLANDIELLA

I SLAND I ELLA

ISL ANDI IELLA

I SLANDIELLA

ROBERTINOIDES

CASSIDUL INA

CASSIDULINA

CASSIDULINA

CASS IDULINA

CASSIDUL INA

CASSIDUL INA

CASSIDUL INA

CASSIDULINA

CASSIDULINA

CASSIDUL INA

CASSIDUL INA

CASSIDUL INA

CASSIDULINA

CASSIDULINA

CASSIDULINA

CASSIDULINA

CASSIDULINA

CASSIDULINA

CASSIDUL INA

CASSIDULINA

CASSIDULINA

CASSIDUL INA

CASSI DUL INA

CASSIDUL INA

CASSIDULINA

CASSIDULINA

CASSIDUL INA

CASSIDUL INA

CASSIDUL INA

CASSIDULINA

CASSIDULINA

CASSIDUL INA

ISLANDIELLA

ISLAND IELLA

ISLANDIELLA

ISLANDIELLA

I SLANDIELL

I SLANDIELLA

ISLANDIELLA

I SL ANDIELLA

ISLAND IELLA

I SL AN D IELLA

I SL AND IELLA

I SLAND IELLA

I SLAND IELLA

ISLANDIELLA

ISLANDIELLA

I SL AND IELLA

ISLAND IELLA

ISLANDIELLA
SUBFUSI FORMIS

SUB FUS I FORMI S

TENUATA

TENUATA

TENUATA

TENUATA

TENUATA

TENUATA

TENUATA

IENUATA

TENUATA

TENUATA

TENUATA

CAL I FORNI CA

CAL IFORNICA

CALIFORNICA

CAL IFORNICA

CAL IFORNICA

CAL IFORNICA

C AL I FORNICA

C AL I F ORNICA

C AL IFORNICA

CAL IFORNICA

CAL I FORNICA

CAL I F ORNICA

CAL I FORNICA

C AL I FORNI CA

CAL IFORNICA

C AL I FORNICA

CAL IFORNICA

CAL I FORNI CA

C AL I FORNI CA

CAL I FORNICA

CAL IFORNICA

CAL I FORNICA

CAL IFORNICA

C AL IF ORNI CA

CHARLOTTENSIS

CRASSA

CUS HMAN I

CUS HMAN I

CUS HMAN I

C US HMAN I

CUS HMAN I

CUSHMANI

CUSHMANI

C US HMANI

DEL I CATA

DEL ICATA

DEL I CATA

DEL I CATA

DEPRESSA

DEPRESSA

BARBARA

BAR BARA

BARBARA

BAR BARA

BARBARA

BARBARA

BARBARA

BARBARA

BAR BARA

BARBARA

BARBARA

BARBARA

BARBARA

BAR BARA

BARBARA

LIMBATA

LIMBATA

LIMBATA

LIMBATA

LIMBATA

LIMBATA

L IM BATA

LIMBATA

LIMBATA

LIMBATA

LIMBATA

LIMBATA

LIMBATA

LIMBATA

LIMBATA

LIMBATA

LIMBATA

L IMBATA

Locality

Lat. Long.

CLARENCE ST.. ALASKA

GULF OF ALASKA

OF $F$ OREGON

OFF OREGON

OF F OREGON

OFF OREGON

GULF OF ALASKA

GULF OF ALASKA

GULF OF ALASKA

GULF OF ALASKA

GULF OF ALASKA

GULF OF ALASKA

GULF OF ALASKA 


\section{Publication}

10466 BERGEN O NE IL 1979 10467 BERGEN O'NEIL 1979

10468 BERGEN O'NE IL 1979

469 BERGEN OONEIL 1979

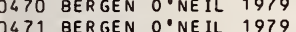

0472 BERGEN O'NEIL 1979

0473 BERGEN OONEIL 1979

0474 BERGEN O'NEIL 1979

10475 BERGEN O'NEIL 1979

10476 BERGEN O'NEIL 1979

10477 BERGEN O'NEIL 1979

0478 CUSHMAN TODD 1947

10479 CUS HMAN TODD 1947

10480 BERGEN O'NEIL 1979

10481 BERGEN O'NEIL 1979

10482 BERGEN O'NEIL 1979

10483 ECHOLS 1969

10484 ECHOLS 1969

10485 TODD LOW 1967

10486 BERGEN O०NEIL 1979

0488 COCKBAIN 1963

10488 COCKBAIN 1963
10489 TODD LOW 1967

10490 BERGEN O.NEIL 1979

0491 BERGEN O०NEIL 1979

10492 BERGEN O N NEIL 1979

0493 BERGEN O'NEIL 1979

10494 BERGEN O'NEIL 1979

10495 BERGEN O'NEIL 1979

10496 BERGEN O'NEIL 1979

10497 BERGEN O NEIL 1979

10498 BERGEN O'NEIL 1979

10499 BERGEN O'NEIL 1979

10500 BERGEN O'NEIL 1979

OSO1 BERGEN O'NEIL 1979

10502 BERGEN O०NEIL 1979

10503 BERGEN O०NEIL 1979

10504 CUSHMAN 1925

0505 CUS HMAN 1925

10506 BERGEN O NE IL 1979

10507 BERGEN O'NEIL 1979

10508 BERGEN O.NEIL 1979

10509 BERGEN O'NEIL 1979

10510 BERGEN O'NEIL 1979

OS11 BERGEN O'NEIL 1979

10512 BERGEN O'NEIL 1979

10513 BERGEN O'NEIL 1979

10514 BERGEN O०NEIL 1979

10515 BERGEN O'NEIL 1979

10516 BERGEN O०NEIL 1979

10517 SMI TH 1973

10518 TODD LOW 1967

10519 BERGEN O.NEIL 1979

10520 BERGEN O'NEIL 1979

10521 TODD LOW 1967

10522 TODD LOW 1967

10523 TODD LOW 1967

10524 BERGEN O'NEIL 1979

10525 BERGEN O०NEIL 1979

10526 BERGEN O०NEIL 1979

10527 BERGEN O'NEIL 1979

10528 BERGEN OONEIL 1979

10529 SMITH 1973

10530 TODD LOW 1967

10531 LANKFORD PHLEGER 1973

10532 SMI TH 1973

10533 TODD LOW 1967

10534 TODD LOW 1967

10535 BERGEN O'NEIL 1979

10536 BERGEN O'NEIL 1979

10536 BERGEN O'NEIL 1979

10538 BERGEN O.NEIL 1979

10538 BERGEN O'NEIL

10540 BERGEN O'NEIL 1979

$\begin{array}{lll}10540 & \text { BERGEN O'NEIL } 1979 \\ 10541 & \text { BERGEN O०NEIL } 1979\end{array}$

10542 BERGEN O०NEIL 1979

10543 CUSHMAN 1925

10544 COOPER 1961

10545 BERGEN O'NEIL 1979

10546 BERGEN O'NEIL 1979

10547 BERGEN O०NE 1979

10547 BERGEN O'NEIL 1979

10549 BERGEN O'NEIL 1979

10550 BERGEN O०NEIL 1979

10551 CUSHMAN 1927

10552 BERGEN O'NEIL 1979

10553 CUSHMAN 1927

10554 BERGEN O'NEIL 1979

10555 BERGEN O'NEIL 1979

10556 BERGEN O०NEIL 1979

10557 CUSHMAN 1927

10558 CUSHMAN 1927
10559 BERGEN O.NEIL 1979

$\begin{array}{lll}10559 & \text { BERGEN O०NEIL } & 1979 \\ 10560 & \text { BERGEN O०NEIL } & 1979\end{array}$
Generic Name Specific Name

I SL AN DIELLA

I SLAND I ELLA

ISLANDI ELLA

ISLANDIELLA

ISLANDIELLA
ISLANDIELLA

I SLANDIELLA

ISLANDIELLA

ISLANDIELLA

ISLANDIELLA

I SLANDI ILLA

ISLANDIELLA

ISLANDIELLA

CASSIDULINA

CASSIDULINA

CASSIDUL INA

CASSI DULINA

CAS SIDUL INA

ISLANDIELLA

ISLANDIELLA

I SLANDI IELLA

ISLANDIELLA

ISLANDIELLA

ISL ANDIELLA

I SLAND IELLA

ISLANDIELLA

ISLANDIELLA

I SLANDIELLA

ISLAND I ELLA

ISLANDIELLA

I LLANDIELLA

ISLANDIELLA

ISLAND IELLA

ISLANDIELLA

I SLAND IELLA

I SL AND I ELLA

I SLANDIELLA

CASSIDULINA

CASSIDUL INA

CASSIDUL INA

CASSIDUL INA

CASSIDULINA

CASSIDULINA

CASSIDULINA

CASSIDULINA

CASSIDUL INA

CASSIDULINA

CASSIDUL INA

CASSIDUL INA

CASSIDULINA

CASSIDULINA

ISLANDI IELLA

ISLANDIELLA

ISLANDIELLA

ISLANDI ILLLA

I SLANDIELLA

I SL AND I ELLA

I SLANDI ELLA

ISL AND IELLA

ISL AND IELLA

I SLANDI I LLLA

I SLANDIELLA

I SLAN DIELLA

I SLANDI ELLA

I SL AND I ELLA

I SLANDI ELLA

I SL AND IELLA

ISLANDIELLA

I SLANDIELLA

ISLANDIELLA

I SLANDIELLA

ISLANDIELLA

I SLANDIELLA

I SLANDIELLA

ISLANDIELLA

I SL AND IELLA

ISLAND IELLA

ISLAND IELLA

I SLAND IELLA

ISLAN D IELLA

I SL AND IELLA

ISLAND I ELLA

I SLANDIELLA

CASSIDUL INOIDES

CH I LOS TOME LL INA

CHILOS TOMELLINA

CHI LOSTOMELLA

CHILOS TOMELLA

CHILOSTOMELLA

CHILOSTOMELLA

CHILOSTOMELLA

CHILOSTOMELLA

CHILOS TOMELLA
LIMBATA

LIMBATA

LIMBATA

LIMBATA

LIMBATA

LIMBATA

LIMBATA

LIMBATA

LIMBATA

LIMBATA

LIMBATA

LIMBATA

LIMBATA

LOM ITENSIS

LOM I TENSIS

LOMITENSIS

MIN UTA

MINUTA

NORCROSSI

NOR CROSSI

NOR CROSSI

NOR CROSS

NOR CROSSI

NOR CROSSI

NOR CROSSI

NOR CROSSI

NOR CROSSI

NOR CROSS

NOR CROSS I

NOR CROSS I

NOR CROSSI

NOR CROSS

NOR CROSSI

NOR CROSSI

NOR CROSSI

NOR CROSS I

PUL CHELLA

PUL CHELLA

SUB CAR INATA

SUBCAR INATA

SUB CAR INATA

SUB CAR INATA

SUBCARINATA

SUBGLOBOSA

SUBGLOBOSA

SUBGLOBOSA

SUBGLOBOSA

SUBGLOB OSA

SUBGLOBOSA

SUB GL OBOSA

SUBGLOBOSA

HELENAE

HEL ENAE

HELENAE

HEL ENAE

HELENAE

HELENAE

HELENAE

HELENAE

HEL ENAE

HEL ENAE

HELENAE

HELENAE

HELENAE
TORTUOSA

TORTUOSA

TOR TUOSA

TOR TUOSA

TORTUOSA

TOR TUOSA

TOR TUOSA

TORTUOSA

TOR TUOSA

TOR TUOSA

TOR TUOSA

TOR TUOSA

TORTUOSA

TOR TUOSA

TRANSLUCENS

TRANSLUCENS

TRANSLUCENS

TRANSLUCENS

TRANSLUCENS

CORNUTA

FIMBRIATA

F IMBR IATA

F IMBR IA TA

O OL INA

O OL INA

OOL INA

OLL INA

OOL INA

OOL INA

OOL INA

OOL INA

Locality

Lat. Long.

GULF OF ALASKA

GULF OF ALASKA

GULF OF ALASKA

GULF OF ALASKA

GULF OF ALASKA

GULF OF ALASKA 


\section{Publication}

10561 BERGEN O'NEIL 1979 10562 CUS HMAN 1927

10563 CUSHMAN 1927

10564 BERGEN O'NEIL 1979

10565 SMITH 1973

10566 SMITH 1973
10567 SMI TH 1973

10568 BERGEN O'NEIL 1979

10569 BERGEN O'NEIL 1979

10570 BERGEN O'NEIL 1979

10571 BERGEN O NEIL 1979

10572 BERGEN O.NEIL 1979

10573 BERGEN OONEIL 1979

10574 BERGEN O'NEIL 1979

10575 BERGEN O'NEIL 1979

10576 BERGEN O'NEIL 1979

10577 BERGEN O'NEIL 1979

10578 BERGEN O'NEIL 1979

10579 BERGEN O'NEIL 1979

10580 COCKBAIN 1963

10581 COCKBAIN 1963

10582 COC KBAIN 1963

10583 COCKBAIN 1963

10584 BERGEN O'NEIL 1979

10585 BERGEN O'NEIL 1979

10586 ECHOLS 1969

10587 BERGEN O'NEIL 1979

10588 BERGEN O'NEIL 1979

10589 BERGEN O'NEIL 1979

10590 BERGEN O'NEIL 1979

10591 BERGEN OONEIL 1979

10592 BERGEN O'NEIL 1979

10593 COCKBAIN 1963

10594 COCKBAIN 1963

10595 COOPER 1961

10596 COOPER 1961

10597 COOPER 1961

10598 BERGEN O'NEIL 1979

10599 BERGEN O.NEIL 1979

10600 COCKBAIN 1963

10601 COCKBAIN 1963

10602 COCKBAIN 1963

10603 COCKBAIN 1963

10604 COCKBAIN 1963

10605 COCKBAIN 1963

10606 COCKBAIN 1963

10607 CUS HMAN TODD 1947

10608 CUS HMAN TODD 1947

10609 CUS HMAN TODD 1947

10610 CUS HMAN TODD 1947

10611 CUS HMAN TODD 1947

10612 CUSHMAN TODD 1947

10613 TODD LOW 1967

10614 TODD LOW 1967

10615 TODD LOW 1967

10616 TODD LOW 1967

10617 TODD LOW 1967

10618 TODD LOW 1967

10619 TODD LOW 1967

10620 SMITH 1973

10621 LANKFORD PHLEGER 1973

10622 BERGEN O'NEIL 1979

10623 BERGEN O'NEIL 1979

10624 BERGEN O'NEIL 1979

10624 BERGEN O'NEIL 1979

10625 BERGEN O'NEIL 1979

10627 BERGEN O०NEIL 1979

10628 BERGEN O N NEIL 1979

10629 ECHOLS 1969

10630 DETLING 1958

10631 BERGEN O'NEIL 1979

10632 BERGEN O'NEIL 1979

10633 BERGEN O'NEIL 1979

10634 BERGEN O.NEIL 1979

10635 BERGEN O'NEIL 1979

10636 BERGEN O'NEIL 1979

10637 BERGEN O'NEIL 1979

10638 COOPER 1961

10639 BERGEN O'NEIL 1979

10640 BERGEN O'NEIL 1979

10649 BERGEN O'NEIL 1979

10642 BERGEN O'NEIL 1979

10643 BERGEN O'NEIL 1979

10644 BERGEN ODNEIL 1979

10645 BERGEN OPNE 1979

10646 BERGEN OONEIL 1979

10647 BERGEN O.NEIL 1979

10648 SMITH 1973

10649 CUS HMAN TODD 1947

10650 COOPER 1961

10651 BERGEN O'NEIL 1979

10652 SMITH 1973

10653 SMI TH 1973

10654 TODD LOW 1967

10655 TODD LOW 1967
Generic Name Specific Name

CHILOSTOMELLA

CHILOSTOMELLA

CHILOSTOMELLINA

CIBICIDES

CIBICIDES

CIBICIDES

CIBICIDES

CIBICIDES

CIBICIDES

CIBICIDES

CIBICIDES

CIBICIDES

CIBICIDES

CIBICIDES

CIBICIDES

CIBICIDES

CIBICIDES

CIBICIDES

CIBICIDES

CIBICIDES

CIBICIDES

CIBICIDES

CIBICIDES

CIBICIDES

CIBICIDES

CIBICIDES

CIBICIDES

CIBICIDES

CIBICIDES

CIBICIDES

CIBICIDES

CIBICIDES

CIBICIDES

CIBICIDES

CIBICIDES

CIBICIDES

CIBICIDES

CIBICIDES

CIBICIDES

CIBICIDES

CIBICTOES

CIBICIDES

CIBICIDES

CIBICIDES

CIBICIDES

CIBICIDES

CIBICIDES

CIBICIDES

CIBICIDES

CIBICIDES

CIBICIDES

CIBICIDES

CIBICIDES

CIBICIDES

CIBICIDES

CIBICIDES

CIBICIDES

CIBICIDES

CIBICIDES

CIBICIDES

CIBICIDES

CIBICIDES

CIBICIDES

CIBICIDES

CIBICIDES

CIBICIDES

CIBICIDES

CIBICIDES

CIBICIDES

CIBICIDES

CIBICIDES

CIBICIDES

CIBICIOES

CIBICIDES

CIBICIDES

CIBICIDES

CIBICIDES

CIBICIDES

CIBICIDES

CIBICIDES

CIBICIOES

CIBICIDES

CIBICIDES

CIBICIDES

CIBICIDES

CIBICIDES

CIBICIDOIDES

CYCLOGYRA

CYCLOGYRA

CYCLOGYRA
O OL INA

OOL INA

F IMBRIATA

BRADYI

BRA DYI

BRA OYI

FLETCHERI

LETCHERI

FLETCHERI

FLETCHER I

FLE TCHERI

FLETCHERI

FLE TCHER I

FLETCHERI

F LET CHERI

FLETCHERI

FLETCHERI

FLETCHERI

FLE TCHER

FLETCHERI

FLE TCHER I

FLETCHERI

FLETCHERI

FLETCHERI

FLE TCHERI

FLE TCHER I

FLETCHERI

FLE TCHERI

FLETCHERI

FLETCHERI

FLETCHER I

FLETCHER I

FLE TCHER I

FLETCHERI

LOBATULUS

LOBATULUS

OBATULUS

LOBATULUS

LOBATULUS

LOBATULUS

LOBATULUS

LOBATULUS

LBATULUS

LBATULUS

LOBATULUS

LOBATULUS

LOBATULUS

$\angle O B A T U L U S$

LOBATULUS

LOBATULUS

LOBATULUS

LBATULUS

$\angle O B$ ATULUS

LOBATULUS

LOBATULUS

LOBATULUS

LOBATULUS

LOBATULUS

LOBATULUS

OBATULUS

LOBATULUS

LOBATULUS

$\angle O B A T$ OL US

LOBATULUS
LOBATULUS

LOBATULUS

LOBATULUS

LOBATULUS

LOBATULUS

OBATULUS

$\angle O B A T U L U S$

$\angle O B A T U L U S$

LOBATULUS

LOBATULUS

LOBATULUS

LOBATULUS

LOBATULUS

MCKANNA

MCK ANNAI

MCK ANNA I

MCK ANNAI

MCK ANNA I

MCKANNAI

MCK ANNA I

MCK ANNA I

MCK ANNA I

REF ULGENS

REFULGENS

REFULGENS

SPIRAL IS

MUNDULUS

INC ERT A

I NV OL VENS

I NV OL VENS

Locality

Lat. Long.

GULF OF ALASKA

OFF OREG ON

OFF OREGON

GULF OF ALASKA

OFF ALASKA PENINSULA

NORTH PACIFIC

OFF ALASKA PENINSULA 


\section{Publication}

10656 DETLING 1958

10657 CUS HMAN 1925

10658 CUSHMAN 1925

10659 BERGEN O.NEIL 1979

10660 CUSHMAN TODD 1947

10661 CUSHMAN TODD 1947

10662 LANKFORD PHLEGER 1973

10663 SCOTT 1974

10664 SCOTT 1974

10665 LANKFORD PHLEGER 1973

10666 LANKFORD PHLEGER 1973

10667 LANKFORD PHLEGER 1973

10668 LANKFORD PHLEGER 1973

10669 LANKFCRD PHLEGER 1973

10670 CUSHMAN 1910

10671 TODD LOW 1967

10672 TODD LOW 1967

10673 TODD LOW 1967

10674 BERGEN O'NEIL 1979

10675 TODD LOW 1967

10676 TODD LOW 1967

10677 TODD LOW 1967

10678 BERGEN O.NEIL 1979

10679 BERGEN O'NEIL 1979

10680 BERGEN O'NEIL 1979

10681 BERGEN O'NEIL 1979

10682 BERGEN O'NEIL 1979

10683 BERGEN O'NEIL 1979

10684 BERGEN O.NEIL 1979

10685 BERGEN O'NEIL 1979

10686 ECHOLS 1969

10687 TODD LOW 1967

10688 TODD LOW 1967

10689 TODD LOW 1967

10690 TODD LOW 1967

10691 BERGEN O NEIL 1979

10692 BERGEN O'NEIL 1979

10693 BERGEN O'NEIL 1979

10694 BERGEN O'NEIL 1979

10695 BERGEN O.NEIL 1979

10696 TODD LOW 1967

10697 BERGEN O.NEIL 1979

10698 TODD LOW 1967

10699 BERGEN O'NEIL 1979

10700 BERGEN O'NEIL 1979

10701 BERGEN O'NEIL 1979

10702 BERGEN O'NEIL 1979

10703 BERGEN O'NEIL 1979

10704 BERGEN O'NEIL 1979

10705 TODD LOW 1967

10706 WHI TEAVES 1886

10707 CUS HMAN 1913

10708 CUS HMAN 1910

10709 TODD LOW 1967

10710 SMITH 1973

10711 CUSHMAN 1910

10712 CUS HMAN 191

10713 CUSHMAN 191

10714 SMITH 1973
10715 SMITH 1973

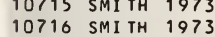

$\begin{array}{ll}10716 & \text { SMI TH } \\ 10717 & \text { SMITH } 1973\end{array}$

10718 SMITH 1973

10719 SMITH 1973

10720 BERGEN O.NEIL 1979

10721 BERGEN O'NEIL 1979

10722 BERGEN O'NEIL 1979

10723 TODD LOW 1967

10724 TODD LOW 1967

10725 CUSHMAN MCCULLOCH 1950

10726 TODD LOW 1967

10727 TODD LOW 1967

10728 BERGEN O.NEIL 1979

10729 TODD LOW 1967

10730 WHITEAVES 1886

10731 WHI TEAVES 1886

10732 WHI TEAVES 1886

10733 CUSHMAN 1925

10734 CUSHMAN 1925

10735 DETLING 1958

10736 CUS HMAN TODD 1947

0737 CUSHMAN TODD 1947

10738 CUSHMAN TODD 1947

10739 COOPER 1961

10740 COOPER 1961

10741 COOPER 1961

10742 COOPER 1961

10743 COOPER 1961

10744 CUSHMAN TODD 1947

10745 CUSHMAN TODD 1947

10746 CUS HMAN TODD 1947

10747 COCKBAIN 1963

10748 COCKBAIN 1963

10749 CUSHMAN 1925
10750 CUSHMAN 1925
Generic Name Specific Name

Locality

Lat. Long.

CYCLOGYRA

CYCLOGYRA INVLVENS

CYCLOGYRA INVOLVENS

CYCLOGYRA INVOLVENS

CYCLOGYRA

CYCLOGYRA

ELPHIDIUM

ELPHIDIUM

ELPHIDIUM

ELPHIDIUM

ELPHIDIUM

ELPHIDIUM

ELPHIDIUM
CRIBROSTOMOIDES

CRIBROSTOMOIDES

CRI BROSTOMOIDES

CRI BROST OMOIDES

CRIBROSTOMOIDES

CRI BR OST OMOIDES

CRIBROSTOMOIDES

CRIBROSTOMOIDES

CRIBROSTOMOIDES

CRI OROSTOMOIDES

CRIBR OS TOMOIDES

CRIBROSTOMOIDES

CRI BROSTOMOIDES

CRIBROSTOMOIDES

CRI BROSTOMOIDES

CRIBROST OMOIDES

CRI BROSTOMOIDES

CRIBROSTOMOIDES

CRIBROSTOMOIDES

CRI IROSTOMOIDES

CRI BROSTOMOIDES

HAPLOPHRAGMOIDES

HAPLOPHRAGMOIDES

HAPLOPHRAGMOIDES

HAPLOPHRAGMOIDES

HAPLOPHRAGMOIDES

HAPLOPHRAGMOIDES

HAPLOPHRAGMOIDES

HAPLOPHRAGMOIDES

HAPLOPHRAGMOIDES

HAPLOPHRAGMOIDES

HAPLOPHRAGMOIDES

HAPLOPHRAGMOIDES

HAPLOPHRAGMOIDES

HAPLOPHRAGMOIDES

CRIBROSTOMOIDES

LENTI CUL INA

ASTACOLUS

CRITHIONINA

CRUCILOCULINA

CYCLAMMINA

CYCLAMM INA

CYCLAMMINA

CYCLAMMINA

CYCLAMMINA

CYCLAMM INA

CYSTAMMINA

CYS TAMMINA

CYSTAMMINA

DENTAL INA

DENTALINA

DENTALINA

DENTALINA

DENTAL INA

DENTAL INA

DENTALINA

DENTALINA

DENTAL INA

GLABRATELLA

GLABRATELLA

ANGULODISCORBIS

ANGULODI

ROSALINA

ROSALINA

ROSALINA

ROSALINA

ROSAL INA

ROSALINA

ROSALINA

ROSALINA

GLALINA

NEOCONORBINA

GLABRATELLA

GLABRATELLA

GLABRATELLA

GLABRATELLA

GLABRA TELLA

GLABRATELLA
SUNSET BAY, OREGON

VIRAGO SD.. B.C.

GULF OF ALASKA

WASHINGTON COAST

WASHINGTON COAST

CRESCENT CITY, CALIF.

SAMISH-PADI LLA BAYS

SAMISH-PAOILLA BAYS

CRESCENT CITY, CALIF.

LAPUSH, WASHINGTON

CRESCENT CITY, CALIF .

DEPOE BAY, OREGON

OFF. WASHINGTON

GULF OF ALASKA

EXCURSIOND. ALASKA

TAKU HARBOUR, ALASKA

GULF OF ALASKA

KASAAN BAY, ALASKA

GAMBIER BAY, ALASKA

LYNN CANAL. ALASKA

GULF OF ALASKA

GULF OF ALASKA

GULF OF ALASKA

GULF OF ALASKA

GULF OF ALASKA

GULF OF ALASKA

GULF OF ALASKA

WASHINGTON COAST

GAMBIER BAY. ALASKA

GULF OF ALASKA

TAKU HARBOUR. ALASKA

EXCURSION, ALASKA

GULF OF ALASKA

GULF OF ALASKA

GULF OF ALASKA

CLARENCE ST.. ALASKA

GULF OF ALASKA

GULF OF ALASKA

GULF OF ALASKA

GULF OF ALASKA

GULF OF ALASKA

GULF OF ALASKA

GULF OF ALASKA

GULF OF ALASKA

STRAIT OF GEORGIA, B. C

OFF ALASKA PENINSULA

OFF OREGON

GULF OF ALASKA

OFF ALASKA PENINSULA

OF F WASHINGTON

OFF BRIT. COLUMBIA

OFF OREGON

OFF ALASKA PENINSULA

OFF ALASKA PENINSULA

OFF ALASKA PENINSULA

OFF ALASKA PENINSULA

OFF ALASKA PENINSULA

OFF ALASKA PEN

GULF OF ALASKA

GULF OF ALASKA

KASAAN BAY, ALASKA

GULF OF ALASKA

OFF ALASKA

TAKU HARBOUR, ALASKA

GULF OF ALASKA

DISCOVERY PASSAGE, $B . C$

QUEEN CHARLOTTE SD

QUEEN CHARLOTTE SD.. B.C.

Q. CHARLOTTE SD.. B.C.

Q. CHARLOTTE SD.. B.C.

SUNSET BAY, OREGON

WASHINGTON COAST

WASHINGTON COAST

WASHINGTON COAST

CALIFORNIA COAST

OREGON COAST

OREGON COAST

OREGON COAST

OREGON COAST

WASHINGTON COAST

WASHING ION COAST

WASHINGTON COAS

BRITISH COLUMEIA

BRITISH COLUMBIA

VIRAGO SD.. B.C.
Q. CHARLOTTE SD.. B.C.

GULF OF ALASKA

Q. CHARLOTTE SD.. B.C.

$4321 \mathrm{~N} 12420 \mathrm{~W}$

$5220 N 13100 W$

$5930 N 14255 \mathrm{~W}$

$4832 \mathrm{~N} 12310 \mathrm{~W}$

$4833 \mathrm{~N} 12300 \mathrm{~W}$

$\begin{array}{llll}41 & 45 N & 124 & 13 W \\ 48 & 36 N & 122 & 29 W\end{array}$

$4836 \mathrm{~N} 12229 \mathrm{~W}$

$44 \quad 47 \mathrm{~N} 12405 \mathrm{~W}$

$4145 \mathrm{~N} 12413 \mathrm{~W}$

$4755 \mathrm{~N} 12739 \mathrm{~W}$

$4145 \mathrm{~N} 12413 \mathrm{~W}$

$44 \quad 47 \mathrm{~N} 12405 \mathrm{~W}$

45
59
$52 N$ $14236 W$ 


\section{Publication}

10751 CUSHMAN TCDD 1947 10752 DETLING 1958

10753 COOPER 1961

10754 COOPER 1961

10755 COOPER 1961

10756 COOPER 1961

10757 BERGEN O'NEIL 1979

10758 BERGEN O'NEIL 1979

10759 BERGEN O'NEIL 1979

10760 DETLING 1958

10761 CUSHMAN TODD 1947

10762 CUSHMAN TODD 1947

10763 CUSHMAN TODD 1947

10764 TODD LOW 1967

10765 BERGEN O'NEIL 1979

10766 BERGEN O.NEIL 1979

10767 SMITH 1973

10768 SMI TH 1973

10769 LANKFORD PHLEGER 1973

10770 BERGEN O'NEIL 1979

10771 BERGEN O'NEIL 1979

10772 BERGEN O'NEIL 1979

10773 CUSHMAN TODD 1947

10774 CUSHMAN TODD 1947

10775 CUSHIAN TODD 1947

10776 TODD LOW 1967

10777 TODD LOW 1967
10778 TODD LOW 1967

10779 LANKFORD PHLEGER 1973

10780 LANKFORD PHLEGER 1973

10781 LANKFORD PHLEGER 1973

10782 DETLING 1958

10783 SCOTT 1974

10784 ECHOLS 1969

10785 ECHOLS 1969

10786 COCKBAIN 1963

10787 COCKBAIN 1963

10788 COCKBAIN 1963

10789 COCKBAIN 1963

10790 COCKBAIN 1963

10791 BERGEN O'NEIL 1979

10792 BERGEN O'NEIL 1979

10793 BERGEN O'NEIL 1979

10794 BERGEN O'NEIL 1979

10795 BERGEN O'NEIL 1979

10796 BERGEN O'NEIL 1979

10797 BERGEN O'NEIL 1979

10798 COOPER 1961

10799 COOPER 1961

10800 COOPER 1961

10801 ECHOLS 1969

10802 COCKBAIN 1963

10803 COCKBAIN 1963

10804 COCKBAIN 1963

10805 TODD LOW 1967

10806 TODD LOW 1967

10807 TODD LOW 1967

10808 TODD LOW 1967

10809 CUSHMAN TODD 1947

10810 TODD LOW 1967

10811 CUSHMAN TODD 1947

10812 CUSHMAN TODD 194

10813 CUSHMAN MCCULLOCH 1939

10814 CUSHMAN MCCULLOCH 1939

10815 CUS HMAN MCCULLOCH 1939

10816 CUSHMAN MCCULLOCH 1939

10817 SMI TH 1973

10818 SMI TH 1973

10819 SMI TH 1973

10820 SMI TH 1973

10821 SMITH 1973

10822 BERGEN O'NEIL 1979

10823 BERGEN O'NEIL 1979

10824 BERGEN O'NEIL 1979

10825 BERGEN O'NEIL 1979

10826 BERGEN O'NEIL 1979

10827 BERGEN O'NEIL 1979

10828 BERGEN O'NEIL 1979

10829 BERGEN O.NEIL 1979

10830 BERGEN O'NEIL 1979

10831 BERGEN O'NEIL 1979

10832 BERGEN O'NEIL 1979

10833 SMITH 1973

10834 SMI TH 1973

10835 SMITH 1973

10836 SMI TH 1973

10837 BERGEN O.NEIL 1979

10838 TODD LOW 1967

10839 SMITH 1973

10840 TODD LOW 1967

10841 TODD LOW 1967

10842 TODD LOW 1967

10843 TODD LOW 1967

10844 TODD LOW 1967

10845 TODD LOW 1967

\section{Generic Name Specific Name}

ORN AT IS SIMA

ORNATISSIMA

ORNATISSIMA

ORNATISSIMA

ORNAT I S IIMA

PRAEGERI

PRAEGERI

PRAEGER I

SAN JUANENSIS

SANJUANENSIS

SANJUANENSIS

BRADYANA

BRA DYANA

EXILIS

EXILIS

BISERIALIS

BISERIALIS

B ISERIALIS

BISERIALIS

BISERIALIS

BISERIALIS

B IS ERIALIS

BISER I ALIS

BISERIALIS

BISERIALIS

PERFORATA

A DVENA

A DVENA

ADVENA

ADVENA

A DVENA
A DVENA

A DVENA

ADVENA

ADVENA

ADVENA

ADVENA

ADVENA

A DVENA

A DVENA

A DVENA

A DVENA

ADVENA

ADVENA

A DVENA

ADVENA

A DVENA

ADVENA

DV ENA

A DVENA

A DVENA

ADVENA

A DVENA

A DVENA

ADV ENA

A DVENA

ADVENA

A DVENA

A DVENA

A DVENA

A DVENA

ADVENA

A DVENA

ADVENA

ADV ENA

BRADYI

BRADYI

BRADYI

BRA DYI

BRADYI

BRADYI

BRADYI

BRADYI

BRADYI

HUMBOLDTI

HUMBOLDTI
PUS ILLA

PUS ILLA

PUS ILLA

PUS ILLA

SCABRA

SCABRA

SCABRA

S CABRA

COMPRESSA

COMPRESSA

HYSTRIX

ARC TICA

ARC TICA

ARC TICA

ARC TICA

ARCTICA
Locality

Lat. Long.

WASHINGTON COAST

SUNSET BAY, OREGON

OREGON COAST

OREGON COAST

OREGON COAST

CALIFORNIA COAST

GULF OF ALASKA

GULF OF ALASKA

GULF OF ALASKA

SUNSET BAY, OREGON

WASHINGTON COAST

WASHINGTON COAST

GULF OF ALASKA

GULF OF ALASKA

GULF OF ALASKA

OFF ALASKA PENINSULA

NORTH PACIFIC

CRESCENT CITY, CALIF.

GULF OF ALASKA

GULF OF ALASKA

GULF OF ALASKA

WASHINGTON COAST

WASHINGTON COAST

WASHINGTON COAST

GAMBIER BAY, ALASKA

KASAAN BAY, ALASKA

EXCURSION, ALASKA

CRESCENT CITY, CALIF.

DEPOE BAY, OREGON

LAPUSH, WASHINGTON

SUNSET BAY,OREGON

SAMISH-PADILLA BAYS

WASHINGTON COAST

WASHINGTON COAST

BR ITISH COLUMBIA

BR IT ISH COL UMBIA

BRITISH COLUMBIA

BRITISH COLUMBIA

BR ITISH COL UMBIA

GULF OF ALASKA

GULF OF ALASKA

GULF OF ALASKA

GULF OF ALASKA

GULF OF ALASKA

GULF OF ALASKA

GULF OF ALASKA

OREGON COAST

OREGON COAST

OREGON COAST

WASHINGT ON COAST

BRITISH COL UMBIA

BRITISH COLUMBIA

BR ITISH COLUMBIA

EXCURSION, ALASKA

KASAAN BAY, ALASKA

TAKU HARBOUR, ALASKA

WAMBIER BAY ALA

LYNN CANAL ALASKA

WASHINGTON COAST

WASHINGTON COAS

OFF ALASKA

OFF ALASKA

KETCHIKAN, ALASKA

OFF ALASKA PENINSULA

OFF ALASKA PENINSULA

OFF ALASKA PENINSULA

OFF ALASKA PENINSULA

OFF ALASKA PENINSULA

GULF OF ALASKA

GULF OF ALASKA

GULF OF ALASKA

GULF OF ALASKA

GULF OF ALASKA

GULF OF ALASKA

GULF OF ALASKA

GULF OF ALASKA

GULF OF ALASKA

GULF OF ALASKA

GULF OF ALASKA

OFF ALASKA PENINSULA

NORTH PACIFIC

OFF ALASKA PENINSULA

KODIAK, ALASKA

GULF OF ALASKA

GULF OF ALASKA

OFF ALASKA PENINSULA

KASAAN BAY, ALASKA

LYNN CANAL, ALASKA

LYNN CANAL, ALASKA

EXCURSION, ALASKA

TAKU HARBOUR, ALASKA

KASAAN BAY, ALASKA

$4833 \mathrm{~N} 12300 \mathrm{~W}$

$4321 \mathrm{~N} 12420 \mathrm{~W}$

$4225 \mathrm{~N} 12426 \mathrm{~W}$

$4536 \mathrm{~N} 12357 \mathrm{~W}$

$4136 \mathrm{~N} 124 \quad 13 \mathrm{~W}$

$5625 \mathrm{~N} 15245 \mathrm{~W}$ 


\section{Publication}

10846 TODD LOW 1967

10847 SMITH 1973

10848 LANKFORD PHLEGER 1973

10849 LANKFORD PHLEGER 1973

10850 LANKFORD PHLEGER 1973

10851 CUSHMAN TODD 1947

10852 CUS HMAN TODD 1947

10853 CUS HMAN TODD 1947

10854 CUSHMAN TODD 1947

10855 CUSHMAN TODD 1947

10856 CUSHMAN TODD 1947

10857 CUS HMAN MCCULLOCH 1940

10858 CUSHMAN MCCULLOCH 194

10859 CUSHMAN MCCULLOCH 194

10860 CUSHMAN MCCULLOCH 1940

10861 CUSHMAN MCCULLOCH 1940

10862 CUSHMAN MCCULLOCH 1940

10863 CUSHMAN MCCULLOCH 1940

10864 SCOTT 1974

10865 COOPER 1961

10866 COOPER 1961

10867 COOPER 1961

10868 COOPER 1961

10869 COOPER 1961

10870 COOPER 1961

10871 COOPER 1961

10872 COOPER 1961

10873 ECHOLS 1969

10874 ECHOLS 1969

10875 COCKBAIN 1963

10876 COCKBAIN 1963
10877 COCKBAIN 1963

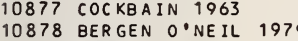

10879 BERGEN O'NEIL 1979

10880 BERGEN O'NEIL 1979

10881 BERGEN O'NEIL 1979

10882 COCKBAIN 1963

10883 COCKBAIN 1963

10884 COCKBAIN 1963

10885 BERGEN O.NEIL 1979

10886 BERGEN O'NEIL 1979

10887 BERGEN O'NEIL 1979

10888 DETLING 1958

10889 NATLAND 1933

10890 BERGEN O'NEIL 1979

10891 BERGEN O'NEIL 1979

10892 BERGEN O'NE IL 1979

10893 CUS HMAN MCCULLOCH 1940

10894 CUS HMAN MCCULLOCH 1940

10895 CUSHMAN MCCULLOCH 1940

10896 CUSHMAN TODD 1947

10897 CUSHMAN TODD 1947

10898 BERGEN O'NEIL 1979

10899 BERGEN O'NEIL 1979

10900 BERGEN O'NEIL 1979

10901 BERGEN O'NEIL 1979

10902 TODD LOW 1967

10902 TODD LOW 1967
10903 TODD LOW 1967

10903 TODD LOW 1967
10904 TODD LOW 1967

10904 TODD LOW 1967
10905 SMI TH 1973

10905 SMI TH 1973
10906 SMI TH 1973

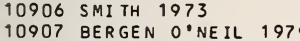

10908 BERGEN O'NEIL 1979

10909 TODD LOW 1967

10910 BERGEN O'NEIL 1979

10911 BERGEN O'NEIL 1979

10912 BERGEN O०NEIL 1979

10913 BERGEN O.NE IL 1979

10914 COCKBAIN 1963

10915 COCKBAIN 1963

10916 COCKBAIN 1963

10917 COCKBAIN 1963

10918 COCKBAIN 1963

10919 COCKBAIN 1963

10920 BERGEN O'NEIL 1979

10921 BERGEN O'NEIL 1979

10922 BERGEN O०NEIL 1979

10923 BERGEN O.NEIL 1979

10924 BERGEN O'NEIL 1979

10925 BERGEN O.NEIL 1979

10926 TODD LOW 1967

10927 TODD LOW 1967

10928 TODD LOW 1967

10929 TODD LOW 1967

10930 BERGEN O'NEIL 1979

10931 BERGEN O'NEIL 1979

10932 BERGEN O.NEIL 1979

10933 SMITH 1973

10934 SMI TH 1973

10935 COCKBAIN 1963

10936 CUSHMAN TODD 1947

10937 CUS HMAN TODD 1947

10938 CUSHMAN TODD 1947

10939 NICOL 1944

10940 COOPER 1961
Generic Name Specific Name

Locality

Lat. Long.

ELPHIDIELLA

ELPHIDIELLA

ELPHIDIELLA

ELPHIDIELLA

ELPHIDIELLA

ELPHIDIELLA

ELPHIDIELLA

ELPHIDIELLA

ELPHIDIELLA

ELPHIDIELLA

ELPHIDIELLA

ELPHIDIELLA

ELPHIDIELLA

ELPHIDIELLA

ELPHIDIELLA

ELPHIDIELLA

ELPHIDIELLA

ELPHIDIELLA

ELPHIDIELLA

ELPHIDIELLA

ELPHIDIELLA

ELPHIDIELLA

ELPHIDIELLA

ELPHIDIELLA

ELPHIDIELLA

ELPHIDIELLA

ELPHIDIELLA

ELPHIDIELLA

ELPHIDIELLA

ELPHIOIELLA

ELPHIDIELLA

ELPHIDIELLA

ELPHIDIELLA

ELPHIDIELLA

ELPHIDIELLA

ELPHIDIELLA

ELPHIDIUM

ELPHIDIUM

ELPHIDIUM

ELPHIDIUM

ELPHIDIUM

ELPHIDIUM

ELPHIDIUM

ELPHIDIUM

ELPHIDIUM

ELPHIDIUM

ELPHIDIUM

ELPHIDIUM

ELPHIDIUM

ELPHIDIUM

ELPHIDIUM

ELPHIDIUM

ELPHIDIUM

ELPHIDIUM

ELPHIDIUM

ELPHIDIUM

ELPHIDIUM

ELPHIDIUM

ELPHIDIUM

ELPHIDIUM

ELPHIOIUM

ELPHIDIUM

ELPHIDIUM

ELPHIDIUM

ELPHIDIUM

ELPHIDIUM

ELPHIDIUM

ELPHIDIUM

ELPHIDIUM

ELPHID IUM

ELPHIDIUM

ELPHIDIUM

ELPHIDIUM

ELPHIDIUM

ELPHIDIUM

ELPHIDIUM

ELPHIDIUM

ELPHIDIUM

ELPHIDIUM

ELPHIDIUM

ELPHIDIUM

ELPHIDIUM

ELPHIDIUM

ELPHIDIUM

ELPHIDIUM

ELPHIDIUM

ELPHIDIUM

ELPHIDIUM
ARC IICA GAMBIER BAY, ALASKA

GROENLANDICA OFF ALASKA PENINSULA

HANNAI LAPUSH, WASHINGTON

HAN NA I

HANNAI

HANNAI

HANNAI

HANNAI

HANNAI

HANNAI

HANNAI

HANNA

HANNAI

HANNA I

HANNAI

HANNAI

HANNA I

HANNA I

HAN NA I

HANNAI

HANNAI

HANNAI

HANNAI

HANNA I

HANNAI

HANNAI

HANNAI

HANNA

HANNAI

NIT IDA

N IT I DA

$N$ IT I DA

NIT I DA

NIT I DA

N IT I DA

NIT I DA

N IT I DA

NITI IDA

NIT I DA

$N$ IT I DA

$N$ IT I DA

NIT I DA

ART I CULATUM

ART I CULATUM

ART I CULATUM

ART I CULATUM

ART I CULATUM

ART I CULATUM

ART I CULATUM

ART I CULATUM

ART I CULATUM

BAR TLETTI

BAR TLETTI

BAR TLETTI

BAR TLETTI

BAR TLETTI

BAR TLETTI

BAR TLETT

BAR TLETTI

BAR TLETT

BAR TLETT

BAR TLETTI

EXC AVATUM

EXCAVATUM

EXCAVATUM

EXCAVATUM

EXC AVATUM

EXCAVATUM

EXCAVATUM

EXCAVATUM

EXC AVATUM

EXC AVATUM

EXC AVATUM

EXCAVATUM

EXCAVATUM

EXCAVATUM

EXCAVATUM

EXCAVATUM

EXC AVATUM

EXCAVATUM

EXCAVATUM

EXCAVATUM

EXC AVATUM

EXC AVATUM

EXCAVATUM

EXCAVATUM

EXC AVA TUM

EXCAVATUM

CRISPUM

CRI SPUM

CRISPUM

CRISPUM

CRI SPUM

CRISPUM

CRISPUM

DEPOE BAY, OREGON

WASH ING TON COAST

WASHINGTON COAST

WASHINGTON COAST

WASH ING TON COAST

WASHINGTON COAST
CRESCENT CITY, CALIF. 


\section{Publication}

10941 PHLEGER 1967

10942 BERGEN O'NEIL 1979

10943 BERGEN O'NEIL 1979

10944 BERGEN O'NEIL 1979

10945 BERGEN O'NEIL 1979

10946 BERGEN O'NEIL 1979

10947 BERGEN O'NEIL 1979

10948 TODD LOW 1967

10949 TODD LOW 1967

10950 TODD LOW 1967

10951 TODD LOW 1967

10952 TODD LOW 1967

10953 TODD LOW 1967

10954 TOD D LOW 1967

10955 CUSHMAN MCCULLOCH 1940

10956 CUSHMAN MCCULLOCH 1940

10957 CUSHMAN MCCULLOCH 1940

10958 CUS HMAN MCCULLOCH 1940

10959 CUS HMAN MCCULLOCH 1940

10960 CUS HMAN TODD 1947

10961 CUSHMAN TODD 1947

10962 BERGEN O.NEIL 1979

10963 BERGEN O.NEIL 1979

10964 BERGEN O'NEIL 1979

10965 BERGEN O'NEIL 1979

10966 JONES ROSS 1979

10967 NATLAND 1933

10968 NATLAND 1933

10969 BERGEN O'NEIL 1979

10970 BERGEN O'NEIL 1979

10971 BERGEN O'NEIL 1979

10972 BERGEN O'NEIL 1979

10973 BERGEN O'NEIL 1979

10974 BERGEN O०NEIL 1979

10975 COOPER 1961

10976 COCKBAIN 1963

10977 COCKBAIN 1963

10978 COCKBAIN 1963

10979 COCKBAIN 1963

10980 DETLING 1958

10981 SMITH 1973

10982 CUSHMAN TODD 1947

10983 CUSHMAN TODD 1947

10984 CUSHMAN TODD 1947

10985 CUSHMAN TODD 1947

10986 CUSHMAN MCCULLOCH 1940

10987 CUSHMAN MCCULLOCH 1940

10988 CUSHMAN MCCULLOCH 1940

10989 PHLEGER 1967

10990 SMI TH 1973

10991 ECHOLS 1969

10992 ECHOLS 1969

10993 ECHOLS 1969

10994 ECHOLS 1969

10995 ECHOLS 1969

10996 ECHOLS 1969

10997 COOPER 1961

10998 COOPER 1961

10999 COOPER 1961

11000 COOPER 1961

11001 COOPER 1961

11002 COOPER 1961

11003 COOPER 1961

11004 COOPER 1961

11005 SMITH 1973

11006 TODD LOW 1967

11007 JONES ROSS 1979

11008 COC KBAIN 1963

11009 COCKBAIN 1963

11010 COCKBAIN 1963

11011 COC KBAIN 1963

11012 COCKBAIN 1963

11013 PHLEGER 1967

11014 PHLEGER 1967

11015 DETLING 1958

11016 COOPER 1961

11017 CODPER 1961

11018 COOPER 1961

11019 CUS HMAN TODD 1947

11020 CUSHMAN TODD 1947

11021 CUSHMAN TODD 1947

11022 CUSHMAN MCCULLOCH 1940

11023 CUSHMAN MCCULLOCH 1940

11024 CUSHMAN MCCULLOCH 1940

11025 CUSHMAN MCCULLOCH 1940

11026 CUSHMAN TODD $194 ?$

11027 CUSHMAN TODD 1947

11028 CUSHMAN TODD 1947

11029 CUS HMAN TODD 1947

11030 CUSHMAN TODD 1947

11031 CUS HMAN TODD 1947

11032 LANKFORD PHLEGER 1973

11033 SMITH 1973

11034 SMI TH 1973

11035 SMI TH 1973

\section{Generic Name Specific Name}

FRI GI DUM

FRIGIDUM

FRIGIDUM

FRIGIDUM

FRI GIDUM

FRI GIDUM

FRI GIDUM

FRI GI DUM

FRI GIDUM

FRI GI DUM

FRIGIDUM

FRIGIDUM

FRI GI DUM

FRIGIDUM

FRI GI DUM

FRI GIDUM

FRI GIDUM

FRIGIDUM

FRIGIDUM

FRIGIDUM

FRI GIDUM

FRIGIDUM

EXCAVATUM

HANNAI

HUGHES I

HUGHESI

HUGHES

HUG HES I

HUGHESI

HUGHES I

HUGHESI FORAMINOSUM

I NCERTUM

INCERTUM

INCERTUM

INCERTUM

INCERTUM

INCERTUM

LENE

LENE

LENE

LENE

LENE

LENE

LENE

LENE

MAGELLANI CUM

MAGELLANI CUM

MAGELLANI CUM

MAGELLANI CUM

MIC ROGR ANULO SUM

MIC ROGRANUL O SUM

MICROGRANULO SUM

MICROGRANULO SUM

MICROGRANULO SUM

MICROGRANULO SUM

MICROGR ANULO SUM

MICROGRANULO SUM

MIC ROGRANULO SUM

MICROGRANULO SUM

MICROGRANUL O SUM

ORB I CULARE

ORE GONENSE

EXC AVATUM

SUBARCTICUM

SUBARCTI I UM

SUBARCTI CUM

SUBARCTICUM

SUBARCTI CUM

SUBARCT I CUM

SUBARCT ICUM

SUB ARCT I CUM

TRANSLUCENS

TRANSLUCENS

TRANSLUCENS

TUM I DUM

TUM I DUM

TUM I DUM

TUMIDUM

TUM IOUM

TUM I DUM

TUM I DUM

MEL 0

MEL O

MEL O

COMPRESSA

COMPRESSA

LUC IDA

BRA DYANA

EXI GUA

EXIGUA

EXIGUA

\section{Locality}

Lat. Long.

GULF OF ALASKA

GULF OF ALASKA

GULF OF ALASKA

GULF OF ALASKA

GULF OF ALASKA

GULF OF ALASKA

GAMBIER BAY, ALASKA

LYNN CANAL, ALASKA

EXCURSION, ALASKA

KASAAN BAY, ALASKA

CLARENCE ST.. ALASKA

GULF OF ALASKA

TAKU HARBOUR, ALASKA

KETCHIKAN, ALASKA

OFF ALASKA

OFF BRIT. COLUMBIA

OFF WASHINGTON

OF F WASHINGTON

WASH INGTON COAST

WASH INGT ON COAST

GULF OF ALASKA

GULF OF ALASKA

GULF OF ALASKA

GULF OF ALASKA

SAMISH BAY, WASH.

CRESCENT CITY, CALIF.

CRESCENT CITY, CALIF.

GULF OF ALASKA

GULF OF ALASKA

GULF OF ALASKA

GULF OF ALASKA

GULF OF ALASKA

GULF OF ALASKA

CALIFORNIA COAST

BRITISH COL UMBIA

BRITISH COL UMBIA

BRITISH COLUMBIA

BRITISH

SUNSET BAY, OREGON

OFF ALASKA PENINSULA

WASH INGTON COAST

WASHINGTON COAST

WASHINGTON COAST

WASHINGTON COAST

KETCHIKAN, ALASKA

OFF ALASKA

OFF BRIT. COLUMBIA

GRAY 'S HARBOUR, WASH.

OFF ALASKA PENINSULA

WASHINGTON COAST

WASHINGTON COAST

WASHINGTON COAST

WASHINGTON COAST

WASH INGTON COAST

WASHINGTON COAST

OREGON COAST

OREGON COAST

CALI FORNIA COAST

OREGON COAST

OREGON COAST

CALIFORNIA COAST

OREGON COAST

OREGON COAST

KODIAK, ALASKA

KASAAN BAY, ALASKA

SAMISH BAY, WASH.

BRITISH COL UMBIA

BRITISH COL UMBIA

BR IT ISH COL UMBIA

BR IT ISH COL UMB IA

BR ITISH COLUMBIA

GRAY'S HARBOUR, WASH.

COOS, BAY, OREGON

SUNSET BAY, OREGON

CALIFORNIA COAST

OREGON COAST

OREGON COAS

WASH INGTON COAST

WASHINGTON COAST

WASH INGTON COAST

OFF BRIT. COLUMBIA

OFF ALASKA

OF F ALASKA

WASHINGTON COAST

WASHINGTON COAST

WASHINGTON COAST

WASH ING TON COAST

WASHINGTON COAST

WASH INGTON COAST

CRES CENT CI YY CALIF

OFF ALASKA PENINSULA

OFF ALASKA PENINSULA

OF F ALASKA PENINSULA

47 OON $12408 \mathrm{~W}$

$5950 N 14547 W$ 


\section{Publication}

11036 SMITH 1973

11037 BERGEN O'NEIL 1979 11038 BERGEN O'NEIL 1979 11039 BERGEN O INEIL 1979

11040 BERGEN O.NEIL 1979

11041 TODD LOW 1967

11042 TODD LOW 1967

11043 BERGEN O'NEIL 1979

11044 BERGEN O'NEIL 1979

11046 BERGEN O'NEIL 1979

11047 BERGEN O'NEIL 1979

11048 BERGEN O'NEIL 1979

11049 BERGEN O'NEIL 1979

11050 BERGEN O'NEIL 1979

11051 BERGEN O'NEIL 1979

11052 BERGEN O'NEIL 1979

11053 COCKBAIN 1963

11054 COCKBAIN 1963

11055 COCKBAIN 1963

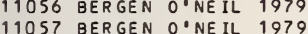

11058 BERGEN O.NEIL 1979

11059 SMITH 1973

11060 TODD LOW 1967

11061 TODD LOW 1967

11062 TODD LOW 1967

11063 TODD LOW 1967

11065 BERGEN O'NEIL 1979

11065 BERGEN O'NEIL 1979

11066 BERGEN O'NEIL 1979

11068 BERGEN O'NEIL 1979

11069 BERGEN O'NEIL 1979

11070 BERGEN O'NEIL 1979

11071 BERGEN O'NEIL 1979

11072 BERGEN O'NEIL 1979

11073 BERGEN O'NEIL 1979

11074 BERGEN O'NEIL 1979

11075 BERGEN O'NEIL 1979

11076 BERGEN O'NEIL 1979

11077 BERGEN O'NEIL 1979

$\begin{array}{lll}11078 & \text { BERGEN O'NEIL } & 1979 \\ 11079 & \text { BERGEN O'NEIL } 1979\end{array}$

11080 BERGEN O'NEIL 1979

11081 COOPER 1961

11082 COOPER 1961

11083 COOPER 196

11084 CUSHMAN TODD 1947

11085 DETLING 1958

11086 CUS HMAN TODD 1947

11087 CUSHMAN TODD 1947

11088 CUSHMAN TODD 194

11089 CUSHMAN TODD 1947

11090 CUSHMAN TODD 1947

11091 CUSHMAN TODD 1947

11092 BERGEN O'NEIL 1979

11093 BERGEN O'NEIL 1979

11094 BERGEN O'NEIL 1979

11095 BERGEN O'NEIL 1979

11096 BERGEN O'NEIL 1979

11097 BERGEN O'NEIL 1979

11098 TODD LOW 1967

11099 BERGEN O'NEIL 1979

11100 BERGEN O'NEIL 1979

11101 BERGEN O'NEIL 1979

$\begin{array}{lll}11102 & \text { BERGEN O'NEIL } 1979 \\ 11103 & \text { BERGEN O'NEIL } 1979\end{array}$

11104 BERGEN O'NEIL 1979

11105 BERGEN O'NEIL 1979

11106 BERGEN O'NEIL 1979

11107 BERGEN O'NEIL 1979

11108 BERGEN O'NEIL 1979

11109 BERGEN O'NEIL 1979

11110 BERGEN O'NEIL 1979

11111 BERGEN O'NEIL 1979

11112 BERGEN O'NEIL 1979

11113 BERGEN O'NEIL 1979

11114 NATLAND 1933

11115 BERGEN O'NEIL 1979

11116 BERGEN O०NEIL 1979

11117 BERGEN O'NEIL 1979

11118 BERGEN O'NEIL 197

11119 CUSHMAN TODD 194

11120 CUSHMAN TODD 1947

11121 BERGEN O'NEIL 1979

11122 BERGEN O'NEIL 1979

11123 BERGEN O'NEIL 1979

11124 BERGEN O'NEIL 1979

11125 BERGEN O'NEIL 1979

11126 BERGEN ODNEIL 1979

11127 BERGEN O'NEIL 1979

11128 BERGEN O'NEIL 1979

11129 BERGEN O'NEIL 1979
11130 BERGEN O'NEIL 1979
Generic Name

EPISTOMINELLA EP ISTOMI NELLA EPISTOMI NELLA EP I STOM I NEL LA EP I STOM I NELLA EPISTOMI NELLA EPISTOMI NELLA EP I S TOMI NELLA EPISTOMI NELLA EPISTOMINELLA EP I STOM I NELLA EP I STOMI NELLA EPISTOMINELLA EP ISTOMINELLA EP I STOMI NELLA EP I STOM I NELLA EPISTOMINELLA EP I STOM I NELLA EP I STOM I NELLA EP I STOM I NELLA EPISTOMI NELLA EPISTOMINELLA EP I STOMI NELLA EP I ST OM I NELLA EP I STOMI NELLA EP I STOMI NELLA EPISTOMI NELLA EP I STOMI NELLA EP IS TOMI NELLA EP I STOM I NELLA EP ISTOM I NELLA EP I STOM I NELLA EP I STOM I NELLA EP I S TOM I NELLA EP ISTOMI NELLA EP I STOMI NELLA EP I S TOM I NELLA EP I S TOM I NELLA EP I S TOM I NELL A EP I STOM I NELLA EPISTOMINELLA EPISTOMINELLA EP I STOM I NELLA EP I STOM I NELLA EPISTOMI NELLA EPONIDES

EPONIDES

EPONIDES

EPONIDES

EPONIDES

EPONIDES

EPONIDES

EPONIDES

BUCCELLA

BUCCELLA

EPONIDES

EPONIDES

EPONIDES

EPONIDES

EPONIDES

EPONIDES

ROSALINA

EPONIDES

EPONIDES

EPONIDES

EPONIDES

EPONIDES

EPONIDES

EPONIDES

EPONIDES

EPONIDES

EPONIDES

EPONIDES

EPONIDES

EPONIDES

EPONIDES

EPONIDES

EPONIDES

EPONIDES

EPONIDES

EPONIDES

EPONIDES

EPONIDES

EPONIDES

EPONIDES

EPONIDES

EPONIDES

EPONIDES

EPONIDES

EPONIDES

EPONIDES
Specific Name

EXI GUA

EXIGUA

PAC IFICA

$P A C$ IF ICA

PAC IFICA

PACIFICA

PAC IFICA

PACIFICA

PACIFICA

PACIFICA

PACIFICA

PACIFICA

PAC IFICA

PACIFICA

PACIFICA

PAC IFICA

PAC IF ICA

PAC I F ICA

$P A C$ IF ICA

PAC IF ICA

VITREA

VITREA

VITREA

VITREA

VITREA

VITREA

VITREA

VITREA

VITREA

VITREA

VITREA

VITREA

VITREA

VITREA

VITTEA

VITREA

VITREA

VITREA

VITREA

COLUMB I ENSIS

C OL UMB IENS IS

COL UMB I ENS IS

COL UMBIENSIS

C OL UMB IENSIS

COL UMB IENS IS

COL UMBIENSIS

COL UMB IENSIS

FRI GIDA

FRI GIDA

FRI GIDA

HEALDI

HEALDI

HEALDI

HEALDI

HEALD

HEALDI

I SABELLEANA

LEV I CULUS

LEV I CULUS

LEV I CULUS

LEVICULUS

LEVICULUS

LEVICULUS

LEVI CULUS

LEV I CULUS

LEV I CULUS

LEV I CULUS

LEV I CULUS

LEVICULUS

LEV ICULUS

LEVICULUS

LEV ICUL US

ORN ATA

REPANDUS

REPANDUS

REPANDUS

REPANDUS

REPANDUS

REPANDUS

REPANDUS

REPANDUS

REPANDUS

SUB TENERA

SUB TENERA

SUB TENERA

SUB TENERA

SUB TENERA

SUB TENERA
SUB TENERA

Locality

Lat. Long.

NORTH PACIFIC

GULF OF ALASKA

GULF OF ALASKA

GULF OF ALASKA

GULF OF ALASKA

CLARENCE ST.. ALASKA

GULF OF ALASKA

GULF OF ALASKA

GULF OF ALASKA

GULF OF ALASKA

GULF OF ALASKA

GULF OF ALASKA

GULF OF ALASKA

GULF OF ALASKA 


\section{Publication}

11131 CUSHMAN 1927

11132 CUS HMAN 1927

11133 BERGEN O NE IL 1979

11134 BERGEN O N NE IL 1979

11135 BERGEN O०NEIL 1979

11136 BERGEN O'NEIL 1979

11137 BERGEN O'NEIL 1979

11138 BERGEN OONEIL 1979

11139 TODD LOW 1967

11140 TOD D LOW 1967

11141 BERGEN O NEIL 1979

11142 BERGEN O'NEIL 1979

11143 BERGEN O'NEIL 1979

11144 DETLING 1958

11145 COOPER 1961

11146 COCKBAIN 1963

11147 COCKBAIN 1963

11148 BERGEN O'NEIL 1979

11149 BERGEN O'NEIL 1979

11150 BERGEN O'NEIL 1979

11151 BER GEN O'NEIL 1979

11151 BER GEN O'NE IL 1979

11152 BERGEN O'NEIL 1979

11154 BERGEN O'NE IL 1979

11155 BERGEN O'NEIL 1979

11156 BERGEN O'NEIL 1979

11157 BERGEN O'NEIL 1979

11158 BERGEN O'NE II 1979

11159 BERGEN O NEE IL 1979

11160 BERGEN O NE IL 1979

19161 BERGEN O०NEIL 1979

11162 BERGEN O'NEIL 1979

11163 TODD LOW 1967

11164 TODD LOW 1967

11165 TODD LOW 1967

11166 LANKFORD PHLEGER 1973

11167 LANKF ORD PHLEGER 1973

11168 COOPER 1961

11169 BERGEN O NEIL 1979

11170 COOPER 1961

11171 COOPER 1961

11172 BERGEN O'NEIL 1979

11173 BERGEN O'NEIL 1979

11174 BERGEN O'NEIL 1979

11175 BERGEN O'NEIL 1979

11176 BERGEN O NE IL 1979

11177 BERGEN O'NEIL 1979

11178 BERGEN O०NEIL 1979

11179 BERGEN O०NEIL 1979

11180 BERGEN O'NEIL 1979

11181 BERGEN O'NE IL 1979

11182 BERGEN O'NEIL 1979

11183 BERGEN O०NE IL 1979

11184 BERGEN O'NEIL 1979

11185 BERGEN O NEE IL 1979

11186 BERGEN O'NEIL 1979

11186 BERGEN O'NE IL 1979

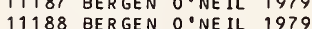

11189 BERGEN O'NEIL 1979

11190 BERGEN O'NEIL 1979

11191 BERGEN O ${ }^{\circ}$ NE IL 1979

11192 LANKFORD PHLEGER 1973

11193 ECHOLS 1969

11194 ECH OLS 1969

11195 ECHOLS 1969

11196 BERGEN O'NEIL 1979

11197 BERGEN OONEIL 1979

11198 BERGEN O'NEIL 1979

11199 BERGEN O०NE IL 1979

11200 BERGEN O'NEIL 1979

11201 BERGEN O'NEIL 1979

11202 BERGEN O'NEIL 1979

11203 BERGEN O'NE IL 1979

11204 BERGEN O'NEIL 1979

11205 BERGEN O'NE IL 1979

11206 BERGEN O०NE IL 1979

11207 TODD LOW 1967

11208 TODD LOW 1967

11209 TOD D LOW 1967

11210 TODD LOW 1967

11211 BERGEN O'NE IL 1979

11212 WHITEAVES 1886

11213 WHI TEAVES 1886

11214 TODD LOW 1967

11215 TODD LOW 1967

11216 TODD LOW 1967

11217 TODD LOW 1967

11218 TODD LOW 1967

11219 TODD LOW 1967

11220 CUSHMAN MCCULLOCH 1939

11221 COCKBAIN 1963

11222 COOPER 1961

11223 COCKBAIN 1963

11224 COC KBAIN 1963

11225 COCKBAIN 1963
Generic Name Specific Name

TENERA

UMB ONATUS

UMBONATUS

UMB ONATUS

UMB ONATUS

UMB ONATUS

AGASSI Z I

AGASSIZ

UC URBI TASEMA

CUCURBITASEMA

LUC IDA

LUC IDA

LUC I DA

LUC IDA

L UC I DA

LUC IDA

LUC IDA

LUC IDA

LUC IDA

LUC IDA

LUC IDA

LUC IDA

LUC I DA

LUC IDA

LUC IDA

LUC I DA

LUC IDA

LUC IDA

LUC IDA

$L U C I D A$

UC I DA

LUC IDA

LUC I DA

LUC IDA

LUC I DA

LUC IDA

MAR G INATA

MAR GINATA

MARGINATA

MAR G INATA

MAR GINATA

MARG INATA

MAR GINATA

A UR I CULA

A UR I CULA

A UR I CULA

A UR I CULA A

A UR I CULA

AUR I CUL A

A UR I CULA

A UR I CULA

A UR I CULA

A UR I CUL A

A UR I CULA

A UR I CULA

A UR I CULA

A UR I CULA

A UR I CULA

BAS ISPINATA

J AP ONICA

JAP ONICA

JAP ONICA

$\angle A B R A D O R I C A$

LABRADORICA

$\angle A B R A D O R I C A$

$\angle A B R A D O R I C A$

LABRADORICA

LABRADORICA

$\angle A B R A D O R I C A$

LABRADORICA

LABRAD

$\angle A B R A D O R I C A$

LABRADORICA

LABRADORICA

LABRADORICA

ABRADORICA

$\angle A B R A D O R I C A$

LABRADORICA

CANALI CULATA

CANALICULATA

GIGAS

GIGAS

ARE NARIA

A RE NARIA

ARE NAR IA

A RE NAR I A

ARE NAR I A

A RE NAR IA

ARE NAR IA

ARENARIA

ARENARIA

ARE NAR IA
Locality

Lat. Long.

OFF OREGON

OFF OREGON

GULF OF ALASKA

GULF OF ALASKA

GULF OF ALASKA

GULF OF ALASKA

GULF OF ALASKA

GULF OF ALASKA

GULF OF ALASKA

GULF OF ALASKA

GULF OF ALASKA

GULF OF ALASKA

SUNSET BAY, OREGON

CALIFORNIA COAST

BR ITISH COL UMBIA

BRITISH COL UMBIA

GULF OF ALASKA

GULF OF ALASKA

GULF OF ALASKA

GULF OF ALASKA

GULF OF ALASKA

GULF OF ALASKA

GULF OF ALASKA

GULF OF ALASKA

GULF OF ALASKA

GULF OF ALASKA

GULF OF ALASKA

GULF OF ALASKA

GULF OF ALASKA

GULF OF ALASKA

GULF OF ALASKA

EXCURSION, ALASKA

CLARENCE ST.. ALASKA

GULF OF ALASKA

CRESCENT CITY, CALIF.

LAPUSH, WASHINGTON

CALIFORNIA COAST

GULF OF ALASKA

OREGON COAST

OREGON COAS

GULF OF ALASKA

GULF OF ALASKA

GULF OF ALASKA

GULF OF ALASKA

GULF OF ALASKA

GULF OF ALASKA

GULF OF ALASKA

GULF OF ALASKA

GULF OF ALASKA

GULF OF ALASKA

GULF OF ALASKA

GULF OF ALASKA

GULF OF ALASKA

GULF OF ALASKA

GULF OF ALASKA

GULF OF ALASKA

GULF OF ALASKA

GULF OF ALASKA

GULF OF ALASKA

GULF OF ALASKA

CRESCENT CITY, CALIF.

WASHINGT ON COAST

WASHINGTON COAST

WASHINGTON COAST

GULF OF ALASKA

GULF OF ALASKA

GULF OF ALASKA

GULF OF ALASKA

GULF OF ALASKA

GULF OF ALASKA

GULF OF ALASKA

GULF OF ALASKA

GULF OF ALASKA

GULF OF ALASKA

GULF OF ALASKA

CLARENCE ST.. ALASKA

KASAAN BAY, ALASKA

EXCURSION, ALASKA

GULF OF ALASKA

GULF OF ALASKA

STRAIT OF GEORGIA, B. C.

STRAIT OF GEORGIA, B. $C$.

GULF OF ALASKA

KASAAN BAY, ALASKA

EXCURSION, ALASK

GULF OF ALASKA

KASAAN BAY, ALASKA

TAKU HARBOUR, ALASKA

OFF ALASKA

BRITISH COLUMBIA

OREG ON COAST

BRITISH COLUMBIA

BR IT ISH COLUMBIA

BRITISH COLUMBIA

$4315 \mathrm{~N} 12453 \mathrm{~W}$

$4308 \mathrm{~N} 12459 \mathrm{~W}$

$5930 \mathrm{~N} 14520 \mathrm{~W}$

$\begin{array}{llll}59 & 30 N & 145 & 40 \mathrm{~W} \\ 59 & 00 N \quad 141 & 20 \mathrm{~W}\end{array}$ 


\section{Publication}

11226 BERGEN O'NEIL 1979 11227 BERGEN O'NEIL 1979 11228 BERGEN O'NEIL 1979 11229 BERGEN O.NEIL 1979 11230 CUSHMAN TODD 1947 11231 CUSHMAN TODD 1947 11232 COOPER 1961

11233 COOPER 1961

11234 COCKBAIN 1963
11235 COOPER 1961

11236 COCKBAIN 1963

11237 COCKBAIN 1963

11238 COCKBAIN 1963

11239 CUSHMAN TODD 1947

11240 ECHOLS 1969

11241 COOPER 196

11242 SCOTT 1974
11243 ECHOLS 1969

11244 BERGEN O'NEIL 1979

11245 BERGEN O.'NE IL 1979

11246 BERGEN O'NEIL 1979

11247 BERGEN O'NEIL 1979

11248 BERGEN O'NEIL 1979

11249 BERGEN O'NEIL 1979

11250 BERGEN O'NEIL 1979

11251 TODD LOW 1967

11252 COCKBAIN 1963

11253 COCKBAIN 1963

11254 COCKBAIN 1963

11255 COCKBAIN 1963

11256 BERGEN O'NEIL 1979

11257 BERGEN O'NEIL 1979

11258 BERGEN O'NEIL 1979

11259 TODD LOW 1967

11260 TODD LOW 1967

11261 TODD LOW 1967

11262 TODD LOW 1967

11263 TODD LOW 19

11265 SERGEN O'NEIL 1979

11266 COCKBAIN 1963

11267 BERGEN O'NEIL 1979

11268 BERGEN O'NE IL 1979

11269 BERGEN O'NEIL 1979

11270 BERGEN O'NEIL 1979

11271 BERGEN O'NEIL 1979

11272 BERGEN O'NEIL 1979

11273 BERGEN O'NEIL 1979

11274 BERGEN O'NEIL 1979

11275 COCKBAIN 1963

11276 SMITH 1973

11277 SMITH 1973

11278 SMITH 1973

$\begin{array}{lll}11279 & \text { SMI TH } & 1973 \\ 11280 & \text { SMI TH } & 1973\end{array}$

11280 SMI TH 1973
11281 TODD LOW 1967

11282 CUSHMAN 1910

11283 CUS HMAN 1910

11284 TODD LOW 1967

11285 BERGEN O'NEIL 1979

11286 BERGEN O'NEIL 1979

11287 BERGEN O'NEIL 1970

11288 BERGEN O'NEIL 1979

11289 BERGEN O'NEIL 1979

11290 BERGEN O'NEIL 1979

11291 BERGEN O'NEIL 1979

11292 BERGEN O'NEIL 1979

11293 BERGEN O'NEIL 1979

11294 BERGEN O'NEIL 1979

11295 BERGEN O'NEIL 1979

11296 BERGEN O'NE IL 1979

11297 BERGEN O.NEIL 1979

11298 BERGEN O'NEIL 1979

11299 BERGEN O'NEIL 1979

11300 BERGEN O'NE IL 1979

11301 BERGEN O'NEIL 1979

11302 BERGEN O'NEIL 1979

11303 SMITH 1973

11304 BERGEN O'NEIL 1979

11305 WHITEAVES 1886

11306 WHITEAVES 1886

1307 WHI TEAVES 1886

1308 WHITEAVES 1886

11309 CUS HMAN 1925
11310 CUSHMAN 1925

11311 CUSHMAN MCCULLOCH 1939

11312 CUSHMAN TODD 1947

11313 CUSHMAN TODD 194

11314 TODD LOW 1967

11315 TODD LOW 1967

11316 SMITH 1973

11317 SMI TH 1973

11318 CUSHMAN 1910

11319 CUSHMAN MCCULLOCH 1939

11320 DETLING 1958
Generic Name Specific Name

\section{Locality}

Lat. Long.

GAUDRYINA

ARENARIA

GAUDRYINA

GAUDRY INA

GAUDRYINA

GAUDRYINA

AUDRY INA

GAUDRYINA

GAUDRY INA

GAUDRYINA

ANGULODISCORBIS

GLABRATELLA

GLABRATELLA

GLABRATELLA

GLANDUL INA

GLOBOBUL IMINA

GLOBOBUL IMINA

GLOBOBUL IMINA

GLOBOBUL IMINA

GLOBOBUL IMINA

GLOBOBUL IM I NA

GLOBOBUL IM INA

GL OBOBUL IM INA

GLOBOBUL IMINA

GLOBOBUL IM I NA

GLOBOBUL IMINA

GLOBOBUL IM INA

GLOBOBUL IM INA

GLOBOBUL IMINA

GLOBOBUL IM INA

GLOBOBUL IMINA

GL OBOBUL IMINA

GLOBOBUL IMINA

GLOBOBULIMINA

GLOBOBUL IMINA

GLOBOBUL IM INA

GLOBOBUL IMINA

GL OBOBUL IMINA

GLOBOBUL IMINA

GLOBOBUL IMINA

GLOBOBUL IMINA

GLOBOBUL IMINA

GLOBOBUL IMINA

GLOBOBUL IMINA

GLOBOBUL IMINA

GLOBO UL IMINA

GLOBOTEXTULARIA

GLOMOSPIRA

GLOMOSPIRA

GLOMOSPI RA

GLOMOSPIRA

GOE SELLA

GLOMOSPIRA

GLOMOSPIRA

GUTTUL INA

GUTTUL INA

GYROIDINA

GROIDINA

GYROIDINA

GYROIDINA

GYROIDINA

GYROIDINA

GYROIDINA

GYROIDINA

GYROIDINA

GYROIDINA

GYROIDINA

GYROIDINA

GYROIDINA

GYROIDINA

GYROIDINA

GYROIDINA

GYROI DINA

GYROIDINA

HAPL OPHRAGMOIDES

HAPLOPHRAGMOIDES

HAPLOPHRAGMOIDES

HAPLOPHRAGMOIDES

HAPLOPHRAGMOIDES

HAPLOPHRAGMOIDES

HAPLOPHRAGMOIDE

HAPLOPHRAGMOIDES

HAPLOPHRAGMOIDE

HAPLOPHRAGMOIDES

HAPLOPHRAGMOIDES

HAPLOPHRAGMOIDES

HAPLOPHRAGMOIDES

HAPLOPHRAGMOIDE

HAPLOPHRAGMOIDE

HAPLOPHRAGMOIDE

HAPLOPHRAGMOIDES
A RE NAR I A

A TL ANTI I A

A TLANTICA PACIF I CA

SUBGLABRATA

SUBGLABRATA

SUB GLABRATA

SUBGLABRATA

SUBGLABRATA

CHARLOTTENSIS

LAURIE I

OPER CULARIS

ORNAT I SSIMA

LAE VIGATA

A UR I CULATA

A UR I CULATA

A UR I CULATA

A UR I CULATA

A UR I CULATA

A UR I CULATA

A UR I CULATA

A UR I CULATA

A UR I CULATA

AUR I CULATA

A UR I CULATA

A UR I CULATA

A UR I CULATA

A UR I CULATA

A UR I CULATA

A UR I CULATA

A UR I CULATA

AUR I CULATA

PAC I I I CA

PACIFICA

PAC IF I CA

PAC IF I CA

PAC IFICA

PAC IFICA

PACIFICA

PAC IFICA

PAC IF I CA

PAC IFICA

ANCEPS

GOR DIALIS

GORDIALIS

GOR DIALIS

GOR D IALIS

FL I NT I I

CHAROIDES

GORDIAL IS

ORIENTALIS

PROBLEMA

ALT IFORMIS

ALT IFORMIS

ALT IFORMIS

ALT I FORMIS

GEMMA

GEMMA

GEMMA

GEMMA

I $O$

I 0

10
10

10
10

10

10

I 0

LAM ARCKI ANA

LAMARCKI ANA

ORB I CULAR IS

CANARIENSIS

CANARIENSIS

CANARIENSIS

CANARIENSIS

A DVENUM

A DVENUM

A DVENUM

ADV ENUM

A DVENUM

BRA DYI

ANARI ENS IS

CANARIENSIS

CANARIENSIS

COL UMB I ENS IS
COL UMBIENSIS
A UR I CULATA

GULF OF ALASKA

GULF OF ALASKA

GULF OF ALASKA

GULF OF ALASKA

WASHINGTON COAST

OREGON COAST

OREGON COAS

BRITISH COL UMBIA

OREGON COAST

BR ITISH COLUMBIA

BR IT ISH COL UMB IA

WASHINGTON COAST

WASH INGT ON COAST 


\section{Publication}

11321 CUSHMAN TODD 1947

11322 CUS HMAN TODD 1947

11323 CUSHMAN MCCULLOCH 1939

11324 CUS HMAN MCCULLOCH 1939

11325 CUS HMAN MCCULLOCH 1939

11326 CUSHMAN 1925

11327 CUSHMAN TODD 1947

11328 CUS HMAN TODD 1947

11329 CUSHMAN MCCULLOCH 1939

11330 CUS HMAN MCCULLOCH 1939

11331 COC KBAIN 1963

11332 COC KBAIN 1963

11333 COCKBAIN 1963

11334 COCKBAIN 1963

11335 SMITH 1973

11336 BERGEN O'NE IL 1979

11337 BERGEN O'NEIL 1979

11338 OERGEN O'NEIL 1979

11338 BERGEN OONEIL 1979

11340 BERGEN O'NEIL 1979

11341 BERGEN OPNE IL 1979

11342 BERGEN O'NEIL 1979

11343 BERGEN O'NEIL 1979

11344 BERGEN O'NEIL 1979

11345 TODD LOW 1967

11346 TODD LOW 1967

11347 TODD LOW 1967

11348 BERGEN O'NEIL 1979

11349 TODD LOW 1967

11350 CUSHMAN MCCULLOCH 1939

11351 PHLEGER 1967

11352 PHLEGER 1967

11353 PHLEGER 1967

11354 CUS HMAN 1927

11355 CUSHMAN 1927

11356 BERGEN O'NEIL 1979

11357 SMITH 1973

11358 SMI TH 1973

11359 SMITH 1973

11360 SMITH 1973

11361 SMITH 1973

11362 TODD LOW 1967

11363 BERGEN O'NEIL 1979

11364 BERGEN O'NEIL 1979

11365 BERGEN O'NEIL 1979

11366 BERGEN O'NEIL 1979

11367 BERGEN O'NEIL 1979

11368 SMITH 1973

11369 SMITH 1973

11370 COC KBAIN 1963

11371 COCKBAIN 1963

11372 COCKBAIN 1963

11373 COCKBAIN 1963

11374 COC KBAIN 1963

11375 SMITH 1973

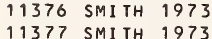

11377 SMITH 1973

11378 SMITH 1973
11379 SMITH 1973

11380 SMITH 1973

11381 PHLEGER 1967

11382 PHLEGER 1967

$\begin{array}{lll}11382 & \text { PHLEGER } & 1967 \\ 11383 & \text { PHLEGER } & 1967\end{array}$

11384 BERGEN O'NEIL 1979

11385 BERGEN O 'NEIL 1979

11386 BERGEN O'NEIL 1979

11387 BERGEN O'NEIL 1979

11388 BERGEN O'NEIL 1979

11389 BERGEN O'NEIL 1979

11390 BERGEN O'NEIL 1979

11391 BERGEN O'NEIL 1979

11392 BERGEN O'NE IL 1979

11393 BERGEN O'NEIL 1979

11394 BERGEN O'NEIL 1979

11395 TODD LOW 1967

11396 TODD LOW 1967

11397 BERGEN O'NEIL 1979

11398 BERGEN O'NEIL 1979

11399 BERGEN O.NE IL 1979

11400 BERGEN O'NEIL 1979

11401 BERGEN $0^{\circ}$ NEIL 1979

11402 BERGEN O'NEIL 1979

11403 BERGEN O'NEIL 1979

11404 CUSHMAN MCCULLOCH 1950

11405 CUSHMAN MCCULLOCH 1950

11406 CUSHMAN TODD 1947

11407 TODD LOW 1967

11408 TODD LOW 1967

11409 BERGEN O'NEIL 1979

11410 BERGEN O'NEIL 1979

11411 BERGEN O'NEIL 1979

11412 BERGEN O.NEIL 1979

11413 GER GEN OINEIL 1979

11414 BERGEN O.NEIL 1979

11415 BERGEN O'NEIL 1979
Generic Name

HAPLOPHRAGMOIDES HAPLOPHRAGMOIDES HAPLOPHRAGMOIDES HAPLOPHRAGMOIDES HAPLOPHRAGMOIDES HAPLOPHRAGMOIDES ADERCOTRYMA

ADERCOTRYMA

CRIBROSTOMOIDES

CRIBROSTOMOIDES

HAPLOPHRAGMOIDES

HAPLOPHRAGMOIDES

HAPLOP HRAGMOIDES

HAPLOPHRAGMOIDES

CRIBROS TOMOIDES

THALMANNAMM INA

THALMANNAMMINA

THALMANNAMMINA

THALMANNAMMINA

THALMANNAMM INA

THALMANNAMM INA

THALMAN NAMM INA

THALMANN AMM I NA

HAPLOPHRAGMOIDES

HAPLOPHRAGMOIDES

HAPLOPHRAGMOIDES

HAPLOPHRAGMOIDES

HAPLOPHRAGMOIDES

CRI BROSTOMOIDES

HAPLOPHRAGMOIDES

HAPL OPHR AGMOIDES

HAPL OPHRAGMOIDE

HAPLOP HRAGMOIDES

HAPLOPHRAGMOIDES

HOE GLUND INA

HOE GLUND INA

HOE GLUND INA

HORMOS INA

HYPERAMM INA

HYPERAMM INA

HYPERAMM INA

HYPERAMM INA

HYPERAMMINA

HYPERAMM INA

HYPERAMM INA

HYPERAMM INA

HYPERAMMINA

HYPERAMM INA

AMMODISCUS

AMMODISCUS

AMMODISCUS

AMMODISCUS

AMMODISCUS

AMMODISCUS

AMMODISCUS

JAC ULELLA

JACULELLA

JACULELLA

JACULELLA

JADAMMINA

J AD AMM INA

KARRERIELLA

KARRERIELLA

KARRER IELLA

KARRERIELLA

KARRERIELLA

KARRER IELLA

KARRER IELLA

KARRERIELLA

KARRER IELLA

KARRER IELLA

KARRERIELLA

KARRERIELLA

KARRERIELLA

KARRER IELLA

KARRER IELLA

KARRER IELLA

KARRER I ELLA

KARRER IELLA

KARRERIELLA

KARRERIELLA

LAGENA

LAGENA

LAGENA

LAGENA

LAGENA

LAGENA

LAGENA

LAGENA

LAGENA

LAGENA

LAGENA
Specific Name

COL UMB IENSIS

C OL UMB IENSIS

C OL UMBIENSIS

COL UMB IENSIS

COL UMBIENSIS

COL UMBIENSIS

GLOMERATA

GLOMERATA

JEFFREYS I I

JEFFREY

BRADYI

BRA DYI

BRADYI

NIT I DUM

PARKERAE

PARKERAE

PARKERAE

PARKERAE

PAR KERAE

PARKERAE

PARKERAE

PARKERAE

PARKERAE

PLANISSIMUM

PLANISSIMUM

PLANISS IMUM

PLANISSIMUM

SPHAERI LOCUL US

SUB GLOBOSUM

SUB INVOLUTUM

SUB INVOLUTUM

SUB INVOLUTUM

TENUIS

ELEGANS

ELEGANS

ELEGANS

GLOBULIFERA

CYL INDR ICA

CYL INDR ICA

ELONGATA

ELONGATA

ELONGATA

ELONGATA

ELONGATA

ELONGATA

FRI ABIL IS

FRIABILIS

GULLMARENSIS

GULLMARENS IS

GULL LMARENSIS

GUL LMARENSIS

GULLMARENSIS

TENUIS

TENUIS

ACUTA

ACUTA

A CUTA

ACUTA

POL YSTOMA

POL YSTOMA

POL YSTOMA

BAC CATA

BAC CATA

BAC CATA

BAC CATA

BAC CATA

BAC CATA

QAC CATA

BAC CATA

BAC CATA

BAC CATA

GAC CATA

BAC CATA

BACCATA

BAC CATA

PARKERAE

PARKERAE

PARKERAE

PARKERAE

PARKERAE

PARKERAE

PARKERAE

API OPLEURA

API OPLEURA

API OPLE URA

AMP HORA

AMP HORA

APIOPLEURA

APIOPLEURA

API OPLEURA

API OPLEURA

API OPLEURA

API OPLEURA

API OPLEURA

Locality

Lat. Long.

WASHINGTON COAST

WASHINGTON COAST

KETCHIKAN, ALASKA

OFF BRIT. COLUMBIA 


\section{Publication}

11416 CUSHMAN TODD 1947

11417 CUSHMAN TODD 1947

11418 CUSHMAN TODD 1947

11420 COCKBAIN 1963

11421 COCKBAIN 1963

11422 COCKBAIN 1963

11423 COCKBAIN 1963

11424 COCKBAIN 1963

11425 TODD LOW 1967

11426 TODD LOW 1967

11427 TODD LOW 1967

11428 CUSHMAN MCCULLOCH 1950

11429 CUSHMAN MCCULLOCH 1950

11430 TODD LOW 1967

11431 TODD LOW 1967

11432 BERGEN O'NEIL 1979

11433 BERGEN O.NEIL 1979

11434 COCKBAIN 1963

11435 CUSHMAN 1927

11436 BERGEN O N NEIL 1979

11437 BERGEN O'NEIL 1979

11438 BERGEN O'NEIL 1979

11439 BERGEN O'NEIL 1979

11440 BERGEN O'NEIL 1979

11441 BERGEN O'NEIL 1979

11442 BERGEN O'NEIL 1979

11443 BERGEN O.NEIL 1979

11444 BERGEN O N NEIL 1979

11445 CUSHMAN TODD 1947

11446 CUS HMAN TODD 1947

11447 COCKBAIN 1963

11448 CUSHMAN 1927

11449 CUSHMAN 1927

11450 COCKBAIN 1963

11451 BERGEN O०NEIL 1979

11452 BERGEN 0. NEIL 1979

11453 BERGEN O'NEIL 1979

11454 BERGEN O.NEIL 1979

11455 BERGEN O'NEIL 1979

11456 CUSHMAN 1927

11457 CUSHMAN 1927

11458 TODD LOW 1967

11459 TODD LOW 1967

11660 BERGEN O NEIL 1979

11461 BERGEN O'NEIL 1979

11462 BERGEN O ONEIL 1979

11463 BERGEN O'NEIL 1979

11464 BERGEN O.NEIL 1979

11465 CUSHMAN MCCULLOCH 1950

11466 CUS HMAN 1927

11467 BERGEN O'NEIL 1979

11468 BERGEN O'NEIL 1979

11469 BERGEN O'NEIL 1979

11470 TODD LOW 1967

11471 TODD LOW 1967

11472 BERGEN O.NEIL 1979

11473 BERGEN O०NEIL 1979

11474 CUSHMAN 1925

11475 BERGEN O'NEIL 1979

11476 BERGEN O'NEIL 1979

11477 BERGEN O०NEIL 1979

11478 CUSHMAN TODD 1947

11479 CUSHMAN MCCULLOCH 1950

11480 CUS HMAN MCCULLOCH 1950

11681 CUS HMAN TODD 1947

11482 CUSHMAN TODD 1947

11483 CUSHMAN TODD 1947

11484 COCKBAIN 1963

11485 COCKBAIN 1963

11486 COCKBAIN 1963

11487 BERGEN O NEIL 1979

11488 TODD LOW 1967

11489 CUSHMAN MCCULLOCH 1950

11490 BERGEN O०NEIL 1979

11491 BERGEN O०NEIL 1979

11492 BERGEN O०NEIL 1979

11493 BERGEN O०NEIL 1979

11494 BERGEN O.NEIL 1979

11495 CUSHMAN MCCULLOCH 1950

11496 BERGEN O'NEIL 1979

11497 BERGEN O'NEIL 1979

11498 BERGEN O'NEIL 1979

11499 BERGEN O'NEIL 1979

11500 BERGEN O.NEIL 1979

11501 BERGEN OPNEIL 1979

11502 BERGEN $0^{\circ} \mathrm{NE}$ IL 1979

11503 BERGEN O०NEIL 1979

11504 BERGEN O'NEIL 1979

11505 LANKFORD PHLEGER 1973

11506 TODD LOW 1967

11507 TODD LOW 1967

11508 CUSHMAN MCCULLOSH 1950

11509 BERGEN O'NEIL 1979

11510 BERGEN OPNEIL 1979
Generic Name Specific Name

LAGENA

OOL INA

OOL INA

LAGENA

LAGENA

LAGENA

LAGENA

LAGENA

LAGENA

LAGENA

LAGENA

LAGENA

LAGENA

LAGENA

LAGENA

LAGENA

LAGENA

LAGENA

A GENA

AGENA

LAGENA

LAGENA

$\angle A G E N A$

LAGENA

LAGENA

LAGENA

LAGENA

AGENA

LAGENA

LAGENA

LAGENA

LAGENA

LAGENA

LAGENA

LAGENA

LAGENA

LAGENA

LAGENA

LAGENA

LAGENA

LAGENA

LAGENA

LAGENA

LAGENA

LAGENA

LAGENA

LAGENA

LAGENA

LAGENA

LAGENA

LAGENA

FIS SUR INA

LAGENA

LAGENA

LAGENA

LAGENA

LAGENA

LAGENA
LAGENA

LAGENA

LAGENA

LAGENA

LAGENA

LAGENA

LAGENA

LAGENA

LAGENA

LAGENA

LAGENA

LAGENA

LAGENA

LAGENA

LAGENA

LAGENA

LAGENA

LAGENA

LAGENA

LAGENA

LAGENA

LAGENA

LAGENA

LAGENA

LAGENA

LAGENA

LAGENA

LAGENA

LAGENA
CLAVATA

BOREALIS

BOREALIS

BOREALIS

DEN TAL I FORMIS

D IS TOMA

DIS TOMA

DIS TOMA

D IS TOMA

DISTOMA

DIS TOMA

DIS TOMA

D IS TOMA

ELONGATA

ELONGATA

ELONGATA

ELONGATA

ELONGATA

ELONGATA

ELONGATA

E LONGATA

ELONGATA

ELONGATA

ELONGATA

ELONGATA

ELONGATA

ELONGATA

ELONGATA

GRACILIS

GRA C ILIS

GRACILIS

GRACILIS

GRACILIS

GRACILIS

GRA C IL IS

GRACILIS

GRACILIS

GRACILIS

CLAVATA

CLAVATA

LAE VIS

LAE VIS

LAE VIS

LAE V I S

LAE VIS

LAE VIS

PAR R I

LAMELLATA

MER IDIONALIS

MER I DIONALIS

MER IDIONAL IS

MER I DI ONAL IS

MER IDIONALIS

MOL LIS

NEB ULOSA

ORB I GNYANA

PAR R I

PARRI

PAR R I

PERLUCIDA

PER LUCIDA

PERLUCIDA

PERLUCIDA

PERLUCIDA

PLI OCENICA

PLIOCENICA

PLI OCENICA

PLI OCENICA

PLIOCENICA

PLI OCENICA

PLIOCENICA

SEM IL INEA TA

SEM ILINEATA

SEMILINEATA

SEMILINEATA

SEM I L INEA TA

SEM IL INEA TA

SEM ILINEATA

SEM IL INEATA

STR IATA

STR IATA

STR IATA

STR IATA

STR IATA

STR IATA

STR I ATA

STR I ATA

STR IATA

STR IATA

STR IATA

STRIATA

Locality

Lat. Long.

WASH INGTON COAST

WASHINGTON COAST

WASHINGT ON COAST

OFF ALASKA

BRITISH COL UMBIA

BRITISH COLUMBIA

BRITISH COLUMBIA

BRITISH COLUMBIA

BRITISH COLUMBIA

CLARENCE ST.., ALASKA

KASAAN BAY, ALASKA

GULF OF ALASKA

OFF ALASKA

OFF ALASK 


\section{Publication}

11511 BERGEN O'NEIL 1979

11512 BERGEN O'NEIL 1979

11513 CUSHMAN TODD 1947
11514 CUS HMAN MCCULLOCH 1950

11515 CUSHMAN MCCULLOCH 1950

11516 CUS HMAN 1927

11517 CUSHMAN 1927

11518 CUS HMAN 1927

11519 CUSHMAN 1927

11520 COCKBAIN 1963

11521 COCKBAIN 1963

11522 WHITEAVES 1886

11523 CUS HMAN TODD 1947

11524 CUSHMAN MCCULLOCH 1950

11525 BERGEN O'AE IL 1979

11526 BERGEN O'NEIL 1979

11527 BERGEN O'NEIL 1979

11528 BERGEN O NEIL 1979

11529 CUS HMAN MCCULLOCH 1950

11530 CUS HMAN MCCULLOCH 1950

11531 LANKFORD PHLEGER 1973

11532 LANKFORD PHLEGER 1973

11533 TODD LOW 1967

11534 BERGEN O'NEIL 1979

11535 TODD LOW 1967

11537 CERGEN O NE 1979

11537 BERGEN O'NE IL 1979

11538 BERGEN O'NEIL 1979

11540 BERGEN O'NEIL 1979

11541 BERGEN O'NEIL 1979

11542 BERGEN O'NEIL 1979

11543 BERGEN O'NEIL 1979

11544 BERGEN O'NEIL 1979

11545 BERGEN 0'NEIL 1979

11546 SMITH 1973

11547 CUS HMAN TODD 1947

11548 TODD LOW 1967

11549 BERGEN O'NEIL 1979

11550 BERGEN O'NEIL 1979

11551 HAMLIN 1960

11552 HAMLIN 1960

11553 LANKFORD PHLEGER 1973

11554 CUS HMAN TODD 1947

11555 CUSHMAN TODD 1947

11556 SMITH 1973

11557 BERGEN O.NEIL 1979

11558 PHLEGER 1967

11559 JONES ROSS 1979

11560 SCOTT 1974

11561 PHLEGER 1967

11562 PHLEGER 1967

11563 PHLEGER 1967

11564 CUSHMAN TODD 1947

11565 CUS HMAN TODD 1947

11566 WHITEAVES 1886

11567 WHITEAVES 1886

11568 WHITEAVES 1886

11569 WHI TEAVES 1886

11570 LANKFORD PHLEGER 1973

11571 COOPER 1961

11572 COOPER 1961

11573 SMI TH 1973

11574 SMITH 1973

11575 SMITH 1973

11576 SMITH 1973

11577 SMITH 1973

11578 BERGEN O'NEIL 1979

11579 WHITEAVES 1886

11580 WHI TEAVES 1886

11581 CUS HMAN TODD 1947

11582 CUSHMAN TODD 1947

11583 CUSHMAN TODD 1947

11584 CUSHMAN MCCULLOCH 1950

11585 CUS HMAN MCCULLOCH 1940

11586 DETLING 1958

11587 CUSHMAN 1927

11588 CUS HMAN 1927

11589 CUSHMAN 1927

11590 CUS HMAN 1927

11591 CUSHMAN 1927

11592 CUSHMAN 1927

11593 CUSHMAN 1927

11594 SMITH 1973

11595 SMITH 1973

11596 CUSHMAN MCCULLOCH 1940

11597 CUSHMAN TODD 1947

11598 CUSHMAN TODD 1947

11599 CUS HMAN TODD 1947

11600 CUSHMAN 1927

11601 CUS HMAN 1927

11602 CUSHMAN 1927

11603 CUS HMAN 1927

11604 NATLAND 193

11605 SMITH 1973
Generic Name Specific Name

STR IATA

STRIATOPUNCTATA

STRIATOPUNCT ATA

STRIATOPUNCTATA GEMMA

SUBSTRIATA

SUBSTRIATA

SUBSIRIATA

SULCATA

SUL CATA

SUL CATA

SPI CATA

SUL CATA

SPICATA

SPICATA

SPI CATA

SPICATA

SPICATA

A TLANTICA

A TL ANTI I A

SCALARIS

SCALARIS

SCALARIS

SCALARIS

SCALARIS

HYALASCIDIA

NIC OBARENSIS

PORRECTA

ALATA

ALATA

ALATA

ALATA

ALATA

AMY GDALIFORM IS

NS TABILE

GLABRA

OCC I DENTAL IS

OCCIDENTALIS

CRES CENTENS IS

CRE SCENTENSIS

PUL CHRA

PULL CHRA

PUL CHRA

BARLEEANUS

POMPILIOIDES

F US CA

FUSCA

FUS CA

FUSCA

FUS CA

FUS CA

FUSCA

$F$ US C

$F$ US C

FERUSSACHII

SEM I NULA

SEM INUL A

SEM INULA

CALIFORNICA

CIR CULARIS

CIRCULARIS

SUBROTUNDA

SUBROTUNDA

SUBROTUNDA

SUBROTUNDA

MEM BRANACEUM

CAL OMORPHA

PAUPERATA

PAUPERATA

SUBSCALARIS

SUBSCALARIS

SUBSCALARIS

SUBSCALARIS

GRATELOUPI

GRATELOUPI

LABRADORICA

LABRADORI CA

LABRADORICA

LABRADORICA

$\angle A B R A D O R I C A$

$\angle A B R A D O R I C A$

$\angle A B R A D O R I C A$

$\angle A B R A D O R I C A$

LABRADORICA

LABRADORICA

BAS ISP INATA

BAS ISPINATA

BAS ISPINATA

BAS ISPINA TA

PUNCTATA

PUNCTATA

PUNCTATA

SCAPHUM

SCA PHUM
Locality

Lat. Long.

GULF OF ALASKA

GULF OF ALASKA

WASHINGTON COAST

OFF ALASKA

OFF ALASKA

OFF OREGON

OFF OREGON

OFF OREGON

OFF OREGON

BRITISH COLUMBIA

BRITISH COL UMB IA

DISCOVERY PASSAGE, 8.C.

WASHINGTON COAST

OFF ALASKA

GULF OF ALASKA

GULF OF ALASKA

GULF OF ALASKA

GULF OF ALASKA

KETCHIKAN, ALASKA

OFF ALASKA

DEPOE BAY, OREGUN

CRESCENT CITY, CALIF.

TAKU HARBOUR, ALASKA

GULF OF ALASKA

GULF OF ALASKA

GULF OF ALASKA

GULF OF ALASKA

GULF OF ALASKA

GULF OF ALASKA

GULF OF ALASKA

GULF OF ALASKA

GULF OF ALASKA

GULF OF ALASKA

GULF OF ALASKA

OFF ALASKA PENINSULA

WASHINGTON COAST

GULF OF ALASKA

GULF OF ALASKA

GULF OF ALASKA

CRESCENT CITY, CALIF.

PORT ORFORD, OREGON

CRESCENT CITY, CALIF.

WASHINGTON COAST

WASHINGTON COAST

OFF ALASKA PENINSULA

GULF OF ALASKA

GRAY'S HARBOUR, WASH.

SAMISH BAY, WASH.

SAMISH-PADILLA BAYS

COOS, BAY, OREGON

PHILLIPS ARM, B. C.

FRASER R.. B.C.

WASHINGION COAST

WASHINGTON COAST

QUEEN CHARLOTTE SD.. B.C. DISCOVERY PASSAGE, B. C. QUEEN CHARLOTTE SD.. B.C. STRAIT OF GEORGIA, B. C

CRESCENT CITY, CALIF.

OREGON COAST

OREGON COAST

OFF ALASKA PENINSULA

OFF ALASKA PENINSULA

OFF ALASKA PENINSULA

NORTH PACIFIC

OFF ALASKA PENINSULA

GULF OF ALASKA

STRAIT OF GEORGIA, B. C.

STRAIT OF GEORGIA, B. C.

WASHINGTON COAST

WASH INGT ON COAST

WASHINGTON COAST

OFF ALASKA

OFF ALASKA

SUNSET BAY, OREGON

OFF OREGON

OFF OREGON

OFF OREGON

OFF OREGON

OFF OREGON

OFF OREGON

OFF OREGON

OFF OREGON

KODIAK, ALASKA

OF $F$ ALASKA PENINSULA

OIF ALASKA

WASHINGTON COAST

WASHINGTON COAST

WASHINGTON COAST

OFF OREGON

OFF OREGON

OFF OREGON

OFF OREGON

CRESCENT CITY, CALIF.

OFF ALASKA PENINSULA

$5955 \mathrm{~N} 14540 \mathrm{~W}$

$5955 N 14225 \mathrm{~W}$

$4832 \mathrm{~N} 12310 \mathrm{~W}$

$5628 N 13223 W$ 


\section{Publication}

11606 CUSHMAN TODD 1947

11607 SMITH 1973

11608 CUSHMAN TODD 1947

11609 COCKBAIN 1963

11610 COOPER 1961

11611 COCKBAIN 1963
11612 COCKBAIN 1963

11612 COCKBAIN 1963

11614 BERGEN O.NEIL 1979

11615 BERGEN O'NEIL 1979

11616 BERGEN O'NEIL 1979

11617 BERGEN O'NEIL 1979

11618 BERGEN O०NEIL 1979

11619 BERGEN O'NEIL 1979

11620 BERGEN O.NEIL 1979

11621 BERGEN O'NEIL 1979

11622 BERGEN O'NEIL 1979

11623 BERGEN O'NEIL 1979

11624 BERGEN O'NE IL 1979

11625 COCKBAIN 1963

11626 COCKBAIN 1963

11627 COCKBAIN 1963

11628 COCKBAIN 1963

11629 CUS HMAN 1927

11630 CUS HMAN MCCULLOCH 1940

11631 CUSHMAN MCCULLOCH 1940

11632 CUSHMAN MCCULLOCH 1940

11633 CUSHMAN MCCULLOCH 194

11634 CUS HMAN TODD 1947

11635 COCKBAIN 1963

11636 COCKBAIN 1963

11637 COCKBAIN 1963

11638 COCKBAIN 1963

11639 ECHOLS 1969
11640 DETLING 1958

11640 DETLING 1958
11641 ECHOLS 1969

11642 ECHOLS 1969

11643 BERGEN O'NEIL 1979

11644 TODD LOW 1967

11645 TODD LOW 1967

11646 TODD LOW 1967

11647 BERGEN O.NEIL 1979

11648 BERGEN O.NEIL 1979

11649 BERGEN O'NEIL 1979

11650 BERGEN O'NEIL 1979

11651 BERGEN O'NEIL 1979

11652 BERGEN O'NEIL 1979

11653 BERGEN O'NEIL 1979

11654 BERGEN O'NEIL 1979

11655 BERGEN O'NEIL 1979

11656 BERGEN O.NEIL 1979

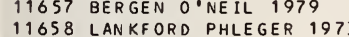

11658 LANKFORD PHLEG
11659 TODD LOW 1967

11659 TODD LOW
11660 SMITH 1973

11661 TODD LOW 1967

11662 COCKBAIN 1963

11663 COCKBAIN 1963

11664 TODD LOW 1967

11665 TODD LOW 1967

11666 CUS HMAN 1925

11667 CUSHMAN 1925

11668 WHI TEAVES 1886
11669 WHITEAVES 1886

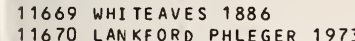

11671 CUSHMAN TODD 1947

11672 SMITH 1973

11673 TODD LOW 1967

11674 TODD LOW 1967

11675 TODD LOW 1967

11676 TODD LOW 1967

11677 COCKBAIN 1963

11678 COCKBAIN 1963

11679 COCKBAIN 1963

11680 COCKBAIN 1963

11681 BERGEN O'NEIL 1979

11682 COCKBAIN 1963

11683 TODD LOW 1967

11684 BERGEN O'NEIL 1979

11685 BERGEN O.NEIL 1979

11686 BERGEN O'NEIL 1979

11687
11688 BERGEN O'NEIL 1979
11689

11688 BERGEN O'NEIL 1979

11689 BERGEN O'NEIL 1979

11690 BERGEN O'NEIL 1979

11691 BERGEN O'NE IL 1979

11692 BERGEN O'NEIL 1979

11693 BERGEN O'NEIL 1979

11694 BERGEN O'NEIL 1979

11695 BERGEN O'NEIL 1979

11696 BERGEN O.NEIL 1979

11697 BERGEN O.NEIL 1979

11698 TODD LOW 1967

11699 BERGEN O.NEIL 1979

11700 BERGEN O.NEIL 1979
Generic Name Specific Name

A UR I CULA

A UR I CUL

AUR I CULA

BAS ISPINATA

BAS I SPINATA

BASISPINATA

BRADYI

TURGIDA DIGITATA

TURGIDA DIGITATA

TURGIDA DIGITATA

TURGIDA DIGITATA

TURGIDA DIGITATA

TURGIDA DIGITATA

TURGIDA DIGITATA

TURGIDA DIGITATA

TUR GIDA DIGITATA

TUR GIDA DIGITATA

TURGIDA DIGITATA

$\angle A B R A D O R I C A$

LABRADORICA

$\angle A B R A D O R I C A$

LABRADORICA

LAB RADORI
MIOCENICA

MIOCENICA

STELLA

STELLA

STE LLA

STELLA

STELLA

STELLA

STELLA

STELLA

STE LLA

STELLA

STE LLA

STELLA

STE LLA

STELLA

STELLA

STELLA

STELLA

STELLA

STE LLA

STE LLA

STELLA

STELLA

STELLA

STELLA

STELLA

STELLA

T UR GIDA

TUR GIDA

TUR GIDA

TURGIDA DIGITATA

TURGIDA DIGITATA

TURGIDA DIGITATA

TURGIDA DIGITATA

AUR IS

AUR IS

SCAPHUM

SCAPHUM

POL YMORPH INOIDES

BRADYI

LINEAR IS

APIOPLEURA

API OPLEURA

API OPLEURA

APIOPLEURA

API OPLEURA

API OPLEURA

API OPLEURA

BOREALIS

BOREALIS

BOREALIS

BOREALIS

BOREALIS

BOREALIS

EAL I

BOREALIS

BOREALIS

BOREALIS

BOREALIS

BOREALIS

BOREALIS

BOREALIS

GLOBOSA

GLOBOSA

GLOBOSA

HEX AGONA

HEXAGONA

HEX AGONA

HEXAGONA

HEXAGONA
Locality

Lat. Long.

WASH ING TON COAST

OFF ALASKA PENINSULA

WASHINGTON COAST

BRITISH COLUMBI

OREGON COAST

BRITISH COLUMBIA

BRITISH COLUMBIA

OFF ALASKA PENINSULA

GULF OF ALASKA

GULF OF ALASKA

GULF OF ALASKA

GULF OF ALASKA

GULF OF ALASKA

GULF OF ALASK'

GULF OF ALASKA

GULF OF ALASKA

GULF OF ALASKA

BRIT ISH COLUMBIA

BR IT ISH COLUMBIA

BRIT ISH COLC

BRITISH COLUMBIA

OFF OREGON

OFF ALASKA

OFF ALASKA

KETCHIKAN, ALASKA

OFF ALASKA

WASHINGTON COAST

BRITISH COL UMBIA

BRITISH COLUMBIA

BRITISH COLUMBIA

BRIIISH COLUMBIA

WASHINGTON COAST

SUNSET BAY, OREGON

WASHINGTON COAS

WA SHINGTON COAST

CLARENCE ST.. ALASKA

GULF OF ALASKA

KASAAN BAY, ALASKA

GULF OF ALASKA

GULF OF ALASKA

GULF OF ALASKA

GULF OF ALASKA

GULF OF ALASKA

GULF OF ALASKA

GULF OF ALASKA

GULF OF ALASKA

GULF OF ALASKA

GULF OF ALASKA

OF ALASKA

CRESCENT CITY, CALIF.

GULF OF ALASKA

OFF ALASKA PENINSULA

CLARENCE ST $\because$ ALASKA

BR ITISH COLUMBIA

BRITISH COLUMB I A

GULF OF ALASKA

CLARENCE ST.. ALASKA

VIRAGO SDO B.C.

Q. CHARLOTTE SD.. B.C

JOHNSTONE STRAIT, B.C

QUEEN CHARLOTTE SD.. B.C.

CRESCENT CITY, CALIF.

WASHINGTON COAS

OFF ALASKA PENINSULA

GULF OF ALASKA

KASAAN BAY, ALASKA

EXCURSION, ALASKA

TAKU HARBOUR, ALASKA

BRITISH COLUMBIA

BR IT ISH COLUMBIA

BRITISH COLUMBIA

BRITISH COLUMBI

GULF OF ALASKA

BRITISH COLUMBIA

EXCURSION. ALASKA

GULF OF ALASKA

GULF OF ALASKA

GULF OF ALASKA

GULF OF ALASKA

GULF OF ALASKA

GULF OF ALASKA

GULF OF ALASKA

GULF OF ALASKA

GULF OF ALASKA

GULF OF ALASKA

GULF OF ALASKA

GULF OF ALASKA

GULF OF ALASKA

GULF OF ALASKA

GULF OF ALASKA

GULF OF ALASKA

GULF OF ALASKA

$4833 \mathrm{~N} 12300 \mathrm{~W}$

$5623 \mathrm{~N} 15427 \mathrm{~W}$

$4832 \mathrm{~N} 12310 \mathrm{~W}$

$12300 \mathrm{~W}$

$4814 \mathrm{~N} 12330 \mathrm{~W}$

$4820 \mathrm{~N} 12300 \mathrm{~W}$

$5623 \mathrm{~N} 15427 \mathrm{~W}$

$5945 \mathrm{~N} 14455 \mathrm{~W}$ 


\section{Publication}

11701 TODD L.OW 1967

11702 BERGEN O.NEIL 1979

11703 TOUD LOW 1967

11704 BERGEN O NEIL 1979

11705 TODD LOW 1967

11706 LANKFORD PHLEGER 1973

11707 TODD LOW 1967

11708 DETLING 1958

11709 BERGEN O'NEIL 1979

11710 COCKBAIN 1963

11711 COCKBAIN 1963

11712 COCKBAIN 1963

11713 COCKBAIN 1963

11714 COCKBAIN 1963

11715 BERGEN O'NEIL 1979

11716 BERGEN O'NEIL 1979

11717 BERGEN O'NEIL 1979

11718 TODD LOW 1967

11719 BERGEN O'NEIL 1979

11720 BERGEN O'NEIL 1979

11721 BERGEN O'NEIL 1979

11722 TODD LOW 1967

11723 SMITH 1973

11724 BERGEN O'NEIL 1979

11725 LANKFORD PHLEGER 1973

11726 CUS HMAN TODD 1947

11727 COCKBAIN 1963

11728 COCKBAIN 1963

11729 BERGEN O.NEIL 1979

11730 PALMER 1929

11731 BERGEN O'NEIL 1979

11732 DETLING 1958

11733 TODD LOW 1967

11734 TODD LOW 1967

11735 BERGEN O N NEIL 1979

11736 BERGEN O०NEIL 1979

11737 SMITH 1973

11738 SMI TH 1973

11739 DETLING 1958

11740 COOPER 1961

11741 CUSHMAN 1927

11742 BERGEN O'NEIL 1979

11743 BERGEN O.NEIL 1979

11744 CUSHMAN 1925

11745 TODD LOW 1967

11746 TODD LOW 1967

11747 TODD LOW 1967

11748 BERGEN O'NEIL 1979

11749 CUSHMAN TODD 1947

11750 WHITEAVES 1886

11750 WHITEAVES 1886
11751 WHI TEAVES 1886

11752 WHITEAVES 1886

11753 WHITEAVES 1886

11754 BERGEN O'NEIL 1979

11755 BERGEN ONNEIL 1979

11756 COCKBAIN 1963

11757 COCKBAIN 1963

11758 BERGEN O'NEIL 1979

11759 CUS HMAN TODD 1947

11760 CUSHMAN TODD 1947

11761 TODD LOW 1967

11762 WHITEAVES 1886

11763 WHI TEAVES 1886

11764 WHI TEAVES 1886

11765 WHITEAVES 1886

11766 WHITEAVES 1886

11767 WHITEAVES 1886

11768 COCKBAIN 1963

11769 COOPER 1961

11770 COCKBAIN 1963

11771 COCKBAIN 1963

11772 DETLING 1958

11773 CUSHMAN MCCULLOCH 1939

11774 CUSHMAN TODD 1947

11775 CUSHMAN TODD 1967

11776 CUS HMAN 1927

11777 CUSHMAN 1927

11778 CUS HMAN 1927

11779 CUS HMAN 1927

11780 CUS HMAN TODD 1947

11781 CUSHMAN MCCULLOCH 1939

11782 PHLEGER 1967

11783 PHLEGER 1967

11784 PHLEGER 1967

11785 COCKBAIN 1963

11785 COCKBAIN 1963
11786 TODD LOW 1967

11787 CUSHMAN TODD 1947

11788 CUSHMAN TODD 1947

11789 TODD LOW 1967

11790 COCKBAIN 1963

11791 COCKBAIN 1963

11792 COCKBAIN 1963

11793 COCKBAIN 1963

11794 COCKBAIN 1963

11795 BERGEN O'NEIL 1979
Generic Name

OOLINA

OOLINA

OOL INA

OOL INA

OOL INA

OOLINA

OOL INA

OOLINA

OOL INA

OOLINA

OOLINA

OOLINA

DOLINA

OOL INA

OOL INA

OOLINA

OOL INA

OOL INA

OOL INA

OPHTHALMIDIUM

PARAFIS SURINA

PATELLINA

PATELLINA

PATELLINA

PATELLINA

PATELLINA

PATELLINA

PATELLINA

PATEORIS

PELOSINA

PELOSINA

PELOSINA

PLACOPS I LINA

PLACOPSILINA

PLANORBULINA

PLANORBULINA

PLANULINA

PLANULINA

PLANUL INA

PSEUDOPOLYMORPHINA

PSEUDOPOLYMORPHINA

PSEUDOPOLYMORPHINA

PSEUDOPOLYMORPHINA

PSEUDOPOLYMORPH INA

PSEUDOPOLYMORPHINA

PSEUDOPOLYMORPHINA

PSEUDOPOLYMORPHINA

PSEUDOPOLYMORPHINA

PSEUDOPOLYMORPHINA

POLYMORPHINA

POLYMORPHINA

POLYMORPHINA

POLYMORPHINA

POLYMORPHINA

POLYMORPHINA

POLYMORPHINA

POLYMORPHINA

LARYNGOSIGMA

ELPHIDIUM

ELPHIDIUM

ELPHIDIUM

ELPHIDIUM

ELPHIDIUM

POROEPONIDES

POROEPONIDES

POROEPONIDES

POROEPONIDES

POROEPONIDES

SACCAMMINA

SACCAMMINA

SACCAMMINA

SACCAMM

SACCAMMINA

SACCAMMINA

SACCAMMINA

SACCAMM I NA

SACCAMM I NA

PROTOSCHISTA

PROTOSCHISTA

PROTOSCHISTA

DENDROPHYRA

DENDROPHYRA

DENDROPHYRA

DENDROPHYRA

DENDROPHYRA

DENDROPHYRA

DENDROPHYRA

DENDROPHYRA

DENDROPHYRA

DENDROPHYRA

DENDROPHYRA

DENDROPHYRA
Specific Name

LAE VIGATA

LAE VIGATA

LINEATA

LINEATOPUNCTATA

MELO

MELO

MELO

MELO

MELO

MELO

MELO

MEL $O$

MELO

STR I ATOPUNCT ATA

STR IATOPUNCTATA

STR IATOPUNCTATA

STR IA TOPUNCTATA

WIL LIAMSONI

ACUTIMARGO

FUS ILIFORMIS

C ORRUGATA

C CRRUGATA

CORRUGATA

CORRUGATA

CORRUGATA

C CRRUGATA

C ORRUGATA

HAUERINOIDES

VAR IAB IL IS

VAR IABIL

D IDERA

DIDERA

BRA DY

BRADY

ACERVALIS

MED I TERRANENSIS

ORNATA

WUE LLERSTORF I

WUELLERSTORF I

CHARLOTTENSIS

CHARLOTTENSIS

CHARLOTTENSIS

CHARLOTTENSIS

CHARLOTTENSIS

LIGUA

LIGUA

LIGUA

LIGUA

KINCAIOI

$K I N C A I D I$

KINCAIDI

KINCAIDI

KINCAIDI

KINCAIDI

$K$ INCAIDI

LAC TEA

CRISPUM

CRI SPUM

CRI SPUM

STR I A TOPUNCT ATA

STRIATOPUNCT ATA

CRI BROREPANDUS

CRI BROREPANDUS

CRI BROREPANDUS

CRIBROREPANDUS

LATERALIS

HANCOCKI

A TL ANT I CA

A TL ANTI CA

A TL ANTI CA

A TLANTI CA

A TLANTICA

A TLANTI CA

L AGENAR IA

LAG ENAR I A

FINDENS

FINDENS

A RB ORE SCENS

ARBORESCENS

ARBORE SCENS

ARBORE SCENS

ARBORES CENS

A RB ORE SCENS

A RB ORE SCENS

A RB ORE SCENS

ARBORESCENS

ARB ORESCENS
ARB ORESCENS

\section{Locality}

GAMBIER BAY, ALASKA

GULF OF ALASKA

GULF OF ALASKA

GULF OF ALASKA

GULF OF ALASKA

CRESCENT CITY, CALIF.

GULF OF ALASKA

SUNSET BAY OREGON

GULF OF ALASKA 


\section{Publication}

11796 BERGEN O'NEIL 1979

11797 TODD LOW 1967

11798 SMITH 1973

11799 TODD LOW 1967

11800 TODD LOW 1967

11801 TODD LOW 1967

11802 TODD LOW 1967

11803 TODD LOW 1967

11804 COCKBAIN 1963

11805 COCKBAIN 1963

11806 TODD LOW 1967

11807 TODD LOW 1967

11809 COCKBAIN 1963

11809 COCKBAIN 1963
11810 COC KBAIN 1963

11811 COCKBAIN 1963

11812 SMITH 1973

11813 BERGEN O'NEIL 1979

11814 BERGEN O'NEIL 1979

11815 BERGEN O'NEIL 1979

11816 BERGEN O'NEIL 1979

11818 CUSHMAN AND TODD 1943

11818 CUSHMAN AND
11819 CUSHMAN 1927

11820 COCKBAIN 1963

11821 COC KBAIN 1963

11822 COCKBAIN 1963

11823 COCKBAIN 1963

11824 COCKBAIN 1963

11825 COCKBAIN 1963

11826 CUSHMAN TODD 1947

11827 CUSHMAN TODD 1947

11828 TODD LOW 1967

11829 BERGEN O'NEIL 1979

11830 BERGEN O'NEIL 1979

11831 BERGEN O०NEIL 1979

11832 BERGEN O'NEIL 1979

11833 BERGEN O'NEIL 1979

11834 BERGEN O'NEIL 1979

11835 BERGEN O'NEIL 1979

11836 BERGEN O'NEIL 1979

11837 BERGEN O'NEIL 1979

11838 BERGEN O'NEIL 1979

11839 BERGEN O'NEIL 1979

11840 BERGEN O'NEIL 1979

11841 BERGEN O'NEIL 1979

11842 BERGEN O'NEIL 1979

11843 BERGEN O'NEIL 1979

11844 BERGEN O'NEIL 1979

11845 SMITH 1973

11846 SMI TH 1973

11847 SMITH 1973

11848 CUSHMAN 1925

11849 CUSHMAN 1925
11850 WHITEAVES 1886

11850 WHITEAVES 1886
11851 WHITEAVES 1886

11851 WHITEAVES 188
11852 CUSHMAN 1925

11853 CUSHMAN 1925

11854 CUS HMAN 1915

11855 CUSHMAN 1927

11856 CUSHMAN 1927

11857 CUSHMAN 1927
11858 CUSHMAN 1927

11858 CUSHMAN 1927
11859 CUS HMAN 1927

11860 TODD LOW 1967

11861 TODD LOW 1967

11862 TODD LOW 1967

11863 TODD LOW 1967

11864 TODD LOW 1967

11866 BERGEN O'NEIL 1979

$\begin{array}{lll}11866 & \text { BERGEN O'NEIL } 1979 \\ 11867 & \text { BERGEN O'NEIL } 1979\end{array}$

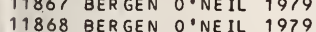

11869 BERGEN O'NEIL 1979

11870 BERGEN O'NEIL 1979

11871 BERGEN O'NEIL 1979

11872 BERGEN O'NEIL 1979

11873 TODD LOW 1967

11874 TODD LOW 1967

11875 COCKBAIN 1963

11876 COCKBAIN 1963

11877 TODD LOW 1967

11878 TODD LOW 1967
11879 TODD LOW 1967

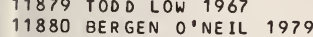

11881 BERGEN O'NEIL 1979

11882 BERGEN O'NEIL 1979

11883 BERGEN O'NEIL 1979

11884 BERGEN O'NEIL 1979

11885 BERGEN O'NEIL 1979

11886 BERGEN O'NE IL 1979

11887 BERGEN O'NEIL 1979

11888 TODD LOW 1967

11889 TODD LOW 1967

11890 TODD LOW 1967
Generic Name

DEN DROPHYRA

GAUDRYINA

PSEUDONODOSARIA

NONIONELLA

NONIONELLA

NON IONELLA

NONIONELLA

NONIONELLA

NONIONELLA

NON IONELLA

NONIONELLA

PSEUDOPOLYMORPHINA

PSEUDOPOLYMORPHINA

PSEUDOPOLYMORPHINA

PULLENIA

PULL.ENIA

PULLENIA

PULLENIA

PULLENIA

PULLENIA

PULLENIA

PULLENIA

PULLEN IA

PULLENIA

PULLENIA

PULLENIA

PULLENIA

PULLENIA

PULLEN IA

PULLENIA
PULLEN I A

PULLENIA

PULLENIA

PULLENIA

PULLENIA

PULLENIA

PULLENIA

PULLENIA

PULLENIA

PULLENIA

PULLENIA

PULLENIA

PULLENIA

PULLEN IA

PULLENIA

PULLENIA

PULLENIA

EPONIDES

BUCCELLA

BUCCELLA

EPONIDES

EPONIDES

EPISTOMINELLA

EPISTOMINELLA

EPISTOMINELLA

EPISTOMINELLA

EPISTOM I NELLA

PYRGO

PYRGO

PYRGO

PYRGO

PYRGO

PYR GO

PYRGO

PYRGO

PYRGO

PYRGO

PYRGO

PYRGO

PYRGO

PYRGO

PYRGO

PYRGO

PYRGOELLA

QUINQUEL OCULINA

QUINQUEL OCUL INA

QUINQUEL OCULINA

QU INQUEL OCUL INA

QUINQUEL OCULINA

QUINQUEL OCUL INA

QUINQUEL OCULINA

QUINQUEL CCULINA

QUINQUELOCULINA

QU INQUEL OCULINA

QUINQUEL OC UL INA

QUINQUEL OCULINA

QUINQUEL OCULINA
Specific Name

Locality

Lat. Long.

ARB ORESCENS

FUS CA

A TL ANTICA

RADICULA

AUR ICULA

A UR I CULA

A UR I CULA

A UR I CULA A

AUR I CULA

A UR I CULA

A UR I CULA

CHARLOTTENSIS

CHARLOTTENSIS

CHARLOTTENSIS

LIGUA

BULLOIDES

BULLOIDES

BULLOIDES

BULLOIDES

BULLOIDES

MUL TILOBATA

SAL I SBURYI

SAL I SBURYI

SALI ISBURY I

SAL ISBURY

SAL I SBURY

SAL ISBURYI

SAL ISBURYI

SALISBURY

SAL ISBURYI

SAL I SBURY I

SAL I SBURYI

SAL I SBURY I

SAL ISBURY

SAL I SBURYI

SAL ISBURY

SAL I SBURYI

SAL I SBURYI

SAL I SBURY

SAL I SBURY

SAL I SBURY I

SAL I SBURYI

SAL I SBURYI

QUINQUELOBA

QUINQUELOBA

QUINQUELOBA

COL UMBIENSIS

C OL UMBIENSIS

FRIGIDA

FRIGIDA

REPANDUS

REPANDUS

UMBONATUS

PACIFICA

PAC IFICA

PAC IFICA

PACIFICA

PAC IFICA
ABY SSORUM

DEPRESSA

DEPRESSA

LUCERNULA

LUCERNULA

LUCERNULA

MURRHINA

MURRHINA

MURR HINA

MURRHINA

MURRHINA

MURRHINA

ROT ALARIA

VESPERTILIO

WILLIAMSONI

WILLIAMSON

SPHAERA

A GGLUTINATA

A GG LUT INATA

AGGLUTINATA

AGGLUTINATA

SP.

$S P$.

SP.

$S P$.

AKNERI ANA

AKNERI ANA

AKNERIANA

GULF OF ALASKA

OFF ALASKA PENINSULA

GULF OF ALASKA

GAMBIER BAY, ALASKA

LYNN CANAL, ALASKA

KASAAN BAY, ALASKA

TAKU HARBOUR, ALASKA

BRITISH COLUMBIA

BRITISH COL UMBI

GULF OF ALASKA

EXCURSION, ALASKA

BR ITISH COLUMBIA

BRITISH

BRITISH COLUMBIA

OFF ALASKA PENINSULA

GULF OF ALASKA

GULF OF ALASKA

GULF OF ALASKA

GULF OF ALASKA

GULF OF ALASKA

OFF OREGON

OFF OREGON

BR IT ISH COL UMBIA

BRITISH COL UMBIA 


\section{Publication}

11891 TODD LOW 1967 11892 TODD LOW 1967

11893 TODD LOW 1967

11894 COOPER 196

11895 BERGEN O'NEIL 1979

11896 BERGEN O'NEIL 1979

11897 COOPER 1961

11898 COOPER 1961

$\begin{array}{llll}11899 & \text { LANKF ORD } & \text { PHLEGER } & 1973 \\ 11900 & \text { LANKFORD } & \text { PHLEGER } & 1973\end{array}$

11901 TODD LOW 1967

11902 TODD LOW 1967

11903 BERGEN O'NEIL 1979

11904 BERGEN O'NEIL 1979

11905 CUSHMAN TODD 1947

11906 CUSHMAN TODD 1947

11907 CUS HMAN TODD 1947

11908 CUS HMAN TODD 1947

11909 CUS HMAN TODD 1947

11910 CUSHMAN TODD 1947

11911 LANKFORD PHLEGER 1973

11912 TODD LOW 1967

11913 CUS HMAN TODD 1947

11914 CUSHMAN 1917

11915 LANKFORD PHLEGER 1973

11916 NATLAND 1933

11917 BERGEN O'NEIL 1979

11918 TODD LOW 1967

11919 TODD LOW 1967

11920 TODD LOW 1967

11921 TODD LOW 1967

11922 TODD LOW 1967

11923 TODD LOW 1967

11924 TODD LOW 1967

11925 TODD LOW 1967

11926 BERGEN O'NEIL 1979

11927 BERGEN O'NEIL 1979

11928 BERGEN O'NEIL 1979

11929 BERGEN O'NEIL 1979

11930 BERGEN O'NEIL 1979

11931 BERGEN O'NEIL 1979

11932 BERGEN O'NEIL 1979

11933 TODD LOW 1967

11934 BERGEN O NEIL 1979

11935 LANKFORD PHLEGER 1973

11936 LANKFORD PHLEGER 1973

11937 CUSHMAN TODD 1947

11938 CUSHMAN TODD 1947

11939 TODD LOW 1967

11940 TODD LOW 1967

11941 TODD LOW 1967

11942 TODD LOW 1967

11943 COCKBAIN 1963

11944 COCKBAIN 1963

11945 COCKBAIN 1963

11946 COCKBAIN 1963

11947 COCKBAIN 1963

11948 ECHOLS 1969

11949 COCKBAIN 1963

11950 COCKBAIN 1963

11951 ECHOLS 1969

11952 ECHOLS 1969

11953 BERGEN O NNEIL 1979

11954 COCKBAIN 1963

11955 COCKBAIN 1963

11956 COCKBAIN 1963

11957 COCKBAIN 1963

11958 COCKBAIN 1963

11959 COCKBAIN 1963

11960 COCKBAIN 1963

11961 SMI TH 1973

11962 SMI TH 1973

11963 SMI TH 1973

11964 SMI TH 1973

11965 SMI TH 1973

11966 SMI TH 1973

11967 SMI TH 1973

11968 SMI TH 1973

11969 SMITH 1973

11970 CUS HMAN 1927

11971 SMITH 1973

11972 SMI TH 1973

11973 CUSHMAN MCCULLOCH 1939

11974 BERGEN O'NEIL 1979

11975 BERGEN O'NEIL 1979

11976 BERGEN O'NEIL 1979

11977 BERGEN O.NEIL 1979

11978 BERGEN O'NEIL 1979

11979 TODD LOW 1967

11980 SMI TH 1973

11981 SMITH 1973

11982 BERGEN O'NEIL 1979

11983 BERGEN O'NEIL 1979

11984 COCKBAIN 1963

11985 COCKBAIN 1963
Generic Name

QUI NQUEL OCULINA

QUINQUELOCULINA

QUINQUELOCULINA

QUINQUEL OCULINA

QUINQUEL OCUL IN

QU INQUEL OCUL INA

QUINQUEL OCUL INA

QU INQUEL OCUL INA

QUINQUEL OCUL INA

QUINQUEL OCUL INA

QUINOUELOCUINA

QU INQUEL OCUL INA

QUINQUEL OCUL INA

QUINQUE LOCULINA

QU INQUEL OCUL INA

MI LIOL INELLA

MILIOL INELLA

MILIOL INELLA

QUINQUEL OCUL INA

QUINQUELOCULINA

QU INQUEL OCUL INA

QUINQUEL OCUL INA

MIL I AMM I NA

QUINQUEL OCUL INA

SIPHONAPERTA

QUINQUEL OCULINA

QUINQUEL OCUL INA

QUINQUEL OCULINA

QUINQUEL OCUL INA

QUINQUEL OCULINA

QU INQUEL OCUL INA

QUINQUEL OCULINA

QUINQUEL OCULINA

QU I NQUEL OCUL INA

QUINQUEL OCUL INA

QU I NQUEL OCUL INA

QUINQUEL OCUI INA

QUINQUEL OCUL INA

QUINQUEL OCUL INA

QUINQUE LOCUL INA

QUINQUEL OCUL INA

MILIOLINELLA

MILIOL INELLA

MILIOL INELLA

QUINQUEL OCUL INA

QUINQUEL OCUL INA

QUINQUELOCULINA

QUINQUEL OCUL INA

RECURVOIDES

RECURVOIDES

RECURVOIDES

REC URVOIDES

RECURVOIDES

RECURVOIDES

REC URVOIDES

RECURVOIDES

RECURVOIDES

RECURVOIDES

RECURVOIDES

RECURVOIDES

RECURVOIDES

RECURVOIDES

RECURVOIDES

RECURVOIDES

REOPHAX

REOPHAX

RE OPHAX

REOPHAX

REOPHAX

REOPHAX

RE OPHAX

REOPHAX

SAC CAMMINA

SACCAMMIN

SACCAMMINA

SAC CAMMINA

SACCAMMINA

SACCAMMINA

REOPHAX

REOPHAX

REOPHAX

REOPHAX

REOPHAX

REOPHAX

REOPHAX

REOPHAX

REOPHAX

REOPHAX

RE OPHAX

REOPHAX

REOPHAX

REOPHAX

REOPHAX

REOPHAX

REOPHAX

REOPHAX
Specific Name

AKNERIANA

AKNERIANA

A KNER I ANA

A KNERI ANA

$S P$.

AKNER I ANA

AKNERI ANA

BELLATULA

BEL LATULA

ARC TICA

ARC TICA

ARC TICA

ARC TICA

CAT ALINENS IS

CIRCULARIS

CIR CULARIS

C IR CULAR IS

E LONGATA

ELONGATA

FRI GID

FUS CA

PROCERA

SAB ULOSA

SEM INULA

SEM I NUL A

SEM INULA

SEM INULA

SEM INULA

SEM INULA

SEM INULA

STALKERI

STALKERI

STALKER I

STALKER I

STA LKERI

STALKER I

STALKER I

STALKERI

STA LKER I

SUBROTUNDA

SUBROTUNDA

SUBROTUNDA

TENAGOS

VUL GAR IS

VUL GARIS

VUL GAR IS

CON TORTUS

CON TORTUS

CONTORTUS

TURB INATUS

TURBINATUS

TURBINATUS

TURBINATUS

TURB INATUS

TUR BINATUS

TURB INATUS

TUR BINATUS

TURBINATUS

TURBINATUS

TURBINATUS

TURBINATUS

TUR BINATUS

CURTUS

CURTUS

C UR TUS

CURTUS

CURTUS

CUR TUS

DEN TAL INIFORMIS

DEN TALINIFORMIS

A TL ANTI CA

A TLANTI CA

A TL ANTICA

A TL ANTI CA

A TL ANTICA

A TLANTI CA

DISTANS

EXCENTRICUS

EXCENTRICUS

EXCENTRICUS

EXCENTRI CUS

GUTTIFER

GUTTIFER

GUTTIFER

GUTTIFER

GUT TIFER

INSECTUS

NODULOSUS

PILULIFER

PILULIFER

PILULIFER

PILULIFER

Locality

GAMBIER BAY, ALASKA

KASAAN BAY ALASKA

TAKU HARBOUR, ALASKA 


\section{Publication}

11986 BERGEN O'NEIL 1979 11987 BERGEN O'NEIL 1979 11988 BERGEN O'NEIL 1979 11989 BERGEN O'NEIL 1979 11990 BERGEN O.NEIL 1979

11991 TODD LOW 1967

11992 SMI TH 1973

11993 BERGEN O'NEIL 1979

1994 BERGEN O'NEIL 1979

11995 BERGEN O'NEIL 1979

11996 BERGEN O.NEIL 1979

11997 TODD LOW 1967

11998 TODD LOW 1967

11999 TODD LOW 1967

12000 TODD LOW 1967

12001 TODD LOW 1967

12002 SMITH 1973

12003 CUSHMAN MCCULLOCH 1939

12004 CUSHMAN MCCULLOCH 1939

12005 CUSHMAN MCCULLOCH 1939

12006 CUSHMAN MCCULLOCH 1939

12007 TODD LOW 1967

12008 WHITEAVES 1886

12009 WHITEAVES 1886

12010 WHITEAVES 1886

12011 WHITEAVES 1886

12012 BERGEN O'NEIL 1979

12013 BERGEN O'NEIL 1979

12014 BERGEN O'NEIL 1979

12015 TODD LOW 1967

12016 TODD LOW 1967

12017 CUSHMAN MCCULLOCH 1948

12018 COOPER 1961

12019 BERGEN O'NEIL 1979

12020 CUS HMAN TODD 1947

12020 CUSHMAN TODD 1947

12022 BERGEN O NEIL 1979

12023 BERGEN O'NEIL 1979

12024 BERGEN O'NEIL 1979

12025 CUS HMAN MCCULLOCH 1948

12026 DETLING 1958

12027 CUSHMAN TODD 1947

12028 TODD LOW 1967

12029 TODD LOW 1967

12030 TODD LOW 1967

12031 CUSHMAN TODD 1947

12032 TODD LOW 1967

12033 COCKBAIN 1963

12034 ECHOLS 1969

12035 COCKBAIN 1963

12036 COCKBAIN 1963

12037 LANKFORD PHLEGER 1973

12038 LANKFORD PHLEGER 1973

12039 LANKFORD PHLEGER 1973

12040 TODD LOW 1967

12041 TODD LOW 1967

12042 TODD LOW 1967

12043 WHITEAVES 1886

12044 WHITEAVES 1886

12045 WHI TEAVES 1886

12048 CUSHMAN 1915
12049 CUS HMAN 1915

12049 CUS HMAN 1915
12050 TODD LOW 1967

12051 BERGEN O.NEIL 1979

12052 BERGEN O'NEIL 1979

12053 COCKBAIN 1963

12054 COCKBAIN 1963

12055 COCKBAIN 1963

12056 TODD LOW 1967

12057 COCKBAIN 1963

12058 COCKBAIN 1963

12060 COCKBAIN 1963

12060 COCKBAIN 1963
12061 COCKBAIN 1963

12061 COCKBAIN 1963
12062 COCKBAIN 1963

12063 COCKBAIN 1963

12064 COCKBAIN 1963

12065 BERGEN O'NEIL 1979

12066 BERGEN O'NEIL 1979

12067 BERGEN O'NEIL 1979

12068 SMITH 1973

12069 BERGEN O'NEIL 1979

12070 BERGEN O'NEIL 1979

12071 TODD LOW 1967

12072 BERGEN O'NEIL 1979

12073 CUSHMAN TODD 1947

12074 BERGEN O'NE IL 1979

12075 TODD LOW 1967

12076 CUSHMAN 1917

12077 BERGEN O'NEIL 1979

12078 TODD LOW 1967

12079 CUS HMAN TODD 1947

12080 CUSHMAN TODD 1947
Generic Name Specific Name

REOPHAX

REOPHAX

REOPHAX

REOPHAX

REOPHAX

REOPHAX
REOPHAX

REOPHAX

REOPHAX

REOPHAX

REOPHAX

REOPHAX

REOPHAX

REOPHAX

REOPHAX

REOPHAX
REOPHAX

REOPHAX

REOPHAX

REOPHAX

RHABDAMMINA

RHABDAMMINA

RHABDAMMINA

RHABDAMM INA

RHABDAMMINA

RHI ZAMMINA

RHI ZAMMINA

RHIZAMMINA

RHIZAMMINA

ROBERTINA

ROBERTINOIDES

ROBERTINOIDES

ROBERTINOIDES

ROBERTINOIDES

ROBERTINOIDES

ROBERTINOIDES

ROBERT INOIDES

ROBERTINOIDES

ROBERTINOIDES

ROBERTINOIDES

LENT I CUL INA

LENTICULINA

LENTICULINA

ASTACOLUS

LENTI CULINA

LENTICULINA

GAVELINOPSIS

ROSALINA

ROSALINA

ROSALINA

ROSALINA

ROSALINA

ROSAL INA

GLABRATELLA

GLABRATELLA

GLABRATELLA

AMMONIA

AMMONIA

AMMONIA

ROSALINA

GYROIDINA

GYROIDINA

RUPERTIA

RUPERTIA

RUPERTIA

SAC CAMMINA

SACCAMMINA

SAC CAMMINA

SAC CAMM I NA

SAC CAMM I NA

SACCAMM INA

SAC CAMMINA

SACCAMMINA

SAC CAMMINA

LAGENAMM INA

LAGENAMM INA

LA GENAMM INA

SAC CAMMI NA

SACCAMMINA

SACCAMMINA

SAC CAMM INA

SAC CORHIZA

SAC CORHIZA

SACCORHIZA

SAC CORHIZA

SACCORHIZA

MARTINOTTIELLA

MARTINOTTIELLA

SIGMOILOPSIS

SP I ROS IGMO IL INA

SPIROS IGMOILINA

SPIROSIGMOILINA

SI GMOMORPHINA
SCORPIURUS

SCORPIURUS

SCORPIURUS

SCORPIURUS

SCORPIURUS

SCORPIURUS

SCORPIURUS

SCORPIURUS

SCORPIURUS

SCORPIURUS

SCORPIURUS

SCORPIURUS

SCORPIURUS

SCORPIURUS

SCORPIURUS

SCOTTII

SCOTTII

SUB FUSI FORMIS

SUB FUS I FORMI S

SUB FUSI FORMI S

ABY SSORUM

ABYSSORUM

ABYSSORUM

ALGAEFORMIS

ALG AEF ORMIS

A LG AEFORMI

I ND IV ISA

A RC TI CA

CHARLOTTENSIS

CHARLOTTENSIS

CHARLOTTENSIS

CHARLOTTENSIS

CHARLOTTENSI S

CHARLOTTENSIS

CHARLOTTENSIS

CHARLOTTENSIS

CHARLOTTENSIS

D'ORB IGNYI

NIC OBARENSIS

N IC OBARENSIS

OCCIDENTALIS

ORBICULARIS

STRONGI

CAMPANULATA

COL UMBIENSIS

COL UMBIENSIS

COL UMB IENSIS

COL UMBIENSIS

COLUMBIENSIS

COL UMB I ENS IS

ORNATISSIMA

ORNATISSIMA

WRI GHTII

BEC CAR I I

BECCAR II

COLCARB IENSIS

ORB I CUL ARIS

SOL DANII

STABILIS

STABILIS

STABILIS

A TL ANTICA

A TLANTICA

A TL ANTICA

A TLANTICA

A TLANTICA

A TLANTICA

A TL ANTI CA

A TLANTICA

LONGICOLLIS

LONGICOLLIS

LONGICOLLIS

SPHAERICA

SPHAER ICA

SPHAERICA

SPHAERICA

RAMOSA

R AM OSA

R AM OSA

RAMOSA

RAMOSA

PRIMAEVA

SCHLUMBERGER I

DISTORTA

DISTORTA

GALLOWAYI

GALLOWAYI
ABY SSORUM

\section{Locality}

Lat. Long.

GULF OF ALASKA

GULF OF ALASKA

GULF OF ALASKA

GULF OF ALASKA

GULF OF ALASKA

GULF OF ALASKA

GULF OF ALASKA

GULF OF ALASKA

GULF OF ALASKA

GULF OF ALASKA 


\section{Publication}

12081 BERGEN O'NEIL 1979

12082 BERGEN O'NEIL 1979

12083 BERGEN O'NEIL 1979

12084 BERGEN O'NEIL 1979

12085 CUSHMAN TODD 1947

12086 COCKBAIN 1963

12087 COCKBAIN 1963

12088 LANKFORD PHLEGER 1973

12089 TODD LOW 1967

12090 CUSHMAN TODD 1947

12091 TODD LOW 1967

12092 COCKBAIN 1963

12093 BERGEN O'NEIL 1979

12094 CUSHMAN 1925

12095 SCOTT 1974

12096 CUSHMAN TODD 1947

12097 CUSHMAN TODD 1947

12098 BERGEN O'NEIL 1979

12099 COC KBAIN 1963

12100 BERGEN O.NEIL 1979

12101 BERGEN O'NEIL 1979

12102 BERGEN O'NEIL 1979

12103 COCKBAIN 1963

12104 COCKBAIN 1963

12105 COCKBAIN 1963

12106 COC KBAIN 1963

12107 BERGEN O'NEIL 1979

12108 BERGEN O NE IL 1979

12109 ECHOLS 1969

12110 ECHOLS 1969

12111 ECHOLS 1969

12112 SMI TH 1973

12113 SMITH 1973

12114 SMITH 1973

12116 TODD LOW 1967

12117 COCKBAIN 1963

12118 COCKBAIN 1963

12119 COCKBAIN 1963

12120 BERGEN O.NEIL 1979

12121 LANKFORD PHLEGER 1973

12122 BERGEN O'NEIL 1979

12123 BERGEN O'NEIL 1979

12124 BERGEN O'NEIL 1979

12125 BERGEN O NEIL 1979

12126 BERGEN O'NEIL 1979

12127 ECHOLS 1969

12128 COCKBAIN 1963

12129 COCKBAIN 1963

12130 HAMLIN 1960

12131 LANKFORD PHLEGER 1973

12132 BERGEN O'NEIL 1979

12133 BERGEN O.NEIL 1979

12134 BERGEN O.NEIL 1979

12135 BERGEN O'NEIL 1979

12136 BERGEN O'NEIL 1979

12137 BERGEN O'NEIL 1979

12138 BERGEN O'NEIL 1979

12139 BERGEN O'NEIL 1979

12140 BERGEN O'NEIL 1979

12141 BERGEN O'NEIL 1979

12142 BERGEN ODNEIL 1979

12143 BERGEN O'NEIL 1979

12144 BERGEN O'NEIL 1979

12145 BERGEN O'NEIL 1979

12146 BERGEN O'NEIL 1979

12147 BERGEN O'NEIL 1979

12148 BERGEN O'NEIL 1979

12149 BERGEN O'NEIL 1979

12150 BERGEN O'NEIL 1979

12151 BERGEN O'NEIL 1979

12152 BERGEN O'NEIL 1979

12153 BERGEN O'NEIL 1979

12154 BERGEN O.NEIL 1979

12155 BERGEN O'NEIL 1979

12156 BERGEN O'NEIL 1979

12157 CUSHMAN TODD 1947

12158 CUSHMAN TODD 1947

12159 TODD LOW 1967

12160 TODD LOW 1967

12160 TODD LOW 1967

12162 CUSHMAN TODD 1947

12163 BERGEN O'NEIL 1979

12164 BERGEN O'NEIL 1979

12165 BERGEN O'NEIL 1979

12166 BERGEN O'NEIL 1979

12167 BERGEN O'NEIL 1979

12168 BERGEN O'NEIL 1979

12169 BERGEN O'NEIL 1979

12170 BERGEN O'NEIL 1979

12171 TOOD LOW 1967

12172 BERGEN O.NEIL 1979

12173 TODD LOW 1967

12174 COCKBAIN 1963

12175 COCKBAIN 1963
Generic Name

SIGMOMORPHINA

SIGMOMORPHINA

SI GMOMORPHINA

SI GMOMORPHINA

SI GMOMORPHINA

SI GMOMORPHINA

SI GMOMORPHINA

SIGMOMORPHINA

SIGMOMORPHINA

SIGMOMORPHINA

SIGMOMORPHINA

SI GMOMORPHINA

SPHAEROI DINA

SEJUNCTELLA

SPIRILLINA

SPIROPLE CTAMMINA

SPIROPLE CTAMMINA

SPIROPLECTAMMINA

SPIROPLECTAMMINA

SPIROPLE CT AMM INA

SPIROPLECTAMMINA

SPI ROPLE CTAMM INA

SPIROPLECTAMMINA

SP I ROPLECTAMM INA

SPIROPLE CTAMMINA

SPI ROPLECTAMM INA

SP I ROPLECTAMM INA

SPI ROPLECTAMM INA

SPIROPLECTAMMINA

SPIROPLECTAMM INA

SPI ROPLECTAMMINA

SPIROPLECTAMMINA

SPI ROPLE CTAMM INA

SPI ROPLE CTAMMINA

SPIROPLE CTAMMINA

SPI ROPLECTAMMINA

SPI ROPLECTAMM INA

SP I ROPLE CTAMM INA

SPIROPLE CTAMM INA

SUGGRUNDA

TEXTULARIA

TEXTULARIA

TEX TULAR I A

TEXTULARIA

TEXTULARIA

TEXTULARIA

ROSALINA

ROS AL INA

ROSAL INA

TRICHOHYALUS

GLABRATELLA

GLABRAT ELLA

GLABRATELLA

GLABRATELLA

GLABRATELLA

GLABRATELLA

GLABRATELLA

TR I FAR INA

TRI FAR INA

TRIFARINA

TRIFAR INA

TRIFAR INA

TRI FAR INA

TR IFAR INA

TRIFAR INA

TRI FARINA

TRI FAR INA

TR I FAR INA

TRI FARINA

TR I FARINA

TR IFARINA

TR I FAR INA

TRI FAR INA

TR I FAR INA

TRIFARINA

MILIOLINELLA

MI LIOLINELLA

TRILOCULINA

TR I LOCULINA

TRILOCULINA

TRILOCULINA

IRILOCULINA

TRI LOCULINA

TR I LOCULINA

TRI LOCULINA

TRILOCULINA

TRILOCULINA

TRILOCUL INA

TRILOCULINA

TROC HAMM INA

TROCHAMM INA

TROCHAMMINA

TROCHAMM INA

TROCHAMM INA
Specific Name

GAL LOWAYI

GALLOWAYI

GAL LOWAYI

GALL LWAYI

TRI LOCULARIS

TRI LOCULARIS

TRI LOCULARIS

TRI LOCULARIS

TRILOCULARIS

TRI LOC ULARIS

TRI LOCULAR IS

BULLOIDES

SPINIGERA REDUCTA

VIVIPARA

BIF ORMIS

BIF ORMIS

BIFORMIS

BIFORMIS

BIF ORMIS

BIF ORMIS

B IF ORM IS

B IF ORMIS

IF ORMIS

BIF ORMIS

BIFORMIS

B IF ORMIS

BIFORMIS

B IF ORMIS

B IF ORMIS

BIFORMIS

BIFORMIS

BIF ORMIS

BIFORMIS

BIF ORMIS

BIFORMIS

BIFORMIS

ECK IS I

EAR LAND

TOR QUATA

TOR QUATA

IOR QUATA

T OR QUATA

TOR QUATA

COL UMBIENSIS

COL UMB IENSIS

COLUMBIENSIS

ORF ORDENSIS

ORNATI SSIMA

ORNATI S SIMA

ORNATISSIMA

ORNATISSIMA

ORNATISSIMA

ORNATISSIMA

ORNATISSIMA

FLUENS

FLUENS

FLUENS

FLUENS

FLUENS

FLUENS

FLUENS

FLUENS

FLUENS

FLUENS

FLUENS

FLUENS

FLUENS

FLUENS

F LUEN

FLUENS
FLUENS

FLUENS

FLUENS

FLUENS

CIRCULARIS

CIR CULARIS

ROTUNDA

ROT UNDA

ROT UNDA

TRI GONULA

TRI HEDRA

TRI HEDRA

TRI HEDRA

TRI HEDRA

TRI HEDRA

TRIHEDRA

TRIHEDRA

TRI HEDRA

A DVENA

A OVENA

A DVENA

CHARLOTTENSIS

CHARLOTTENSIS

Locality

GULF OF ALASKA

GULF OF ALASKA

GULF OF ALASKA

GULF OF ALASKA

WASHINGTON COAST 


\section{Publication}

12176 COCKBAIN 1963

12177 COCKBAIN 1963

12178 CUS HMAN 1925
12179 CUSHMAN TODD 1947

12180 LANKFORD PHLEGER 1973

12181 LANKFORD PHLEGER 1973

12182 LANKFORD PHLEGER 1973

2183 CUSHMAN TODD 1947

12184 DETLING 1958

12185 ECHOLS 1969

12186 PHLEGER 1967

12187 COCKBAIN 1963

12189 COCKBAIN 1963

12190 COCKBAIN 1963

12191 CUS HMAN TODD 1947

12192 CUSHMAN TODD 1947

12193 DETLING 1958

12194 COCKBAIN 1963

12195 COCKBAIN 1963

12196 COCKBAIN 1963

12197 COCKBAIN 1963

12199 COCKBAIN 1963

12199 COCKBAIN 1963
12200 COCKBAIN 1963

12200 COCKBAIN 1963
12201 COCKBAIN 1963

12202 CUS HMAN MCCULLOCH 1939

12203 BERGEN O०NEIL 1979

12204 BERGEN O'NEIL 1979

12205 BERGEN O'NEIL 1979

12206 BERGEN O'NEIL 1979

12207 BERGEN O NEIL 1979

12208 BERGEN $0^{\circ} \mathrm{NE}$

12209 SMI TH 1973
12210 SMI TH 1973

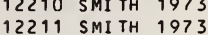

12212 SMITH 1973

12213 SMI TH 1973

12214 SMITH 1973

12215 SMITH 1973

12216 SMI TH 1973

12218 SMITH 1973

12219 SMITH 1973

12220 SMITH 1973

12221 PHLEGER 1967

12222 PHLEGER 1967

12223 PHLEGER 1967

12224 PHLEGER 1967
12225 PHLEGER 1967

12226 SCOTT 1974

12227 BERGEN O'NEIL 1979

12228 BERGEN O'NEIL 1979

12229 BERGEN O'NEIL 1979

12230 BERGEN O'NEIL 1979

12231 DETLING 1958

12232 LANKFORD PHLEGER 1973

12233 COOPER 1961

12234 COOPER 1961

12235 COOPER 1961

12236 COOPER 1961

12237 COOPER 1961
12238 PHLEGER 1967
12239 PHLEGER 1967

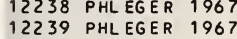

12240 PHLEGER 1967

12241 SMITH 1973

12242 COCKBAIN 1963

12243 COCKBAIN 1963

12244 COCKBAIN 1963

12246 COCKBAIN 1963

12247 COCKBAIN 1963

12247 COCKBAIN 1963
12248 COCKBAIN 1963

12249 SMITH 1973

12250 SMITH 1973
12251 SMITH 1973

12251 SMITH 1973
12252 CUSHMAN MCCULLOCH 1939

12253 CUS HMAN MCCULLOCH 1939

12254 CUSHMAN MCCULLOCH 1939.

12255 JONES ROSS 1979

12256 CUS HMAN 1925

12257 CUSHMAN 1925

12258 COOPER 1961

12259 COCKBAIN 1963

12260 COCKBAIN 1963

12261 COCKBAIN 1963

12262 COCKBAIN 1963
12263 COCKBAIN 1963

12263 COCKBAIN 1963

12264 COCKBAIN 1963
12265 COCKBAIN 1963

12266 COCKBAIN 1963

12267 COCKBAIN 1963

12268 LANKFORD PHLEGER 1973

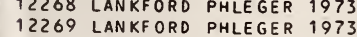

12270 SCOTT 1974
Generic Name Specific Name

CHARLOTTENSIS

CHARLOTTENSIS

CHARLOTTENSIS

CHARLOTTENSI

CHA RLOTTENS IS

CHARLOTTENS I

CHARLOTTENSIS

CHARLOTTENSIS

CHARLOTTENSIS

CHARLOTTENSI

CHARLOTTENSI

C HARLOTTENSI

CHARLOTTENSIS

C HARLOTTENSIS

DISCORBIS

DIS CORBIS

DISCORBIS

DISCORBIS

DIS CORBIS

DISCORBIS

DISCORBIS

DIS CORBIS

DIS CORBIS

DIS CORBIS

DIS CORBIS

DIS CORBIS

GLOBIGERINIF ORMIS

GLOBIGERIN I F ORMIS

GLOBIGER IN IF ORM IS

GLOBIGER IN IF ORM IS

GLOBIGERINIF ORM IS

GLOBIGER IN IF ORM IS

GLOBIGERINIF ORM IS

GLOBIGERIN IF ORM IS

GLOBIGERIN I F ORM I S

GLOBIGERINIF ORM I S

GLOBIGER IN I F ORM I S

GLOBIGERINIF ORM I S

GLOBIGERINIF ORM IS

GRISEA

GRISEA

GRISEA

GRI SEA

I NF L ATA

I NF LATA

INF LATA

INF LATA

INFLATA

I NF LATA

INF LATA

INF LATA

INF LATA

I NF LATA

I NF LATA

KELLETTAE

KELLETTAE

KELLETTAE

ELLET TAE

KELLETTAE

MACRESCENS

MACRESCENS

MACRESCENS

NANA

NAN

NANA

NAN A

NANA

NAN

NANA

NAN

NIT I DA

NIT I DA

N I T I DA

NIT I DA

NITI DA

NITI I DA

PAC IFICA

PAC IFICA

PAC IFICA

PAC IF ICA

PAC I F I CA

PAC IFICA

PACIFICA

PAC IFICA

PAC IFICA

PACIFICA

PAC IFICA

PACIFICA

PACIFICA

PAC IFICA

PAC IF I CA

PAC IFICA

\section{Locality}

BRITISH COLUMBIA

BR IT ISH COL UMBIA

Q. CHARLOTTE SD.. B.C.

WASHINGTON COAST

LAPUSH, WASHINGTON

DEPOE BAY, OREGON

CRESCENT CITY, CALIF.

WASHINGTON COAST

SUNSET BAY, OREGON

WASHINGTON COAST

GRAY 'S HARBOUR, WASH.

BRITISH COLUMBIA

BRITISH COLUMBIA

BRITISH COLUMBIA

BRITISH COL UMBIA

WASHINGTON COAST

WASHINGTON COAST

SUNSET BAY, OREGON

BRITISH COLUMBIA

BRIT ISH COLUMBIA

BR ITISH COL UMBIA

BRIT ISH COL UMBIA

BRITISH COLUMBIA

BR IT ISH COLUMBIA

BRITISH COL UMBIA

BR IT ISH COLUMBIA

KETCHIKAN, ALASKA

GULF OF ALASKA

GULF OF ALASKA

GULF OF ALASKA

GULF OF ALASKA

GULF OF ALASKA

GULF OF ALASKA

OFF ALASKA PENINSULA

OFF ALASKA PENINSULA

OFF ALASKA PENINSULA

OFF ALASKA PENINSULA

OFF ALASKA PENINSUL

OFF ALASKA PENINSULA

NORTH PACIFIC

OFF ALASKA PENINSULA

OFF ALASKA PENINSULA

OFF ALASKA PENINSULA

NORTH PACIF IC

OFF ALASKA PENINSULA

GRAY'S HARBOUR, WASH.

COPPER R.. ALASKA

FRASER R.. B. C.

COOS, BAY, OREGON

PHILLIPS ARM, B.C.

SAMISH-PADILLA BAYS

GULF OF ALASKA

GULF OF ALASKA

GULF OF ALASKA

GULF OF ALASKA

SUNSET BAY, OREGON

DEPOE BAY, OREGON

OREGON COAST

OREGON COAST

CALIFORNIA COAST

OREGON COAST

OREGON COAST

FRASER R B,

COOS, BAY, OREGON

GRAY'S HARBOUR, WASH.

OFF ALASKA PENINSULA

BRITISH COLUMBIA

BR IT ISH COLUMBIA

BR ITISH COL UMBIA

BRITISH COLUMBIA

BR IT ISH COL UMBIA

BRIT ISH COLUMBIA

BR IT ISH COLUMBIA

OFF ALASKA PENINSULA

NORTH PACIFIC

OFF ALASKA PENINSULA

OFF ALASKA

OFF ALASKA

SAMISH BAY, WASH.

Q. CHARLOTTE SD.. B.C.

VIRAGO SD.. B.C.

OREGON COAST

BR ITISH COLUMBIA

BRITISH COLUMBIA

BRITISH COLUMBIA

BRITISH COLUMBIA

BRITISH COL UMBIA

BR IT ISH COLUMBIA

BRITISH COLUMBIA

BR ITISH COLUMBIA

CRESCENT CITY. CALIF.

DEPOE BAY OREGON

SAMISH-PADILLA BAYS

Lat. Long.

$4835 \mathrm{~N} 12251 \mathrm{~W}$

$4900 \mathrm{~N} 12315 \mathrm{~W}$

$5220 \mathrm{~N} 131$ 0OW

$4755 \mathrm{~N} 12739 \mathrm{~W}$

$44 \quad 47 \mathrm{~N} 12405 \mathrm{~W}$

$4145 N 12413 \mathrm{~W}$

$4833 \mathrm{~N} 12300 \mathrm{~W}$

$4321 \mathrm{~N} 12420 \mathrm{~W}$

$4710 \mathrm{~N} 12414 \mathrm{~W}$

$\begin{array}{llll}47 & 00 N 12408 W\end{array}$

$4820 \mathrm{~N} 12300 \mathrm{~W}$

$4820 \mathrm{~N} 124 \quad 10 \mathrm{~W}$

$4814 \mathrm{~N} 12330 \mathrm{~W}$ 


\section{Publication}

12271 CUSHMAN MCCULLOCH 1939 12272 CUSHMAN MCCULLOCH 1939 12273 CUSHMAN MCCULLOCH 1939 12274 CUSHMAN TODD 1947

12275 TODD LOW 1967

12276 TODD LOW 1967

12277 TODD LOW 1967

12278 TODD LOW 1967

12279 CUSHMAN TCDD 1947
12280 BERGEN O'NEIL 1979

12281 BERGEN O'NEIL 1979

12282 DETLING 1958

12283 BERGEN O'NEIL 1979

12284 BERGEN O'NEIL 1979

12285 BERGEN O'NEIL 1979

12286 BERGEN O'NEIL 1979

12287 BERGEN O'NEIL 1979

12288 TODD LOW 1967

12289 CUS HMAN MCCULLOCH 1939

12290 COCKBAIN 1963

12291 COCKBAIN 1963

12292 COCKBAIN 1963

12293 COCKBAIN 1963

12294 WHITEAVES 1886

12295 WHITEAVES 1886

$\begin{array}{lll}12296 & \text { BERGEN O'NEIL } & 1979 \\ 12297 & \text { BERGEN O'NEIL } & 1979\end{array}$

12298 COCKBAIN 1963

12299 COCKBAIN 1963

12300 COCKBAIN 1963

12301 COCKBAIN 1963

12302 TODD LOW 1967

12303 COCKBAIN 1963

12304 COCKBAIN 1963

12305 COCKBAIN 1963
12306 COOPER 1961

12306 COOPER 1961
12307 CUSHMAN 1910

12308 WHITEAVES 1886

12309 WHITEAVES 1886

12310 WHITEAVES 1886

12311 WHITEAVES 1886

12312 WHI TEAVES 1886

12313 WHITEAVES 1886

12314 WHITEAVES 1886

12315 WHITEAVES 1886

12316 WHITEAVES 1886

12317 WHITEAVES 1886

12318 WHITEAVES 1886

12319 WHITEAVES 1886

12320 WHITEAVES 1886

12321 WHITEAVES 1886

12322 WHITEAVES 1886

12323 WHITEAVES 1886

12324 WHITEAVES 1886

12325 WHITEAVES 1886

12326 WHITEAVES 1886

12327 WHITEAVES 1886

12328 CUSHMAN 1915

12329 COOPER 1961

12330 BERGEN O'NEIL 1979

12331 BERGEN O.NEIL 1979

12332 BERGEN O'NEIL 1979

12333 BERGEN O'NEIL 1979

12334 BERGEN O'NEIL 1979

12335 SMITH 1973

12336 BERGEN O'NEIL 1979

12337 BERGEN O'NEIL 1979

12338 BERGEN O'NEIL 1979

12339 BERGEN O'NEIL 1979

12340 BERGEN O'NEIL 1979

12341 BERGEN O'NEIL 1979

12342 BERGEN O'NEIL 1979

12343 BERGEN O'NEIL 1979

12344 BERGEN O'NEIL 1979

12345 BERGEN O'NEIL 1979

12346 BERGEN O'NEIL 1979

12347 BERGEN O'NEIL 1979

12348 BERGEN O'NEIL 1979

12349 CUSHMAN TODD 1947

12350 CUSHMAN TODD 1947

12351 COCKBAIN 1963

12352 COCKBAIN 1963

12353 COCKBAIN 1963

12354 COCKBAIN 1963

12355 BERGEN O'NEIL 1979

12356 BERGEN O'NEIL 1979

12357 BERGEN O'NEIL 1979

12358 BERGEN O'NEIL 1979

12359 BERGEN O'NEIL 1979

12360 COCKBAIN 1963

12361 COCKBAIN 1963

12362 SERGEN O'NEIL 1979

12363 BERGEN O'NEIL 1979

12364 BERGEN O'NEIL 1979

12365 BERGEN O'NEIL 1979
Generic Name

TROCHAMMINA

TROCHAMM INA

TROCHAMM INA

TROCHAMM INA

TROCHAMM INA

TROCHAMMINA

TROCHAMMINA

TROCHAMM INA

TROCHAMM INA

TROCHAMM INA

TROCHAMMINA

TROCHAMMINA

TROCHAMM INA

TROCHAMM INA

IROCHAMMINA

TROCHAMMINA

TROCHAMMINA

TROCHAMM INA

TROCHAMM INA

TROCHAMMINA

TROCHAMM INA

TROCHAMM INA

TROCHAMMINA

TROCHAMM

TROCHAMM INA

TROCHAMM INA

TROCHAMM INA

TROCHAMMINA

TROCHAMM INA

TROCHAMM INA

TROCHAMM INA

RECURVOIDES

CIBICIDES

CIBICIDES

CIBICIDES

CIBICIDES

CIBICIDES

CIBICIDES

CIBICIDES

CIBICIDES

CIBICIDES

CIBICIDES

CIBICIDES

CIBICIDES

CIBICIDES

CIBICIDES

CIBICIDES

CIBICIDES

C IBICIDES

CIBICIDES

CIBICIDES

CIBICIDES

PLANUL INA

TURRISP I RILLINA

UVI GER INA

UV I GER INA

UV I GER INA

UVI GER INA

UV I GER INA

UV I GER INA

UVI GER INA

UVI GER INA

UV I GER INA

UVI GER INA

UVIGER INA

UVI GER INA

UVI GER INA

UVI GER INA

UVI GER INA

UVI GER INA

UVI GER I NA

UV I GER IN A

UVI GER INA

UVI GER INA

UVI GER INA

UVIGERINA

UVIGER INA

UV I GER INA

UV I GER INA

UVIGERINA

UVI GER INA

UVI GER INA

UV I GER INA

UVIGERINA

UVI GER INA

UV I GER INA

UVI GER INA

UVI GER INA

UV I GER INA

UV I GER INA
Specific Name

PACIFICA

PACIFICA

PACIFICA SIMPLISSIMA

ROT ALIFORMIS

ROTALIFORMIS

ROT ALI FORMIS

ROTALI FORMIS

ROTALIFORMIS

ROTALIFORMIS

ROTALIFORMIS

ROTALIFORMIS

ROT ALIFORMIS

ROTALIFORMIS

ROT ALI IFORMIS

ROT AL I FORMIS

ROTALI IFORMIS

SQUAMATA

SQU AMATA

SQUAMATA

SQUAMATA

SQUAMATA

SQU AMATA

SQUAMATA

SQU AMATA

SQUAMATA

SQU AMATA

SQUAMATA

SQUAMATA

SQU AMATA

SQU AMI FORMIS

SQUAMI FORMIS

SQUAMI FORMIS

SQUAMIF ORMIS

TURBINATUS

LOBATULUS

LOBATULUS

LOBATULUS

LOBATULUS

LOBATULUS

LOBATULUS

$\angle O B A T U L U S$

LOBATULUS

LOBATULUS

LOBATULUS

LOBATULUS

LOBATULUS

LOBATULUS

LOBATULUS

LOBATULUS

LOB ATULUS

LOBATULUS
LOBATULUS

LOBATULUS
LOBATULUS

LOBATULUS

LOBATULUS

WUELLERSTORF I

A RC TICA

A UB ER I ANA

A UBERI ANA

A UBERI ANA

A UBERIANA

A UBER I ANA

JUN CEA

PEREGRINA DI RUPT A

PEREGRINA DIRUPTA

PEREGRINA DIRUPT

PEREGRINA DIRUPT

PEREGRINA DI RUPTA

PEREGRINA DIRUPTA

PEREGRINA DIRUPTA

PEREGRINA DI RUPTA

JUN CEA

J UN CEA

JUN CEA

JUNCEA

JUNCEA

UN CEA

$J U N C E A$

JUN CEA

JUNCEA

JUN CEA

JUN CEA

JUN CEA

JUN CEA

JUNCEA

JUNCEA

J UN CEA

JUN CEA

JUNCEA

JUNCEA

J UN CEA

JUN CEA

Locality

Lat. Long.

KETCHIKAN, ALASKA

OFF ALASKA

WASHINGTON COAST

EXCURSION, ALASKA

TAKU HARBOUR, ALASKA

KASAAN BAY, ALASKA

WASHINGTON COAST

GULF OF ALASKA 


\section{Publication}

12366 BERGEN O'NEIL 1979 12367 BERGEN O'NEIL 1979 12368 BERGEN O'NEIL 1979 12369 BERGEN O'NEIL 1979 12370 BERGEN O'NEIL 1979 12371 BERGEN O'NEIL 1979 12372 BERGEN O'NEIL 1979 12372 BERGEN O'NEIL 1979 13374 BERGEN O'NEIL 1979 $\begin{array}{lll}12374 & \text { BERGEN O'NEIL } 1979 \\ 12375 & \text { BERGEN O'NEIL } 1979\end{array}$ 12376 BERGEN O'NEIL 1979 12377 SMITH 1973

12378 TODD LOW 1967

12379 TODD LOW 1967

12380 TODD LOW 1967

12381 TODD LOW 1967

12382 BERGEN O N NEIL 1979

12383 BERGEN O'NEIL 1979

12384 BERGEN O'NEIL 1979

12385 BERGEN O'NEIL 1979

12386 CUS HMAN MCCULLOCH 1948

12387 BERGEN O'NEIL 1979

12388 TODD LOW 1967

12389 BERGEN O'NEIL 1979

12390 BERGEN O'NEIL 1979

12390 BERGEN O NEIL 1979

12391 BERGEN O'NEIL 1979

12393 BERGEN O'NEIL 1979

12394 BERGEN O'NEIL 1979

12395 BERGEN O'NEIL 1979

12396 BERGEN O'NEIL 1979

12397 BERGEN O'NEIL 1979

12398 BERGEN O'NEIL 1979

12399 BERGEN O'NEIL 1979

12400 BERGEN O'NEIL 1979

12401 BERGEN O'NEIL 1979

12402 BERGEN O'NEIL 1979

12403 BERGEN O'NEIL 1979

12404 BERGEN O'NEIL 1979

12405 BERGEN O'NEIL 1979

12406 CUSHMAN 1927

12407 BERGEN $0^{\circ}$ NEIL 1979

12408 BERGEN O'NEIL 1979

12409 BERGEN O'NEIL 1979

12410 BERGEN O'NEIL 1979

12411 BERGEN O'NEIL 1979

12412 BERGEN O'NEIL 1979

12413 BERGEN O'NEIL 1979

12414 BERGEN O'NEIL 1979

12415 BERGEN O'NEIL 1979

12416 BERGEN O'NEIL 1979

12417 BERGEN O N NE IL 1979

12418 SMITH 1973

12619 SMI TH 1973

12420 TODD LOW 1967

2421 TODD LOW 1967

12422 SMITH 1973

12423 BERGEN O'NEIL 1979

12424 BERGEN O'NEIL 1979

12425 BERGEN O'NEIL 1979

12426 BERGEN O'NEIL 1979

12427 BERGEN O'NEIL 1979

12428 BERGEN O.NEIL 1979
Generic Name Specific Name

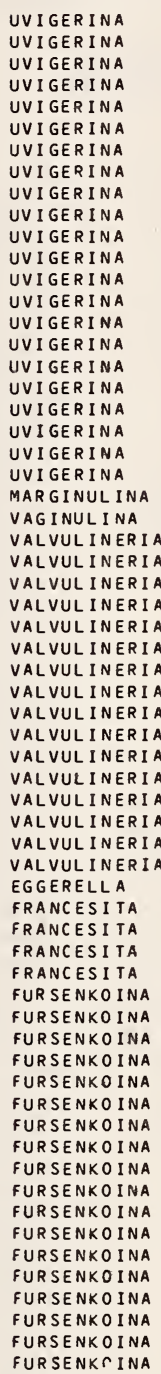

SEM I NUDA

SEM I NUDA

\section{Locality}

GULF OF ALASKA

GULF OF ALASKA

GULF OF ALASKA

GULF OF ALASKA

GULF OF ALASKA

GULF OF ALASKA

GULF OF ALASKA

GULF OF ALASKA

GULF OF ALASKA

GULF OF ALASKA

GULF OF ALASKA

OFF ALASKA PENINSULA

CLARENCE ST.. ALASKA

GULF OF ALASKA

KASAAN BAY, ALASKA

TAKU HARBOUR, ALASKA

GULF OF ALASKA

GULF OF ALASKA

GULF OF ALASKA

GULF OF ALASKA

KETCHIKAN, ALASKA

GULF OF ALASKA

GULF OF ALASKA

GULF OF ALASKA

GULF OF ALASKA

GULF OF ALASKA

GULF OF ALASKA

GULF OF ALASKA

GULF OF ALASKA

GULF OF ALASKA

GULF OF ALASKA

GULF OF ALASKA

GULF OF ALASKA

GULF OF ALASKA

GULF OF ALASKA

GULF OF ALASKA

GULF OF ALASKA

GULF OF ALASKA

GULF OF ALASKA

GULF OF ALASKA

OFF OREGON

GULF OF ALASKA

GULF OF ALASKA

GULF OF ALASKA

GULF OF ALASKA

GULF OF ALASKA

GULF OF ALASKA

GULF OF ALASKA

GULF OF ALASKA

GULF OF ALASKA

GULF OF ALASKA

GULF OF ALASKA

OFF ALASKA PENINSULA

CLARENCE ST.. ALASKA

EXCURSION, ALASKA

OFF ALASKA PENINSULA

GULF OF ALASKA

GULF OF ALASKA

GULF OF ALASKA

GULF OF ALASKA

GULF OF ALASKA

GULF OF ALASKA
Lat. Long.

$5955 \mathrm{~N} 14225 \mathrm{~W}$ $5945 \mathrm{~N} 14455 \mathrm{~W}$ $5925 \mathrm{~N} 14525 \mathrm{~W}$ $5930 \mathrm{~N} 14520 \mathrm{~W}$ $5930 \mathrm{~N} 14300 \mathrm{~W}$ $5930 N 14545 W$ $5635 \mathrm{~N} 15150 \mathrm{~W}$ $5930 \mathrm{~N} 14540 \mathrm{~W}$ $5900 N 14120 W$ $5850 \mathrm{~N} 14115 \mathrm{~W}$ $5935 \mathrm{~N} 14300 \mathrm{~W}$ $5353 \mathrm{~N} 16140 \mathrm{~W}$ $5521 \mathrm{~N} 13158 \mathrm{~W}$ $5932 \mathrm{~N} 14236 \mathrm{~W}$ $5526 \mathrm{~N} 13214 \mathrm{~W}$ $5802 \mathrm{~N} 13400 \mathrm{~W}$ $5930 \mathrm{~N} 14520 \mathrm{~W}$ $5900 \mathrm{~N} 14120 \mathrm{~W}$ $5930 \mathrm{~N} 14300 \mathrm{~W}$ $5920 \mathrm{~N} 14538 \mathrm{~W}$ $5521 \mathrm{~N} 13139 \mathrm{~W}$ $5925 \mathrm{~N} 14525 \mathrm{~W}$ $5932 \mathrm{~N} 14236 \mathrm{~W}$ $5925 \mathrm{~N} 14525 \mathrm{~W}$ $5920 \mathrm{~N} 14538 \mathrm{~W}$ $5930 \mathrm{~N} 14520 \mathrm{~W}$ $5930 \mathrm{~N} 14545 \mathrm{~W}$ $5930 \mathrm{~N} 14255 \mathrm{~W}$ $5930 N 14540 W$ $5930 \mathrm{~N} 14300 \mathrm{~W}$ 59 OON $14120 \mathrm{~W}$ $5930 \mathrm{~N} 14300 \mathrm{~W}$ 58 50N $14115 \mathrm{~W}$ $5930 \mathrm{~N} 14545 \mathrm{~W}$ $5930 \mathrm{~N} 14255 \mathrm{~W}$ 59 OON $14120 \mathrm{~W}$ $5925 \mathrm{~N} 14525 \mathrm{~W}$ $5920 \mathrm{~N} 14538 \mathrm{~W}$ $5930 \mathrm{~N} 14520 \mathrm{~W}$ $5930 \mathrm{~N} 14540 \mathrm{~W}$ $4314 \mathrm{~N} 12443 \mathrm{~W}$ $5935 \mathrm{~N} 14300 \mathrm{~W}$ $5930 N 14540 W$ $5930 \mathrm{~N} 14300 \mathrm{~W}$ $5920 \mathrm{~N} 14538 \mathrm{~W}$ $5930 \mathrm{~N} 14520 \mathrm{~W}$ $\begin{array}{llll}59 & 00 N & 141 & 20 W \\ 59 & 20 N & 145 & 38 W\end{array}$ $5930 \mathrm{~N} 14540 \mathrm{~W}$ $5900 \mathrm{~N} 14120 \mathrm{~W}$ $5930 \mathrm{~N} 14520 \mathrm{~W}$ $5925 \mathrm{~N} 14525 \mathrm{~W}$ $5749 \mathrm{~N} 15230 \mathrm{~W}$ $5623 \mathrm{~N} 15427 \mathrm{~W}$ $5825 \mathrm{~N} 13530 \mathrm{~W}$ $\begin{array}{llll}58 & 25 \mathrm{~N} & 135 & 30 \mathrm{~W} \\ 53 & 53 \mathrm{~N} & 161 & 40 \mathrm{~W}\end{array}$ 59 DON $14120 \mathrm{~W}$ $5930 \mathrm{~N} 14520 \mathrm{~W}$ $5925 \mathrm{~N} 14525 \mathrm{~W}$ $5930 \mathrm{~N} 14255 \mathrm{~W}$ $5900 \mathrm{~N} 14120 \mathrm{~W}$ 


\section{Catalog 3}

\section{Unsynonymized Species}

ADERCOTRYMA

A LVEOLOPHR AGMIUM

ALVEOLOPHR AGM IUM

ALVEOLOPHR AGMIUM

ALVEOLOPHRAGMIUM

ALVEOLOPHRAGMIUM

ALVEOLOPHRAGMIUM

ALVEOLOPHR AGM IUM

ALVEOLOPHR AGM IUM

AMMOBACULITES

AMMOBACULITES

AMMOBACULITES

AMMOBACULITES

AMMOBAC ULITES

AMMOBACULITES

AMMOBACULITES

AMMOB AC ULITES

AMMOD IS CUS

AMMOD IS CUS

AMMOD IS CUS

AMMOD IS CUS

A MMOM AR G IN UL INA

AMMOM AR G INULI INA

AMMON IA

AMMON IA

AMMOS CAL AR IA

AMMOT IUM

AMMOT IUM

AMMOT IUM

ANGUL ODISCORBIS

ANGUL OGER INA

AN GUL OGERINA

ANGUL OGERINA

ANGUL OGERINA

ANOMALINA

ANOMA LINA

ASTACOLUS

ASTAC OLUS

ASTRONONION

ASTRONONION

ASTRONONION

BATHYSIPHON

B I LOC UL INA

BILOC UL INA

BILOC UL INA

B IL OC UL INELLA

BOL IV IN A

BOLIVINA

BOL IV INA

BOLIVINA

BOLIVINA

BOLIVINA

BOLIVINA

BOL IV INA

BOLIVINA

BOL IV INA

BOL IV INA

BOLIVINA

BOLIVINA

BOLIVINA

BOLIV INA

BOLIVINA

BUCCELLA

BUCCELLA

BUCCELLA

BUL IM INA

BUL IM INA

BUL IM IN A

BUL IM IN A

BUL IM INA

BUL IM INA

BULIMINA

BUL IM INA

BUL IM IN A

BUL IM INELLA

BUL IM INELL

BUL IM INELLA

BUL IM INELLA

BUL IM INELL A

BUL IM INELLA

CASSI DULINA

CASSI DULINA

CASSI DULINA
GLOMERATA

GLOMERATUM

COLUMBIENSE

COLUMB IENS IS

NIT I DUM

RINGENS

ROBUSTUM

SCI TULUM

SUBGLOBOSUM

WE I SNER I

AGGLUTINANS

AGGLUTINANS FILAFORMIS

AMERICANUS

ARENARIUS

CASSIS

DILATATUS

EXIGLUS

FILAFORMIS

ARENACEUS

GULL MARENS IS

PACIFICUS

FOL IACEA

SANDIEGOENSIS

BECCAR II

BECCARII TEPIDA

PSEUDOSPIRALIS

CASSIS

PLANISSIMUM

SALSUM

CHARLOTTENSIS

ANGULOSA

FLUENS

HUGHESI

SEMITRIGONA

ARIMINENS I

SCHMITTI

HYALACRULUS

PLANULATUS

GALL OWAYI

STELLAT UM

VIRAGOENS

ARENACEA

DEPRESSA

LUCERNULA

SERRAT A

GLOBULA

ACEROSA PACIFICA

ALA TA

BARBATA

COMPACTA

DECUSSATA

MINUTA

OCEANICA

PACIFICA

PAULA

PORRECTA

PSEUDOPLICATA

PUNCTATA

SEMINUDA HUMILIS

SPIS SA

SUBADVENA

SUBAENARIENSIS

FRI GIDA

I NUS IT ATA

IENERR IMA

AFF INIS

A UR I CULATA

BAR BATA

INFLATA

MEXICANA

OVATA

OVULA

OVULA

PORULA

SUBACUMINATA

BAS I COSTATA

ELE GANTISS IMA

ELEGANT IS S IMA LIMBOSA

SUBFUSIFORMIS

SUBFUSIFORMIS TENUATA

TENUAT A

C AL I FORNICA

CHARLOTTENSIS

CRASSA
CASSI DULINA

CASSI DULINA

CASSI DULINA

CASSI DUL INA

CASSIDULINA

CASSI DULINA

CASSIDULINA

CASSI DULINA

CASSIDULINA

CASSIDULINA

CASSI DULINA

CASSI DULINA

CASSIDULINA

CASSI DULINA

CASSIDULINOIDES

CHILOSTOME LLA

CHILOSTOME LLA

CHILOSTOME LL INA

CIBICIDES

CIBICIDES

CIBICIDES

CIBICIDES

CIBICIDES

CIBICIDES

CIBICIDES

CIBICIDOIDES

CORNUSPIRA

CORNUSPIRA

CORNUSPIRA

CRIBR OE LPH IDIUM

CRI BR OE LPH ID IUM

CRI IRR OELPH ID IUM

CRIBROELPHIDIUM

CRIBR ON ONI ON

CRIBRON ONION

CRIBR OS TOMOIDES

CRI BR OSTOMOIDES

CRIBR OSTOMOIDES

CRIBR OS TOMOIDES

CRIBROSTOMOIDES

CRI BR OSTOMOIDES

CRISTELLAR IA

CRISTELLARIA

CRITHIONINA

CRUCILOCUL INA

CYCLAMMINA

CYCL AMM INA

CYCLAMMINA

CYSTAMMINA

DENTALINA

DENTALINA

DENTALINA

DENTALINA

DISCORBINA

DISCORBIS

DISCORBIS

DISCORBIS

DISCORBIS

DISCORBIS

DISCORBIS

DISCORBIS

DISCORBIS

DISCORBIS

DOROTHIA

DOROTHIA

DOROTHIA

DYOCIBICIDES

EGGERELLA

EGGERELLA

EGGERELLA

EGGERELLA

EGGERELLA

EHRENBERGINA

EHRENBE RG INA

ELPHIDIELLA

ELPHIDIELLA

ELPHIDIELLA

ELPHIDIUM

ELPHIDIUM

ELPHIDIUM

ELPHIDIUM

ELPHIDIUM

ELPHIDIUM

ELPHIDIUM

C USHMAN

DELI CATA

DEPRESSA

I SLANDICA

IMBATA IS

LOMITEN

NORCROSSI

PULC HELLA

SUBC ARINATA

SUBGLOBOSA

TERE TIS

TORTUOSA

TRAN SLUCENS

CORNUTA

F IMBR IATA

O OL INA

F IMBRIATA

BRADYI

FLET CHER I

LOBATULUS

LOBATUS

MCKANNAI

REFULGENS

SPIRALIS

MUNDULUS

I NCE RTA

INVOLVENS

PLANORBIS

CLAVATUM

FRIG IDUM

SELSEYENSE

TUMI DUM

FRIGIDUM

LENE 
ELPHIDIUM

ELPHI DI UM

ELPHIDIUM

ELPHI DIUM

ELPHIDIUM

ELPHI DIUM

ELPHIDIUM

ELPHI DIUM

ELPHIDIUM

ELPHIDIUM

ELPHIDIUM

ELPHIDIUM

ELPHI DIUM

ELPHIDIUM

ENTOS OLENIA

ENTOS OL ENIA

ENTOS OLENIA

EPIST OM INELLA

EP IST OM I NELLA

EPI ST OM I NE LLA

EPIST OM INELLA

EPI STOMI NELL LA

EPONIDES

EPONI DES

EPONIDES

EPONIDES

EPONI DES

EPONI DES

EPONIDES

EPONIDES

EPONI DES

EPONIDES

FISSURINA

FISSURINA

FISSURINA

FISSURINA

F ISSURINA

FLORILUS

FLORILUS

FLORILUS

FLORILUS

FROND ICULAR IA

FROND IC ULAR IA

GAUDR YINA

GAUDR YINA

GAUDR YINA

GAUDRYINA

GLABR AT ELLA

GLABR ATELLA

GLABR AT ELLA

GLABRATELLA

GLAND UL INA

GLOBO BUL IM INA

GLOBOBUL IMINA

GLOBOTEXTULAR IA

GL OMO SP IRA

GOESELLA

GORDI AMM INA

GORDI AMM INA

GUTTULINA

GUTTULINA

GYROI DINA

GYROI DINA

GYROI DINA

GYROI DINA

GYROI DINA

HAPLOPHRAGMIUM

HAPLOPHRAGMOIDES

HAPLOPHRAGMOIDES

HAPLOPHRAGMOIDES

HAPLOPHRAGMOIDES

HAPLOPHRAGMOIDES

HAPLOPHRAGMOIDES

HAPLOPHRAGMOIDES

HAPLOPHRAGMOI DES

HAPLOPHRAGMOIDES

HAPLOPHRAGMOI DES

HAPLOPHRAGMOIDES

HAPLOPHRAGMOIDES

HAPLOPHRAGMOIDES

HAPLOPHRAGMOIDES

HAPLOPHRAGMOIDES

HAPLOPH RAGMOIDES

HAPLOPHAGMOIDES

HAPLOPHRAGMOIDES

HAPLOPHRAGMOI DE

HOE GL UN DINA

HORMOSINA

HYPER AMM INA

HYPER AMM INA

HYPER AMMINA

INVOL UT INA

INVOL UT INA

JACULELLA

JADAMMINA

KARRERIELLA
HANNAI

HUGHESI

HUGHESI FORAMINOSUM

INCERT UM

INCERTUM LENE

LENE

MAGELLANI C UM

MICROGRANULOSUM

ORB I CULARE

OREGONENSE

SELSEYENSE

SUBARC TICUM

TRANSL UCENS

TUM I DUM

CATENULATA

COMPRESSA

LUC IDA

BRADYANA

EXI GUA

PACIFICA

UMBONIFERA

VITREA

COLUMB IENS IS

FRIGIDUS

HEALDI

I SABELLEANUS

LEVI CULUS

ORNATA

REPANDUS

SUBTENER

TENERA

UMBONATUS

AGASSIZI

CUCURBITASEMA

CUCURB I TOSEMA

LUC IDA

MAR G INA TA

A UR I CUL US

BASI SPINATUS

JAPONICUS

LABRADORIC US

GIGAS

ARENARIA

ATLANTICA PACIFICA

ATLANTICA PAC

SUBGLABRATA
CHARLOTTENSIS

CHARLOT

OPERCULAR IS

ORNATI SSIMA

LAEVIGATA

AUR I CULATA

PACIFICA

ANCEPS

GORDIALIS

FLINTII

CHAROIDES

GORDIALIS

ORIENTALIS

PROBLEMA

ALTI FORMIS

GEMMA

IO

LAMARC KIANA

ORBI CULAR IS

CANARI ENSE

ADVENA

ADVENUM

BRADYI

CANARIENSIS

COLUMBIENSE

COLUMBIENSE EVOLUTUM

COLUMBIENS IS

GLOMERATUM

HANC OCKI

NEOBRADY

NIT I DA

PARKERAE

PLANISSIMUS

SPHAERILOCULUS

SUBGLOBOSUM

SUBI NVOLUT UM

SUBI NVOLUTUS

TENUIS

ELEGANS

GLOBUL IFERA

CYL I NDR ICA

ELONGATA

FRIABILIS

GULLMARENS IS

TENUIS

TENUIS

POLYSTOMA

BAC CATA
KARRERI ELLA
LAGENA

LAGENA

LAGENA

LAGENA

LAGENA

LAGENA

LAGENA

LAGENA

LAGENA

LAGENA

LAGENA

LAGENA

LAGENA

LAGENA

LAGENA

LAGENA

LAGENA

LAGENA

LAGENA

LAGENA

LAGENA

LAGENA

LAGENA

LAGENA

LAGENA

LAGENA

LAGENA

LAGENA

LAGENA

LAGENA

LAGENA

LAGENA

LAGENA

LAGENAMM INA

LAGENONODOSARIA

LARYNGOS IGMA

LENTI CUL INA

LOXOSTOMA

LOXOSTOMA

LOXOSTOMUM

LOXOSTOMUM

MARGINUL INA

MARTINOTTIELLA

MASSILINA

MASSILINA

MELON IS

MELONIS

M IL I AMM INA

MIL I OL I NA

MILIOLINA

MILIOLINELLA

MILIOL INELLA

MIL IOL INELLA

NODELLUM

NODOSAR IA

NOD OSAR I A

NODOSARIA

NODOSAR I A

NONION

NONION

NONION

NONION

NON ION

NONION

NONION

NONION

NONION

NONIONELLA

NONI ONE LLA

NONI ONE LLA

NONIONELLA

NONIONELLA

NONI ONELLA

NONIONELLA

NONIONELLA

NONIONINA

NONI ON I NA

NOUR I A

NUBE CULARI A

OCULOSIPHON

OOL INA

OOL INA

OOL INA

OOL INA

OOL INA

OOL INA

OOL INA

OOL INA

OOL INA

OOL INA

OOLINA

OPHTHALMIDIUM

PARAFISSUR INA

PARKERAE 


\begin{tabular}{|c|c|}
\hline PATELLINA & CORRUGATA \\
\hline PATEORIS & HAUERI NOI DES \\
\hline PELOS INA & VARIABILIS \\
\hline PELOS INELLA & DIDERA \\
\hline PLACOPS IL INA & BRADYI \\
\hline PLANORBULINA & ACERVALIS \\
\hline PLANO RBULINA & MEDI TERRANENSIS \\
\hline PLANULI NA & ORNATA \\
\hline PLANULINA & WUELLERSTORFI \\
\hline POLYM ORPHINA & CHARLOTIENSIS \\
\hline POL YMORPHINA & COMPRESSA \\
\hline POLYM ORPHINA & KINCAIDI \\
\hline POL YMORPHINA & LACTEA \\
\hline POLYSTOMELLA & CRISPA \\
\hline POLYS TOMELLA & STR I ATOPUNCTATA \\
\hline $\begin{array}{l}\text { POROE PONIDES } \\
\text { POR OE PONIDES }\end{array}$ & $\begin{array}{l}\text { CRIBROREPANDUS } \\
\text { LATERALIS }\end{array}$ \\
\hline PROTE ON INA & COMPRESSA \\
\hline PROTE ON I NA & DIF FLUG IF ORMIS \\
\hline PROTE ONINA & DIFFLUGIFORMIS LAGENARIUM \\
\hline PROTOSCHISTA & FINDENS \\
\hline PSAMM AT ODE NDRON & ARB ORESCENS \\
\hline P SAMM OSPHAERA & FUSCA \\
\hline PSEUD OG AUDRY I NA & A TLANTI CA \\
\hline PSEUD ONODOSARIA & RADI CULA \\
\hline P SEUD ON ONION & AUR I CULA \\
\hline PSEUD OPOLYMORPH INA & CHARLOTTENSIS \\
\hline PSEUD OP OLYMORPH INA & LINGUA \\
\hline PULLENI A & BULLOIDES \\
\hline PULLENIA & MULTILOBATA \\
\hline PULLENIA & QUINQUELOBA \\
\hline PULLENIA & SALISBURYI \\
\hline PULLENIA & SUBCAR I NATA \\
\hline PULVINULINA & COLUMB I ENSIS \\
\hline PULVI NUL INA & KARSTEN I \\
\hline PULVI NULINA & REPANDA \\
\hline PUL VI NUL INA & UMBONATA \\
\hline PULVI NUL INELLA & PACIFICA \\
\hline PYRGO & ABYSSORUM \\
\hline PYRGO & DEPRESSA \\
\hline PYRGO & LUCERNULA \\
\hline PYRGO & MURRHINA \\
\hline PYRGO & ROTALARIA \\
\hline PYRGO & VESPERTILIO \\
\hline PYRGO & WILLI AMSON I \\
\hline PYRGOELLA & SPHAERA \\
\hline QUI NQ UE LOCUL I NA & AGGLUT I NATA \\
\hline QU INQ UEL OCCUL I NA & AKNERI ANA \\
\hline QUI NQ UE L OCULI INA & AKNERIANA BELLATULA \\
\hline QUINQ UEL OCULINA & ARCTICA \\
\hline QUINQ UEL OCUL INA & CATALINENS IS \\
\hline QUINQUELOCULINA & DISCIFORMIS \\
\hline QUINQ UEL OCULINA & ELONGATA \\
\hline QUING UEL OCULINA & FRIGIDA \\
\hline QUINQ UE LOCULINA & FUSCA \\
\hline QUINQ UEL OCULINA & PROCERA \\
\hline QUINQUE LOCULINA & SABULOSA \\
\hline QUI NQ UEL OCUL I NA & SEMI NULA \\
\hline QUINQ UE LOCULINA & SEM I NULUM \\
\hline QUINQ UEL OCUL INA & STALKERI \\
\hline QU I NQ UE L OCUL I NA & SUBROTUNDA \\
\hline QUINQ UE L OCUL I NA & TENAGOS \\
\hline QUINQ UE LOC UL INA & VULGARIS \\
\hline RECUR VO I DES & CONTORTUS \\
\hline RECUR VO IDES & TURBINATUS \\
\hline REOPHAX & CURTUS \\
\hline REOPHAX & DENTAL INAFORMIS \\
\hline REOPHAX & DIF FLUG IF ORMIS \\
\hline REOPHAX & DISTANS \\
\hline REOPHAX & EXCENTRICUS \\
\hline REOPHAX & GUTTIFER \\
\hline REOPHAX & INSECTUS \\
\hline REOPHAX & NODULOSUS \\
\hline REOPHAX & PILULI FER \\
\hline REOPHAX & PILULIFERA \\
\hline REOPHAX & SCORPIURUS \\
\hline REOPHAX & SCOTII \\
\hline REOPH AX & SCOTTII \\
\hline REOPHAX & SUBFUS IFORMIS \\
\hline RHABD AMM IN A & ABYSSORUM \\
\hline RHI Z A MM I N A & ALGAEF ORMIS \\
\hline RH I ZA MM I NA & I ND I V I SA \\
\hline ROBER TINA & ARCTICA \\
\hline
\end{tabular}

ROBERTINA

ROBULUS

ROBULUS

ROBULUS

ROBULUS

ROBULUS

ROSALINA

ROSAL INA

ROSALINA

ROSALINA

ROTAL IA

ROTAL IA

ROT AL IA

RUPERTIA

RUPERTINA

SACC AMM INA

SACCAMMINA

SACC AMMINA

SAC CORHIZA

S CHENCKIEL LA

SIGMOIL INA

SIGMOMORPHINA

SIGMOMORPH INA

SPHAEROIDINA

SPIRILL INA

SPI RI LLINA

SPIROPLECT AMMINA

SUGGRUNDA

TEXTULARIA

TEXTULARIA

TRI CHOHYAL US

TRICHOHYAL US

TRI CHOHYALUS

TRI CHOHYALUS

TRI FARINA

TRILOCULINA

TRILOCULINA

TRIL OCULINA

TRILOCUL INA

TROCHAMMINA

TROCHAMMINA

TROCHAMMINA

TROCHAMMINA

TROCHAMMINA

TROC HAMMINA

TROCHAMMINA

TROCHAMMINA

ROCHAMMINA

TROCHAMMINA

TROCHAMMIN A

TROCHAMMINA
IROCHAMMINA

TROCHAMMINA
TROCHAMMINA

TROCHAMMINA

TROCHAMMINA

TROCHAMMINA

TRUNCATULI NA

TRUNCATULI NA

TURR ISPIRI LLINA

UVIGERINA

UVIGERINA

UVIGERINA

UVIGERINA

UVIGERINA

UVIGERINA

UVIGERINA

VAGINULINOPS IS

VAG INUL INOPS IS

VALVULINER IA

VAL VULINER IA

VERNEUILINA

VIRGULINA

VIRGULINA

VIRGULINA

VIRGULINA

VIRGULINA

VIRGULINA

VIRGULINA

VIRGULINA
CHARLOTTENS IS

CHARLOTTENSIS

D'ORBIGNYI

NI COBARENSIS

OCCIDENTALIS

ORBI CULARIS

STRONG I

C AMPANULATA

COLUMBIENSIS

ORNATISSIMA

WRIGHTII

BECCARII

COLUMBIENSIS

ORBI CULARIS

SOLDANII

STABILIS

STABILIS

DIFFLUGIFORMIS

LONG I COLLIS

SPHAERICA

RAMOSA

PRIMAEVA

CELATA

DISTORTA

GALLOWAYI

TRILOCULARIS

BULLOIDES

SPINIGERA REDUCTA

VIVIPARA

BIFORMIS

ECKISI

EARL ANDI

ORQUATA

COLUMBIENSIS

ORFORDENSIS

ORNAT IS SIMA

ORNATISSIMUS

FLUENS

CIRCULARIS

ROTUNDA

TRIG ONULA

TRIHEDRA

ADVENA

CHARLOTTENSIS

DISCORBIS

GLOB IGER INI FOR MIS

GRISEA

INFL A TA

KELLETTAE

MACRESCENS

NANA

NITI DA

PACIFICA

ACIFICA SIMPLEX

ROTALIFORMIS

SQUAMATA

S QUAMI FORMIS

TURB INATA

LOBA TULA

WUELLERSTORFI

ARCT I CA

AUBERIANA

CUSHMANI

DIRUPTA

JUNCEA

PEREGRINA

PROBOSCIDEA

SENT I COSA

BACHEI I

EGUMEN

RAU CANA

GLABRA

A DVENA

A DVENA

BRAMLETTEI

COMPLANATA

FUSIFORM I S

PAUCI LOCULATA

SANDIEGOENS IS

SEMINUDA 


\section{Synonymized Species}

ADERC OTRYMA

AMMOB AC ULITES

AMMOBACULI TES

AMMOBAC ULITES

AMMOBAC ULI TES

AMMOB ACULITES

AMMOD IS CUS

AMMODISCUS

AMMOD IS CUS

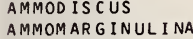

AMMOM AR G INUL INA

AMMONIA

AMMOS CALARI A

AMMOT IUM

AMMOT IUM

AMPHI CORYNA

ANGUL ODI SCORBIS

ANOMALINA

ASTAC OLUS

ASTAC OLUS

ASTAC OLUS

ASTAC OLUS

ASTRONONION

ASTRONONION

BATHY SIPHON

B ILOC UL I NELLA

BOL IV INA

BOLIVINA

BOLIVINA

BOL IVINA

BOL IV INA

BOL IV IN A

BOL IV INA

BOLIVINA

BOL IV IN A

BOLIVINA

BOL IVINA

BOL IV INA

BOLIVINA

BOL IV INA

BU C CE LLA

BUCCELLA

BULIMINA

BUL IMINA

BUL IM INA

BUL IM INA

BUL IM INA

BUL IM INA

BUL IM INA

BULIM INA

BUL IMINA

BUL IM INA

BUL IM INELLA

BUL IM INELLA

CASSI DULINA

CASSI DULINA

CASSI DULINA

CASSI DULINA

CASSI DULINA

CASSI DULINA

CASSI DULINA

CASSI DULINA

CASSI DULINA

CASSIDULINA

CASSI DULINOIDES

CHILOSTOME LLA

CHILOST OMELLINA

CIBICIDES

CIBIC IDES

CIBICIDES

CIBICIDES

CIBIC IDES

CIBIC IDES

CIBICIDOIDES

CRIBR OS TOMOIDES

CRIBR OSTOMOIDES

CRIBR OS TOMOIDES

CRIBR OST OMOIDES

CRIBR OS TOMOIDES

CRIBR OS TOMOIDES

CRITHIONINA

CRUCI LOCUL INA
GLOMERATA

AGGLUTINANS

AMER I C ANUS

ARENARI US

EXIGUUS

FILIFORMIS

ARENACEUS

GULLMARENSIS

MINUTISSIMUS

PACIFICUS

TENUIS

FOL I ACEA
SANDIEGOENSIS

BEC CAR I I

PSEUDOSPIRALIS

CASS IS

SALSUM

SCALARIS

CHARLOTTENSIS

SCHMITTI

HYALACRULUS

LATUS

OCC I DENTALIS

PLANULATUS

GALLOWAYI

VIRAGOENS IS

ARENACEA

GLOBULA

ALATA

BARBATA

COMPACTA

DECUSSATA

HUM I L I S

MINUTA

OCEAN I CA

PACIFICA

PAULA

PSEUDOPLICATA

PUNCTATA

SPISSA

SUBADVENA

SUBAENARIENSIS

FRIGIDA

I NUS I TATA

TENERR I MA

AFFINIS

BARBATA

INFLATA

INFLATA MEXICANA

OVATA

PYRULA

ROSTRATA

SUBACUMINATA

SUBFUS I FORMIS

TENUATA

BAS I COSTATA

ELEGANTISS IMA

BARBARA

CRASSA

CUSHMANI

DELICATA

DEPRESSA

LOMITENSIS

MINUTA

PULCHELLA

SUBCARINATA

SUBGLOBOSA

CORNUTA

OOL INA

FIMBRIATA

BRADYI

FLETCHERI

LOBATULUS

MCKANNAI

REFULGENS

REFULGENS

SPIRALIS

CRASSIMARG

JEFFREYSI I

NITI DUM

SUBGLOBOSUM

VELERONIS

WIESNER I

PISUM

TRIANGULAR IS
CYCLAMMINA

CYCLAMMINA

CYCLAMMINA

CYCLOGYRA

CYCLOGYRA

CYCLOGYRA

DENDROPHYRA

DENTALINA

DENTALINA

DENTALINA

DENTALINA

DENTALINA

DOROTHIA

DOROTHIA

DYOCIBICIDES

DYOCIBICIDES

EGGERELLA

EGGERELLA

EGGERELLA

EGGERELLA

EHRENBERG I NA

E HRE NBER GI NA

ELPHIDIELLA

ELPHIDIELLA

ELPH I DIELLA

ELPHIDIELLA

ELPHIDIUM

ELPH I DIUM

ELPHIDIUM

ELPHIDIUM

ELPHIDIUM

ELPHIDIUM

ELPHIDIUM

ELPHIDIUM

ELPHIDIUM

ELPHIDIUM

ELPHIDIUM

ELPH I DIUM

ELPHIDIUM

ELPHIDIUM

ELPHIDIUM

ELPHI DIUM

EP ISTOM INELLA

EPISTOM INE LLA

EPISTOMINE LLA

EPISTOMINELLA

EPISTOMINELLA

EPONIDES

EPONIDES

EPONIDES

EPONIDES

EPONIDES

EPONIDES

EPONIDES

EPONIDES

EPONIDES

FISSURINA

FISSUR INA

F ISSURINA

FISSURINA

F ISSURINA

F I SSURINA

FLORILUS

FLORILUS

FRANCESITA

FRONDICULARIA

FRONDICULARI

FURSENKOINA

FURSENKOINA

FURSENK OINA

FUR SENKOINA

FURSENKOINA

FUR SENKOINA

GAUDRYINA

GAUDRY

GAUDRYINA

GAUDRYINA

GAVELINOPS IS

GAVELINOPS IS

GLABRATELLA

GLABRATELLA

GLABRATELLA

GLABRATELLA

CANCELLATA

PUSILLA

TRULLISSATA

INCERTA

I NVOLVENS

GALE ATA

ARBORESCENS

BAGG I

C ALOMORPHA

DECEPTA

PAUPERATA

SUBSOLUTA

BRADYANA

EXIL IS

BISERIALIS

PERF ORATA

A DVENA

BRADYI

HUMBOLDTI

PUSILLA

COMPRESSA

HYSTRIX

GROENLANDICA

HANN AI

NITIDA

ARTI CULATUM

BARTLETT I

CRISPUM

EXCAVATUM

FRIGIDUM

HUGHESI FORAMI NOSUM

I NCERTUM

LENE

MAGE LLANI CUM

MI CR OGRANULOSUM

OREG ONENSE

STRIATOPUNCTATA

SUBARCTI CUM 


\begin{tabular}{|c|c|}
\hline GLABRAT ELLA & WR IGHTI I \\
\hline GLAND UL INA & LAEVIGATA \\
\hline GLOBOBULIMINA & A UR I CULATA \\
\hline GLOBOBUL IM INA & OVULA \\
\hline GLOBOBUL IM INA & PACIFICA \\
\hline GLOBO TE X TULAR IA & ANCEPS \\
\hline GLOMO SP I RA & CHAROIDES \\
\hline GLOMOSP I RA & GORDIALIS \\
\hline GOESE LLA & FLINTII \\
\hline GUTTULINA & ORIENTALIS \\
\hline GUTTULINA & PROBLEMA \\
\hline GYROI DINA & ALTI FORMIS \\
\hline GYROI DINA & GEMMA \\
\hline GYROI DINA & 10 \\
\hline GYROI DINA & LAMARCKIANA \\
\hline GYROI DINA & ORBICULARIS \\
\hline GYROI DINA & SOLDANII \\
\hline HAPLOPHRAGMOIDES & ADVENUM \\
\hline HAPLOPHRAGMOIDES & BRADYI \\
\hline HAPLOPHRAGMOIDES & CANARIENS IS \\
\hline HAPLOPHRAGMOIDES & COLUMBIENSIS \\
\hline HAPLOPHRAGMOIDES & PLANISSIMUM \\
\hline HAPLOPHRAGMOIDES & RINGENS \\
\hline HAPLOPHRAGMOIDES & SCITULUM \\
\hline HAPLOPHRAGMOIDES & SPHAE R ILOCUL \\
\hline HAPLOPHRAGMOIDES & SUBINVOLUT UM \\
\hline HAPLOPHRAGMOIDES & TENUIS \\
\hline HAYNE SINA & ORBICULARE \\
\hline HOE GL UND INA & ELEGANS \\
\hline HORMOSINA & GLOBUL I FERA \\
\hline HYPER AMM IN A & CYLINDRICA \\
\hline HYPER AMM IN A & ELONGATA \\
\hline HYPER AMM IN A & FRIABILIS \\
\hline I SLANDI ELLA & CALIFORNICA \\
\hline I SLAN DI ELLA & HELENAE \\
\hline I SLANDI ELLA & LIMBATA \\
\hline I SLAN DI ELLA & NORCROSSI \\
\hline I SLANDI ELLA & TORT UOSA \\
\hline I SLANDI ELLA & TRANSLUCENS \\
\hline JACUL ELLA & ACUTA \\
\hline J A DAMMI NA & POLYSTOMA \\
\hline KARRERI ELLA & BACCATA \\
\hline KARRERI ELLA & PARKERAE \\
\hline LAGENA & A MPHORA \\
\hline LAGENA & APIOPLEURA \\
\hline LAGENA & CLAVATA \\
\hline LAGENA & DENTAL I FORMIS \\
\hline LAGENA & DISTOMA \\
\hline LAGENA & ELONGATA \\
\hline LAGENA & GRACILIS \\
\hline LAGENA & HISPIDULA \\
\hline LAGENA & LAEVIS \\
\hline LAGENA & LAME LLA TA \\
\hline LAGENA & MERIDIONALIS \\
\hline LAGENA & MOLLIS \\
\hline LAGENA & NEBULOSA \\
\hline LAGENA & PARRI \\
\hline $\begin{array}{l}\text { LAGEN A } \\
\text { LAGENA }\end{array}$ & $\begin{array}{l}\text { PERLUCIDA } \\
\text { PLIOCENICA }\end{array}$ \\
\hline LAGENA & SEMI LINEATA \\
\hline LAGENA & SPICATA \\
\hline LAGENA & STRIATA \\
\hline LAGENA & SUBSTRIATA \\
\hline LAGENA & SULCATA \\
\hline LAGEN AMM IN A & LONG I COLL IS \\
\hline LARYNGOSIGMA & HYALASCIDIA \\
\hline LARYNGOS I GMA & LACTEA \\
\hline LENTI CULINA & D'ORB I GNYI \\
\hline LENTI CUL INA & IОTA \\
\hline LENTI CULINA & NICOBARENSIS \\
\hline LENTI CUL INA & ORB I CULAR IS \\
\hline LENTI CUL INA & STRONG I \\
\hline MARGI NUL INA & BACHEI I \\
\hline MARGI NUL INA & GLABRA \\
\hline MARTI NOTTIELLA & OCCIDEN TALIS \\
\hline MARTI NOTTIELLA & PRIMAEVA \\
\hline MASSI LINA & CRESCENTENSIS \\
\hline MASSILINA & PULCHRA \\
\hline MELON IS & BARLEEANUS \\
\hline MELON IS & POMP ILIOIDES \\
\hline MILIAMM INA & FUSCA \\
\hline MILIOLINELLA & CALIFORNICA \\
\hline MILIOLINELLA & CIRCULARIS \\
\hline MILIOLI NELLA & SUBROTUNDA \\
\hline NEOCONORBINA & TERQUEM I \\
\hline NODEL LUM & MEMBRANACEUM \\
\hline NODOS AR I A & SUBS CALARIS \\
\hline NON I O NE LLA & AUR I CULA \\
\hline NONIONE LLA & AUR I S \\
\hline NONIONE LLA & BAS I SPINATA \\
\hline NONIO NE LLA & BRADYI \\
\hline NON IO NE LLA & GRATELOUP I \\
\hline NON IO NE LLA & JAPON I CA \\
\hline NON I O NE L LA & MIOCEN I CA \\
\hline NONIONE L LA & PUNCTATA \\
\hline
\end{tabular}

NONIONELLA

NONIONELLA

NONI ONELLINA

NOUR IA

OOL INA

OOL INA

OOL INA

OOL INA

OOL INA

OOL INA

OOL INA

OOL INA

OOL INA

OOL IN

OPHTHALMIDIUM

PARAF I S SUR INA

PARRINA

PATELLINA

PELOSINA

PELOSINA

PLACOPSILINA

PLANORBULINA

PLANORBULINA

PLANULINA

PLANULINA

PLANULINA

POLYMORPHINA

POROEPONIDES

POROEPONIDES

PROTOSCHISTA

PSAMMOSPHAERA

PSEUDONODOSARIA

PSEUDOPOLYMORPHINA

PSEUDOPOLYMORPHINA

PULLENIA

PULLENIA

PULLENIA

PULLENIA

PYRGO

PYRGO

PYRGO

PYRGO

PYRGO

PYRGO

PYRGO

PYRGO

QUINQUEL OCUL INA

QUINQUE L OC UL IN

QUINQUELOC UL INA

QUINQUELOC UL INA

QUINQUELOC UL INA

QUINQUELOC UL INA

QUINQUELOCUL INA

QUINQUELOC UL INA

QUINQUELOC UL INA

QUIN QUE LOC UL INA

QUINQUELOC UL INA

QUINQUEL OC UL IN

QUINQUELOC UL INA

QUINQUELOC UL INA

RECTOBOL IV INA

RECTOBOLIVINA

REC TOBOL IV INA

RECURVOIDES

RECURVOIDES

REOPHAX

REOPHAX

REOPHAX

REOPHAX

REOPHAX

REOPHAX

REOPHAX

REOPHAX

REOPHAX

REOPHAX

REOPHAX

RHABDAMMINA

RHABDAMM IN A

RHI Z AMM INA

RHI Z AMMINA

ROBERTINA

ROBERTINOIDES

ROSAL INA

ROSALINA

ROSALINA

RUPERTI A

SACCAMM INA

SACCAMM INA

SACCAMM INA

SACCAMM INA

SAC CORHIZA

SEJUNCTELL
STELLA

TURG I DA

TURG I DA DIGITATA

$\angle A B R A D O R I C A$

POLYMORPHINOIDES

BOREALIS

GLOBOSA

HEXAGONA

L INEATA

L INEATOPUNCTAT A

STRIA TOPUNCTATA

STRIATOPUNCTATA GEMMA

WILL I AMSON I

A CUT IMARGO

FUSILIFORMIS

BRADYI

CORRUGATA

HAUE RINOIDES

DIDERA

VARIABILIS

BRAD Y I

ACERVALIS

MEDITERRANENSIS

ARIMINENSIS

ORNATA

WUELLERSTORF I

KINCA IDI

CRIBROREPANDUS

LATERALIS

FINDENS

F USC A

RADI CULA

CHARLOTTENSIS

LIGUA

BULLOIDES

MULT ILOBATA

QUINQUELOBA

SALI SBURY

ABYSSORUM

DEPRESSA

LUCE RNULA

MURR H INA

ROTALARIA

SERRATA

VESPERTILIO

WILL IAMSON I

SPHAERA

A GGLUTINATA

A KNER IANA

A RC T I CA

BELLATULA

CATALINENSIS

ELONGATA

FERUSSACHII

FRIGIDA

PROCERA

SEMINULA

SP.

STALKERI

TENAGOS

VULGARIS

AMYGDALIFORMIS

I NST ABILE

PORRECTA
CONTORTUS

CONTORTUS

CURTUS

DENT AL I N I FORMIS

DISTANS

EXCENTRICUS

GUTT IFER

IN SE CTUS

NODULOSUS

PILULIFER

SCORPIURUS

SCOTTII

SUBFUSIF ORMIS

ABYS SORUM

L INEAR IS

ALGAEFORMIS

I NDI VISA

A RCT ICA

CHARLOTTENSIS

C OLUMBIENSIS

I SABELLEANA

MONICANA

STABILIS

A TLANTI CA

HANC OCKI

LAGENARIA

SPHAERICA

RAMOSA

SPINIGERA REDUCTA 


$\begin{array}{ll}\text { SIGMOILOPSIS } & \text { SCHLUMBERGERI } \\ \text { SIGMOMORPHINA } & \text { GALLOWAYI } \\ \text { SIGMOMORPHINA } & \text { TRILOCULARIS } \\ \text { SIPHONAPERTA } & \text { SABULOSA } \\ \text { SPHAEROIDINA } & \text { BULLOIDES } \\ \text { SPIRILLINA } & \text { VIVIPARA } \\ \text { SPIROPLECTAMMINA } & \text { BIFORMIS } \\ \text { SPIROSIGMOILINA } & \text { DISTORTA } \\ \text { SUGGRUNDA } & \text { ECKISI } \\ \text { TEXTULARIA } & \text { EARLANDI } \\ \text { TEXTULARIA } & \text { TORQUATA } \\ \text { THALMANNAMMINA } & \text { PARKERAE } \\ \text { TRICHOHYALUS } & \text { ORFORDENSIS } \\ \text { TRIFARINA } & \text { ANGULOSA } \\ \text { TRIFARINA } & \text { FLUENS } \\ \text { TRIFARINA } & \text { HUGHESI } \\ \text { TRIFARINA } & \text { SEMITRIGONA } \\ \text { TRILOCULINA } & \text { ROTUNDA } \\ \text { TRILOCULINA } & \text { TRIGONULA } \\ \text { TRILOCULINA } & \text { TRIHEDRA } \\ \text { TROCHAMMINA } & \text { ADVENA } \\ \text { TROCHAMMINA } & \text { CHARLOTTENSIS } \\ \text { TROCHAMMINA } & \text { DISCORBIS } \\ & \end{array}$

$\begin{array}{ll}\text { TROCHAMMINA } & \text { GLOBIGERINIFORMIS } \\ \text { TROCHAMMINA } & \text { GRISEA } \\ \text { TROCHAMMINA } & \text { INFLATA } \\ \text { TROCHAMMINA } & \text { KELLETTAE } \\ \text { TROCHAMMINA } & \text { MACRESCENS } \\ \text { TROCHAMMINA } & \text { NANA } \\ \text { TROCHAMMINA } & \text { NITIDA } \\ \text { TROCHAMMINA } & \text { PACIFICA } \\ \text { TROCHAMMINA } & \text { PACIFICA SIMPLISSIMA } \\ \text { TROCHAMMINA } & \text { ROTALIFORMIS } \\ \text { TROCHAMMINA } & \text { SQUAMATA } \\ \text { TROCHAMMINA } & \text { SQUAMIFORMIS } \\ \text { TURRISPIRILLINA } & \text { ARCTICA } \\ \text { UVIGERINA } & \text { AUBERIANA } \\ \text { UVIGERINA } & \text { JUNCEA } \\ \text { UVIGERINA } & \text { PEREGRINA } \\ \text { UVIGERINA } & \text { PEREGRINA DIRUPTA } \\ \text { UVIGERINA } & \text { PROBOSCIDEA } \\ \text { UVIGERINA } & \text { SENTICOSA } \\ \text { VAGINULINA } & \text { LEGUMEN } \\ \text { VALVULINERIA } & \text { ARAUCANA } \\ \text { VALVULINERIA } & \text { GLABRA } \\ & \end{array}$




\section{CATAlog 5}

\section{Latitude and Longitude of Synonymized Species}

\section{Lat. Long. \\ $4114 N 12407 \mathrm{~W}$ \\ $41 \quad 36 \mathrm{~N} 124 \quad 13 \mathrm{~W}$}

$4142 \mathrm{~N} 12413 \mathrm{~W}$ $41 \quad 45 N \quad 124 \quad 13 W$

$4146 \mathrm{~N} 12414 \mathrm{~W}$

$4225 \mathrm{~N} 12426 \mathrm{~W}$

$4245 \mathrm{~N} 12431 \mathrm{~W}$

$4254 \mathrm{~N} 12443 \mathrm{~W}$

$4254 \mathrm{~N} 12444 \mathrm{~W}$

$4254 \mathrm{~N} 12448 \mathrm{~W}$

\section{Locality}

CALIFORNIA COAST CALIFORNIA COAST CALIFORNIA COAST CALIFORNIA COAST CALIFORNIA COAST CALI FORNIA COAST CALIFORNIA COAST CALIFORNIA COAST CALIFORNIA COAST CALIFORNIA COAST CALIFORNIA COAST CALIFORNIA COAST CALIFORNIA COAST CALI IFORNIA COAST CALIFORNIA COAST CALIFORNIA COASI CALIFORNIA COAST CALIFORNIA COAST OF $F$ CALIFORNIA CRESCENT CITY, CALIF. CRESCENT CITY, CALIF CRESCENT CITY, CALIF. CRESCENT CITY, CALIF CRESCENT CITY, CALIF. CRESCENT CITY, CALIF. CRESCENT CITY CALIF. CRESCENT CITY, CALIF. CRESCENT CITY, CALIF. CRESCENT CITY, CALIF. CRESCENT CITY, CALIF. CRESCENT CITY, CALIF. CRESCENT CITY, CALIF CRESCENT CITY, CALIF. CRESCENT CITY, CALIF. CRESCENT CITY, CALIF CRESCENT CITY, CALIF. CRESCENT CITY, CALIF CRESCENT CITY, CALIF . CRESCENT CITY, CALIF. CRESCENT CITY, CALIF CRESCENT CITY, CALIF. CRESCENT CITY, CALIF. CRESCENT CITY, CALIF CRESCENT CITY, CALIF. CRESCENT CITY, CALIF CRESCENT CITY, CALIF. CRESCENT CITY, CALIF CRESCENT CIIY, CALIF. CRESCENT CITY, CALIF CRESCENT CITY, CALIF. CRESCENT CITY, CALIF. CRESCENT CITY, CALIF CRESCENT CITY, CALIF. CRESCENT CITY, CALIF. CRESCENT CITY, CALIF. CRESCENT CITY, CALIF CRESCENT CITY, CALIF CRESCENT CITY, CALIF CRESCENT CITY, CALIF CRESCENT CITY, CALIF. OREGON COAST OREGON COAST OREGON COAST OREGON COAST OREGON COAST OREGON COAST OREGON COAST OREGON COAST OREGON COAST PORT ORFORD, OREGON PORT ORFORD, OREGON OF F OREGON OFF OREGON OFF OREGON OF F OREGON OFF OREGON OFF OREGON OF $F$ OREGON OFF OREGON OFF OREGON OFF OREGON OF F OREGON OF F OREGON OFF OREGON
Generic Name

BUL IMINELLA

ELPHIDIELLA

ELPHIDIUM

ELPHI DIUM

TROCHAMMINA

BUCCELLA

CIBICIDES
ELPHIOIELLA

ELPHIDIUM

ELPHIDIUM

ELPHIDIUM

EPONIDES

FIS SUR INA

FISSUR INA

GLABRATELLA

I SLANDIELLA

POROEPONIDES

ROSALINA

BUL IM INELLA

ANGULODIS CORB IS

BOL IVINA

BUCCELLA

BUL IM INELLA

CIBICIDES

DYOCIBICIDES

OYOCIBICIDES

ELPHIOIELLA

ELPHIDIUM

ELPHIOIUM

ELPHIDIUM

EPISTOMINEL LA

FISSURINA

HAPLOPHRA GMOI DES

LAGENA

MASSIL INA

MILIOL INELLA

NONIONELLA

NONIONELLA

NOUR I A

OOL INA

PATELLINA

QUINQUELOCULINA

QUINQUELOCULINA

QUINQUELOCULINA

QUINQUEL OCULINA

ROSALINA

SACCAMMINA

SI GMOMORPHINA

SIPHONAPERTA

TEXTULARI A

TROC HAMMINA

TROCHAMMINA

ANOMALINA

ELPHIOIELLA

ELPHIOIUM

ELPHIDIUM

EPONIDES

FLORILUS

MASSILINA

QUINQUELOCULINA

BOL I VINA

BUCCELLA

CIBICIDES

ELPHIDIELLA

ELPHIDIUM

ELPHIDIUM

ELPHIDIUM

GLABRA TELLA

GLABRATELLA

TROCHAMM INA

MASS IL INA

TRICHOHYALUS

BUL IM INA

NON IONELLA

NONI ONEL L INA

BUL IMINA

LAGENA

LAGENA

NONI ONELLA

NONIONELL IN

SACCAMMINA

BOL IV INA

GLOBOBUL I MINA

HAPLOPHRAGMOI DES

LAGENA
Specific Name

Publication

ELEGANTIS SIMA

HANNAI

CR ISPUM

MI CROGRANUL OS UM

KELLET TAE

TENERR IMA

FLETCHERI

HANNA:

HUGHESI FORAMINOSUM

MI CROGRANUL OSUM

TRANSLUCENS

COLUMBIENSIS

LUCIDA

MARGINATA

ORNAT ISSIMA

LIMBATA

CRIBR OREPANDUS

MONICANA

ELEGANTIS SIMA

CHARL OTTENSIS

COMPACTA

ELEGANTIS SIMA

LOBATULUS

BISER IALIS

PERFORAT

HANNAI

EXCAVATUM

LENE

BRADYANA

LUCIDA

COLUMBIENSIS

STRIATA

PULCHRA

CALIFORNICA

BAS ISPINATA

ST ELLA

POLYMORPHINOIDES

MELO

CORRUGATA

BELLATULA

ELONGATA

TENAGOS

VULGARIS

COLUMBIENSIS

A T LANTICA

TRILOCULARIS

SA BULOSA

EA RLAN DI

CHARL OTTENSIS

PACIFICA

SCHMITTI

HANNAI

ART IC ULA TUM

HU GHES I

ORNATA

ORNATA

SCAPHUM
CRESCENTENSIS

CRESCENTENS

SEMINULA
HUMILIS

TE NER R IMA

FLETCHER I

HANNAI

MI CROGRANUL OSUM

TRANSLUCENS

COLUMBIENSIS

LAURIEI

ORNAT ISS IMA

KELLETTAE

CRESCENTENS IS

OR FORDENSIS

OVATA

PUNCTATA

LA BRA DOR ICA

OVATA

GRACILIS

SUBSTRIATA

PUNCTATA

LA BRADORICA

A TLANTICA

PUNCTATA

OVULA

TENUIS

CLAVATA

COOPER 196

COOPER 1961

COOPER 1961

COOPER 1961

COOPER 1961

COOPER 1961

COOPER 1961

COOPER 1961

COOPER 1961

COOPER 1961

COOPER 1961

COOPER 1961

COOPER 1961

COOPER 1961

COOPER 1961

COOPER 1961 
Lat. Long. Locality

$4254 \mathrm{~N} 12448 \mathrm{~W}$

$4255 N 12445 W$

OF F OREGOH OFF OREGON OF F OREGON OFF OREGON OFF OREGON OFF OREGON OFF OREGON OF F OREGON OFF OREGON $4300 N 12507 W$ OFF OREGON OF F OREGON OFF OREGON OFF OREGON OFF OREGON

$4302 \mathrm{~N} 12500 W$ OFF OREGON OFF OREGON OFF OREGON
OFF OREGON

$4303 \mathrm{~N} 12452 \mathrm{~W}$ OFF CALIFORNIA

43 OSN $12501 \mathrm{~W}$ OFF OREGON

$4306 \mathrm{~N} 12508 \mathrm{~W}$ OFF OREGON OFF OREGON OFF OREGON OFF OREGON OFF OREGON

$4307 N 12426 \mathrm{~W}$ OREGON COAST OREGON COAST OREGON COAST OREGON COAST OREGON COAST ORE GON COAST OREGON COAST OREGON COAST OREGON COAST OREGON COAST OREGON COAST OREGON COAST OREGON COAST OREGON COAST OREGON COAST OREGON COAST OREGON COAST OREGON COAST OREGON COAST OREGON COAST OREGON COAST OREGON COAST ORE GON COAST OREGON COAST

$4307 N 12441 \mathrm{~W}$ OFF OREGON OFF OREGON OFF OREGON OFF OREGON

$4308 \mathrm{~N} 1245 \mathrm{SW}$ OFF OREGON OF F OREGON OFF OREGON OFF OREGON OFF OREGON OFF OREGON
OFF OREGON OFF OREGON
OFF OREGON OFF OREGON OFF OREGON

$4308 \mathrm{~N} 12459 \mathrm{~W}$ OFF OREGON OFF OREGON OFF OREGON OFF OREGON OFF OREGON

$4309 N 12442 W$

$4312 \mathrm{~N} 12501 \mathrm{~W}$ OF F OREGON OF F OREGON OF F OREGON OFF OREGON OFF OREGON OF F OREGON OFF OREGON OFF OREGON OFF OREGON OFF OREGON OF F OREGON OF F OREGON

$4315 N 12453 W$

$4321 \mathrm{~N} 12420 \mathrm{~W}$
SUNSET BAY, OREGON SUNSET BAY, OREGON SUNSET BAY, OREGON SUNSET BAY, OREGON SUNSET BAY, OREGON SUNSET BAY, OREGON SUNSET BAY, OREGON SUNSET BAY, OREGON SUNSET BAY, OREGON SUNSET BAY, OREGON SUNSET BAY, OREGON SUNSET BAY, OREGON SUNSET BAY, OREGON SUNSET BAY, OREGON SUNSET BAY, OREGON SUNSET BAY, OREGON SUNSET BAY, OREGON
Generic Name Specific Name

Publication

NONIONELLA

SACCAMMINA

BULIMIAA

CHILOSTOMELLA

$\begin{array}{ll}\text { LAGENA } & \text { SUBSTRIATA } \\ \text { NONIONELLINA } & \text { LABRADORICA }\end{array}$

REOPHAX EXCENTRICUS

SACCAMMINA

BULIMINA

BULIMIAA

EPISTOMINELLA

FLORILUS

NONIONELLINA

PYRGO

CHILOSTOMELLA

EPISTOMINELLA

GLOBOBUL IMINA

PYRGO

PULLENIA

BULIMINA

BULIMINA

GLOBOBUL IMINA

LAGENA

LAGENA

BUCCELLA
BULIMINELLA

BULIMINELLA

CIBICIDES

EGGERELLA

ELPHIDIELLA

ELPHIDIUM

ELPHIDIUM

FISSURINA

GAUDRYINA

GAUDRY INA

GAUDRYINA

GAUDRYINA

GLABRATELLA

HAPLOPHRAGMOI DES

I SLANDIELLA

MILIOLINELLA

NONI ONELLA

QUINQUEL OCULINA

ROBERT INOIDES

ROSAL INA

TRI FAR INA

TROCHAMMINA

BOL I V INA

BUL IMINA

NONIONELLA

BATHYSIPHON

BULIMIAA

CASSI DUL INA

CHILOSTOMELLA

EPISTOMINELLA

GLOBOBUL I MINA

HAPLOPHRAGMOI DES

PLANULINA

PYRGO

BUL IMINA

EPONIDES

LAGENA

NONIONELL INA

PULLENIA

BUL IMINA

BUL IM INA

BULIMINA

CHILOSTOMELLA

CHILOS TOMELLINA

EPIST OMINELLA

EGGERELLA

EPISTOMINELLA

LAGENA

LAGENA

CHILOSTOMELLINA

EPONIDES

ASTRONONION

BUCCELLA

BUL I M INELLA

CIBICIDES

CIBICIDES

CYCLOGYRA

EGGERELLA
ELPHIDIELLA

ELPHI DIUM

ELPHIDIUM

EPONIDES

FIS SUR INA

GLABRATELLA

GLABRA TELLA

HAPL OPHRAGMOI DES

I SLAN DIELLA

NONIONELLA

NONIONELLA

OOL INA

MI OCENICA

A TLANTICA

OVATA

SUBSTRIATA

A TLANTICA

AF F IN I

OVATA

PACIFICA

SCAPHUM

LABRADORICA

DEPRE S SA

OOLINA

PACIFI I A

OVULA

SERRATA

MULTILOBATA

OVATA

TENUATA

OVULA

CLAVATA

ELONGATA

ELEGANTIS SIMA

FLETCHERI

LOBAT ULUS

ADVENA

ADVENA
HANNAI

MI CROGRANULOSUM

TRANSLUCENS

MARGINATA

ARENARIA

ATLANTICA

ATLANTICA PACIFICA

SUBGLABRATA

ORNAT ISS IMA

TORTUOSA

CI RCUL ARIS

BASISPINATA

AKNER I ANA

CHARL OTTENSIS

MONICANA

HU GHES I

KELLETTAE

PUNCTATA

OVATA

PUNCTATA

LABRADORICA

ARENACEA

TENUATA

DELICATA

OOL INA

PACIFICA

OVULA

TENUIS

ORNAT A

DEPRESSA

TE NUA TA

TENERA

SUBSTRIATA

LABRADORICA

QUINQUELOBA

I N FLATA

OVATA

TENUA TA

OOLINA

F I MBR I ATA

PACIFICA

AD VENA

PACIFICA

LAMELLATA

SUBSTRIATA

FI MBR I AT A

TENER A

GALLOWAYI

ELEGANTIS SIMA

LOBAT ULUS

IN VOL VENS

AD VENA

NIT IDA

INCER T UM

SUBARCTI CUM

COLUMBIENSIS

LUCIDA

ORNAT I SS IMA

SANJU ANENSIS

COLUMBIENSIS

LIMBATA

GRATELOUPI

STELLA

MELO 


\section{Lat. Long. Locality}

$4321 \mathrm{~N} 12420 \mathrm{~W}$

$4324 \mathrm{~N} 12414 \mathrm{~W}$

$4325 \mathrm{~N} 12408 \mathrm{~W}$

$4347 N 16036 \mathrm{~W}$

$4358 \mathrm{~N} 12436 \mathrm{~W}$

(2)

$402 N 12408 \mathrm{~W}$

0
04

$4447 N 12405 W$

$4449 \mathrm{~N} 12404 \mathrm{~W}$

$4530 \mathrm{~N} 12452 \mathrm{~W}$

$4536 \mathrm{~N} 12357 \mathrm{~W}$
SUNSET BAY, OREGON SUNSET BAY, OREGON SUNSET BAY, OREGON SUNSET BAY, OREGON SUNSET BAY, OREGON SUNSET BAY, OREGON SUNSET BAY, OREGON SUNSET BAY, OREGON SUNSET BAY, OREGON SUNSET BAY, OREGON COOS BAY, OREGON COOS, BAY, OREGON COOS, BAY, OREGON COOS, BAY OREGON COOS BAY OREGON COOS, BAY, OREGON COOS, BAY, OREGON
COOS, BAY, OREGON COOS, BAY, OREGON COOS, BAY, OREGON COOS, BAY, OREGON COOS, BAY, OREGON NORTH PACIFIC NORTH PACIFIC NORTH PACIFIC NORTH PACIFIC NORTH PACIFIC NORTH PACIFIC NORTH PACIFIC NORTH PACIFIC NORTH PACIFIC NORTH PACIFIC NORTH PACIFIC NORTH PACIFIC NORTH PACIFIC NORTH PACIFIC NORTH PACIFIC
NORTH PACIFIC NORTH PACIFIC OFF OREGON OFF OREGON OFF OREGON OFF OREGON OFF OREGON OFF OREGON OFF OREGON
OFF OREGON OFF OREGON
OFF OREGON OF F OREGON OFF OREGON OREGON COAST OREGON COAST OREGON COAST OREGON COAST OREGON COAST OREGON COAST OREGON COAST OREGON COAST OREGON COAST OREGON COAST DEPOE BAY, OREGON DEPOE BAY, OREGON DEPOE BAY, OREGON DEPOE BAY, OREGON DEPOE BAY, OREGON DEPOE BAY, OREGON DEPOE BAY, OREGON DEPOE BAY, OREGON DEPOE BAY, OREGON DEPOE BAY, OREGON DEPOE BAY, OREGON DEPOE BAY, OREGON DEPOE BAY, OREGON DEPOE BAY, OREGON OREGON COAST OREGON COAST OREGON COAST OREGON COAST OREGON COAST OREGON COAST OREGON COAST OREGON COAST OREGON COAST OREGON COAST OREGON COAST OREGON COAST OREGON COAST OFF WASHINGTON OFF WASHINGTON OFF WASHINGTON OFF WASHINGTON OFF WASHINGTON OREGON COAST OREGON COAST OREGON COAST OREGON COAST OREGON CO OREGON COAST

\section{Generic Name Specific Name}

PATEORIS

PLANORBUL INA

POROEPONIDES

ROBERT INO IDES

ROSAL INA

TR IFAR INA

TROCHAMMINA

TROC HAMMINA

TROCHAMMINA

TROCHAMMINA

AMMON I A

AMMOBACULITES

AMMOT I UM

HAPLOPHRAGMOI DES

HAPLOPHR A

MILI AMMINA

PROTOSCHISTA

TROCHAMM INA

TROCHAMM I NA

ADERCOTRYMA

AMMOBACUL ITES

CIBICIDES

CRIBROSTOMOIDES

DOROTHIA

DOROTHIA

EPISTOMINELLA

GLOMOSPIRA

MIL IOL INELLA

OPHTHALMI DIUM

PLACOPSILINA

PULLENIA

SACC AMMINA

SPIROPLEC TAMM INA

TROCHAMM INA

TROCHAMM INA

TROCHAMMINA

CRITHIONINA

CYCLAMMINA

EPONIDES

GLOMOSPIRA

GLOMOSPIRA

GYROIDINA

GYROIDINA

HAPLOPHRAGMOIDES

PYRGO

SIGMOILOPSIS

BUCCELLA

BUL I M I NELLA

EGGERELLA

ELPHI DIELLA

ELPHIO IUM

FISSUR INA

HAPLOPHRAGMOI DES

QUINQUELOCULINA

ROSAL INA

TROCHAMMINA

ANGUL ODIS CORB IS

BUCCELLA

BUL IM INELLA

EGGERELLA

ELPHIDIELLA

ELPHIDIUM

ELPHIDIUM

GLABRATELLA

HAPLOPHRAGMOI DES

ROSAL INA

SACCAMMINA

TROCHAMMINA

TROCHAMM INA

TROCHAMM I NA

BOL IVINA

BUCCELLA

ELPHIDIELLA

ELPHIDIUM

EPONIDES

GLABRA TELLA

MILIOL INELLA

PLANORBUL INA

QUINQUELOCULINA

ROSAL INA

TRO CHAMM INA

TROCH AMM I NA

TURR I SPIRILLINA

CRIBROSTOMOIDES

CYCLAMMIN A

PLANUL INA

PLANULINA

QUINQUELOCULINA

BUCCELLA

CIBICIDES

EGGERELLA

ELPHIDIELLA

ELPHI DIUM

GLABRATELLA

HA UER I NO I DES

ACERVALIS

CHARLOTTENSIS

COLUMBIENSIS

FL UENS

CHARL OTTENS IS

DISCORBI

IN F LATA

RO TAL I FORMIS

BECCAR I I

SALSUM

SUBARCTI CUM

SUBINVOLUTUM

POLYSTOMA

FUSCA

FI NDENS

INFLATA

MACRESCENS

GL OMERATA

AG GLUTINANS

BRADY I

NI TIDUM

EXILIS

SC ABRA

EXIGUA

GORDIALIS

SUBROTUNDA

AC UTI MARGO

BRADY I

QUINQUELOBA

A TL AN T ICA

BI FORMIS

GL OBI GER INIFORMIS

GR ISEA

NITIDA

PI SUM

PUSILLA

UMBONATUS

CHAROIDES

GORDIALIS

ORBICULARIS

SOLDANII

CANARIENSIS

LU CERNULA

SC HLUMBER GER I

TENERR IMA

ELEGANTISSIMA

AD VENA

HANNA

MI CROGRANUL OSUM

MARGINATA

COLUMBIENSIS

AKNER I ANA

MONIC ANA

KELLETTAE

CHARL OTTENSIS

TENERR IMA

EL EGAN TIS SIMA

AD VEN A

HANNA I

LENE

ORNAT ISSIMA

COLUMBIENSIS

COLUMBIENSIS

ATLAN I ICA

CHARL OTTENSIS

KELLETTAE

PACIF ICA

PSEUDOPL ICATA

TE NER R IMA

HANNAI

MI CROGRANULOSUM

COLUMBIENSIS

OPERCULARIS

CI RCULARIS

MEDITERRANENS IS

AKNER I ANA

MONICANA

PACIFICA

SQUAMI FORMIS

ARCTICA

SUBGL OBOSUM

CANCELLATA

AR IMINENS IS 


\section{Lat. Long. \\ Locality}

$4536 \mathrm{~N} 12357 \mathrm{~W}$

$4554 \mathrm{~N} 12448 \mathrm{~W}$

$4555 \mathrm{~N} 12448 \mathrm{~W}$ $4611 \mathrm{~N} 12359 \mathrm{~W}$

$4700 \mathrm{~N} 12408 \mathrm{~W}$

$4700 \mathrm{~N} 12410 \mathrm{~W}$

$4700 \mathrm{~N} 12412 \mathrm{~W}$

$4710 \mathrm{~N} 12414 \mathrm{~W}$

$4730 \mathrm{~N} 12229 \mathrm{~W}$

$4730 \mathrm{~N} 12420 \mathrm{~W}$

$4740 N 12225 \mathrm{~W}$

$4741 N 12428 W$

$4748 N 12243 W$

$749 \mathrm{~N} 12528 \mathrm{~W}$

$4755 \mathrm{~N} 12739 \mathrm{~W}$

$4759 \mathrm{~N} 12215 \mathrm{~W}$ $4807 \mathrm{~N} 12255 \mathrm{~W}$

$4814 \mathrm{~N} 12330 \mathrm{~W}$
OREGON COAST OREGON COAST OFF OREGON OF F OREGON OFF OREGON OREGON COAST OREGON COAST GRAY'S HARBOUR, WASH. GRAY'S HARBOUR, WASH GRAY'S HARBOUR, WASH GRAY'S HARBOUR, WASH. GRAY'S HARBOUR, WASH. GRAY'S HARBOUR, WASH. GRAY'S HARBOUR, WASH GRAY'S HARBOUR, WASH. GRAY'S HARBOUR, WASH. GRAY'S HARBOUR, WASH. GRAY'S HARBOUR, WASH. GRAY'S HARBOUR, WASH. GRAY'S HARBOUR, WASH. GRAY'S HARBOUR, WASH. WASHINGTON COAST WASHINGTON COAST WA SHINGTON COAST WASHINGTON COAST WASHINGTON COAST WASHINGTON COAST WASHINGTON COAST WASHINGTON COAST WA SHING TON COAST WASHINGTON COAST WA SHINGTON COAST WASHINGTON COAST WA SHINGTON COAST WA SHINGTON COAST OFF WASHINGTON WASHINGTON COAST WASHINGTON COAST WASHINGTON COAST WASHINGTON COAST WASHINGTON COAST WA SHINGTON COAST WASHINGTON COAST WASHINGTON COAST WASHINGTON COAST WA SHINGTON COAS WA SHINGTON COAST WASHINGTON COAST WASHINGTON COAST WASHINGTON COAS WASHINGTON COAST WASHINGTON COAST WASHINGTON COAST WASHINGTON COAST WASHINGTON COAST ON COAST GTON COAST WASHINGTON COAS WASHINGTON COAS WASHINGTON COAST WASHINGTON COAST WASHINGTON COAST WASHINGTON COAST WASHINGTON COAST WASHINGTON COAST WA SHINGTON COAST OFF WASHINGTON OF F WASHINGTON
LAPUSH, WASHINGTON LAPUSH, WASHINGTON
LAPUSH, WASHINGTON LAPUSH, WASHINGTON LAPUSH, WASHINGTON LAPUSH, WASHINGTON LAPUSH, WASHINGTON LAPUSH WA SHINGTON LAPUSH, WA SHINGTON LAPUSH, WASHINGTON LAPUSH, WASHINGTON LAPUSH, WASHINGTON OF F WASHINGTON WASHINGTON COAST WASHINGTON COAST BRITISH COLUMBIA BR ITISH COLUMEIA BRITISH COLUMBIA BR ITISH COLUMBIA BRITISH COLUMBIA BR ITISH COLUMBIA OR ITISH COLUMBIA OR ITISH COLUMBIA
BRITISH COLUMBIA OR ITISH COLUMBIA ORITISH COLUMBIA OR ITISH COLUMBIA ORITISH COLUMBIA BR ITISH COLUMBIA OR ITISH COLUMBIA BR ITISH COLUMBIA ORITISH COLUMBIA OR ITISH COLUMBIA
Generic Name Specific Name

LI MBA TA

KELLETTAE

LA BRADORICA

ATLANTICA

GRACILIS

HANNAI

MI CROGRANULOSUM

EXIGUUS

SALSUM

ELEGANTISSIMA

FR IGI DUM

LENE

SUBARCTI CUM

SUBINVOLUTUM

POLYSTOMA

FUSCA

FI NDENS

CHARLOTTENSIS

INFLATA

MACRESCENS

FR IGIDA

TENER R IMA

EL EGANTIS SIMA

ADVEN A

MA GELLANI CUM

MI CROGRANULOSUM

JAPONICA

STELLA

TURBINATUS

QI FORMIS

MINUTA

JEFFREYSII

HANNA I

CHARLOTTENSIS

FR IGI DUM

FRIGIDA

TENERR IMA

ELEGANTIS SIMA

ADVENA

MAGELLANI CUM

MI CROGRANULOSUM

JAPONICA

STELLA

TURBINATUS

BI FORMIS

LOBATULUS

HANNA I

CRISPUM

LENE

COLUMBIENSIS

LIMBATA

SUBSC ALARIS

MELO

LOBAT ULUS

I SER IALIS

HANNA I

REPANDUS

CALIFORNICA

LIMBATA

ORNAT I SS IMA

PL IOCENICA

BA SISPINATA

SEMITR IGONA

JUNCEA

TURBINATUS

TENERR IMA

ELEGANTIS SIMA

ADVENA

HANNA

LENE

LUCIDA

TORTUOSA

BELLATULA

COLUMBIENSIS

CHARLOTTENSIS

FR I GI OUM

LOBATULUS

REPANDUS

GL OMERAT A

GULLMARENSIS

GALLOWAYI

PACIFICA

ELEGANTIS SIMA

FLETCHER I

LOBATULUS

ARBORESCENS

ADVENA

NI TIDA

EX CAVA TUM

I N CERT UM

SUBARC TICUM

PACIFICA

PACIFICA

SUBGLABRATA

AURICULATA

\section{Publication}

COOPER 196

COOPER 1961

CUSHMAN 1927

CUSHMAN 1927

CUSHMAN 1927

COOPER 196

COOPER 196

COOPER 1961

PHLEGER 1967

PHLEGER 196

PHLEGER 1967

PHLEGER 1967

PHLEGER 1967

PHLEGER 1967

PHLEGER 1967

PHLEGER 1967

PHLEGER 1967

PHLEGER 1967

PHLEGER 1967

PHLEGER 1967

ECHOLS 1969

ECHOLS 1969

ECHOLS 1969

ECHOLS 1969

ECHOLS 1969

ECHOLS 1969

ECHOLS 196

ECHOLS 196

ECHOLS 1969

ECHOLS 1969

ECHOLS 1969

ECHOLS 1969

ECHOLS 1969

ECHOLS 1969

CUSHMAN MCCULLOCH 1940

ECHOLS 1969

ECHOLS 1969

ECHOLS 1969

ECHOLS 1969

ECHOLS 1969

ECHOLS 1969

ECHOLS 1969

ECHOLS 1969

ECHOLS 1969

ECHOLS 1969

CUSHMAN TODD 1947

CUSHMAN TODD 1947

CUSHMAN TODD 1947

CUSHMAN TODD 1947

CUSHMAN TODD 1947

CUSHMAN TODD 1947

CUSHMAN TODD 1947

CUSHMAN TODD 1947

CUSHMAN TODD 1947

CUSHMAN TODD 1947

CUSHMAN TODD $194 ?$

CUSHMAN TODD 1947

CUSHMAN TODD 1947

CUSHMAN TODD 1947

CUSHMAN TODD 1947

CUSHMAN TODD 1947

CUSHMAN TODD 1947

CUSHMAN TODO 1947

CUSHMAN TODO T947

CUSHMAN 1910

CUSHMAN 1910
LANKFORD PHLEGER 1973

$\begin{array}{lll}\text { LANKFORD } & \text { PHLEGER } & 1973 \\ \text { LANKFORD } & \text { PHLEGER } & 1973\end{array}$

$\begin{array}{lll}\text { LANKFORD } & \text { PHLEGER } & 1973 \\ \text { LANKFORD } & \text { PHLEGER } & 1973\end{array}$ 


\section{Lat. Long. \\ Locality}

$4814 \mathrm{~N} 12330 \mathrm{~W}$

$4816 \mathrm{~N} 12247 \mathrm{~W}$

$\begin{array}{llll}48 & 16 N & 124 & 16 W \\ 48 & 18 N & 124 & 43 W\end{array}$

$4820 \mathrm{~N} 12300 \mathrm{~W}$
BR ITISH COLUMBIA BRITISH COLUMBIA BRITISH COLUMBIA BRITISH COLUMBIA BRITISH COLUMBIA BR ITISH COLUMBIA BRITISH COLUMBIA BR ITISH COLUMBIA BR ITISH COLUMBIA BRITISH COLUMBIA BR ITISH COLUMBIA BRITISH COLUMBIA BR ITISH COLUMBIA BRITISH COLUMBIA BR ITISH COLUMBIA BRITISH COLUMBIA BRITISH COLUMBIA BRITISH COLUMBIA BR ITISH COLUMBIA BRITISH COLUMBIA BRITISH COLUMBIA BR ITISH COLUMBIA BR ITISH COLUMBIA BRITISH COLUMBIA BRITISH COLUMBIA BRITISH COLUMBIA BRITISH COLUMBIA BR ITISH COLUMBIA BR ITISH COLUMBIA BRITISH COLUMBIA BRITISH COLUMBIA WASHINGTON COAST WASHINGTON COAST WASHINGTON COAST WASHINGTON COAST WASHINGTON COAST WASHINGTON COAST WASHINGTON COAST WASHINGTON COAST WASHINGTON COAST WASHINGTON COAST WASHINGTON COAST WASHINGTON COAST BRITISH COLUMBIA BRITISH COLUMBIA BRITISH COLUMBIA BRITISH COLUMBIA BRITISH COLUMBIA BR ITISH COLUMBIA BRITISH COLUMBIA BRITISH COLUMBIA BRITISH COLUMBIA BRITISH COLUMBIA BRITISH COLUMBIA BRITISH COLUMBIA BRITISH COLUMBIA BRITISH COLUMBIA BR ITISH COLUMBIA BRITISH COLUMBIA BRITISH COLUMBIA BRITISH COLUMBIA BRITISH COLUMBIA BRITISH COLUMBIA BRITISH COLUMBIA BR ITISH COLUMBIA BRITISH COLUMBIA BRITISH COLUMBIA BRITISH COLUMBIA BRITISH COLUMBIA BR ITISH COLUMBIA BR ITISH COLUMBIA BR ITISH COLUMB IA BRITISH COLUMBIA BRITISH COLUMBIA BRITISH COLUMBIA BRITISH COLUMBIA BRITISH COLUMBIA BRITISH COLUMBIA BR ITISH COLUMBIA BR ITISH COLUMBIA BR ITISH COLUMBIA BRITISH COLUMBIA BRITISH COLUMBIA BR ITISH COLUMBIA BR ITISH COLUMBIA BRITISH COLUMBIA BR ITISH COLUMBIA BR ITISH COLUMBIA BRITISH COLUMBIA BR ITISH COLUMBIA BRITISH COLUMBIA BRITISH COLUMBIA BRITISH COLUMBIA BR ITISH COLUMBIA BRITISH COLUMBIA BRITISH COLUMBIA
Generic Name Specific Name

Publication

HAPLOPHRAGMOI DES HAPLOPHRAGMOIDES HAPLOPHRAGMOI DES

ISLANDIELLA

I SLANDIELLA

LAGENA

LAGENA

LAGENA

NONIONELLA

NONIONELLA

OOL INA

PATELLINA

POROEPONIDES

PULLENIA

RECURVOIDES

REOPHAX

REOPHAX

SACCAMMINA

SIGMOMORPHINA.

SPIROPLECTAMM INA

TRIFARINA

TRIFAR INA

TROCHAMMINA

TROCHAMMINA

TROCHAMMINA

TROCHAMM I NA

TROCHAMM INA

TROCHAMM INA

UVIGERINA

ELPHIDIELLA

I SLANDIELLA

EPONIDES

ANGULODIS CORB IS

CASSIDULINA

CIBICIDES

CIBICIDES

ELPH I DIELLA

GLABRATELLA

ROSAL INA

ROSALINA

ADERCOTRYMA

AMPHI CORYNA

BOLIVI A A

BULIM I NELLA

CASSIDULINA

CIBICIDES

CIBICIDES

DENDROPHYRA

EGGERELLA

ELPHIOIELLA

ELPHIDIUM

ELPHIDIUM

ELPHIDIUM

ELPHIDIUM

EPISTOMINELLA

F IS SUR INA

GAUDRYINA

GAUDRYINA

GAVEL I NOPSIS

GLABRATELLA

GLOBOBUL IMINA

GLOBOBUL IMINA

HAPLOPHRAGMOI DES

HAPLOPHRAGMOI DES

HAPLOPHRAGMOI DES

HAPLOPHRAGMOI DES

ISLANDIELLA

I SLAND IELLA

I SLANDIELLA

LAGENA

LAGENA

LAGENA

LAGENA

$\angle A G E N A$

LAGENA

LAGENA

LAGENAMM I NA

NONIONELLA

NON IONELLA

NONIONELLA

NONIONELLA

NONIONELL INA

OOL INA

OOLINA

PATELLINA

POL YMORPHINA

POROEPONIDES

PSEUDOPOL YMORPHINA

PULLENIA

PYRGO

RECURVOIDES

REOPHAX

ROSALINA

COLUMBIENSIS

COLUMBIENSIS

PLANISSIMUM

CALIFORNICA

LIMBATA

AP IOPLEURA

DISTOMA

PLIOCENICA

AURICULA

BASISPINATA

LABRADORICA

MELO

CORRUGATA

CRIBR OREPANDUS

SALISBURYI

TURBINATUS

CURTUS

PI LUL I FER

TRILOCULARIS

BI FORMIS

ANGULOSA

SEMITR IGONA

CHARL OTTENSIS

DISCORBIS

NANA

PACIFICA

SQUAMI FORMIS

JUNCEA

HANNA I

IMBATA

COLUMBIENSIS

CHARLOTTENSIS

MI NUTA

FLETCHER I

LOBATULUS

HA NNA I

ORNAT ISS IMA

COLUMBIENSIS

COLUMBIENSIS

GL OMERATA

SCALARIS

GALLOWAYI

PACIF ICA

ELEGANTISSIMA

DEPRESSA

FLETCHERI

LOBATULUS

ARBORESCENS

AD VENA

NI TIDA

CR ISPUM

EXCAVATUM

INCERT UM

SUBARCTICUM

PACIFICA

LUCIDA

ARENARIA

SUBGLABRATA

CAMPANULATA

ORNAT ISSIMA

AURICULATA

PACIFICA

BRADY I

COLUMBIENSIS

COLUMBIENSIS

PLANISSIMUM

CALIFORNICA

LI MBATA

APIOPLEURA

DENTALIF ORMIS

DISTOMA

ELONGATA

GRACILIS

PL IOCENICA

SULCATA

LONGICOLLIS

AURI CULA

BASISPINATA

STELLA

TURGIDA DIGITATA

LABRADORICA

BOREAL IS

MELO

CORRUGATA

KI NCA I D I

CRIBR OREPANDUS

CHARL OTTENS IS

SALISBURYI

WILLI AMS ON I

TURBINATUS 
Lat. Long. Locality

$4820 \mathrm{~N} 12300 \mathrm{~W}$

$4820 \mathrm{~N} 12410 \mathrm{~W}$

$4820 \mathrm{~N} 12428 \mathrm{~W}$

$4820 N 12442 W$

$4820 N 12444 \mathrm{~W}$

$4832 \mathrm{~N} 12300 \mathrm{~W}$

$4832 \mathrm{~N} 12310 \mathrm{~W}$
BR ITISH COLUMBIA BRITISH COLUMBIA BRITISH COLUMBIA BRITISH COLUMBIA BRITISH COLUMBIA BRITISH COLUMBIA BRITISH COLUMBIA BR I ISH COLUMBIA BRITISH COLUMBIA BRITISH COLUMBIA BR ITISH COLUMBIA BRITISH COLUMBIA BRITISH COLUMBIA BRITISH COLUMBIA BRITISH COLUMBIA BRITISH COLUMBIA BRITISH COLUMBIA BRITISH COLUMBIA BRITISH COLUMBIA BRITISH COLUMBIA BR ITISH COLUMBIA BRITISH COLUMBIA BRITISH COLUMBIA BRITISH COLUMBIA BRITISH COLUMEIA BRITISH COLUMBIA BR ITISH COLUMBIA BRITISH COLUMBIA BRITISH COLUMBIA BRITISH COLUMBIA BRITISH COLUMBIA BRITISH COLUMBIA BRITISH COLUMBIA BRITISH COLUMBIA BRITISH COLUMBIA BRITISH COLUMBIA BRITISH COLUMBIA BRITISH COLUMBIA BRITISH COLUMBIA BRITISH COLUMBIA BRITISH COLUMBIA BRITISH COLUMBIA BRITISH COLUMBIA BRITISH COLUMBIA

JUAN DE FUCA STRAITS

WASHINGTON COAST WASHINGTON COAST WA SHINGTON COAST WASHINGTON COAST WA SHING TON COAST
WA SHINGTON COAST WASHINGTON COAST WASHINGTON COAST WASHINGTON COAST WA SHINGTON COAST WASHINGTON COAST WASHINGTON COAST WASHINGTON COAST WASHINGTON COAST WASHINGTON COAST
WASHINGTON COAST WA SHINGTON COAST WASHINGTON COAST WASHINGTON COAST WASHINGTON COAST WA SHINGTON COAST WASHINGTON COAST WASHINGTON COAST WASHINGTON COAST WASHINGTON COAST WA SHINGTON COAST WASHINGTON COAST
WASHINGTON COAST WASHINGTON COAST WA SHINGTON COAST WASHINGTON COAST WA SHINGTON COAST WASHINGTON COAST WASHINGTON COAST WASHINGTON COAST WASHINGTON COAST WASHINGTON COAST
WASHINGTON COAST WASHINGTON COAST WASHINGTON COAST WASHINGTON COAST WASHINGTON COAST WASHINGTON COAST WA SHINGTON COAST WA SHINGTON COAST WA SHINGTON COAST WASHINGTON COAST WASHINGION COAST WASHINGTON COAST WASHINGTON COAST WA SHINGTON COAST WASHINGTON COAST WA SHINGTON COAST WASHINGTON COAST WA SHINGTON COAST WASHINGTON COAST WASHINGTON COAST

\section{Generic Name Specific Name}

Publication

ROSAL IN

SACCAMMINA

SI GMOMCRPHINA

SPI ROPLEC TAMM INA

TRIFARINA

TRIFARINA.

TROCHAMMINA

TROCHAMMINA

TROCHAMM INA

TROCHAMM INA

TROCHAMMINA

TROCHAMMINA

UVIGERINA

ADERCOTRYMA

AMMODISCUS

BOL IVINA

BULIMINELLA

CIBICIDES

C IBICIDES

DENDROPHYRA

EGGERELLA

ELPHIDIELLA

ELPHIDIUM

ELPHIDIUM

GAUDRYINA

GAUDRYINA

GLOBOBUL I MINA

HAPL OPHRAGMOI DES

HAPLOPHRAGMOI DES

HAPLOPHRAGMOI DES

I SLANDIELLA

NON IONELLA

PULLENIA

RECURVOIDES

REOPHAX

SACCAMMINA

SPIROPLECTAMM INA

TRI FAR INA

TROCHAMMINA

TROCHAMMINA

TROCHAMM I NA

TROCHAMM I NA

TROCHAMM INA

UVI GER INA

ELPHIDIUM

BUCCELLA

BUCCELLA

BUL IMINELLA

EGGERELLA

ELPHIDIUM

NONIONELLA

NONIONELLA

RECURVOIDES

SPIROPLECTAMMINA

BOLIVINA

QUINQUELUCULINA

A DERCOTRYMA

A STRONONION

BUCCELLA

BUL I M I NELLA

CIBICIDES

CYCLOGYRA

DENDROPHYRA

DYOCIBICIDES

EGGERELLA

ELPHIDIELLA

ELPHIDIUM

ELPHIDIUM

ELPHIDIUM

ELPHIDIUM

ELPHIDIUM

EPONIDES

FIS SUR INA

GAUDRYINA

GAUDRYINA

GLABRATELLA

GLABRA TELLA

HAPLOPHRA GMOI DES

HAPLOPHRAGMOI DES

I SLANDIELLA

ISLANDIELLA

LAGENA

LAGENA

LAGENA

LAGENA

LAGENA

LENTI CULINA

MASSILINA

MILIAMMINA

MILIOL INELLA

MILIOL INELLA

NODOSARIA

NONIONELLA

NONIONELLA

COLUMBIENSIS

A TLANTICA

TR ILOCULAARIS

BI FORMIS

ANGUL OSA

SEMITRIGONA

CHARLOTTENSIS

DI SCORBIS

NANA

PACIFICA

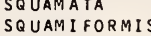

JUNCEA

GL OMERATA

GULLMARENSIS

PACIFICA

ELEGANTISSIMA

FLETC HER I

LOBATULUS

ARBORESCENS

A D VENA

NITIDA

EX CAVATUM

SUBARCTI CUM

ARENARIA

SUBGLABRATA

AURICULATA

BRADYI

COLUMBIENSIS

COLUMBIENSIS

LI MBATA

STELLA

SALISBURYI

TURBINATUS

CURTUS

A TLAN TICA

BI FORMIS

ANGUL OSA

CHARLOTTENSIS

DISCORBIS

NANA

PACIFICA

SQUAMATA

JUNCEA

CR ISPUM

FRIGIDA

TENERR IMA

ELEGANTIS SIMA

ADVEN

MA GELLAN I CUM

MI CROGRANUL OSUM

JAPON I CA

STELLA

TURBINATUS

B I FORMIS

PACIFICA

EL ONGATA

GL OMERATA

GALLOWAY

FRIGIDA

ELEGANTIS SIMA

LOBATULUS

PLANORBIS

ARBORESCENS

BISERIALIS

A DVENA

HANNA

ARTI CULATUM

CRISPUM

FRIGIDUM

LENE

TUMIDUM

COLUMBIENSIS

COMPRESSA

ARENARIA

SUBGLABRATA

ORNAT ISS IMA
SANJUANENSIS

ADVENUM

COLUMBIENSIS

CALIFORN I CA

LIMBATA

APIOPLEURA

CLAVATA

GRACILIS

PERLU CIDA

D. ORB I GNYI 


\section{Lat. Long. Locality}

$4832 \mathrm{~N} 12310 \mathrm{~W}$

$4833 N 12300 W$

$4833 \mathrm{~N} 12301 \mathrm{~W}$

$4835 \mathrm{~N} 12228 \mathrm{~W}$

$4835 N 12251 \mathrm{~W}$
WASHINGTON COAST WA SHINGTON COAST WA SHINGTON COAST WA SHINGTON COAST WASHINGTON COAST WA SHINGTON COAST WA SHINGTON COAST WASHINGTON COAST WASHINGTON COAST WASHINGTON COAST WA SHINGTON COAST WASHINGTON COAST WASHINGTON COAST WA SHINGTON COAST WA SHINGTON COAST COST WASHINGTON COAST WASHINGTON COAST WASHINGTON COAST WA SHINGTON COAST WASHINGTON COAST WASHINGTON COAST WASHINGTON COAST WASHINGTON COAST WASHINGTON COAST WASHINGTON COAST WA SHINGTON COAST WA SHINGTON COAST WASHINGTON COAST WASHINGTON COAST WASHINGTON COAST WA SHINGTON COAST WA SHINGTON COAST WA SHINGTON COAST WASHINGTON COAST WASHINGTON COAST WASHINGTON COAST WASHINGTON COAST WA SHINGTON COAST WASHINGTON COAST WASHINGTON COAST WASHINGTON COAST WA SHINGTON COAST WASHINGTON COAST WASHINGTON COAST WASHINGTON COAST WASHINGTON COAST WASHINGTON COAST WASHINGTON COAST WA SHINGTON COAST WASHINGTON COAST WA SHINGTON COAST WASHINGTON COAST WA SHINGTON COAST WASHINGTON COAST WASHINGTON COAST WASHINGTON COAST WASHINGTON COAST WASHINGTON COAST WA SHINGTON COAST WA SHINGTON COAST WASHINGTON COAST WASHINGTON COAST WASHINGTON COAST WASHINGTON COAST WASHINGTON COAST WASHINGTON COAST WA SHINGTON COAST WASHINGTON COAST WASHINGTON COAST WA SHINGTON COAST WASHINGTON COAST WASHINGTON COAST WASHINGTON COAST WASHINGTON COAST WASHINGTON COAST WASHINGTON COAST WASHINGTON COAST WA SHINGTON COAST WASHINGTON COAST WASHINGTON COAST WA SHINGTON COAST WASHINGTON COAST WA SHINGTON COAST WASHINGTON COAST WASHINGTON COAST WASHINGTON COAST SAN JUAN IS.. WASH. SAMISH BAY, WASH. SAMISH BAY, WASH SAMISH BAY, WASH SAMISH BAY, WASH SAMISH BAY, WASH. SAMISH BAY, WASH. SAMISH BAY, WASH. SAMISH BAY, WASH. BRIIISH COLUMBIA BR ITISH COLUMBIA BRITISH COLUMBIA

\section{Generic Name Specific Name}

OOL INA

OOL INA

POLYMORPHINA

PULLENIA

QUINQUEL OCULINA

QUINQUELOCULINA

ROBERTINOIDES

ROSALINA

SAC CAMMINA

SACCAMMINA

SIGMOMORPHINA

SIGMOMORPHINA

SPIROPLEC TAMM INA

TRI FARINA

TRI FAR INA

TROCHAMMINA

TROCHAMM INA

TROCHAMM I NA

UVIGERINA

ADERCOTRYMA

ASTRONONION

BOL IV INA

BOLIVIAA

BUCCELLA

CIBICIDES

CIBICIDES

CYCLOGYRA

DENDROPHYRA

DYOCIBICIDES

EGGERELLA

ELPHIDIELLA

ELPHIDIUM

ELPHIDIUM

ELPHIDIUM

ELPHIDIUM

ELPHIDIUM

EPONIDES

F IS SUR INA

FI S SUR INA

GAUDRYINA

GLABRA TELLA

GLABRA TELLA

GLOBOBUL IMINA

HAPLOPHRA GMOI DES

HAPLOPHRA GMOI DES

ISLANDIELLA

LAGENA

LAGENA

LAGENA

LAGENA

MASS I L INA

MIL I AMMIN A

MIL I OL INELLA

MILIOLINELLA

NEOCONORBINA

NODOSARIA

NONI ONELLA

NONI ONELLA

NONI ONELLA

OOL INA

OOL INA

PARRINA

PATELLINA

PSEUDOPOL YMORPHINA PULLENIA

QUINQUEL OCULI NA QUINQUELOCULINA

RECTOBOLIVINA

ROBERTINOIDES

ROSALINA

SACCAMMINA

SI GMOMORPHINA

SI GMOMORPHINA

SPI ROPLEC TAMM INA

TRIFARINA

TRIL OCUL INA

TROCHAMMINA

TROCHAMMI NA

TROCHAMMINA

PATELLINA

AMMOBACULITES

AMMONIA

A AMOT I UM

BUCCELLA

ELPHIDIUM

ELPHIDIUM

MIL IAMMINA

TROCHAMMINA

TROCHAMMINA

ADERCOTRYMA

EGGERELLA

BOREALIS

ME LO

STRIAT OPUNCTATA

KI NCA I DI

SALISBURYI

CATALINENSI
VULGARIS

VULGARIS

COLUMBIENSIS

A TLANTICA

LAGENARI

RAMOSA

GALLOWAYI

TRILOCULARIS

BI FORMIS

HU GHE SI

SEMITRIGONA

CHARLOTTENSIS

DI SCORBIS

PACIF ICA SIMPLISSIMA

JUNCEA

GL OMERATA

VI RAGOENS IS

COMPACTA

PACIFIC

FRIGIDA

ELEGANTISSIMA

LOBATULUS

REFULGENS

PLANORBIS

ARBORESCENS

BISERIALIS

A D VENA

HANNAI

ARTICULA TUM

CR I SPUM

FRIGI DUM

LENE

TUMIDUM

COLUMBIENSIS

COMPRESSA

LUCIDA

ARENARIA

ORNATISSIMA

SANJUANENSIS

AURICULATA

ADVENUM

COLUMBIENSIS

LI MBA TA

GRACILIS

PERLUCIDA
PLIOCENICA

PLIOCENICA

SPICATA

FUSCA

CI RCULARIS

CIRCULARIS

CI RCULARI

SUBSCALARIS

AURICULA

BASISPINATA

STELLA

BOREALIS

MELO

BRADY I

CORRUGATA

KINCA IDI

CHARL OTTENSIS

SALI SBURYI

EL ONGATA

VULGARIS

INSTABILE

CHARLOTTENSIS

COLUMBIENSIS

A TLANTICA

GALLOWAYI

TRILOCULARIS

BI FORMIS

SEMITR IGONA

TRIGONULA

CHARL OTTENSIS

DI SCORBIS

ROTALIFORMIS

CORRUGATA

EXIGUUS

BECCARII

SALSUM

FR IGIDA

EX CAVATUM

EX CAVATUM

FU SCA

PACIFI CA 
Lat. Long. Locality

$4835 N 12251 \mathrm{~W}$ BRITISH COLUMBIA BRITISH COLUMBIA BRITISH COLUMBIA BR ITISH COLUMBIA BRITISH COLUMBIA BRITISH COLUMBIA BRITISH COLUMBIA BRITISH COLUMBIA BRITISH COLUMBIA BRITISH COLUMBIA

$4836 N 12229 w$ SAMISH-PADILLA BAYS SAMISH-PADILLA BAYS SAMISH-PADILLA BAYS SAMISH-PADILLA BAYS SAMISH-PADILLA BAYS SAMISH-PADILLA BAYS SAMISH-PADILLA BAYS SAMISH-PADILLA BAYS SAMISH-PADILLA BAYS SAMISH-PADILLA BAYS SAMISH-PADILLA BAYS SAMISH-PADILLA BAYS SAMISH-PADILLA BAYS

$4840 \mathrm{~N} 12331 \mathrm{~W}$ $\begin{array}{llll}48 & 40 N & 123 & 31 W \\ 48 & 43 N & 122 & 55 W\end{array}$ DIS COVERY PASSAGE, B.C. WASHINGTON COAST WASHINGTON COAST WASHINGTON COAST WA SHINGTON COAST WASHINGTON COAST WASHINGTON COAST WASHINGTON COAST WASHINGTON COAST WASHINGTON COAST WA SHINGTON COAST BR ITISH COLUMBIA BRITISH COLUMBIA BRITISH COLUMBIA BRITISH COLUMBIA BRITISH COLUMBIA BR ITISH COLUMBIA BRITISH COLUMBIA BRITISH COLUMBIA BRITISH COLUMBIA BR ITISH COLUMBIA BRITISH COLUMBIA BR ITISH COLUMBIA BRITISH COLUMBIA BRITISH COLUMBIA BRITISH COLUMBIA BRITISH COLUMBIA BRITISH COLUMBIA BRITISH COLUMBIA BR ITISH COLUMBIA BR ITISH COLUMBIA BRITISH COLUMBIA BRITISH COLUMBIA BRITISH COLUMBIA BRITISH COLUMBIA BRITISH COLUMBIA BRITISH COLUMBIA BR ITISH COLUMBIA BRITISH COLUMBIA BRITISH COLUMBIA BR ITISH COLUMBIA BRITISH COLUMBIA BRITISH COLUMBIA BRITISH COLUMBIA BRITISH COLUMBIA BRITISH COLUMBIA BRITISH COLUMBIA BRITISH COLUMBIA BRITISH COLUMBIA BR ITISH COLUMBIA BR ITISH COLUMBIA BRITISH COLUMBIA BRITISH COLUMBIA BR ITISH COLUMBIA BR ITISH COLUMBIA BRITISH COLUMBIA BRITISH COLUMBIA BR ITISH COLUMBIA BRITISH COLUMBIA BRITISH COLUMBIA BRITISH COLUMBIA BRITISH COLUMBIA BRITISH COLUMBIA BRITISH COLUMBIA BRITISH COLUMBIA BR ITISH COLUMBIA

DIS COVERY PASSAGE, B.C. DISCOVERY PASSAGE, B.C. DISCOVERY PASSAGE, B.C. DISCOVERY PASSAGE, B.C DISCOVERY PASSAGE, B. C DISCOVERY PASSAGE, B.C.

\section{Generic Name Specific Name}

HAPLOPHRAGMOI DES RECURVOIDES

REOPHAX

SACCAMMINA

SPIROPLECTAMM INA

TROCHAMMINA

TROCHAMMINA

TROCHAMMINA

TROCHAMM INA

TROCHAMM I NA

AMMONIA

AMMOT IUM

BUCCELLA

EGGERELLA

LPHIDIELLA

ELPHIOIUM

ELPHIDIUM

GLABRATELLA

SPIRILLINA

TROCHAMM I NA

TROCHAMM INA

CIBICIDES

BUCCELLA

CIBICIDES

ELPHIDIELLA

ELPHID IUM

ELPHIDIUM

GLABRATELLA

MILIAMMINA

MIL IOL INELLA

ROSALINA

ADERCOTRYMA

AMMODISCUS

A STRONONION

BOL IVINA

BOLIVINA

BUL IMINELLA

CASSI DUL INA

CIBICIDES

CIBICIDES

DENDROPHYRA

EGGERELLA

ELPHIDIELLA

ELPH I DIUM

ELPHIDIUM

ELPHIDIUM

EPISTOMINEL LA

FIS SURINA

GAUDRYINA

GAUDRYINA

GLABRATELLA

GLOBOBUL IMINA

HAPLOPHRAGMOI DES

HAPLOPHRAGMOI DES

I SLANDIELLA

ISLANDIELLA

LAGENA

LAGENA

LAGENA

LAGENA

AGENA

LAGENAMMINA

NON IONELLA

NONIONELLA

NONIONELLA

OOL INA

OOL INA

POLYMORPHINA

PSEUDOPOL YMORPHINA PULLENIA

PYRGO

RECURVOIDES

ROSAL INA

ROSALINA

SACCAMMINA

SIGMOMORPHINA

SPIROPLECTAMMINA

TRIFAR INA

TRIFARINA

IROCHAMMINA

TROCHAMMINA

TROCHAMMINA

TROCHAMMI NA

TROC HAMM INA

TROCHAMM INA

UVIGERINA

AMM ON I A

BUCCELLA

CIBICIDES

GLABRATELLA

HAPLOPHRAGMOI DES

LAGENA
PLANISSIMUM

CURTUS

ATLANTICA

BIFOR IIS

CHARLOTTENSIS

DISCORBIS

NANA

PACIFICA

SQUAMATA

EXIGUUS

BECCARII

SALSUM

FRIGIDA

ADVENA

HANNAI

EXCAVATUM

FRIGIDUM

OPERCULARIS

FUSCA

VIVIPARA

IN FLATA

PACIFICA

LOBATULUS

FR IGIDA

LOBATULUS

AD VENA

HANNA

LENE

TUMIDUM

SANJUANENSIS

FUSCA

CIRCULARIS

COLUMBIENSIS

GL OMERATA

GULLMARENSIS

GALLOWAYI

COMPACTA

PACIFICA

ELEGANTISSIMA

DEPRESSA

FLETC HER I

LOBATULUS

ARBORESCENS

AD VENA

NITIDA

EXCAVATUM

IN CERTUM

SUBARCTICUM

PACIFICA

LUCIDA

ARENARIA

SUBGLABRATA

ORNAT ISS IMA

PACIFICA

COLUMBIENSIS

PLANISSIMUM

CALIFORNICA

LIMBATA

API OPLEURA

DISTOMA

GRACILIS

PLIOCENICA

SULCATA

LONGI COLLIS

BASISPINATA

STELLA

TURGIDA DIGITATA

BOREALIS

MELO

KINCAI OI

CHARL OTTENSIS

SALIS BURYI

WI LLIAMSON

TURBI NATUS

COLUMBIENSIS

ATLAN TICA

TR ILOCULARIS

BI FORMIS

ANGULOSA

SEMITRIGONA

CHARL OTTENSIS

DISCORBIS

NANA

PACIF I CA

SQUAMATA

SQUAMI FORMIS

JUNCEA

BECCARII

FR IGIDA

LOBAT ULUS

PARISIENS IS

CANARI ENSIS

SULCATA 


\section{Lat. Long. Locality}

$4845 \mathrm{~N} 12333 \mathrm{~W}$

$4861 \mathrm{~N} 16107 \mathrm{~W}$

$4900 \mathrm{~N} 12315 \mathrm{~W}$

$4905 N 12306$

$49 \quad 15 N \quad 12325 \mathrm{~W}$

$49 \quad 15 N 12330 w$ OFF ALASKA
DISCOVERY PASSAGE, B.C. DISCOVERY PASSAGE, B.C OF F ALASKA PENINSULA OFF ALASKA PEN INSULA OF F ALASKA PENINSULA OFF ALASKA PENINSULA OFF ALASKA PENINSULA OFF ALASKA PENINSULA OF F ALASKA PENINSULA OF F ALASKA PENINSULA OF F ALASKA PENINSULA OFF ALASKA PENINSULA OFF ALASKA PENINSULA OF F ALASKA PEN OF F ALASKA PENINSULA OF F ALASKA PENINSULA OF F ALASKA PENINSULA OFF ALASKA PENINSULA OF F ALASKA PENINSULA OF $F$ ALASKA PENINSULA OF F ALASKA PENINSULA OF F ALASKA PENINSULA OFF ALASKA PENINSULA OFF ALASKA PENINSULA OFF ALASKA PENINSULA OF F ALASKA PENINSULA OFF ALASKA PENINSULA BRITISH COLUMBIA BRITISH COLUMBIA BRITISH COLUMBIA BRITISH COLUMBIA BRITISH COLUMBIA BR ITISH COLUMBIA BR ITISH COLUMBIA BRITISH COLUMBIA BR ITISH COLUMBIA BRITISH COLUMBIA BRITISH COLUMBIA BRITISH COLUMBIA BR ITISH COLUMBIA BRITISH COLUMBIA BR ITISH COLUMBIA BRITISH COLUMBIA BRITISH COLUMBIA BRITISH COLUMBIA BR ITISH COLUMBIA BR I IISH COLUMBIA BRITISH COLUMBIA BRITISH COLUMBIA BR ITISH COLUMBIA BR ITISH COLUMBIA BRITISH COLUMBIA BR ITISH COLUMBIA BR ITISH COLUMBIA BR ITISH COLUMBIA BRITISH COLUMBIA BRITISH COLUMBI BR ITISH COLUMBIA FRASER R.. B.C. FRASER R.. B. C FRASER R.. B.C. FRASER R.. B.C. FRASER R.. B.C. FRASER R.. B.C. OFF BRIT COLUMBI OFF BRIT. COLUMBIA OF F BRIT. COLUMBIA OFF BRIT. COLUMBI OF F BRIT. COLUMBIA OF F BRIT . COLUMB I A BRITISH COLUMBIA BRITISH COLUMBIA BR ITISH COLUMBIA BR ITISH COLUMBIA BRITISH COLUMBIA BRITISH COLUMBIA BRITISH COLUMBIA BRITISH COLUMBIA BRITISH COLUMBIA BRITISH COLUMBIA BR I IISH COLUMBIA BRITISH COLUMBIA BRITISH COLUMBIA BR ITISH COLUMBIA BRITISH COLUMBIA BRITISH COLUMBIA BR ITISH COLUMBIA BR ITISH COLUMBIA BR I IISH COLUMBIA BR ITISH COLUMBIA BRITISH COLUMBIA BR ITISH COLUMBIA BRITISH COLUMBIA BRITISH COLUMBIA

\section{Generic Name}

LARYNGOS IGMA QUINQUELOCULINA ADERCOTRYMA AMMOBACULITES AMMOBACUL ITES AMMOMARG INULI NA CRIBROSTOMOIDES CRIBR OSTOMOIDES CRIBROSTOMOIDES CYCLAMMINA CYCLOGYRA CYSTAMNINA DOROTHIA

EGGERELLA GLOMOSPIRA HAPLOPHRAGMOI DES HYPERAMMINA JACULELLA REOPHAX REOPHAX

REOPHAX

SACCAMMINA

SPIROPLECTAMMINA

TROCHAMMINA

TROCHAMMINA

TROCHAMMINA

TROCHAMMINA

ADERCOTRYMA

AMIMOOISCUS

BOL IVINA

BOLIVINA

CIBICIDES

CIBICIDES

DENDROPHYRA

EGGERELLA

ELPHIOIELLA

ELPHIOIUM

ELPHIOIUM

GLOBOBUL IMINA

HAPLOPHRAGMOIDES

HAPLOPHRAGMOI DES

ISLANDIELLA

LAGENA

NON IONELLA

NONI ONELL INA

OOL INA

POR OEP ONI DES

PULLENIA

RECURVOIDES

SACCAMMINA

SPIROPLECTAMMINA

TRIFARINA

TRI FAR IN A

TROCHAMMINA

TROCHAMMINA

TROCHAMM I NA

TROCHAMMINA

TR OCHAMMINA

TROCHAMMI NA

UVIGER INA

AMMOT I UM
HAPLOPHRA GMOI OES

JADAMMINA

MIL I AMMIN A

PROTOSCHISTA

TROCHAMMINA

TROCHAMMINA

ELPHIOIELLA

ELPHIDIUM

ELPHIOIUM

ELPHIOIUM

HAPLOPHRAGMOI DES

ADERCOTRYMA

AMMODISCUS

CIBICIDES

EGGERELLA

EGGERELLA

HAPLOPHRA GMOI DES

HAPL OPHR AGMOI DES

HAPLOPHRA GMOI DES

HAPLOPHRAGMOI OES

ISLANDIELLA

I SLANDIELLA

LAGENA MMI NA

NONIONELLINA

PSEUDOPOLYMOR PHINA RECURVOIDES

REOPHAX

REOPHAX

SACCAMMINA SPI ROPLECTAMM INA

TROCHAMM I NA

TROCHAMMINA

T ROCHAMM I NA

Specific Name

LACTEA

SEMINULA

GL OME RATA

AMERI CANUS

FI LIF ORMI

FOLIACEA

NI TIDUM

SUBGL OBOSUM

WI ESNER I

TRULLISSATA

IN CERTA

GALEATA

SCABRA

BRAOY I

GOROIALIS

SCITULUM

CYLINORICA

ACUTA

DENTALIN I FORMIS

DI STANS

SC ORPIURUS

A TLAN TICA

BIFORMIS

GL OBI GER IN IF ORM IS

GR ISEA

INFLATA

NITIDA

GL OMERATA

GULLMARENSIS

COMPACTA

PACIF ICA

FLETCHER I

LOBATULUS

ARBORESCENS

AD VENA

NITIOA

EXCAVATUM

IN CER TUM

AURI CULATA

COLUMBIENSIS

PLANISSIMUM

LIMBATA

OISTOMA

ST ELLA

LA BRA DORI CA

ME LO

CRI BR OREPANDUS

SALISBURYI

TURBINATUS

A T LAN TICA

BIFORMIS

AN GUL OSA

SEMITRIGONA

CHARL OTTENSIS

DI SCORBIS

NANA

PACIFICA

SQUAMATA

JUNCEA

SALSUM
SUBINVOLUTUM

POLYSTOMA

FUSCA

F I NOENS

IN FLATA

MA CRES CENS

HANNA I

FRI GI DUM

LENE

TUMIOUM

COLUMBIENSIS

GL OME RATA

GULLMARENSIS

LOBATULUS

ADVENA

EX CAVATUM

BRAOYI

COLUMBIENSIS

COLUMBIENSIS

PLANISSIMUM

LI MBATA

NORCROSSI

LONGI COLLIS

AURICULA

LABRA DORI CA

CHARL OTTENSIS

TURBINATUS

CURTUS

PILUL I FER 
Lat. Long. Locality

$4915 \mathrm{~N} 12330 \mathrm{~W}$

$4920 N 12428 W$

$4928 \mathrm{~N} 12430 \mathrm{~W}$ $4930 \mathrm{~N} 12422 \mathrm{~W}$

$4940 N 12445 \mathrm{~W}$

$4940 \mathrm{~N} 12456 \mathrm{~W}$ $4950 \mathrm{~N} 12442 \mathrm{~W}$

$4950 N 16057 \mathrm{~W}$

50 OON $12458 \mathrm{~W}$

SO $00 \mathrm{~N} 12507 \mathrm{~W}$

$5010 \mathrm{~N} 12501 \mathrm{~W}$ $5030 \mathrm{~N} 125$ OOW

$5030 \mathrm{~N} 12605 \mathrm{~W}$

$5031 \mathrm{~N} 12655 \mathrm{~W}$ $5033 \mathrm{~N} 126 \quad 40 \mathrm{~W}$ $5034 \mathrm{~N} 12659 \mathrm{~W}$

$5035 \mathrm{~N} 12657 \mathrm{~W}$

$5035 \mathrm{~N} 12800 \mathrm{~W}$
BR ITISH COLIIMBIA BR ITISH COLUIABIA BRITISH COLUMBIA

STRAIT OF GEORGIA, B. C. STRAIT OF GEORGIA, B. C STRAIT OF GEORGIA B $C$ STRAIT OF GEORGIA, B.C. STRAIT OF GEORGIA, B.C. STRAIT OF GEORGIA, B.C. STRAIT OF GEORGIA, B. C.
STRAIT OF GEORGIA, B. BRITISH COLUMBIA BRITISH COLUMBIA BRITISH COLUMBIA BRITISH COLUMBIA BR ITISH COLUMBIA BRIT ISH COLUMBIA BRITISH COLUMBIA BRITISH COLUMBIA BRITISH COLUMBIA BRITISH COLUMBIA BRITISH COLUMBIA BRITISH COLUMBIA BRITISH COLUMBIA BR ITISH COLUMBIA BR ITISH COLUMBIA

COMOX HARBOUR, B.C.

STRAIT OF GEORGIA, B.C. STRAIT OF GEORGIA, B. C. STRAIT OF GEORGIA, B. C. STRAIT OF GEORGIA, B. C.
STRAIT OF GEORGIA, B. $C$. STRAIT OF GEORGIA, B. C.
STRAIT OF GEORGIA, B. C. STRAIT OF GEORGIA, B.C. OFF ALASKA PENINSULA OF $F$ ALASKA PENINSULA OF F ALASKA PENINSULA OFF ALASKA PENINSULA OF F ALASKA PENINSULA OFF ALASKA PENINSULA OFF ALASKA PENINSULA OF F ALASKA PENINSULA
OF F ALASKA PENINSULA OFF ALASKA PENINSULA OF $F$ ALASKA PENINSULA OF F ALASKA PENINSULA OF F ALASKA PEN INSULA OF F ALASKA PENINSULA OFF ALASKA PENINSULA OF F ALASKA PENINSULA OFF ALASKA PENINSULA OF F ALASKA PENINSULA OF F ALASKA PENINSULA OFF ALASKA PENINSULA OF F ALASKA PENINSULA OF F ALASKA PENINSULA OFF ALASKA PENINSULA OFF ALASKA PENINSULA OFF ALASKA PENINSULA INSULA SULA OFF ALASKA PENINSULA OFF ALASKA PENINSULA OF F ALASKA PENINSULA OF F ALASKA PENINSULA OF F ALASKA PENINSULA OFF ALASKA PENINSULA OFF ALASKA PENINSULA OFF ALASKA PENINSULA OFF ALASKA PENINSULA STF ALASKA PENINSULA STRAIT OF GEORGIA, B. C.
STRAIT OF GEORGIA, B.C. BRITISH COLUMBIA BRITISH COLUMBIA BR ITISH COLUMBIA BR ITISH COLUMBIA BR ITISH COLUMBIA BRITISH COLUMBIA BRITISH COLUMBIA BRITISH COLUMBIA BRITISH COLUMBIA BRITISH COLUMBIA BRITISH COLUMBIA BRITISH COLUMBIA

STRAIT OF GEORGIA, B.C. PHILLIPS ARM, B.C. PHILLIPS ARM, B.C. JOHNSTONE STRAIT, B.C. JOHNSTONE STRAIT, B.C. JOHNSTONE STRAIT, B.C. JOHNSTONE STRAIT, B.C.
JOHNSTONE STRAIT, B.C. JOHNSTONE STRAIT, B.C.
JOHNSTONE STRAIT, B.C. JOHNSTONE STRAIT, B.C QUEEN CHARLOTTE SD. B.C. QUEEN CHARLOTTE SD., B.C QUEEN CHARLOTTE SD.. B.C. QUEEN CHARLOTTE SD.. B.C QUEEN CHARLOTTE SD.. B.C. QUEEN CHARLOTTE SD.. QUATSINO SOUND. B.C.
Generic Name Specific Name

Publication

TROCHARMINA

ROCHAMMINA PACIFICA

UVIGERINA

CIBICIDES

HAPLOPHRAGMOI DES

CIBICIDES

CIBICI DES

DENTAL INA

QUINQUELOCULINA

TROCHAMMINA

ADERCOTRYMA

EGGERELLA

HAPLOPHRAGMOI DES

HAPLOPHRAGMOI DES

SQUAMAT

JUNCEA

LOBAT ULUS

CANAL I CULATA

CANARIENSIS

LOEATULUS

LOBATULUS

PAUPERATA

SEMINULA

SQUAMATA

GL CMERAT A

ADVENA

BRADY

COLUMBIENSIS

COLUMBIENSIS

HAPLOPHRA GMOI DES

HAPLOPHRAGMOI DES PLANISSIMUM

RECURVOIDES

REOPHAX

SACCAMMINA

SPI ROPLECTAMM INA

TROCHAMM INA

TROCHAMMINA

TROCHAMMINA

TROCHAMMINA

ELPHIDIUM

CIBICIDES

FRONDI CULAR IA

LEN TI C UL INA

PSEUDOPOL YMORPHINA

RHABDAMMINA

A DER O OTRYMA

AMMOBACULITES

AMMOBACULITES

AMMODISCUS

CIBICIDES

CIBICIDOIDES

CRIBROSTOMOIDES

CRIBROSTOMOIDES

CYCLAMMINA

CYCLAMMINA

DOROTHIA

EGGERELLA

EHRENBER G INA

EP ISTOMINELLA

EPISTOMINEL LA

GLOBOTEXTUL AR IA

GLOMOSPIRA

GYROIDINA

HAPLOPHRAGMOI DES

HOE GLUNDI NA

HORMOS INA

HYPERAMMINA

HYPERAMMINA

JACULELLA

MELONIS

MIL IOL INELLA

PLACOPSILINA

REOPHAX

SACCAMMINA

SPI ROPLE C TAMM INA

TROCHAMMINA

TROCH AMM I NA

TROCHAMMINA

CIBICI DES

RHABDAMMINA

BOL IVINA

BOLIVINA

CIBICIDES

CIBICIDES

ELPHIDIELLA

ELPHIDIUM

HAPL OPHRAGMOI DES

HAPLOPHRAGMOI DES

I SLAND IELLA

OOLINA

PULLENIA

TROCHAMMINA

CIBICIDES

MIL I AMMIN A

TROCHAMM INA

BUCCELLA

CIBICIDES

CIBICIDES

ELPHIDIUM
FLORILUS

CIBICIDES

CIBICIDES

CIBICIDES

AMMONIA

CIBICIDES

ELPHI DIUM

GLABRATELLA

C IBICIDES

TURBINATUS

CURTUS

A TLANTIC

BI FORMIS

CHARL OTTENSIS

DISCORBIS

PACIFICA

SQUAMATA

STRIA TOPUNCTATA

LOBATULUS

PAUPERATA

CA NAL I CUL ATA

IO TA

LI GUA

AB YSS ORUM

GL OMERATA

AG GLUT INANS

FI LIFORMIS

TENUIS

BRADY

MUNDULUS

NITIDUM

SUBGL OBOSUM

CANCELLATA

TRULL IS SATA

EXILIS

BRADYI

HY STR IX

EXIGUA

UMBONI FERA

ANCEPS

GORDIALIS

LAMAR CKIANA

RINGENS

ELEGANS

GLOBUL IFERA

CYLINDRICA

FR IAB ILIS

ACUTA

BA RLEEANUS

SUBROTUNDA

BRADYI

EX CENTRICUS

A T LAN TICA

GL OBIGER INIFORMIS

NANA

NI TID

LOBAT ULUS

ABYSS ORUM

COMPACTA

PACIFICA

FLETCHERI

LOBATULUS

NITIDA

SUBARCTICUM

COLUMBIENSIS

PLANISSIMUM

LIMBATA

ME LO

SALISBURY I

PACIFICA

LOBATULUS 


\section{Lat. Long. \\ $5035 \mathrm{~N} 12800 \mathrm{~W}$}

$5037 \mathrm{~N} 12801 \mathrm{~W}$

$5040 \mathrm{~N} 12636 \mathrm{~W}$ $5050 \mathrm{~N} 12652 \mathrm{~W}$ $5050 N 12655 \mathrm{~W}$

$5055 \mathrm{~N} 12800 \mathrm{~W}$

$5105 N 12736$ $\begin{array}{lllll}51 & 23 N & 130 & 34 \mathrm{~W} \\ 52 & 20 \mathrm{~N} & 131 & 00 \mathrm{~W}\end{array}$
Locality

QUATSINO SOUND, 8.8. QUATSINO SOUND, B. $C$. QUATSINO SOUND, B.C. QUATSINO SOUND, $8 . C$. QUATSINO SOUND, B.C. QUATSINO SOUND, B.C. QUATSINO SOUND, B.C.

QUEEN CHARLOTTE SD., B.C. QUEEN CHARLOTTE SD.. B.C. QUEEN CHARLOTTE SD., B.C. QUEEN CHARLOTTE SD.. QUEEN CHARLOTTE SD., B.C. QUEEN CHARLOTTE SD.. B.C. QUEEN CHARLOTTE SD., B.C. QUEEN CHARLOTTE SD.. B.C. QUEEN CHARLOTTE SD., B.C. QUEEN CHARLOTTE SD., B.C. QUEEN CHARLOTTE SD., B.C. QUEEN CHARLOTTE SD.. B.C. QUEEN CHARLOTTE SD. QUEEN CHARLOTTE SD., B.C. OFF BRIT. COLUMBIA

Q. CHARLOTTE SD.. B.C.

Q. CHARLOTTE SD.. B.C.

Q. CHARLOTTE SD.. B.C.

Q. CHARLOTTE SD., B.C.

Q. CHARLOTTE SO., B.C.

Q. CHARLOTTE SD.. B.C.

Q. CHARLOTTE SD., B.C.

Q. CHARLOTTE SD.. B.C.

Q. CHARLOTTE SD., B.C.

Q. CHARLOTTE SD., B.C.

Q. CHARLOTTE SD.. B.C.

Q. CHARLOTTE SD.. B.C.

Q. CHARLOTTE SD.. B.C.

Q. CHARLOTTE SD., B.C

Q. CHARLOTTE SD., B.C.

Q. CHARLOTTE SD., B.C.

Q. CHARLOTTE SD., B.C.

Q. CHARLOTTE SD.. B.C.

OFF ALASKA PENINSULA

OFF ALASKA PEN INSULA

OFF ALASKA PEN INSULA

OFF ALASKA PENINSULA

OFF ALASKA PENINSULA

OF F ALASKA PENINSULA

OF $F$ ALASKA PENINSULA

OFF ALASKA PENINSULA

OFF ALASKA PENINSULA

OFF ALASKA PENINSULA

OFF ALASKA PENINSULA

OFF ALASKA PENINSULA

OFF ALASKA PENINSULA

OF $F$ ALASKA PENINSULA

OFF ALASKA PENINSULA

OFF ALASKA PENINSULA

OFF ALASKA PENINSULA

OFF ALASKA PENINSULA

OFF ALASKA PENINSULA

OFF ALASKA PEN INS SULA

OFF ALASKA PENINSULA

OFF ALASKA PENINSULA

OFF ALASKA PEN INSULA

OFF ALASKA PENINSULA

OFF ALASKA PENINSULA OFF ALASKA PENINSULA OFF ALASKA PENINSULA OFF ALASKA PENINSULA OFF ALASKA PENINSULA OFF ALASKA PENINSULA OFF ALASKA PENINSULA OFF ALASKA PENINSULA OFF ALASKA PENINSULA OFF ALASKA PENINSULA OFF ALASKA PENINSULA OFF ALASKA PENINSULA OFF ALASKA PEN INSULA OFF ALASKA PEN INSULA OFF ALASKA PENINSULA OFF ALASKA PENINSULA OFF ALASKA PENINSULA OFF ALASKA PENINSULA OFF ALASKA PENINSULA OFF ALASKA PENINSULA OFF ALASKA PENINSULA OFF ALASKA PENINSULA OFF ALASKA PENINSULA OFF ALASKA PENINSULA OFF ALASKA PEN INSULA OFF ALASKA PENINSULA OFF ALASKA PENINSULA
OFF ALASKA PENINSULA OFF ALASKA PENINSULA OFF ALASKA PENINSULA OFF ALASKA PENINSULA

\section{Generic Name Specific Name}

Publication

ELPHIDIUM
TROCHAMMINA
AMMONIA
CIBICIDES
HAPLOPHRAGMOIDES
PSEUDOPOLYMORPHINA
RHABDAMMINA
CIBICIDES
CIBICIDES
CIBICIDES
PSEUDOPOLYMORPHINA
CIBICIDES

R ISPUM

SQUAMATA

BECCARII

LOBATULUS

LI GUA

AB YSSORUM

LOBATULUS

LOBATULUS

CIBICIDES LOBATULUS

ELPHIDIUM CRISPUM

FLORILUS

SCAPHUM

PARISIENSIS

PSEUDOPOLYMORPHINA LIGUA

QUINQUEL OCULINA FERUSSACHII

QUINQUELOCULINA

RHABDAMMINA

C IBICIDES

CYCLAMMINA

ANGULODIS CORBIS

CASSIDULINA

CYCLOGYRA

EPONIDES

EPONIDES

GLABRATELLA

HAPLOPHRAGMOIDES

HAPLOPHRAGMOI DES

I SLAND IELLA

I SLAND IELLA

NONIONELLA

PSEUDOPOL YMORPHINA

ROBERT INOIDES

ROSAL INA

SEJUNCTELLA

TROCHAMMINA

TROCHAMMINA

A DER COTRYMA

AMMOBACUL ITES

AMMOMARG INULI NA

CASSIDUL INA

CIBICIDES

CRIBROSTOMOIDES

CRIBROSTOMOIDES

CRIBROSTOMOIDES

CRIBROSTOMOIDES

CYSTAMMINA

EGGERELLA

GLOMOSPIRA

HAPLOPHRAGMOIDES

HAPLOPHRA GMOI DES

HYPERAMM INA

JACULELLA

MIL IOL INELLA

NODELLUM

PULLENIA

REOPHAX

REOPHAX

RHABDAMMINA

SAC CAMMINA

TROCHAMM INA

TROCHAMMINA

ADERC OTRYMA

AMMOBACULITES

AMMOBA CUL ITES

AMMODISCUS

CRIBROSTOMOIDES

CRIBROSTOMO IDES

J ACUL ELLA

M I L I O L INELLA

PULLENIA

REOPHAX

REOPHAX

REOPHAX

SACCAMMINA

BUL IM I NA

CRIBROSTOMOIDES

EGGERELLA

ELPHIDIELLA

ELPHI DIUM

FURSENKOINA

GL 0808 UL I MI NA

GLOBOBUL I MINA

HOE GLUNDINA

HOE GLUNDINA

NONIONELL INA

NONIONELL INA

TROCHAMMINA

TROCHAMMINA

ASTACOLUS
SEMINULA

ABYSSORUM

LOBATULUS

CANCELLATA

CHARL OTTENSIS

PULCHELLA

COLUMBIENSIS

REPANDUS

ORBIGNYANA

ORNAT ISS IMA

AD VENUM

COLUMBIENSIS

CALIFORNICA

TORTU OSA

AURIS

CHARL OTTENSIS

CHARLOTTENSIS

COLUMBIENSIS

SPINIGERA REDUCTA

CHARL OTTENSIS

PACIFICA

GL OMERATA

F I L IF ORM I S

OLIACEA

CRASSA

BRADYI

NITIDUM

NITIDUM

SUBGL OBOSUM

WIESNERI

GALEATA

GORDIALIS

CANARIENSIS

RI NGENS

FR IAB I LIS

AC UTA

SUBROTUNDA

MEMBR A NA CEUM

QUINQUELOBA

EXCENTRI CU

PILUL IFER

LINEARIS

ATLANTICA

GL OBI GER INIFORMIS

GR I SE A

GL OME RAT A

AMERI CANUS

FILIFORM IS

TENUIS

NITIDUM

SUBGL OBOSUM

ACUTA

SUBROTUNDA

QU INQUELOBA

DENTALIN I FORMIS

NODULOSUS

SCOTTII

A TLANTICA

GL OBI GER INI FORMIS

INFLATA MEXI CANA

SUBGL OBO SUM

BR A DY I

GR OENLAND I CA

IN CERT UM

PAUCILOCULATA

AURICULATA

PACIFICA

ELEGANS

TURGIDA

LA BRA DORICA

GL OBI GER IN I FORM IS

GR I SEA

PEREGR INA

LATUS
WHITEAVES 1886 WHITEAVES 1886 WHITEAVES 1886 WHITEAVES 1886 WHITEAVES 1886 WHITEAVES 1886 WHITEAVES 1886 WHITEAVES 1886 WHITEAVES 1886 WHITEAVES 1886 


\section{Lat. Long. Locality}

$5410 N 13230 \mathrm{~W}$

$5451 N 15524 \mathrm{~W}$

$5455 \mathrm{~N} 15759 \mathrm{~W}$

$5521 \mathrm{~N} 13139 \mathrm{~W}$

$5521 N 13158 \mathrm{~W}$

$5526 \mathrm{~N} 13214 \mathrm{~W}$
VIRAGO SD., B.C.

VIRAGO SD.. B.C.

VIRAGO SD., B.C.

VIRAGO SD., B. C.

VIRAGO SD.. B.C.

VIRAGO SD.. B.C.

VIRAGO SD.. B.C.

VIRAGO SD., B.C.

VIRAGO SD.. B.C.

VIRAGO SD.. B.C.

OFF ALASKA PENINSULA

OFF ALASKA PENINSULA

OF $F$ ALASKA PENINSULA

OF $F$ ALASKA PENINSULA

OF F ALASKA PENINSULA

OF F ALASKA PENINSULA

OF $F$ ALASKA PENINSULA

OFF ALASKA PENINSULA

OF F ALASKA PENINSULA OF $F$ ALASKA PENINSULA OFF ALASKA PENINSULA OF $F$ ALASKA PENINSULA OF $F$ ALASKA PENINSULA OF F ALASKA PENINSULA OF $F$ ALASKA PENINSULA OF F ALASKA PENINSULA OF $F$ ALASKA PENINSULA OF $F$ ALASKA PENINS ULA OF F ALASKA PENINSULA OF $F$ ALASKA PENINSULA OF $F$ ALASKA PENINSULA OF $F$ ALASKA PENINSULA OF $F$ ALASKA PENINSULA OF F ALASKA PENINSULA OFF ALASKA PENINSULA KETCHIKAN, ALASKA KETCHIKAN, ALASKA KE TCHIKAN, ALASKA KETCHIKAN, ALASKA KETCHIKAN, ALASKA KETCHIKAN, ALASKA KETCHIKAN, ALASKA KE TCHIKAN, ALASKA KETCHIKAN, ALASKA KE TCHIKAN, ALASKA KE TCHIKAN, ALASKA KETCHIKAN, ALASKA KETCHIKAN, ALASKA KETCHIKAN, ALASKA KETCHIKAN, ALASKA
KETCHIKAN, ALASKA KETCHIKAN, ALASKA
KETCHIKAN, ALASKA KETCHIKAN, ALASKA KETCHIKAN, ALASKA KETCHIKAN, ALASKA KETCHIKAN, ALASKA KETCHIKAN, ALASKA KETCHIKAN, ALASKA KETCHIKAN, ALASKA
KETCHIKAN, ALASKA
KETCHIKAN, ALASKA CLARENCE ST., ALASKA CLARENCE ST.. ALASKA CLARENCE ST.. ALASKA CLARENCE ST.. ALASKA CLARENCE ST.. ALASKA CLARENCE ST.. ALASKA CLARENCE ST. ALASKA CLARENCE ST., ALASKA CLARENCE ST.. ALASKA CLARENCE ST_. ALASKA CLARENCE ST., ALASKA CLARENCE ST.. ALASKA CLARENCE ST ALASKA CLARENCE ST ALASKA CLARENCE ST ALASKA CLARENCE ST.O ALASKA CLARENCE ST., ALASKA CLARENCE ST.. ALASKA CLARENCE ST.. ALASKA CLARENCE ST.. ALASKA CLARENCE ST.. ALASKA CLARENCE ST.. ALASKA CLARENCE ST.. ALASKA CLARENCE ST.. ALASKA CLARENCE ST ALASKA CLARENCE ST ALASKA CLARENCE ST.. ALASKA CLARENCE ST.. ALASKA CLARENCE ST. ALASKA CLARENCE ST.. ALASKA KASAAN BAY, ALASKA KASAAN BAY, ALASKA KA SAAN BAY, ALASKA KASAAN BAY, ALASKA KASAAN BAY, ALASKA KASAAN BAY, ALASKA KASAAN BAY, ALASKA KASAAN BAY, ALASKA
Generic Name Specific Name

ASTRONONION

BUL I M INE LLA

CASSI DUL INA

CYCLOGYRA

EPONIDES

EPONIDES

GLABRATELLA

HAPLOPHRAGMOI DES

NONIONELLA

TROCHAMMINA

AMMOMARG I NULI INA

CASSI DULINA

CRIBROSTOMOIDES

CRIBR OSTOMOIDES

CYCLAMMINA

CYSTAMMINA

EGGERELLA

SACCAMMINA

SPIR OPLECTAMM INA

TROCHAMMINA

BOLIVINA

CASSI DULINA

CIBICIDES

CIBICIDES

ELPHIDIUM

ELPHIDIUM

EPISTOMINELLA

FLORI LUS

GAUDRYINA

I SLANDIELLA

I SLANDIELLA

PSEUDOPOLYMORPHINA

REC TOBOLIVINA

TRIFARINA

UVI GERINA

AMMOTIUM

BOLIVINA

BOLIVINA

BUL IMINELLA

CRIBR OSTOMOIDES

EGGERELLA

ELPHIDIELLA

ELPHIDIUM

ELPHIDIUM

ELPHIDIUM

HAPLOPHRAGMOI DES

LAGENA

LAGENA

LAGENA

NONI ONELLA

ROBERTINOIDES

SACCAMMINA

SAC CAMMINA

TRIFARINA

TRIFAR INA

TROCHAMMINA

TROCHAMMINA

TROCHAMMINA

UVIGERINA

BOL IV INA

BOL IVINA

BOL IVINA

BUCCELLA

BUCCELLA

CASS I DUL INA

CIBICI DES

ELPHIDIUM

ELPHIDIUM

EPISTONINELLA

F IS SUR INA

FURSENKOINA

GLOBOBUL I MINA

HAPLOPHRAGMOIDES

HAPLOPHRAGMOIDES

HAPLOP

LAGENA

LAGENA

NONIONELLA

NONIONELLA

NONI ONELLA

NONIONELLINA

PYRGO

PYRGO

QUINQUEL OCULINA

QUINQUELOCULINA

QUINQUEL OCULINA

UVI GER INA

AMMODISCUS

BOL IVINA

BOL I VINA

BUCCELLA

CIBICIDES

CRIBROSTOMOIDES

CYCLOGYRA

CYCLOGYRA

VI RAGOENSIS

ELEGANTIS SIMA

PULCHELLA

IN VOL VENS

COLUMBIENSIS

REPAN DUS

ORNAT ISS IMA

ADVENUM

AURIS

PACIFICA

FOLIACEA

SU BGL OBOSA

NI TIDUM

SUBGL OBOSUM

TR ULL ISSATA

GALEA TA

BRADYI

A TLAN TICA

BI FORMIS

GLOBI GERINI FORMIS

DE CUSSATA

CRASSA

LOBATULUS

RE FUL GENS

BARTLEITI

CRISPUM

EXIGUA

SCAPHUM

A T LAN T I C A

HELENAE

TORTUOSA

LI GUA

AM YGD ALI FORMIS

ANGUL OSA

JUNCEA

CASSIS

PACIFICA

PAULA

ELEGANTIS SIMA

JEFFREYSII

AD VENA

HANNA

ARTICULATUM

FR IGIDUM

LENE

COLUMBIENSIS

AP I OPLEURA

PLIOCENICA

SPICATA

STELLA

CHARLOTTENSIS

HANCOCKI

LAGENARI A

ANGULOSA

FLUENS

DI SCORBIS

PACIFICA

ROTALI FORMIS

SENTI COSA

ALATA

DECUSSATA

SUBAENAR IENSIS

FRIGIDA

SUBFUSIF ORMIS

BARBARA

LOBATULUS

EXCAVATUM

FRIGIDUM

PACIF I C

LUCIDA

FUSIFORMIS

AURICULATA

BRADY I

SC I TULUM

AMPHORA

ELONGATA

ELONGATA

STELLA
TURGI DA

TURGI DA DIGITATA

LABRADORICA

DE PRESSA

ROTALARIA

AKNER I ANA

SEMINULA

ST ALKERI

PEREGRINA

ARENACEUS

DE CUSSATA 
Lat. Long.
$5526 \mathrm{~N} 132 \quad 14 \mathrm{~W}$

$5526 \mathrm{~N} 13214 \mathrm{~W}$

$56 \quad 23 N \quad 15427 \mathrm{~W}$

$5625 \mathrm{~N} 15245 \mathrm{~W}$

$5628 \mathrm{~N} 13223 \mathrm{~W}$

$5630 \mathrm{~N} 15240 \mathrm{~W}$

Locality

KASAAN BAY, ALASKA KASAAN BAY, ALASKA KASAAN BAY, ALASKA KASAAN BAY, ALASKA KASAAN BAY, ALASKA KASAAN BAY, ALASKA KASAAN BAY, ALASKA KA SAAN BAY, ALASKA KASAAN BAY, ALASKA KASAAN BAY ALASKA KA SAAN BAY, ALASKA KA SAAN BAY, ALASKA KASAAN BAY, ALASKA KASAAN BAY, ALASKA KASAAN BAY, ALASKA KASAAN BAY, ALASKA KASAAN BAY, ALASKA KASAAN BAY ALASKA KASAAN BAY ALASKA KA SAAN BAY O ALASKA KASAAN BAY, ALASKA KASAAN BAY, ALASKA KASAAN BAY, ALASKA KASAAN BAY, ALASKA KASAAN BAY, ALASKA KASAAN BAY, ALASKA KASAAN BAY, ALASKA KASAAN BAY ALASKA KASAAN BAY ALASKA KA SAAN BAY, ALASKA KASAAN BAY, ALASKA KASAAN BAY, ALASKA KA SAAN BAY, ALASKA KASAAN BAY, ALASKA KA SAAN BAY, ALASKA KASAAN BAY ALASKA KASAAN BAY OLASKA KASAAN BAY ALASKA KASAAN BAY, ALASKA KASAAN BAY, ALASKA OFF ALASKA PENINSULA OF F ALASKA PENINSULA OF F ALASKA PENINSULA OF $F$ ALASKA PENINSULA OF $F$ ALASKA PENINSULA OF $F$ ALASKA OF F ALASKA PENINSULA GULF OF ALASKA GULF OF ALASKA GULF OF ALASKA GULF OF ALASKA GULF OF ALASKA GULF OF ALASKA GULF OF ALASKA GULF OF ALASKA GULF OF ALASKA GULF OF ALASKA
GULF OF ALASKA GULF OF ALASKA GULF OF ALASKA GULF OF ALASKA GULF OF ALASKA GULF OF ALASKA GULF OF ALASKA GULF OF ALASKA GULF OF ALASKA GULF OF ALASKA
GULF OF ALASKA GULF OF ALASKA OFF ALASKA OF F ALASKA OF F ALASKA OF F ALASKA OF ALASKA OFF ALASKA OF $F$ ALASKA OF F ALASKA OF F ALASKA OF F ALASKA OF F ALASKA OF F ALASKA OFF ALASKA OF F ALASKA OF F ALASKA OF F ALASKA OF F ALASKA OF F ALASKA OF F ALASKA OF F ALASKA OF F ALASKA OF F ALASKA OF $F$ ALASKA OF F ALASKA OF ALASKA OF F ALASKA
OF F ALASKA GULF OF ALASKA GULF OF ALASKA GULF OF ALASKA GULF OF ALASKA
Generic Name

Specific Name

Publication

DYOCIBICIDES
EGGERELLA
ELPHIDIELLA
ELPHIDIELLA
ELPHIDIUM
ELPHIDIUM
ELPHIDIUM
EPISIONINELLA
FRONDI CULARIA
GAUDRYINA
GLABRATELLA
GLOBOBULIMINA
HAPLOPHRAGMOI DES
ISLANDIELLA
ISLANDIELLA
ISLANDIELLA
ISLANDIELLA
KARRERIELLA
LAGENA
LAGENA
LAGENA
LAGENA
LENTICULINA
LENTICULINA
NONIONELLA
NONIONELLA
NONIONELLINA
PELOSINA
PSEUDOPOLYMORPHINA
PYRGO

ISER I AL IS

ADVENA

ARCTICA

EX CAVATUM

FR IGI DUM

OREGONENSE

VI TREA

GI GAS

ARENARIA

ORNAT ISS IMA

AURICULATA

BRADYI

CALIFORNICA

HE LENAE

NORCR OSS I

TORTUOSA

BACCATA

AP IOPLEURA

DISTOMA

MERID IONALIS

STRIATA

NI COBARENSIS

STRONG I

STELLA

LABRADORICA

VARIABILIS

PSEUDOPOL YMORPHINA CHARLOTTENSIS

PYRGO

QUINQUELOCULINA

QUINQUELOCULINA

RECURVOIDES

REOPHAX

TR I FAR INA

TROCHAMM INA

TROCHAMM INA

UVIGER INA

BOL IVINA

BUL IM INELLA

DOROTHIA

ELPHIDIUM

EP I STOMINELLA

FUR SENKOIAA

NON IONELLA

NONIONELLA

ADERCOTRYMA

ASTACOLUS

CIBICIDES

CIBICIDES

CRIBROSTOMOIDES

GAVELINOPSIS

GLABRA TELLA

I SLANDIELLA

I SLANDIELLA

I SLANDIELLA

KARRER IELLA

LAGENA

NONIONELLA

OOL INA

OOL INA

PULLENIA

PYRGO

QUINQUEL OCULINA

TROCHAMM INA

UVI GER INA

BOL IV INA

BUL IM INELLA

DENTALINA

ELPHIDIELLA

ELPHIDIUM

ELPHIDIUM

GAUDRYINA

LAGENA

LAGENA

LAGENA

LAGENA

LAGENA

LAGENA

LAGENA

NODOSARIA

NON IONELLA

NONIONELLA

NONIONELLA

NON IONELLA

OOL INA

ROBERT INOIDES

TRIFARINA

TRIFARINA

TROCHAMMINA

ASTRONONION

BUCCELLA

C IBICI DES

LU CERNULA

AK NER I ANA

STALKERI

CONTORTUS

SC ORP I URUS

FLUENS

AD VEN A

ROTALI FORMIS

PEREGR INA

PSEUDOPL I CATA

BASICOSTATA

SC ABRA

MA GEL LAN I CUM

EXIGUA

LOEBL I CH I

AURICULA

BRADYI

GL OMERATA

HYALA CRULUS

FLETCHER I

LOBATULUS

JEFFREYSI

PRAEGERI

ORNAT ISSIMA

CALIFORNICA

LI MBATA

TORTUOSA

BACCATA

STRIATA

AURICULA

BOREALIS

HEXAGONA

SALISBURYI

MURRH INA

$S P$.

SQUAMATA

JUNCEA

PACIFICA

ELEGANTISSIMA

CALOMORPHA

HANNA I

ARTI CULATUM

ARTIC

LENE
ARENARIA

APIOPLEURA

DISTOMA

PARRI

PERLUCIDA

SPICATA

STRIATA

SULCATA

SUBSCALARIS

BASISPINATA

GRATE LOUP I

MI OCENICA

STELLA

BOREALIS

STRIATOPUNCTATA

CHARL OTTENSIS

ANGUL OSA

FLUENS

PACIFICA

GALLOWAYI

FR IGIDA

FLETCHER I

TODD LOW 1967

TODD LOW 1967

TODD LOW 1967

TODD LOW 1967

TODD LOW 1967

TODD LOW 1967

TODD LOW 1967

TODD LOW 1967

TODD LOW 1967

TODD LOW 1967

TODD LOW 1967

TODD LOW 1967

TODD LOW 1967

TODD LOW 1967

TODD LOW 1967

TODD LOW 1967

TODD LOW 1967

TODD LOW 1967

TODD LOW 1967

TODD LOW 1967

TODD LOW 1967

TODD LOW 1967

TODD LOW 1967

TODD LOW 1967 


\section{Lat. Long. Locality}

$5630 \mathrm{~N} 15240 \mathrm{~W}$

$5635 \mathrm{~N} 15150 \mathrm{~W}$

$5635 \mathrm{~N} 15200 \mathrm{~W}$

$5640 \mathrm{~N} 15155 \mathrm{~W}$

$5720 \mathrm{~N} 13402 \mathrm{~W}$

57 $49 \mathrm{~N} 15230 \mathrm{~W}$
GULF OF. ALASKA GULF OF ALASKA GULF OF ALASKA GULF OF ALASKA GULF OF ALASKA GULF OF ALASKA GULF OF ALASKA GULF OF ALASKA GULF OF ALASKA GULF OF ALASKA GULF OF ALASKA GULF OF ALASKA GULF OF ALASKA GULF OF ALASKA GULF OF ALASKA GULF OF ALASKA GULF OF ALASKA GULF OF ALASKA GULF OF ALASKA GULF OF ALASKA GULF OF ALASKA GULF OF ALASKA GULF OF ALASKA GULF OF ALASKA GULF OF ALASKA GULF OF ALASKA. GULF OF ALASKA GULF OF ALASKA GULF OF ALASKA GULF OF ALASKA GULF OF ALASKA GULF OF ALASKA GULF OF ALASKA GULF OF ALASKA GULF OF ALASKA GULF OF ALASKA GULF OF ALASKA GULF OF ALASKA GULF OF ALASKA GULF OF ALASKA GULF OF ALASKA GULF OF ALASKA GULF OF ALASKA GULF OF ALASKA GULF OF ALASKA GULF OF ALASKA GULF OF ALASKA GULF OF ALASKA GULF OF ALASKA GULF OF ALASKA GULF OF ALASKA GULF OF ALASKA GULF OF ALASKA GULF OF ALASKA GULF OF ALASKA GULF OF ALASKA GULF OF ALASKA GULF OF ALASKA GULF OF ALASKA GULF OF ALASKA GULF OF AL ASKA GAMBIER BAY. ALASKA GAMBIER BAY, ALASKA GAMBIER BAY, ALASKA GAMBIER BAY, ALASKA GAMBIER BAY, ALASKA GAMBIER BAY, ALASKA GAMBIER BAY, ALASKA
GAMBIER BAY, ALASKA GAMBIER BAY, ALASKA GAMBIER BAY, ALASKA GAMBIER BAY, ALASKA GAMBIER BAY, ALASKA GAMBIER BAY, ALASKA GAMBIER BAY, ALASKA GAMBIER BAY, ALASKA GAMBIER BAY, ALASKA GAMBIER BAY, ALASKA GAMBIER BAY, ALASKA GAMBIER BAY, ALASKA GAMBIER BAY ALASKA GAMBIER BAY ALASKA GAMBIER BAY, ALASKA GAMBIER BAY, ALASKA GAMBIER BAY, ALASKA GAMBIER BAY, ALASKA GAMBIER BAY, ALASKA GAMBIER BAY. ALASKA GAMBIER BAY. ALASKA GAMBIER BAY, ALASKA KODIAK, ALASKA KODIAK, ALASKA KODIAK, ALASKA KODIAK。 ALASKA KODIAK, ALASKA
Generic Name Specific Name

Publication

EPONIDES

GAUDRYINA

GLABRATELLA

HAPL OPHRAGMOI DES

ISLANDIELLA

ISLANDIELLA

ISLANDIELLA

LAGENA

MILIOL INELLA

NONIONELLA

NONIONELLA

PATELLINA

QUINQUEL OCULINA

QUINQUEL OCULINA

QUINQUELOCULINA

ROBERT INOIDES

TRIFARINA

TROCHAMMINA

TROCHAMMINA

BUCCELLA

BUCCELLA

CIBICIDES

ELPHIDIUM

ELPHIDIUM

EPISTOMINELLA

FISSURINA

ISLANDIELLA

ISLANDIELLA

LAGENA

NONIONELLLA

NONI ONELLLA

OOLINA

TRIFAR INA

UVIGERINA

UVIGERINA

CIBICIDES

CIBICIDES

CRIBROSTOMOIDES

GAVELINOPSIS

ISLANDIELLLA

ISLANDIELLA

CIBICIDES

CIBICIDES

DYOCIBICIDES

ELPHIDIUM

EPONIDES

FISSURINA

GUTTULINA

ISLANDIELLA

ISLANDIELLA

ISLANDIELLLA

KARRER IELLA

LAGENA

LAGENA

OOLINA
POLYMORPHINA

POLYMORPHINA

PULLENIA

REOPHAX

SIGMOMORPHINA

AMMODISCUS

ASTRONONION

BOL IVINA

BUCCELLA

CIBICIDES

CRIBROSTOMOIDES

CRIBROSTOMOIDES

DYOCIBICIDES

EGGERELLA

ELPHIDIELLA

ELPHIDIUM

GLABR A TELL LA

ISLANDIELLA

I SLAND IELLA

MILIOLINELLA

NONIONELLA

NONIONELL

QUINQUEL OCULINA

QUINQUELOCULINA

QUINQUEL OCULI NA

RECURVOIDES

REOPHAX

ROBERTINA

ROBERTINA

TRIFARINA

TRILOCUL INA

IROCHAMMINA

TROCHAMMINA

BUCCELLA

BUCCELLA

BUL IMINELLA

CRIBROSTOMOIDES

DOROTHIA

REPANDUS

ORNAT ISSIMA

ORNATISSIMA

SCITULUM

CALIFORN I CA

LIMBATA

TORTUOSA

SUAROTUNDA

AURICULA

STELLA

CORRUGATA

AG GLUTINAT

ARCYICA

SP.

CHARL OTTENSIS

FLUENS

ROTAL I FORMIS

SQUAMATA

FR IGIDA

INUSI TATA

LOBATULUS

MC KANNAI

AR TICULA TUM

EXCAVATUM

PACIFICA

LUCIOA

CALIFORNICA

NORCR OSS I

APIOPLEURA

AURICULA

TURGI DA DIGITATA

LABRADORICA

MELO

FLUENS

JUNCEA

PEREGR INA

FLETCHER I

LOBATULUS

JEFFREYSII

PRAEGERI

HELENAE

LIMBATA

FLETCHER I

LOBAT ULUS

BISER I ALIS

FR IGI DUM

REPANDUS

MARGINATA

PROBLEMA

CALIFORNI CA

LIMBATA

TORTUOSA

BACCATA

AP IOPLEURA

ELONGATA

BOREALIS

KINCAIDI

SALISBURYI

SP.

SC ORP IURUS

GALLOWAY I

GULLMARENSIS

GALLOWAYI

DE CUSSATA

FR IGIDA

LOBATULUS

CRASS IMARGO

JEFFREYSII

BISER I AL IS

ADVENA

AR CTICA

FR IGI DUM

OR NAT ISSIMA

CALIFORNICA

LIMBATA

SUBROTUNDA

AURICULA

LAEVIGATA

AKNER I ANA

ARCIICA

SEMINULA

CONTORTUS

SCORPIUR

COLUMBIENSIS

COLUMBIE

FLUENS

ROTALIFORMIS

SQUAMATA

FR IGIDA

INUSITATA

ELEGANTISSIMA 


\section{Lat. Long. Locality}

$5749 N 15230 W$

$5802 N 13400 W$

$5819 \mathrm{~N} 13420 \mathrm{~W}$

$5825 N 13530 \mathrm{~W}$

$5830 \mathrm{~N} 13900 \mathrm{~W}$

$5835 \mathrm{~N} 13815 \mathrm{~W}$
KODIAK, ALASKA

KODIAK, ALASKA

KODIAK, ALASKA

KODIAK, ALASKA

KODIAK, ALASKA

TAKU HARBOUR, ALASKA

TAKU HARBOUR, ALASKA

TAKU HARBOUR, ALASKA

TAKU HARBOUR, ALASKA

TAKU HARBOUR, ALASKA

TAKU HARBOUR, ALASKA

TAKU HARBOUR, ALASKA

TAKU HARBOUR. ALASKA

TAKU HARBOUR, ALASKA

TAKU HARBOUR, ALASKA

TAKU HARBOUR, ALASKA

TAKU HARBOUR, ALASKA

TAKU HARBOUR, ALASKA

TAKU HARBOUR, ALASKA

TAKU HARBOUR, ALASKA

TAKU HARBOUR, ALASKA

TAKU HARBOUR, ALASKA

TAKU HARBOUR, ALASKA

TAKU HARBOUR, ALASKA

TAKU HARBOUR, ALASKA

TAKU HARBOUR, ALASKA

TAKU HARBOUR, ALASKA

TAKU HARBOUR, ALASKA

TAKU HARBOUR ALASKA

TAKU HARBOUR, ALASKA

TAKU HARBOUR, ALASKA

TAKU HARBOUR, ALASKA

OF F ALASKA

OF $F$ ALASKA

OF F ALASKA

EXCURSION, ALASKA

EXCURSION, AL ASKA

EXCURSION, ALASKA

EXCURSION. ALASKA

EXCURSION, ALASKA

EXCURSION, ALASKA

EXCURSION, ALASKA

EXCURSION, ALASKA

EXCURSION, ALASKA

EXCURSION, ALASKA

EXCURSION, ALASKA

EXCURSION, ALASKA

EXCURSION, ALASKA

EXCURSION. ALASKA

EXCURSION, ALASKA

EXCURSION, ALASKA

EXCURSION, ALASKA

EXCURSION, ALASKA

EXCURSION, ALASKA

EXCURSION. ALASKA

EXCURSION, ALASKA

EXCURSION, ALASKA

EXCURSION, ALASKA

EXCURSION, ALASKA

EXCURSION, ALASKA

EXCURSION, ALASKA

EXCURSION, ALASKA

EXCURSION, ALASKA

EXCURSION, ALASKA

EXCURSION, ALASKA

EXCURSION, ALASKA

EXCURSION, ALASKA

EXCURSION, ALASKA

EXCURSION, ALASKA

EXCURSION, ALASKA

EXCURSION ALASKA

EXCURSION, ALASKA

EXCURSION, ALASKA

EXCURSION, ALASKA

EXCURSION, ALASKA

EXCURSION, ALASKA

GULF OF ALASKA

GULF OF ALASKA

GULF OF ALASKA

GULF OF ALASKA

GULF OF ALASKA

GULF OF ALASKA

GULF OF ALASKA

GULF OF ALASKA

GULF OF ALASKA

GULF OF ALASKA

GULF OF ALASKA

GULF OF ALASKA

GULF OF ALASKA

GULF OF ALASKA

GULF OF ALASKA

GULF OF ALASKA

GULF OF ALASKA

GULF OF ALASKA

\section{Generic Name Specific Name}

ELPHIDIUM

FURSENKOINA

HAPLOPHRAGMOIDES

HAYNES INA

NONIONELL INA

AMPHI CORYNA

ASTRONONION

BOL IVINA

BUCCELLA

CRIBROSTOMOIDES

CRIBROSTOMOIDES

CRIBROSTOMO

EGGERELLA

ELPHIDIELLA

ELPHIOIUM

ELPHIDIUM

ELPHIDIUM

GAUDRYINA

GLOBOBUL IMINA

HAPLOPHRAGMOI DES

I SLANDIELLA

I SLAND IELLA

LAGENA

NONIONELLA

QUINQUEL OCULINA

QUINOUELOCULINA

REOPHAX

TRIFAR INA

TROCHAMMINA

UVIGER INA

EGGERELLA

ELPHIDIELLA

ELPHI DIUM

AMMODISCUS

AMMOTIUM

ASTRONONION

BOL IVINA

BUCCELLA

BUL IM I NELLA

CASSI DUL INA

CIBICIDES

CRIBROSTOMOIDES

CRIBROSTONOIDES

DYOCIBICIDES

EGGERELLA

ELPHIDIELLA

ELPHIDIUM

ELPHIDIUM

ELPHIDIUM

EP ISTOMINELLA

F ISSUR INA

FUR SENKOINA

GAUDRY INA

GLOBOBUL IMINA

HAPLOPHRAGMOIDES

I SLAND IELLA

I SLANDIELLA

ISLAND IELLA

LAGENA

NONI ONELL A

NONIONELL INA

OOL INA

PSEUDOPOL YMOR PHINA

PYRGO

QUINQUELOCULINA

QUINQUEL OCULINA

QUINQUEL OCULINA

QUINQUELOCULINA

RECURVOIDES

REOPHAX

SIGMOMORPHINA

SPIROPLEC TAMM INA

TRIFAR INA

TROCHAMM INA

BOL IVINA

BUCCELLA

CIBICIDES

CIBICIDES

ISLANDIELLA

I SLANDIELLA

I SLAND IELLA

LAGENA

NON IONELLA

PULLENIA

TR I F AR INA

UVIGER INA

UVIGERINA

ASTACOLUS

BOL IVINA

BUL IM INE LLA

DENTAL INA

BARTLETTI

EX CAVATYM

CANAR IENSIS

ORBICULARE

LABRADORICA

SCALAR IS

GALLOWAY I

FR IGIDA

LOBATULUS

CRASS IMARGO

JEFFREYSII

DE CEPTA

AD VENA

ARCTICA

BARTLETTI

EX CAVATUM

FRIGIDUM

VI TREA

ARENARIA

AURICULATA

PLANISSIMUM

CALIFORNICA

LIMBATA

AP I OPLEURA

AURICULA

AK NER I AN A

FR IGIDA

SC ORPIURUS

FLUENS

ROTAL I FORMIS

PE REGR INA

AD VENA

HANNAI

TUMIDUM

GULLMARENSIS

CASSIS

GALLOWAY I

DE CUSSATA

FR IGIDA

ELEGANTISSIMA

BARBARA

LOBATULUS

CRASS IMARGO

JEFFREYSI I

B I SER I AL IS

AD VENA

ARCTICA

BARTLETTI

EXCAVATUM

FRIGI DUM

VITREA

LUCIDA

FUSIF ORMIS

ARENARIA

AUR I CULATA

PL ANISSIMUM

CALIFORN I CA

HELENAE

LI MBATA

AP IOPLEUR

AU RI CULA

LABRA DORICA

BOREALIS

CHARL OTTENSIS

LUCERNULA

AKNER I ANA

ARCTI CA

SEMINULA

STALKERI

TURBINATUS

SCORPIURUS

TRILOCULARIS

BI FORMIS

FLUENS

RO TAL I FORMIS

DECUSSATA

FR IGI DA

FLETCHER I

LOBATULUS

REPANDUS

CALIF ORNICA

LI MBATA

TORTUOSA

GRACI LIS

AURICULA

SALISBURYI

FLUENS

JUNCEA

PLANULATUS

DE CUSSATA

PACIF I CA

ELEGANTI SSIMA

DECEPTA 
Lat. Long. Locality

$5835 N \quad 138 \quad 15 W$

$58 \quad 35 N \quad 13915 W$

$5840 N 13500 W$

$5850 \mathrm{~N} 14115 \mathrm{~W}$

59 $00 \mathrm{~N} 14120 \mathrm{~W}$
GULF OF ALASKA GULF OF ALASKA GULF OF ALASKA GULF OF ALASKA GULF OF ALASKA GULF OF ALASKA GULF OF ALASKA GULF OF ALASKA GULF OF ALASKA GULF OF ALASKA GULF OF ALASKA GULF OF ALASR GULF OF ALASKA GULF OF ALASKA GULF OF ALASKA GULF OF ALASKA GULF Of ALASKA GULF OF ALASKA GULF OF ALASKA GULF OF ALASKA GULF OF ALASKA GULF OF ALASKA GULF GULF OF ALASKA GULF OF ALASK GULF OF ALASKA GULF OF ALASKA GULF OF ALASKA GULF OF ALASKA GULF OF ALASKA GULF OF ALASKA GULF OF ALASKA GULF OF ALASKA GULF OF ALASKA GULF OF ALASKA GULF OF ALASKA GULF OF ALASKA GULF OF ALASKA GULF OF ALASKA

LYNN CANAL, ALASKA LYNN CANAL, ALASKA LYNN CANAL, ALASKA LYNN CANAL. ALASKA LYNN CANAL, ALASKA LYNN CANAL. ALASKA LYNN CANAL, ALASKA
LYNN CANAL, ALASKA LYNN CANAL, ALASKA LYNN CANAL, ALASKA LYNN CANAL, ALASKA LYNN CANAL, ALASKA LYNN CANAL, ALASKA LYNN CANAL ALASKA LYNN CANALE ALASKA LYNN CANAL, ALASKA LYNN CANAL, ALASKA LYNN CANAL, ALASKA LYNN CANAL, ALASKA LYNN CANAL, ALASKA LYNN CANAL, ALASKA LYNN CANAL ALASKA

GULF OF ALASKA GULF OF ALASKA GULF OF ALASKA GULF OF ALASKA GULF OF ALASKA GULF OF ALASKA GULF OF ALASKA GULF OF ALASKA GULF OF ALASKA GULF OF ALASKA GULF OF ALASKA GULF OF ALASKA GULF OF ALASKA GULF OF ALASKA GULF OF ALASKA GULF OF ALASKA GULF OF ALASKA GULF OF AL ASKA GULF OF ALASKA GULF OF ALASKA GULF OF ALASKA GULF OF ALASKA GULF OF ALASKA GULF OF ALASKA GULF OF ALASKA GULF OF ALASKA GULF OF ALASKA GULF OF ALASKA GULF OF ALASKA GULF OF ALASKA GULF OF ALASKA GULF OF ALASKA GULF OF ALASKA GULF OF ALASKA GULF OF ALASKA GUIF OF ALASKA GULF OF ALASKA GULF OF ALASKA GULF OF ALASKA GULF OF ALASKA GULF OF ALASKA
Generic Name Specific Name

EGGERELLA

ELPHIDIUM

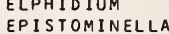

EPONIDES

I SLAND IELLA

I SLANDIELLA
KARRER IELLA

LAGENA

LAGENA

LAGENA

LAGENA

LAGENA

LAGENA

NONI ONELLA

OOL INA

OOLINA

PULLEN IA

PYRGO

QUINQUEL OCULINA

UVIGER INA

ASTRONONI ON

BUCCELLA

CIBICIDES

CIBICIDES

ELPONIDES

FI SSUR INA

I SLANDIELLA

I SLANDIELLA

I SLANDIELLA

KARRERIELLA

PYRGO

SIGMOMORPHINA

TRIFAR INA

TROCHAMMINA

AMMOT IUM

BUL IMINELLA

CASSI DULINA

CIBICIDES

CRIBROSTOMOIDES

DENDROPHYRA

EGGERELLA

ELPHIDIELLA

ELPHIDIUM

ELPHIDIUM

EPISTOMINELLA

GLOBOBULIMINA

I SLANDIELLA

NONIONELLA

QUINQUELOCULINA

QUINQUELOCULINA

REOPHAX

TRI LOCUL INA

AMMODISCUS

ASTRONONION

BOLIVINA

BOL IVINA

BOLIVINA

BUCCELLA

BUL IMINA

BULIMINA

CASSIDUL INA

CASSI DULINA

CASSIDULINA

CIBICIDES

DOROTHIA

EGGERELLA

EHRENBERG INA

EPISTOMINELLA

EPISTOMINELLA

EPONIDES

GYROIDINA

HAPLOPHRAGMOI DES

I SLAND IELLA

LAGENA

LENTI CUL INA

OOL INA

PULLENIA
RECTOBOL IVINA

RUPERTIA

TRIFARINA

UVIGERINA

UVIGERINA

VAL VUL INERIA

AMMOBACUL ITES

AMMODISCUS

AMMODISCUS

AMMOMARGINULINA

BOLIVINA

BOL IVINA

BOL IVINA

BOL IVINA

AD VENA

EX CAVATUM

FRIGIDUM

LEVICULUS

HELENAE

NORCROSSI

BACCATA

ELONGATA

GRACILIS

LAEVIS

PLIOCENICA

SEMIL INEATA

STRIATA

STELLA
BOREALIS

MELO

SALISBURYI

MURRH INA

ST ALKERI

JUNCEA

GALLOWAYI

FRIGIOA

FLETC HER I

LOBATULUS

NI TIDA

REPANDUS

LUCIDA

CALIFORNICA

LIMBATA

TORTUOSA

BACCATA

MURRH INA

GALLOWAY

FLUENS

ROTALI FORMIS

CASSIS

FRIGIDA

EL EGANTIS SIMA

BARBARA

LOBATULUS

CRASS IMARGO

ARBORESCENS

ADVENA

ARCTICA

ARCTICA

BARTLETTI

FRIGI DUM

VI TREA

AURICULATA

HE LENAE

AURICULA

SEMINULA

SC ORP I URUS

ROTUNDA

MINUT I SS IMUS

GALLOWAY I

DE CUSSATA

PACIFICA

SP ISSA

FRIGIDA

BARBATA

SU BAC UMINATA

BARBARA

CUSHMANI

SUBGLOBOSA

FLETCHER I

BRADYANA

BRADY I

COMPRESSA

PACIFICA

VI TREA

LEVICULUS

10

SC I TULUM

TRANSLUCENS

MERIDIONALIS

NI COBARENSIS

BOREALIS

SALISBURY

PORRECTA

STABILIS

FLUENS

JUNCEA

PEREGR INA

GLABRA

A GGLUT INANS

MINUT I SSIMUS

PACIFICUS

SANDIEGOENSIS

AL ATA

BARBATA

DE CUSSATA 


\section{Lat. Long. Locality}

$5900 N 14120 W$ GULF OF ALASKA GULF OF AL ASKA GULF OF ALASKA GULF OF ALASKA GULF OF ALASKA GULF OF ALASKA GULF OF ALASKA GULF OF AL ASKA GULF OF ALASKA GULF OF ALASKA GULF OF ALASKA GULF OF ALASKA GULF OF ALASKA GULF OF ALASKA GULF OF ALASKA GULF OF ALASKA GULF OF ALASKA GULF OF ALASKA GULF OF ALASKA GULF OF ALASKA GULF OF ALASKA GULF OF ALASKA GULF OF ALASKA GULF OF ALASKA GULF OF ALASKA GULF OF ALASKA GULF OF ALASKA GULF OF ALASKA GULF OF ALASKA GULF OF ALASKA GULF OF ALASKA GULF OF ALASKA GULF OF ALASKA GULF OF ALASKA GULF OF AL ASKA GULF OF ALASKA GULF OF ALASKA GULF OF ALASKA GULF OF ALASKA GULF OF ALASKA GULF OF ALASKA GULF OF ALASKA GULF OF ALASKA GULF OF ALASKA GULF OF ALASKA GULF OF ALASKA GULF OF ALASKA GULF OF ALASKA GULF OF ALASKA GULF OF ALASKA GULF OF ALASKA GULF OF ALASKA GULF OF ALASKA GULF OF ALASKA GULF OF ALASKA GULF OF ALASKA GULF OF ALASKA GULF OF ALASKA GULF OF ALASKA GULF OF ALASKA GULF OF ALASKA GULF OF ALASKA GULF OF ALASKA GULF OF ALASKA GULF OF ALASKA GULF OF ALASKA GULF OF ALASKA GULF OF ALASKA GULF OF ALASKA GULF OF ALASKA GULF OF ALASKA GULF OF ALASKA GULF OF ALASKA GULF OF ALASKA GULF OF ALASKA GULF OF ALASKA GULF OF ALASKA GULF OF ALASKA GULF OF ALASKA GULF OF ALASKA GULF OF ALASKA GULF OF ALASKA GULF OF ALASKA GULF OF ALASKA GULF OF ALASKA GULF OF ALASKA GULF OF ALASKA GULF OF ALASKA GULF OF ALASKA GULF OF ALASKA GULF OF ALASKA GULF OF ALASKA GULF OF ALASKA GULF OF ALASKA GULF OF ALASKA

\section{Generic Name Specific Name}

B OL I V INA

BOL IVINA

BULIMINA

BUL IMINA

CASSIDUL INA

CASSI DUL I AA

CAS S I DUL INA

CASSIDUL INA

CASSI DULINA

CHILOSTOMELLA

CHILOSTOMELLINA

CIBICIDES

CIBICIDES

CIBICIDES

EGGERELLA

EGGERELLA

EGGERELLA

ELPHI DIUM

EPISTOMINELLA

EPISTOMINELLA

EPONIDES

EPONIDES

EPONIDES

EPONIDES

FISSURINA

FURSENKOINA

FURSENKOINA

FURSENKOINA

FURSENKOINA

GLANDULINA

GLOBOBULININA

GLOBOBULIMINA

GYROIDINA

GYROIDINA

HAPLOPHRAGMOI DES

HYPERAMMINA

I SLANDIELLA

KARRERIELLA

LAGENA

LAGENA

LAGENA

LAGENA

LAGENA

LAGENA

MAR INOT TIELLA

MELONIS

NON IONELLA

NONIONELLA

NON I ONELLINA

OOL INA

OOLINA

PELOSINA

PLANUL INA

PULLENIA

PULLENIA

REOPHAX

REOPHAX

RHIZAMMINA

SACCAMMINA

TEXTULAR I A

THALMANNAMM IN A

TRIFAR INA

TRI LOCUL INA

TROCHAMM INA

TROCHAMMINA

UVIGER INA

UVI GER INA

UVI GER INA

UVIGER INA

VALVUL INERI A

VALVUL INERIA

ASTRONONION

BOL IVINA

BOL IVINA

BUL IMIAA

BUL IMINA

CASSI DUL INA

EP I S TOMINELLA

EPISTOMINELLA

EPONIDES

EPONIDES

EPONIDES

EPONIDES

FRANCESITA

FURSENKOINA

GYROIDINA

ISLANDIELLA

LAGENA

LAGENA

NON I ONELL A

NONIONELLA

TRIFAR INA

TRI LOCUL INA

UVI GER INA
SP ISS A

SUEADVENA

SUBAC UMINATA

TENUATA

BARBARA
CUSHMANI

LOMITENSIS

SUBCARINATA

SUBGLOBOSA

OOLINA

FI MBR I A T A

FLETCHER I

LOBATULUS

MC KANNA

ADVENA

BR A DYI

PUSILLA

PACIF I CA

VI IREA

HE ALD I

LEVICULUS

SUBTENER

UMBONATUS

LUCIDA

LOEBL I CHI

SANDIEGOENSIS

SEMINUDA

LAEVIGATA

AURICULATA

PACIFICA

AL TIFORMIS

SC I TULUM

CALIFORNICA

PARKERAE

EL ONGATA

GRACILIS

MERIDIONALIS

NE BULOSA

SEMIL I NEATA

STRIATA

OC CIDENTALIS

POMPILIOIDES

AURICULA

TURGIDA DIGITATA

LABRA DORI CA

GLOBOSA

ME LO

DI DER A

WUELLERSTORFI

BULLOIDES

SALIS BURYI

GUTTIFER

SC ORP I URUS

AL GAE FORMIS

SPHAE I I A

TORQUATA

PARKERAE

FLUENS

TR I HE DRA
GL OBI GER INI FORM IS

IN OBI GER

INFLAT A

AUBER I ANA

PEREGRINA

PROBOSCIDEA

AR AUC ANA

GLABRA

GALLOWAY I

DE CUSSATA

PACIFICA

BA RBATA

IN FLATA MEXICANA

CUSHMANI

PACIFICA

VI TREA

HE ALD I

LEVICULUS

SUBTENERA

UMBONAT

ADVENA

LOE

NORCROSS I

ELONGATA

STRIATA

AURICULA

TURGIDA DIGITATA

PARKERAE

FLUENS

TR I HE DRA

PEREGRINA DIRUPTA
BRAMLETTEI

Publication

BERGEN O'NE IL 1979 BERGEN O'NEIL 1979 BERGEN O'NE IL 1979 BERGEN O NE IL 1979 BERGEN O'NE IL 1979 
Lat. Long. Locality

$5920 N 14538 \mathrm{~W}$ GULF OF ALASKA GULF OF ALASKA GULF OF ALASKA

$59 \begin{aligned} 23 \mathrm{~N} 13520 \mathrm{~W} & \text { OF F ALASKA } \\ & \text { OFF ALASKA } \\ & \text { OFF ALASKA }\end{aligned}$

$5925 \mathrm{~N} 14525 \mathrm{~W}$ GULF OF ALASKA GULF OF ALASKA GULF OF ALASKA GULF OF ALASKA GULF OF ALASKA GULF OF ALASKA GULF OF ALASKA GULF OF ALASKA GULF OF ALASKA GULF OF ALASKA GULF OF ALASKA GULF OF ALASKA GULF OF ALASKA GULF OF ALASKA GULF OF ALASKA GULF OF ALASKA GULF OF ALASKA GULF OF ALASKA GULF OF ALASKA GULF OF ALASKA GULF OF ALASKA GULF OF ALASKA GULF OF ALASKA GULF OF ALASKA GULF OF ALASKA GULF OF ALASKA GULF OF ALASKA GULF OF ALASKA GULF OF ALASKA GULF OF ALASKA GULF OF ALASKA GULF OF ALASKA GULF OF ALASKA GULF OF ALASKA GULF OF ALASKA GULF OF ALASKA GULF OF ALASKA GULF OF ALASKA GULF OF ALASKA GULF OF ALASKA GULF OF ALASKA GULF OF ALASKA GULF OF ALASKA GULF OF ALASKA GULF OF ALASKA GULF OF ALASKA GULF OF ALASKA GULF OF ALASKA GULF OF ALASKA GULF OF ALASKA GULF OF ALASKA GULF OF ALASKA GULF OF ALASKA GULF OF ALASKA GULF OF ALASKA GULF OF ALASKA GULF OF ALASKA GULF OF ALASKA GULF OF ALASKA GULF OF ALASKA GULF OF ALASKA GULF OF ALASKA GULF OF ALASKA GULF OF ALASKA GULF OF ALASKA GULF OF ALASKA GULF OF ALASKA GULF OF ALASKA GULF OF ALASKA GULF OF ALASKA GULF OF ALASKA GULF OF ALASKA GULF OF ALASKA GULF OF ALASKA GULF OF ALASKA GULF OF ALASKA GULF OF ALASKA GULF OF ALASKA GULF OF ALASKA GULF OF ALASKA GULF OF ALASKA GULF OF ALASKA GULF OF ALASKA GULF OF ALASKA GULF OF ALASKA GULF OF ALASKA GULF OF ALASKA GULF OF ALASKA GULF OF ALASKA

\section{Generic Name Specific Name}

UVIGER INA

VAL VUL INERIA

AMMOT I UM

ELPHIOIUM

HAPLOPHRAGMOI DES

AMMOMARG I NULI NA

AMMOSCALARIA

ASTRONONION

BOL IVINA

BOL IVINA

BOLIVINA

BOL IVINA

BOLIVINA

BUCCELLA

BUL IMINA

BUL IMINA

BUL IMIAA

BULIMINA

CASSIDUL INA

CASS I DUL INA

CASSIDULINA

CASSI DUL INOIDES

CHILOSTOMELLA

C IBICIDES

DENTAL INA

ELPHI DIUM

EPISTOMINELLA

EPISTOMINELLA

EPONIDES

EPONIDES

F IS SUR INA

FURSENKOINA

FUR SENKO INA

GLOBOBUL IMINA

GYRO I O INA

GYROIDINA

GYROI DINA

HAPLOPHRAGMOI DES

HAPLOPHRAGMOI DES

HOE GLUND INA

HYPER AMM INA

KARRERIELLA

LAGENA

NONIONELLA

NONIONELLINA

PULLENIA

PULLENIA

PYRGO

REOPHAX

REOPHAX

ROBERTINOIDES

SACCORHIZA

SUGGRUNDA

THALMANNAMM IN A

TRIFAR INA

IRILOCULINA

TROCHAMMINA

TROCHAMMINA

TROCHAMM INA

UVIGER INA

UVIGER INA

UVIGER INA

UVIGER INA

VAG INUL INA

VAL VUL INER I A

AMMODISCUS

AMMOMARG INUL INA

AMMOSCALARIA

BOLIVINA

BOL IVINA

BOL IVINA

BULIMINA

BUL IM INA

BUL IMINA

CASSI DUL INA

CASSI DULINA

CASSIDUL INA

CHILOS TOMELLA

CHILOSTOMEL

CIBICIDES
CRIBROSTOMOIDES

CYCLOGYRA

EGGERELLA

EGGERELLA

EGGERELLA

EPISTOMINELLA

EPISTIDIN

EPONIDES

EPONIDES

FISSUR INA

F IS SUR INA

FISSURINA

FUR SENKOINA

GLOBOBUL IMI NA

GL OBOBUL IMINA

GYROIDINA

HAPLOPHRAGMOIDES
PROBOSCIDEA

ARAUC ANA

GL ABR A

CASSIS

FR I GI DUM

ADVENUM

SANDIEGOENS IS

PSEUD OSPIRAL IS

GALLOWAY I

AL ATA

DE CUSSATA

MINUTA

PACIF I CA

SPISSA

FRIGIDA

INFLATA MEXI CANA

ROSTRATA

SUBACUMINATA

TENUATA

BARBARA

CUSHMANI

SUBCARINATA

CORNUTA

OOLINA

MCKANNAI

CALOMORPHA

FRIGIDUM

PACIFICA

VI TREA

HE ALO I

LEVICULUS

LUCIDA

LOEBL I CH I

SEMINUDA

PACIFICA

ALTIFORMIS

GEMMA

10

PLANISSIMUM

SC I TUL UM

ELEGANS

ELONGATA

PARKERAE

EL ONGATA

AURICULA

LABRADORICA

BULLOIDES

SALISBURYI

MURRH INA

GUTTIFER
SCORPIURUS

CHARL OTTENSIS

RAMOSA

ECKISI

PARKERAE

FLUENS

TR IHE DRA

GLOBIGERINIFORMIS

IN FLA TA

AU BER I ANA

PEREGR INA

PEREGR INA DIRUPTA

SENTI COSA

LE GUMEN

GL ABR A

MINUT I SSIMUS

SANDIEGOENSIS

PSEUDOSPIRAL IS

DECUSSATA

PACIFICA

SPISSA

BARBATA

SUBAC UMINATA

TENUAT A

CUSHMANI

SUBCARINATA

SUBGL OBOSA

OOL INA

FLETCHER I

JEFFREYSII

IN VOL VENS

A D VENA

BRAOYI

PUS ILLA

VI TREA

LEVICULUS

SUBTENERA

CUCURBITASEMA

LUCIOA

MARGINAT

SEMINUDA

AURICULATA

PACIFICA

10

PLANISSIMUM

Publication

BERGEN O'NEIL 1979 


\section{Lat. Long. Locality}

$5930 N 14255 \mathrm{~W}$

$5930 \mathrm{~N} 14300 \mathrm{~W}$ GULF OF ALASKA
GULF OF ALASKA GULF OF ALASKA GULF OF ALASKA GULF OF ALASKA GULF OF ALASKA GULF OF ALASKA GULF OF ALASKA GULF OF ALASKA GULF OF ALASKA GULF OF ALASKA GULF OF ALASKA GULF OF ALASKA GULF OF ALASKA GULF OF ALASKA GULF OF ALASKA GULF OF ALASKA GULF OF ALASKA GULF OF ALASKA GULF OF ALASKA GULF OF ALASKA GULF OF ALASKA GULF OF ALASKA GULF OF ALASKA GULF OF ALASKA GULF OF ALASKA GULF OF ALASKA GULF OF ALASKA GULF OF ALASKA GULF OF ALASKA GULF OF ALASKA GULF OF ALASKA GULF OF ALASKA GULF OF ALASKA GULF OF ALASKA GULF OF ALASKA GULF OF ALASKA GULF OF ALASKA GULF OF ALASKA GULF OF ALASKA GULF OF ALASKA GULF OF ALASKA GULF OF ALASKA GULF OF ALASKA GULF OF ALASKA GULF OF ALASKA GULF OF ALASKA GULF OF ALASKA GULF OF ALASKA GULF OF ALASKA GULF OF ALASKA GULF OF ALASKA GULF OF ALASKA GULF OF ALASKA GULF OF ALASKA GULF OF ALASKA GULF OF ALASKA GULF OF ALASKA GULF OF ALASKA GULF OF ALASKA GULF OF ALASKA GULF OF ALASKA GULF OF ALASKA GULF OF ALASKA GULF OF ALASKA GULF. OF ALASKA GULF OF ALASKA GULF OF ALASKA GULF OF ALASKA GULF OF ALASK GULF OF ALASKA GULF OF ALASKA GULF OF ALASKA GULF $O F$ UUF OF ALASKA GULF OF ALASKA GULF OF ALASKA GULF OF ALASKA GULF OF ALASKA GULF OF ALASKA GULF OF ALASKA GULF OF ALASKA GULF OF ALASKA GULF OF ALASKA GULF OF ALASKA GULF OF ALASKA GULF OF ALASKA GULF OF ALASK GULF GULF OF ALASKA GULF OF ALASKA GULF OF ALASKA GULF OF ALASKA GULF OF ALASKA GULF OF ALASKA GULF OF ALASKA GULF OF ALASKA GULF OF ALASKA

\section{Generic Name Specific Name}

HAPLOPHRAGMOI DES

SC ITULUM

HYPERAMMINA

I SLAND IELLA

KARRER IELLA

LAGENA

NONIONELLA

NONIONELLA

OOL INA

OOL INA

OOLINA

PULLENIA

PULLENIA

REOPHAX

REOPHAX

REOPHAX

RHIZAMMINA

SACCAMMINA

TEXTULARIA

THALMANNAMMINA

TR I FAR INA

TRILOCULINA

TROCHAMMINA

IROCHAMMINA

UVIGERINA

VAL VUL INER I A

VALVUL INERIA

AMMODISCUS

AMMOMARG INULINA

BOL IVINA

BOL IVINA

BOLIVINA

BUCCELLA

BUL IMINA

BUL IMINA

BUL IMINA

CASSIDULINA

CASSIDULINA

CASSIDULINA

CAS SI DUL INA

CHILOSTOMELLA

CIBICIDES

CIBICIDES

CIBICIDES

DENTALINA

DOROTHIA

EGGERELLA

EGGERELLA

EPONIDES

EPONIDES

EPONIDES

FRANCESITA

FURSENKOINA

GLOBOBULIMINA

GLOBOBUL IMINA

GYROIDINA

GYROIDINA

GYROIDINA

GYROIDINA

GYROIDINA

HAPL OPHRAGMOI DES

HAPLOPHRA GMOIDES

HYPERAMMINA

I SLANDIELLA

ISLANDIELLA

ISLANDIELLA

KARRERIELLA

LAGENA

MART INOTTIELLA

NONIONELLA

NONIONELLA

NON IONELL INA

PELOSINA

PLANULINA

PULLENIA

PULLEN IA

PYRGO

REOPHAX

REOPHAX

RUPERTIA

SACCAMMINA

SACCORHIZA

SPHAEROIDINA

SPIROPLECTAMM INA

TEXTULARIA

TRIFAR INA

TRI LOCUL INA

TROCHAMMINA

UVI GER INA

UVIGERINA

UVIGERINA

UVIGERINA

UVI GER INA

VALVULINERIA

VALVUL INERIA

NORCROSS I

PARKERAE

ELONGATA

STRIATA

AURICULA

TURGIDA DIGITATA

BOREALIS

GL OBOSA

HEXAGONA

BULLOIDES

SALISBURYI

GUTTIFER

PILUL I FER

SCORPIURUS

AL GAE FORMIS

SPHAERICA

TORQUATA

PARKERAE

FLUENS

TR I HE D RA

GL OBI GERINIFORMIS

ROTAL IFORMIS

PEREGRINA DIRUPTA

ARAUCANA

GLABRA

MINUT I SS IMUS

SANDIEGOENSIS

DE CUSSATA

PACIFICA

SPISSA

FRIGIDA

BARRATA

INFLATA MEXICANA

TENUATA

BARBARA

CUSHMANI

LOMITENSIS

SUBCARINATA

OOLINA

FLETCHERI

MCKANNAI

SPI RALIS

BAGGI

BRADYANA

BRADY I

PUSILLA

LEVICULUS

SUBTENERA

UMBONATUS

ADVENA

SEMINUDA

AURICULATA

PACIFICA

AL TIF ORMIS

GEMMA

10

LAMAR CKI ANA

ORBICULARIS

PLANISSIMUM

SCITULUM

ELONGATA

CALIFORNICA

NORCROSS I

TRANSLUCENS

PARKERAE

ELONGATA

OCCIDENTALIS

AURICULA

TURGIDA DIGITATA

LABRADORICA

DI DERA

WUELLERSTORFI

BULLOIDES

SALISBURYI

MURRH I NA

GUTTIFER

SCORPIURUS

STABILIS

SPHAERICA

RAMOSA

BULLOIDES

BI FORMIS

TORQUATA

TR I HE D RA

GL OBI GER IN I FORM IS

AU BER I ANA

JUNCE A

PEREGRINA

PEREGR INA DIRUPTA

PROBOSCIDEA 
Lat. Long. Locality

$5930 N 14520 W$ GULF OF ALASKA GULF OF ALASKA GULF OF ALASKA GULF OF ALASKA GULF OF ALASKA GULF OF ALASKA GULF OF ALASKA GULF OF ALASKA GULF OF ALASKA GULF OF ALASKA GULF OF ALASKA GULF OF ALASKA GULF OF ALASKA GULF OF ALASKA GULF OF ALASKA GULF OF ALASKA GULF OF ALASKA GULF OF ALASKA GULF OF ALASKA GULF OF ALASKA GULF OF ALASKA GULF OF ALASKA GULF OF ALASKA GULF OF ALASKA GULF OF ALASKA GULF OF ALASKA GULF OF ALASKA GULF OF ALASKA GULF OF ALASKA GULF OF ALASKA GULF OF ALASKA GULF OF ALASKA GULF OF ALASKA GULF OF ALASKA GULF OF ALASKA GULF OF ALASKA GULF OF ALASKA GULF OF ALASKA GULF OF ALASKA GULF OF ALASK GULF OF ALASKA GULF OF ALASKA GULF OF ALASKA GULF OF ALASKA GULF OF ALASKA GULF OF ALASKA GULF OF ALASKA GULF OF ALASKA GULF OF ALASKA GULF OF ALASKA GULF OF ALASKA GULF OF ALASKA GULF OF ALASKA GULF OF ALASKA GULF OF ALASKA GULF OF ALASKA GULF OF ALASKA GULF OF ALASKA GULF OF ALASKA GULF OF ALASKA GULF OF ALASKA GULF OF ALASKA GULF OF ALASKA GULF OF ALASKA GULF OF ALASKA GULF OF ALASKA GULF OF ALASKA GULF OF ALASKA GULF OF ALASKA GULF OF ALASKA GULF OF ALASKA GULF OF ALASKA GULF OF ALASKA GULF OF ALASKA GULF OF ALASKA GULF OF ALASKA GULF OF ALASKA GULF OF ALASKA
Generic Name Specific Name AMMODISCUS ASTRONONION BOLIVINA BOLIVINA BOL IVI $A$ A BUCCELLA BUL IMINA BUL IMINA

BUL IM INA

BUL IM INA

BULIMIAA

CASSIDULINA

CASSIDULI IAA

CASSI DUL INA

CASS I DUL INA

CHILOS TOMELLA

CHILOSTOMELLINA

CIBICIDES

C IBICIDES

EGGERELLA

EGGERELLA

EGGERELLA

EGGERELLA

ELPHIDIUM

ELPHIDIUM

EPISTOMINELLA

EPISTOMINEL LA

EPONIDES

EPONIDES

EPONIDES

EPONIDES

FIS SUR INA

F ISSUR INA

FURSENKOINA

FURSENKOINA

FURSENKOINA

GLOBOBUL IMINA

GLOBOBULIMINA

GYROIDINA

GYROIDINA

GYROIDINA

HAPLOPHRAGMOI DES

HAPLOPHRAGMOI DES

HYPERAMM INA

I SLANDIELLA

I SLANDIELLA

I SLANDIELLA

KARRERIELLA

LAGENA

LAGENA

NONIONELLA

NON I ONELLA

NONIONELLINA

PULLENIA

PULLENIA

RECURVOIDES

REOPHAX

REOPHAX

REOPHAX

RHIZAMMINA

SACCORHIZA

SPIROPLEC TAMM INA

TEXTULARIA

TRIFAR IN A

TRILOCUL INA

TROCHAMMINA

TROCHAMMINA

UVIGERINA

UVI GER INA

UVIGERINA

UVIGERINA

VAL VUL INERI A

VALVUL INERI A

ADERCOTRYMA

ASTRONONION

BOLIVINA

BOL IVINA

BOL IVINA

BOLIVINA

BOLIVINA

BOL IVINA

BUCCELLA

BUL IMINA

BUL IMINA

BUL IMINA

BUL IM I NELLA

CASSIDULINA

CASSIDULINA

CASSIDUL INA

CHILOSTOMELL

CIBICIDES

CIBICIDES

CIBICIDES
MINUT ISSIMUS

PACIFI CUS

GALLOWAY I

DE CUSSATA

PACIFICA

SP ISSA

FRIGIDA

BARBATA

INFLATA MEXICANA

ROSTRATA

SUBACUMINATA

TENUATA

BARBARA

CUSHMANI

LOMITENSIS

SUBGL OBOSA

OOL INA

F I MBR I AT A

FLETCHERI

AD VENA

ADVENA

HUMBOLDT

PUSILLA

EXCAVATUM

FR IGI DUM

PACIFICA

VITREA

HEALDI

LEVICULUS

SUBTENERA

UMBONATUS

LUCIDA

LUCIDA

BRAMLETTE

LOEBL I CH I

SEMINUDA

AURICULATA

PACIFICA

ALTIFORMIS

GEMMA

IO

PLANISSIMUM

SCITULUM

ELONGATA

CALIFORNICA

NORCROSSI

TRANSLUCENS

PARKER AE

ELONGAT

MOLLIS

AURICULA

TURGIDA DIGITATA

$\angle A B R A D O R I C A$

BULLOIDES

SALISBURYI

TURBINATUS

GUTTIFER

PILUL IFER

SCORPIURUS

AL GAE F ORMIS

RAMOSA

BI FORMIS

TORQUATA

TR I HE DRA

GL OBI GER INIFORMIS

GL OBI GER

AUBER I AN A

PEREGRINA

PEREGR INA DIRUPTA

PR OBOSCIDEA

AR AUC ANA

GL ABR A

GL OMERAT A

GALLOWAY I

AL ATA

BARBATA

DE CUSSATA

PACIF I CA

PUNCTATA

SP ISSA

FR IGIDA

INFLATA MEXICANA

SUBACUMINATA

TENUATA

ELEGANTISSIMA

BARBARA

CUSHMANI

SUBGL OBOSA

OOL INA

FLETCHER I

LOBATULUS

MC KANNAI

BAGGI

\section{Publication}

BERGEN O'NEIL 1979 BERGEN O'NEIL 1979 BERGEN O'NE IL 1979 BERGEN O'NE IL 1979 BERGEN O'NEIL 1979 BERGEN O'NEIL 197 BERGEN O'NEIL 1979 BERGEN O'NE IL 1979 BERGEN O'NE IL 1979 BERGEN O'NE IL 1979 BERGEN O'NEIL 1979 BERGEN O०NE IL 1979 BERGEN OONEIL 1979 BERGEN O'NETL 197 BERGEN O'NEIL 197 BERGEN O'NEIL 197 BERGEN O'NE IL 1979 BERGEN O'NEIL 1979 
Lat. Long. Locality

$5930 \mathrm{~N} 14540 \mathrm{~W}$ GULF OF ALASKA GULF OF ALASKA GULF OF ALASKA GULF OF ALASKA GULF OF ALASKA GULF OF ALASKA GULF OF ALASKA GULF OF ALASKA GULF OF ALASKA GULF OF ALASKA GULF OF ALASKA GULF OF ALASKA GULF OF ALASKA GULF OF ALASKA GULF OF ALASKA GULF OF ALASKA GULF OF ALASKA GULF OF ALASKA GULF OF ALASKA GULF OF ALASKA GULF OF ALASKA GULF OF ALASKA GULF OF ALASKA GULF OF ALASKA GULF OF ALASKA GULF OF ALASKA GULF OF ALASKA GULF OF ALASKA GULF OF ALASKA GULF OF ALASKA GULF OF ALASKA GULF OF ALASKA GULF OF ALASKA GULF OF ALASKA GULF OF ALASKA GULF OF ALASKA GULF OF ALASKA GULF OF ALASKA GULF OF ALASKA GULF OF ALASKA GULF OF ALASKA GULF OF ALASKA GULF OF ALASKA GULF OF ALASKA GULF OF ALASKA GULF OF ALASKA GULF OF ALASKA GULF OF ALASKA GULF OF ALASKA GULF OF ALASKA GULF OF ALASKA GULF OF ALASKA GULF OF ALASKA GULF OF ALASKA GULF OF ALASKA GULF OF ALASKA GULF OF ALASKA GULF OF ALASKA GULF OF ALASKA GULF OF ALASKA GULF OF ALASKA GULF OF ALASKA GULF OF ALASKA GULF OF ALASKA GULF OF ALASKA GULF OF ALASKA GULF OF ALASKA GULF OF ALASKA GULF OF ALASKA GULF OF ALASK GULF OF ALASKA GULF OF ALASKA GULF OF ALASKA GULF OF ALASKA GULF OF ALASKA GULF OF ALASKA GULF OF ALASKA GULF OF ALASKA GULF OF ALASKA GULF OF ALASK GULF OF ALASKA GULF OF ALASKA GULF OF ALASKA GULF OF ALASKA GULF OF ALASKA GULF OF ALASKA GULF OF ALASKA GULF OF ALASKA GULF OF ALASKA GULF OF ALASKA GULF OF ALASKA GULF OF ALASKA GULF OF ALASKA GULF OF ALASKA GULF OF ALASKA

Generic Name Specific Name

Publication

EGGERELL

ELPHIDIUM

ELPHIDIUM

ELPHIDIUM

EPISTONINELLA

EPISTOMINELLA

EPISTOMINELL

EPONIDES

EPONIDES

EPONIDES

FIS SUR INA

FIS SUR INA

FRANCESITA

GLOBOBUL IMINA

GLOBOBUL IMINA

GYROIOINA

GYROIDINA

I SLANDIELLA

I SLAND IELLA

I SLANDIELLA

I SLANDIELLA

ISLANDIELLA

KARRER IELLA

KARRERIELLA

LAGENA

LAGENA

LAGENA

LAGENA

LAGENA

LAGENA

NONIONELLA

NONIONELLA

NONI ONELLA

NON IONELL INA

OOL INA

OOL INA

OOL INA

OOLINA

PATELLINA

PULLENIA

QUINQUELOCULINA

QUINQUELOCULINA

SPIROPLECTAMM INA

TRIFARINA

TRILOCUL INA

TROCHAMM INA

TROC HAMMINA

UVIGER INA

UVIGER INA

UVI GER INA

UVIGERINA

VALVUL INERIA

VAL VULINERI

AMMODISCUS

BOLIVIAA

BOLIVINA

BOLIVINA

BOLIVINA

BUL IMINA

BUL IMINA

BUL I I INA

BUL I M INA

CASSIDULINA

CASSI DULINA

CASSIDUL INA

CASSI DUL INA

CIBICIDES

EP I S TOMI NELLA

EPISTOMINELLA

EPONIDES

EPONIDES

EPONIDES

F ISSUR INA

GLOBOBUL IMINA

HAPLOPHRAGMOI DES

I SLAND IELLA

NONIONELLA

SPIROPLECTAMM INA

TEXTULARIA

THALMANNAMM IN A

TRIFAR INA

TR I LOCUL INA

UVIGER INA

UVIGER INA

VALVULINERIA

VAL VUL INER IA

AMMOBACULITES

AMMODISCUS

AMMOD I SCUS

AMPHI CORYNA

ASTACOLUS
NIT.IDA

BARTLETTI

EXCAVATUM

FR IGI DUM

EXIGUA

PACIFICA

VITREA

HE ALD I

LEVICULUS

SUBTENERA

UMBONATUS

CUCURBI TASEMA

LUCIDA

ADVENA

LOEBL I CH I

AURICULATA

PACIFICA

GEMMA

CALIFORNICA

HELENAE

LI MBA TA

NORCR OSS I

TRANSLUCENS

BACCATA

PARKERAE

AP IOPLEURA

ELONGATA

GRACILIS

LAEVIS

SEMIL INEA TA

SPICATA

AURICULA

STELLA

IURGIDA DIGITATA

LABRADORICA

BOREALIS

BOREALIS

LINEATA

STRIATOPUNCTATA

CORRUGATA

SALISBURYI

SP.

STALKERI

BI FORMIS

FLUENS

TR I HE ORA

GL OBI GER IN I F ORM IS

ROTALIFORMIS

AUBER IANA

JUNCEA

PEREGR INA

PEREGR INA DIRUPTA

ARAUC ANA

GL ABR A

MINUT ISSIMUS

ALATA

DE CUSSATA

PACIFICA

SPISSA

BARBATA

INFLATA MEXICANA

SUBAC UMINATA

TE NUA TA

BARBARA

CUSHMANI

SUBCARINATA

SUBGLOBOSA

FLETCHER I

PACIFICA

VI TREA

HE ALO I

LEVICULUS

SUBTENERA

LUCIDA

PACIFICA

SC I TULUM

NORCROSS I

ST ELLA

BI FORMIS

TORQUATA

PARKERAE

FL UEN S

TR I HE DRA

PEREGR INA

PEREGR INA DIRUPTA

ARAUC ANA

GLABRA

ARENARIUS

ARENACEUS

GULLMARENSIS

SCALARIS

OCCIDENTALIS

BERGEN O'NEIL 1979 BERGEN O NE IL 1979 
Lat. Long. Locality

GULF OF ALASKA GULF OF ALASKA GULF OF ALASKA GULF OF ALASKA GULF OF ALASKA GULF OF ALASKA GULF OF ALASKA GULF OF ALASKA GULF OF ALASKA GULF OF ALASKA GULF OF ALASKA GULF OF ALASKA GULF OF ALASKA GULF OF ALASKA GULF OF ALASKA GULF OF ALASKA GULF OF ALASKA GULF OF ALASKA GULF OF ALASKA GULF OF ALASKA GULF OF ALASKA GULF OF ALASKA GULF OF ALASKA GULF OF ALASKA GULF OF ALASKA GULF OF ALASKA GULF OF ALASKA GULF OF ALASKA GULF OF ALASKA GULF OF ALASKA GULF OF ALASKA GULF OF ALASKA GULF OF ALASKA GULF OF ALASKA GULF OF ALASKA GULF OF ALASKA GULF OF ALASKA GULF OF ALASKA GULF OF ALASKA GULF OF ALASKA GULF OF ALASKA GULF OF ALASKA GULF OF ALASKA GULF OF ALASKA GULF OF ALASKA GULF OF ALASKA GULF OF ALASKA GULF OF ALASKA GULF OF ALASKA GULF OF ALASKA GULF OF ALASKA GULF OF ALASKA GULF OF ALASKA GULF OF ALASKA GULF OF ALASKA GULF OF ALASKA GULF OF ALASKA GULF OF ALASKA GULF OF ALASKA GULF OF ALASKA GULF OF ALASKA GULF Of ALASKA GULF OF ALASKA GULF OF ALASKA GULF OF ALASKA GULF OF ALASKA GULF OF ALASKA GULF OF ALASKA GULF OF ALASKA ULF OF ALASKA GULF OF ALASKA GULF OF ALASKA GULF OF ALASKA GULF OF ALASKA GULF OF ALASKA GULF OF ALASKA GULF OF ALASKA GULF OF ALASKA GULF OF ALASKA GUF OF ALASKA GULF OF ALASKA GULF OF ALASKA GULF OF ALASKA GULF OF ALASKA GULF OF ALASKA GULF OF ALASKA GULF OF ALASKA GULF OF ALASKA GULF OF ALASKA GULF OF ALASKA GULF OF ALASKA GULF OF ALASKA GULF OF ALASKA GULF OF ALASKA GULF OF ALASKA
GULF OF ALASKA
Generic Name Specific Name

Publication

ASTACOLUS

ASTRONONION GALLOWAYI

BILOCULINELLA GLOBULA

BOLIVINA DECUSSATA

BOLIVINA

BOL IVINA

BULIMINA

CASSI DUL INA

CIBICIDES

CRIBROSTOMOIDES

CRIBROSTOMOIDES

CRIBROSTOMOIDES

CRUCIL OCULINA

CYCLOGYRA

DENDROPHYRA

DENTAL INA

DENTALINA

DENTALINA

DOROTHIA

EHRENBER GINA

ELPHIDIUM

ELPHIDIUM

EPISTOMINELLA

EPISTOMINELLA

FISSURINA

FISSUR INA

FIS SUR INA

FRONDI CULARIA

GAUDRYINA

GLABRATELLA

GLOBOBULIMINA

GOESELLA

GUESELLA

HAPLOPHRA GMOI DES

HAPLOPHRAGMOI DES

HAPLOPHRAGMOI DES

HYPERAMMINA

ISLANDIELLA

I SLAND IELLA

ISLANDIELLA

I SLANDIELLA

I SLANDIELLA

KARRERIELLA

LAGENA

AGENA

LAGENA

LAGENA

LAGENA

LAGENA

LAGENA

LAGENA

LAGENA

LENTI CUL INA

LENTI C UL INA

MARG INUL INA

MARG INUL INA

NONIONELLA

NONIONELLA

NON IONELLA

NONIONELLA

NON IONELLINA

OOL INA

OOL INA

OOL INA

OOLINA

OOL INA

OOL INA

PELOS IAA

POLYMORPHINA

PSAMMOSPHAERA

PSEUDONODOSAR IA

PSEUDOPOLYMORPHINA

PULLENIA

PYRGO

PYYRGO

PIYRGO

PYRGO

PYRGOELLA

QUINQUELOCULINA

QUINQUELOCULINA

QUINQUELOCULINA

REC TOBOL IVINA

RECURVOIDES

REOPHAX

REOPHAX

RHABDAMMINA

RHIZAMMINA

ROSAL INA

RUPERT IA

SACCAMMINA

SACCAMMINA

SACCORHIZA

SPIROSIGMOILINA

TRI FAR INA

TRI LOCULINA
DE CUSSATA

OCEANICA

SUBFUSIFORMIS

SUBGL OBOSA

LOBATULUS

CRASS I MARGO

JEFFREYS I I

VELERONIS

TR IAN GUL ARIS

IN VOL VENS

ARBORESCENS

BA GG I

DE CEPTA

SUBSOLUTA

BRADYANA.

COMPRESSA

EXCAVATUM

FRIGIDUM

PACIF I CA

VI TREA

AGASSI I I

CUCURBITASEMA

LUCIDA

GI GAS

ARENAR IA

WR I GH TII

AURICULATA

FL INT I I

OR IENT AL IS

PLANISSIMUM

SCI TULUM

SPHAE RILOCULUS

ELONGATA

CA LIF ORN I CA

HELENAE

LI MBATA

NORCROSS

TORTUOSA

BAC CATA

AMPHORA

DI STOMA

ELONGATA

HI SPI DULA

LAEVIS

MERIDIONALIS

PL IOCENICA

STRIATA

NI COBARENSIS

BACHE I I

GLABRA

PR IMAEVA

AUR I CULA

STELLA

TURGIDA

TURGIDA DIGITATA

LABRADORICA

HE XAGONA

LEVIGATA

ME LO

STRIA TOPUNCTATA

WI LL I AMS ON I

VARIABILIS

KINCA I DI

FUSCA

RADICULA

CHARLOTTENSIS

SALIS BURYI

ABYSSORUM

DEPRESSA

LU CERNULA

VESPER TILIO

SPHAERA

AGGLUT INATA

AKNER I ANA

SEMINULA

PORRECTA
CONTORTUS

CONTORTUS

INSECTUS

ABYSSORUM

IN DIV ISA

IS ABE LLEANA

STABILIS

ATLAN TICA

RAMOSA

TRILOCULARIS

DISTORTA

FL UENS

ROTUNDA
LINEA TOPUNCTATA

TODD LOW 1967

TODD LOW 1967

TODD LOW 196 ?

TODD LOW 1967

TODD LOW 9967 


\section{Lat. Long. Locality}

$5932 \mathrm{~N} 14236 \mathrm{~W}$ GULF OF ALASKA GULF OF ALASKA GULF OF ALASKA GULF OF ALASKA GULF OF ALASKA GULF OF ALASKA GULF OF ALASKA GULF OF ALASKA GULF OF ALASKA GULF OF ALASKA GULF OF ALASKA GULF OF ALASKA GULF OF ALASKA GULF OF ALASKA GULF OF ALASKA GULF OF ALASKA GULF OF ALASKA GULF OF ALASKA GULF OF ALASKA GULF OF ALASKA GULF OF ALASKA GULF OF ALASKA GULF OF ALASKA GULF OF ALASKA GULF OF ALASKA GULF OF ALASKA GULF OF ALASKA GULF OF ALASKA GULF OF ALASKA GULF OF ALASKA GULF OF ALASKA GULF OF ALASKA GULF OF ALASKA GULF OF ALASKA GULF OF ALASKA GULF OF ALASKA GULF OF ALASKA GULF OF ALASKA GULF OF ALASKA GULF OF ALASKA GULF OF ALASKA GULF OF ALASKA GULF OF ALASKA GULF OF ALASKA GULF OF ALASKA GULF OF ALASKA GULF OF ALASKA GULF OF ALASKA GULF OF ALASKA GULF OF ALASKA GULF OF ALASKA GULF OF ALASKA GULF OF ALASKA GULF OF ALASKA GULF OF ALASKA GULF OF ALASKA GULF OF ALASKA GULF OF ALASKA GULF OF ALASKA GULF OF ALASKA GULF OF ALASKA GULF OF ALASKA GULF OF ALASKA GULF OF ALASKA GULF OF ALASKA GULF OF ALASKA GULF OF ALASKA GULF OF ALASKA GULF OF ALASKA GULF OF ALASKA GULF OF ALASKA GULF OF ALASKA GULF OF ALASKA GULF OF ALASKA GULF OF ALASKA GULF OF ALASKA GULF OF ALASKA GULF OF ALASKA GULF OF ALASKA GULF OF ALASKA GULF OF ALASKA GULF OF ALASKA GULF OF ALASKA GULF OF ALASKA GULF OF ALASKA GULF OF ALASKA GULF OF ALASKA GULF OF ALASKA GULF OF ALASKA GULF OF ALASKA GULF OF ALASKA GULF OF ALASKA GULF OF ALASKA GULF OF ALASKA GULF OF ALASKA GULF OF ALASKA
Generic Name Specific Name

Publication

TROCHAMM INA

UVIGERINA

AMMODISCUS

ASTRONONION

BOL IV I AA

BOL IVINA

BOLIVINA

BUCCELLA

BUL IMINA

CAS SI DUL INA

CASSI DUL INA

CIBICIDES

C IBICIDES

CIBICIDES

CRIBROSTOMOIDES

DENDROPHYRA

ELPHIDIUM

EPISTOMINELLA

EP I S TOMINELLA

EPONIDES

FISSUR INA

FRANCESITA

GAUDRYINA

GLOBOBUL IMINA

HAPLOPHRAGMOIDES

I SLANDIELLA

I SLAND I ELLA

I SLANDIELLA

KARRER IELLA

KARRER IELLA

LAGENA

LAGENA

LAGENA

LAGENA

MARTINOTTIELLA

NONIONELLA

NONIONELLA

NONIONELLA

NONIONELLINA

OOL INA

OOL INA

PARAF ISS

PULLENIA

QUINQUEL OCULINA

THALMANNAMMINA

TRI FAR INA

UVIGER INA

UVIGERINA

A STRONONION

CIBICIDES

CIBICIDES

DYOCIBICIDES

ELPHIDIUM

ELPHIOIUM

EPISTOMINELLA

EPISTOMINELLA

EPONIDES

GAUDRYINA

GAVELI NOPSIS

GLABRA TELLA

I SLAND IELLA

ISLANDIELLA

I SLAND IELLA

I SLANDIELLA

KARRER IELLA

OOL INA

OOL INA

POLYMORPHINA

PULLENIA

QUINQUEL OCULINA

QUINQUEL.OCULINA

QUINQUEL OCULINA

QUINQUEL OCULINA

ROBERTINOIDES

ADERCOTRYMA

ASTACOLUS

ASTRONONION

BOLIVINA

BUCCELLA

BUL IMINELLA

CASSI DUL INA

CIBICIDES

CIBICIDES

CIBICIDES

CIBICIDES

DYOCIBICIDES

DYOCIBICIDES

ELPHIDIUM

ELPHIDIUM

ELPHIDIUM

ELPHI DIUM

EPISTOMINELLA

EPISTOMINELLA
ADVENA

ROTAL I FORMIS

PEREGR INA

MI NUT I SS I MUS

GALLOWAYI

ALATA

DE CUSSATA

PACIFICA

FR IGIDA

INUSITATA

TENUATA

BARBARA

DELICATA

LOBATULUS

MCKANNAI

JEFFREYSII

ARBORESCENS

EX CAVATUM

PACIFICA

VI TREA

LEVICULUS

LUCIDA

AOVENA

AURICULATA

SC I TULUM

CALIFORN I CA

NORCR OSS I

TRANSLUCENS

BACCATA

PARKERAE

EL ONGATA

PARRI

SPICATA

STRIATA

PR IMAEVA

AURICULA

STELLA

TURGIDA DIGITATA

LA BRA DORICA

HEXAGONA

STRIATOPUNCTATA

FUSIL I FORMIS

SALISBURY I

STALKERI

DISTORTA

PARKERAE

FLUENS

PEREGR INA

GALLOWAYI

FLETCHER I

LOBATULUS

BISERIALIS

EXCAVATUM

HUGHES I

PACIFICA

VITREA

REPANDUS

ARENARIA

PRAEGERI

ORNAT ISSIMA

CALIF ORN I CA

LI MBATA

NORCROSS I

TORTUOSA

BACCATA

BOREALIS

HEXAGONA

KINEA I D I

SA.L.IS BURY I

AGGLUTINATA

ARCTICA

SEMINULA

CHARL OTTENSIS

GL OMERATA

HYALACRULUS

GALLOWAYI

FRIGIDA

ELEGANTIS SIMA

BARBARA

FLETCHER I

LOBATULUS

MCKANNAI

BA GGI

BISER I ALIS

BARTLETTI

EXCAVATUM

FRIGIDUM

HU GHES I

PACIFICA

VITREA
DE CUS SAT

TODD LOW 1967

TODD LOW 1967

TODD LOW 1967 


\section{Lat. Long. Locality}

$5942 \mathrm{~N} 14410 \mathrm{~W}$

$5945 N 14455 \mathrm{~W}$
GULF OF ALASKA GULF OF ALASKA GULF OF ALASKA GULF OF ALASKA GULF OF ALASKA GULF OF ALASKA GULF OF ALASKA GULF OF ALASKA GULF OF ALASKA GULF OF ALASKA GULF OF ALASKA GULF OF ALASKA GULF OF ALASKA GULF OF ALASKA GULF OF ALASKA GULF OF ALASKA GULF OF ALASKA GULF OF ALASKA GULF OF ALASKA GULF OF ALASKA GULF OF ALASKA GULF OF ALASKA GULF OF ALASKA GULF OF ALASKA GULF OF ALASKA GULF OF ALASKA GULF OF ALASKA GULF OF ALASKA GULF OF ALASKA GULF OF ALASKA GULF OF ALASKA GULF OF ALASKA GULF OF ALASKA GULF OF ALASKA GULF OF ALASKA GULF OF ALASKA GULF OF ALASKA GULF OF ALASKA GULF OF ALASKA GULF OF ALASKA GULF OF ALASKA GULF OF ALASKA GULF OF ALASKA GULF OF ALASKA GULF OF ALASKA GULF OF ALASKA GULF OF ALASKA GULF OF ALASKA GULF OF ALASKA GULF OF ALASKA GULF OF ALASKA GULF OF ALASKA GULF OF ALASKA GULF OF ALASKA GULF OF ALASKA GULF OF ALASKA GULF OF ALASKA GULF OF ALASKA GULF OF ALASKA GULF OF ALASKA GULF OF ALASKA GULF OF ALASKA GULF OF ALASKA GULF OF ALASKA GULF OF ALASKA GULF OF ALASKA GULF OF ALASKA GULF OF ALASKA GULF OF ALASKA GULF OF ALASKA GULF OF ALASKA GULF OF ALASKA GULF OF ALASKA GULF OF ALASKA GULF OF ALASKA GULF OF ALASKA GULF OF ALASKA GULF OF ALASKA GULF OF ALASKA GULF OF ALASKA GULF OF ALASKA GULF OF ALASKA GULF OF ALASKA GULF OF ALASKA GULF OF ALASKA GULF OF ALASKA GULF OF ALASKA GULF OF ALASKA GULF OF ALASKA GULF OF ALASKA GULF OF ALASKA GULF OF ALASKA GULF OF ALASKA GULF OF ALASKA GULF OF ALASKA GULF OF ALASKA
Generic Name Specific Name

\section{Publication}

EPONIDES

FISSURINA

FISSURINA

FISSURINA

GLABRA TELLA

GLOBOBUL IMINA

HAPLOPHRAGMOI DES

ISLANDIELLA

I SLAND IELLA

ISLANDIELLA

ISLANDIELLA

KARRERIELLA

LAGENA

LAGENA

LAGENA

LAGENA

LAGENA

LAGENA

LAGENA

LARYNGOS I GMA

NONIONELLA

NONIONELLA

NONIONELLA

NONI ONELLINA

OOL INA

OOL INA

PSEUDOPOLYMORPHINA PULLENIA

PYRGO

QUINQUELOCULINA

QUINQUELOCULINA REOPHAX

ROBERTINOIDES

SIGMOMORPHINA

TRIFAR INA

TROCHAMM INA

UVIGER INA

A DERCOTRYMA

AMMODISCUS

ASTRONONION

BOL IVINA

BOL IVIAA

BUCCELLA

BUL IMINELLA

CASSIDULINA

CIBICIDES

CIBICIDES

CIBICIDES

CRIBROSTOMOIDES

CRIBROSTOMO IOES

EGGERELLA

ELPHIDIELLA

ELPHIOIUM

ELPHIOIUM

ELPHIOIUM

ELPHIDIUM

EPISTOMINELLA

EPISTONINELLA

EPISTOMINELLA

EPONIDES

FISSURINA

FIS SUR INA

FISSUR INA

GLOBOBUL IMINA

GLOBOBUL ININA

HAPLOPHRAGMOIDES

I SLANDIELLA

ISLANOIELLA

I SLANOIELLA

I SLAND IELLA

KARRER IELLA

LAGENA

LAGENA

LAGENA

LAGENA

LAGENA

LAGENA

NONIONELLA

NONIONELLA

NONIONELLA

NON I ONELL INA

OOL INA

OOL INA

PULLENIA

QUINQU

REOPHAX
SPIROPLECTAMM INA

SPIROPLECTAMM I
THALMANNAMM INA

TR I FAR INA

TROCHAMMINA

TROCHAMMINA

TROCHAMMINA

REPANDUS

LUCIOA

LUCIOA

LUCIDA

ORNAT ISS IMA

AURICULATA

SC I TULUM

CALIFORNICA

LIMBATA

NORCROSS I

TORTUOSA

BACCATA

AP I OP LEURA

ELONGAT

GRACILIS

LAEVIS

SEMIL I NEA TA

SPICA TA

STRIATA

AURICULA

STELLA

TURGIDA OIGITATA

LAERADORI CA

HEXAGONA

STRIA TOPUNCTATA

CHARL OTTENSIS

SALISBURYI

MURRH INA

$S P$.

STALKERI

SCORPIURUS

CHARL OTTENSIS

GALLOWAYI

FLUENS

RO TAL I FORMIS

JUNCEA

GL OME RATA

MINUT I SSIMUS

GALLOWAYI

DE CUSSATA

PACIFICA

FRIGIDA

ELEGANTIS SIMA

BARBARA

DELICATA

FLETCHER

LOBATULUS

MCKANNAI

JEFFREYSII

ADVENA

NITIOA

BARTLETTI

EXCAVATUM

FR I G I DUM

HU GHES I

PACIFICA

PACIFICA

VI TREA

LEVICULUS

AGASS I II

LUCIOA

MARGINATA

AURICULATA

PACIFICA

SCITULUM

CALIFORNICA

HELENAE

LIMBA TA

NORCROSS I

BACCATA

AP IOPLEURA

LAEVIS

MERIOIONALIS

PARRI

SEMIL INEA TA

SPICATA

STRIATA

AURICULA

STELLA

TURGIOA DIGITATA

LABRADORICA

BOREALIS

STRIATOPUNCTATA

SALIS BURYI

ST ALKERI

SC ORPIURUS

BI FORMIS

PARKERAE

FLUENS

ADVENA

IN FLATA 


Lat. Long.
$5945 \mathrm{~N} 14455 \mathrm{~W}$
$5945 \mathrm{~N} 14600 \mathrm{~W}$

$5950 N 14547 \mathrm{~W}$

$5955 \mathrm{~N} 14225 \mathrm{~W}$

$5955 \mathrm{~N} 14540 \mathrm{~W}$
Locality

GULF OF ALASKA GULF OF ALASKA GULF OF ALASKA GULF OF ALASKA GULF OF ALASKA GULF OF ALASKA GULF OF ALASKA GULF OF ALASKA GULF, OF ALASKA GULF OF ALASKA GULF OF ALASKA GULF OF ALASKA GULF OF ALASKA GULF OF ALASKA GULF OF ALASKA GULF OF ALASKA GULF OF ALASKA GULF OF ALASKA GULF OF ALASKA GULF OF ALASKA GULF OF ALASKA GULF OF ALASKA GULF OF ALASKA GULF OF ALASKA GULF OF ALASKA GULF OF ALASKA GULF OF ALASKA GULF OF ALASKA GULF OF ALASKA GULF OF ALASKA GULF OF ALASKA GULF OF ALASKA GULF OF ALASKA GULF OF ALASKA GULF OF ALASKA GULF OF ALASKA GULF OF ALASKA GULF OF ALASKA GULF OF ALASKA GULF OF ALASKA GULF OF ALASKA GULF OF ALASKA GULF OF ALASKA GULF OF ALASKA GULF OF ALASKA GULF OF ALASKA GULF OF ALASKA GULF OF ALASKA GULF OF ALASKA GULF OF ALASKA GULF OF ALASKA GULF OF ALASKA GULF OF ALASKA GULF OF ALASKA GULF OF ALASKA GULF OF ALASKA GULF OF ALASKA GULF OF ALASKA GULF OF ALASKA GULF Of ALASKA GULF OF ALASKA GULF Of ALASKA GULF Of ALASKA GULF OF ALASKA GULF OF ALASKA GLLF OF ALASKA GULF OF ALASKA GULF OF ALASKA GULF OF ALASKA GULF OF ALASKA GULF OF ALASKA GULF OF ALASKA GULF OF ALASKA GULF OF ALASKA GULF OF ALASKA GULF OF ALASKA GULF OF ALASSKA GULF OF ALASKA GULF OF ALASKA GULF OF ALASKA GULF OF ALASKA GULF OF ALASKA GULF OF ALASKA GULF OF ALASKA GULF OF ALASKA GULF OF ALASKA GULF OF ALASKA GULF of ALASKA GULF OF ALASKA GULF OF ALASKA GULF OF ALASKA GULF OF ALASKA GULF OF ALASKA GULF OF ALASKA GULF OF ALASKA GULF OF ALASKA
Generic Name Specific Name

Publication

UVIGERINA

UVIGERINA

ADERCOTRYNA

ASTRONONION

BUCCELLA

ELPHIDIUM

EPISTOM INELLA

EPISTOMINELLA

EPONIDES

FIS SUR INA

I SLANDIELLA

I SLAND IELLA

I SLANDIELLA

LAGENA

NONIONELLA

NON I ONELL INA.

THAL MANNAMM INA

TRI F AR INA

UVI GER INA

BUCCELLA

BUL I M INELLA

EGGERELLA

ELPHIDIUM

ELPHIDIUM

EPISTOMINELLA

EPISTOMINELLA

I SLANDIELLA

NONIONELLA

NONI ONEL L INA

UVI GE R INA

AMPHI CCRYNA

ASTRONONION

BUCCELLA

BUL IMINE LLA

CAS SI DUL INA

CIBICIDES

CIBICIDES

CRIBROSTOMOIDES

CRIBR OSTOMOIDE

EGGERELLA

ELPHIDIEL:A

ELPHIDIUM

ELPHI DIUM

EPISTOMINELLA

EPISTOMINELLA

EPONIDES

FISSUR INA

GLOBOBUL I MINA

GLOBOBULIMINA

HAPLOPHRAGMOIDES

I SLAND IELLA

I SLANDIELLA

I SLANDIELLA

I SL ANDIELLA

KARRER IELLA

LAGENA

LAGENA

LAGENA

LAGENA

NONIONELLA

NONIONELLA

NONIONELLA

NON IONELL INA

QUINQUELOCULINA

REOPHAX

REOPHAX
SPI ROPLEC TAMM INA

THALMANNAMM IN A

I? IFAR INA

UVIGER INA

UVI GER INA

AMPHICORYNA

BUCCELLA

CASSI DUL INA

CIBICIDE

CIBICIDES
ELPHIDIELLA

ELPHIDIELLA

ELPHI DIUM

EPONIDES

F ISSUR INA

GAUDRYINA

GLABRA TELLA

I SLAND IEL LA

I SL ANDIELLA

I SLANDIELLA

KARRER IELLA

LAGENA

NONIONELLA

OOL INA

OOL INA

POLYMORPHINA
JUNCEA

PEREGR INA

GL OME RATA

GALLOWAYI

FRIGIDA

EXCAVATUM

HU GHES I

EXIGUA

VI TREA

LEVICULUS

LUCIDA

CALIFORNICA

LI MBATA

NORCROSS I

SEMIL INEATA

STELLA

LA BRA DOR I CA

PARKERAE

FLUENS

JUNCEA

FR IGIDA

ELEGANTISSIMA

ADVENA

EXCAVATUM

FR I GI DUM

PACIFICA

VI TRE A

NORCROSSI

STELLA

LABRADORICA

JUNCEA

GL OMERATA

SCALARIS

GALLOWAYI

FRIGIDA

ELEGANTISSIMA

BARBARA

FLETCHER I

LOBATULUS

CR ASS IMARGO

JEFFREYSI I

ADVENA

NI TIDA

EXCAVATUM

FR I GI DUM

PACIFICA

VITREA

LEVICULUS

LUCIDA

AURICULATA

PACIFICA

SCITULUM

CALIFORNICA

HELENAE

LI MBATA

NORCROSS I

BACCATA

LAEVIS

PARRI

SEMIL INEATA

STRIATA

AURICULA

AURICULA

STELLA

TURGIDA DIGITATA

LABRADORICA

$S P$.

SC ORPI URUS

BI FORMIS

PARKERAE

FLUENS

JUNCEA

PEREGRINA

SCALARIS

FRIGIDA

BARBARA

FLETCHERI

LOBATULU
NITIDA

ARTICULATUM

BARTLETTI

REPAN DU

LUCIDA

ARENARIA

ORNAT ISS IMA

CALIF ORNICA

LIMBATA

NORCROSSI

BACCATA

AP IOPLEURA

STRIATA

AURICULA

BOREALIS

AEVI GAT 
Lat. Long. Locality

$5955 N 14540 \mathrm{~W}$

$5956 \mathrm{~N} 14425 \mathrm{~W}$

$60 \quad 15 N 14531 \mathrm{~W}$ $6028 \mathrm{~N} 14627 \mathrm{~W}$

$6030 \mathrm{~N} 14600 \mathrm{~W}$

$6035 N 14544 \mathrm{~W}$

$6037 \mathrm{~N} 14605 \mathrm{~W}$

$6040 \mathrm{~N} 14553 \mathrm{~W}$

$6107 N 140 \quad 17 \mathrm{~W}$ OF F ALASKA
GULF OF ALASKA GULF OF ALASKA GULF OF ALASKA GULF OF ALASKA GULF OF ALASKA GULF OF ALASKA GULF OF ALASKA GUULF OF ALASKA GULF OF ALASKA GULF OF ALA GULF OF ALASKA GULF $O F$ GULF OF ALASKA GULF OF ALASKA GULF OF ALASKA GULF OF ALASKA GULF OF ALASKA GULF OF ALASKA GULF OF ALASKA GULF OF ALASKA GULF OF ALASKA GULF OF AL ASKA GULF OF ALASKA GULF OF ALASKA GULF OF ALASKA GULF OF ALASKA GULF OF ALASKA GULF OF ALASKA GULF OF ALASKA GULF OF ALASKA GULF OF ALASKA GULF OF ALASKA GULF OF ALASKA
GULF OF ALASKA COPPER R.. ALASKA OF $F$ ALASKA OF F ALASKA OF F ALASKA OF $F$ ALASKA OF F ALASKA OFF ALASKA OF F ALASKA OFF ALASKA OFF ALASKA
OFF ALASKA OF $F$ ALASKA OF $F$ ALASKA OF $F$ ALASKA OFF ALASKA OF F ALASKA OF F ALASKA OFF ALASKA OF $F$ ALASKA OF $F$ ALSAKA OF F ALASKA OF $F$ ALASKA OF $F$ ALASKA OF F ALASKA OF F ALASKA OF F ALASKA OF F ALASKA OF $F$ ALASKA OF $F$ ALASKA

\section{Generic Name Specific Name}

SALIS BURYI

MURRHINA

$S P$.

PYRGO

QUINQUEL OCULINA

QUINQUELOCULINA

SIGMOMORPHINA

TRIFARINA

TROCHAMMINA

UVIGERINA

ELPHIDIELLA

ELPHIDIUM

ELPHIDIUM

ELPHIDIUM

I SLANDIELLA

NONIONELLA

BUCCELLA

BUCCELLA

BUL I M INELLA

CASSI DUL INA

CASSIDUL INA

C IB I CIDES

CIBICIDES

CRIBROSTOMOIDES

DENDROPHYRA

ELPHIDIELLA

ELPHIDIUM

ELPHIDIUM

ELPHIDIUM

ELPHIDIUM

GLABRATELLA

I SLANDIELLA

ISLANDIELLA

MILIOL INELLA

NON I ONELL A

TROCHAMM I NA

ELPHIDIELLA

OOL INA

AMMOTIUM

EGGERELLA

ELPHIDIELLA

HAPLOPHRAGMOI DES

REOPHAX

REOPHAX

TROCHAMMINA

AMMOT I UM

BUL IM I NELL LA

CRIBR OSTOMO IDES

EGGERELLA

ELPHIDIUM

ELPHIDIUM

HAPLOPHRAGMOI DES

NON IONELLA

REOPHAX

TROCHAMMINA

TROCHAMMINA

ELPHIDIELLA

ELPHIDIUM

CRIBR OSTOMOIDES

CRIBROST

LAGENA

LAGENA
LAGENA

LAGENA

REOPHAX

TROCHAMM INA
ST ALKERI

GALLOWAY

FL UENS

ROTAL IFORMIS

JUNCEA

BARTLETTI

EXCAVATUM

HU GHES I

HELENAE

ST ELLA

FRIGIDA

INUSI TATA

ELEGANTISSIMA

BARBARA

DELICATA

FLETCHER I

LOBATULUS

JEFFREYSI I

AR BORESCENS

NI TIDA

AR TIC ULATUM

BARTLETTI

EXCAVATUM

HUGHES I

ORNAT ISS IMA

HELENAE

LI MBAT A

SUBROTUNDA

ST ELLA

IN FLATA

HANNAI

STRIATOPUNCTATA GEMMA

CASSIS

ADVEN A

HANNA I

COLUMBIENSIS

EXCENTRICUS

SUBFUS IFORMIS

NITIDA

CASSIS

ELEGANTISSIMA

JEFFREYSII

AO VENA

ARTICULATUM

TUMIDUM

COLUMBIENSIS

STELL

SUBFUSIFORMIS

NI TIDA

PACIFICA

HANNA I

TUMIDUM

SUBGL OBOSUM

DISTOMA

PERLUCIDA

SEMIL I NEATA

SCOTTII

SUBFUS IFORMIS

NI TIOA
Publication

BERGEN O'NE IL 1979 BERGEN O'NE IL 1979 BERGEN O'NE IL 1979 BERGEN O'NEIL 1979 BERGEN O'NEIL 1979 BERGEN O'NEIL 1979 BERGEN O'NEIL 1970 BERGEN O'NE IL 1979 BERGEN O'NE IL 1979 BERGEN O'NE IL 1979 BERGEN O'NEIL 1979 BERGEN O'NEIL 1979 BERGEN OONE GERGEN O'NEIL 1979 BERGEN O'NEIL 1979 BERGEN O'NEIL 1979 BERGEN O'NEIL 1979 BERGEN O'NE IL 1979 BERGEN O'NE IL 1970 BERGEN O'NE IL 1979 BEREN O'NE IL 1979 BERGEN O'NE IL 1979 GERGEN O'NEIL 1979 BERGEN O'NEIL 1979 BERGEN O'NEIL 1979 BERGEN O'NEIL 1979 BERGEN O'NEIL 1979 BERGEN O'NE IL 1979 BERGEN O'NE IL 1979 BERGEN O'NE IL 1979 BERGEN OONE IL 1979 BERGEN O'NEIL 1979 BERGEN O'NE IL 1979 BERGEN O'NEIL 1979 PHLEGER 196?

C USHMAN MCCULLOCH 1940 CUSHMAN MCC ULLOCH 1950 CUSHMAN MCCULLOCH 1939 CUSHMAN MCC ULLOCH 1939 CUSHMAN MCC ULLOCH 1940 CUSHMAN MCCULLOCH 1939 CUSHMAN MCC ULLOCH 1939 CUSHMAN MCC ULLOCH 1939 CUSHMAN MCC ULLOCH 1939 CUSHMAN MCCULLOCH 1939 CUSHMAN MCCULLOCH 1948 CUSHMAN MCCULLOCH 1939 CUSHMAN MCCULLOCH 1939 CUSHMAN MCC ULLOCH 1940 CUSHMAN MCC ULLOCH 1940 CUSHMAN MCC ULLOCH 1939 CUSHMAN MCCULLOCH 1940 CUSHMAN MCCULLOCH 1939 CUSHMAN MCCULLOCH 1939 CUSHMAN MCCULLOCH 1939 CUSHMAN MCCULLOCH 1940 CUSHMAN MCC ULLOCH 1940 CUSHMAN MCCULLOCH 1930 CUSHMAN MCC ULLOCH 1939 CUSHMAN MCCULLOCH 1950 CUSHMAN MCC ULLOCH 1950 CUSHMAN MCCULLOCH 1939 CUSHMAN MCCULLOCH 1939 CUSHMAN MCC ULLOCH 1939 
MAPS: Figures 1-139

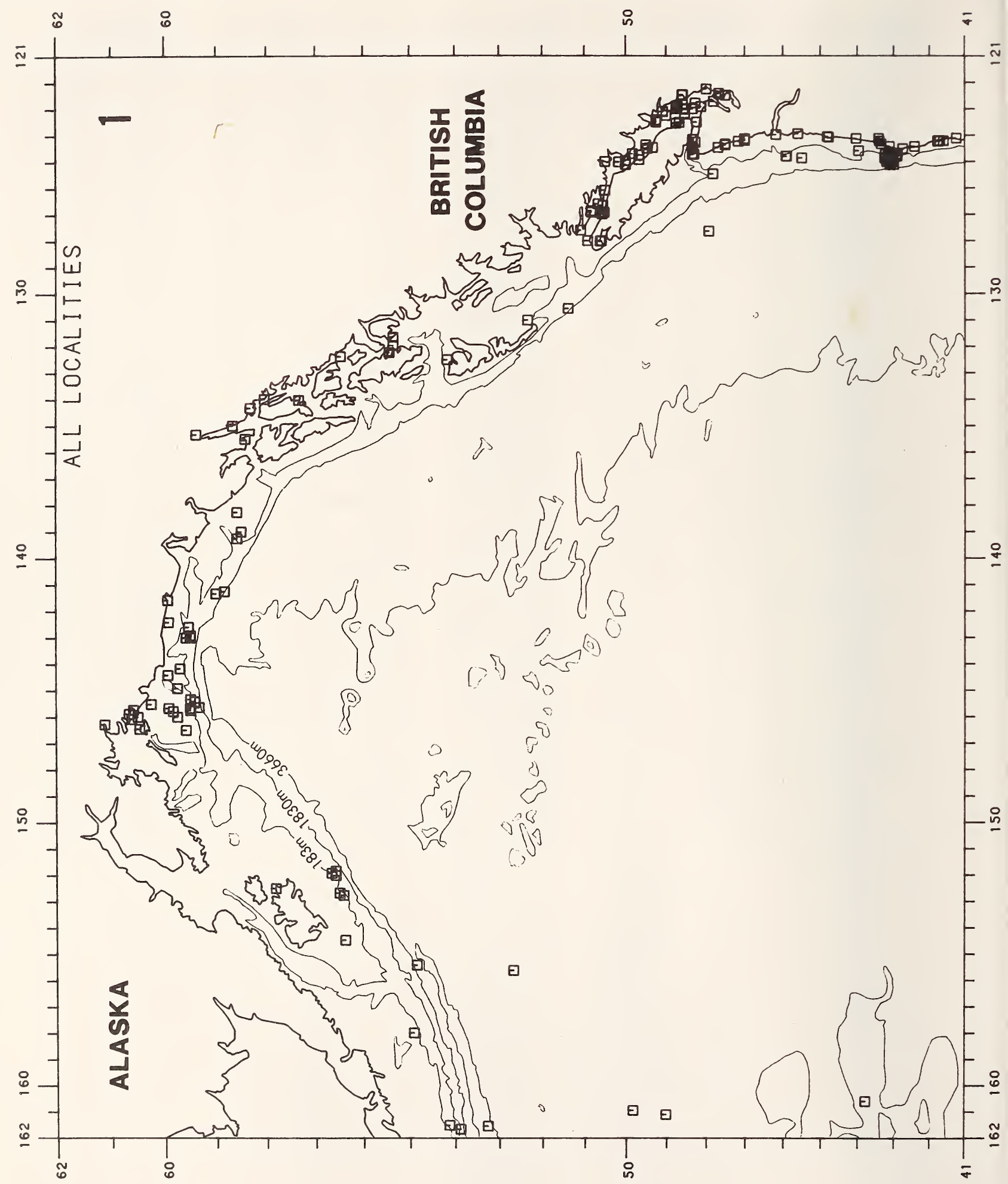




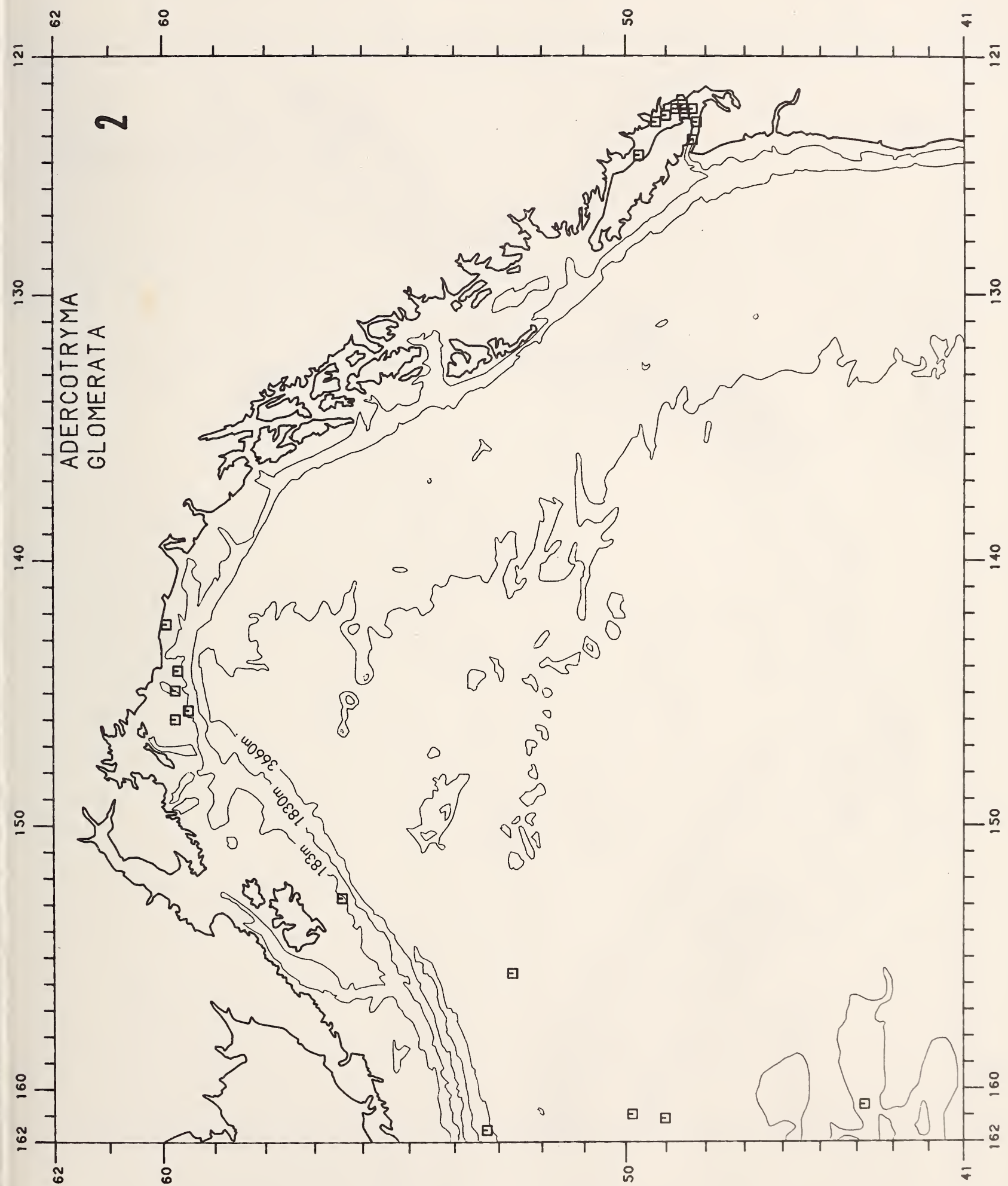




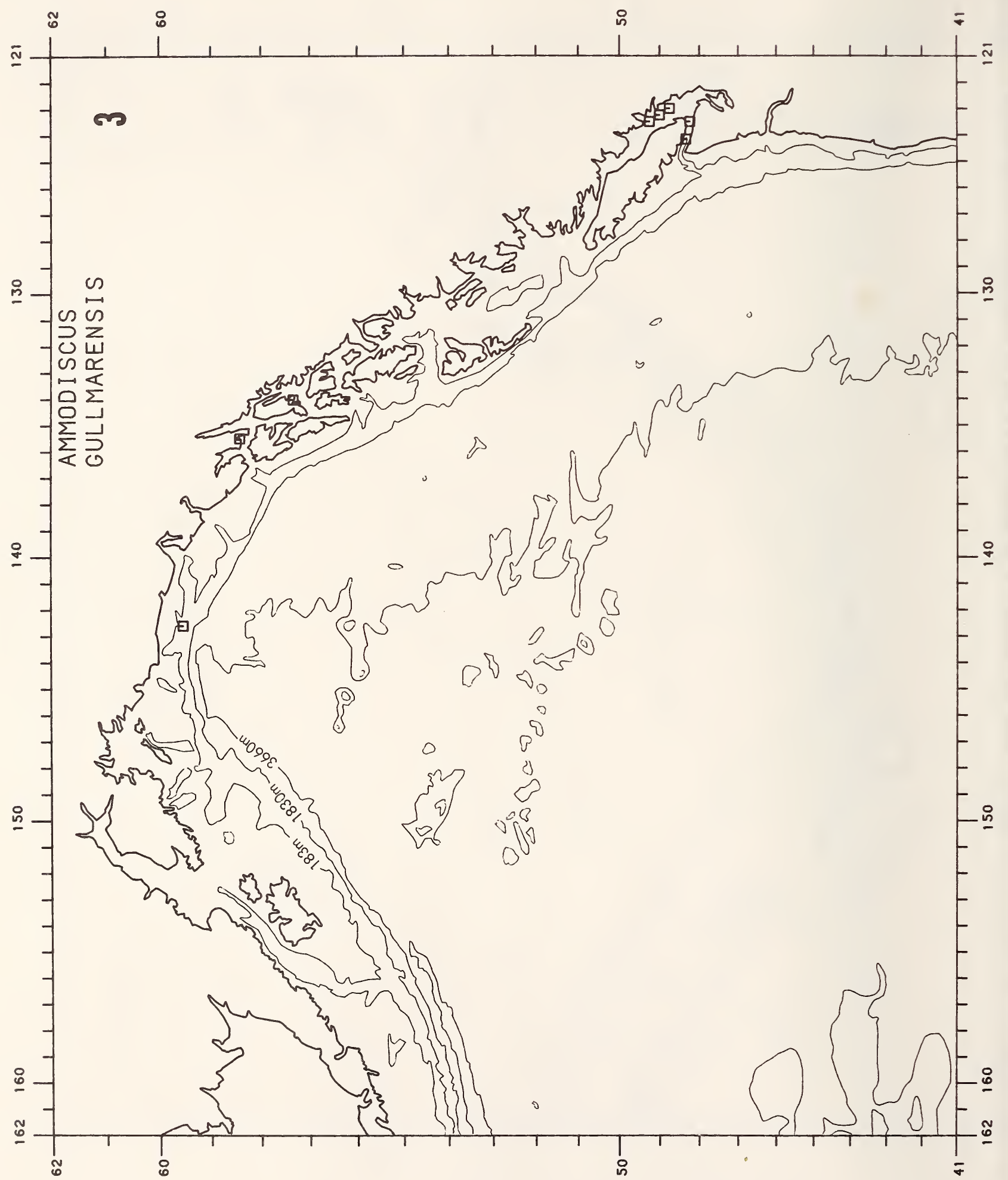




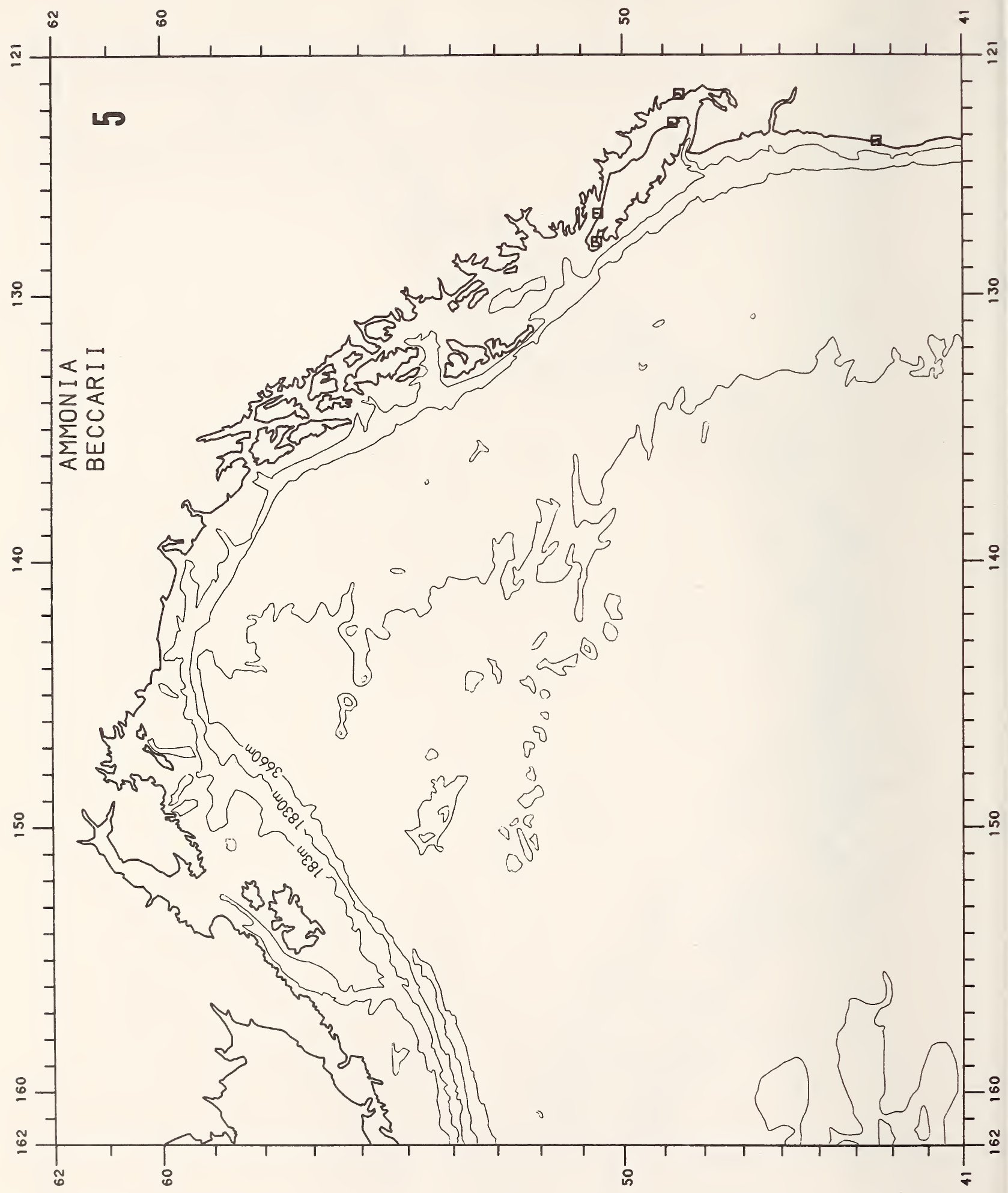




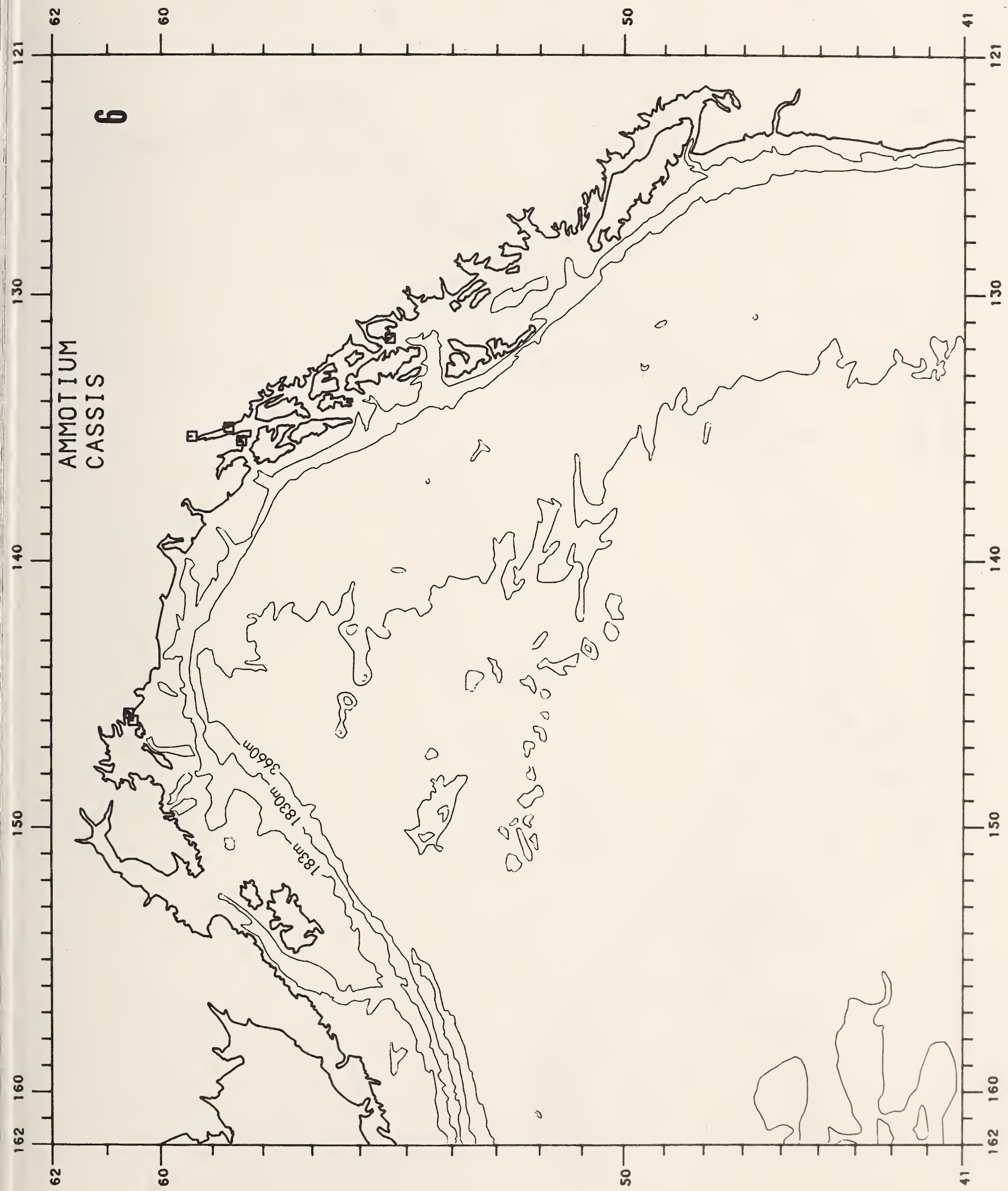




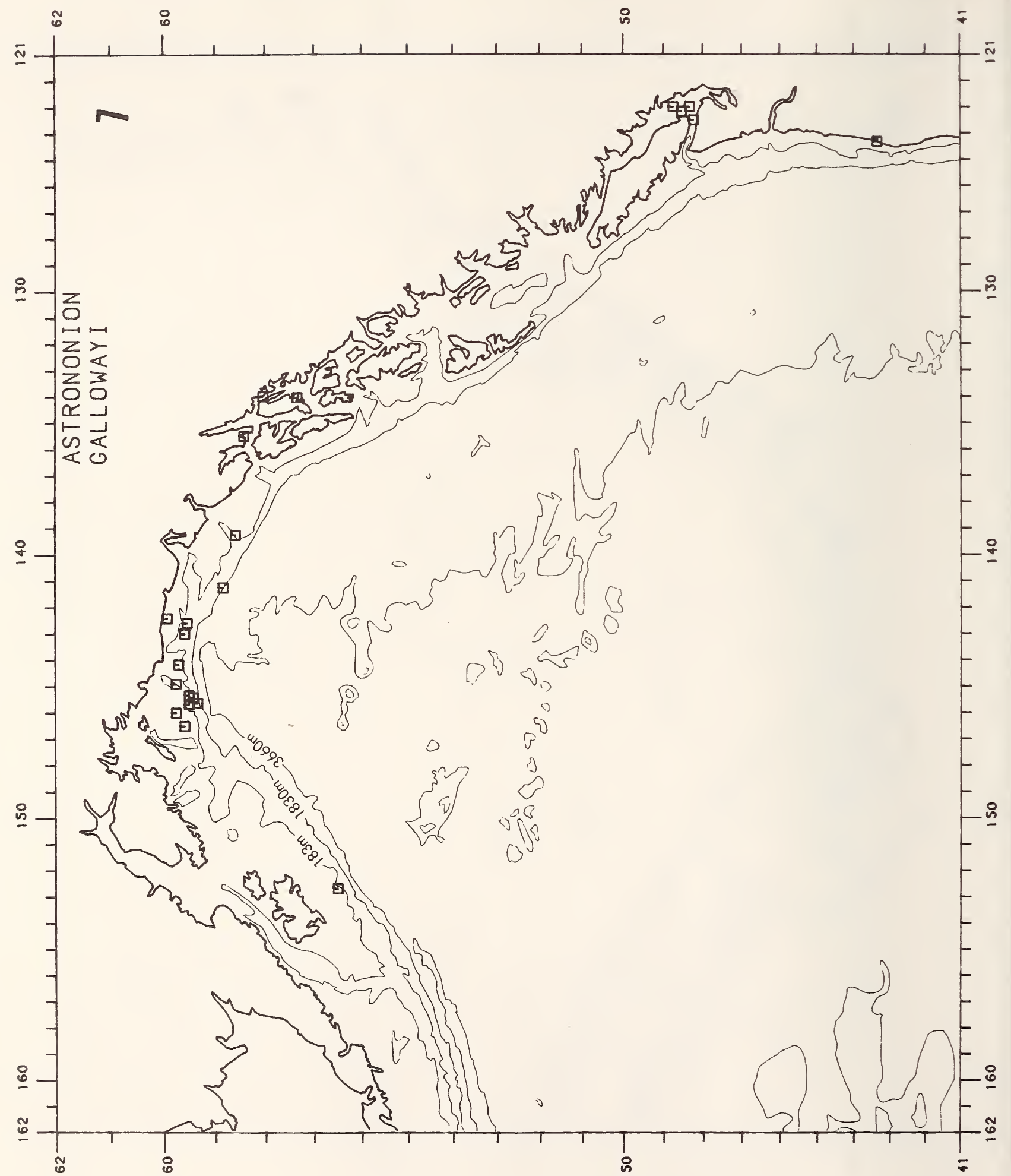




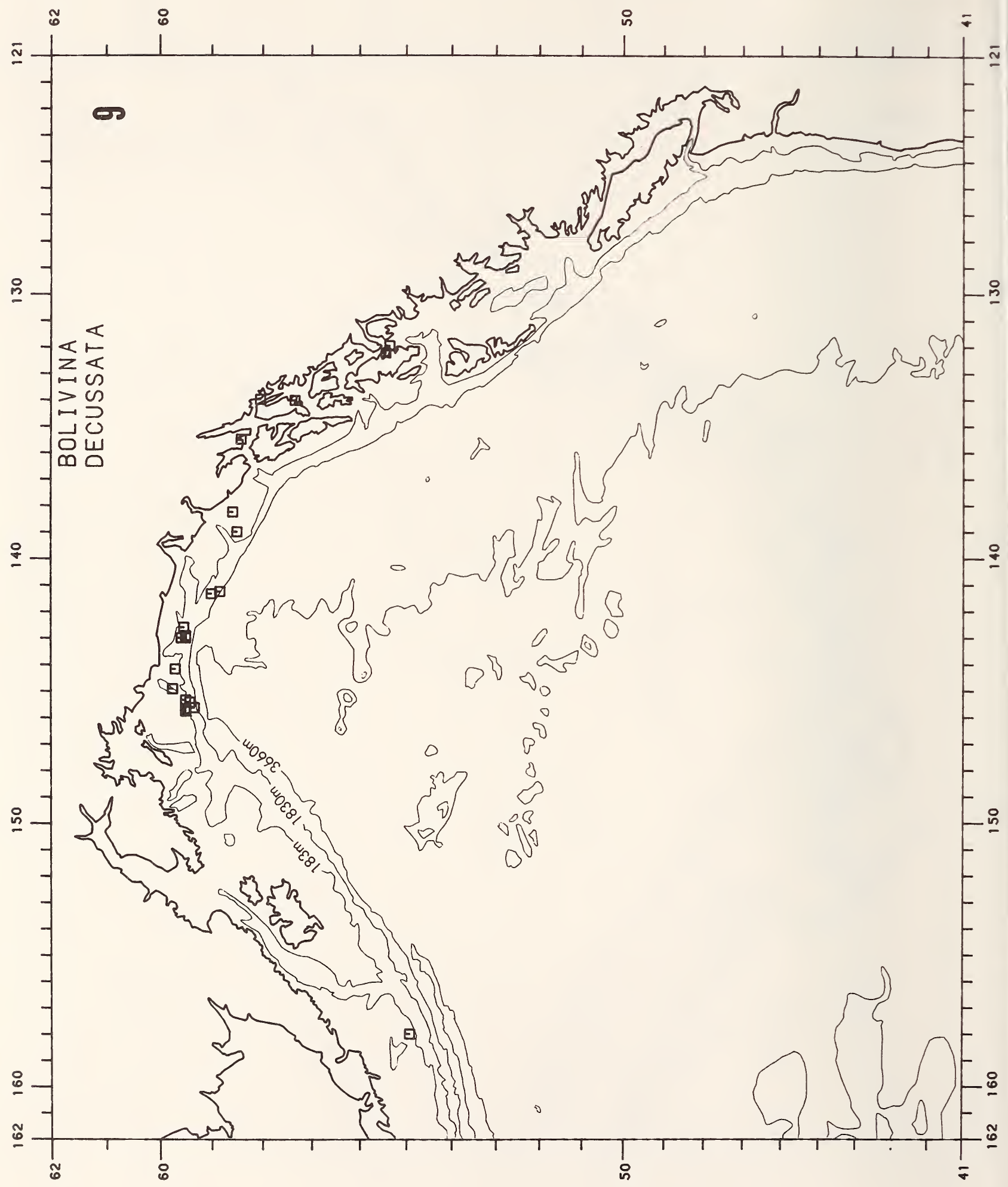




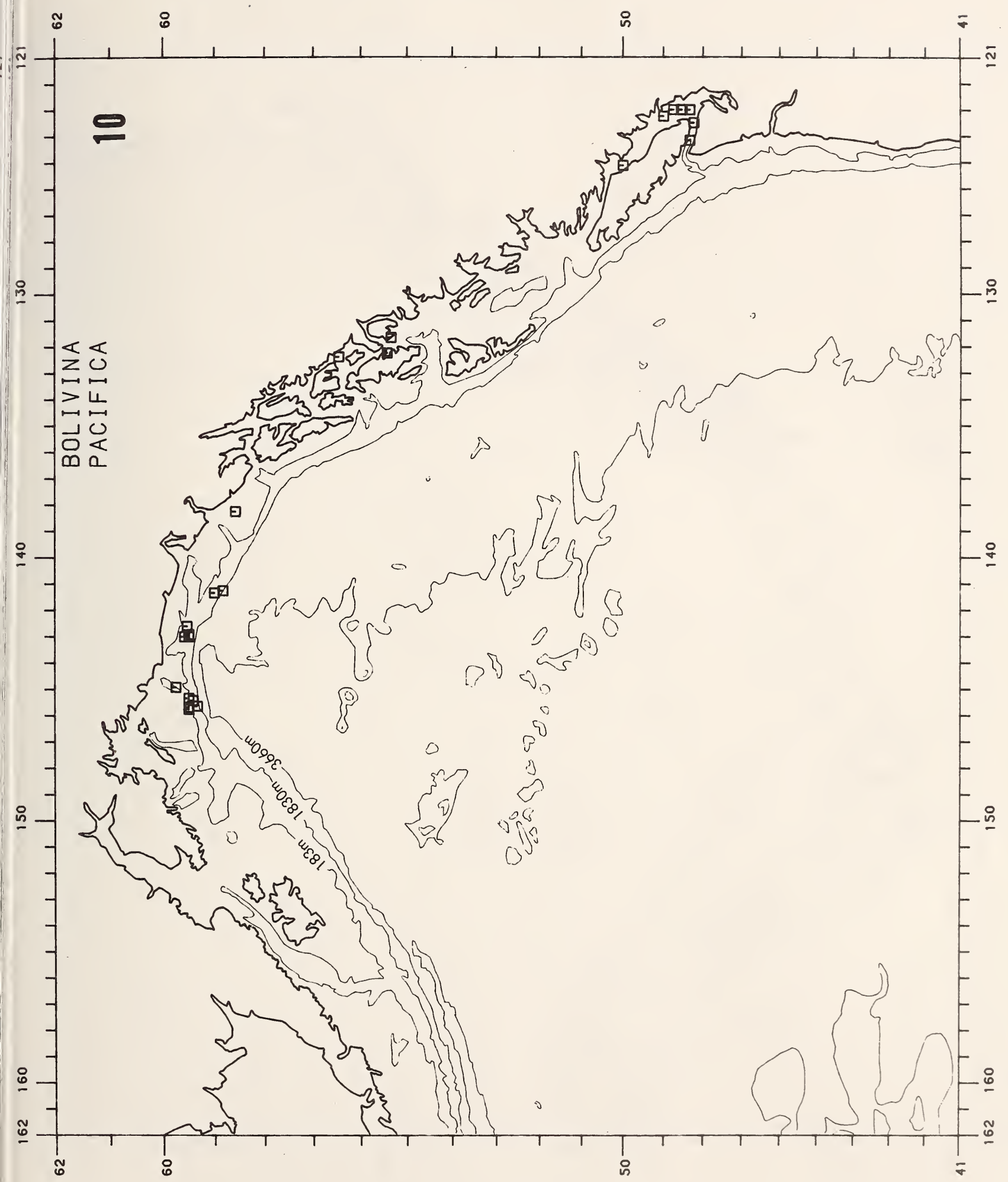




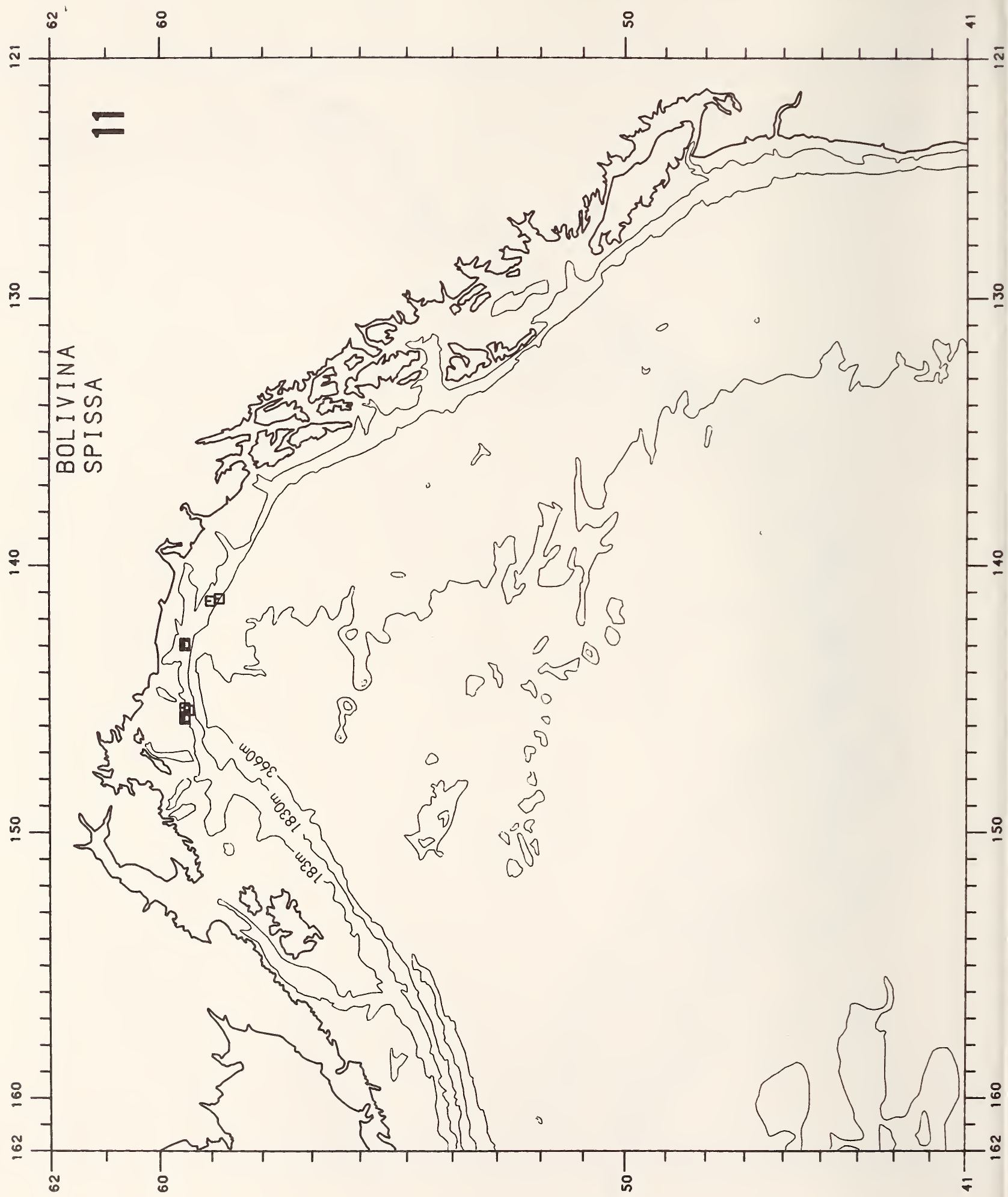




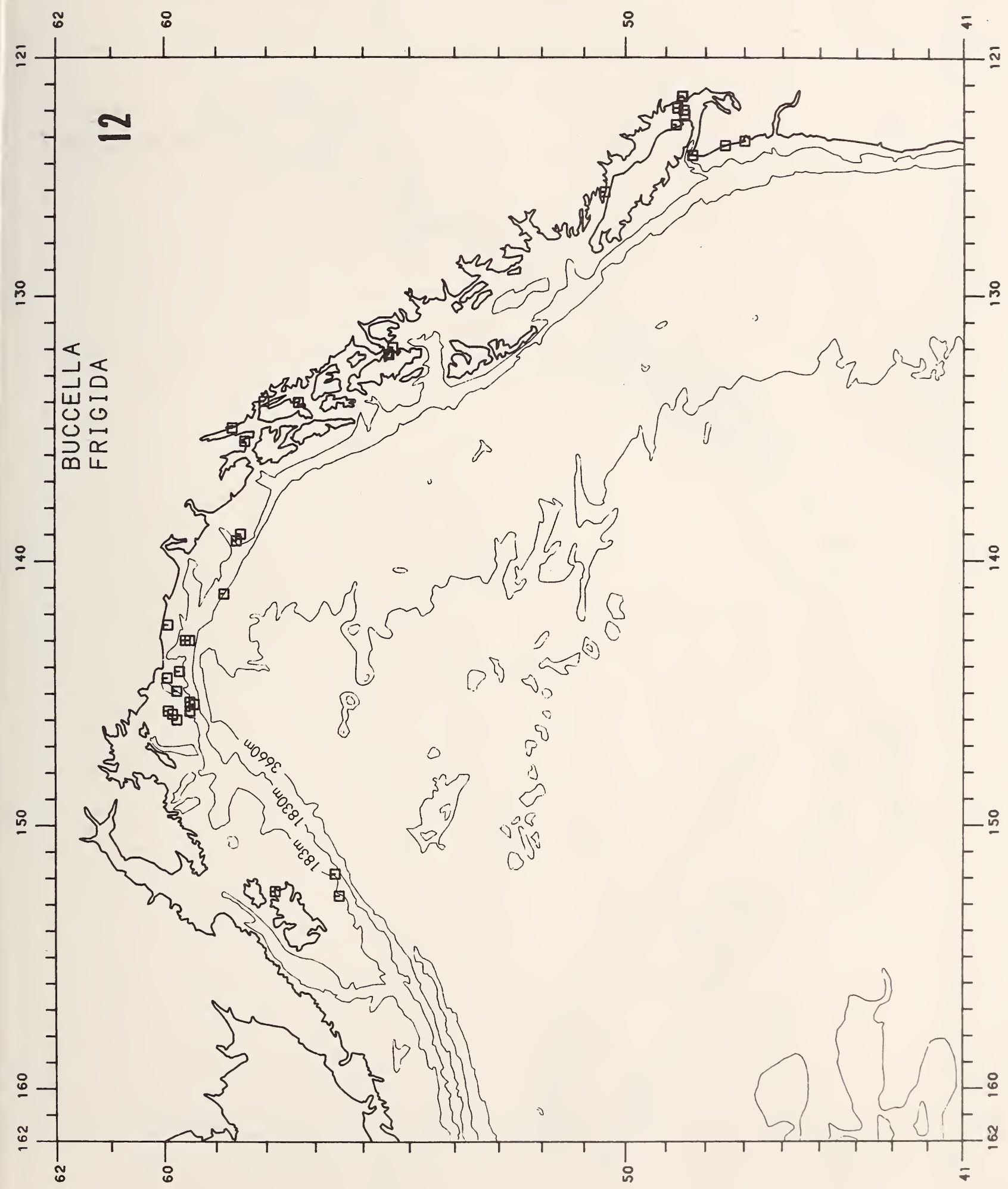




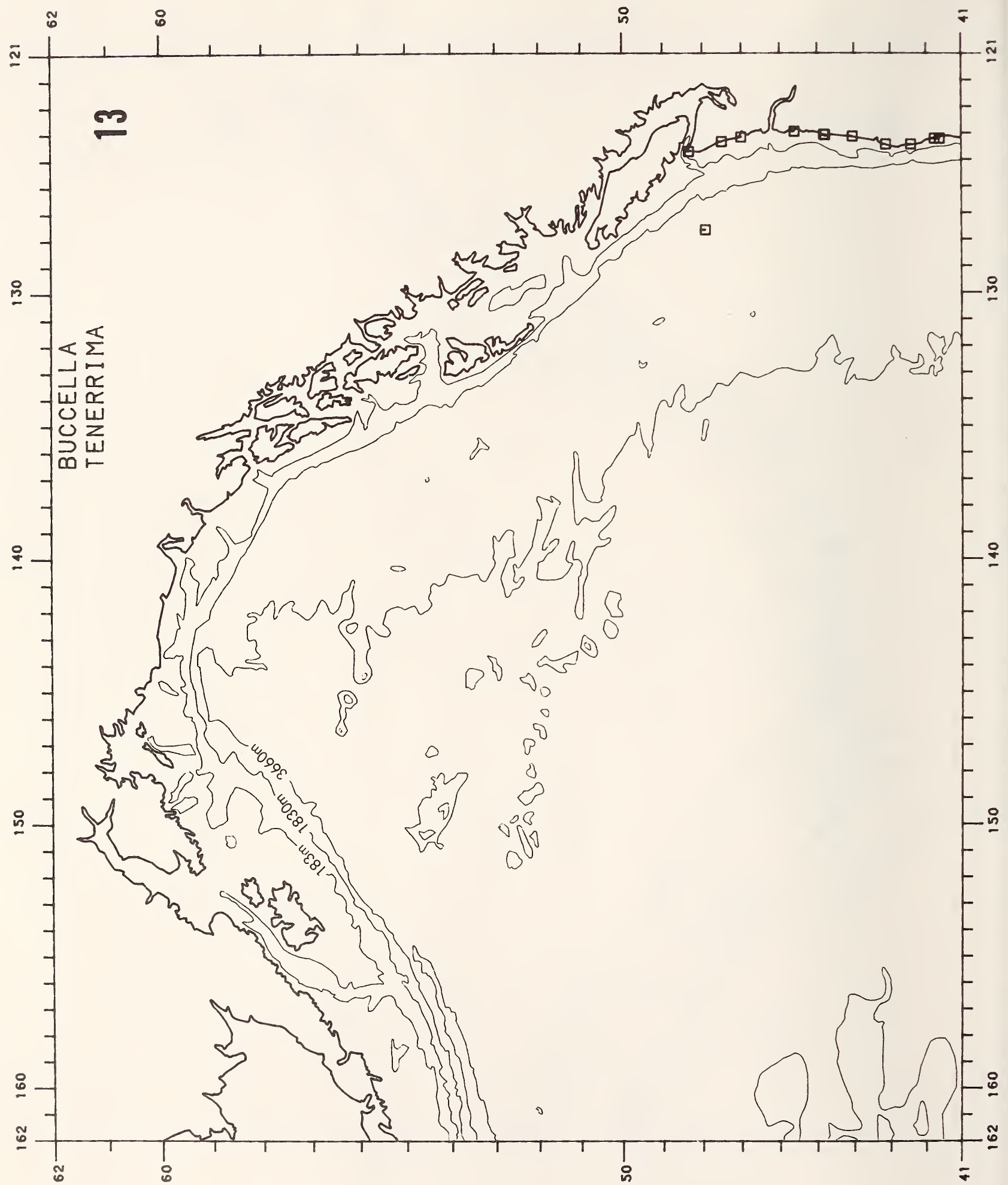




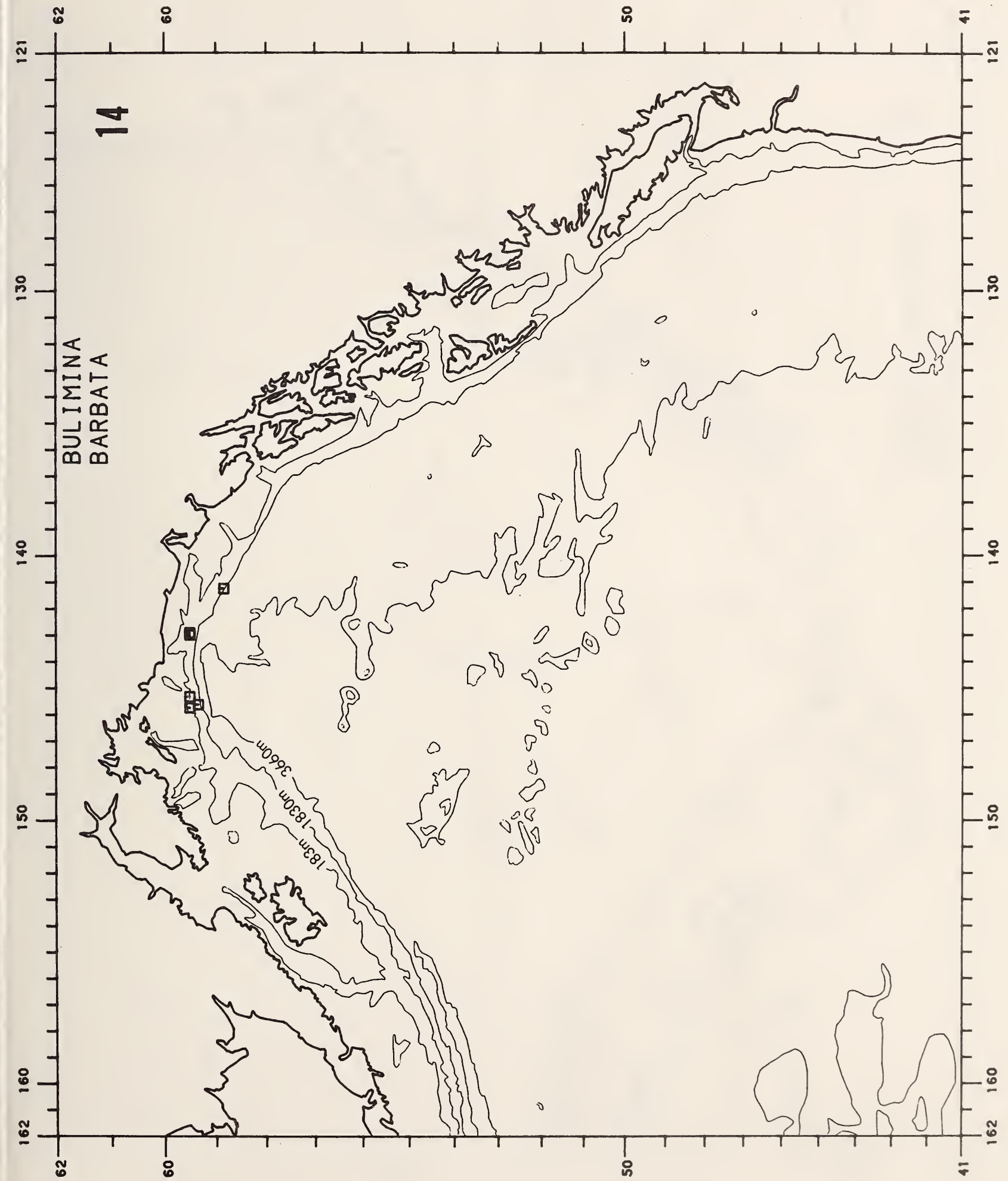




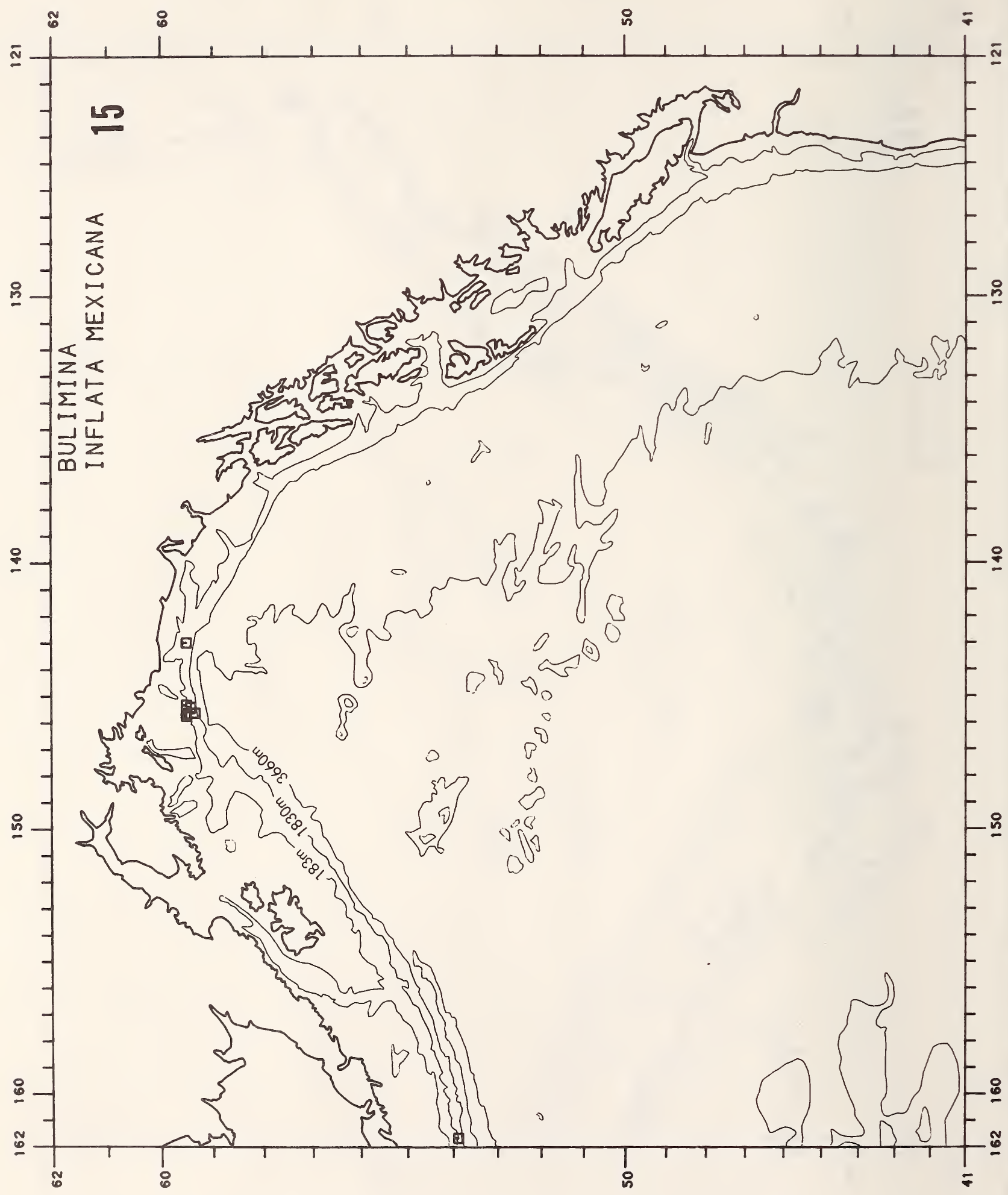




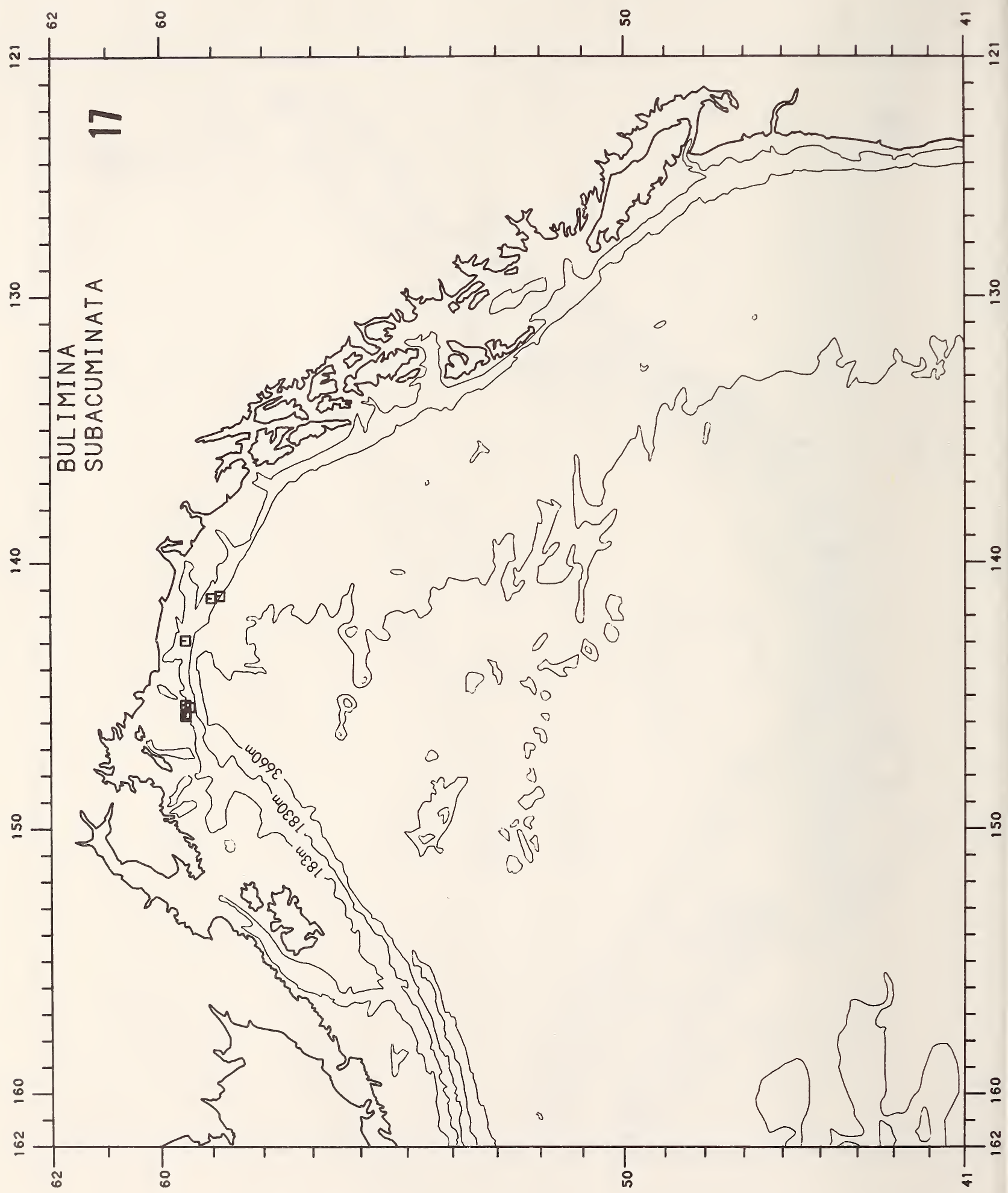




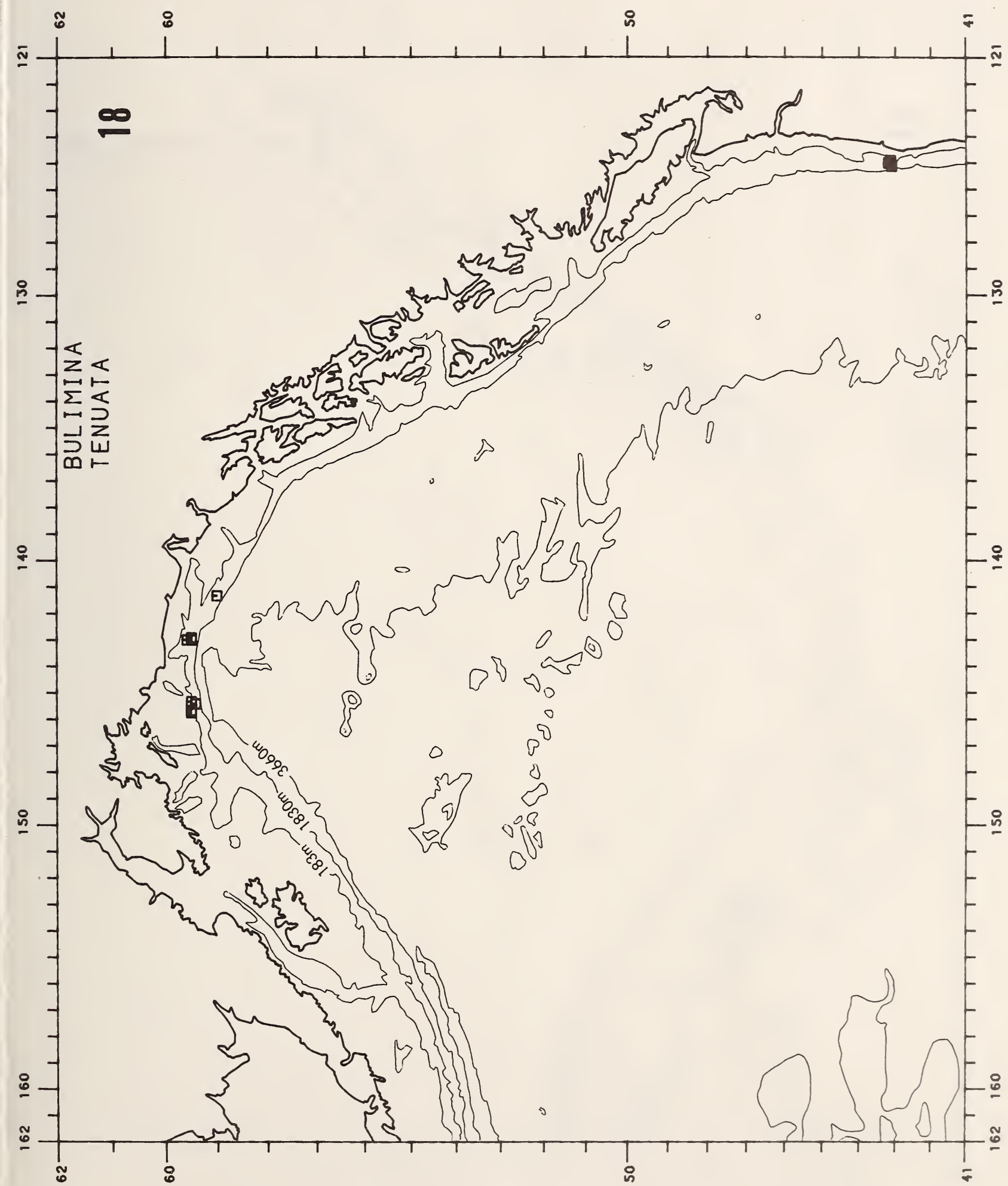




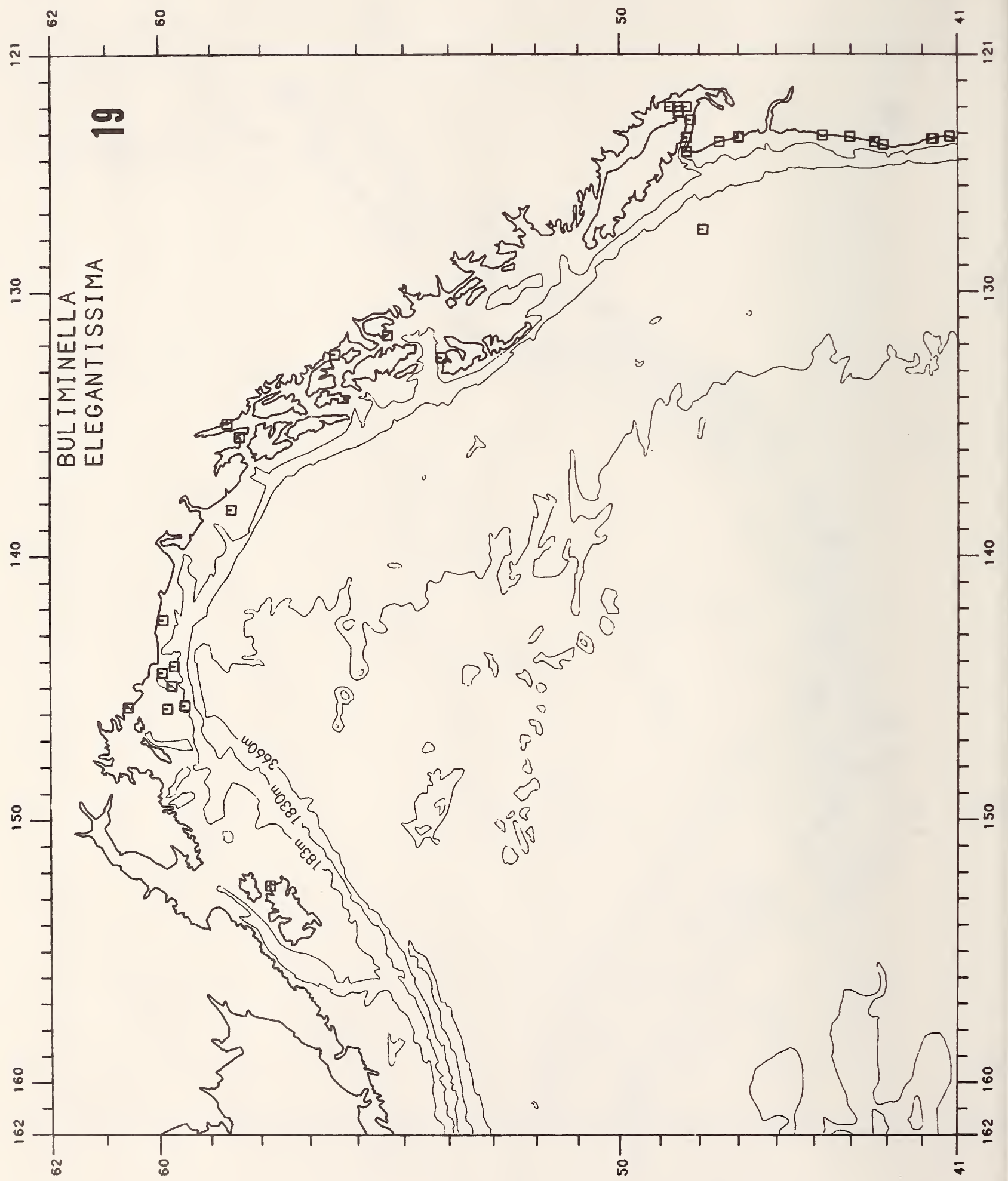




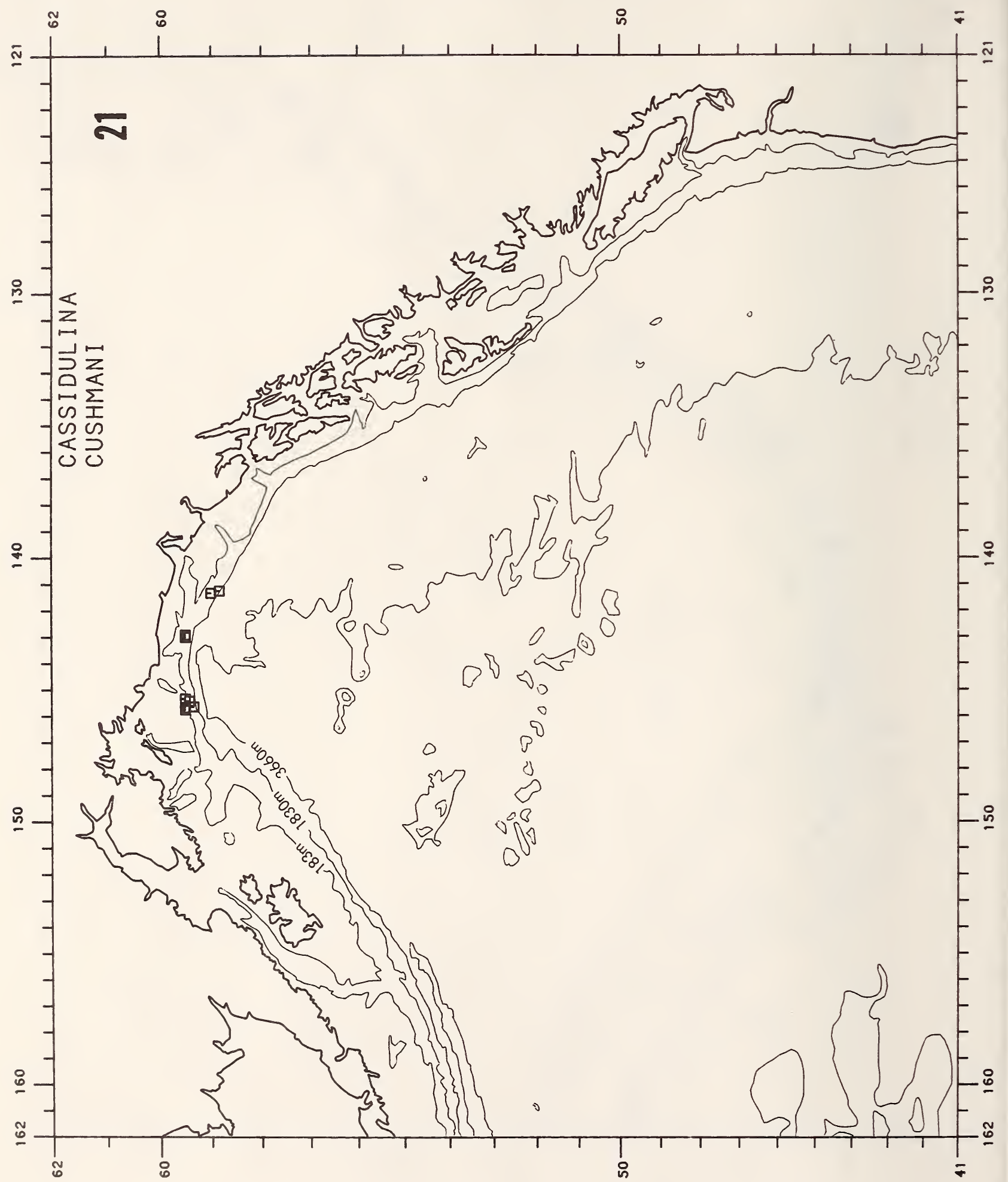




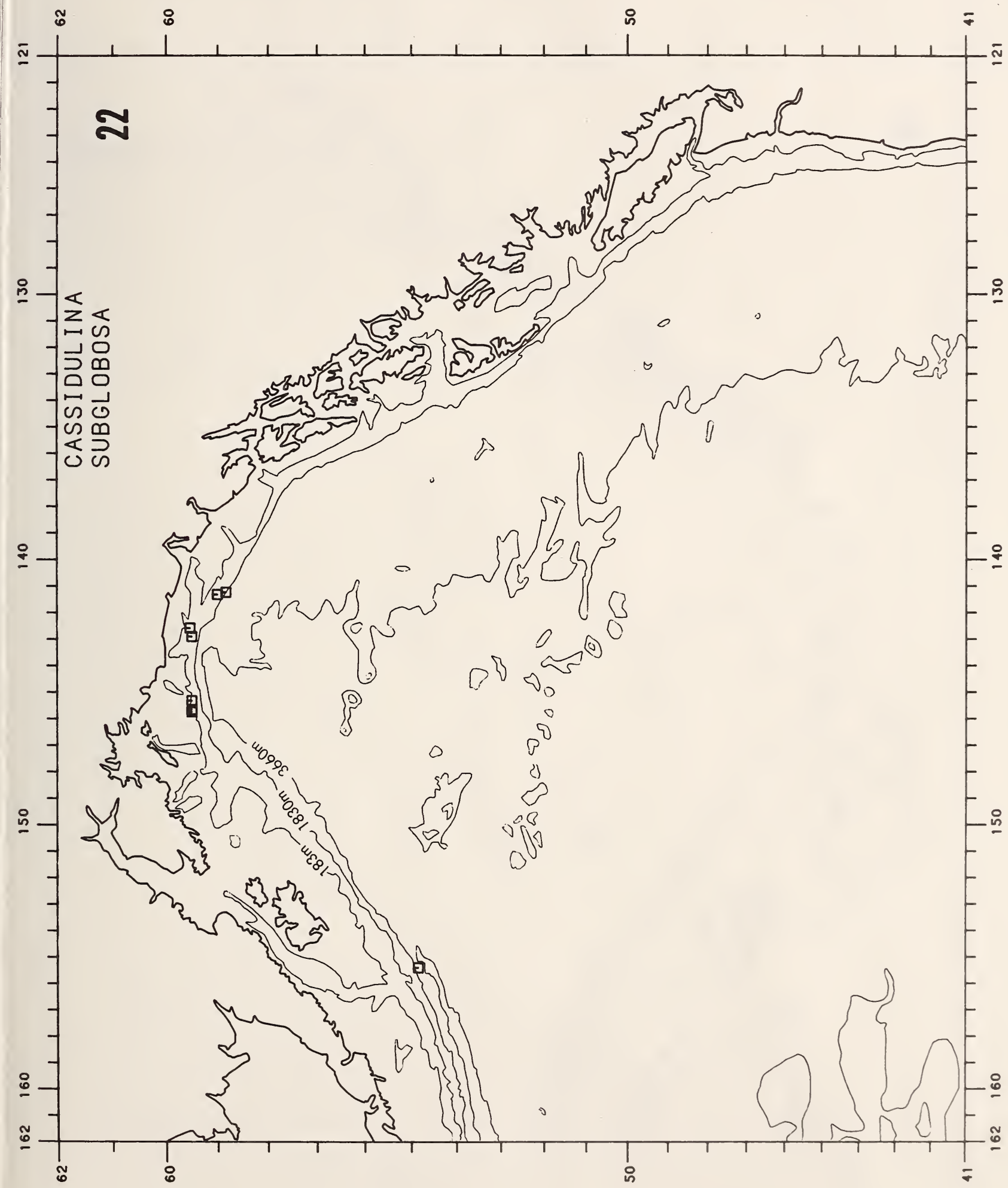




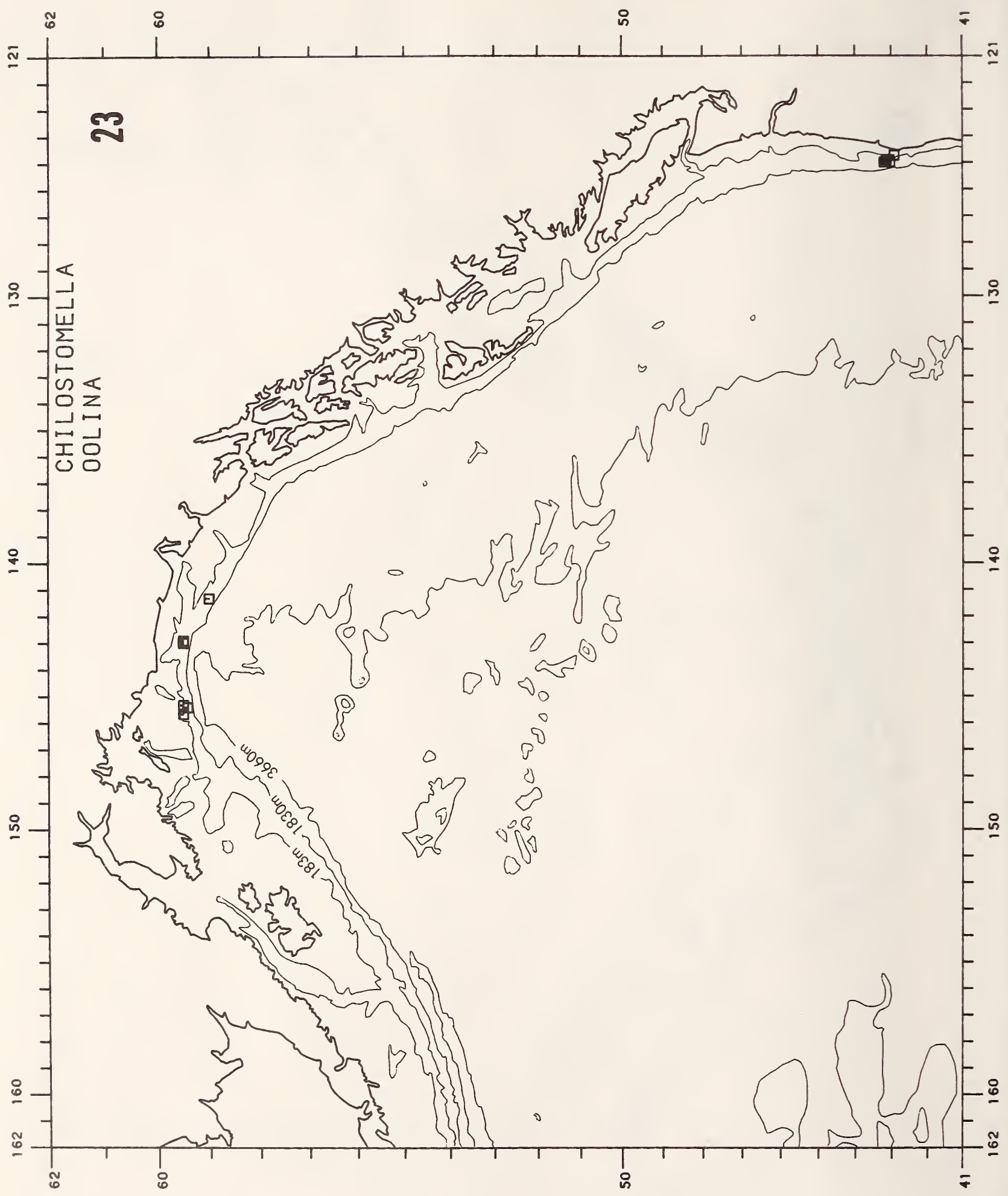




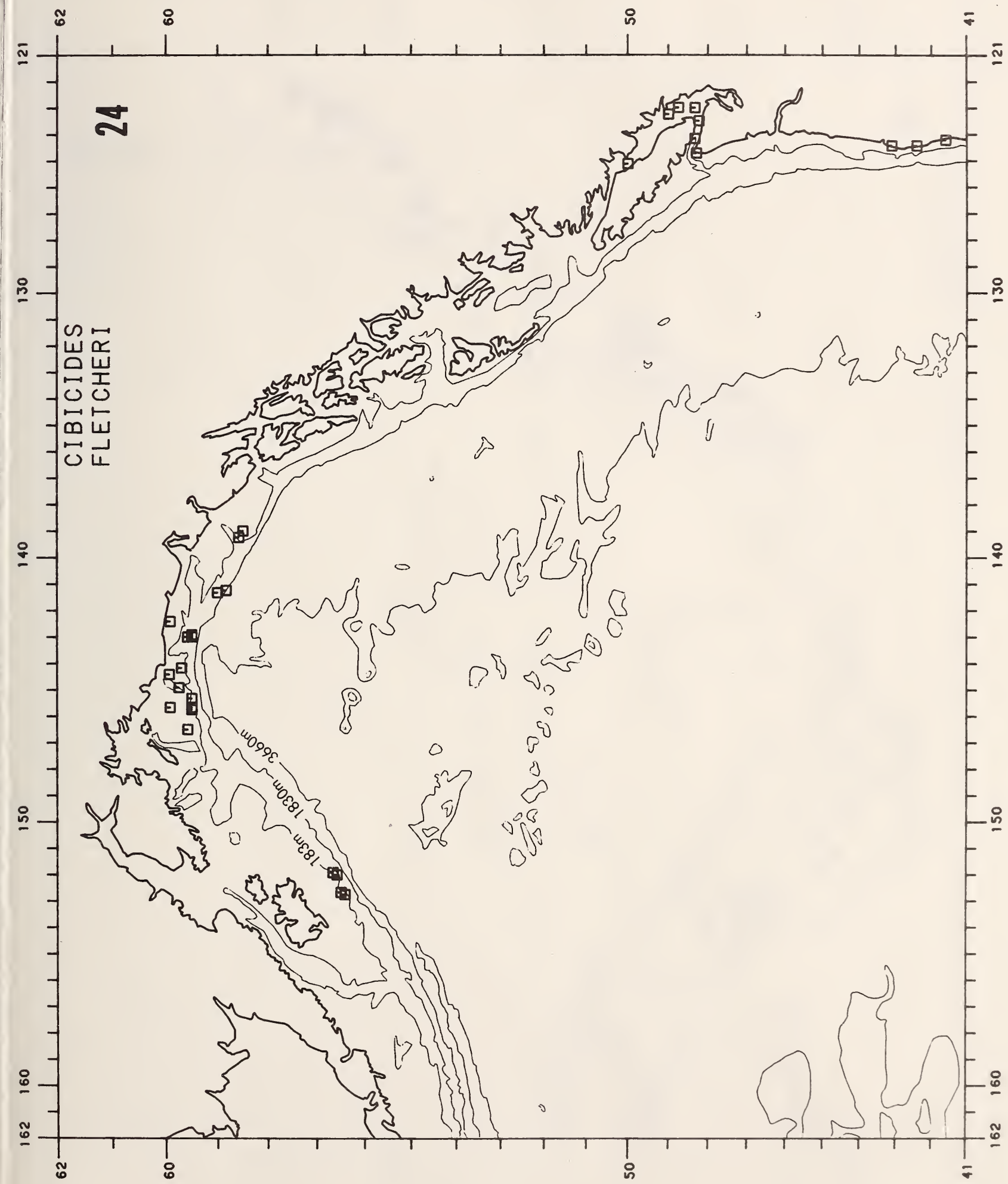




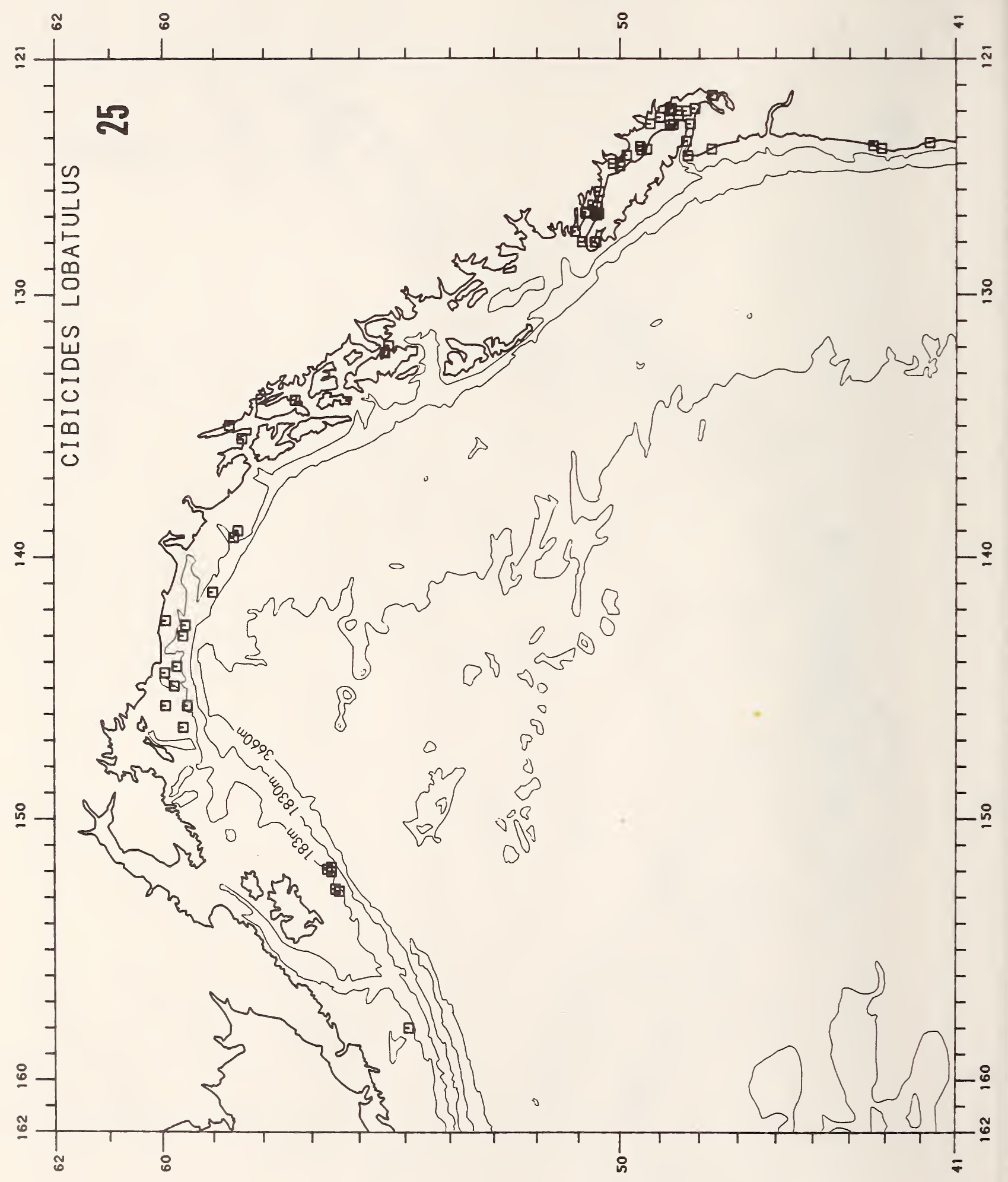




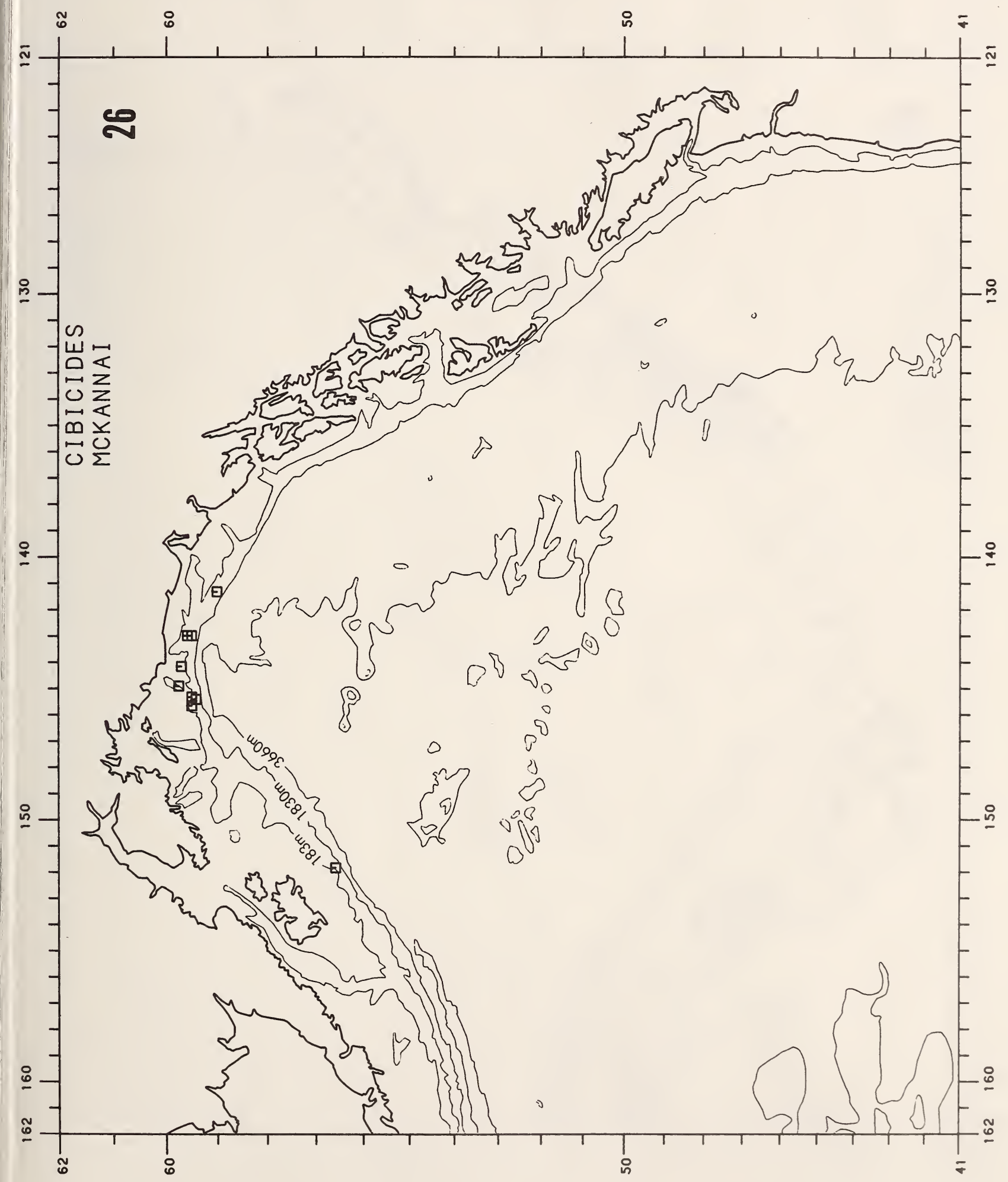




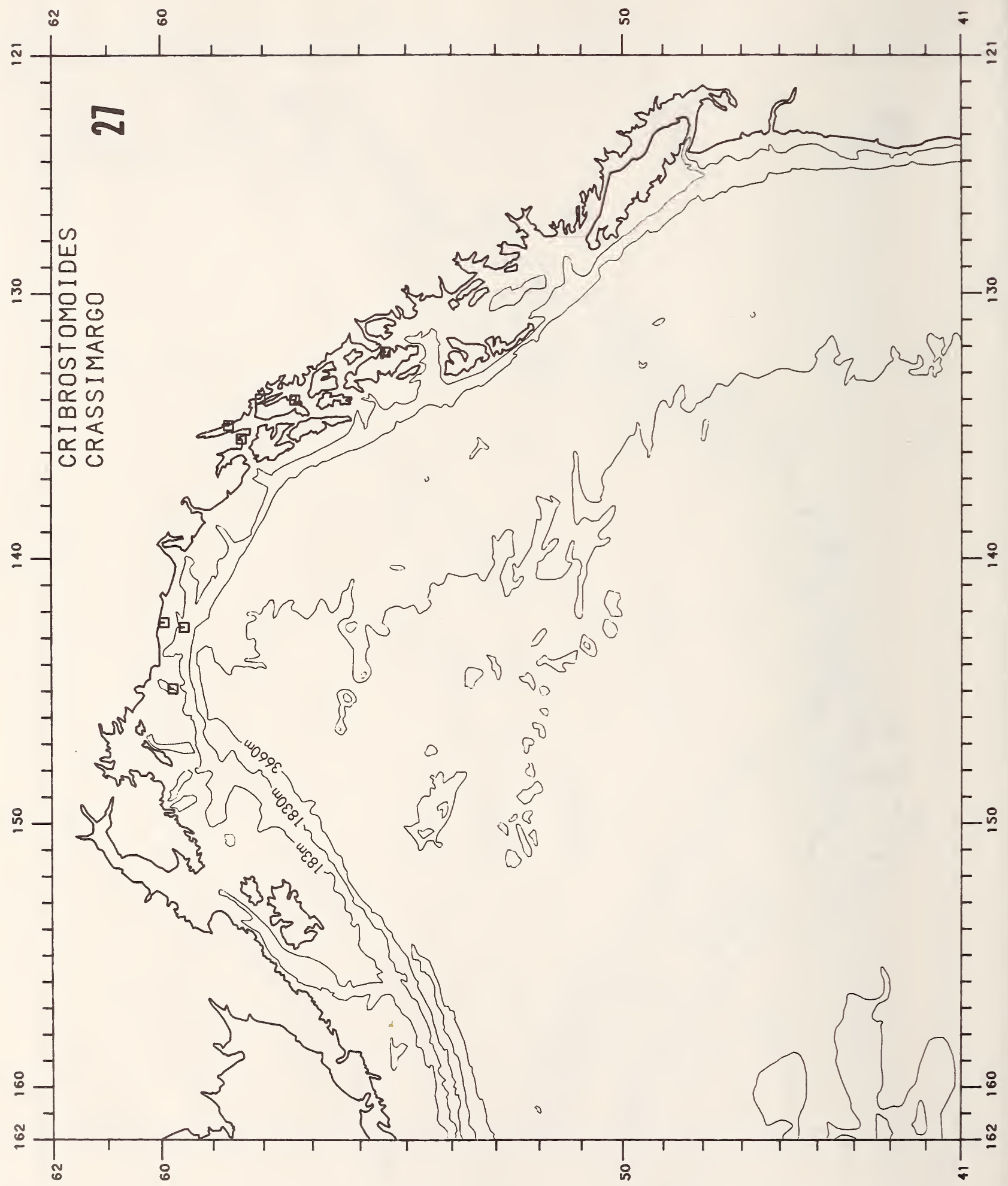


NUMBER 26

123

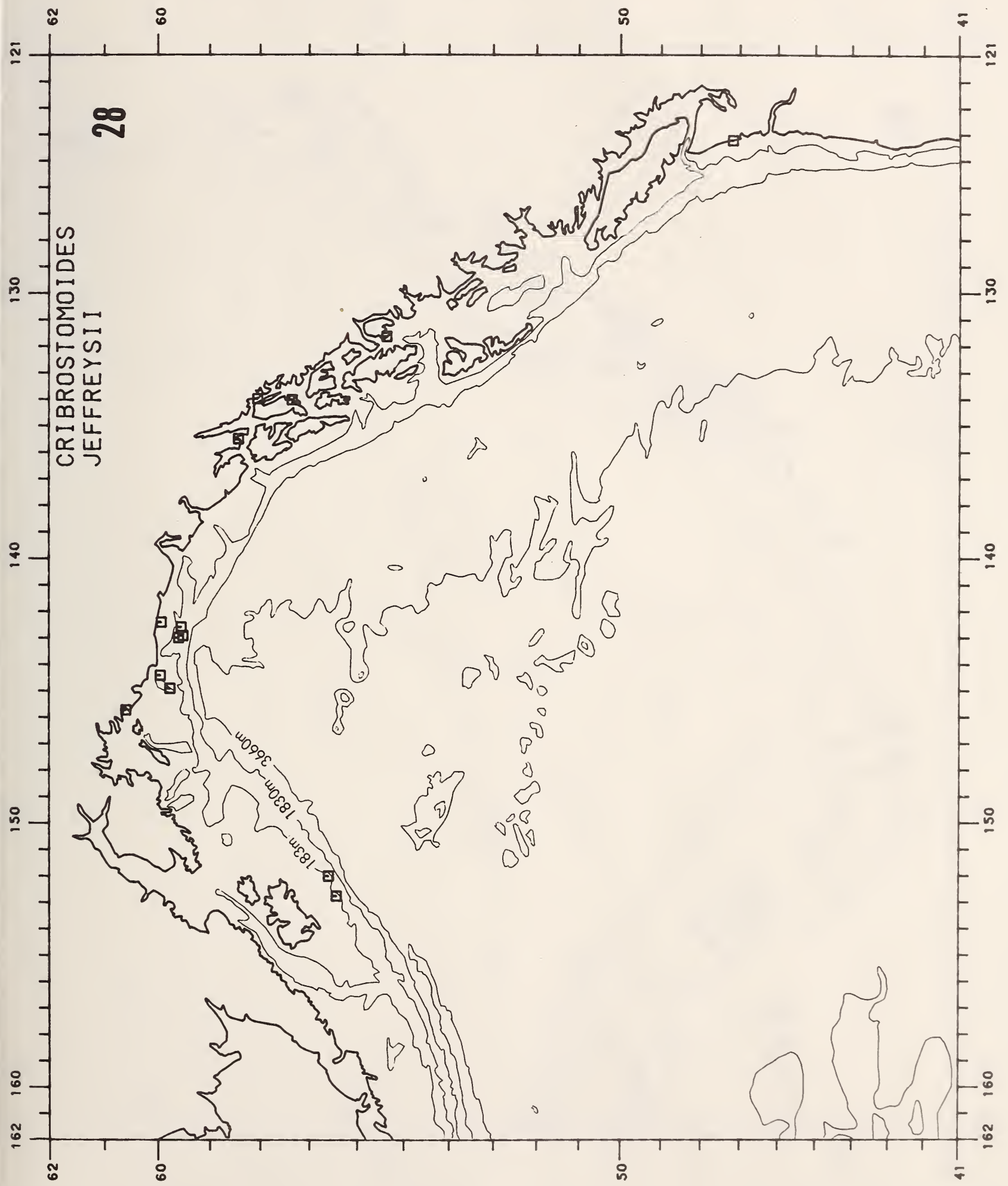




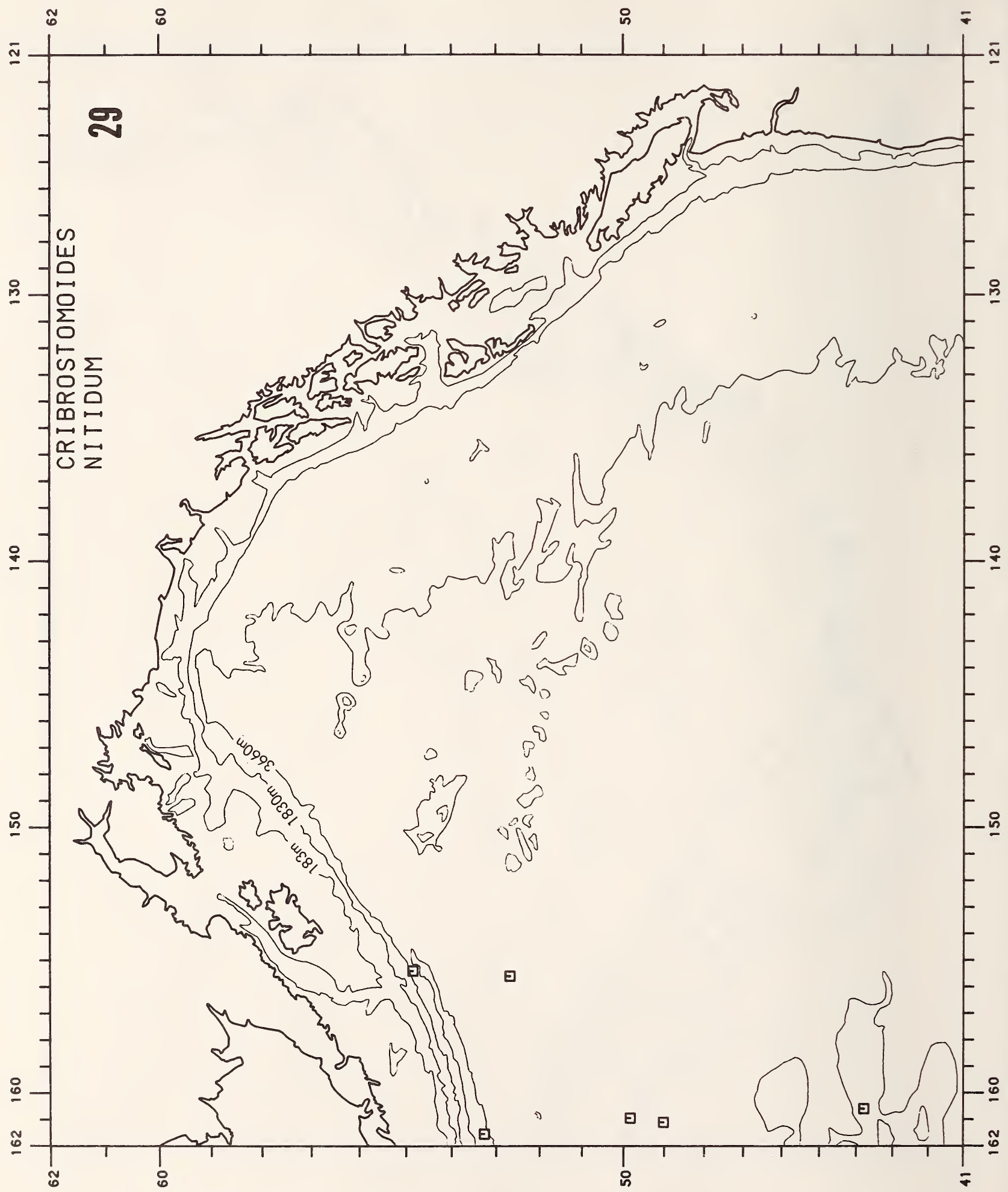




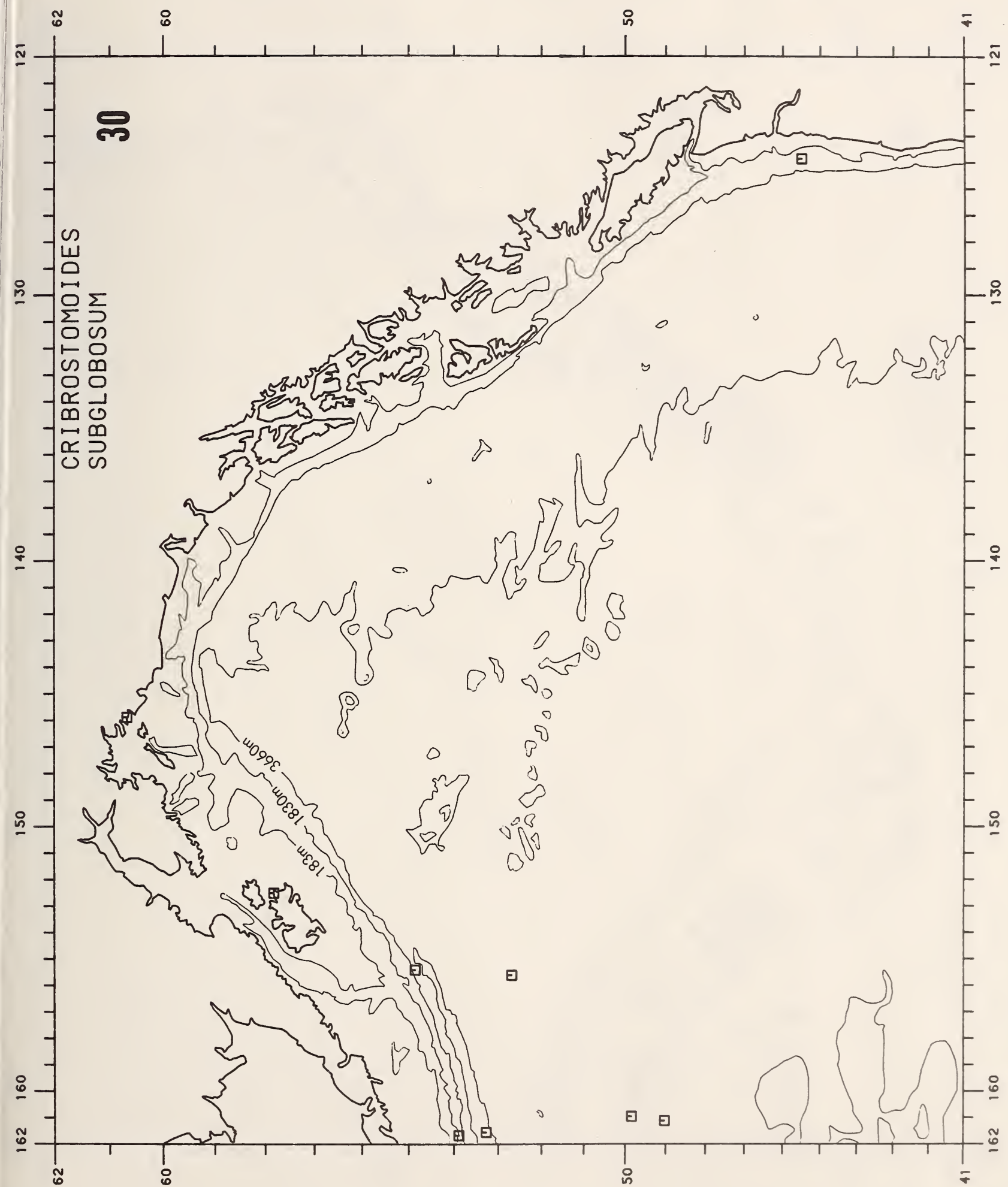




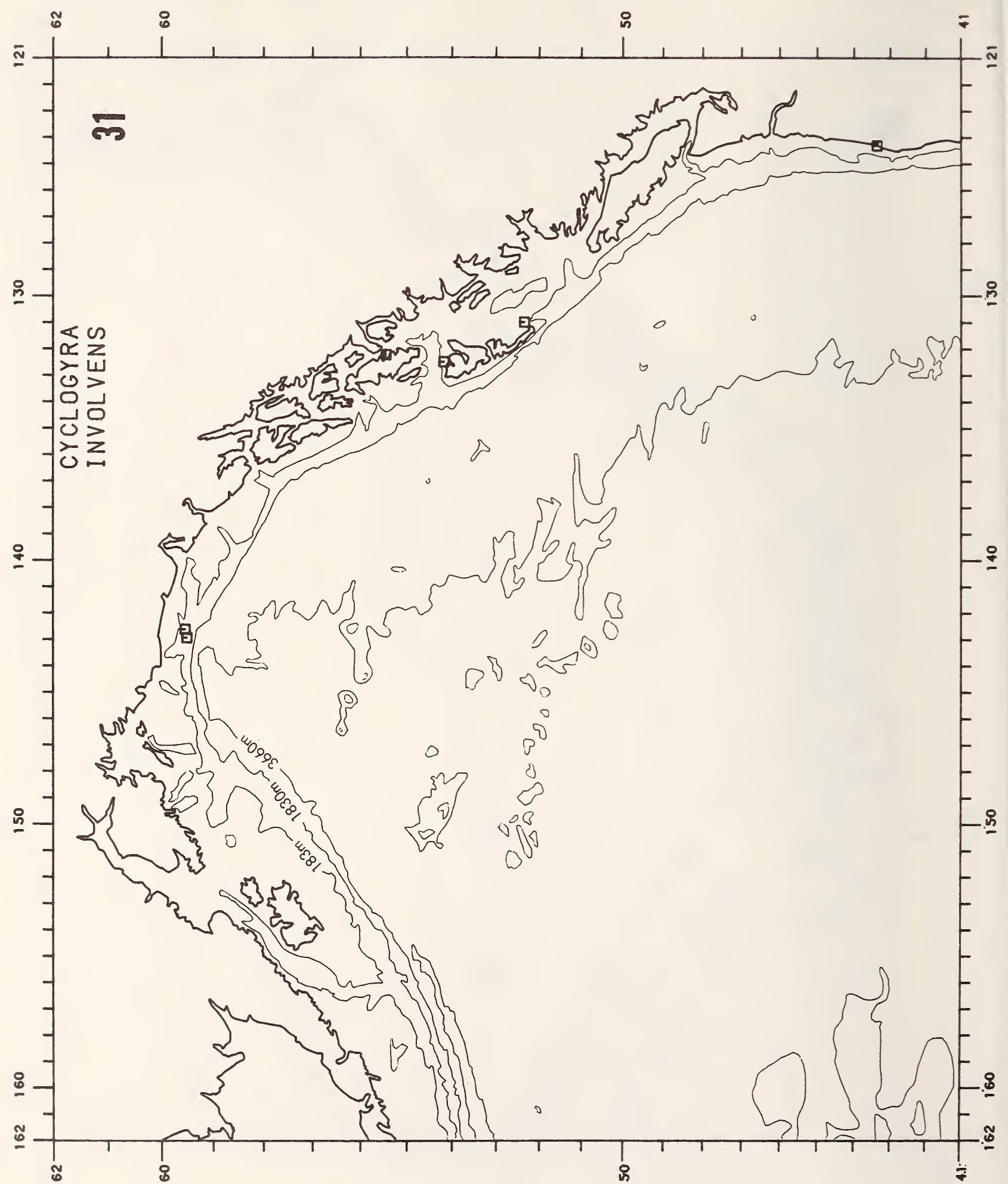


NUMBER 26

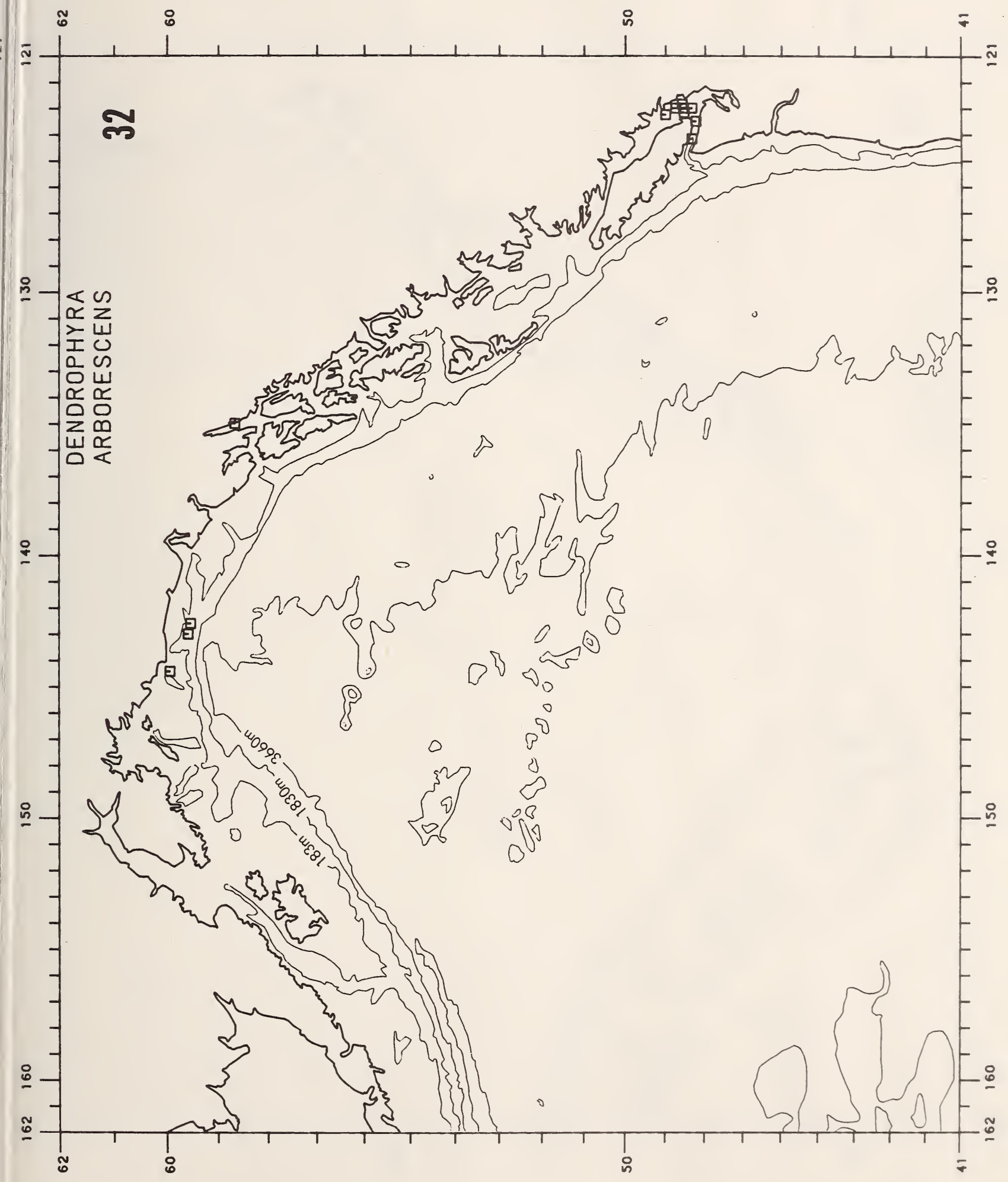




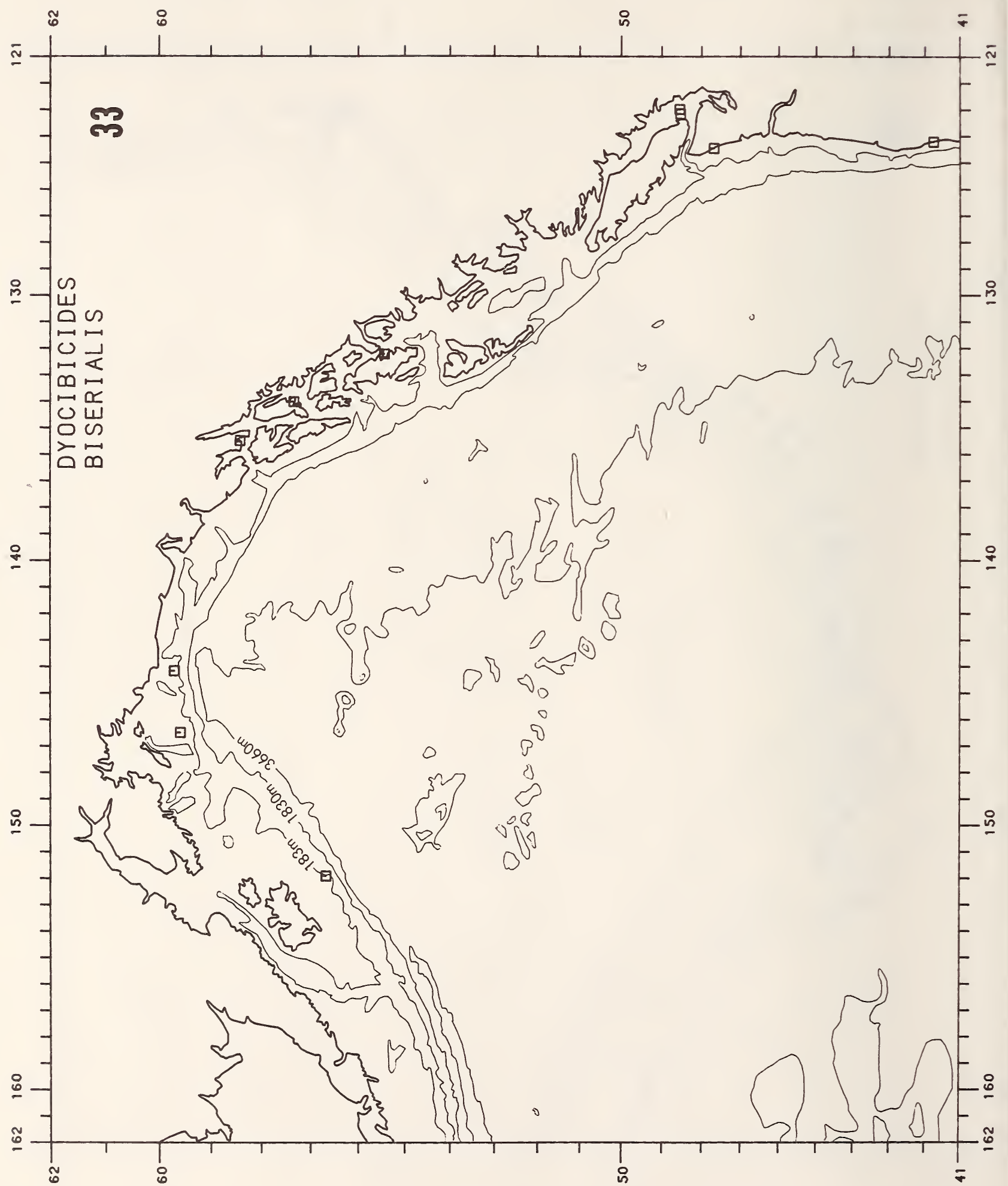




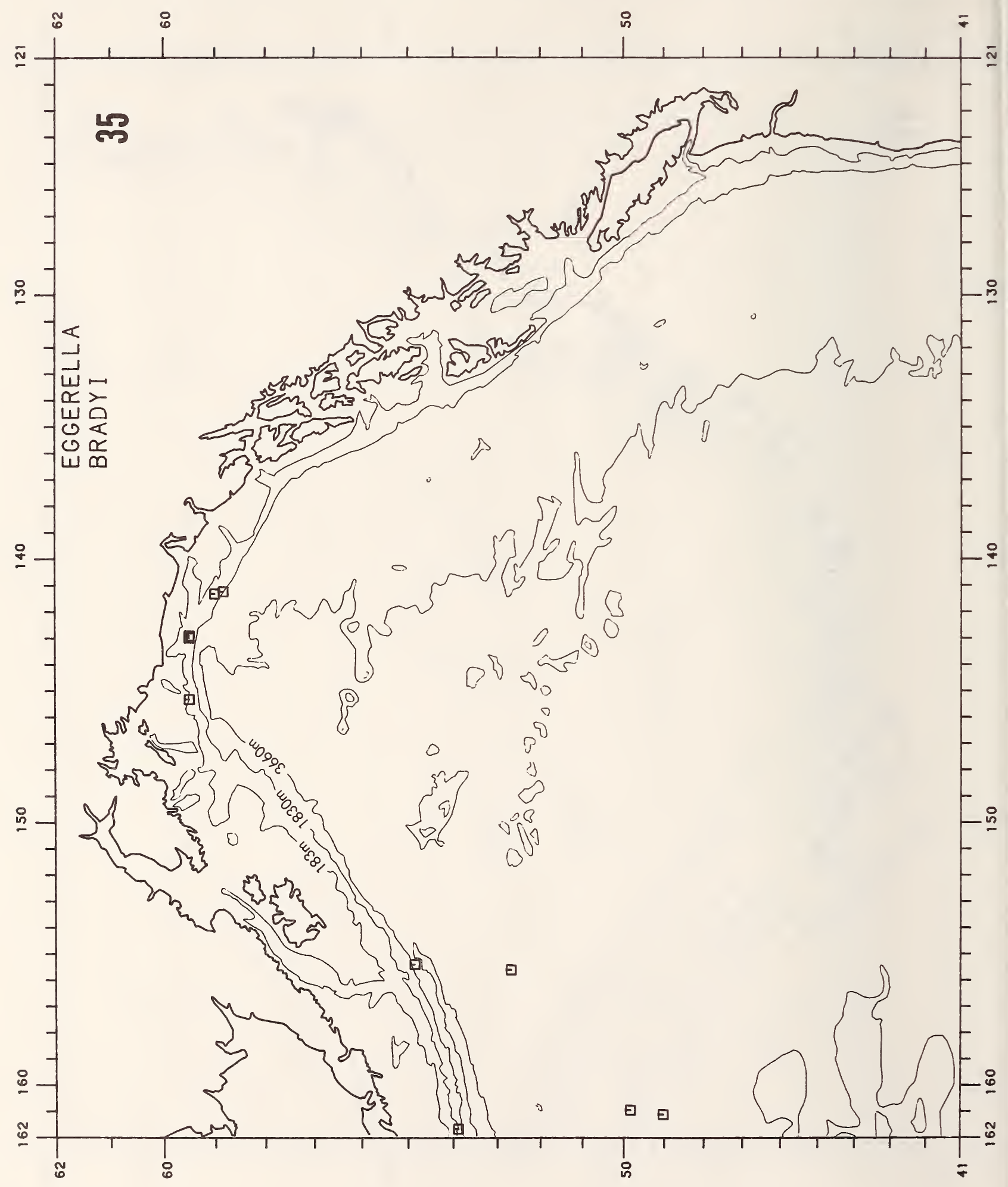




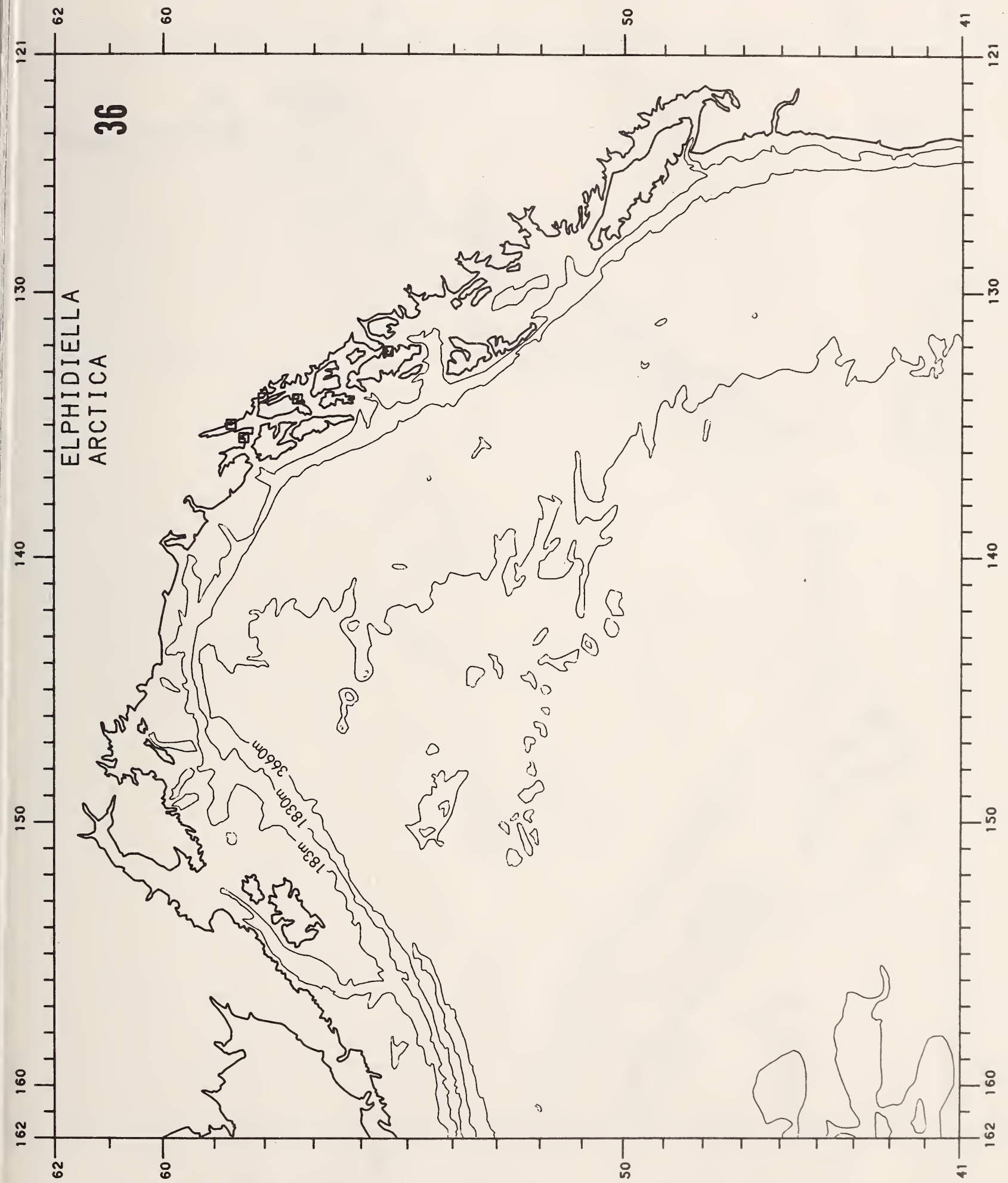




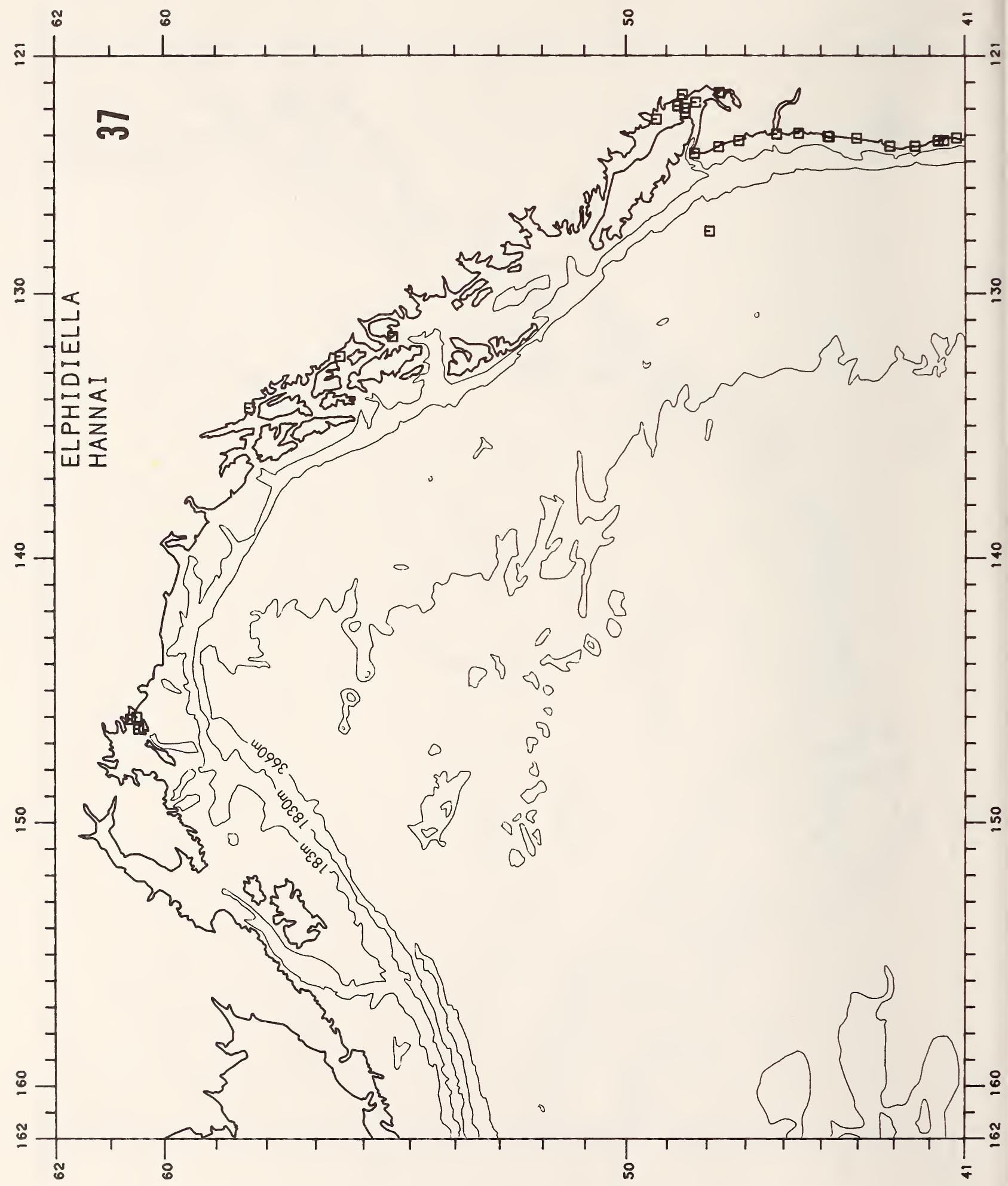




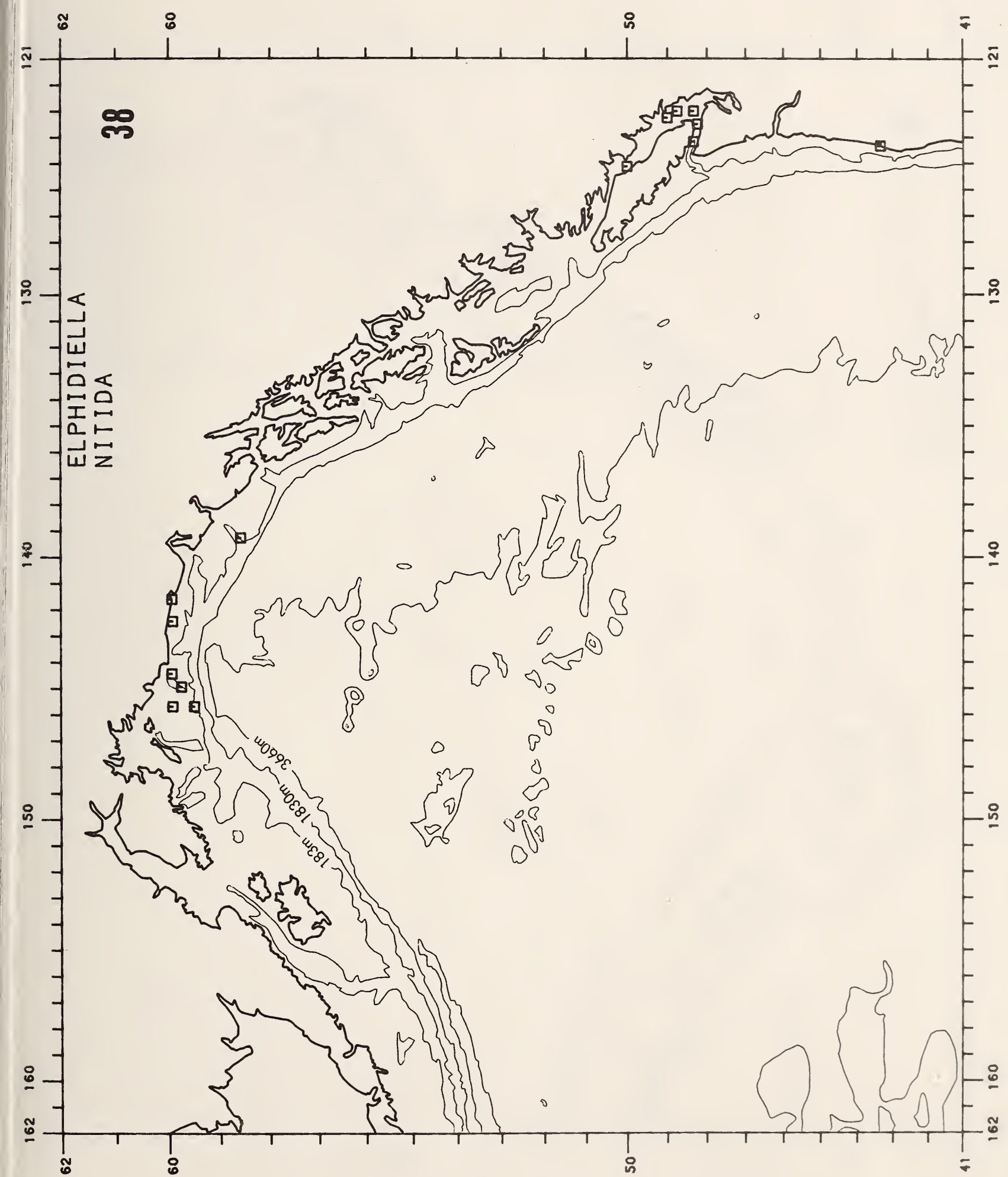




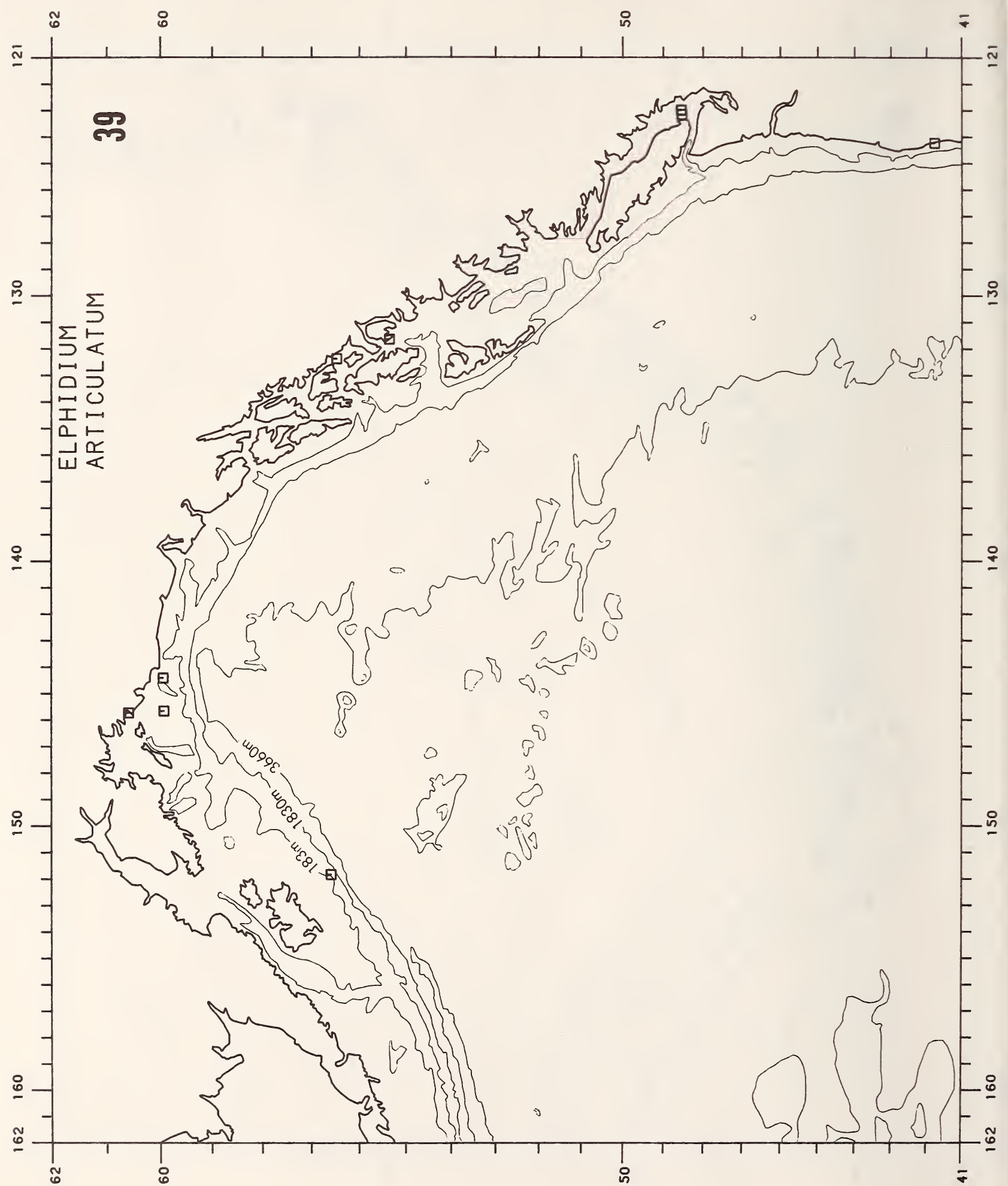




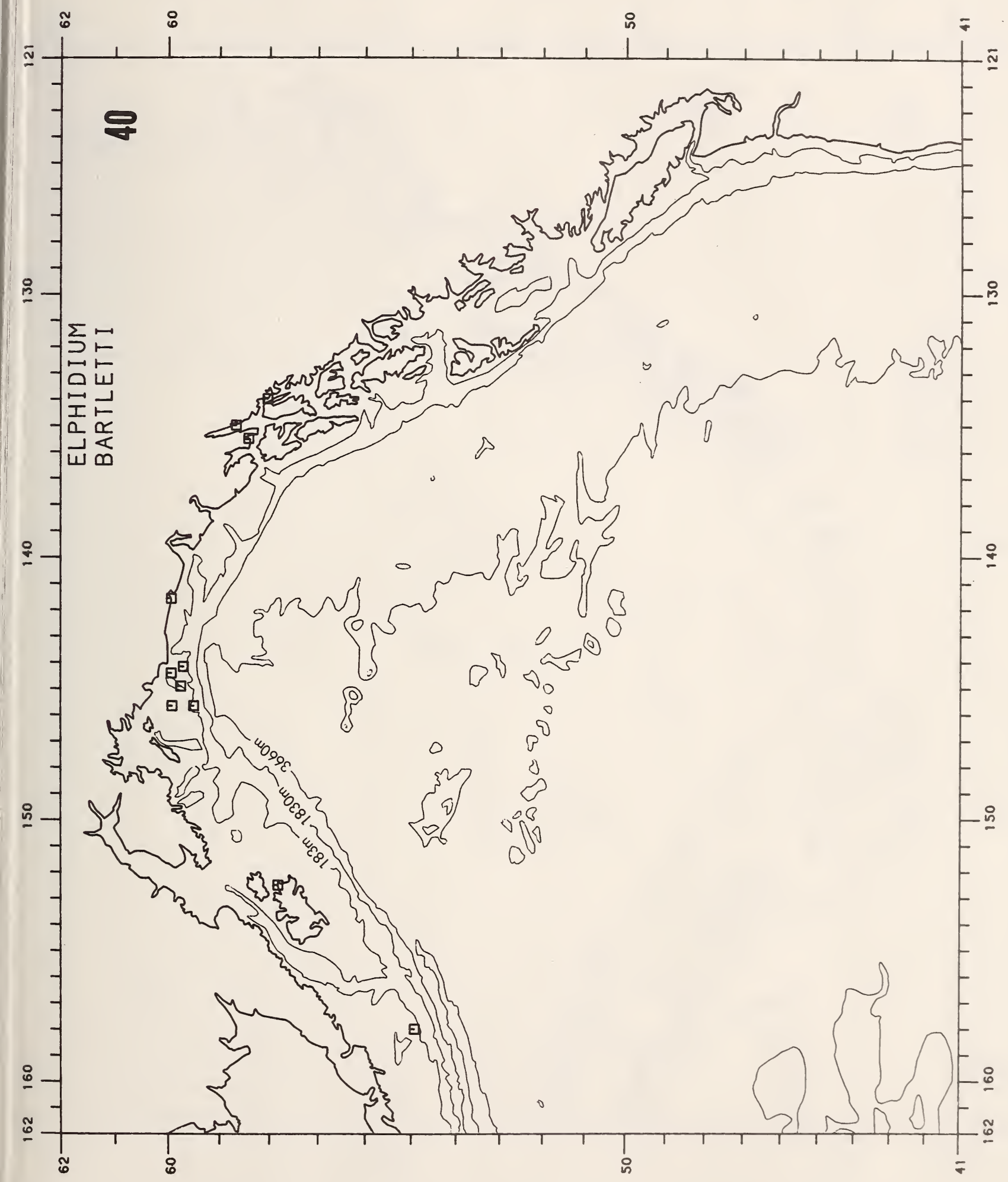




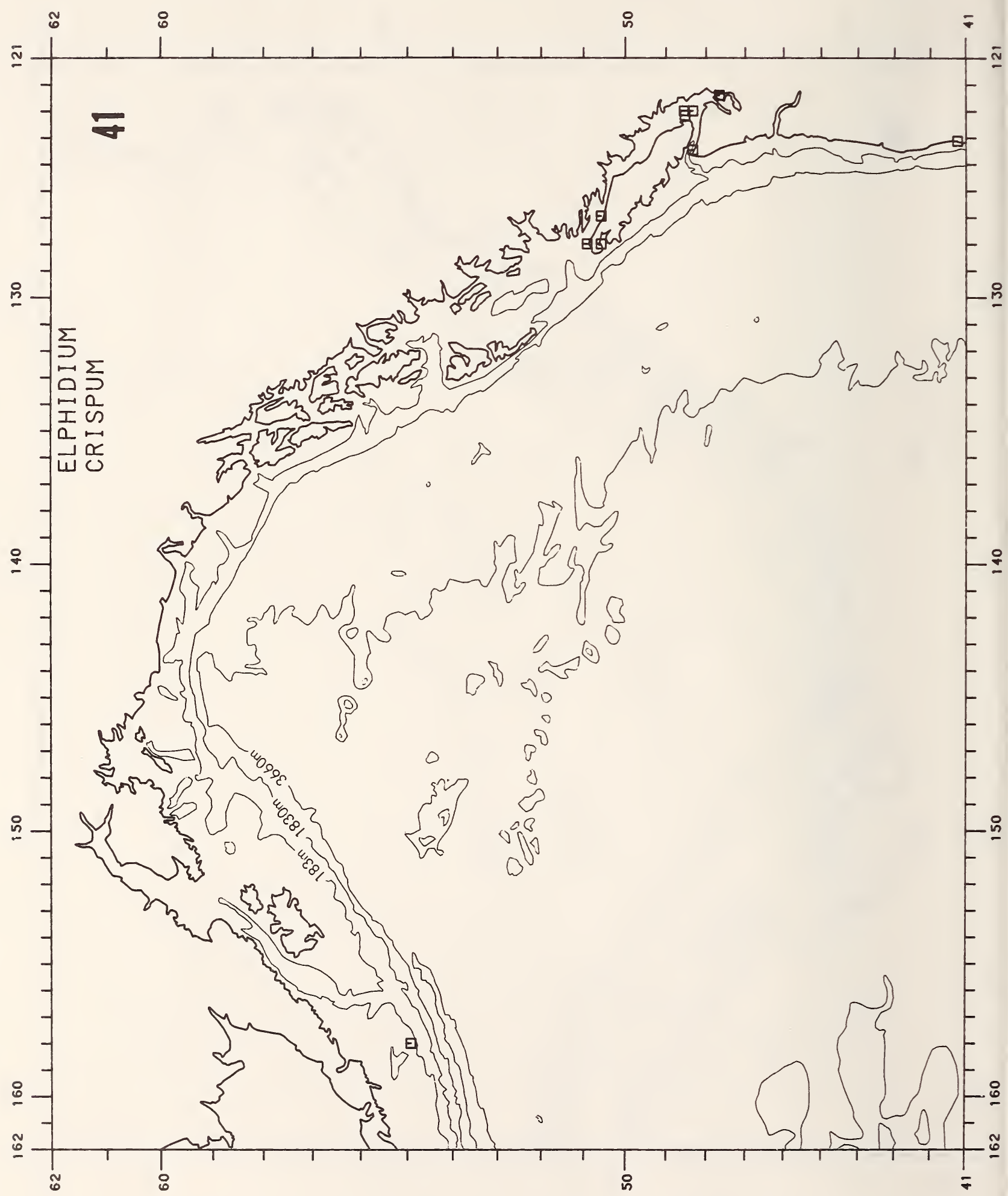




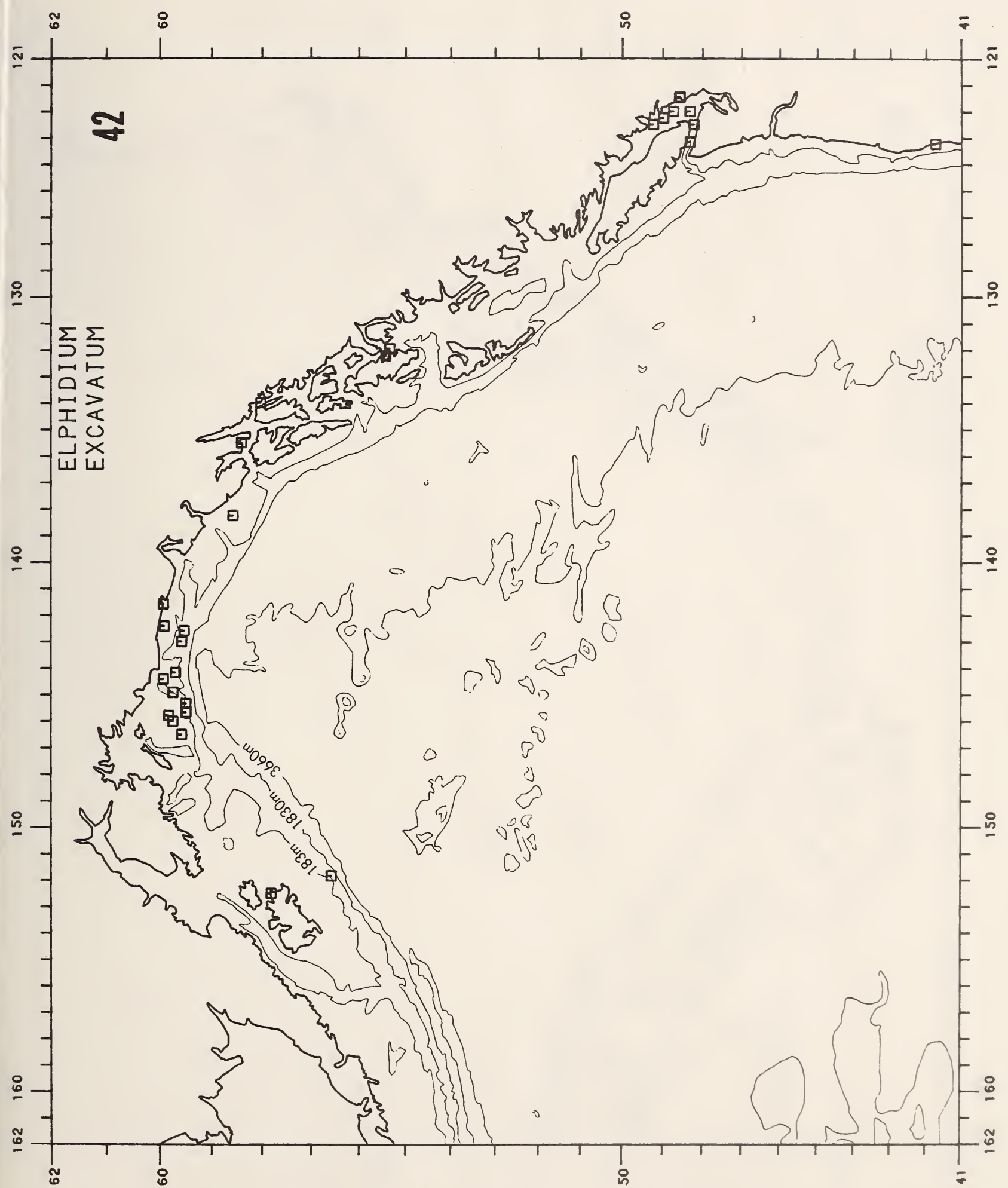




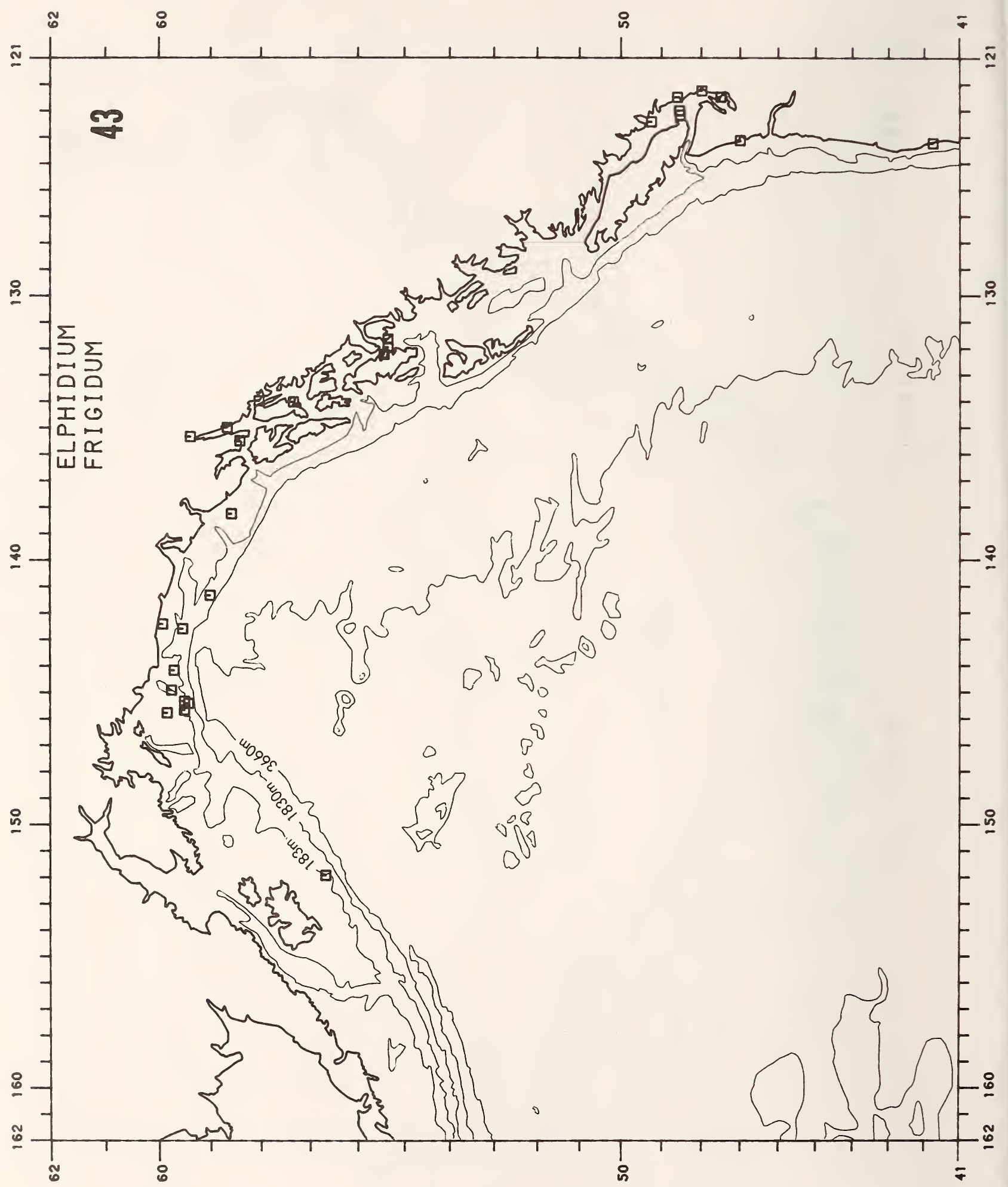




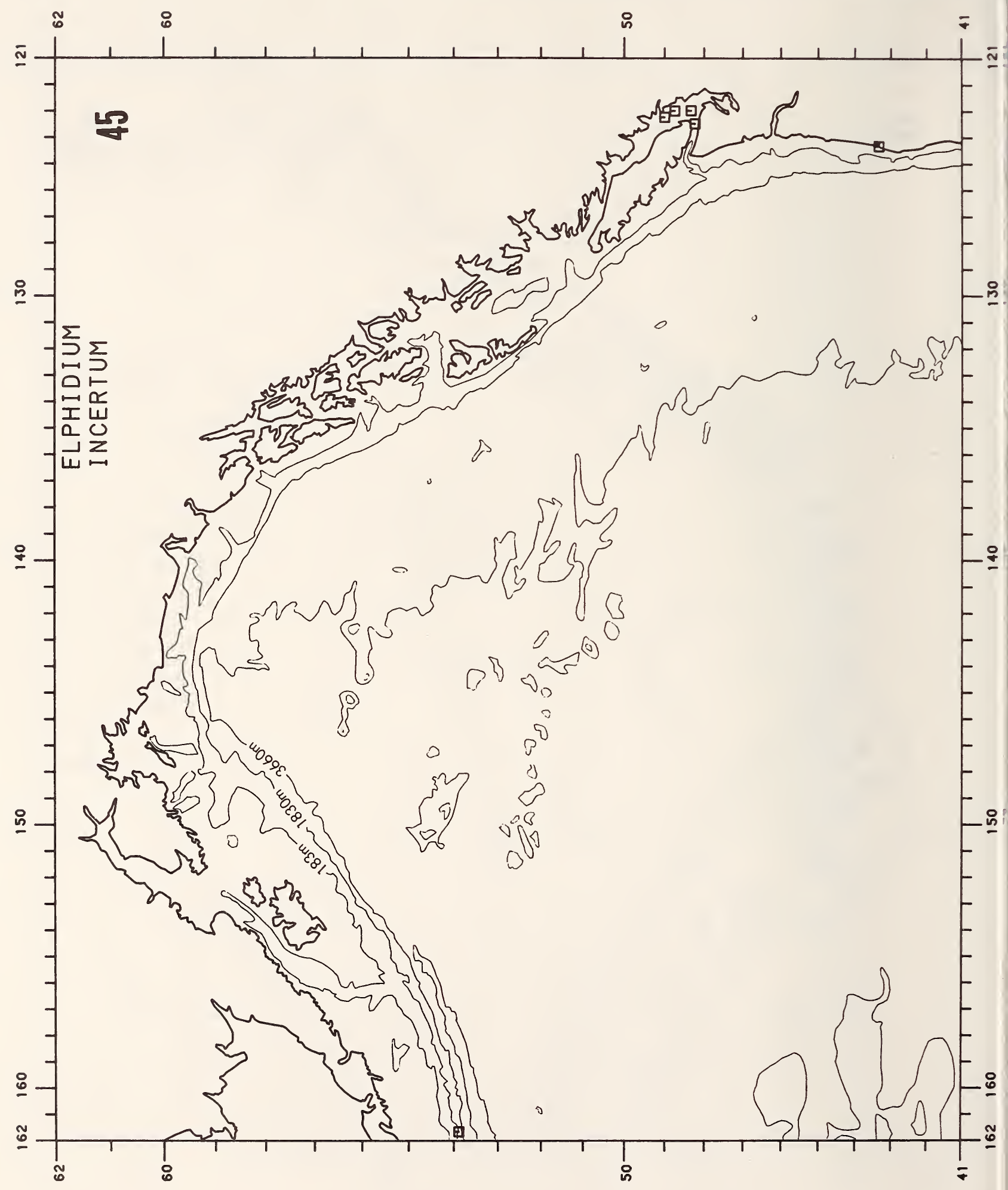




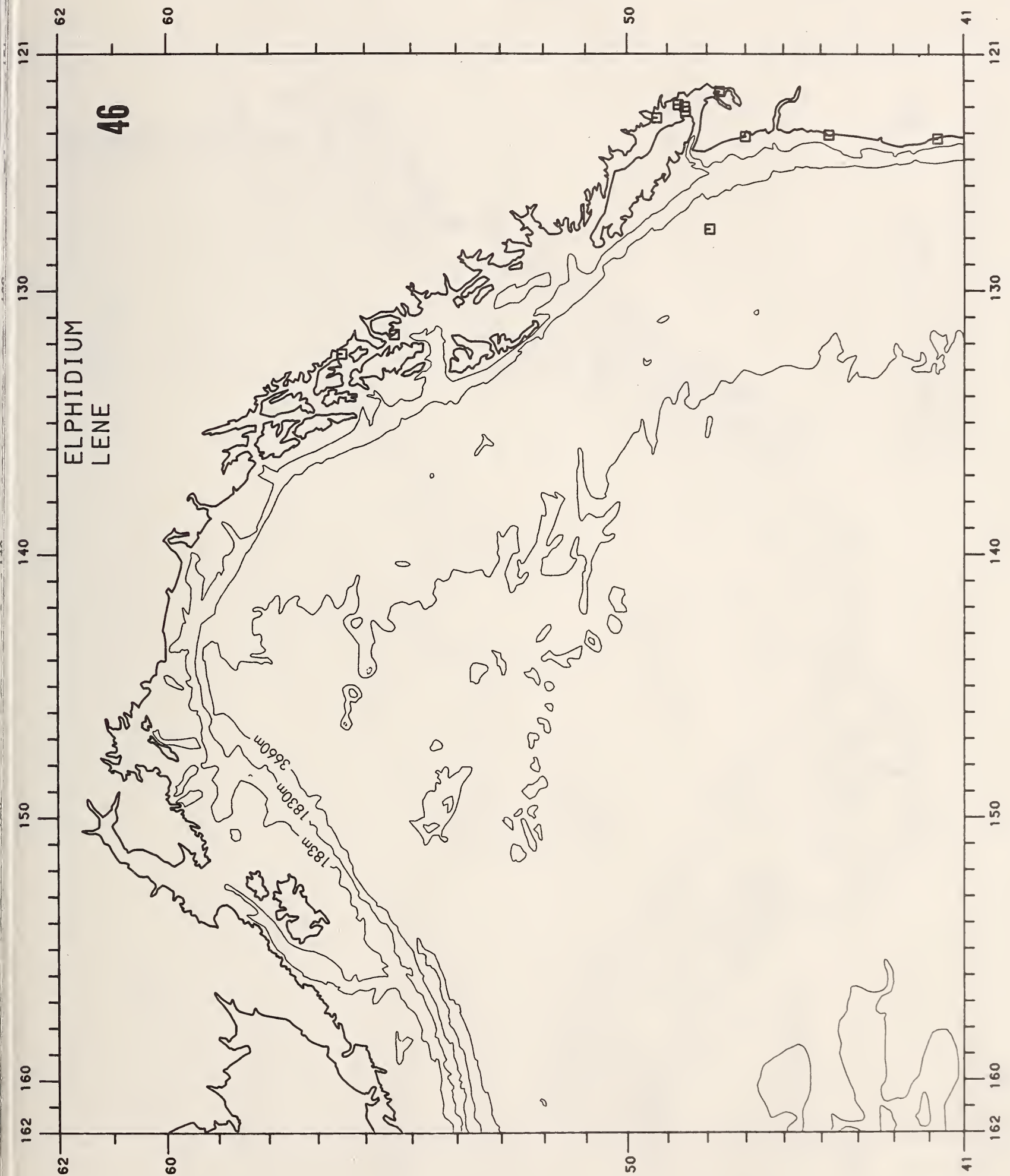




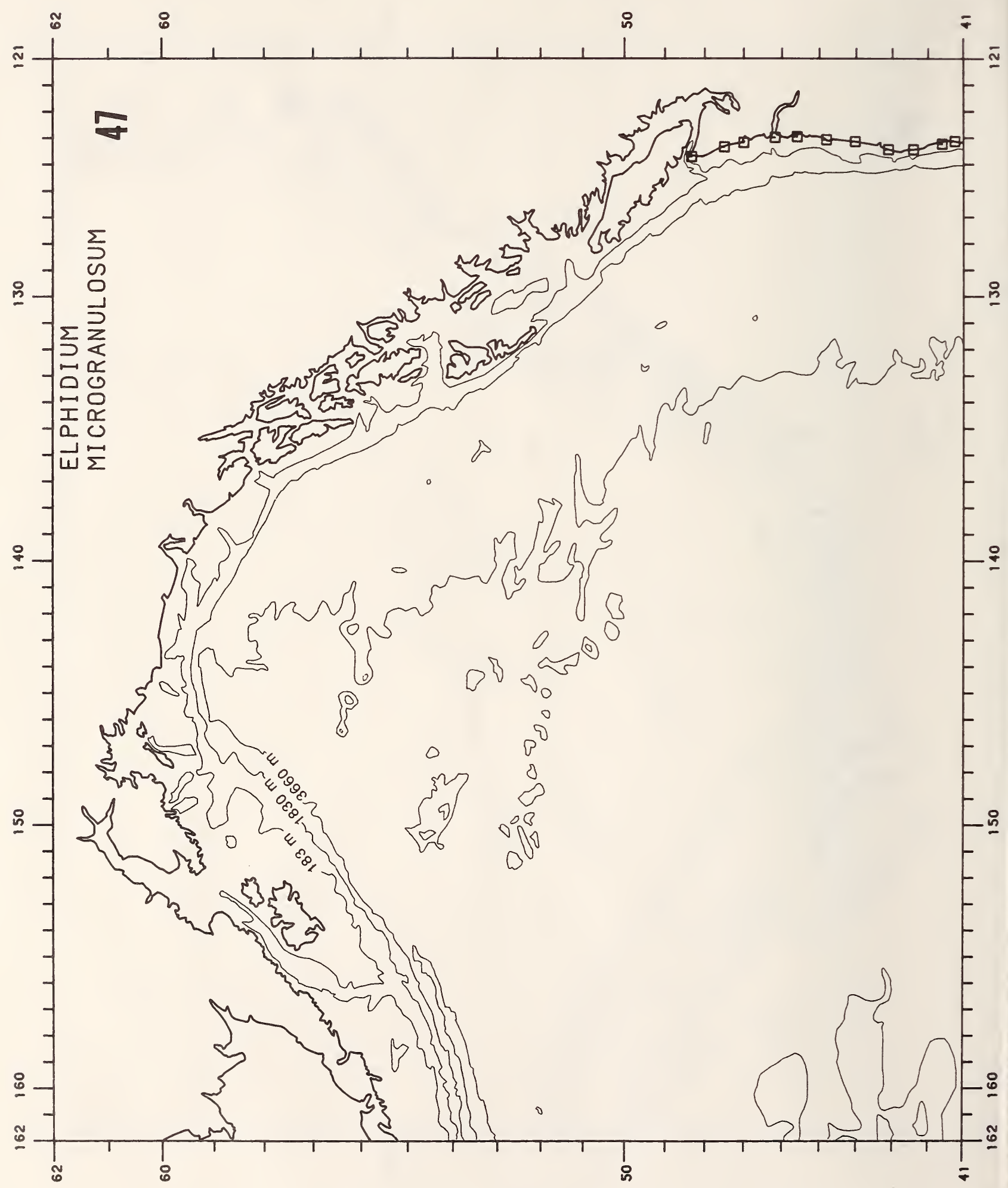


NUMBER 26

143

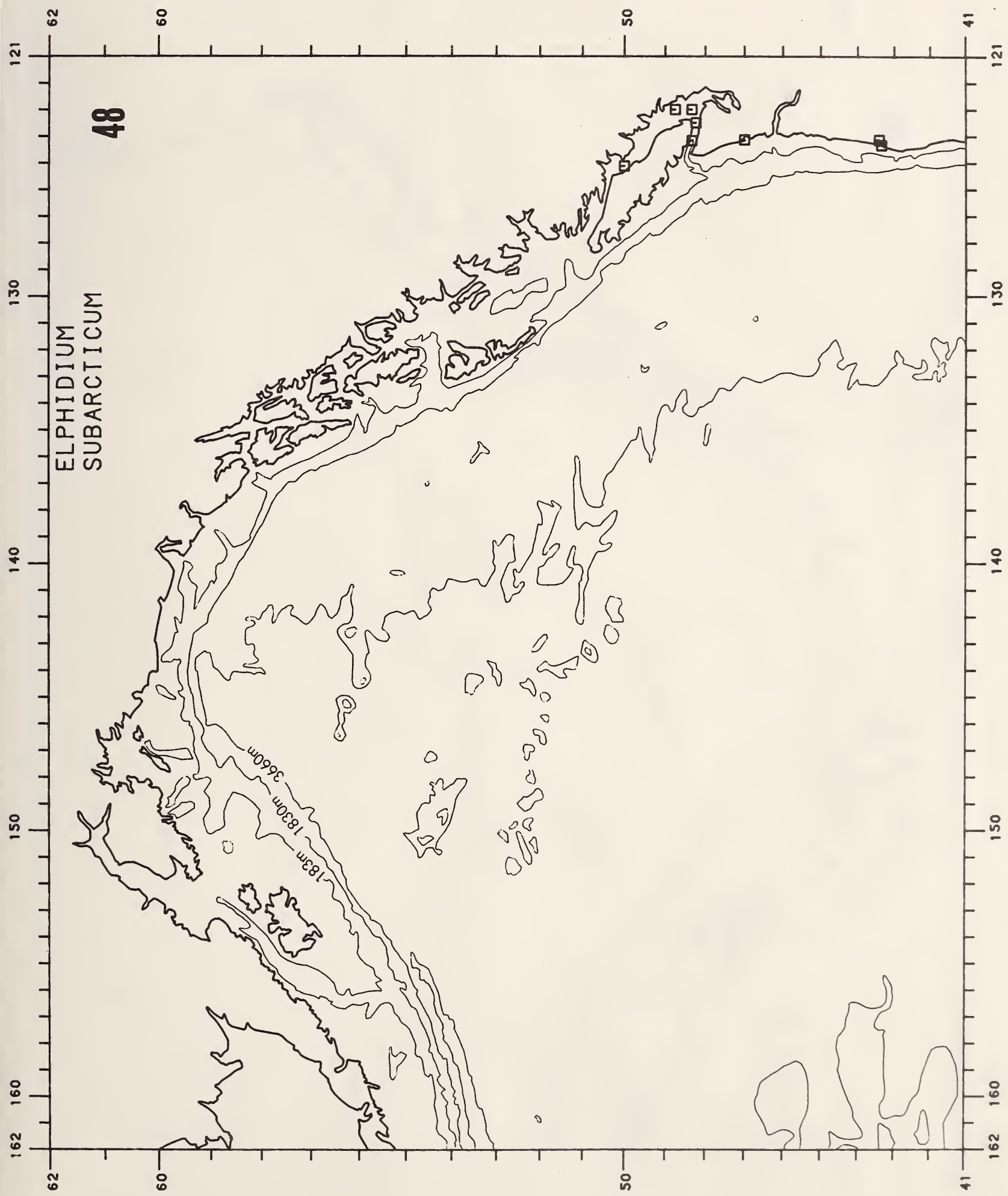




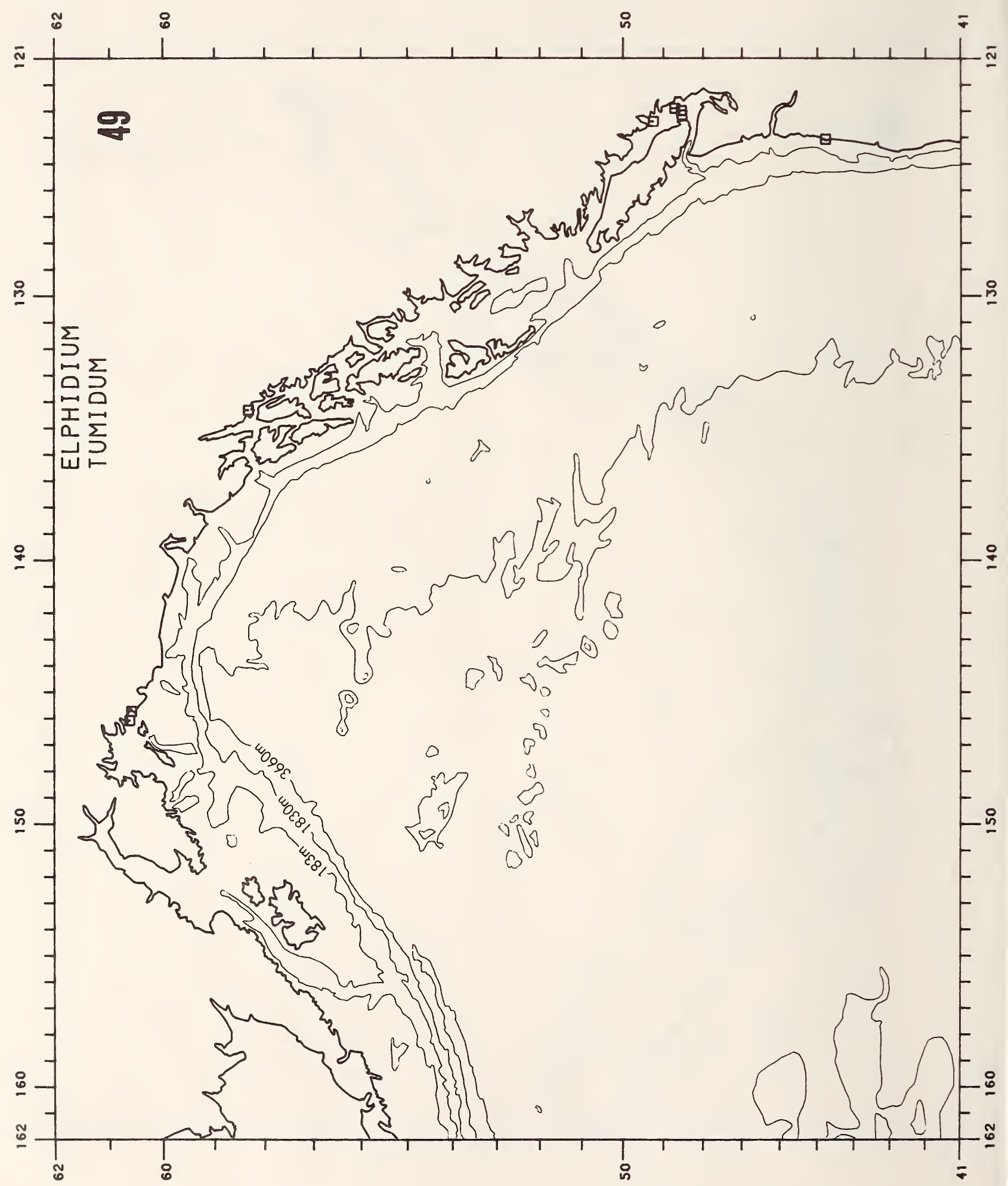




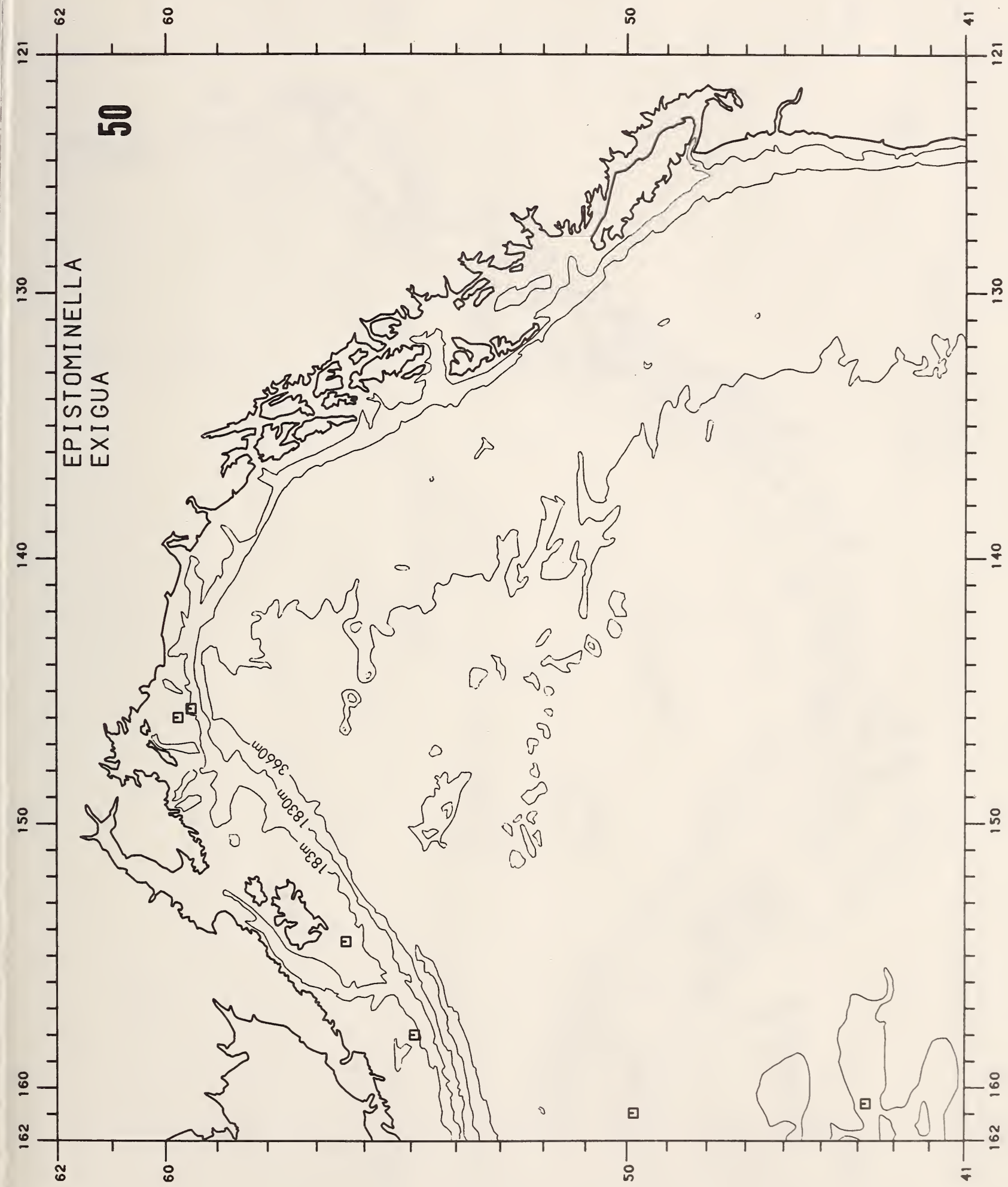




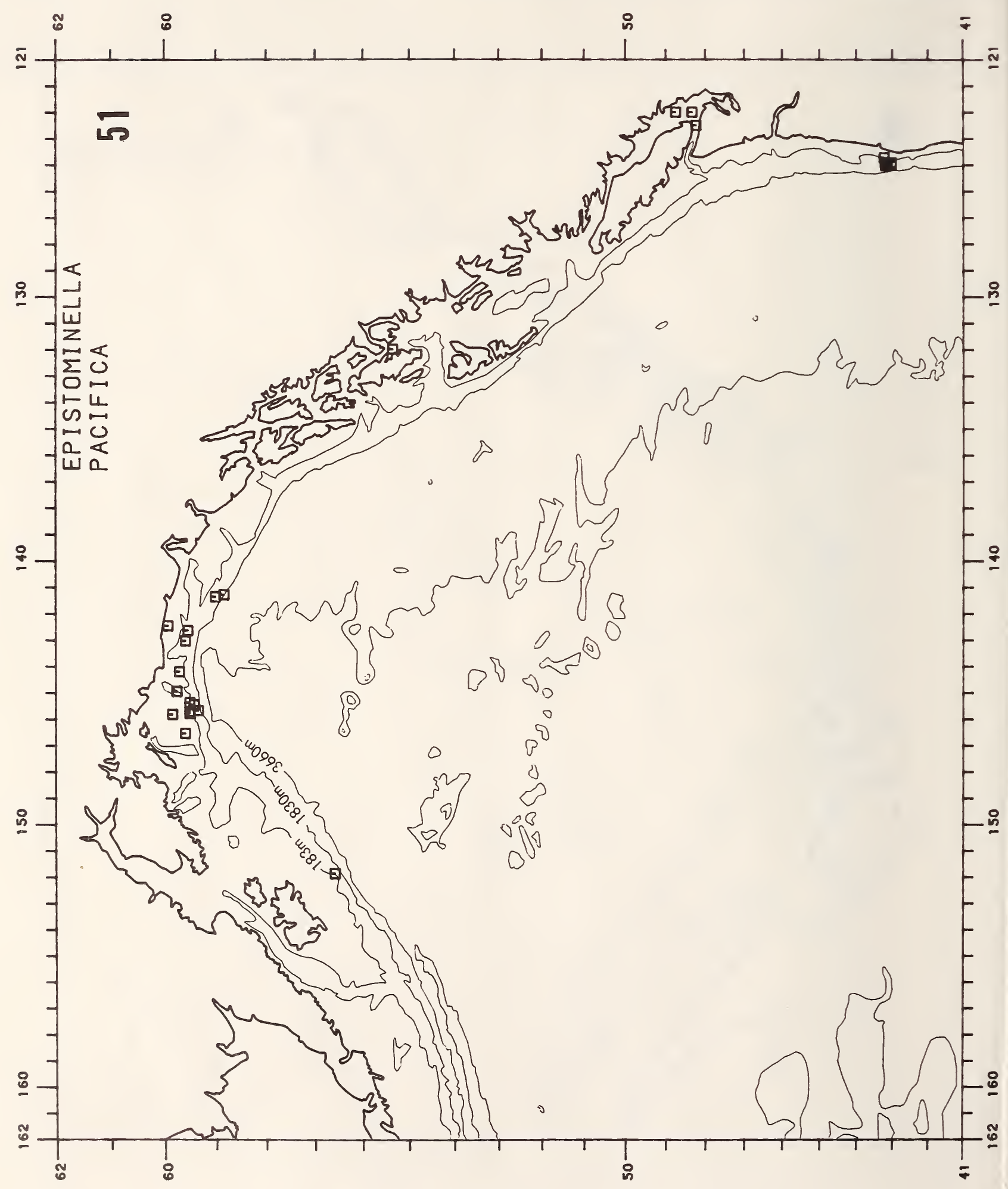




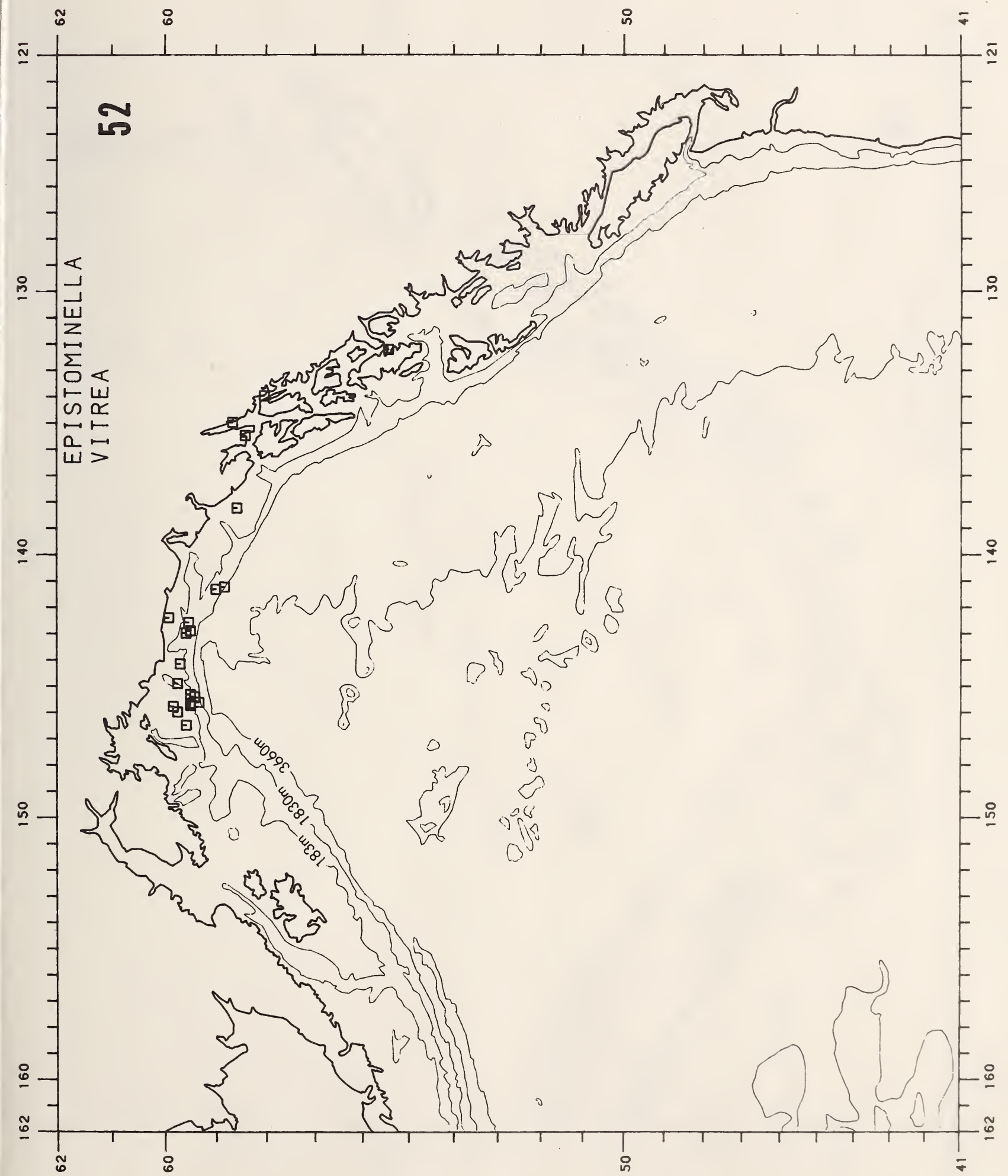




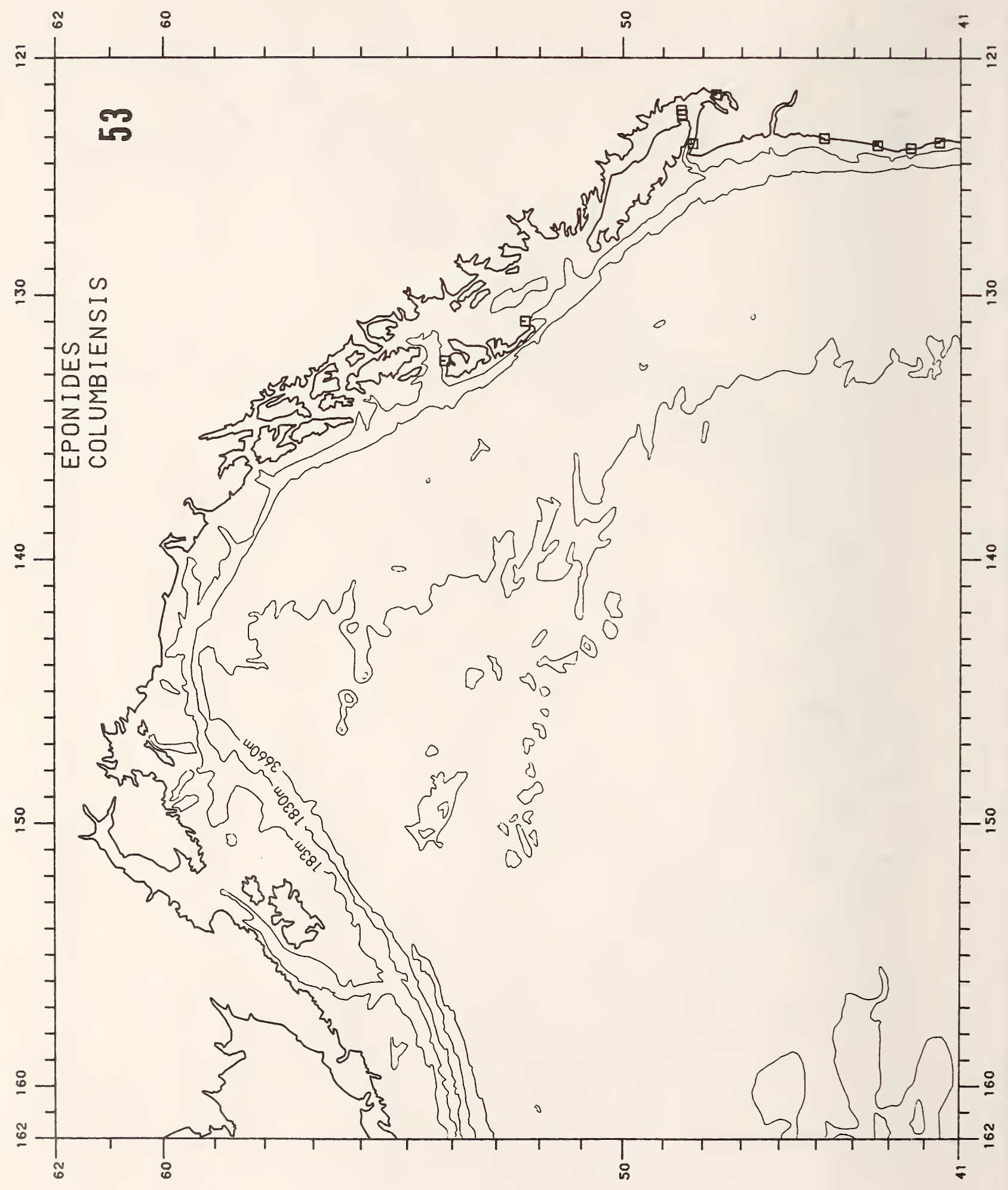




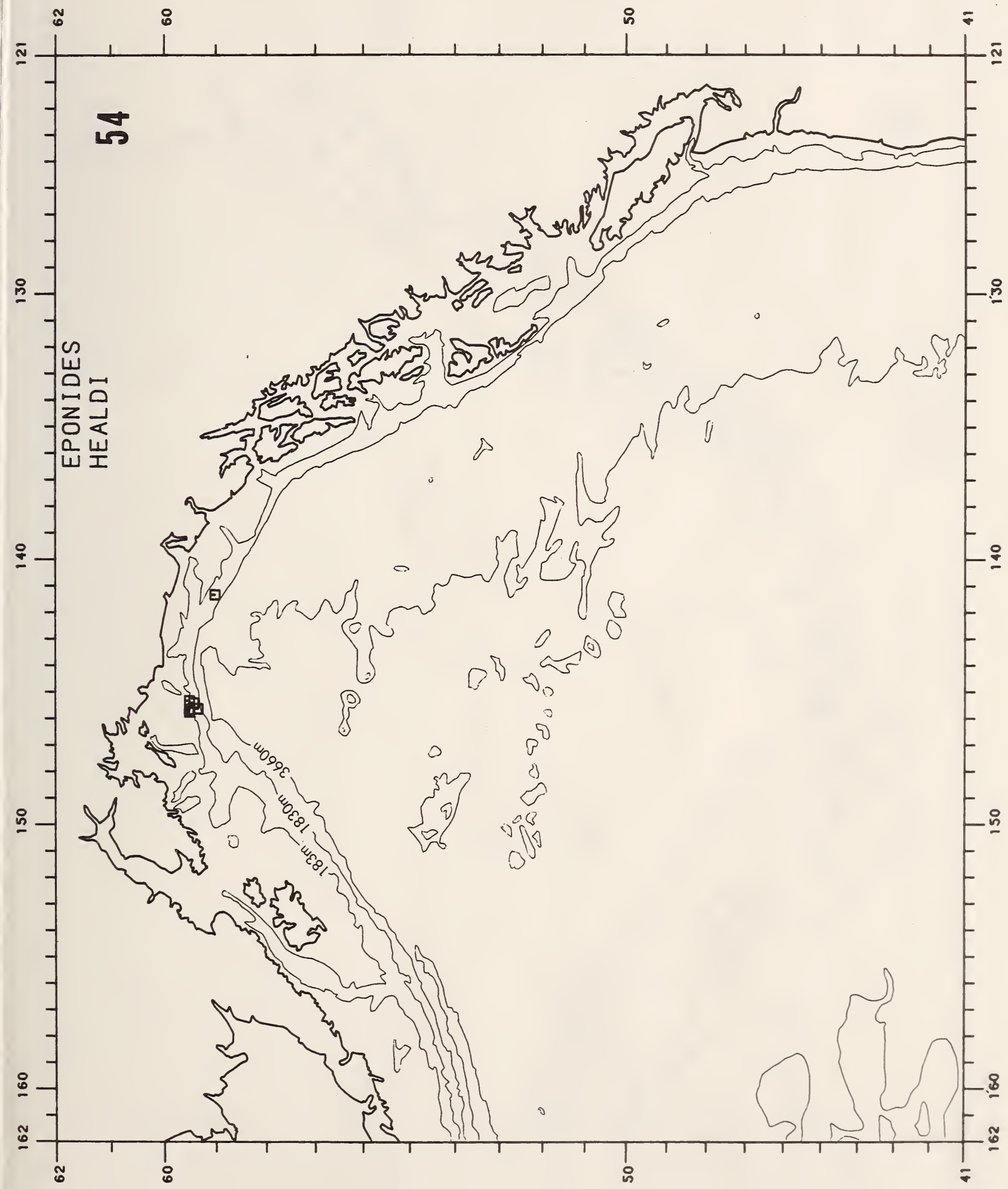




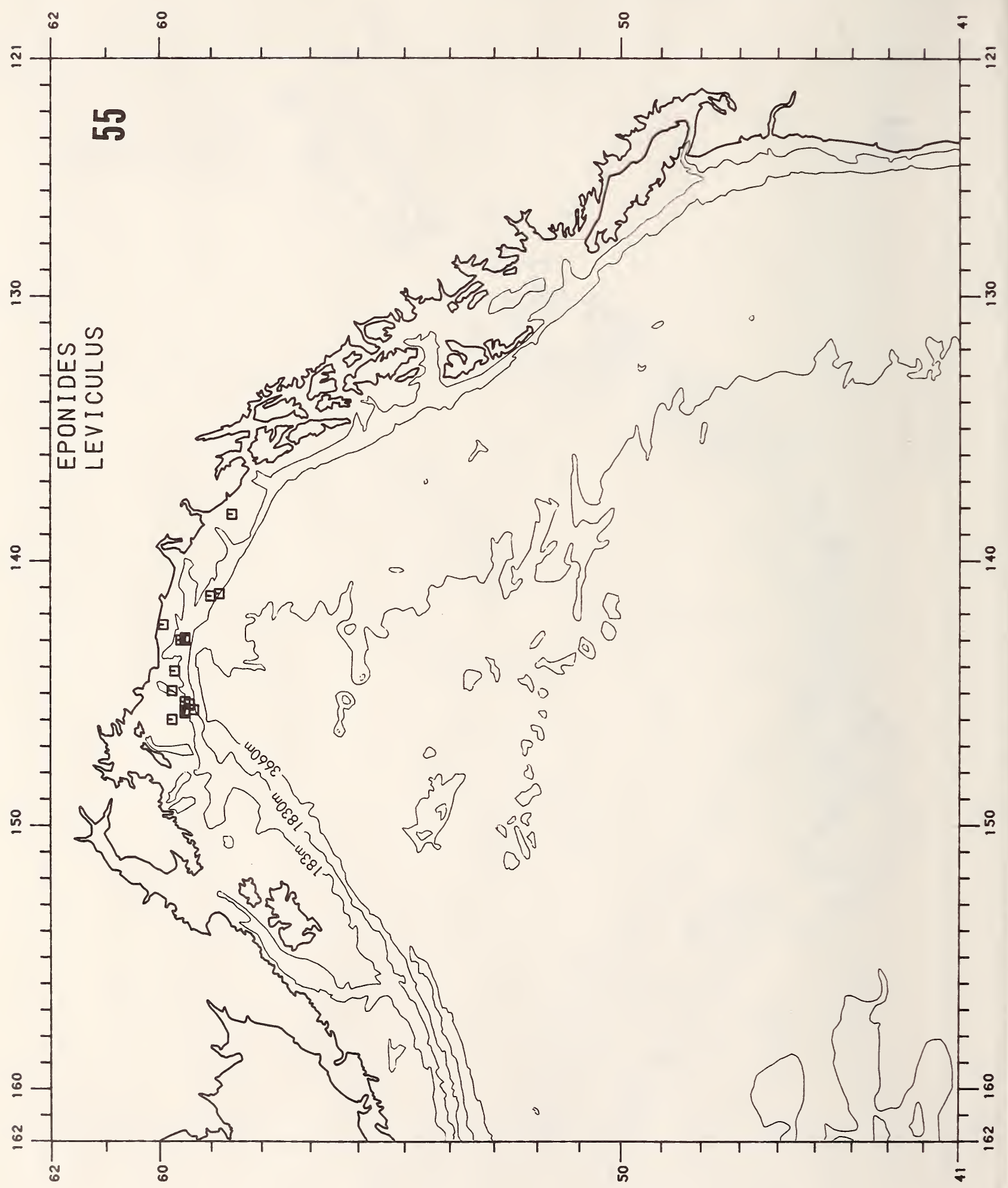




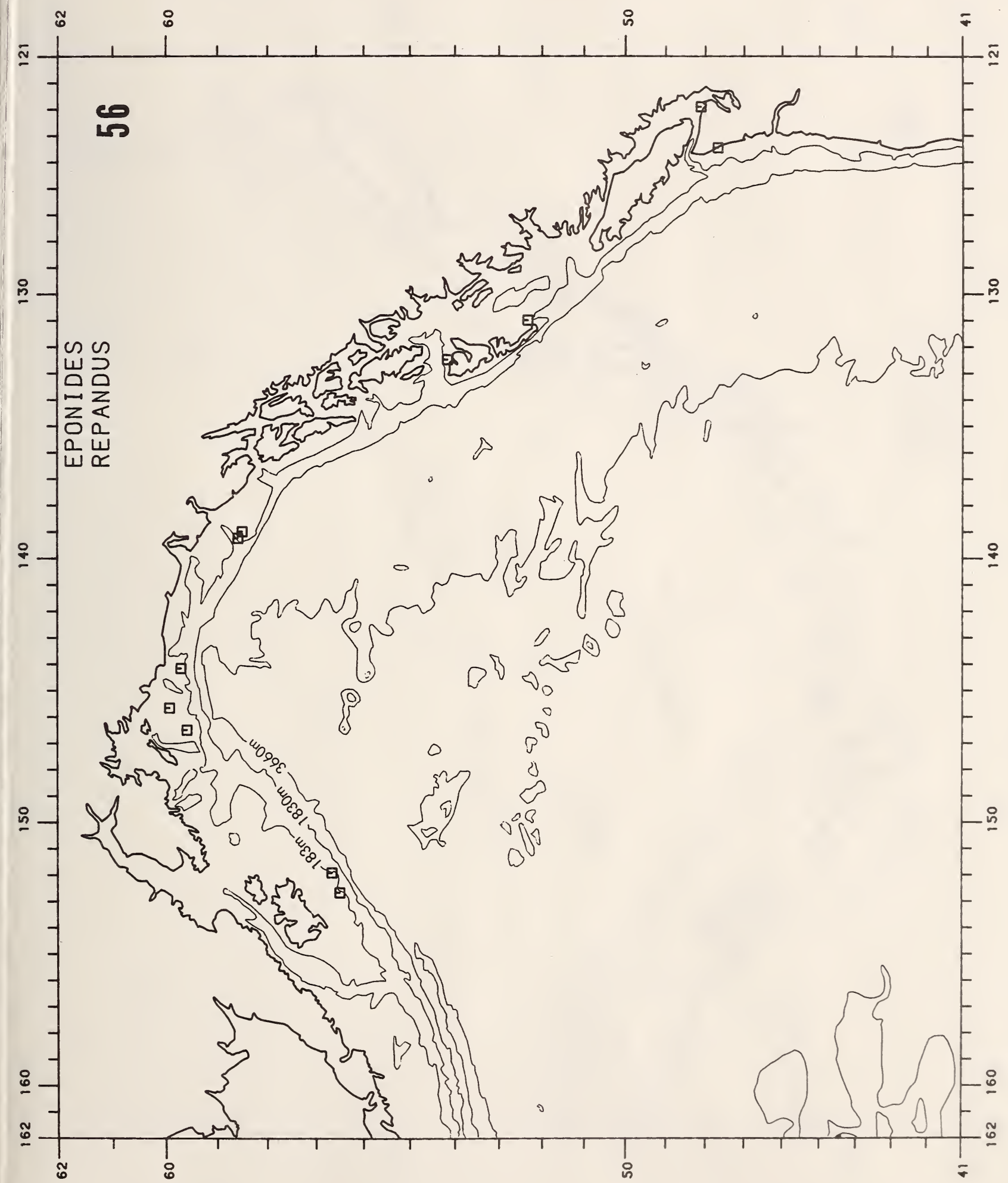




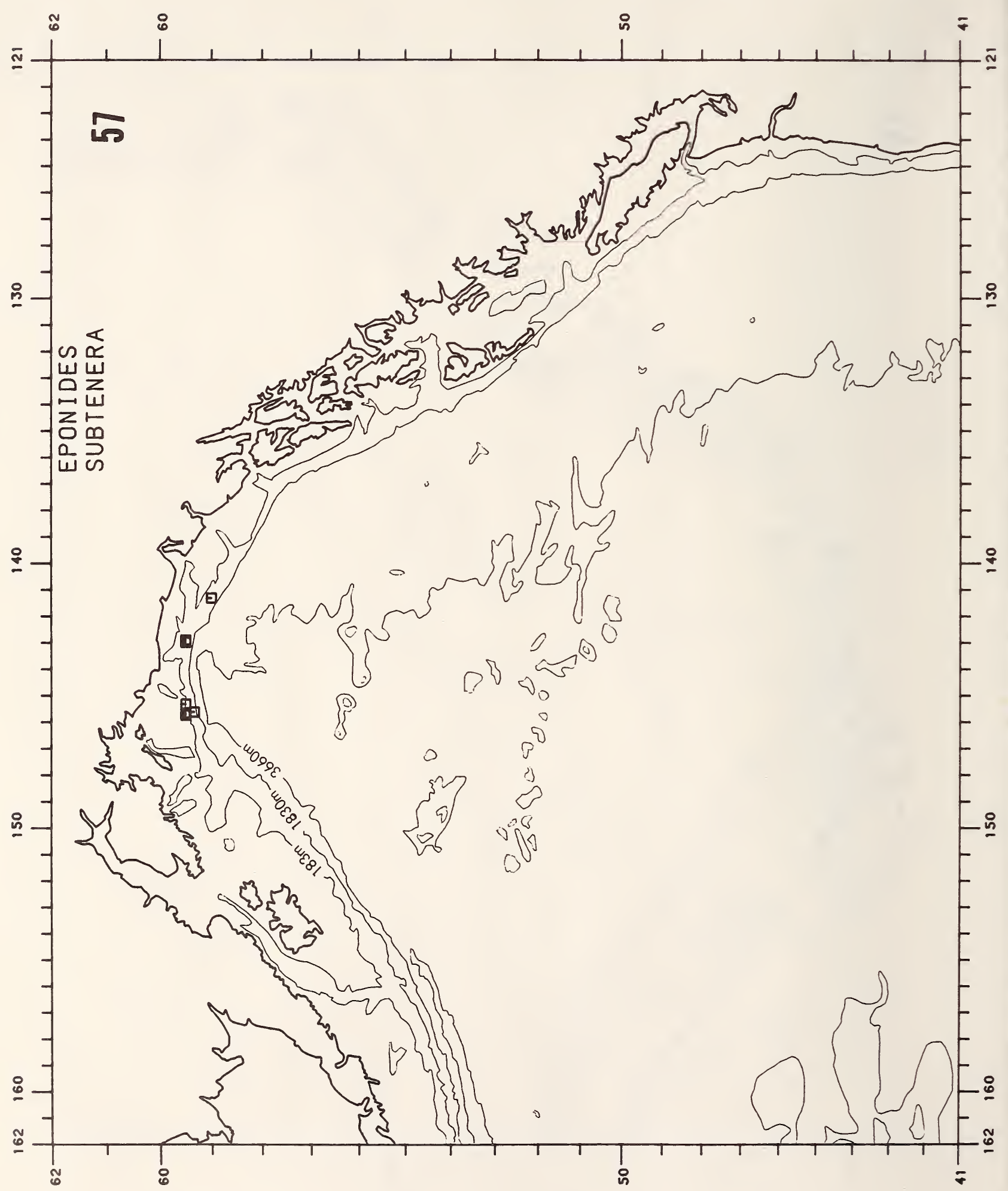




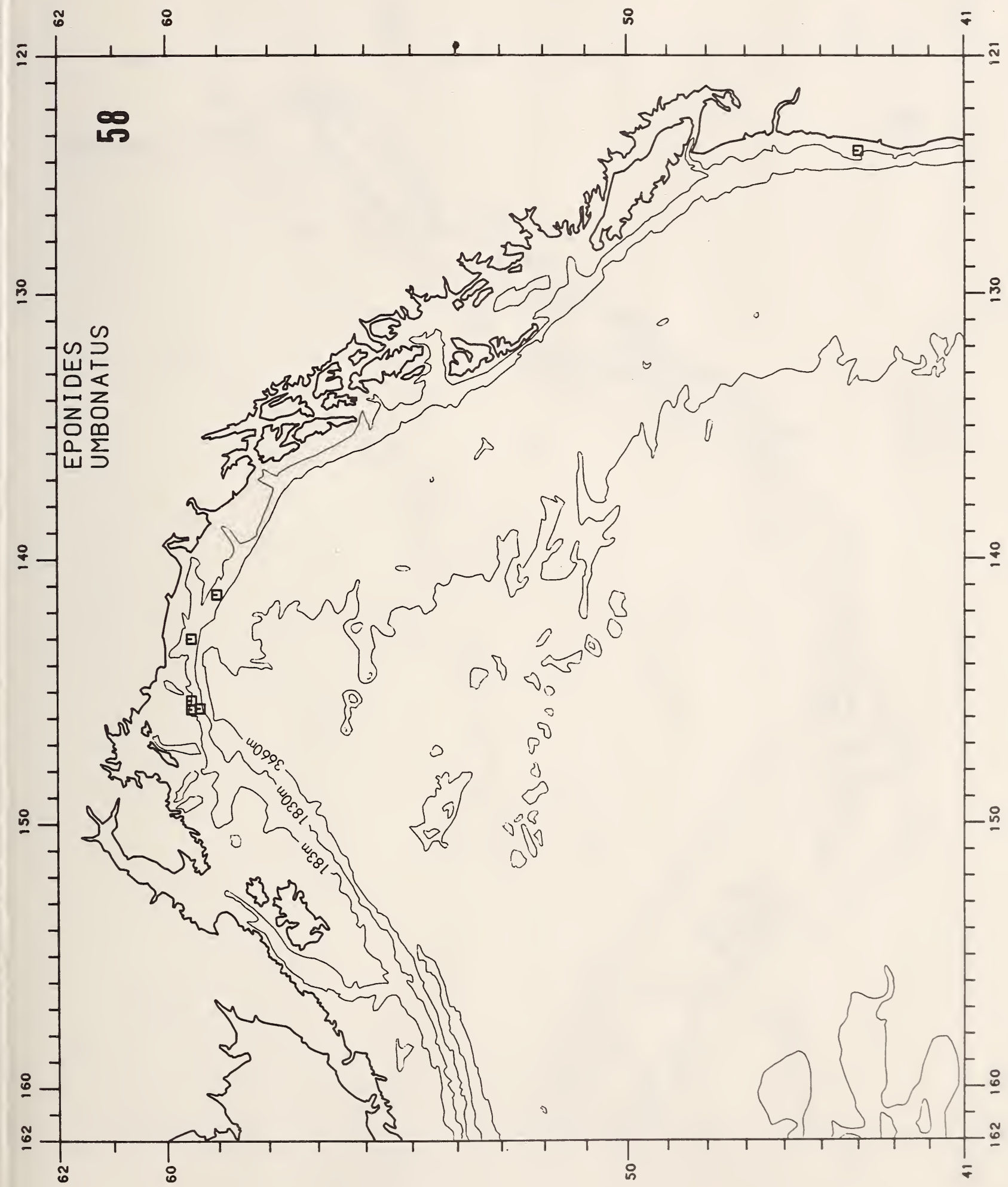




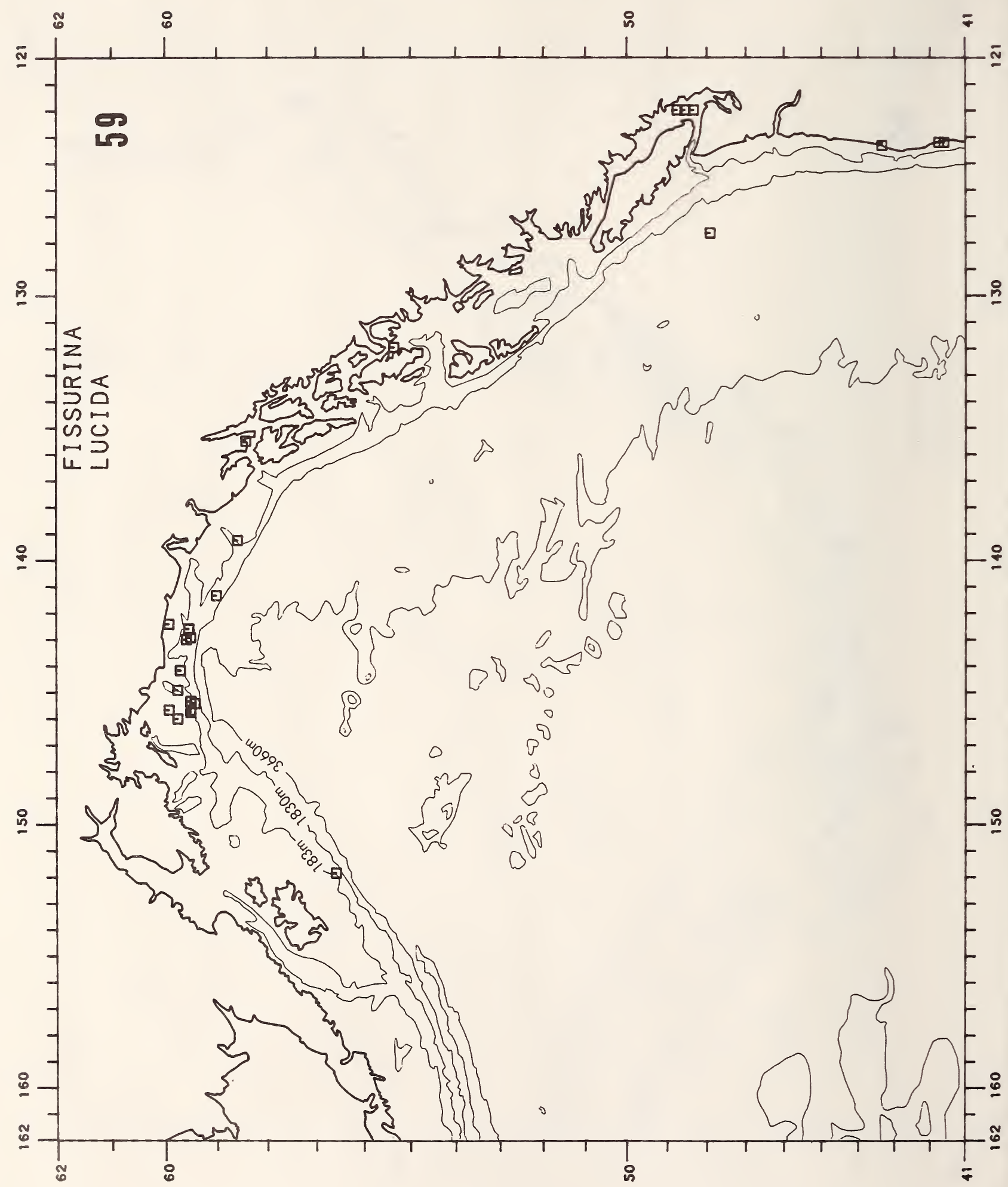




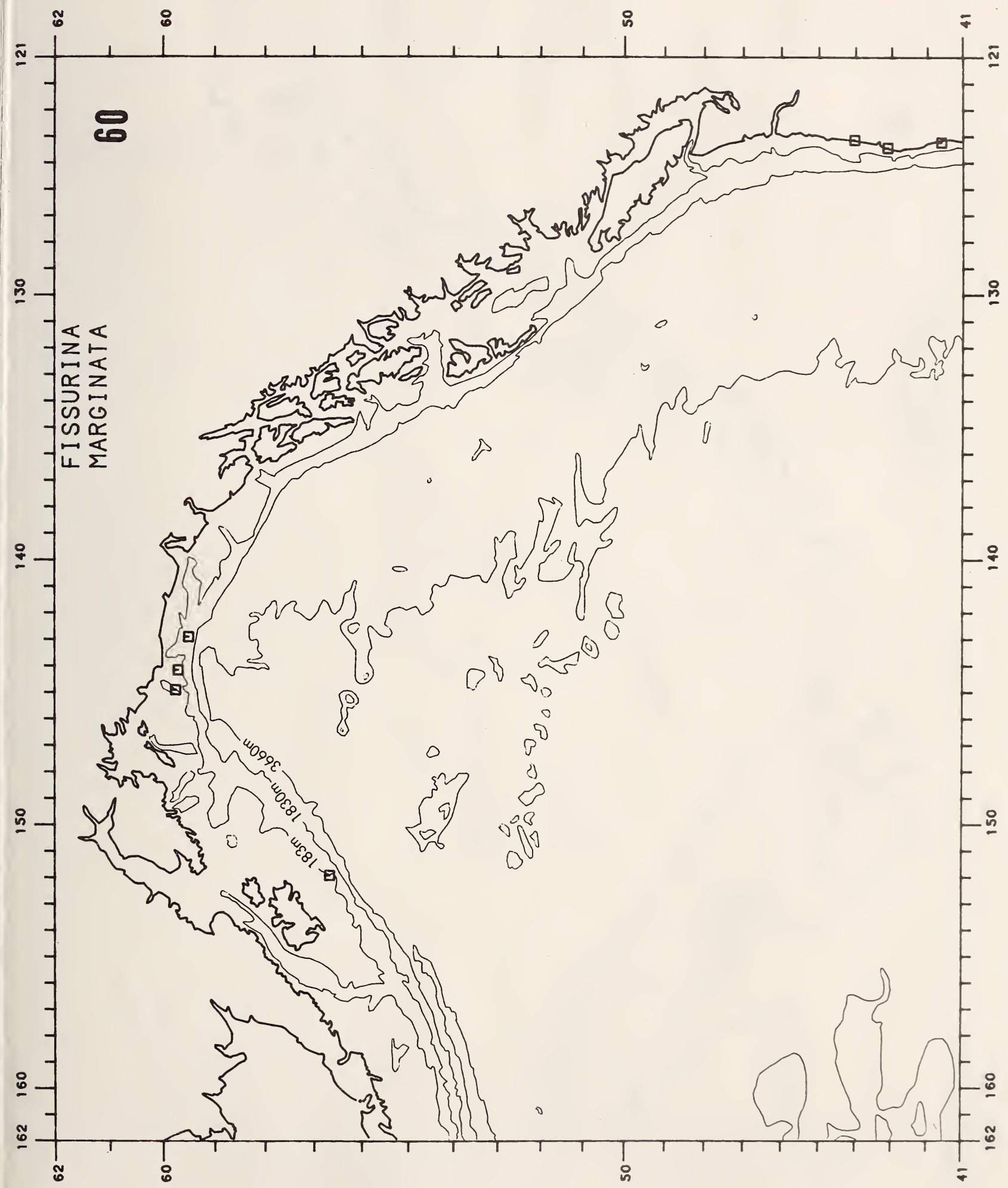




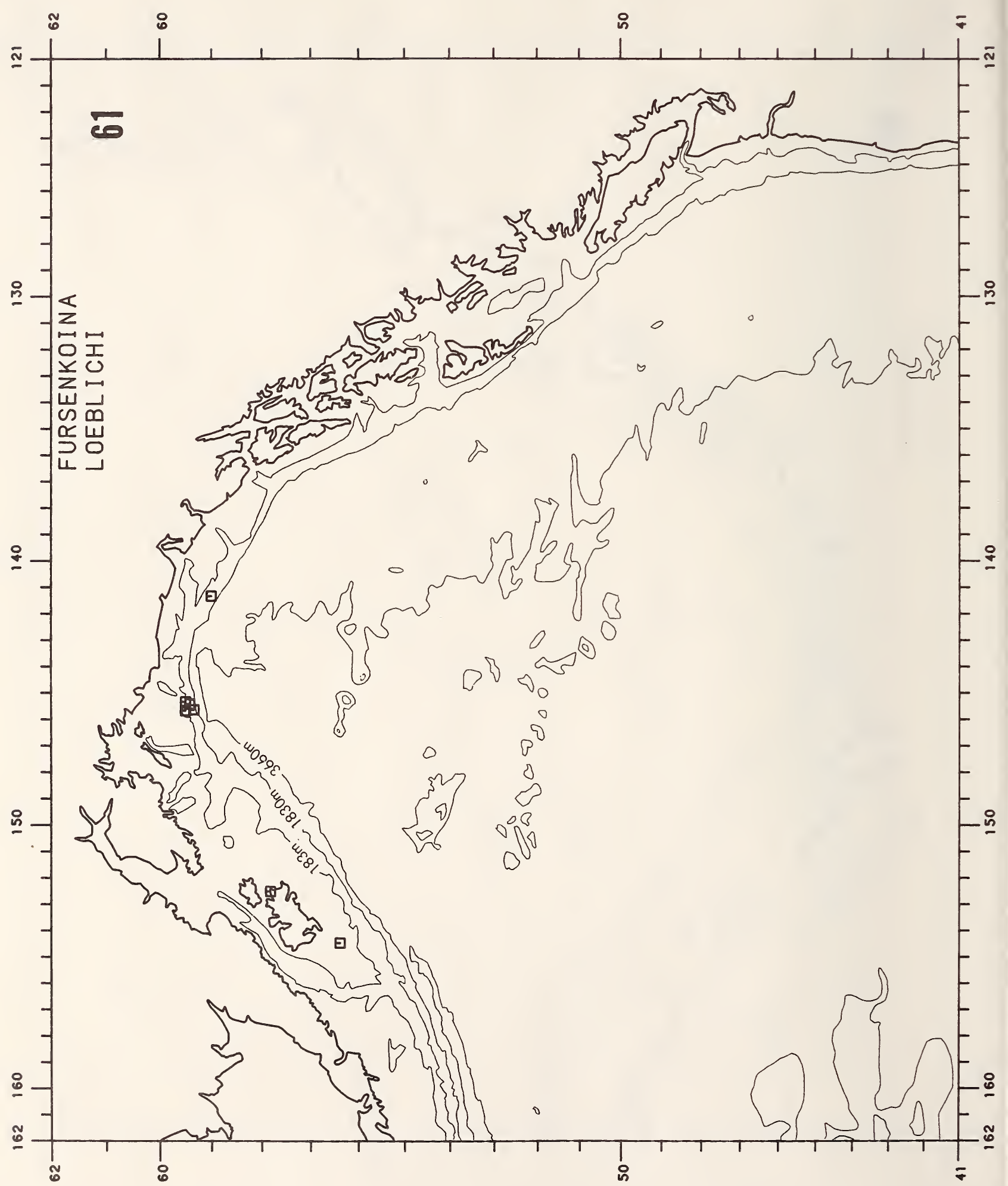




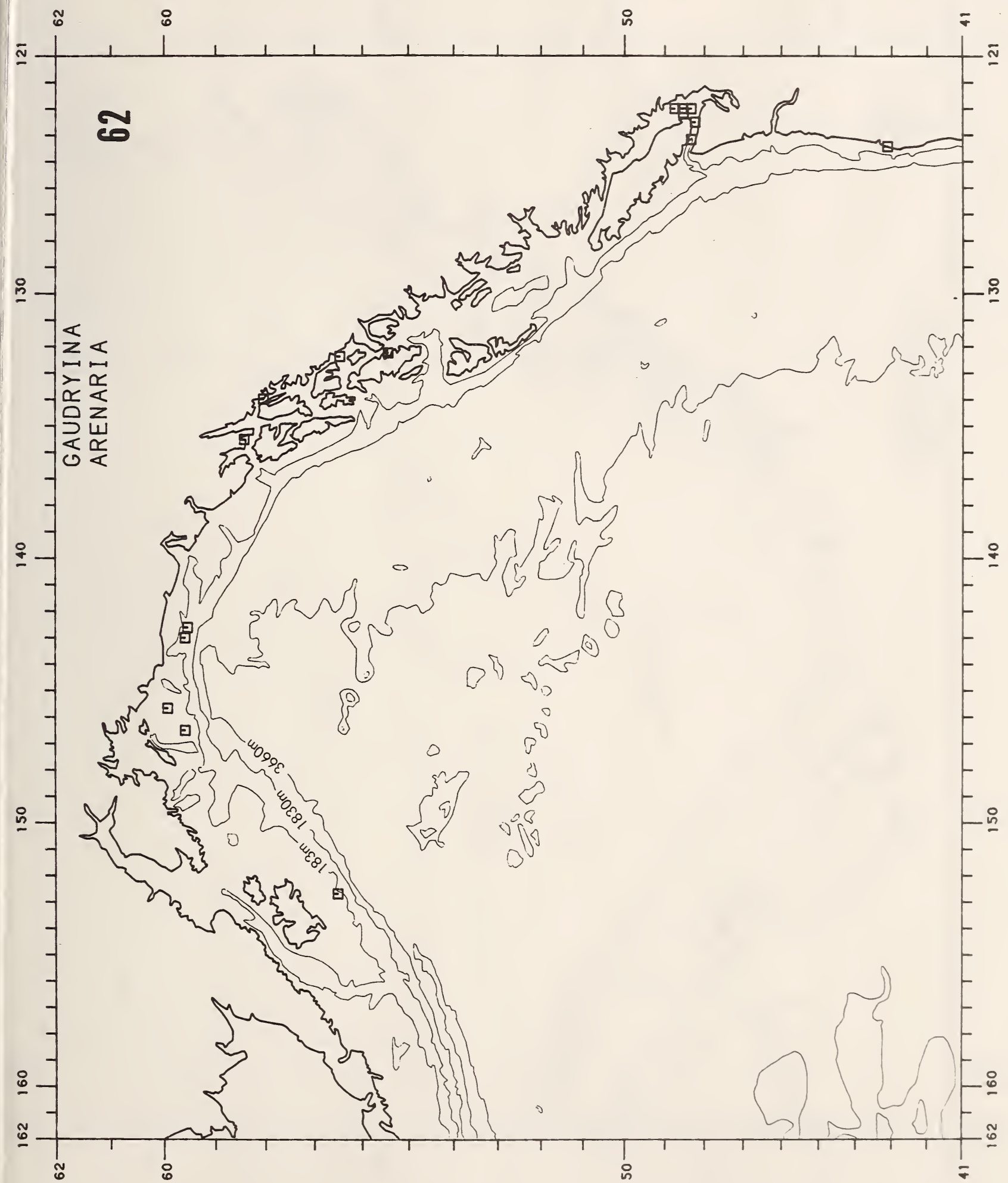




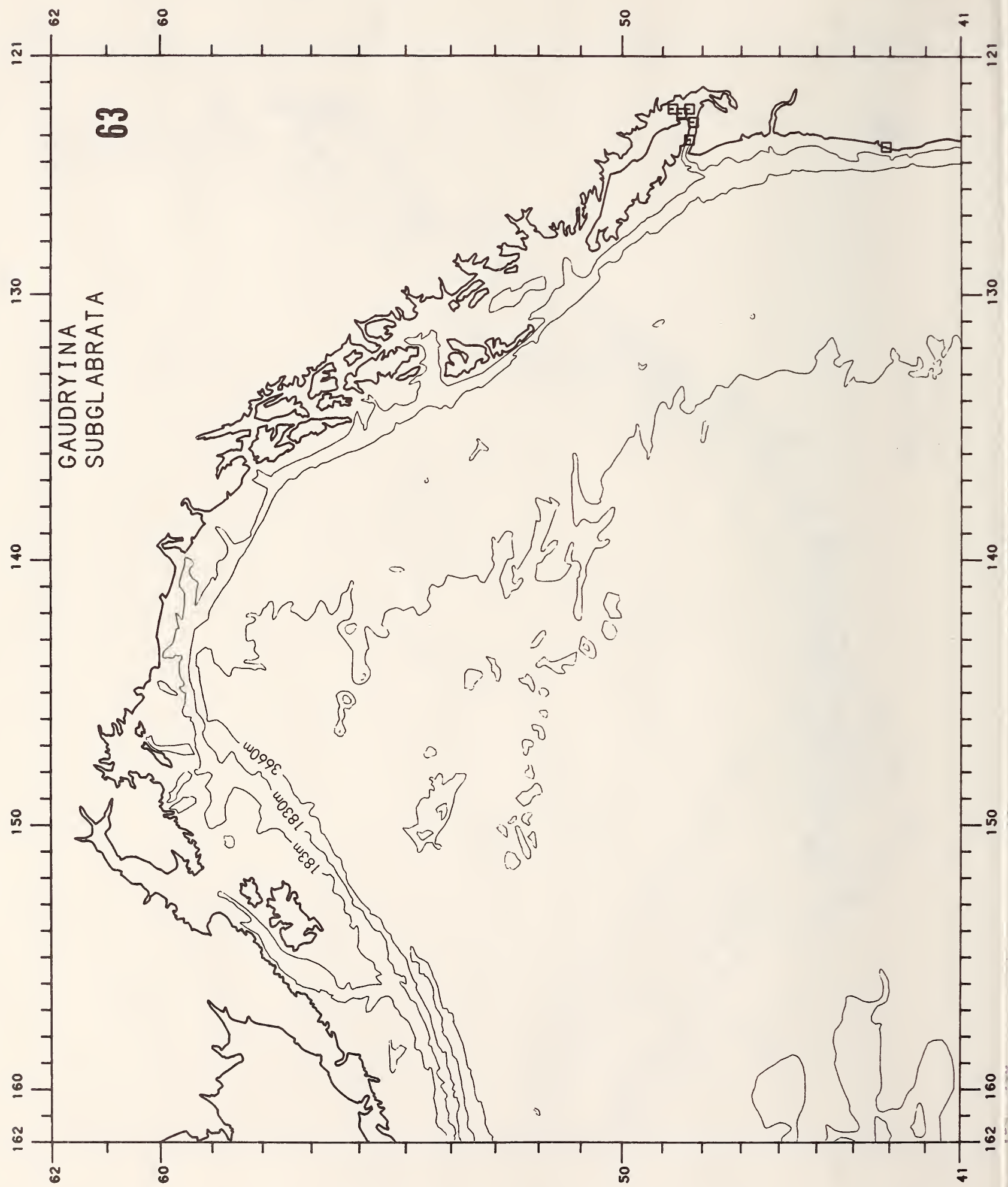


NUMBER 26

159

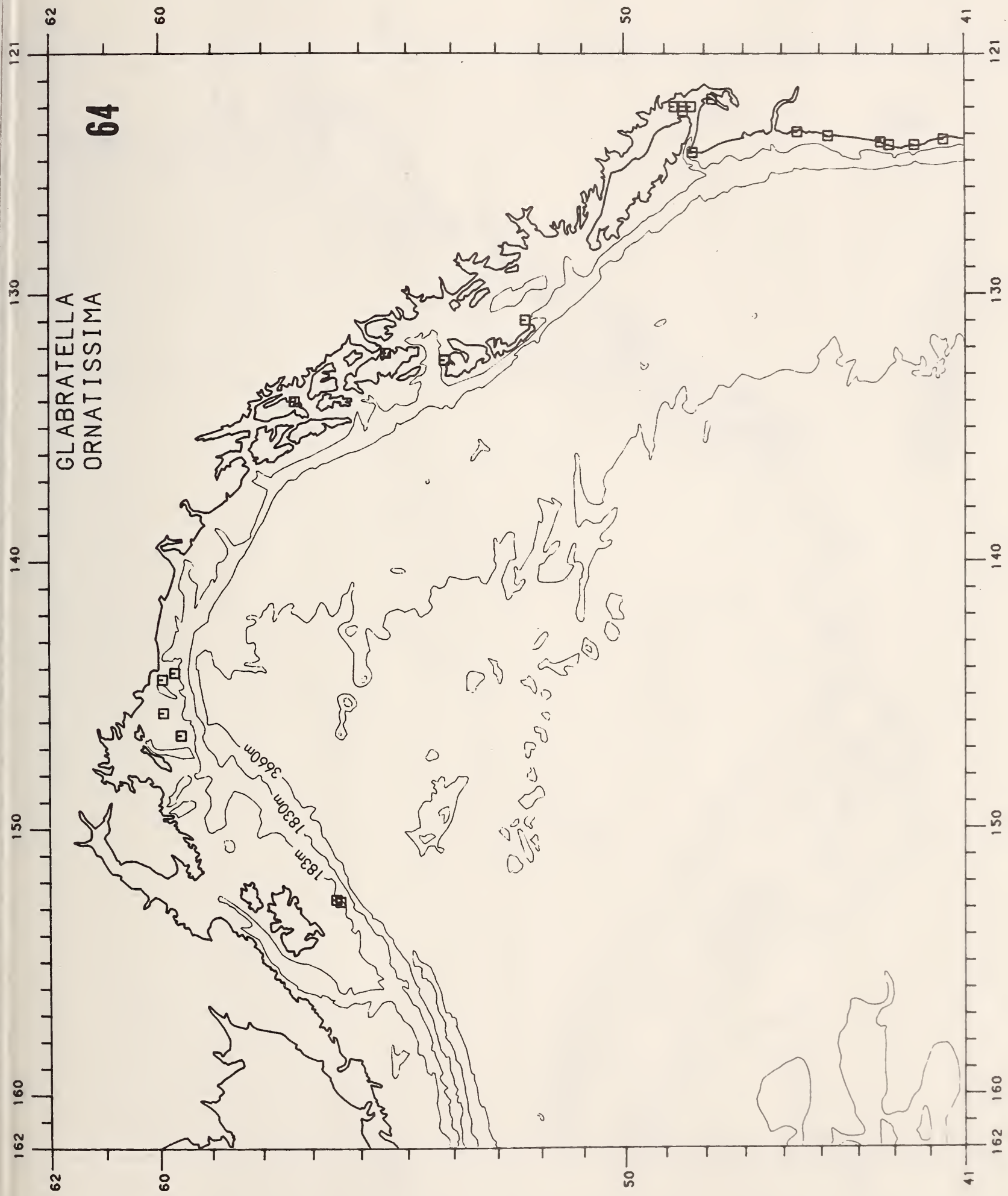




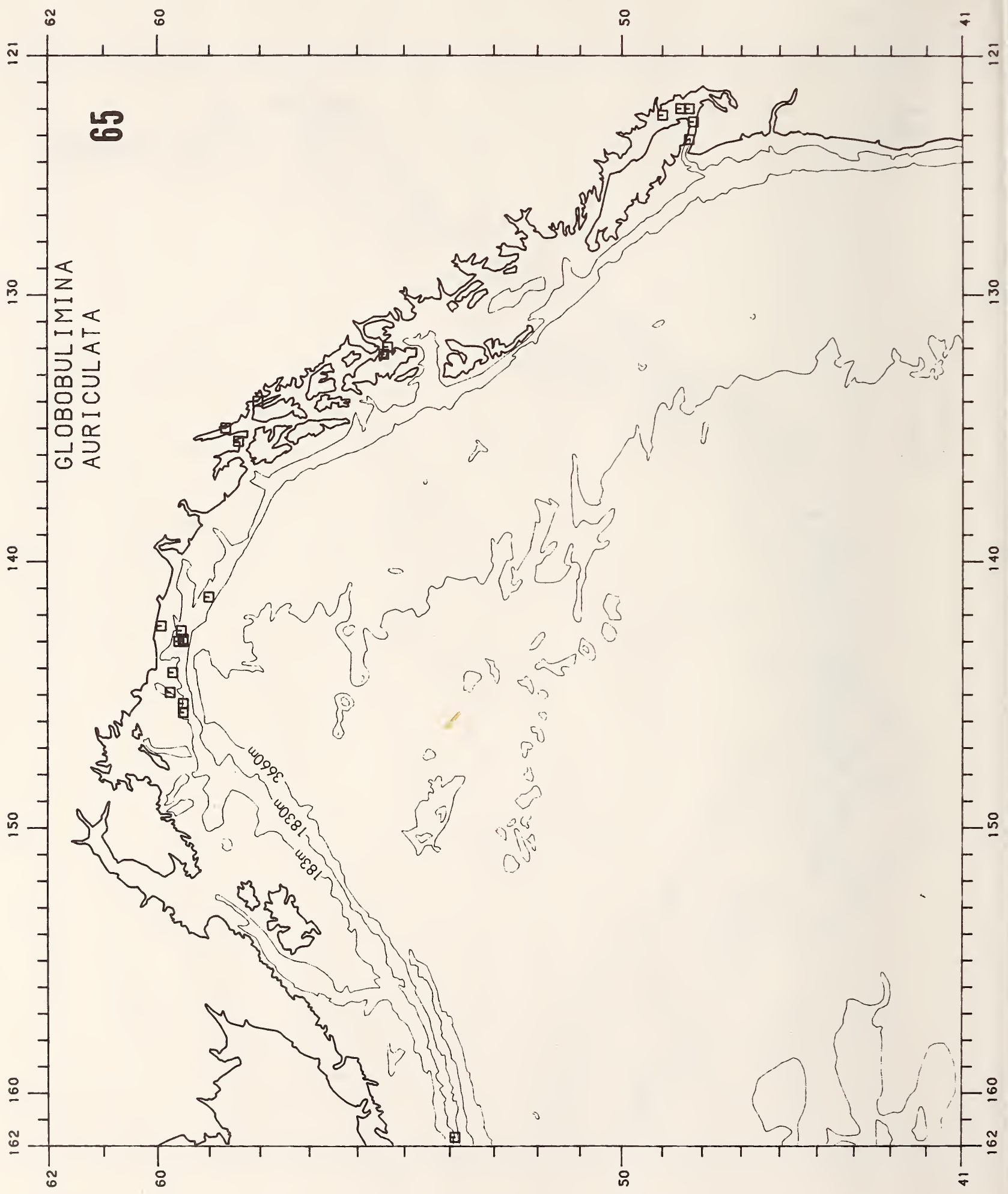




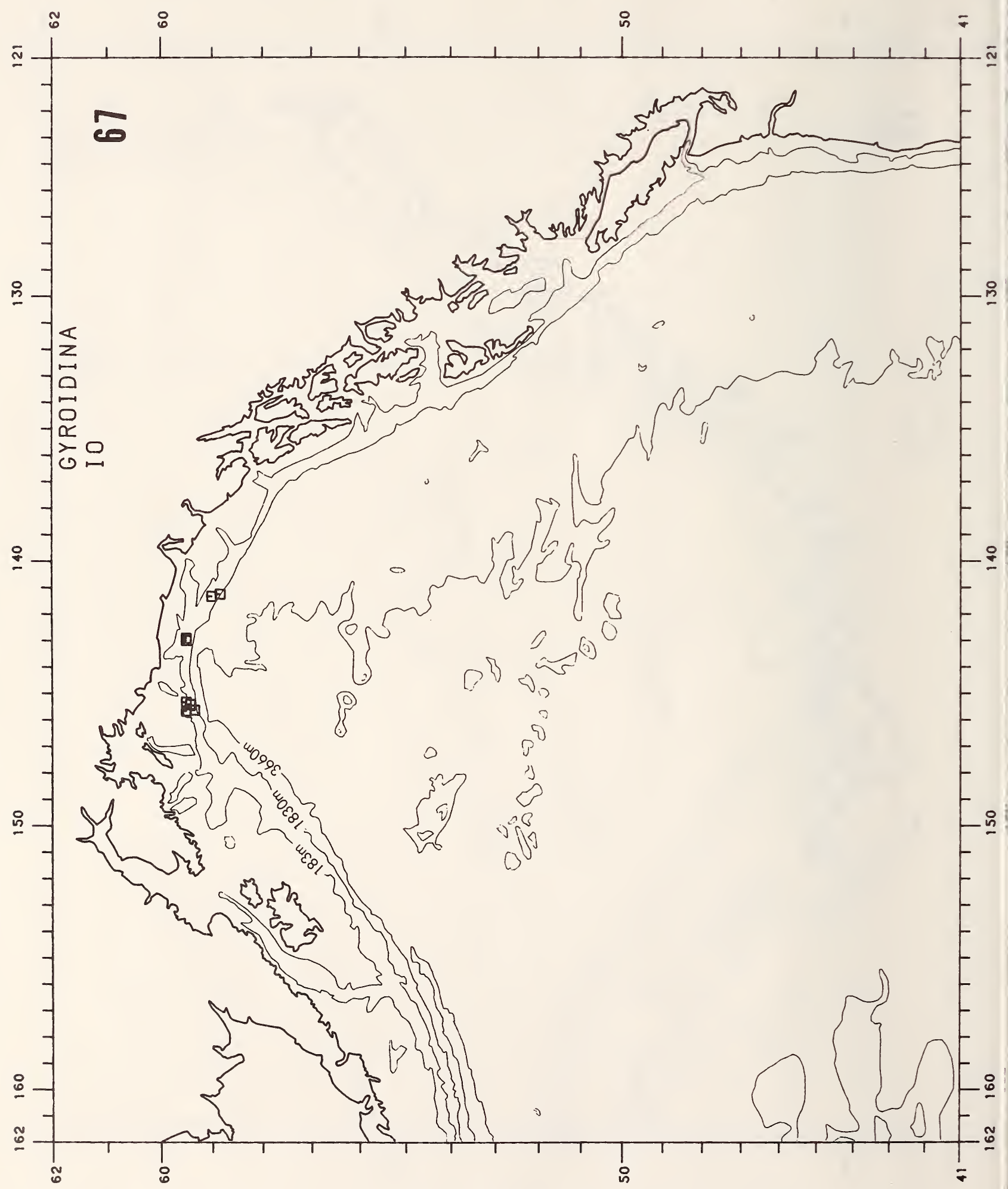




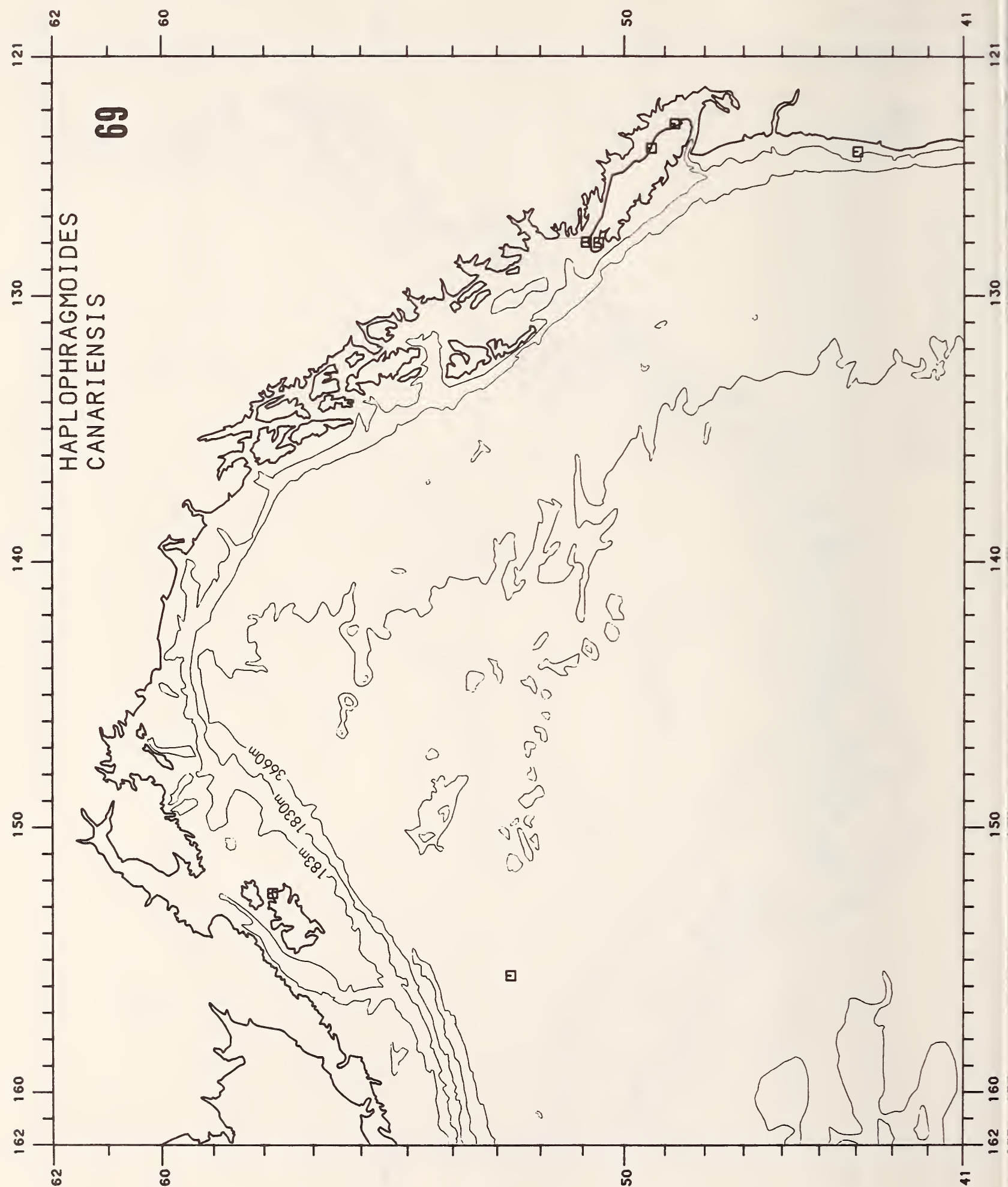




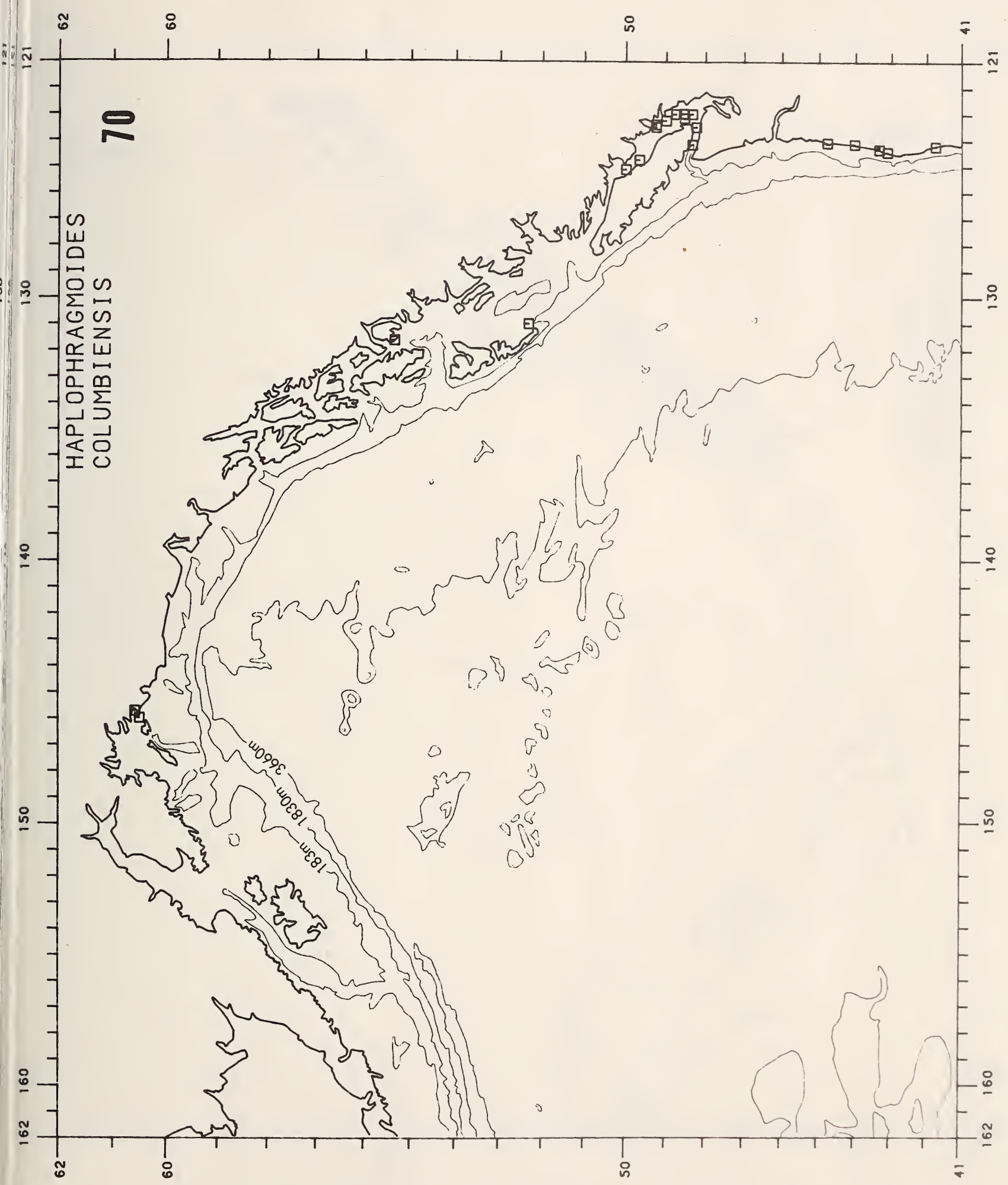


166

SMITHSONIAN CONTRIBUTIONS TO THE MARINE SCIENCES

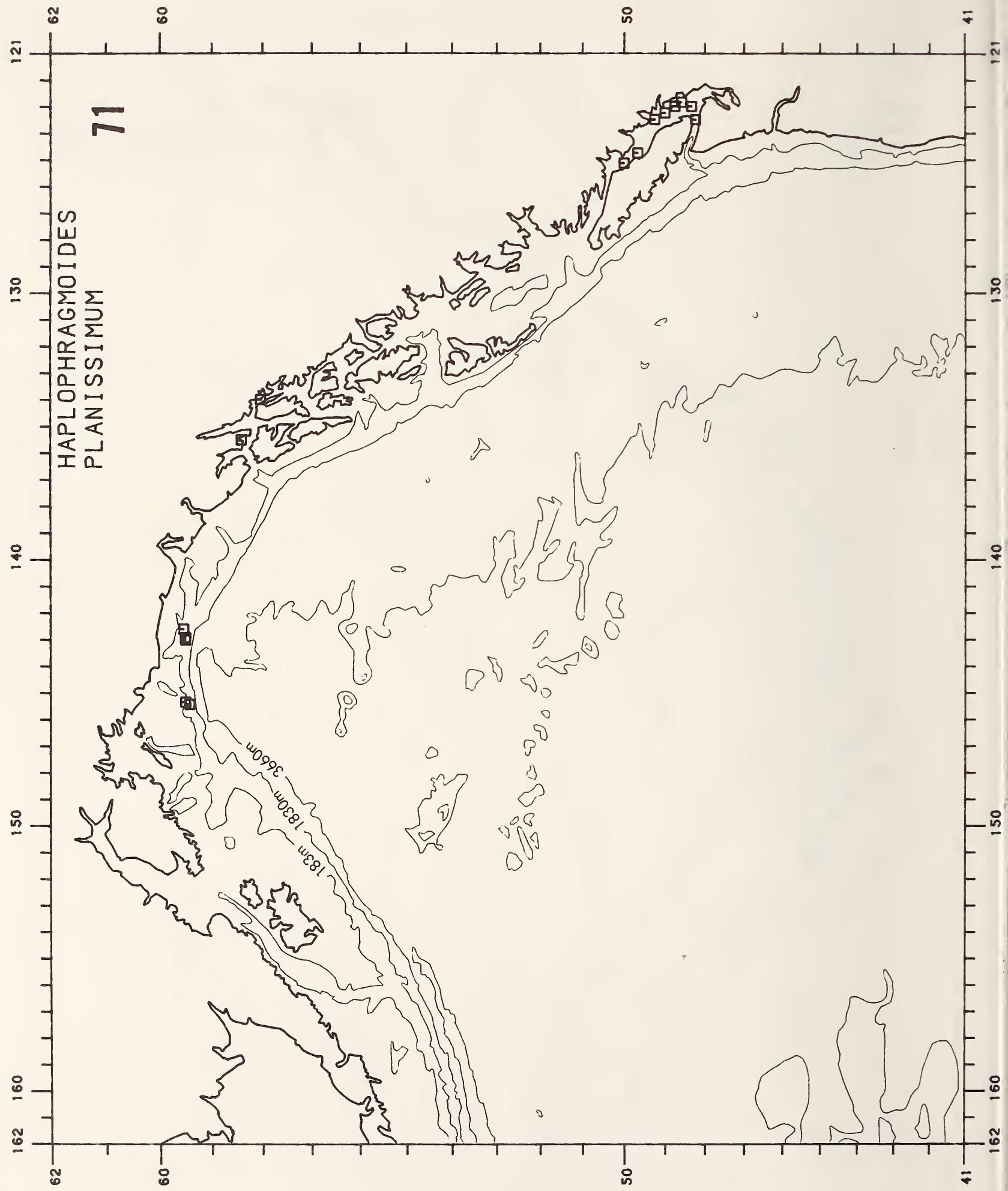




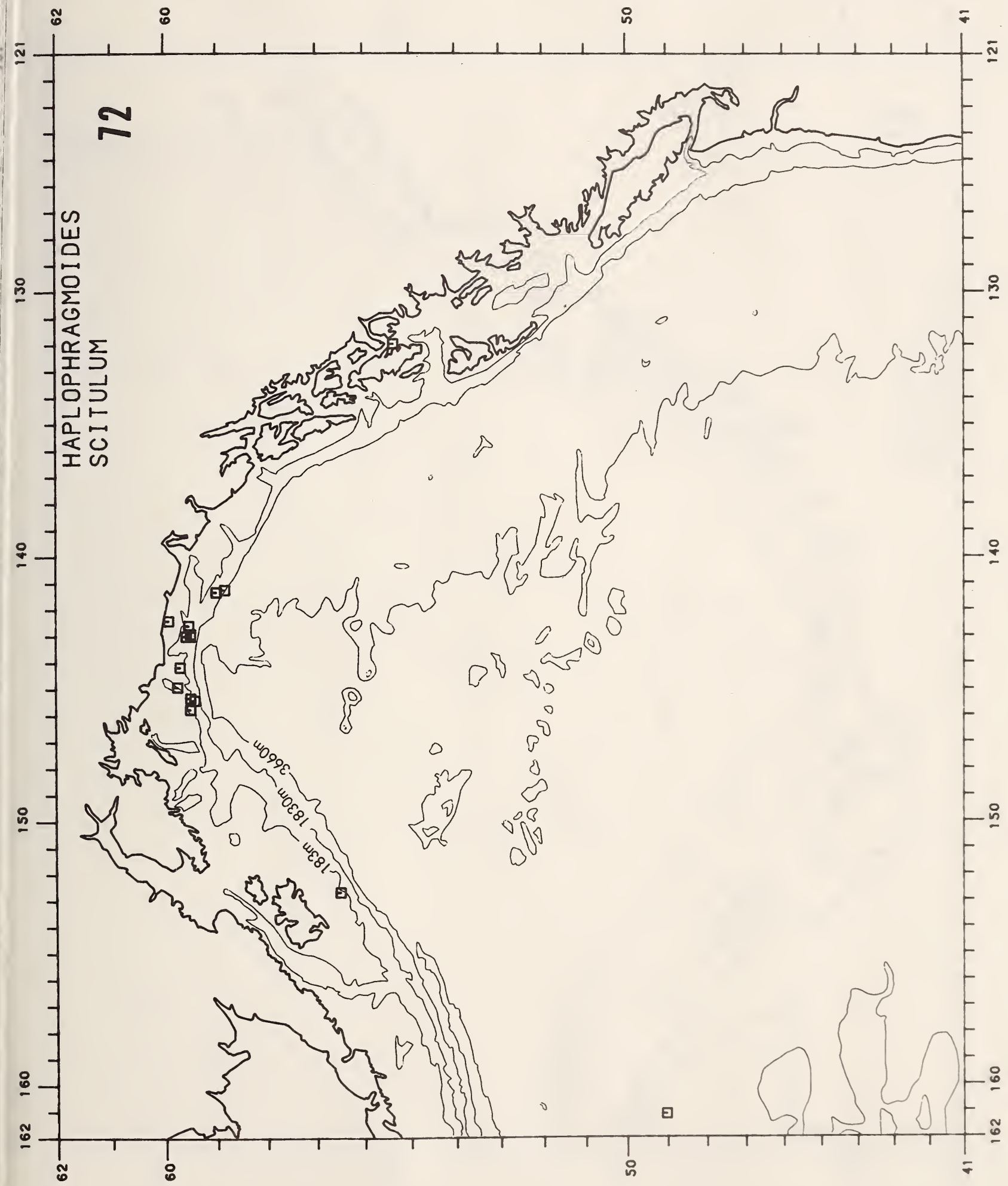




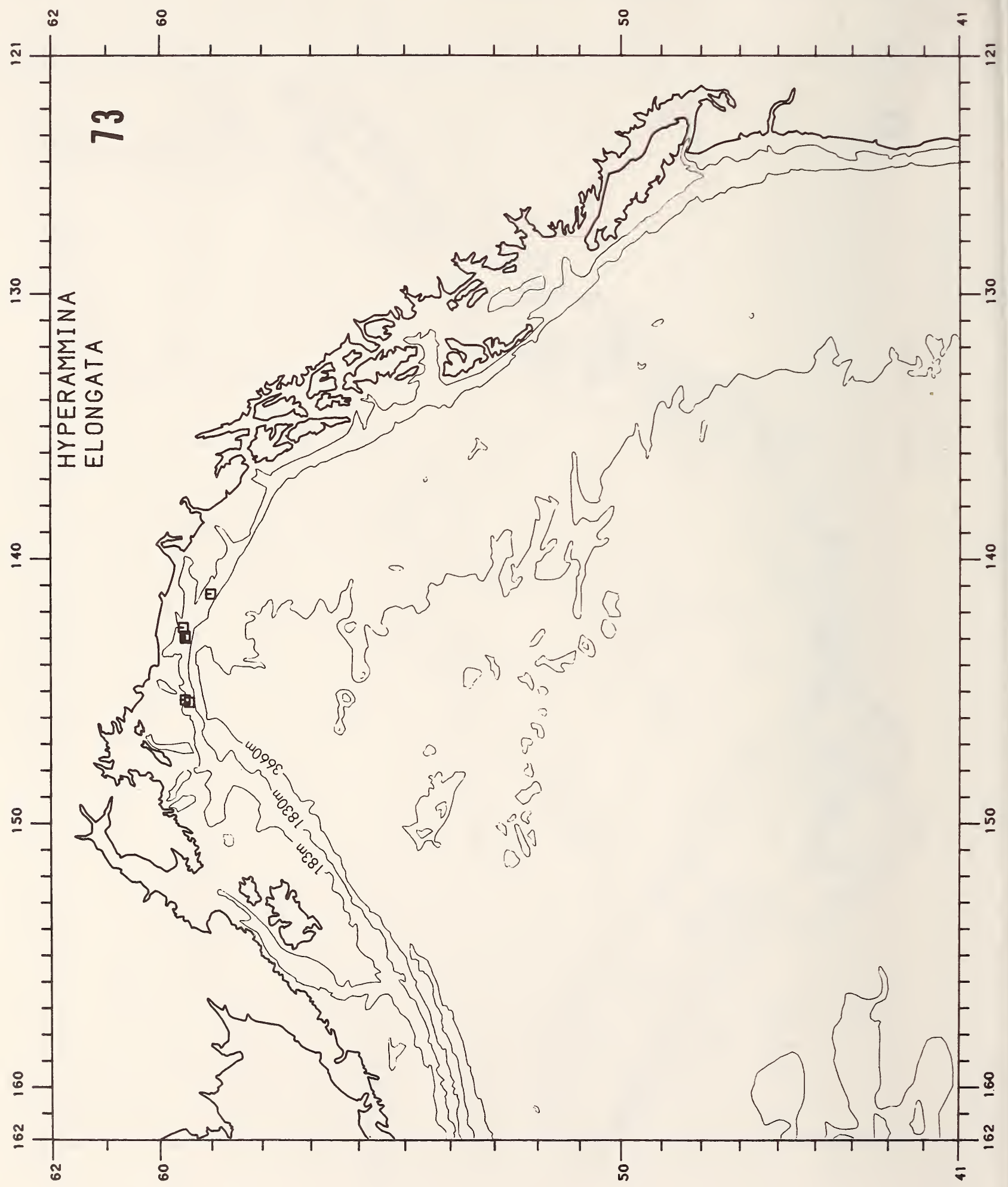




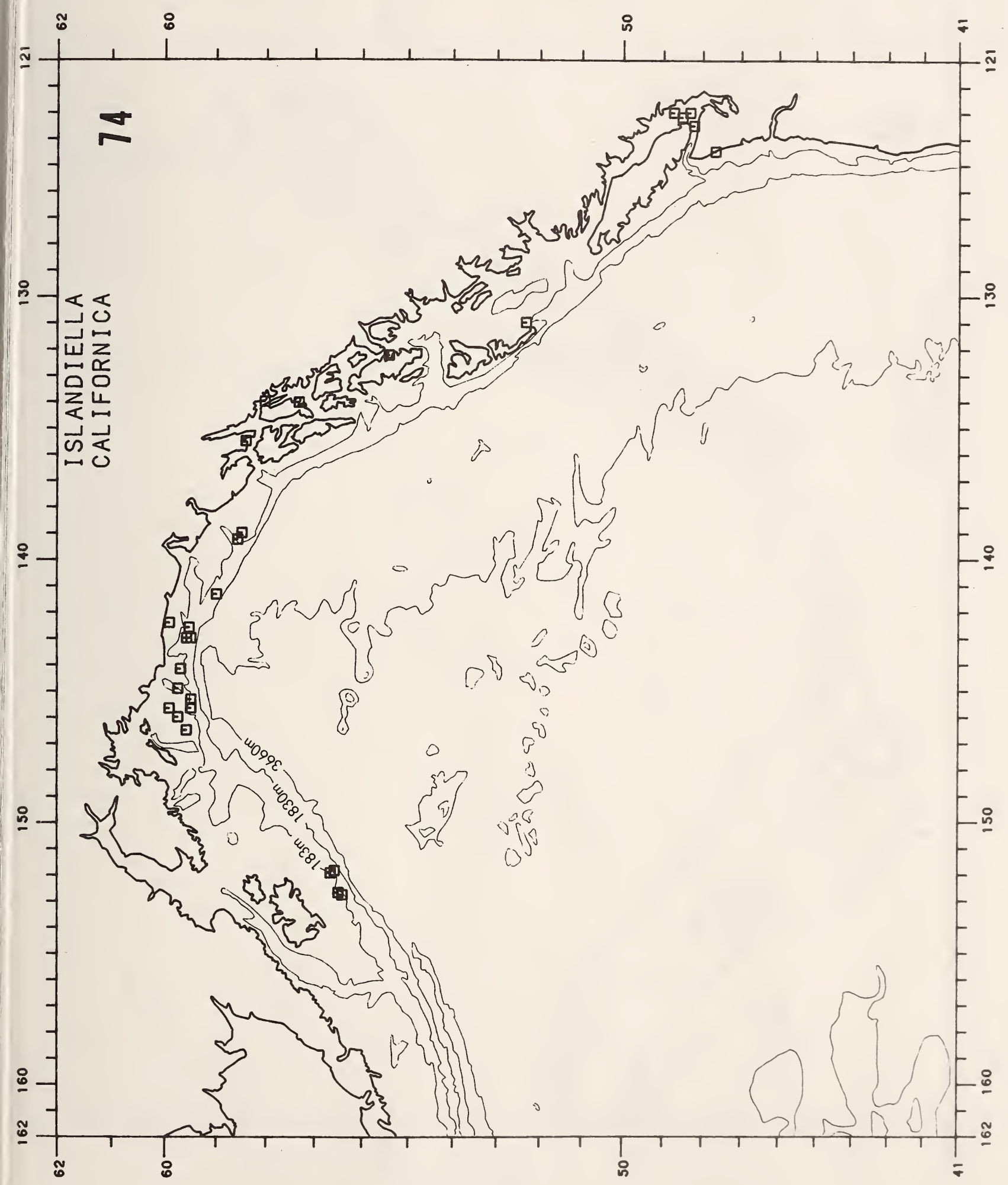




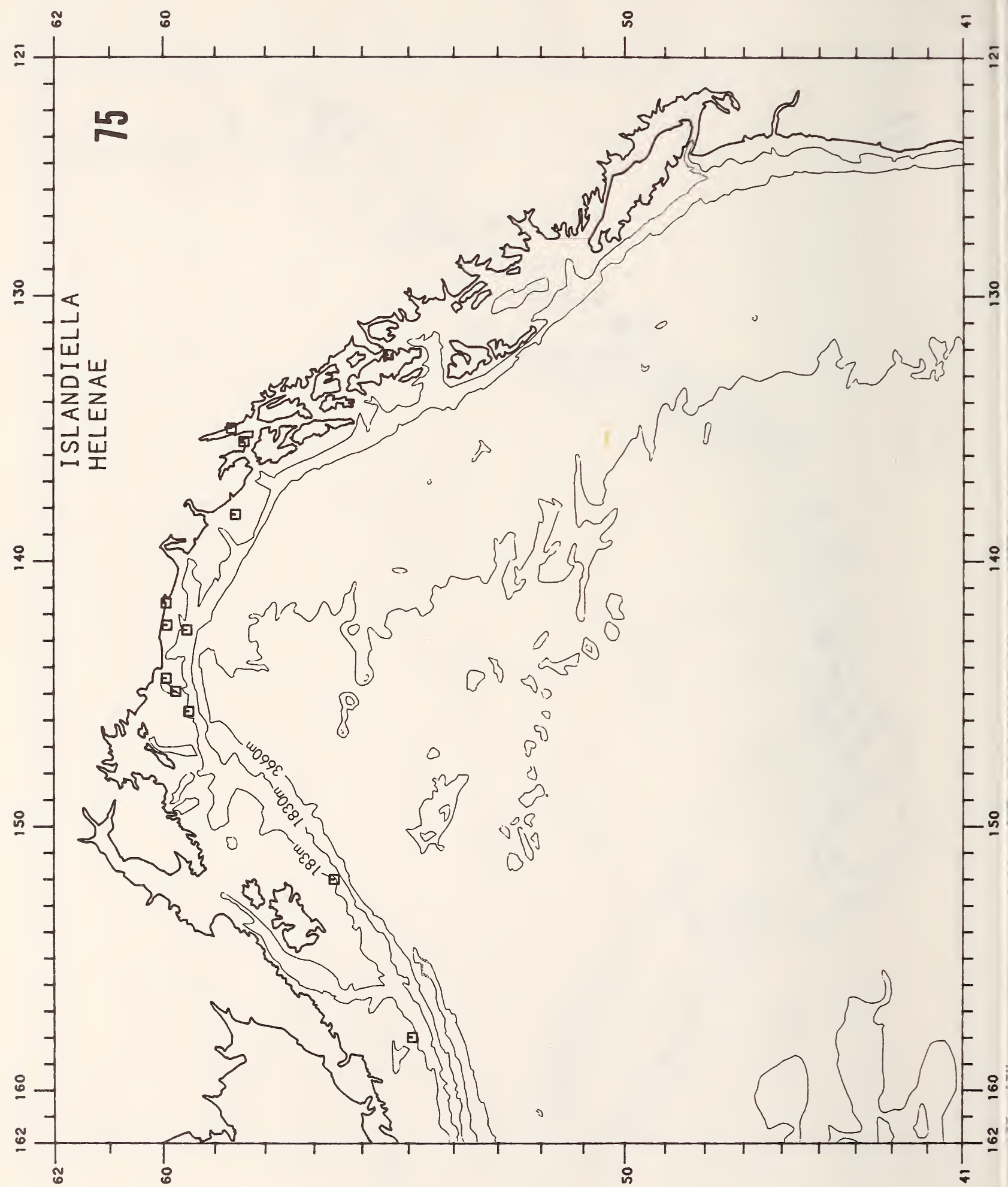


NUMBER 26

171

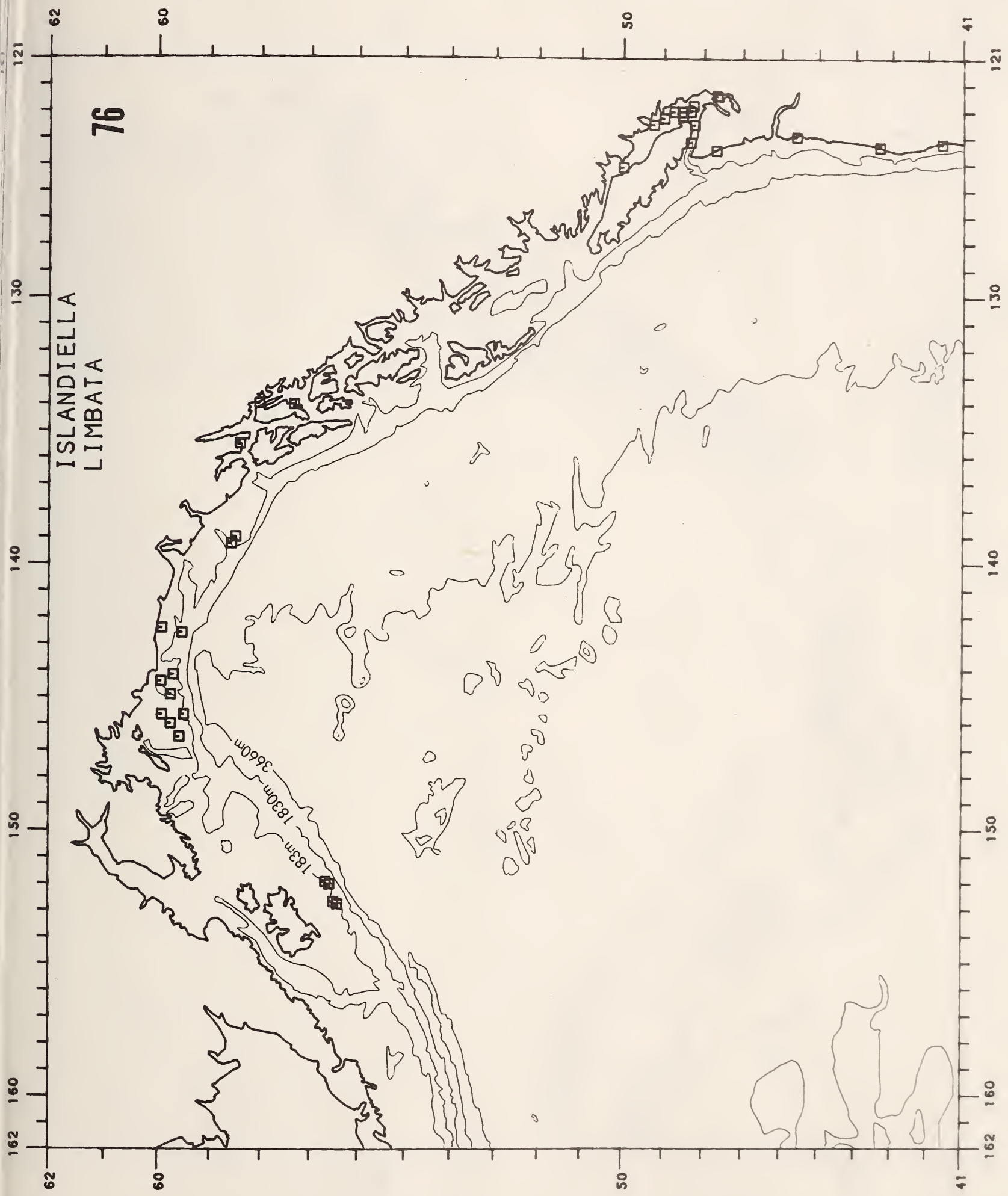




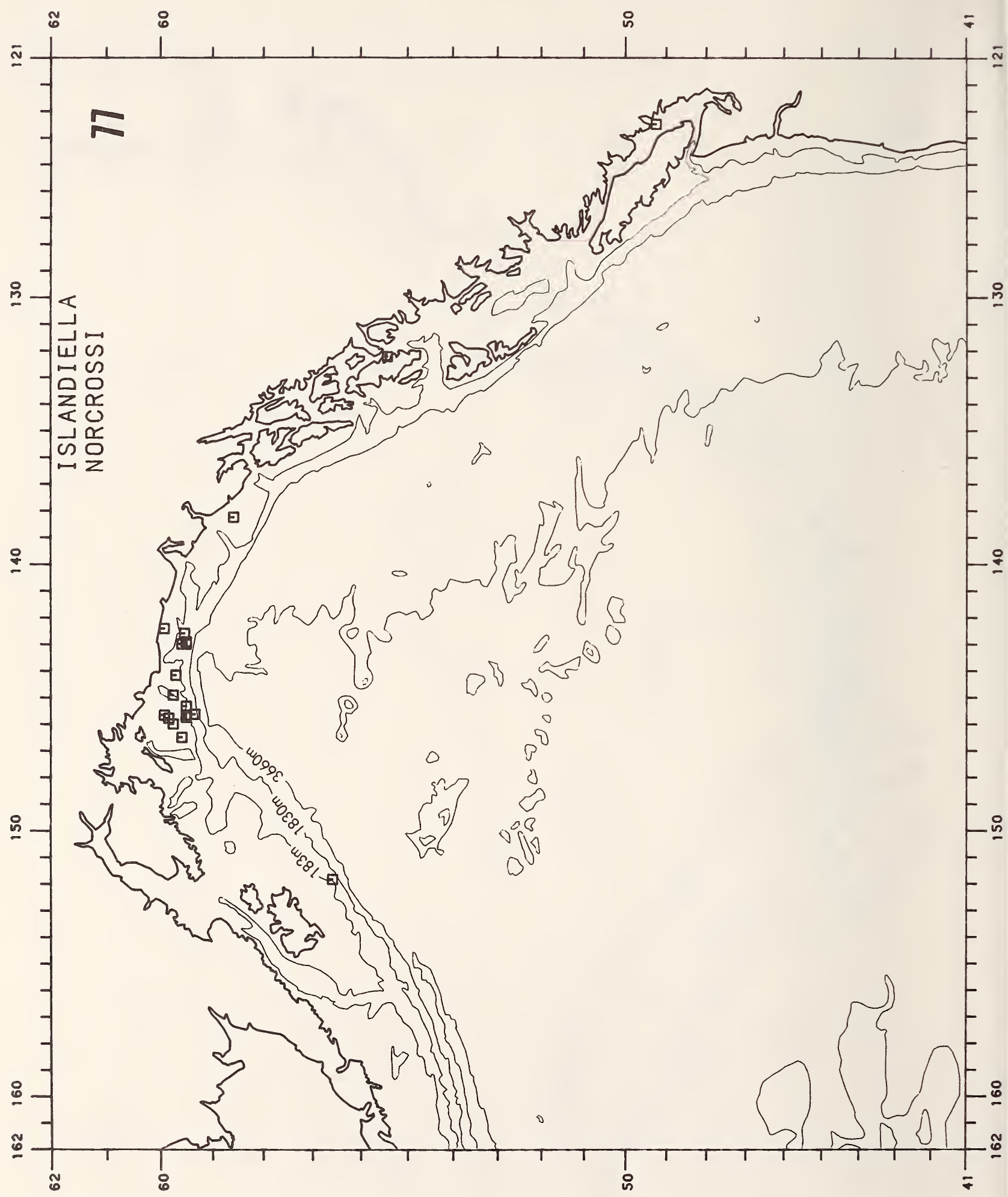




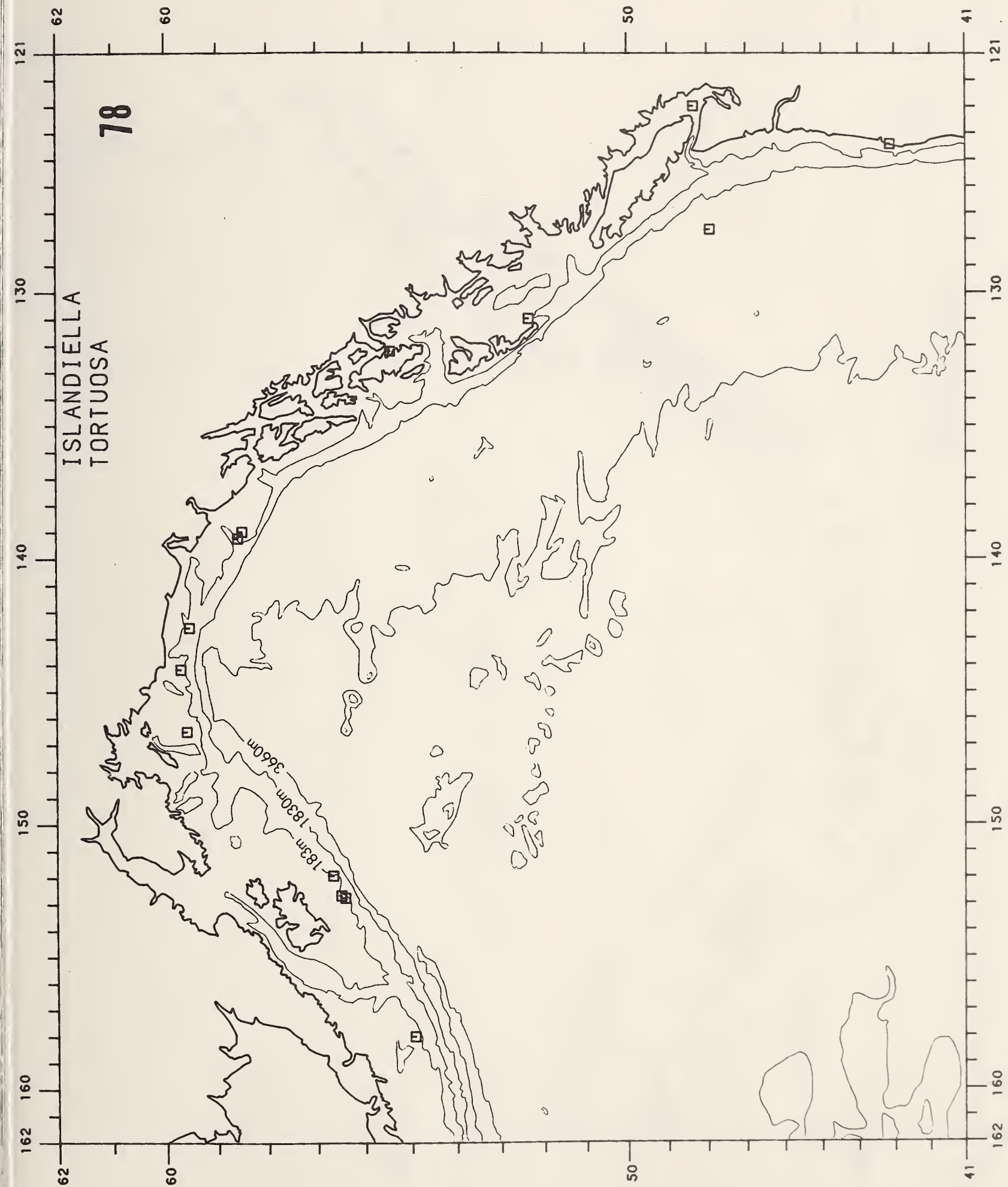




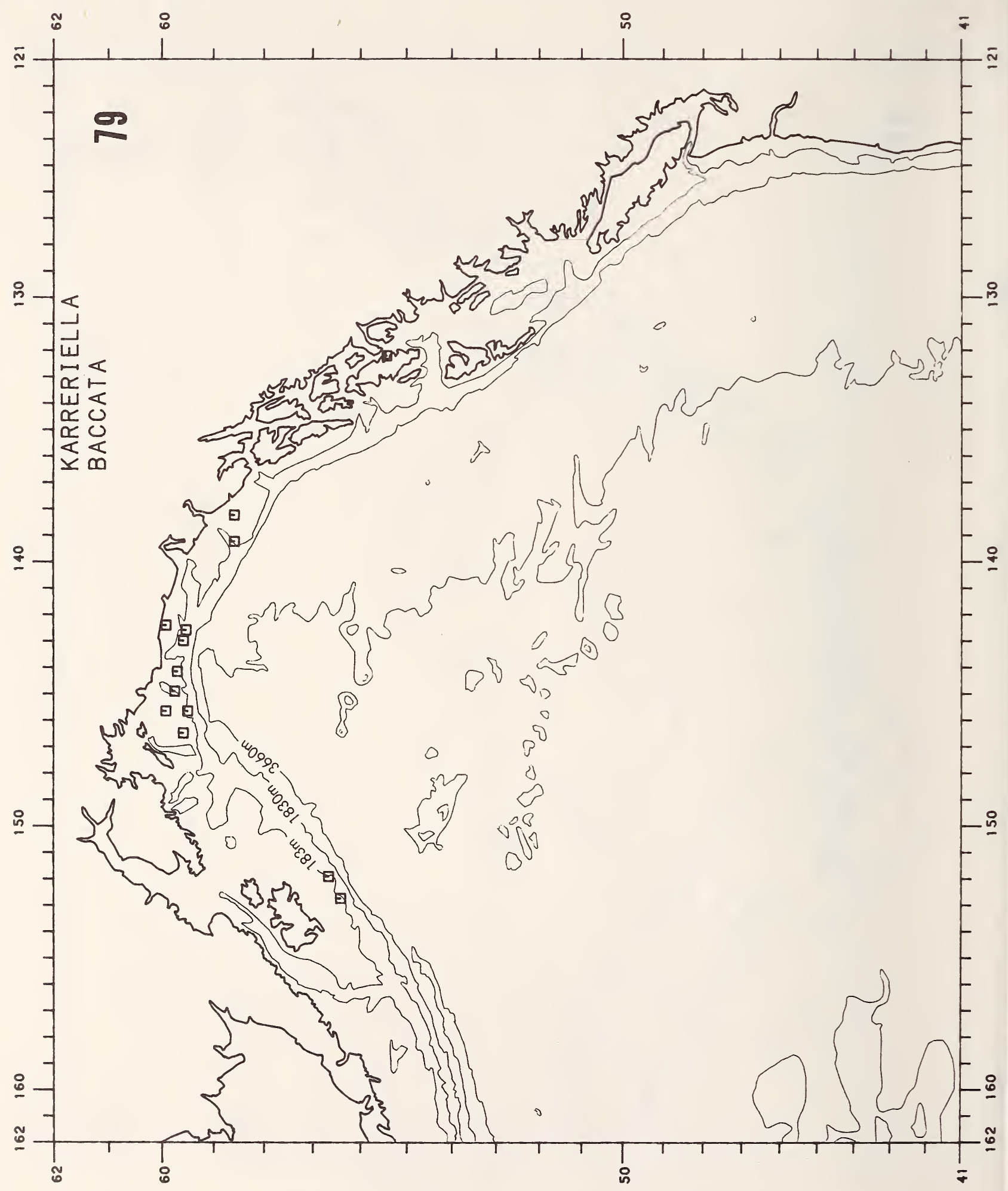




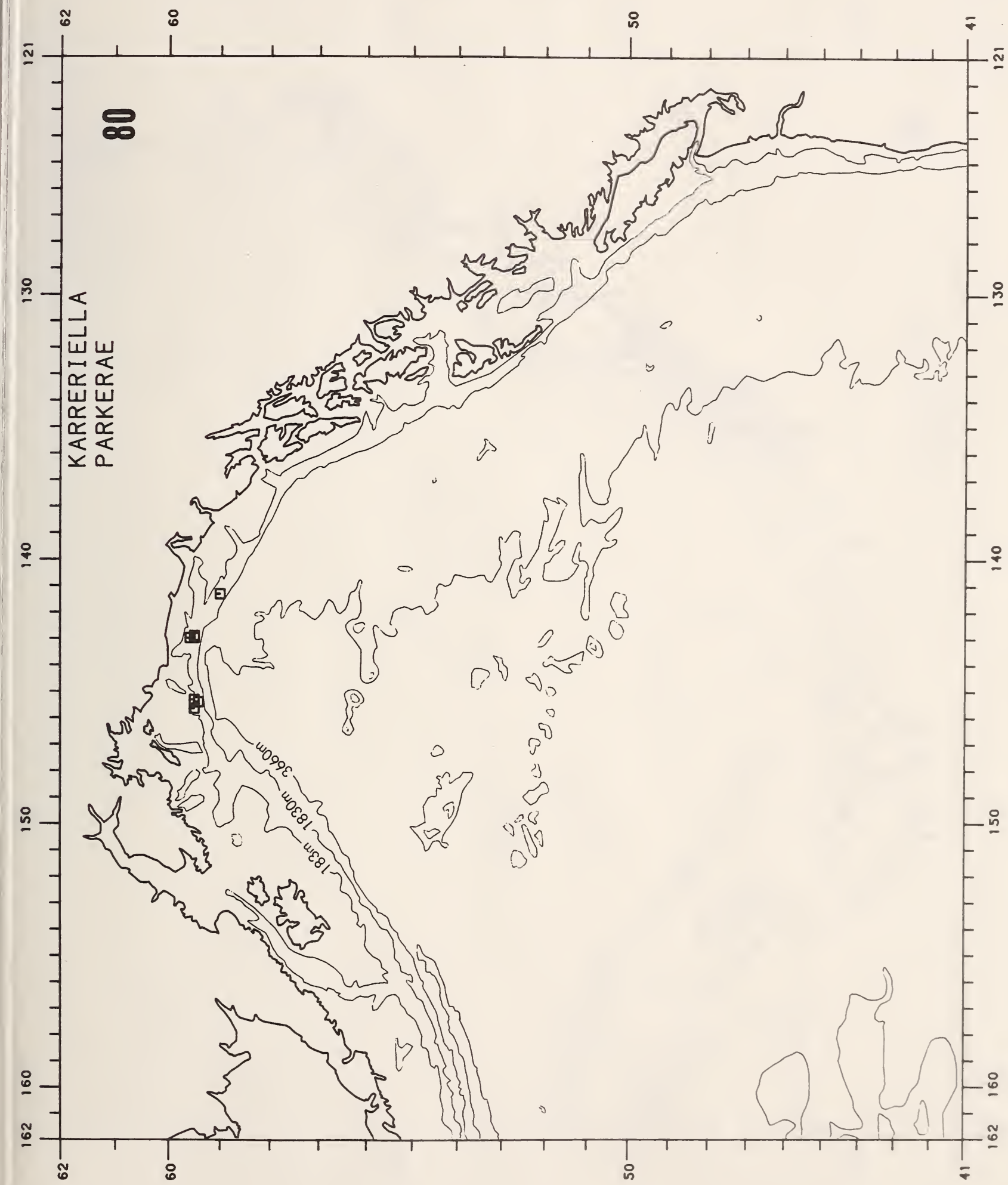




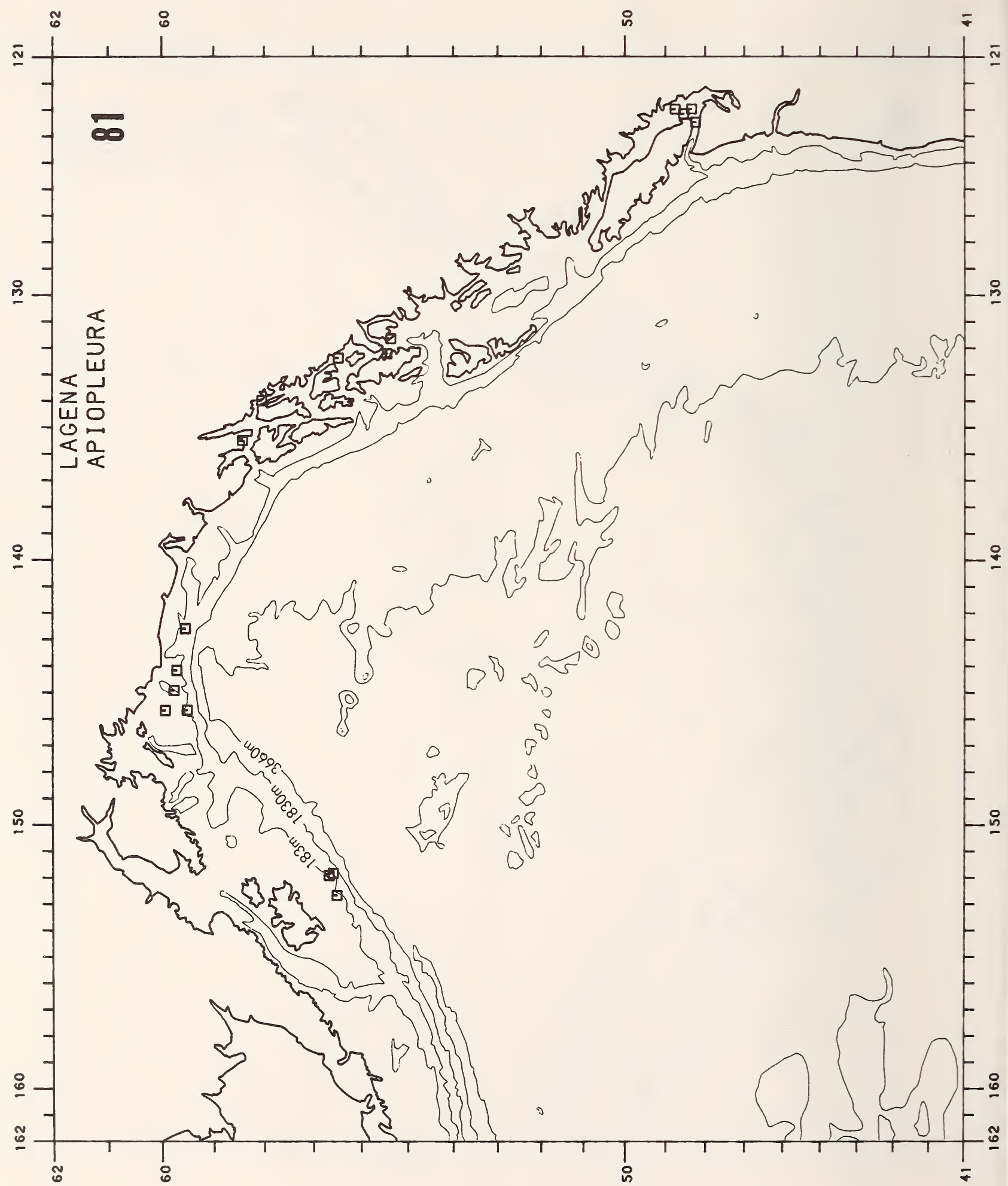




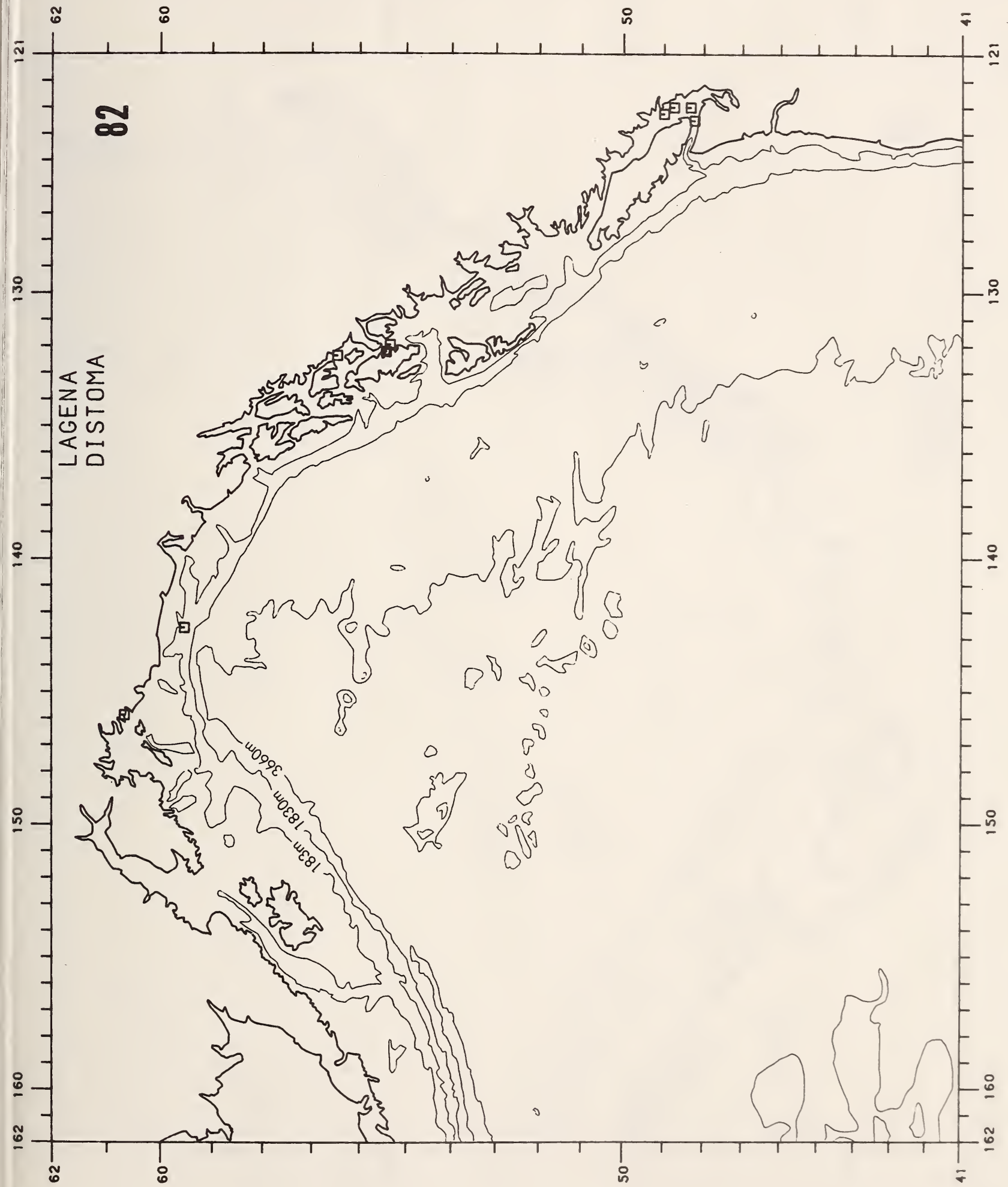




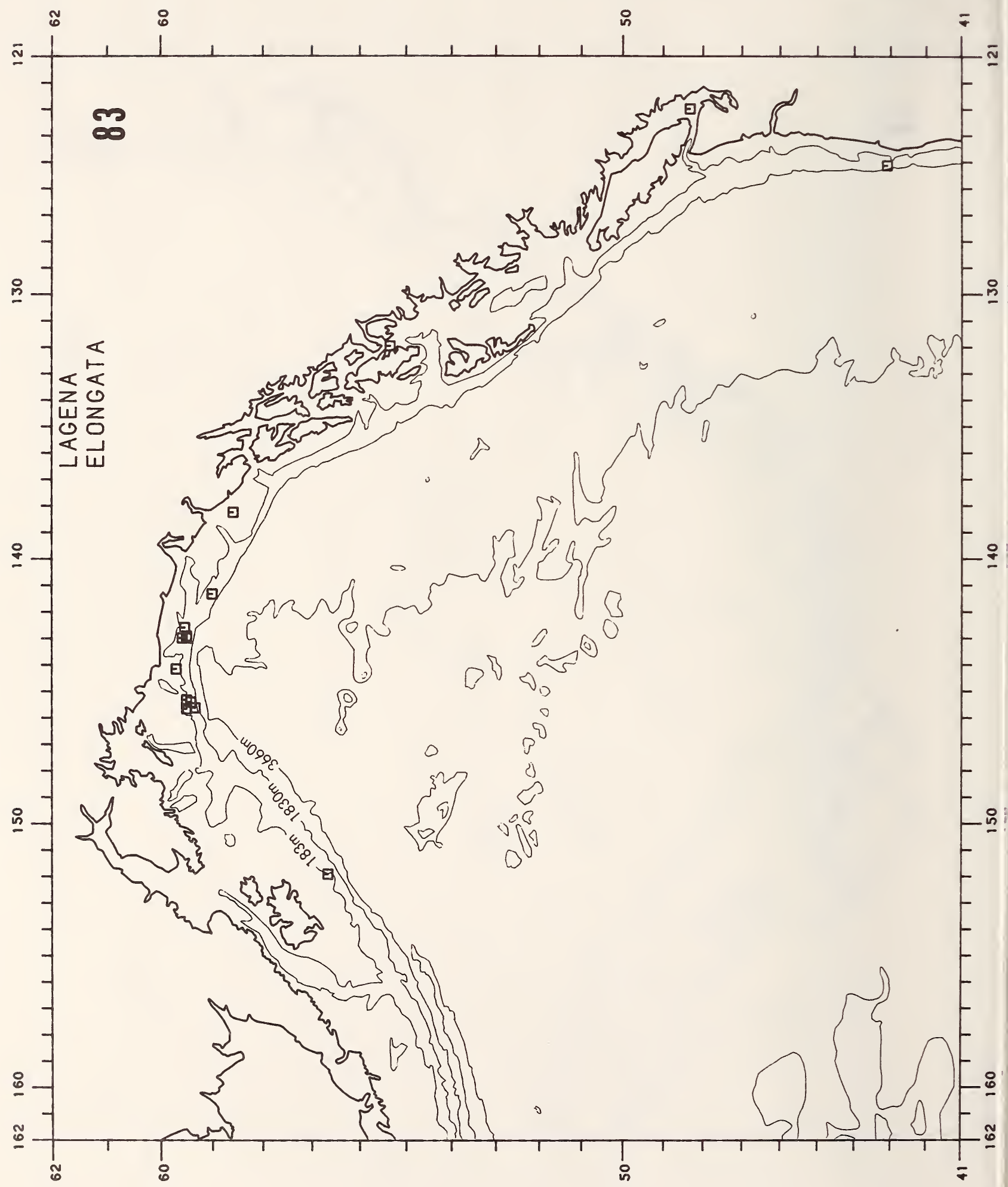




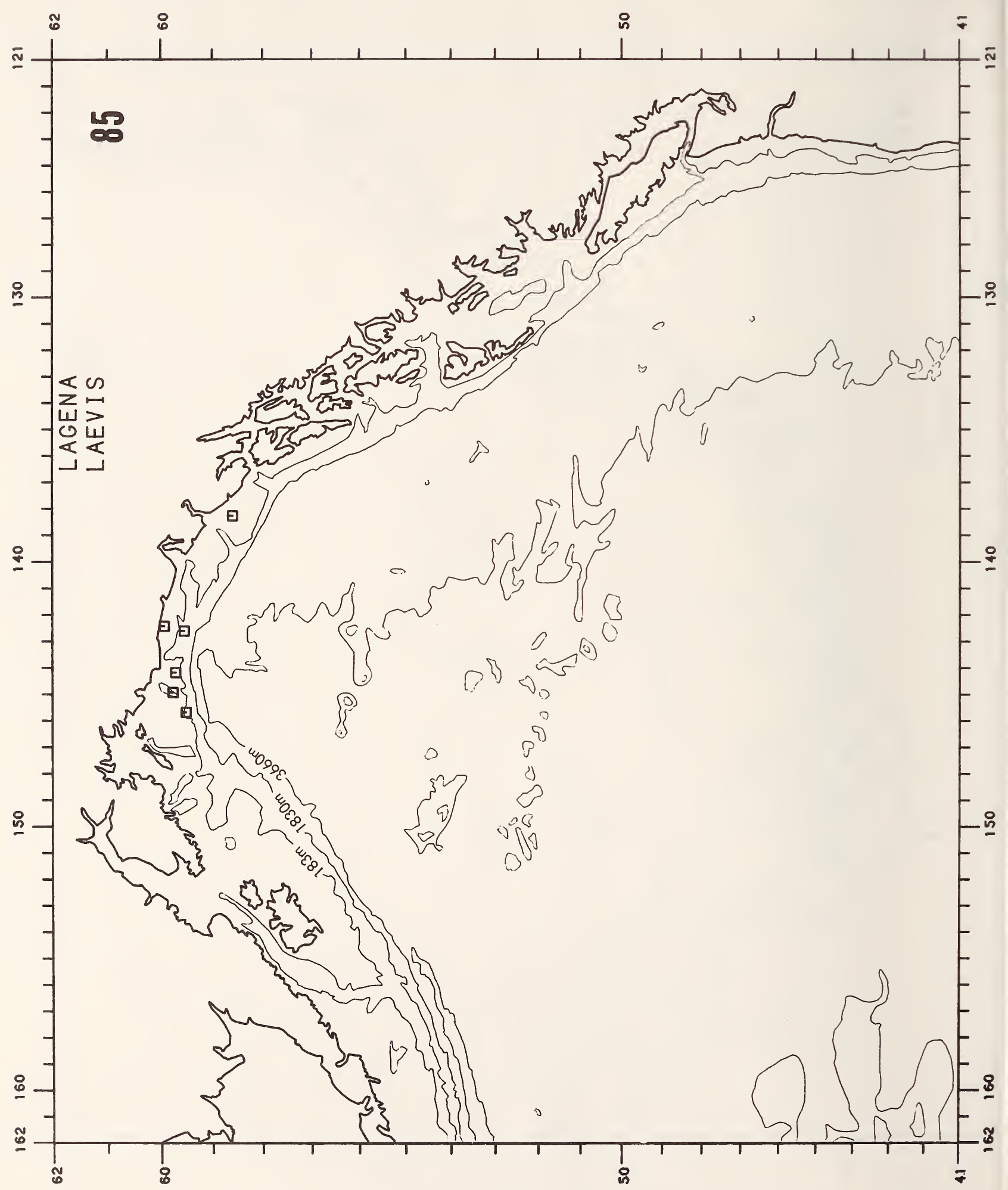




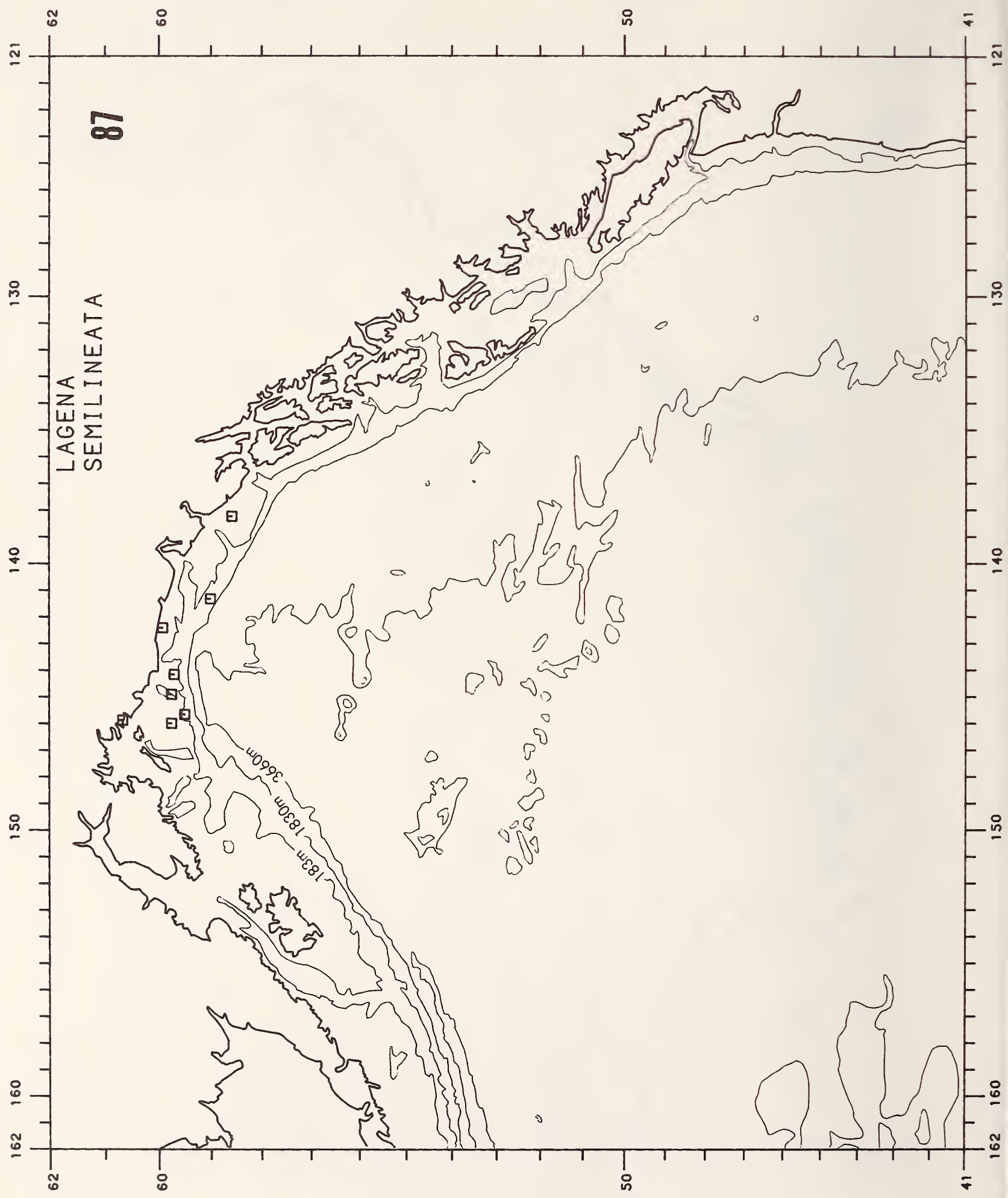




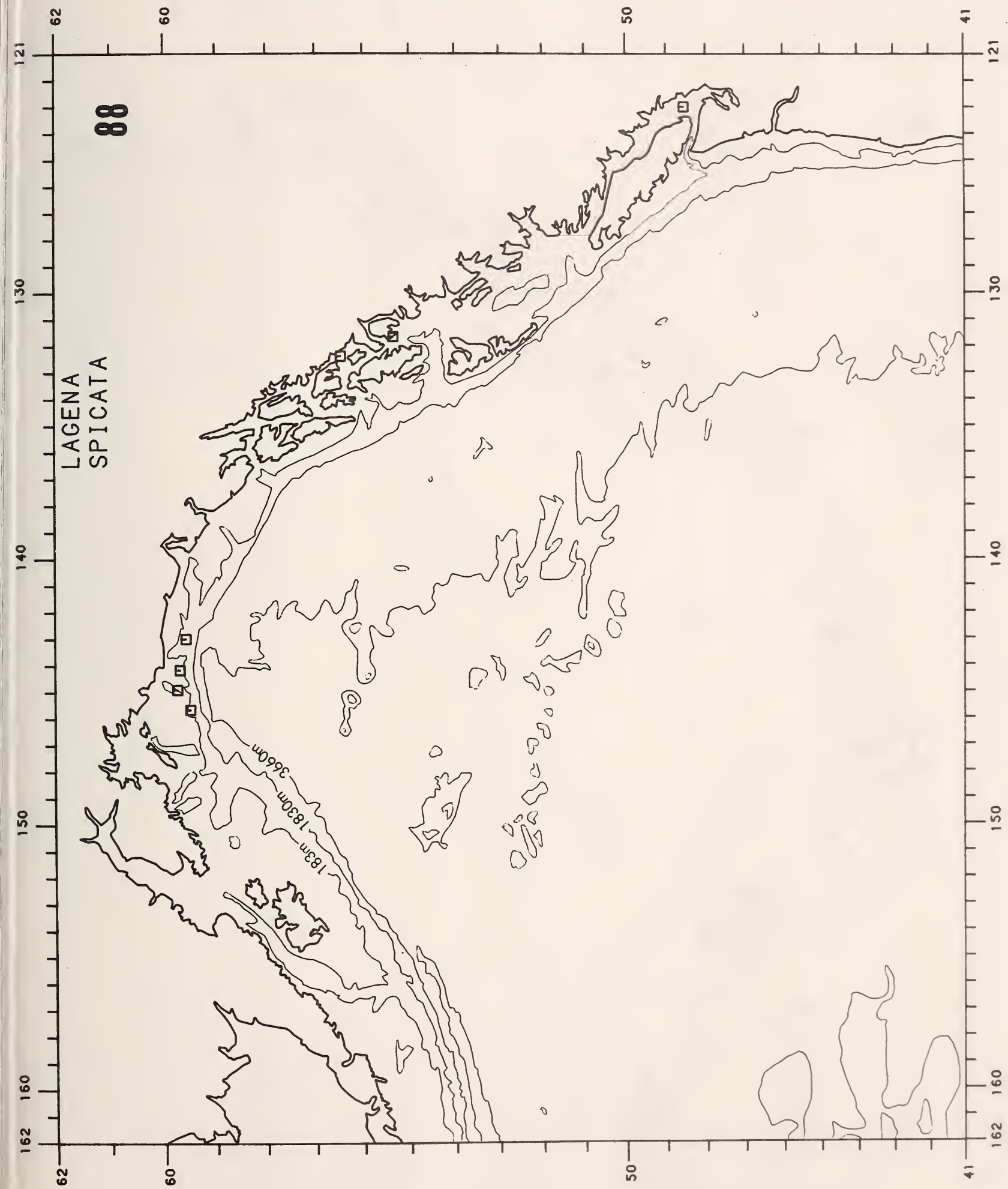




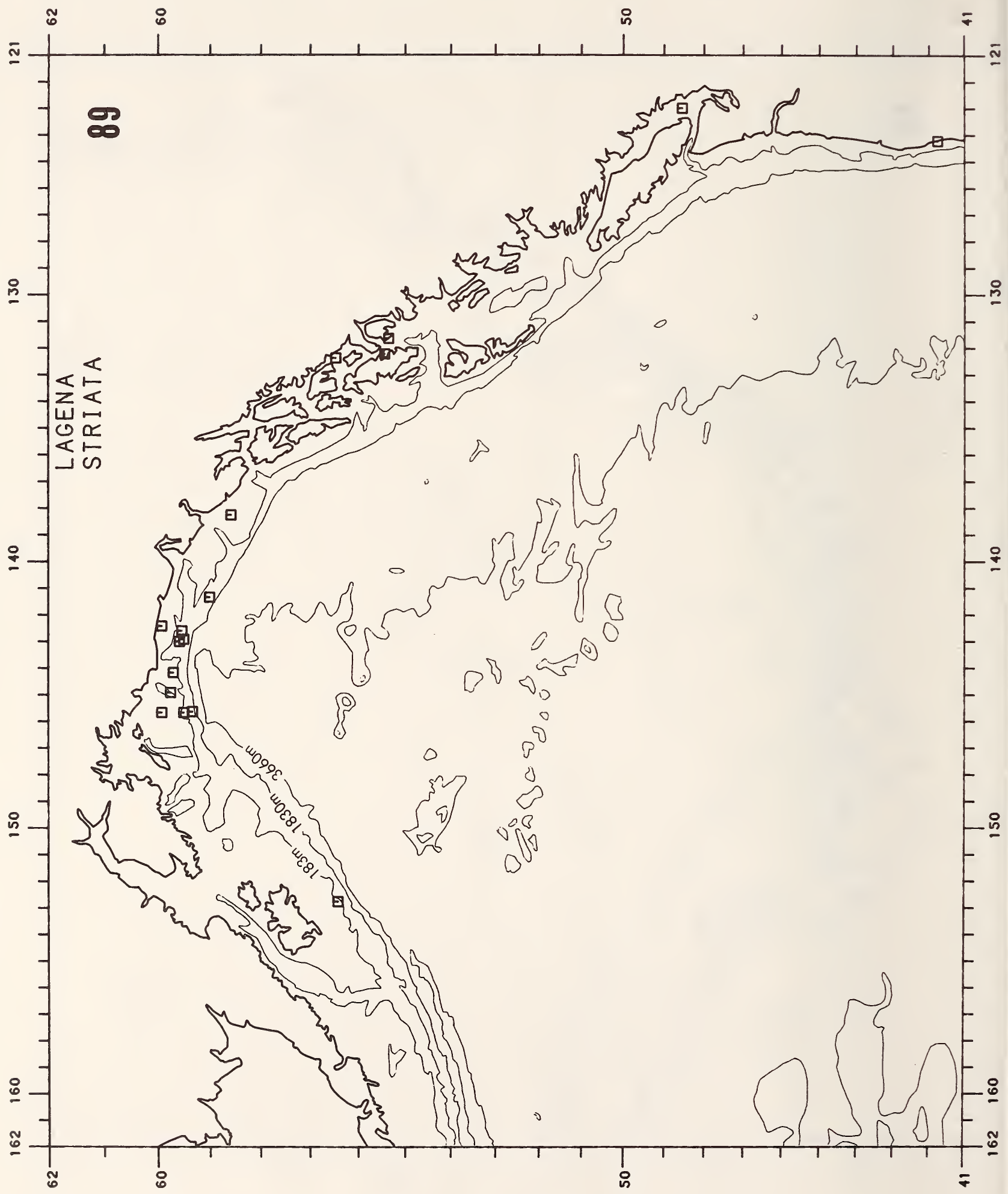




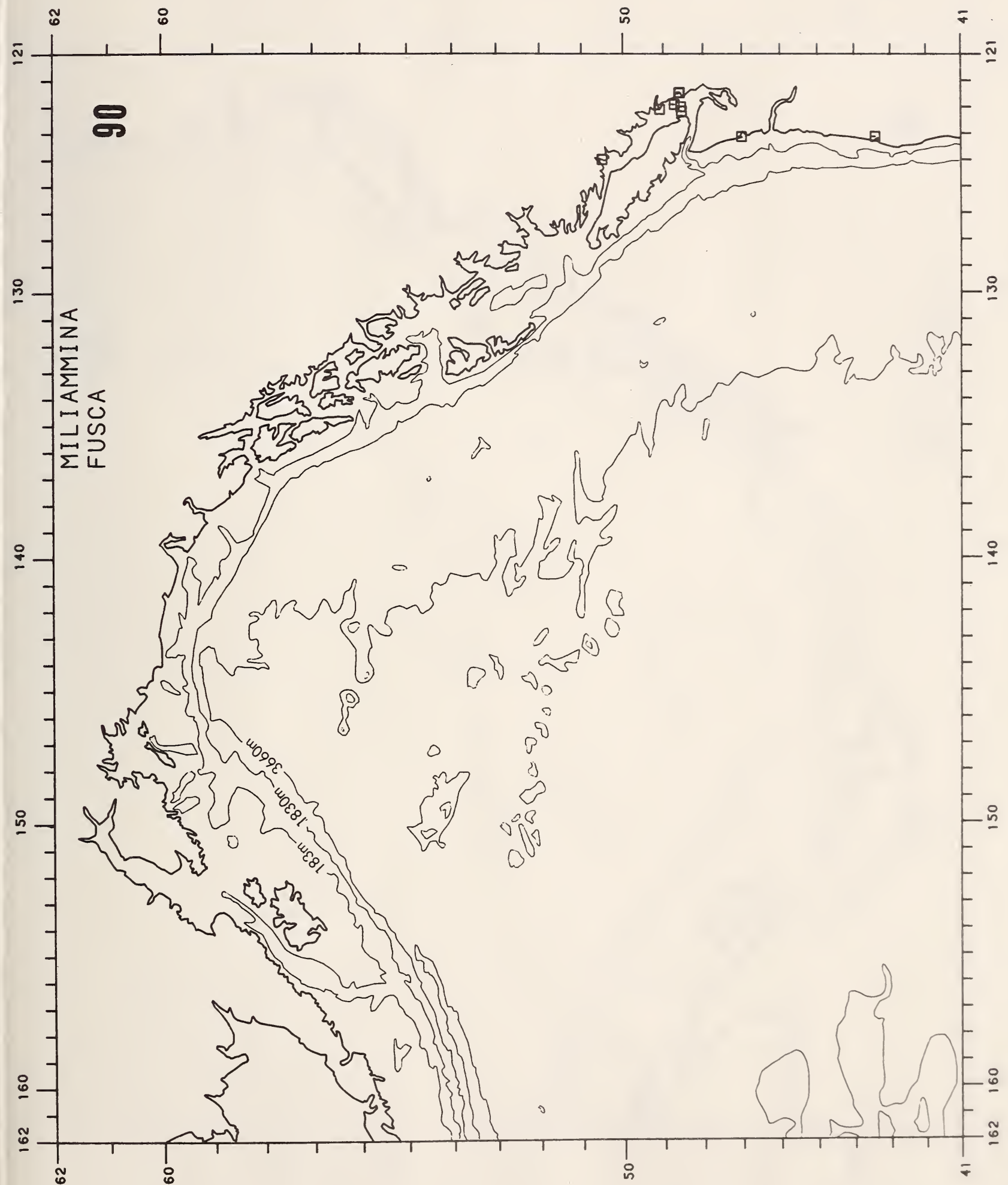




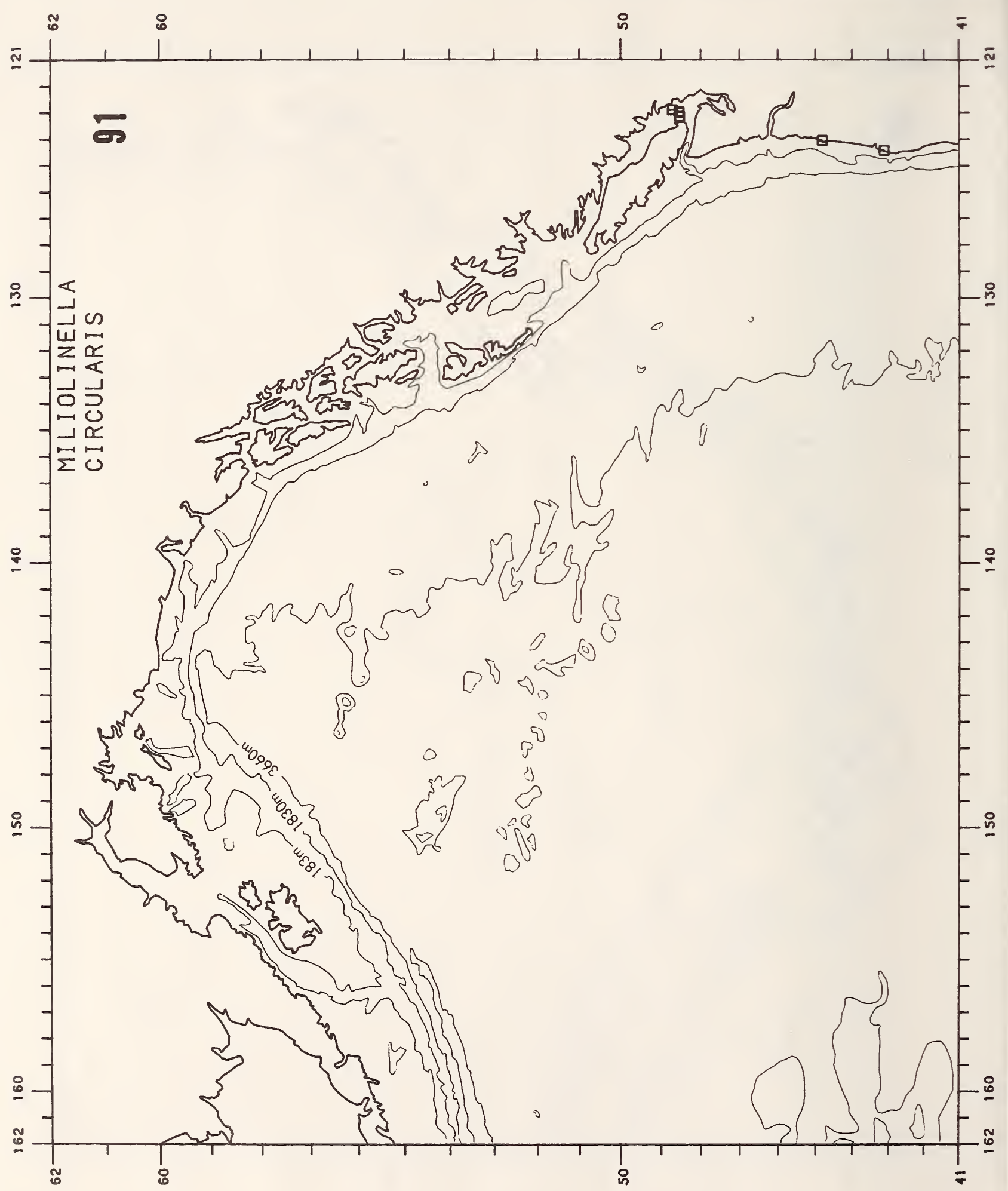




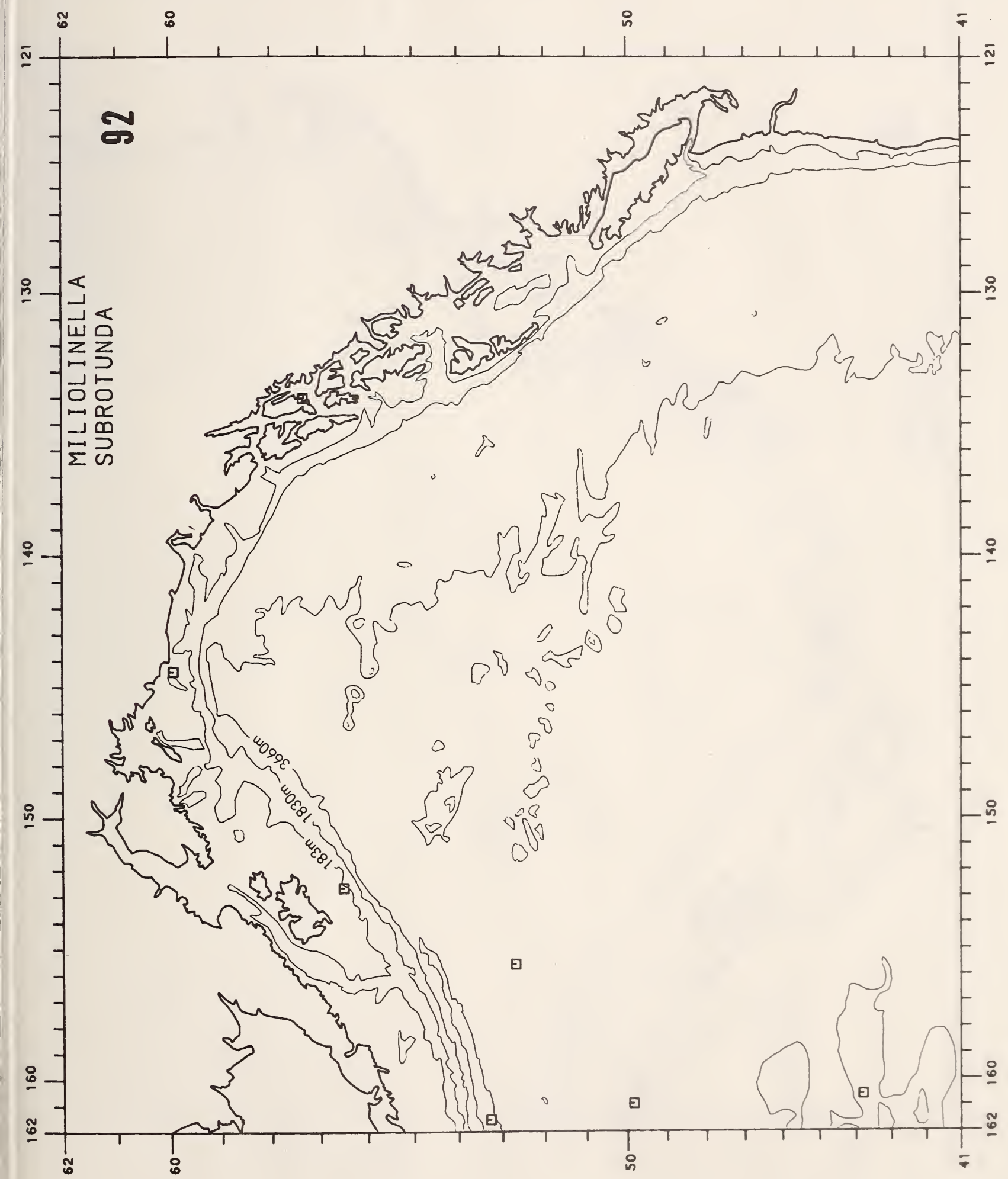




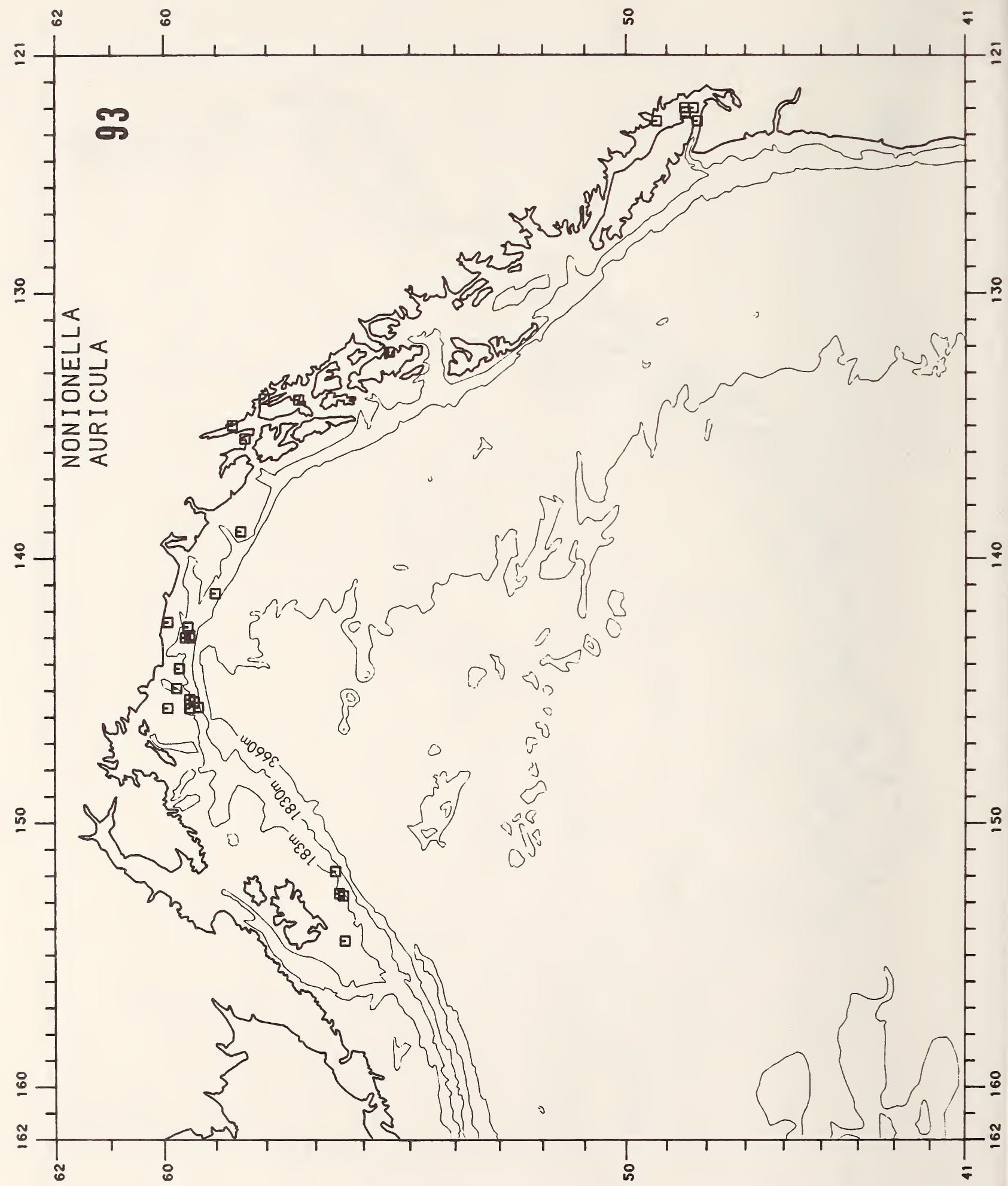




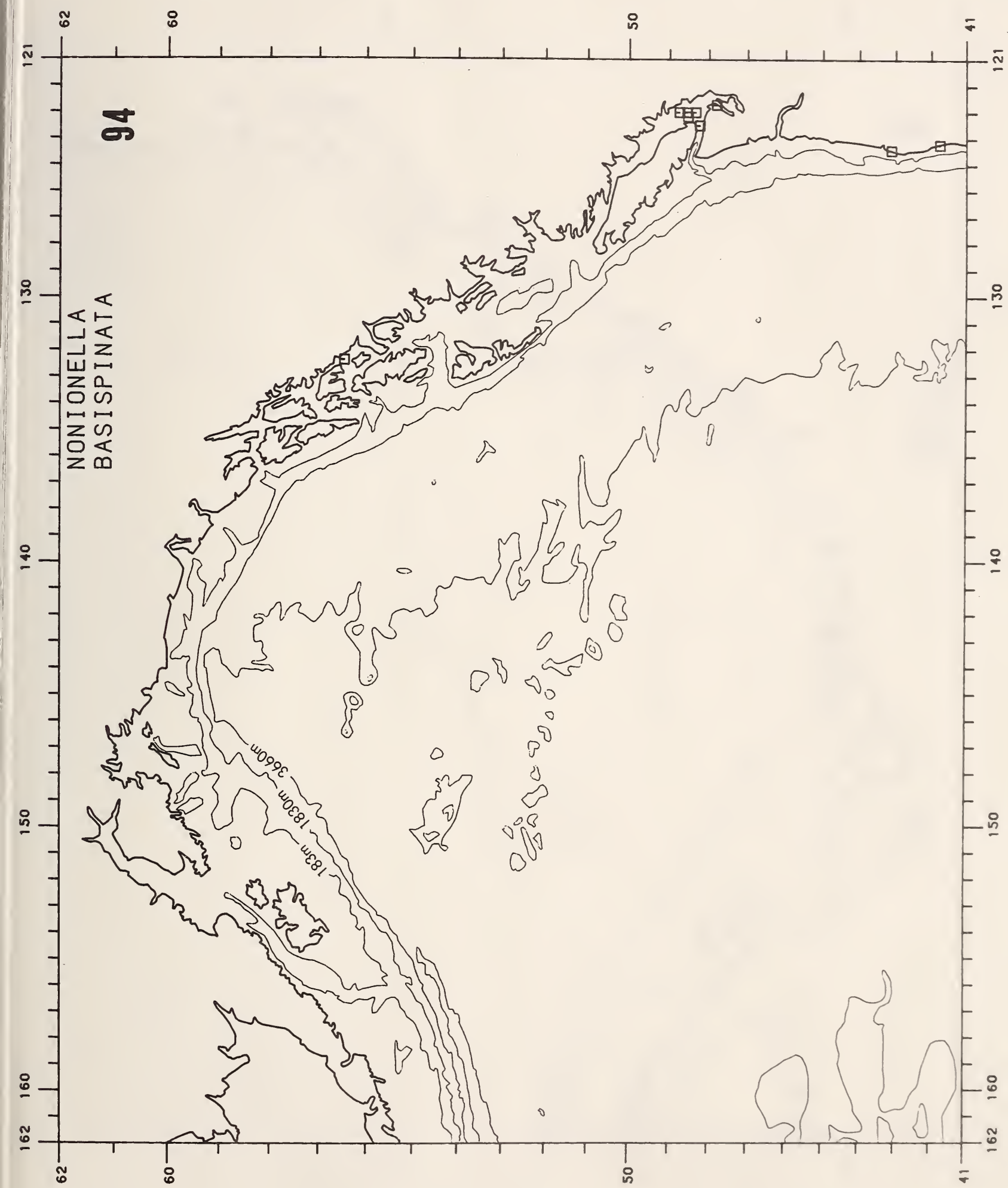




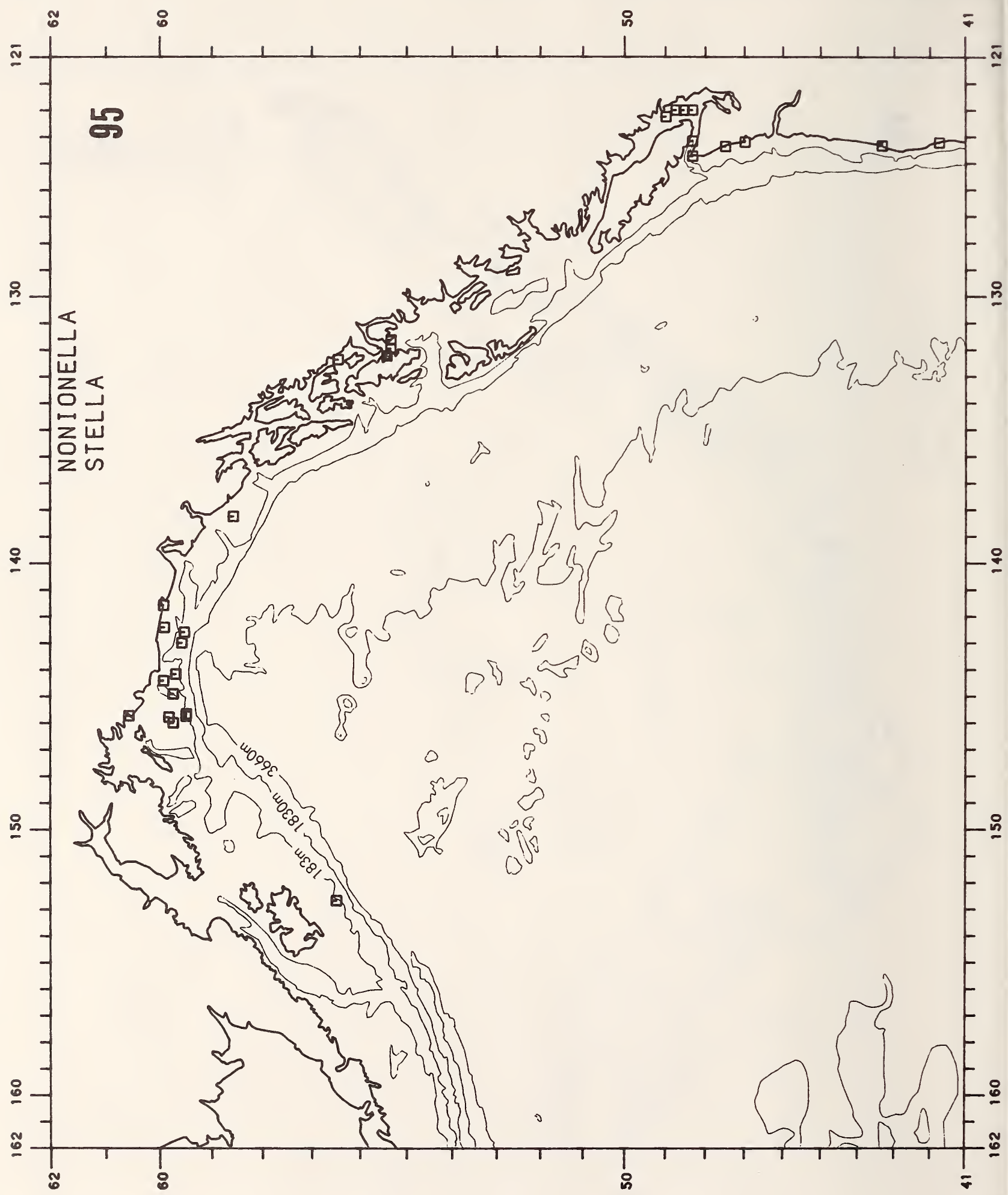




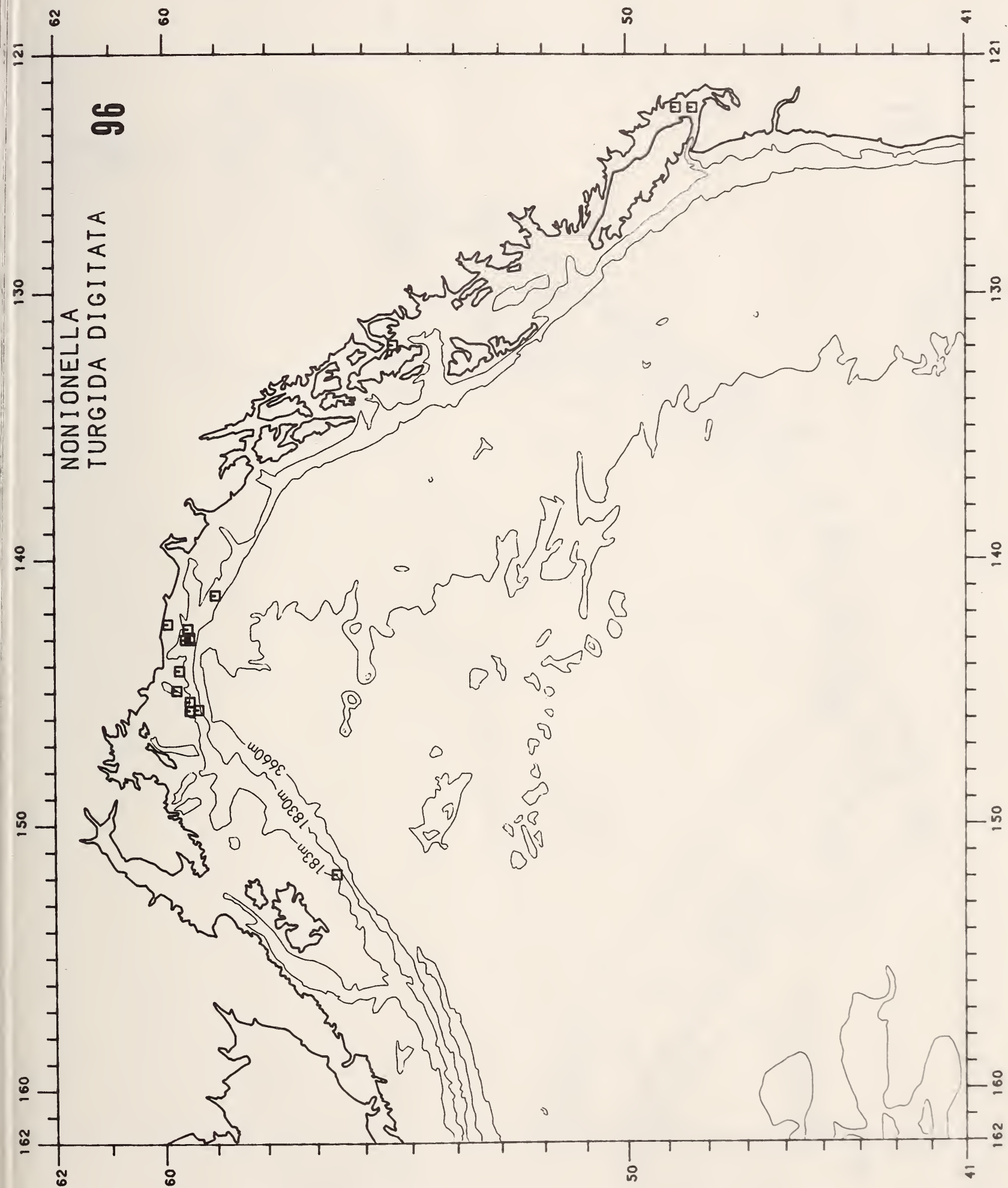




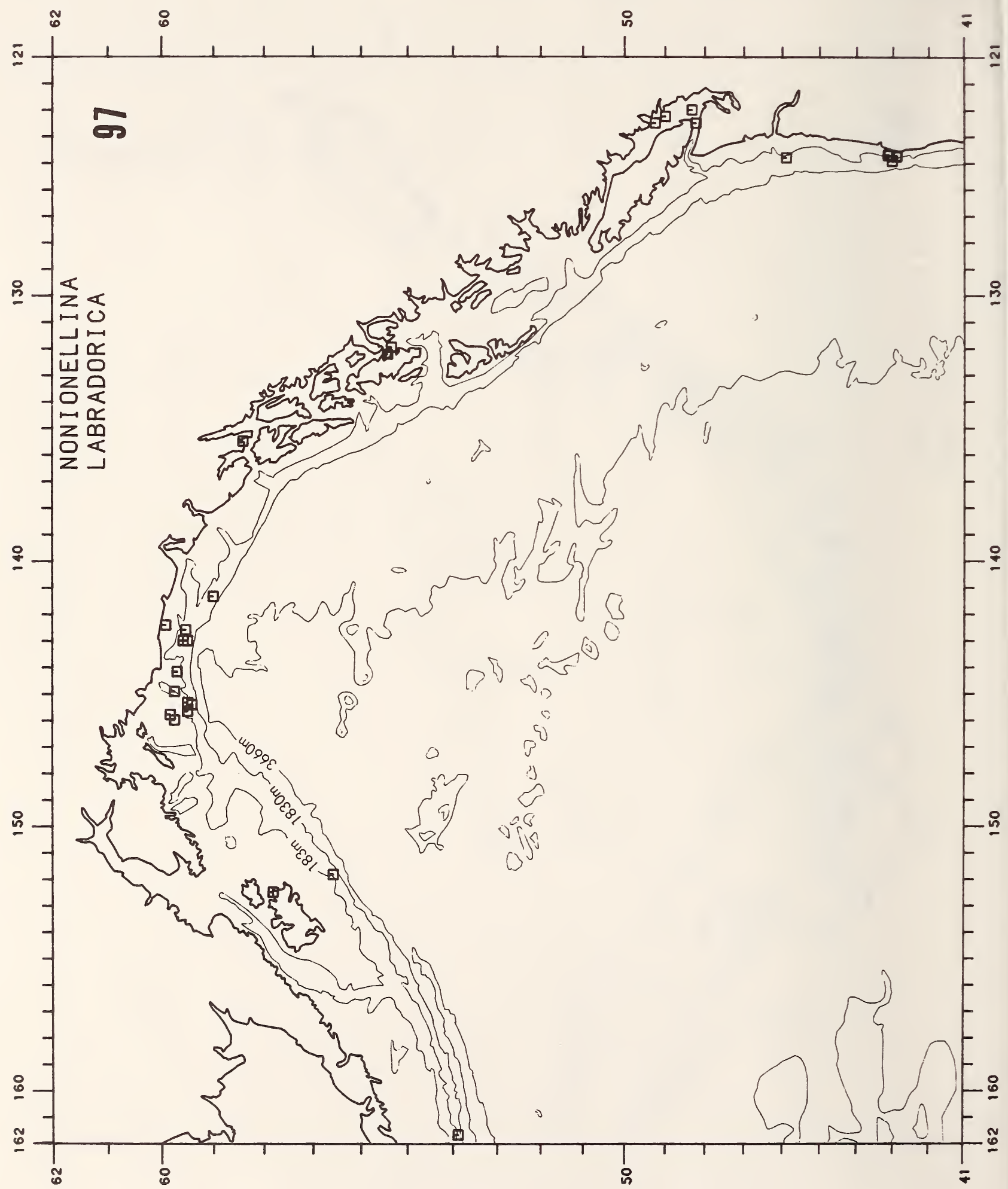




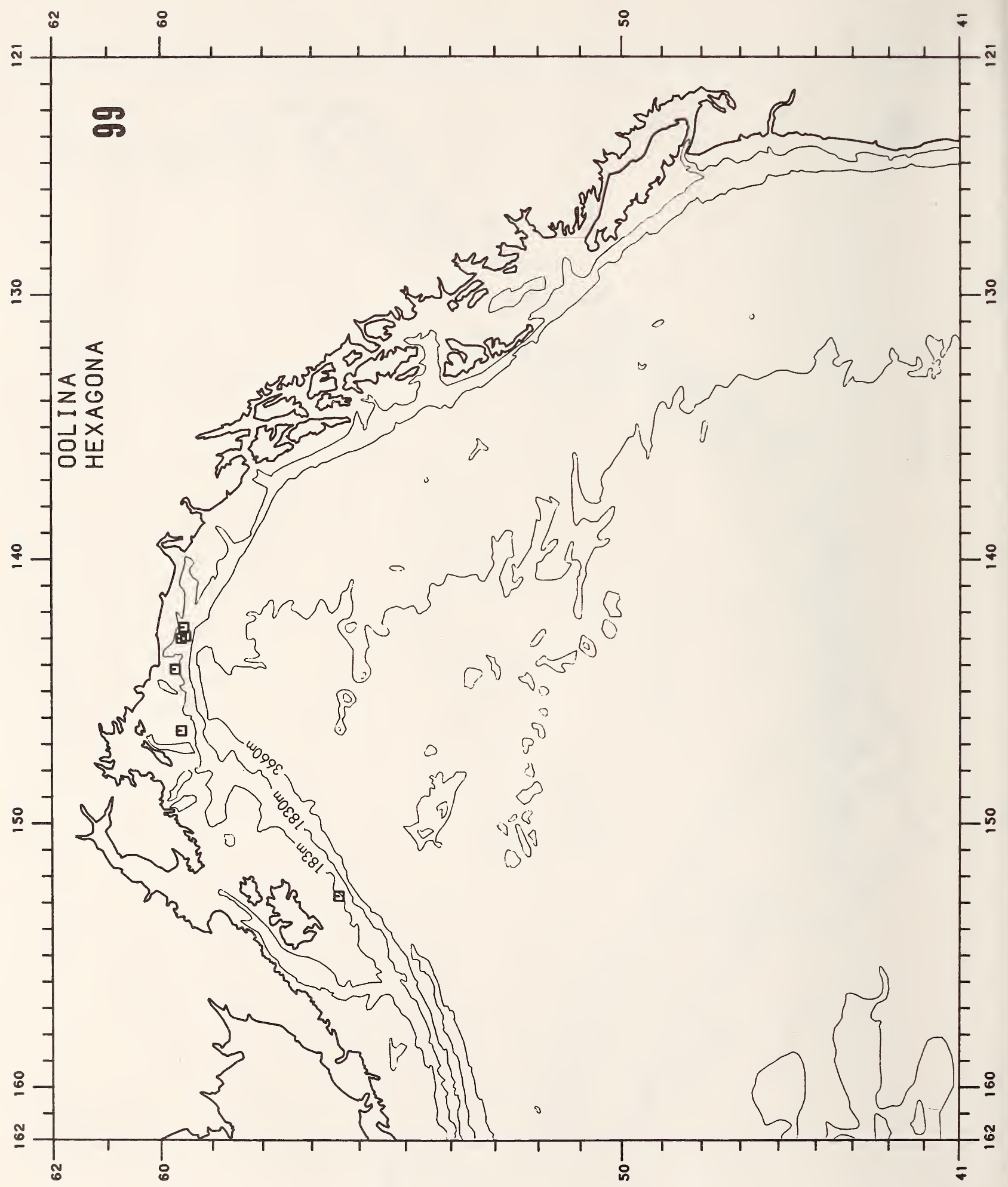




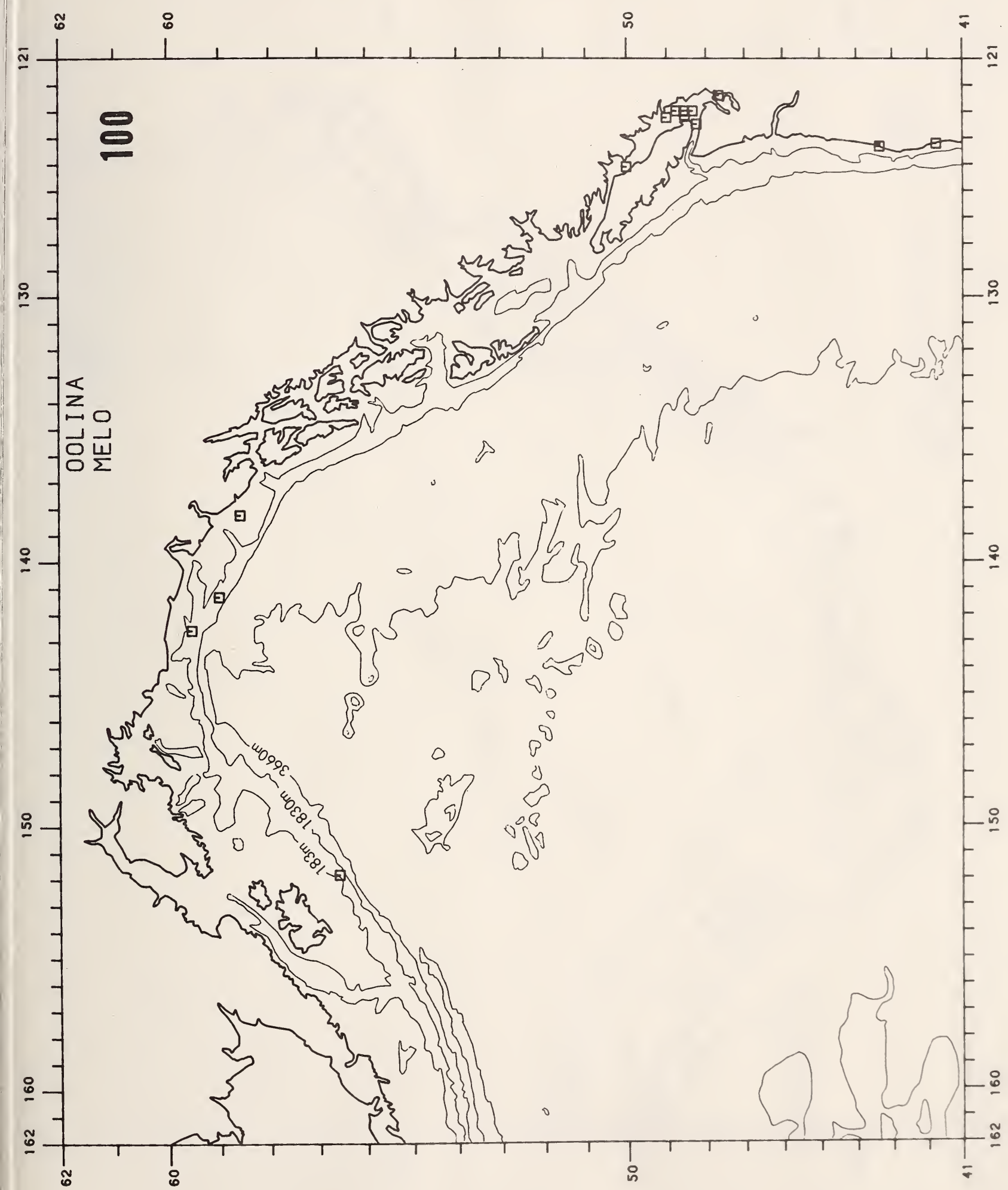




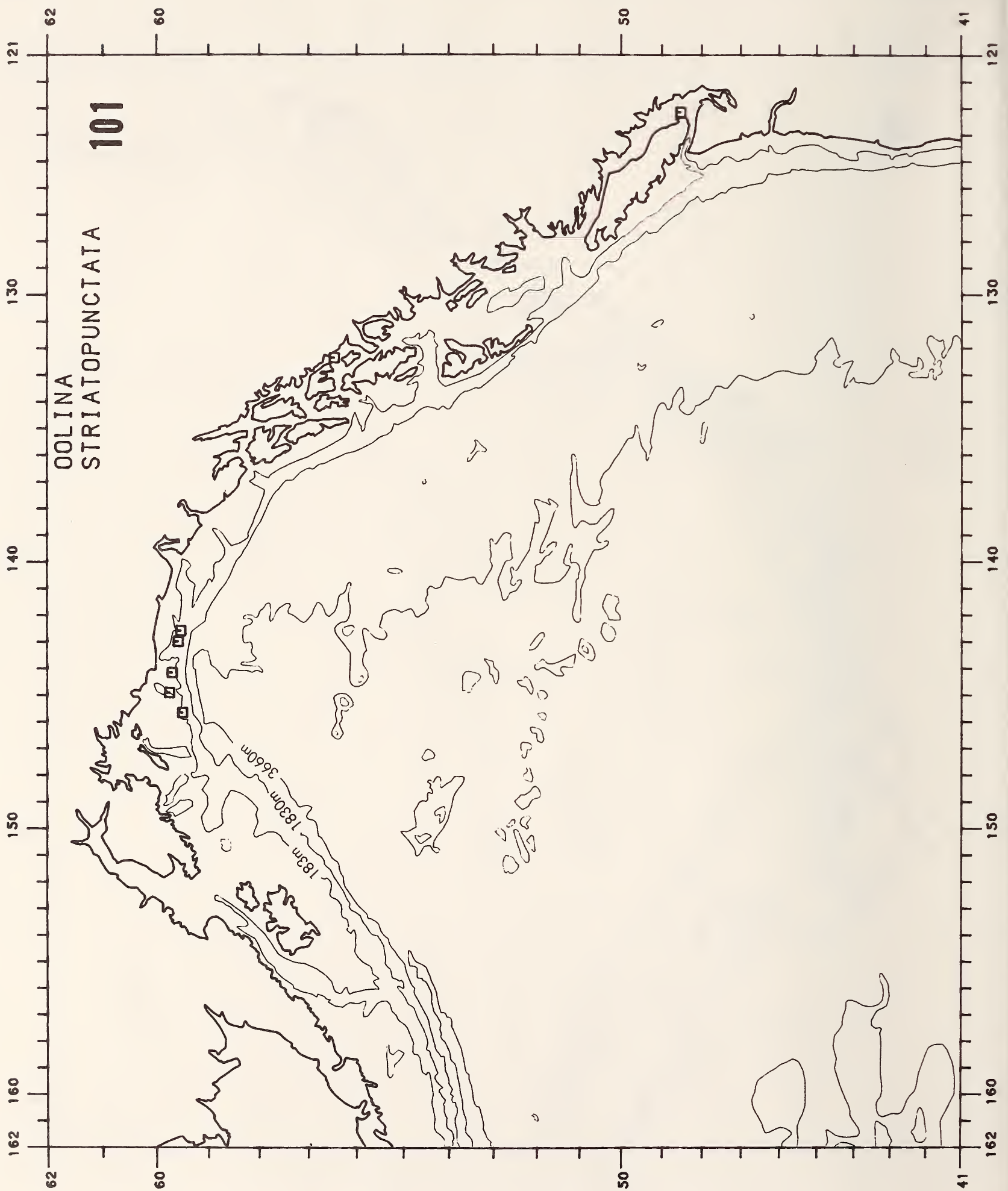




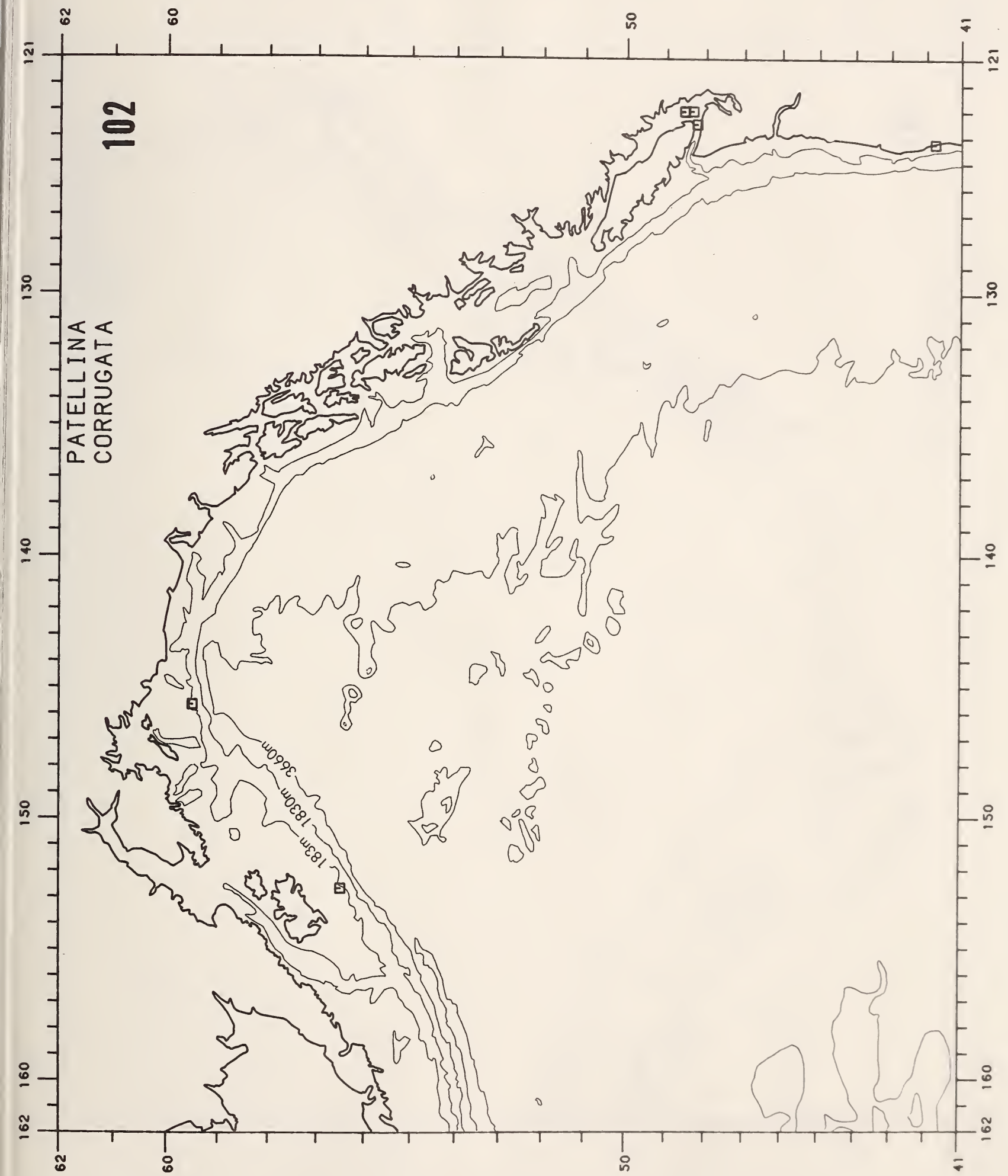




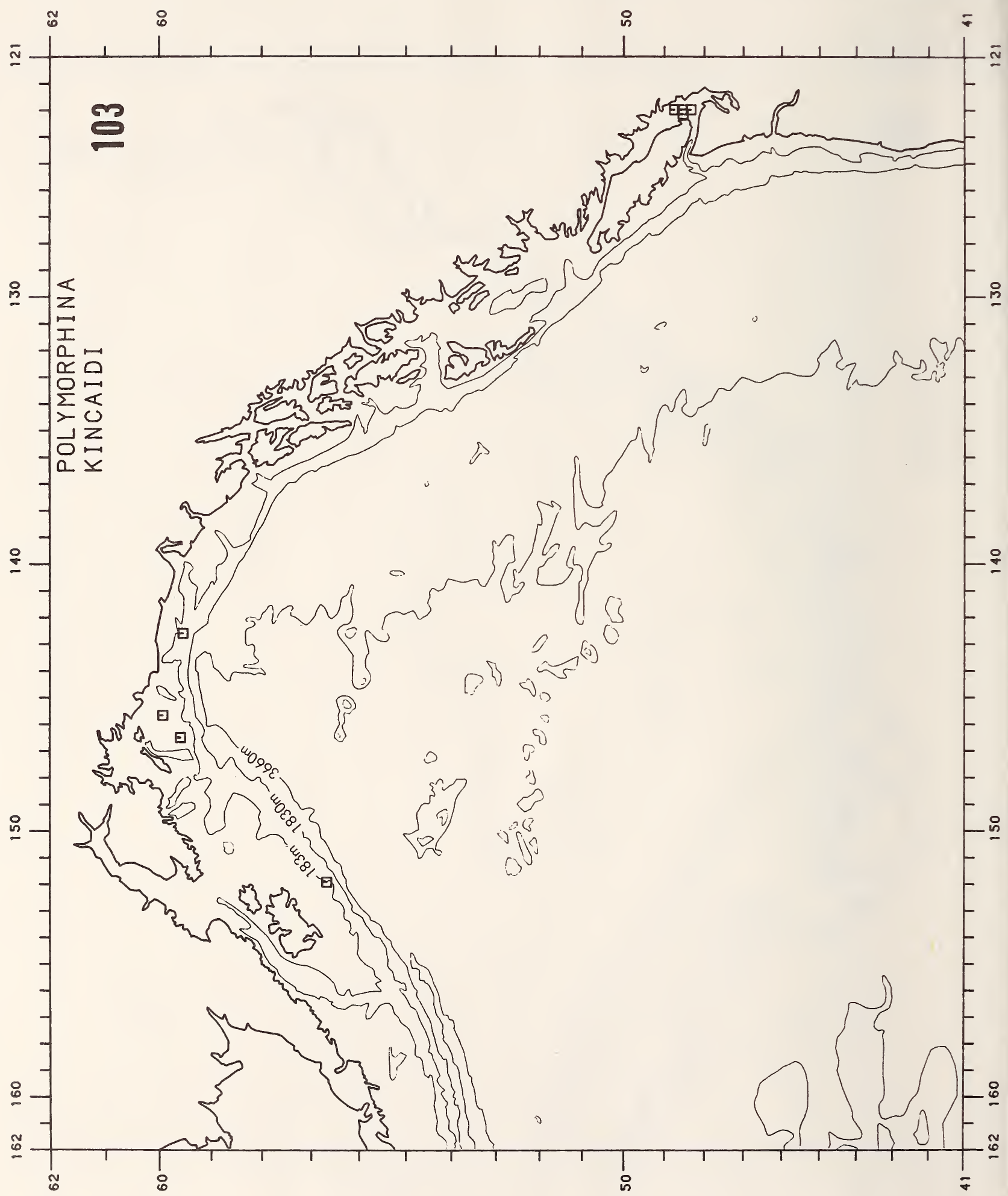




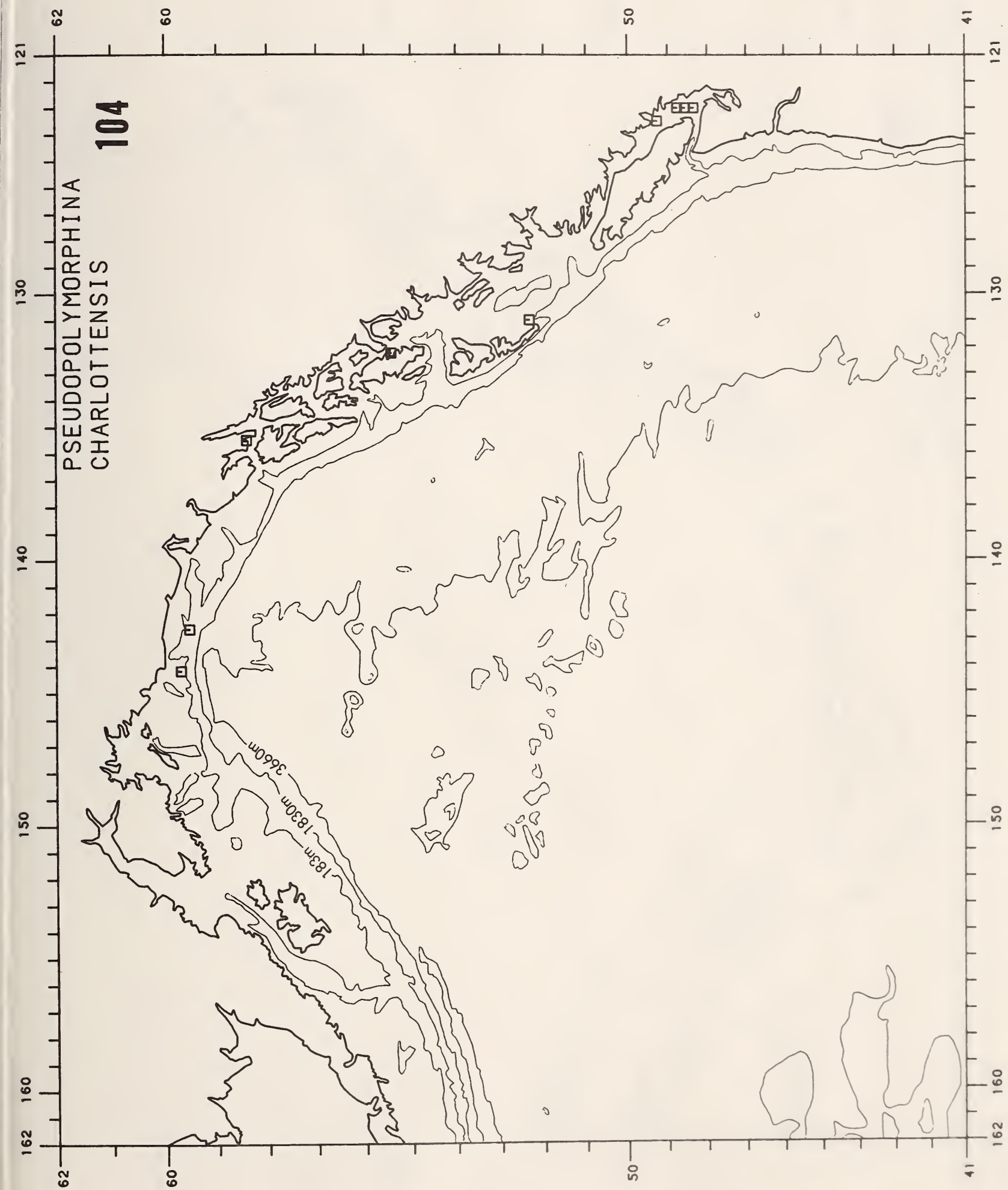




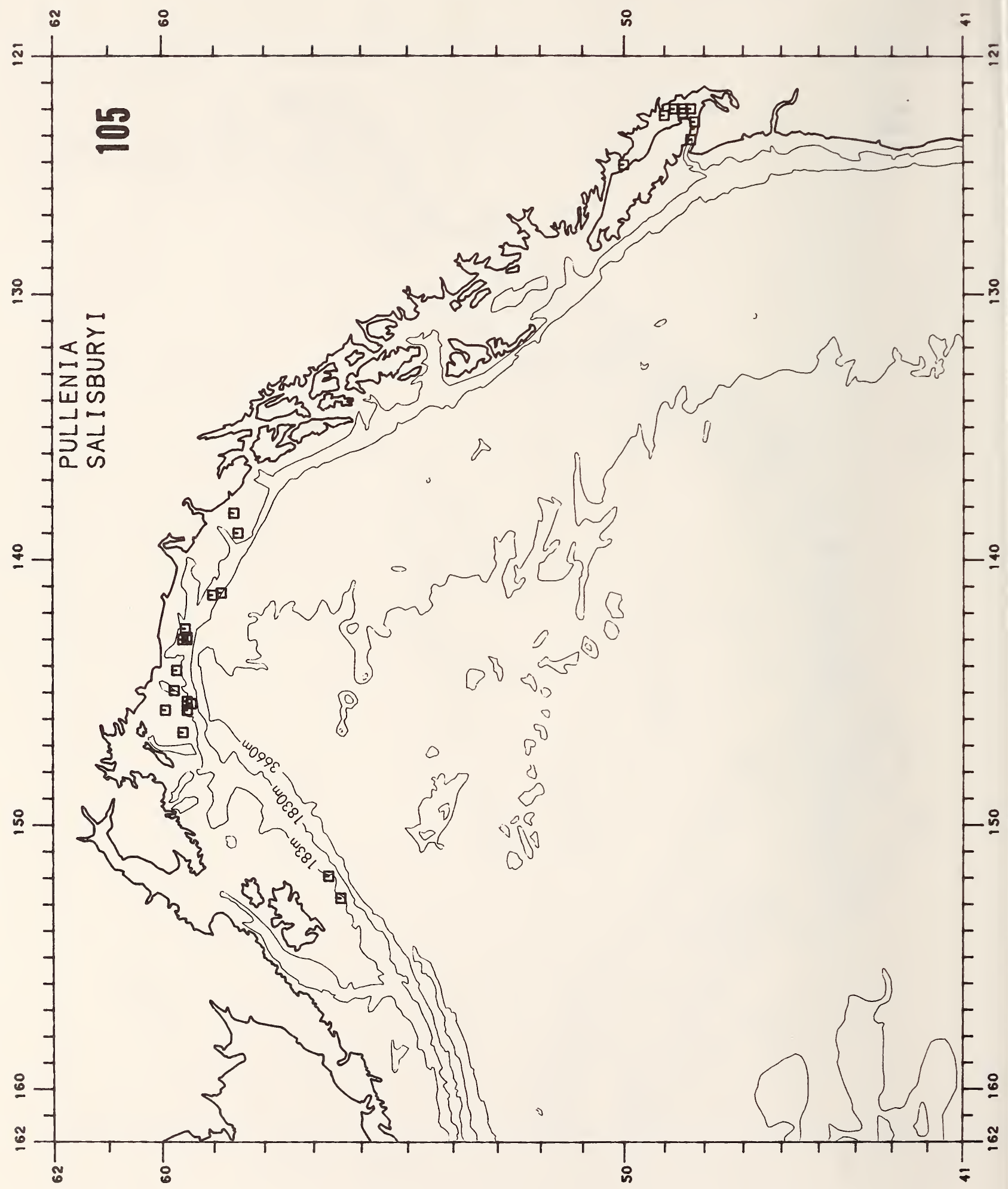




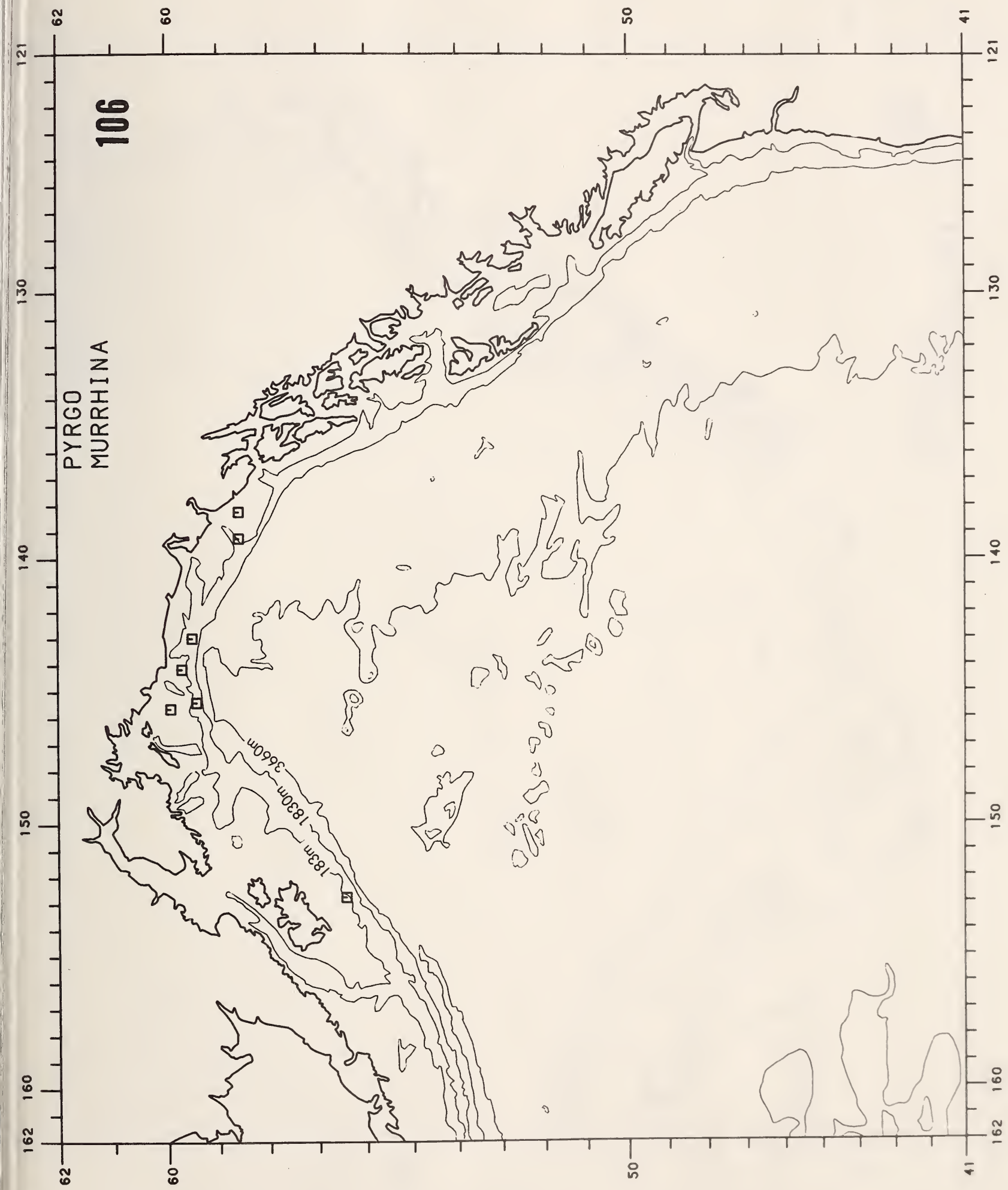




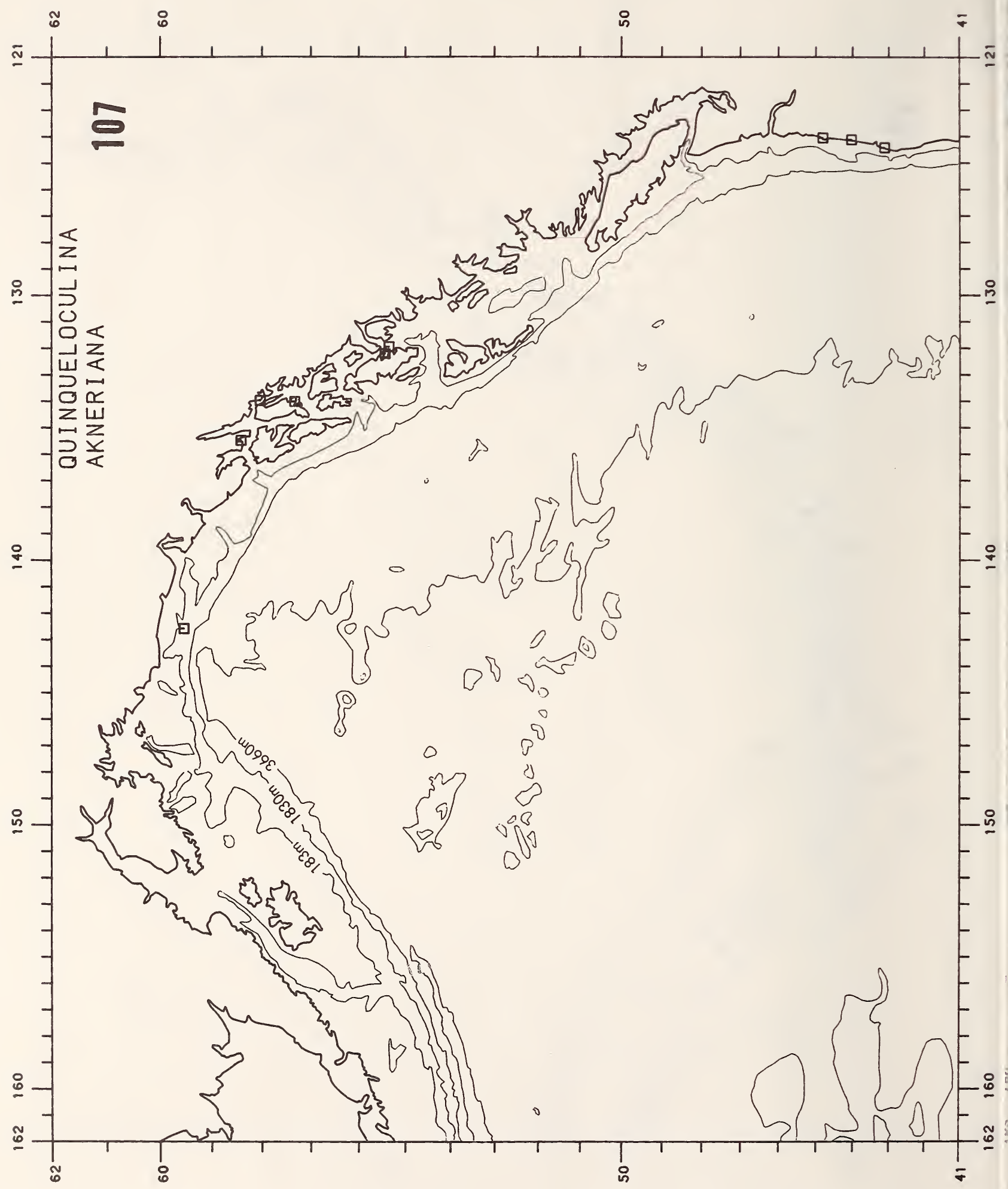




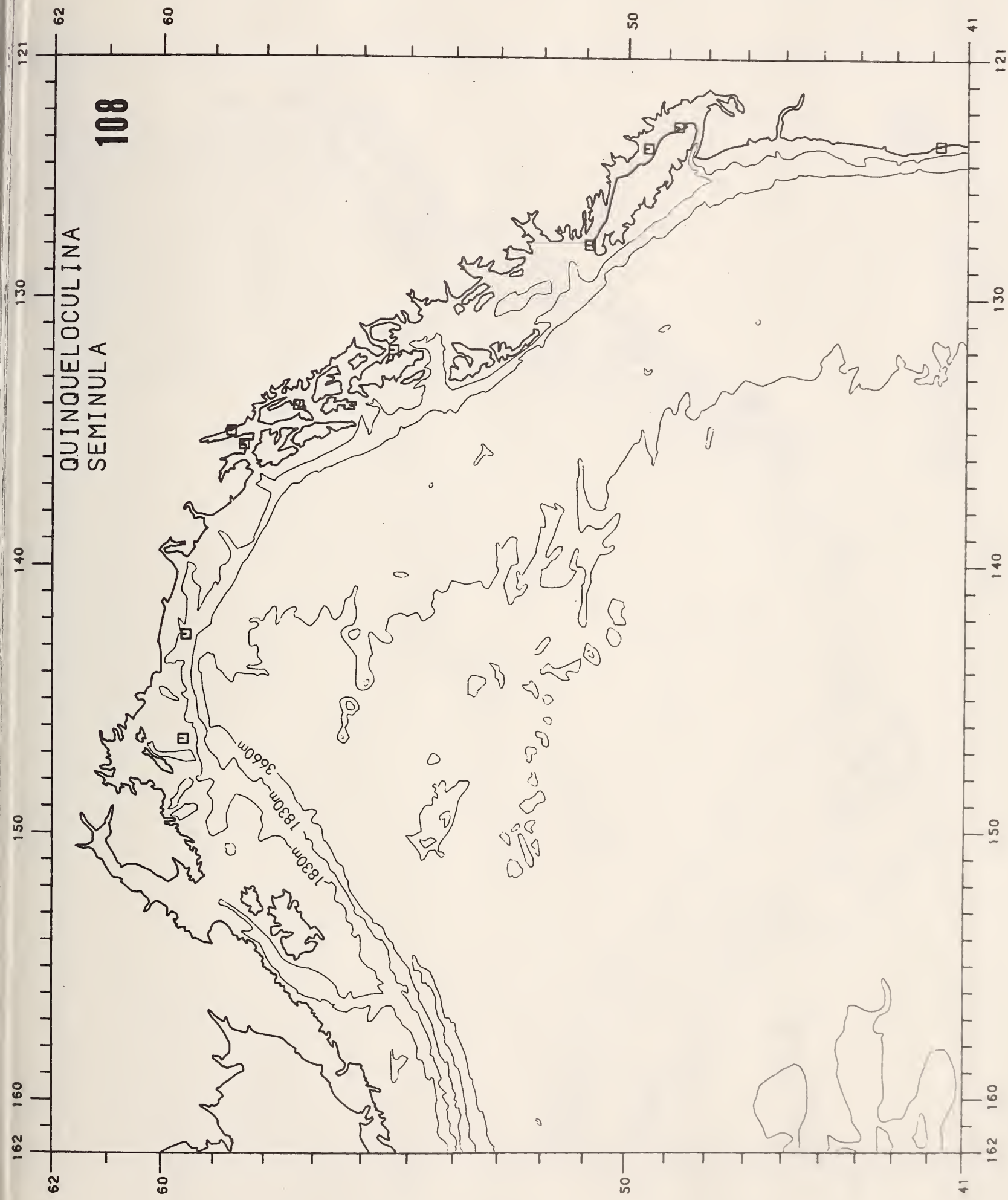




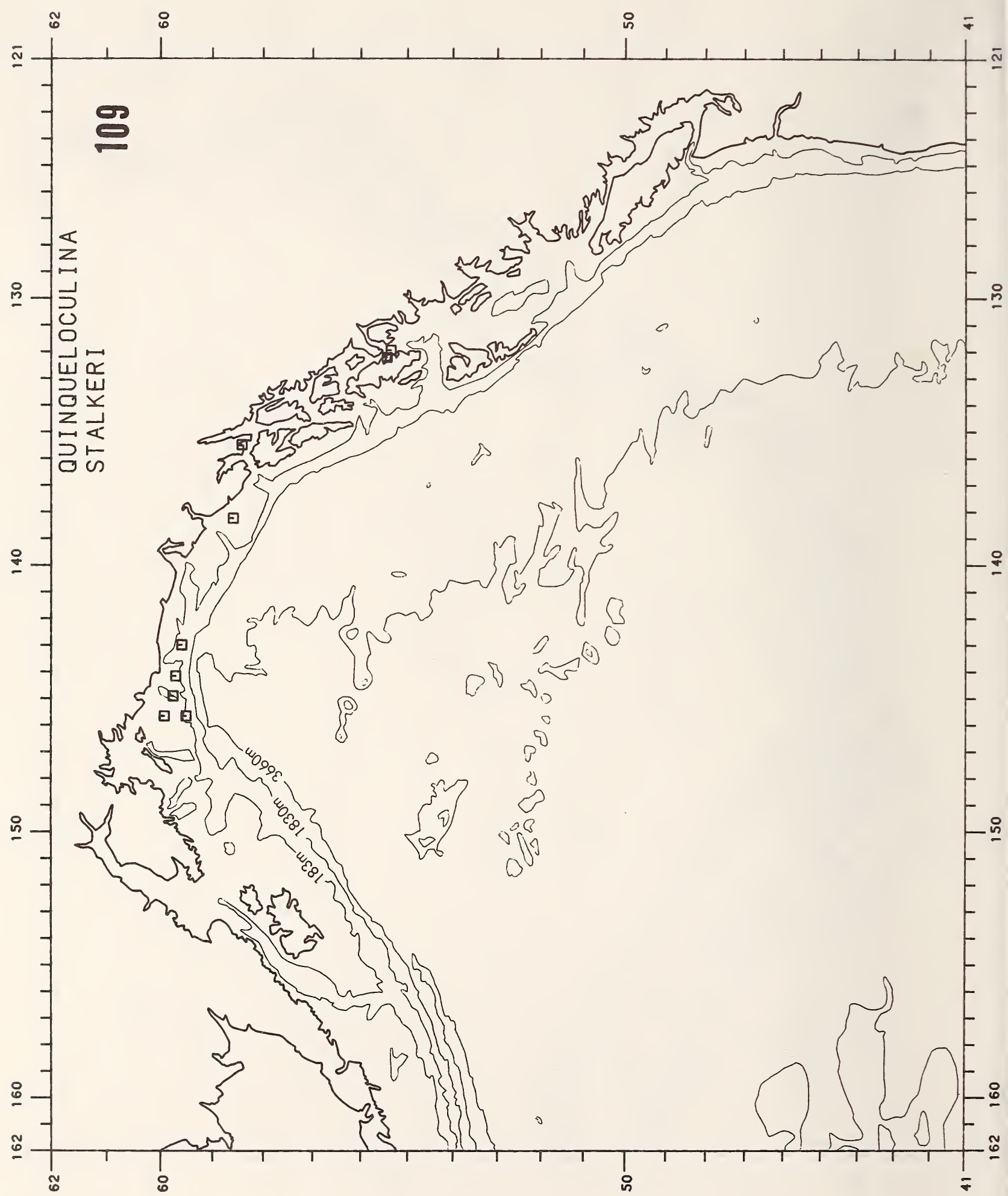




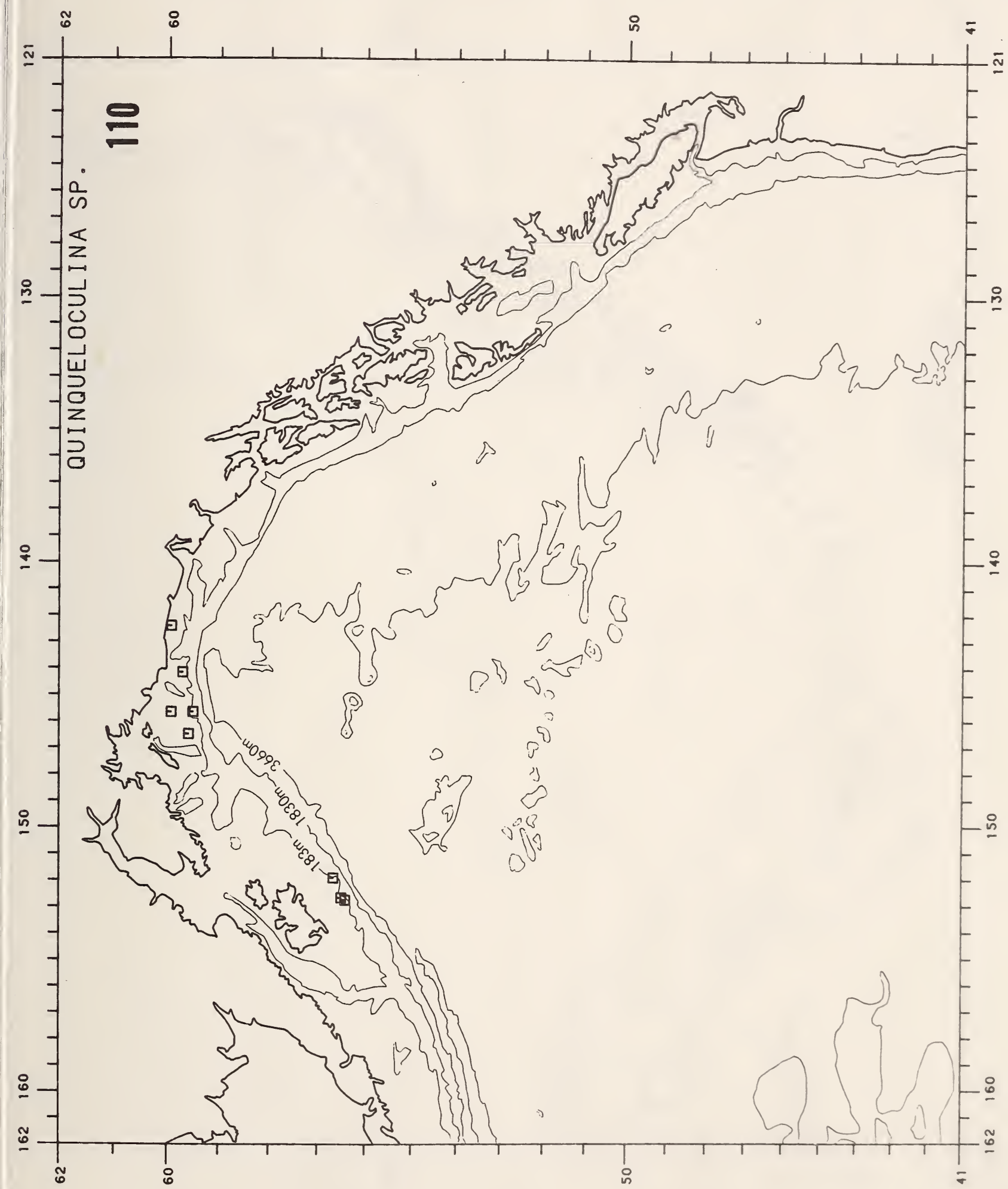




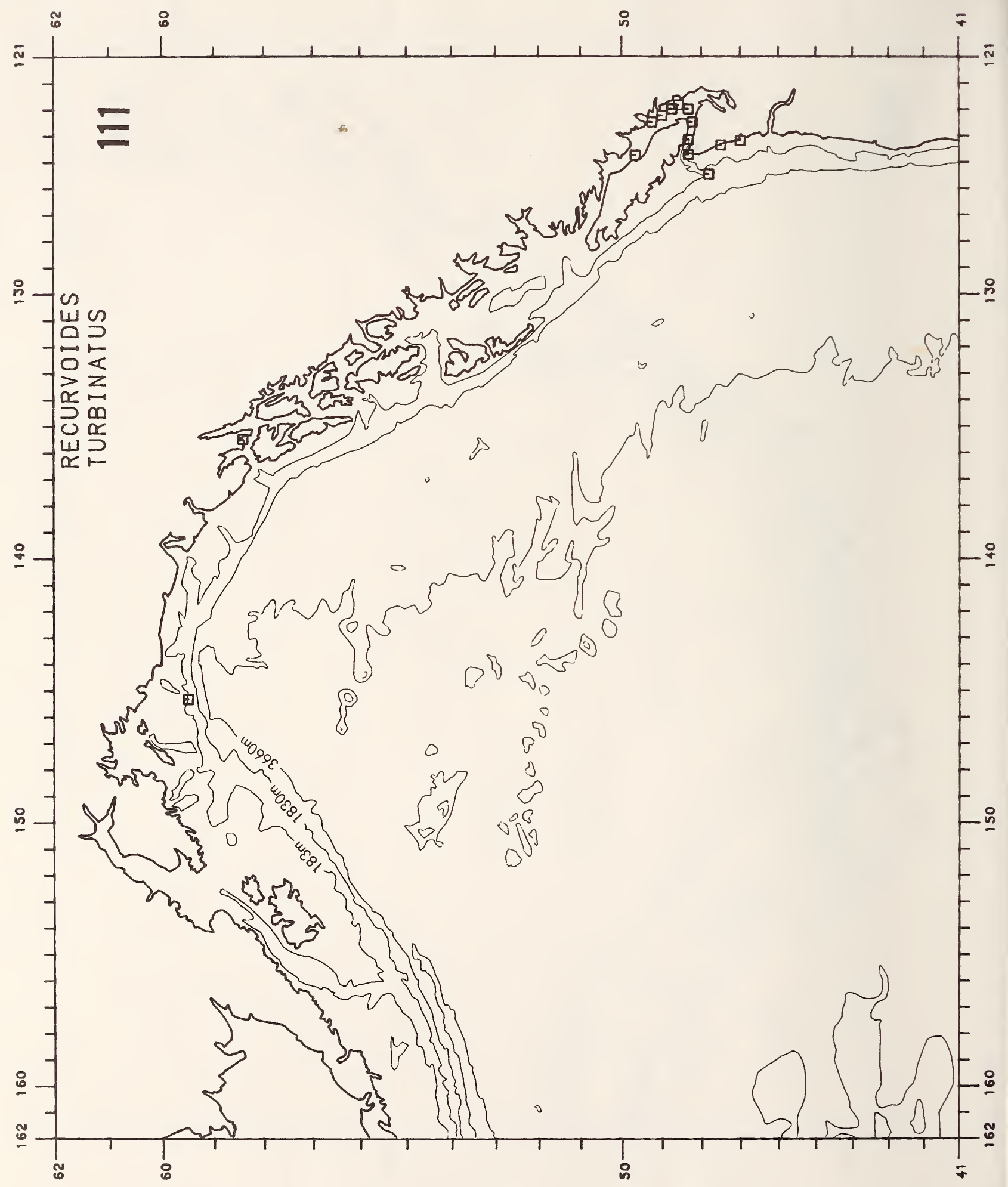




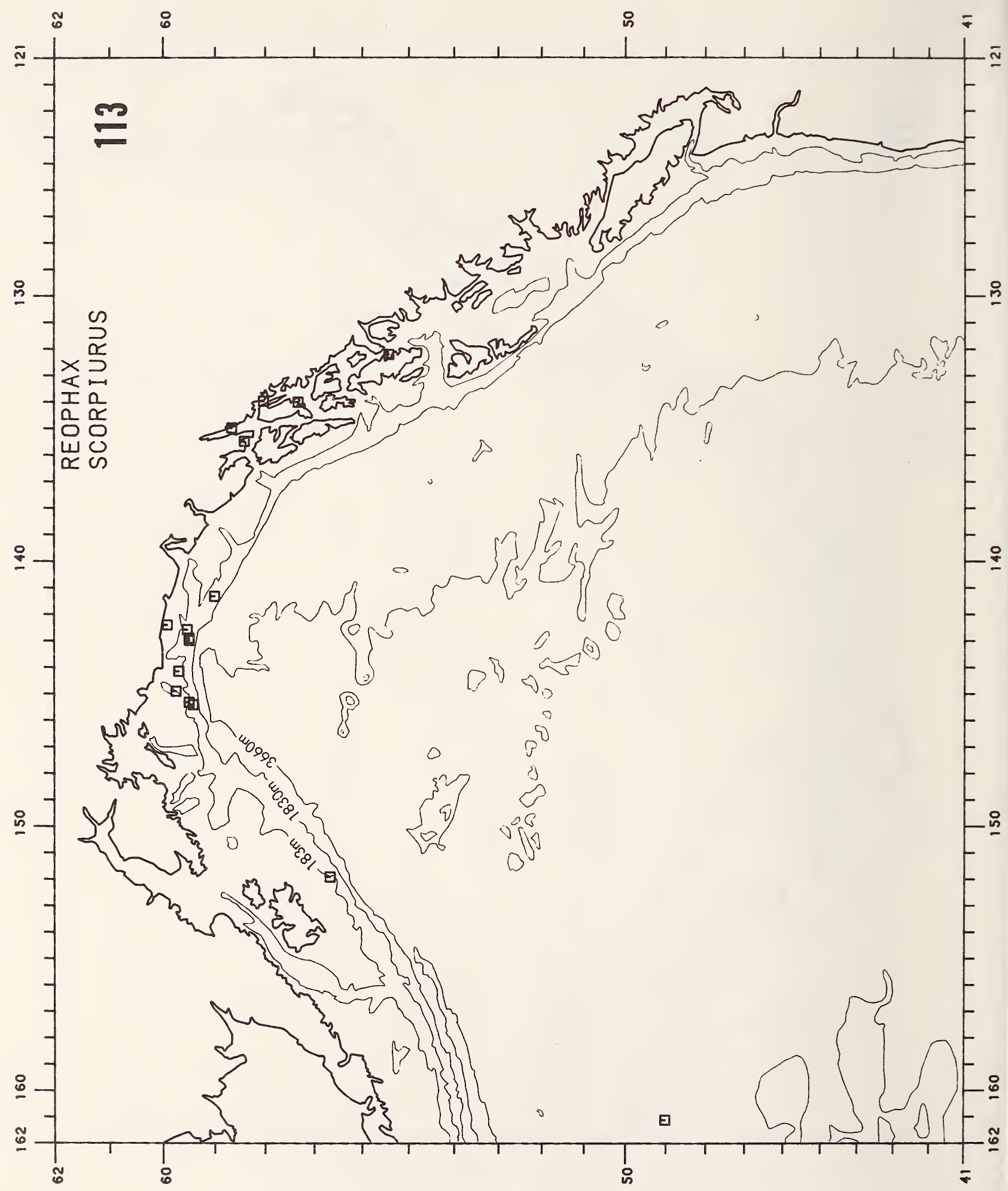




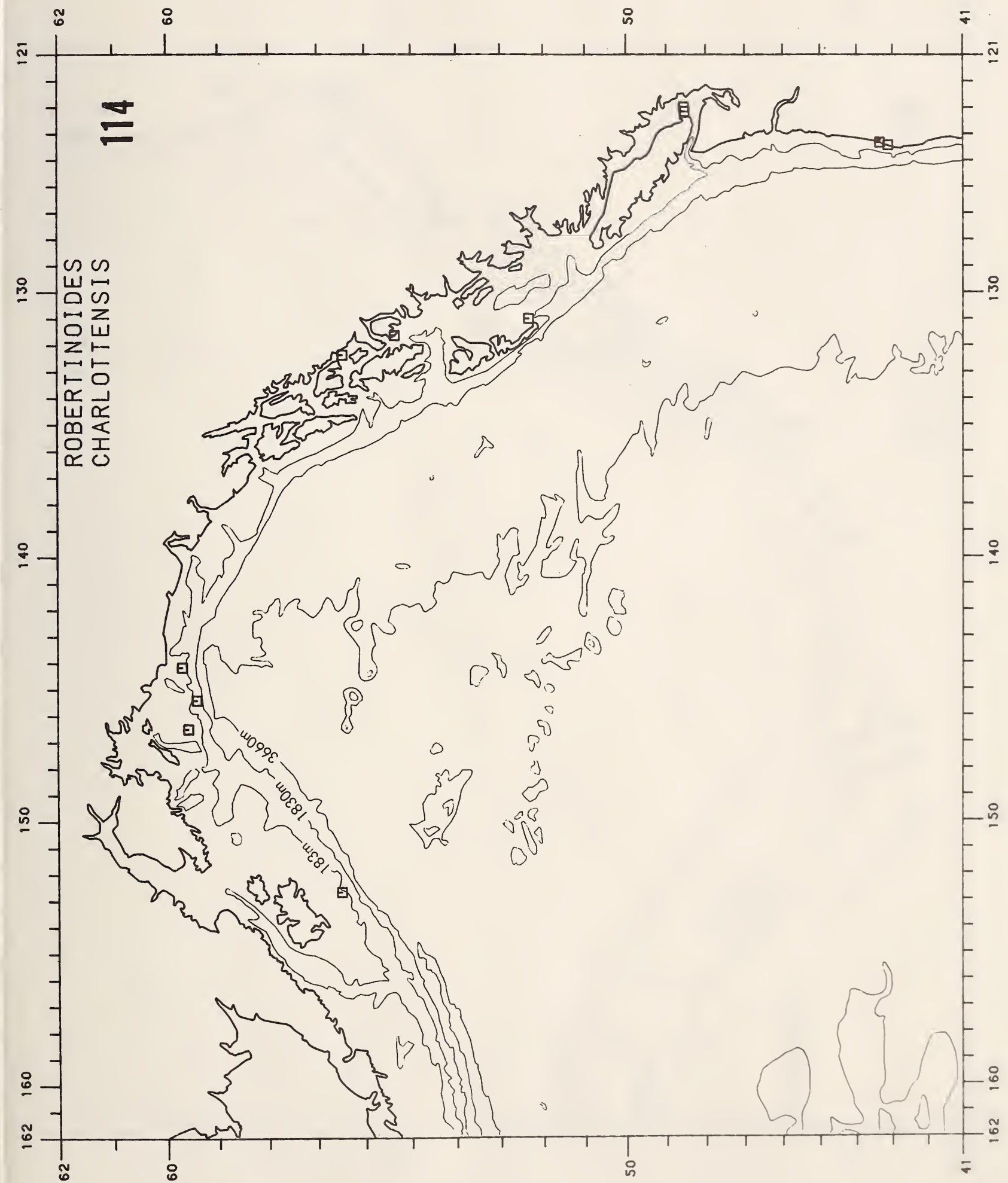




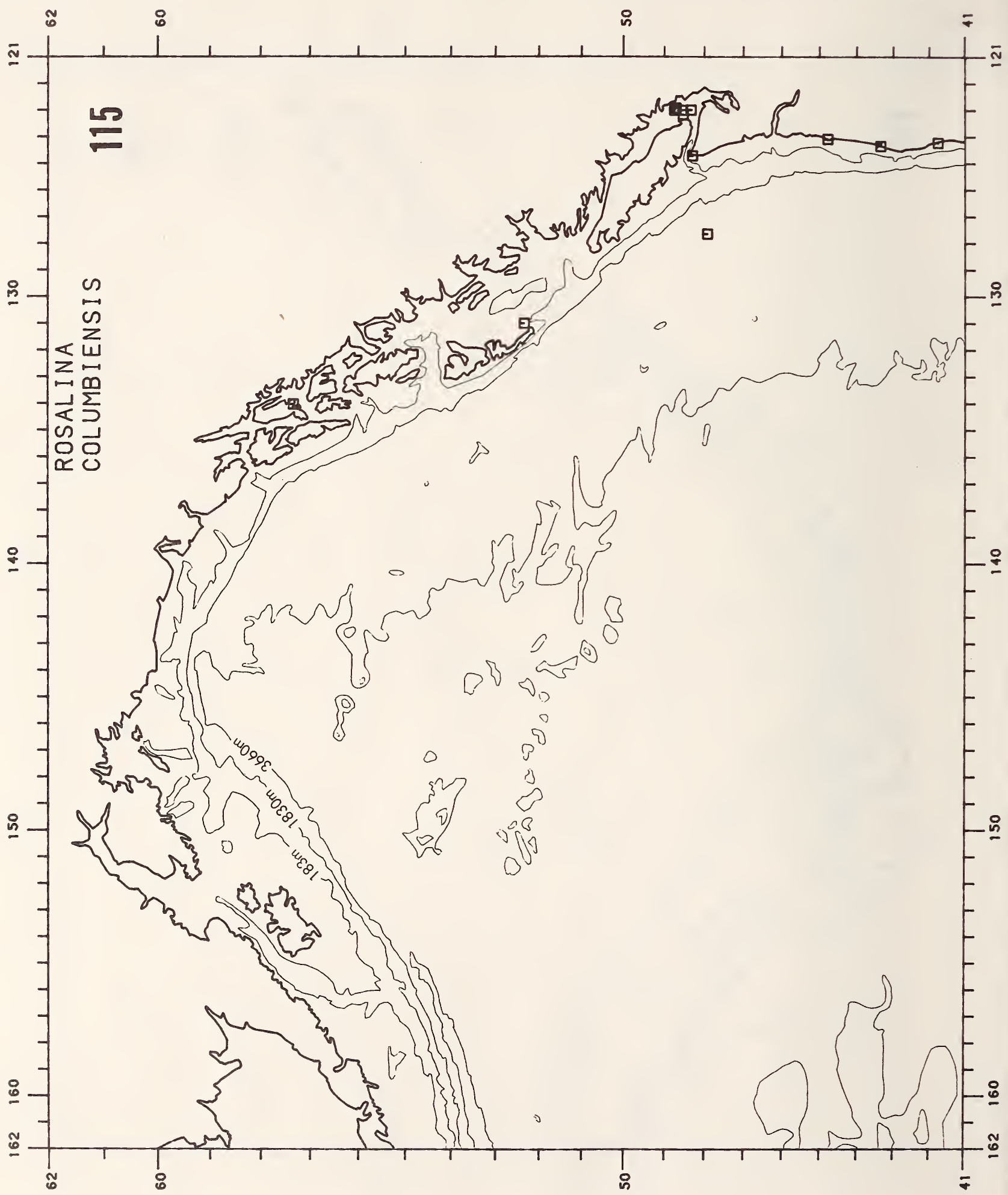




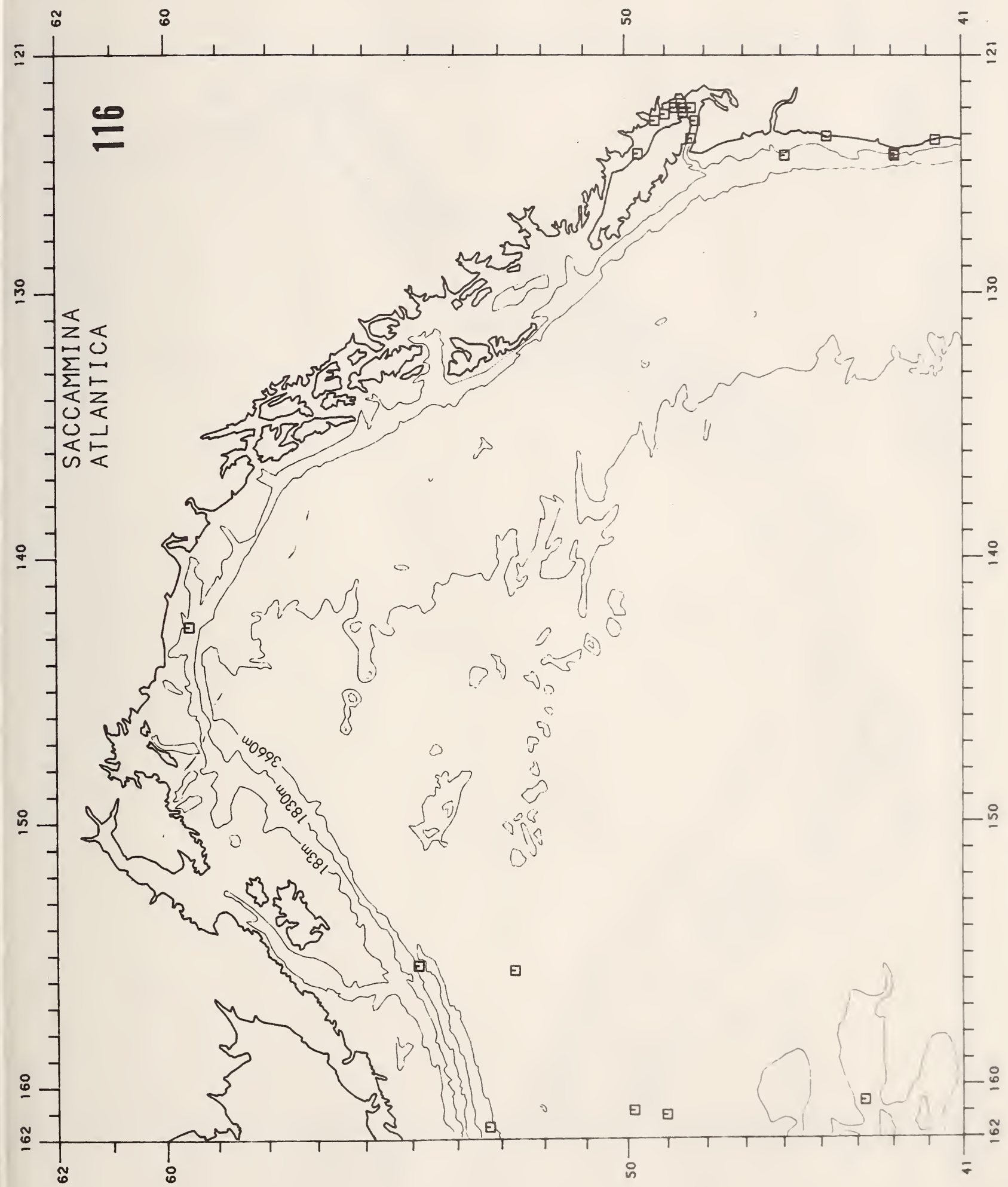




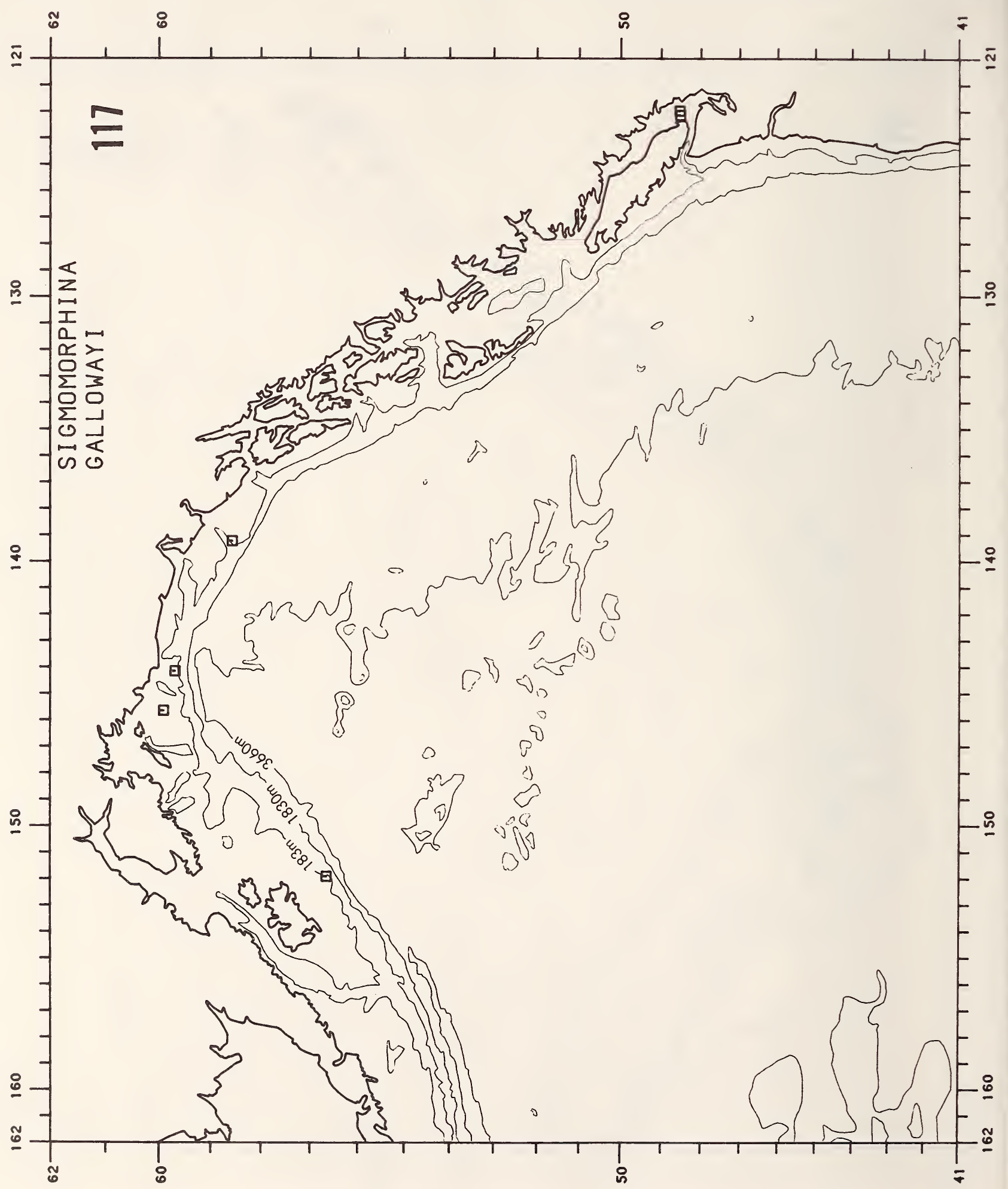


NUMBER 26

213

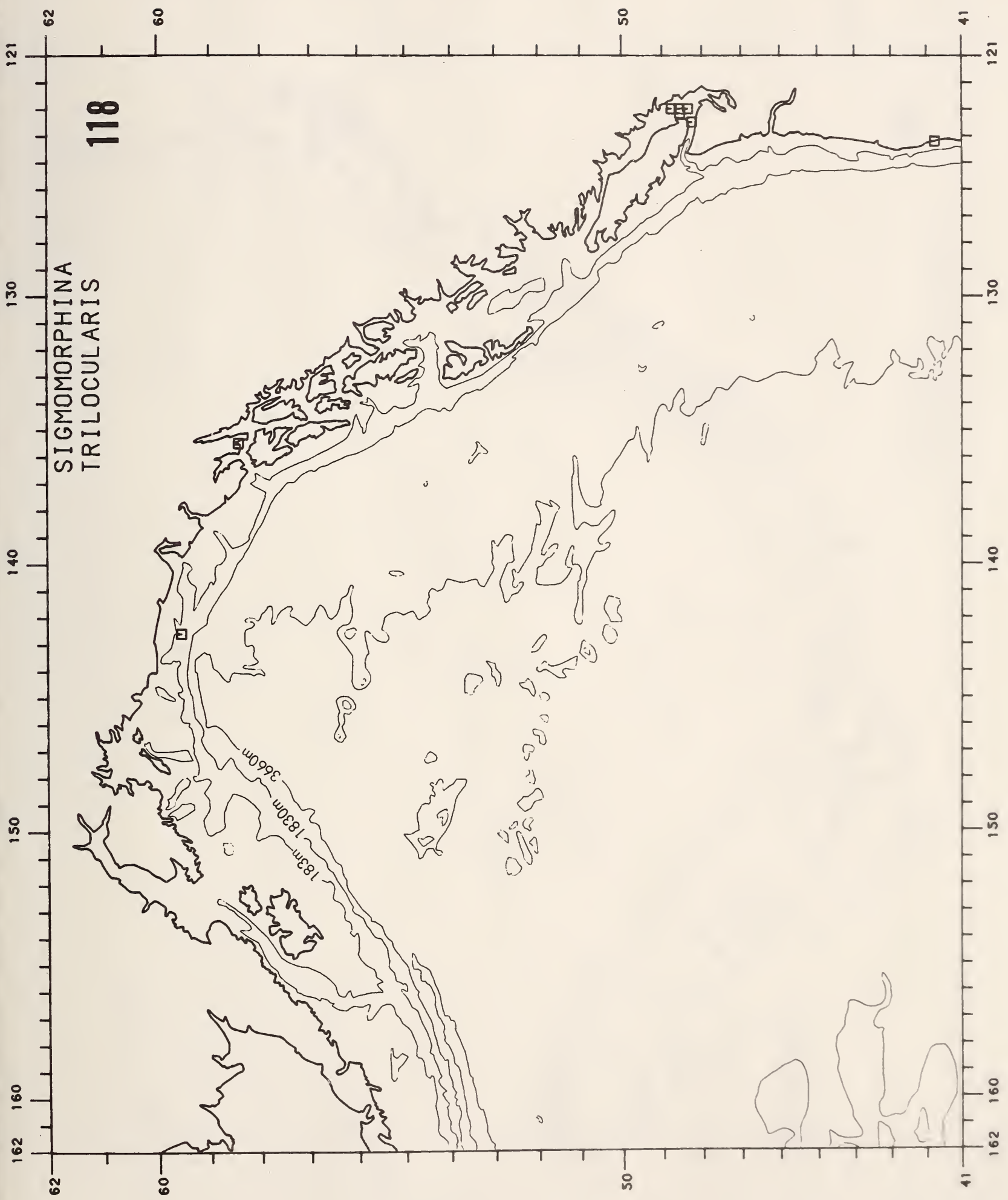




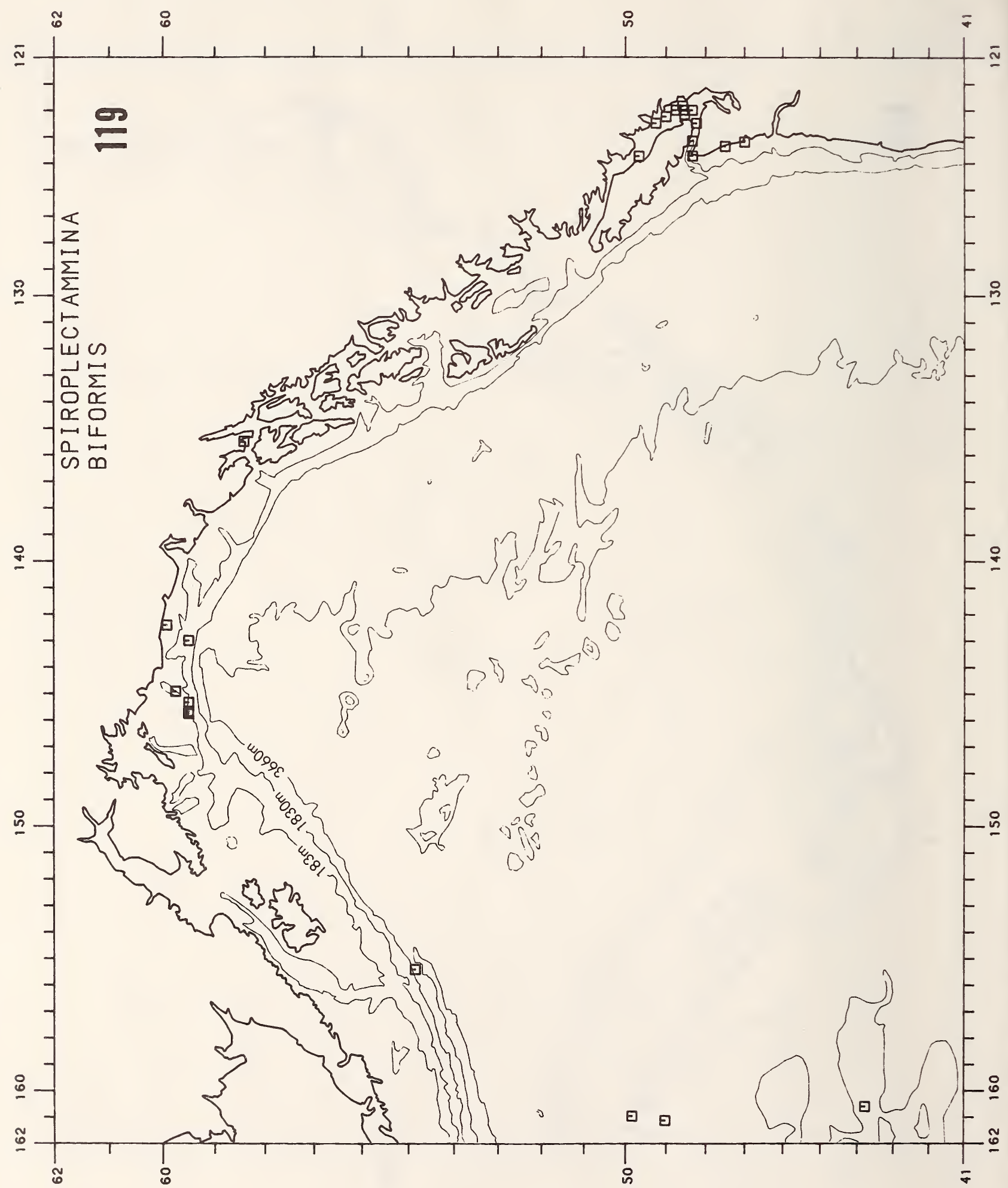




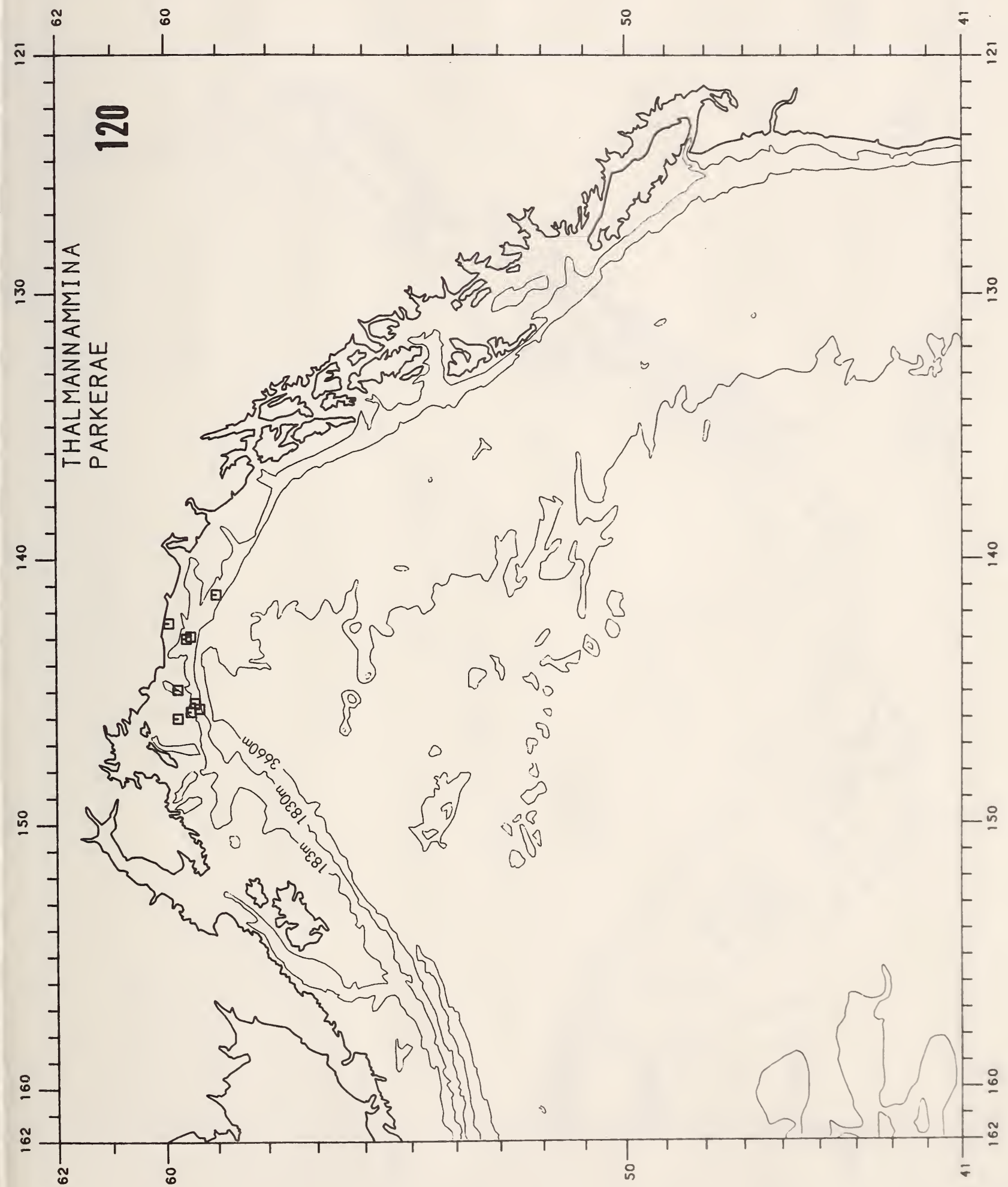




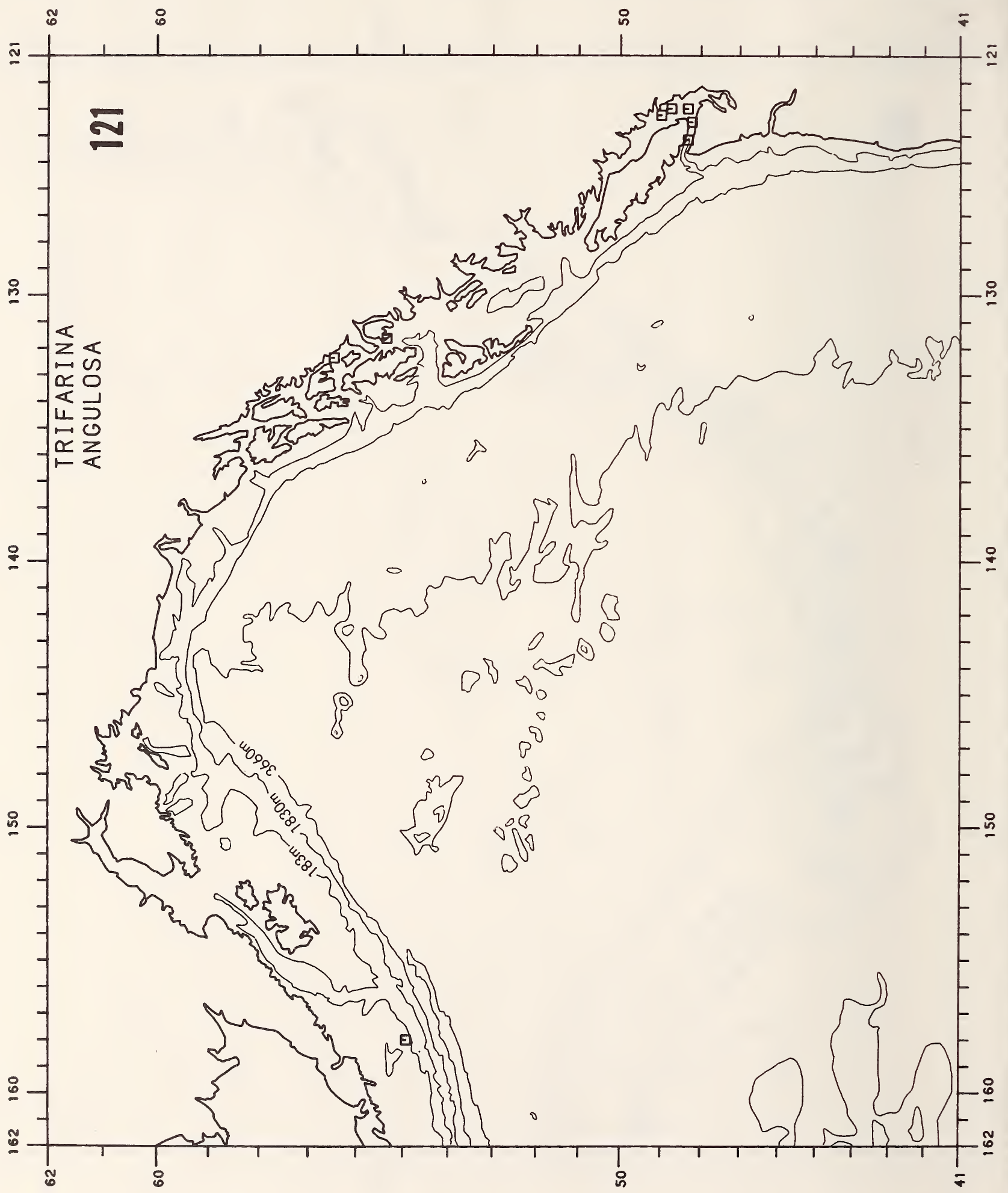




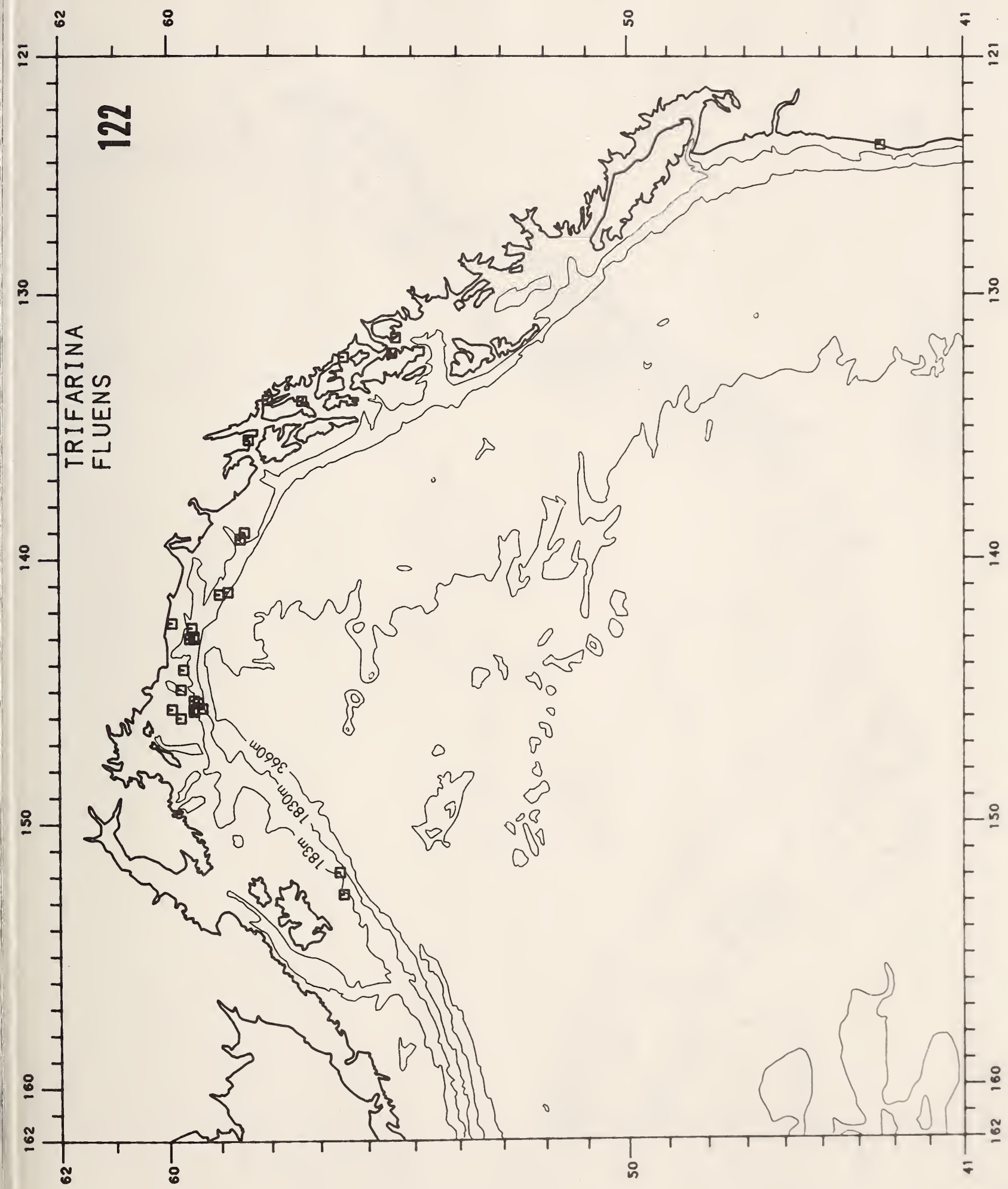




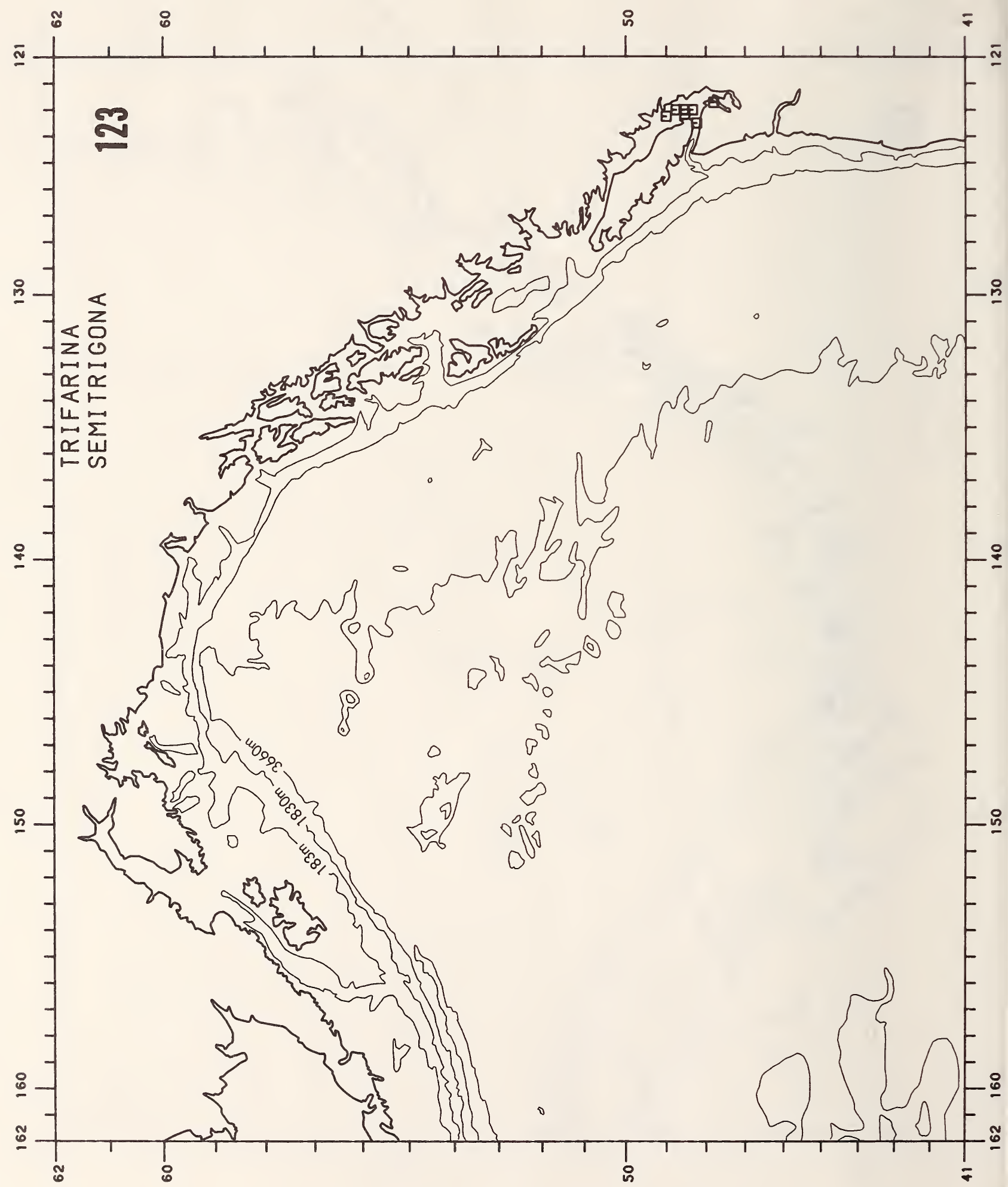




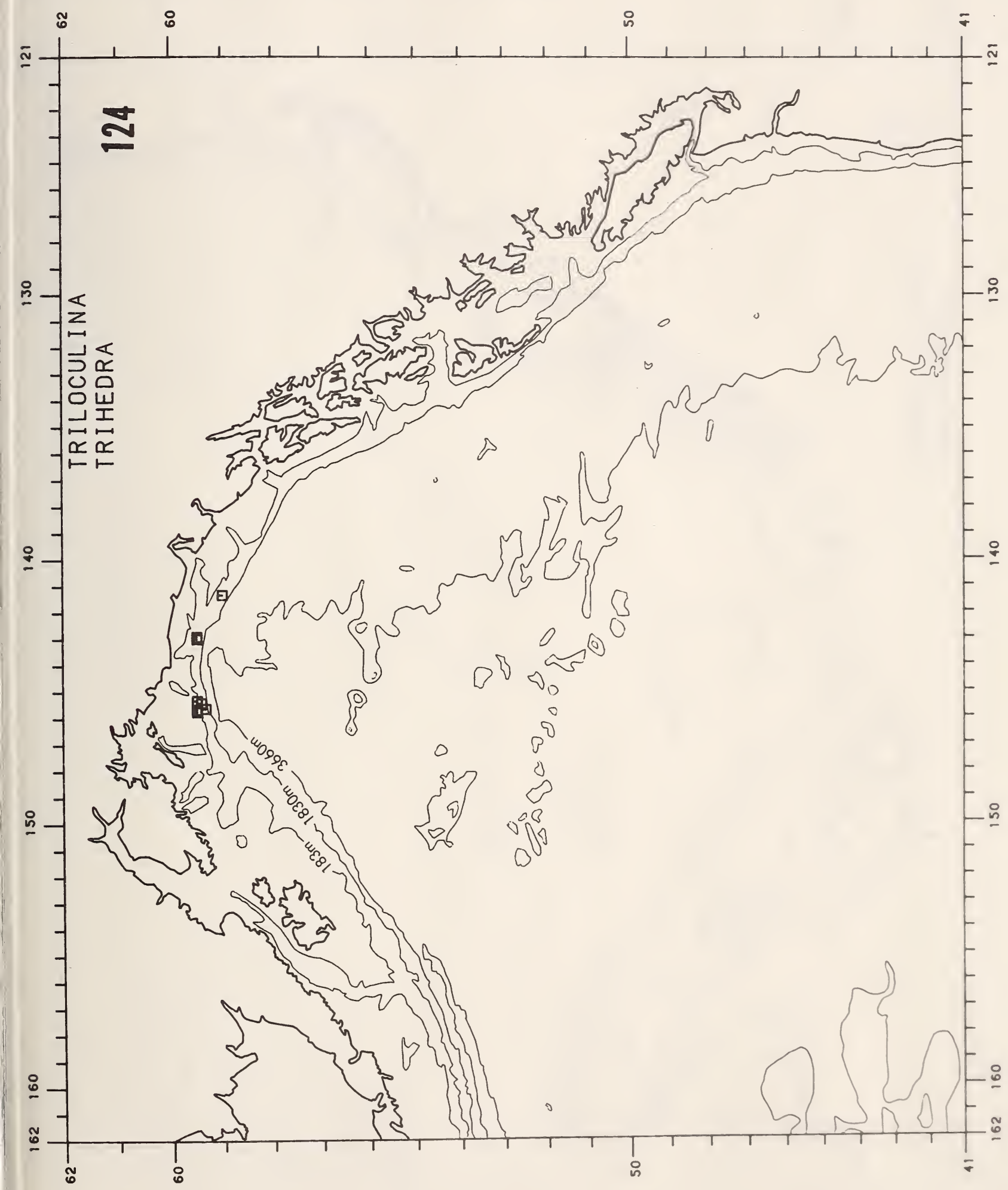




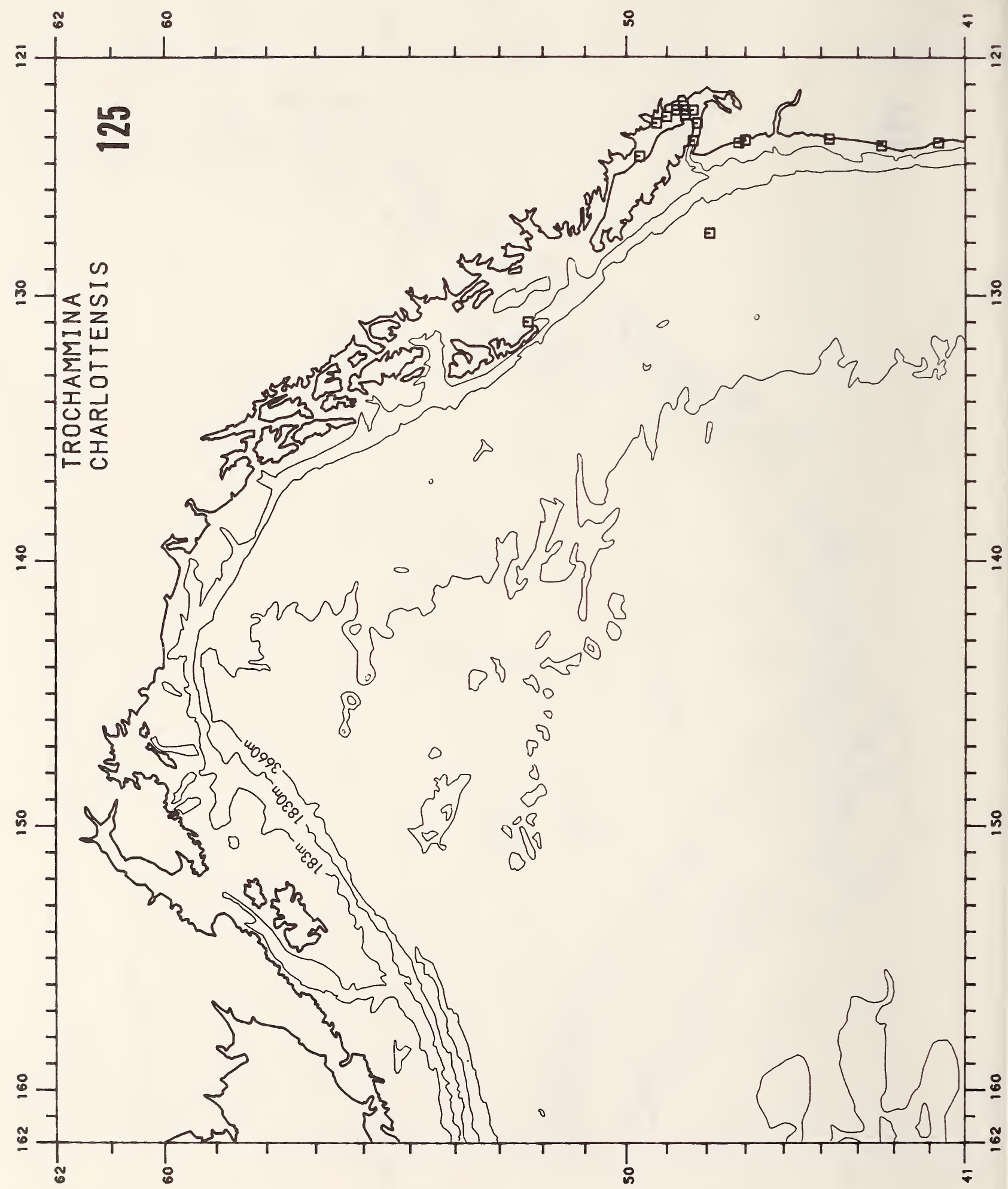




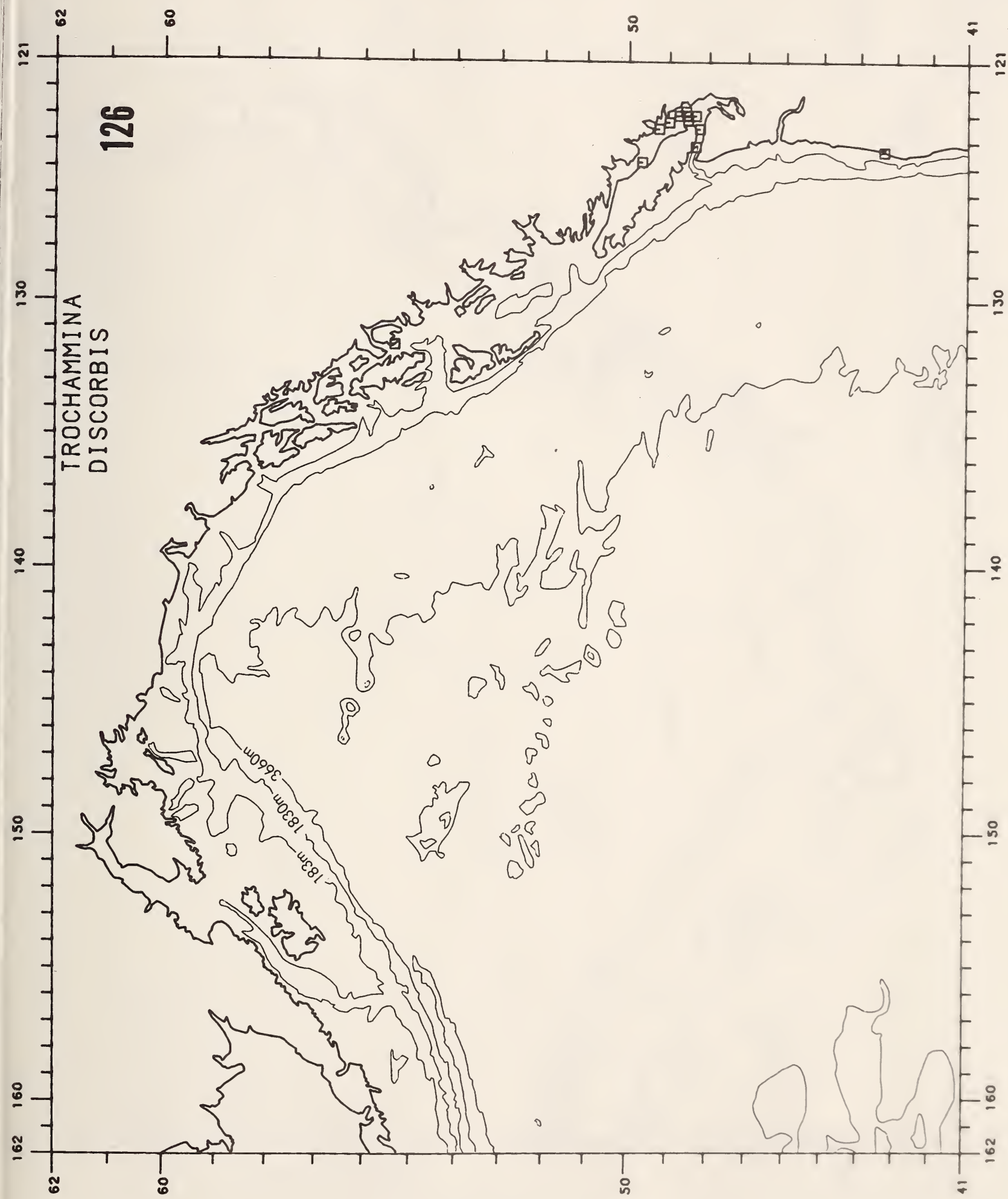




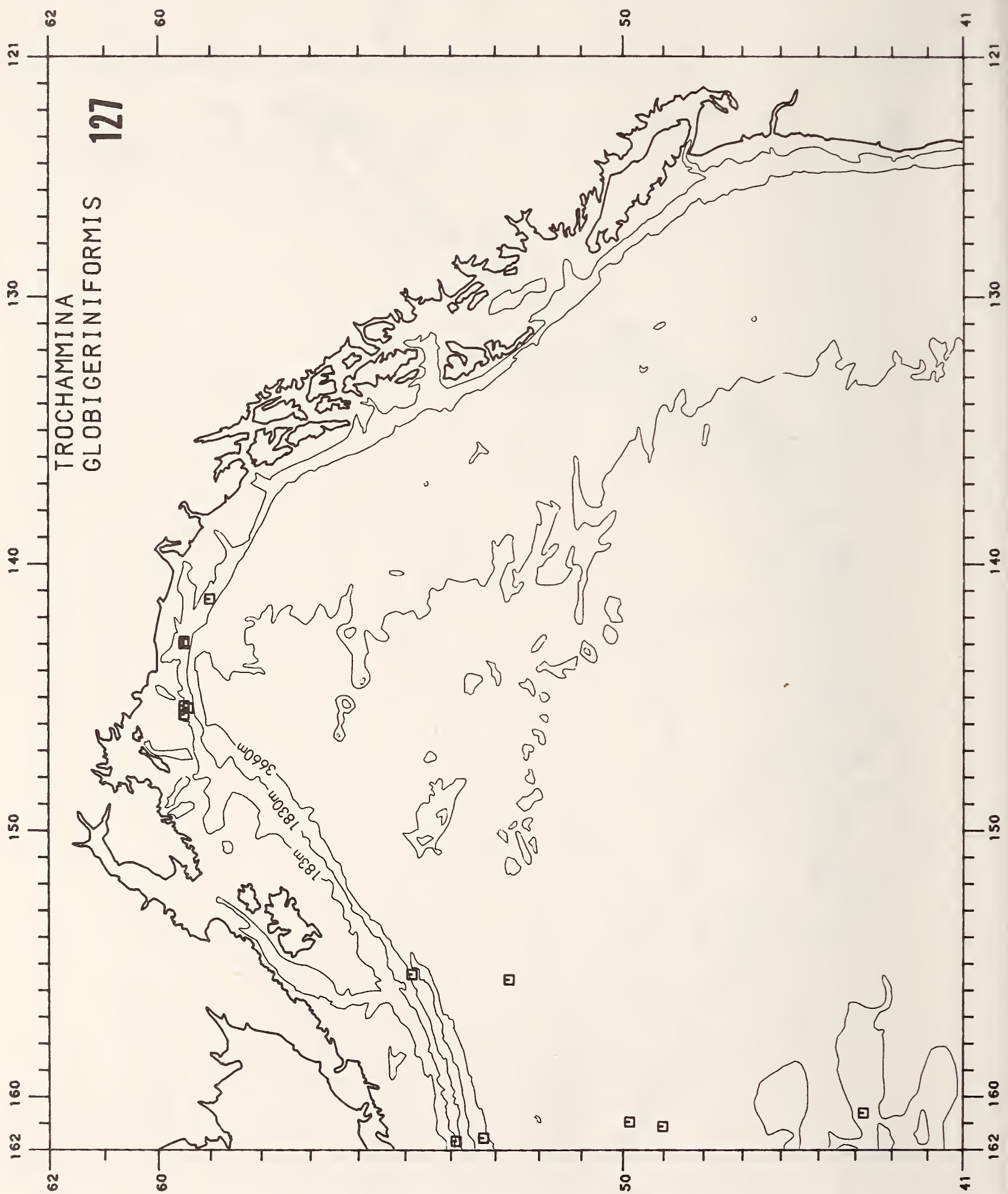




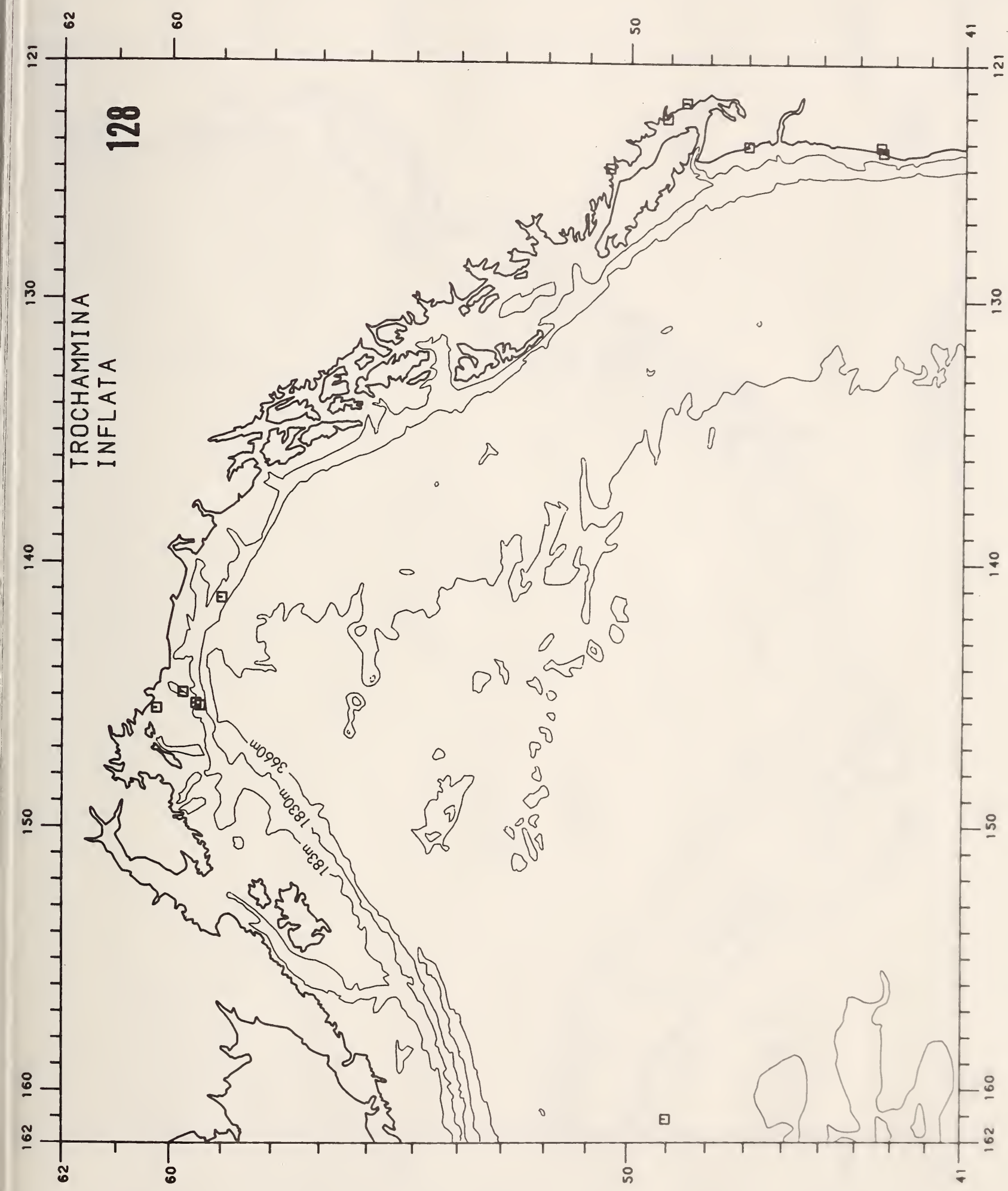




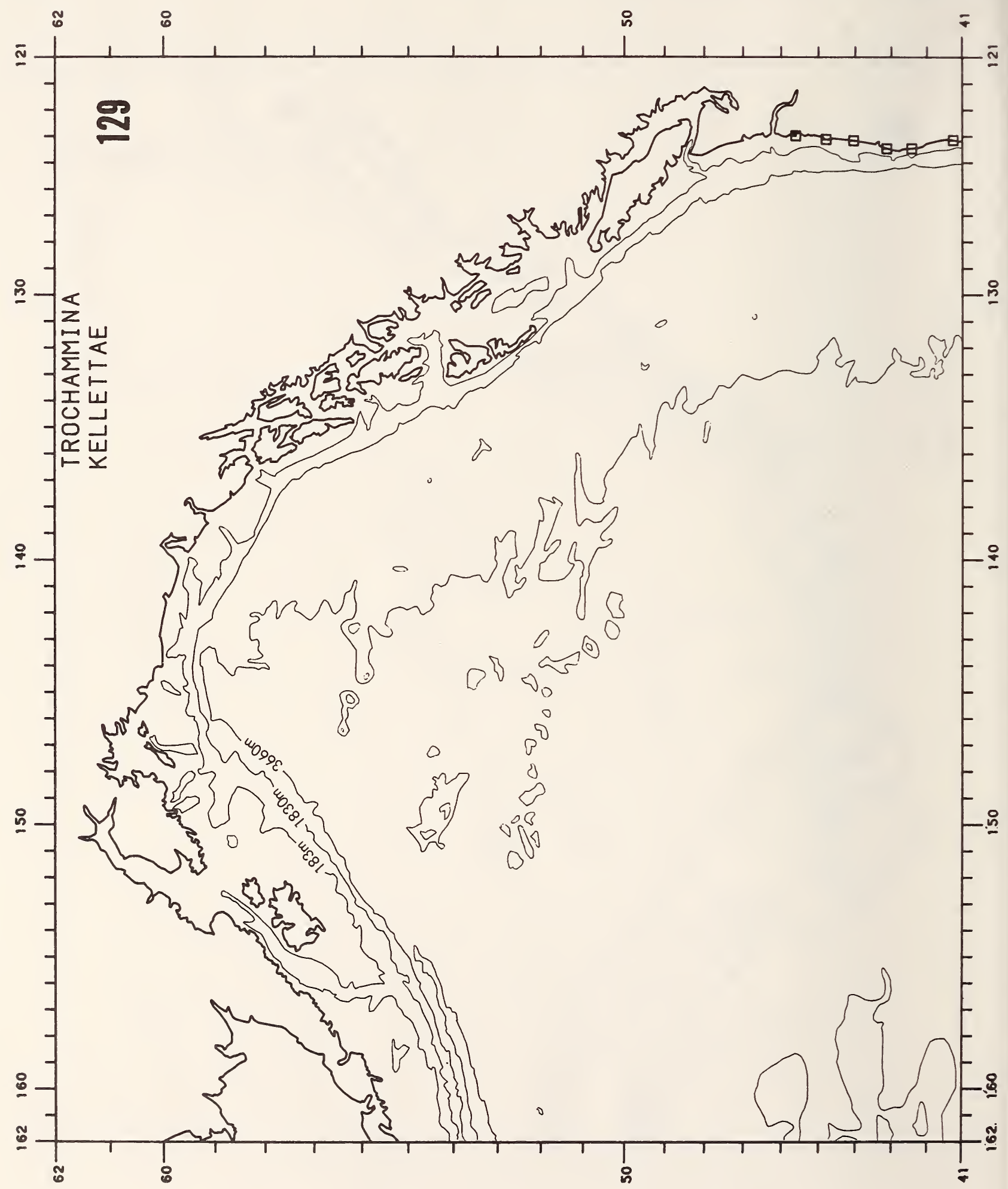




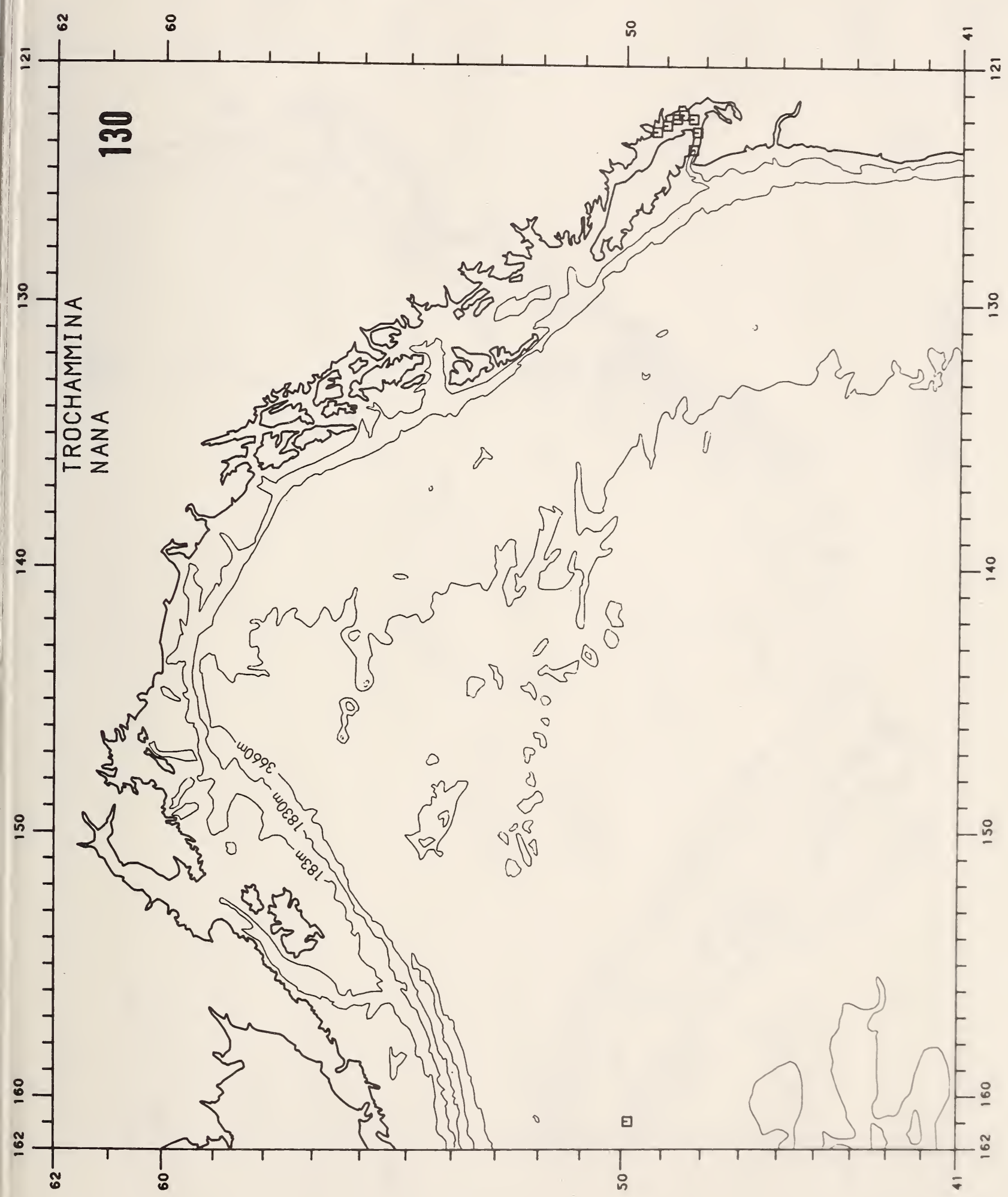




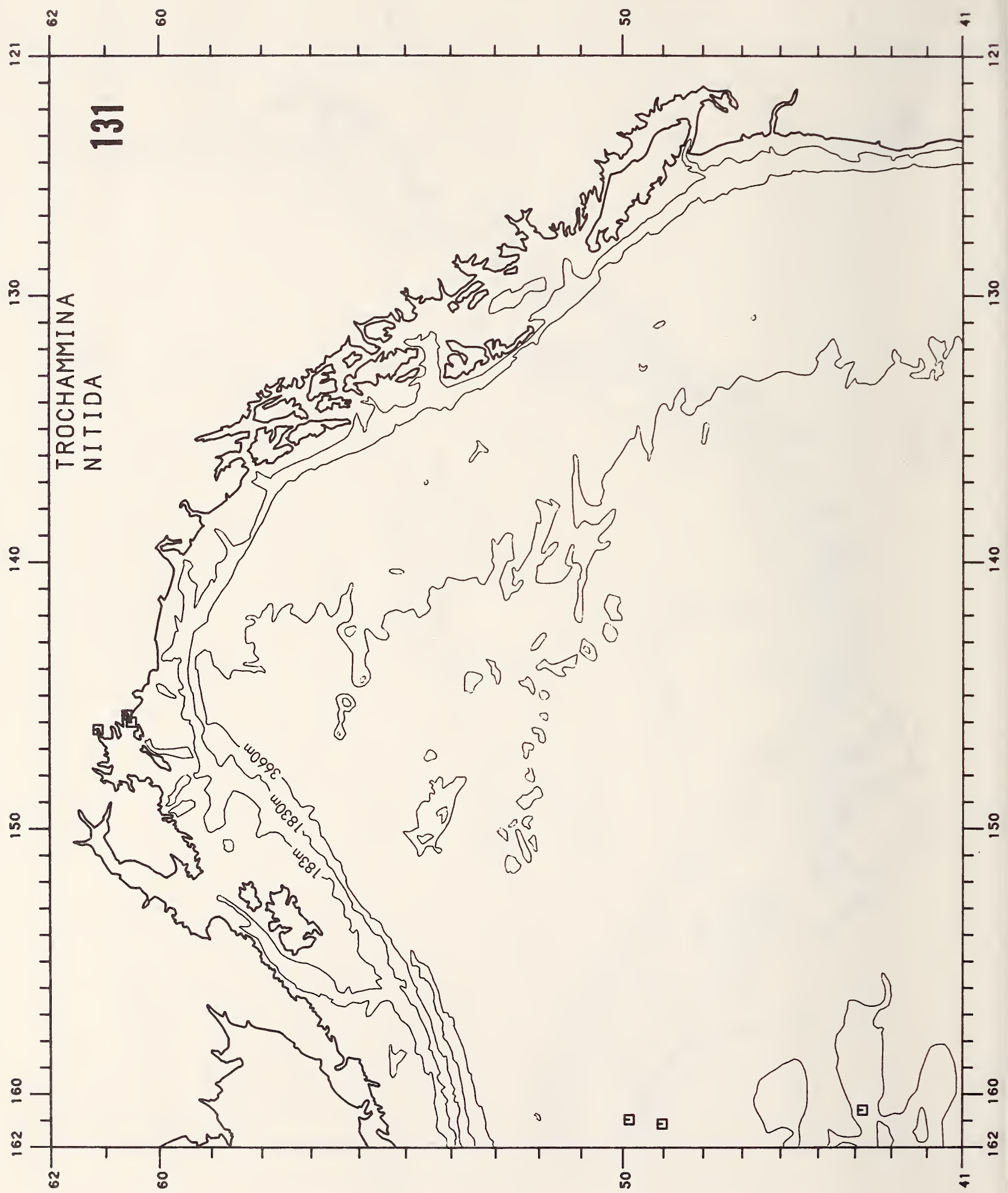




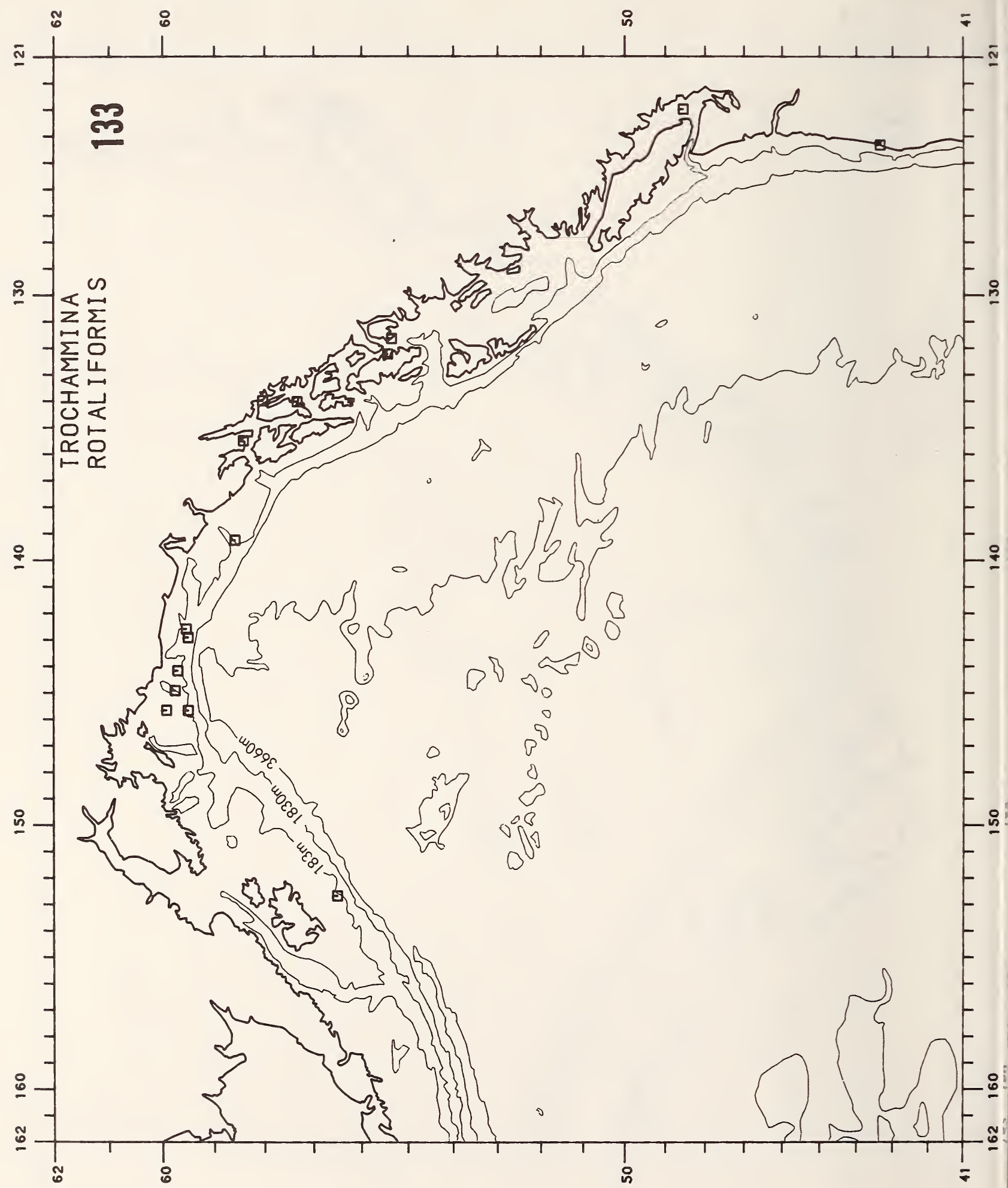




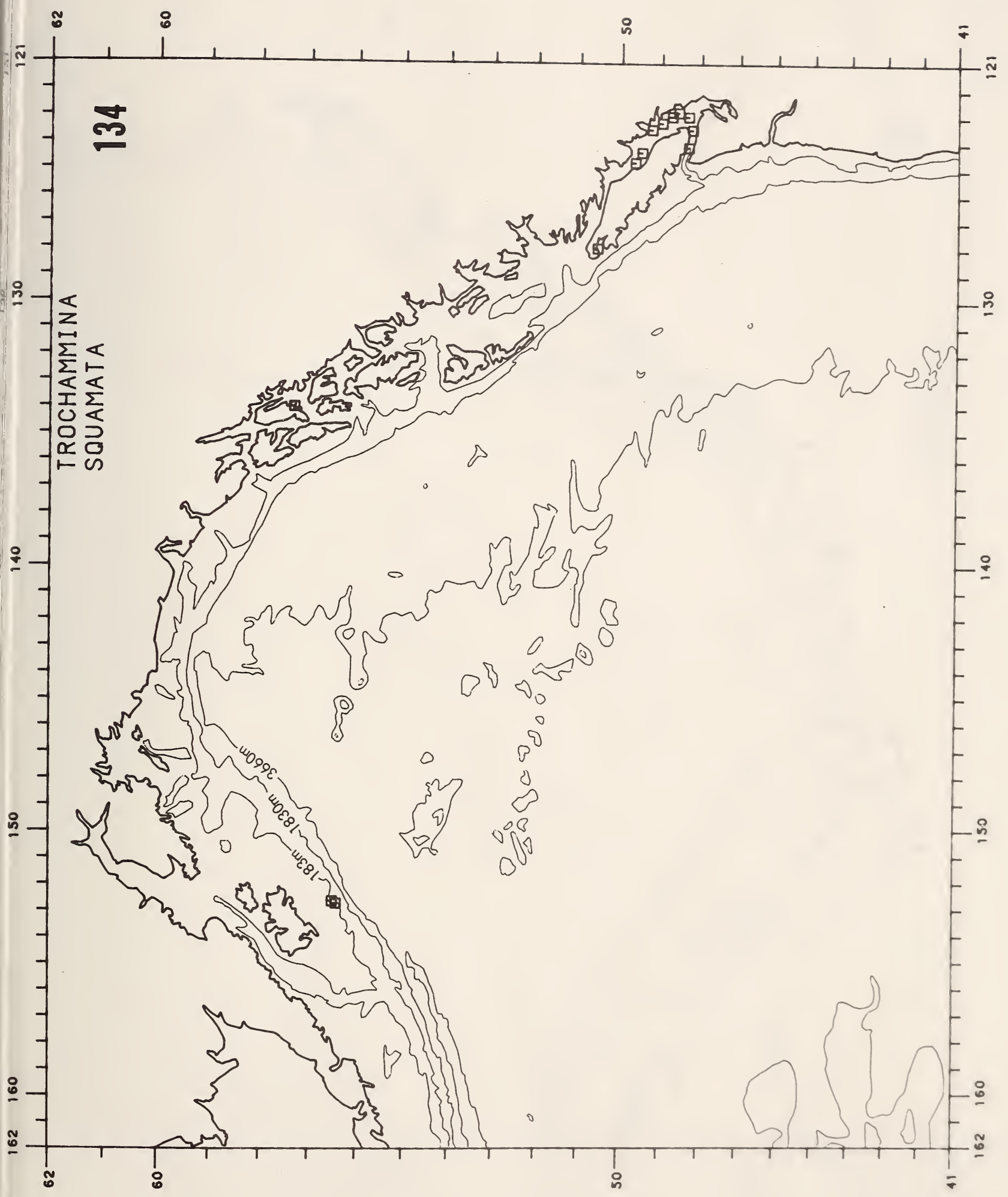




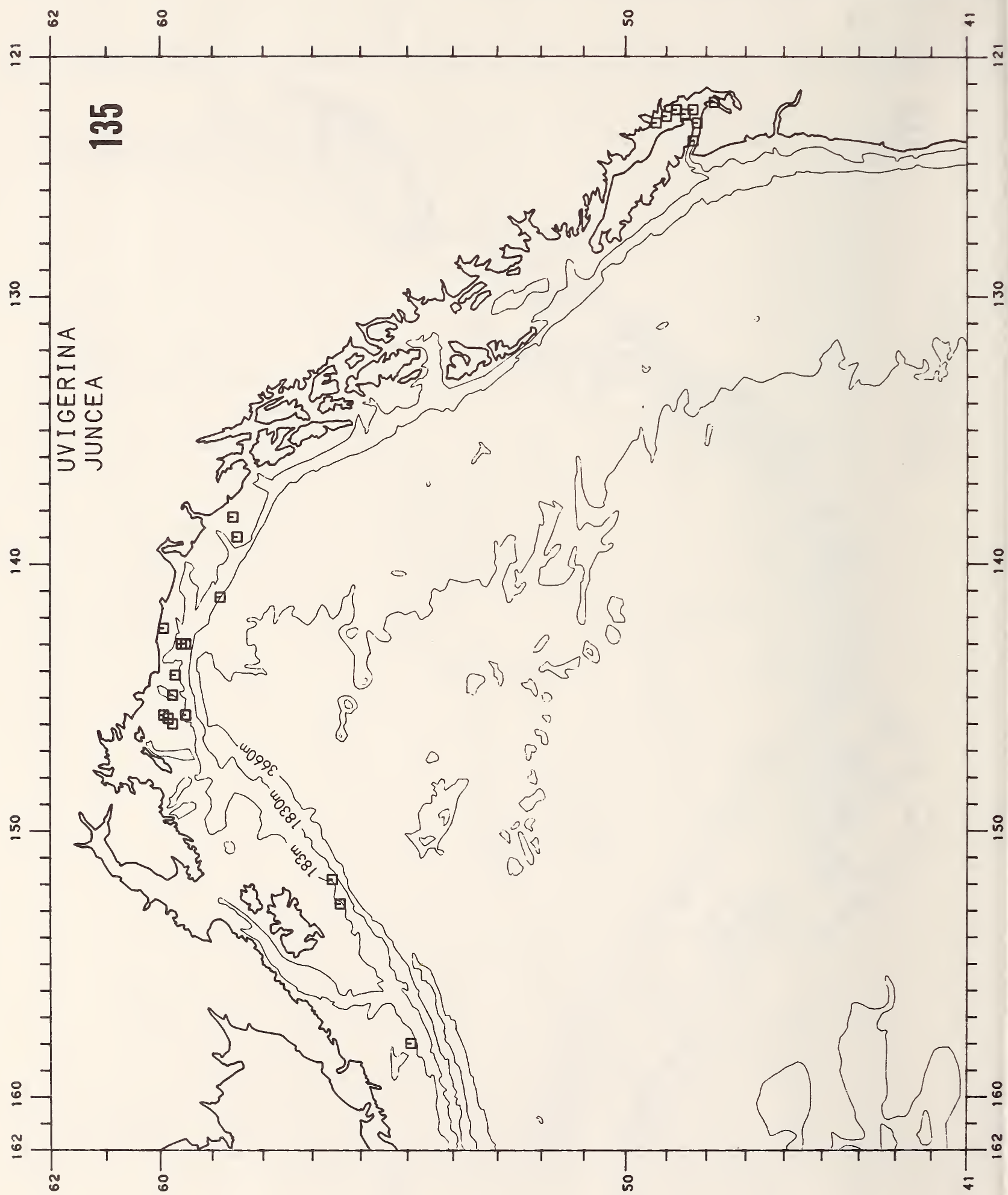




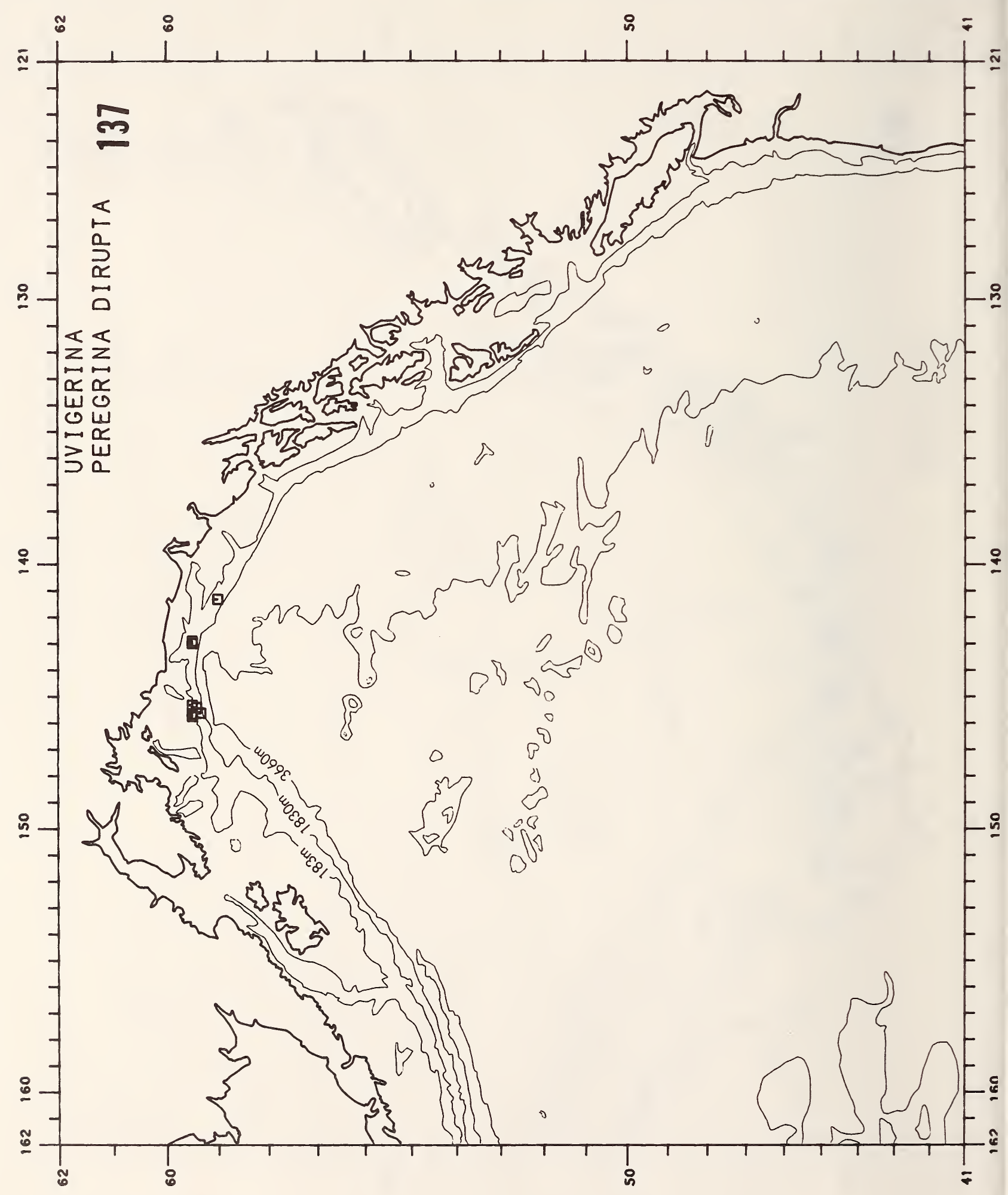




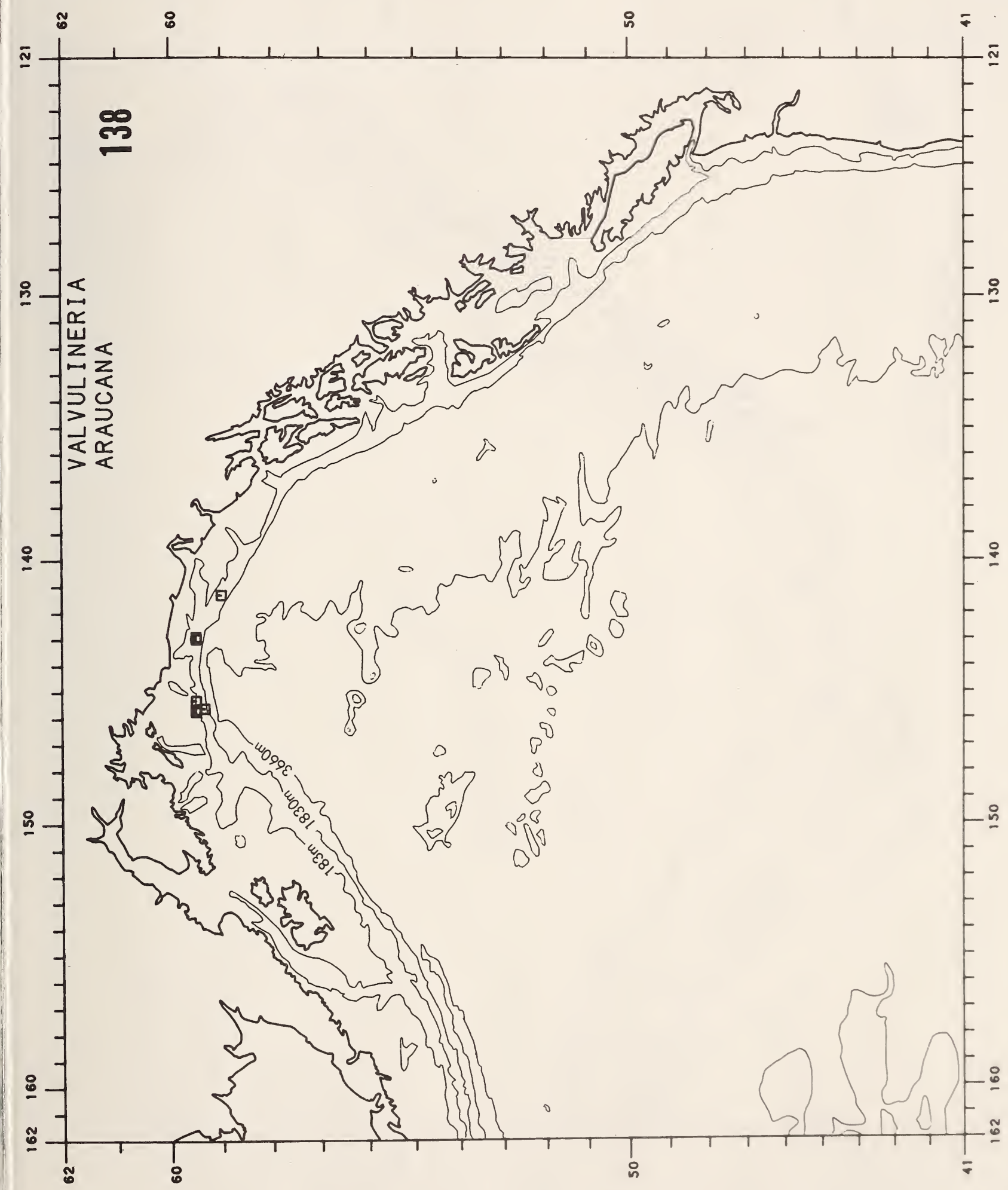




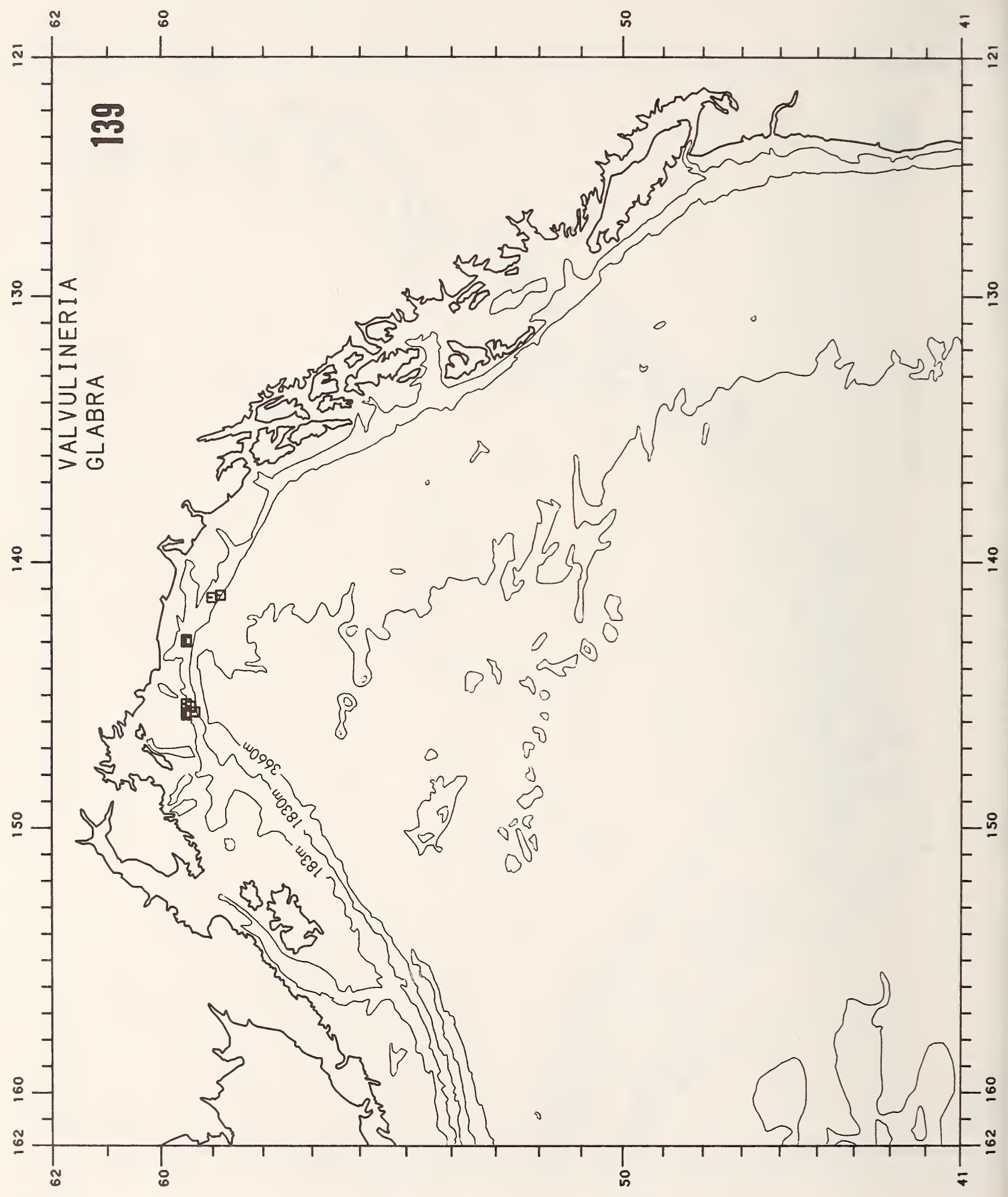





\section{REQUIREMENTS FOR SMITHSONIAN SERIES PUBLICATION}

Manuscripts intended for series publication receive substantive review within their originating Smithsonian museums or offices and are submitted to the Smithsonian Institution Press with Form SI-36, which must show the approval of the appropriate authority designated by the sponsoring organizational unit. Requests for special treatment-use of color, foldouts, casebound covers, etc.- require, on the same form, the added approval of the sponsoring authority.

Review of manuscripts and art by the Press for requirements of series format and style, completeness and clarity of copy, and arrangement of all material, as outlined below, will govern, within the judgment of the Press, acceptance or rejection of manuscripts and art.

Copy must be prepared on typewriter or word processor, double-spaced, on one side of standard white bond paper (not erasable), with 11/4" margins, submitted as ribbon copy (not carbon or xerox), in loose sheets (not stapled or bound), and accompanied by original art. Minimum acceptable length is 30 pages.

Front matter (preceding the text) should include: title page with only title and author and no other information; abstract page with author, title, series, etc., following the established format; table of contents with indents reflecting the hierarchy of heads in the paper; also, foreword and/or preface, if appropriate.

First page of text should carry the title and author at the top of the page; second page should have only the author's name and professional mailing address, to be used as an unnumbered footnote on the first page of printed text.

Center heads of whatever level should be typed with initial caps of major words, with extra space above and below the head, but with no other preparation (such as all caps or underline, except for the underline necessary for generic and specific epithets). Run-in paragraph heads should use period/dashes or colons as necessary.

Tabulations within text (lists of data, often in parallel columns) can be typed on the text page where they occur, but they should not contain rules or numbered table captions.

Formal tables (numbered, with captions, boxheads, stubs, rules) should be submitted as carefully typed, double-spaced copy separate from the text; they will be typeset unless otherwise requested. If camera-copy use is anticipated, do not draw rules on manuscript copy.

Taxonomic keys in natural history papers should use the aligned-couplet form for zoology and may use the multi-level indent form for botany. If cross referencing is required between key and text, do not include page references within the key, but number the keyed-out taxa, using the same numbers with their corresponding heads in the text.

Synonymy in zoology must use the short form (taxon, author, year:page), with full reference at the end of the paper under "Literature Cited." For botany, the long form (taxon, author, abbreviated journal or book title, volume, page, year, with no reference in "Literature Cited") is optional.

Text-reference system (author, year:page used within the text, with full citation in "Literature Cited" at the end of the text) must be used in place of bibliographic footnotes in all Contributions Series and is strongly recommended in the Studies Series: "(Jones, 1910:122)" or ". . . Jones (1910:122)." If bibliographic footnotes are required, use the short form (author, brief title, page) with the full citation in the bibliography.

Footnotes, when few in number, whether annotative or bibliographic, should be typed on separate sheets and inserted immediately after the text pages on which the references occur. Extensive notes must be gathered together and placed at the end of the text in a notes section.

Bibliography, depending upon use, is termed "Literature Cited," "References," or "Bibliography." Spell out titles of books, articles, journals, and monographic series. For book and article titles use sentence-style capitalization according to the rules of the language employed (exception: capitalize all major words in English). For journal and series titles, capitalize the initial word and all subsequent words except articles, conjunctions, and prepositions. Transliterate languages that use a nonRoman alphabet according to the Library of Congress system. Underline (for italics) titles of journals and series and titles of books that are not part of a series. Use the parentheses/colon system for volume(number):pagination: "'10(2):5-9." For alignment and arrangement of elements, follow the format of recent publications in the series for which the manuscript is intended. Guidelines for preparing bibliography may be secured from Series Section, SI Press.

Legends for illustrations must be submitted at the end of the manuscript, with as many legends typed, double-spaced, to a page as convenient.

Illustrations must be submitted as original art (not copies) accompanying, but separate from, the manuscript. Guidelines for preparing art may be secured from Series Section, SI Press. All types of illustrations (photographs, line drawings, maps, etc.) may be intermixed throughout the printed text. They should be termed Figures and should be numbered consecutively as they will appear in the monograph. If several illustrations are treated as components of a single composite figure, they should be designated by lowercase italic letters on the illustration; also, in the legend and in text references the italic letters (underlined in copy) should be used: "Figure 9b. " Illustrations that are intended to follow the printed text may be termed Plates, and any components should be similarly lettered and referenced. Plate 9b." Keys to any symbols within an illustration should appear on the art rather than in the legend.

Some points of style: Do not use periods after such abbreviations as ' $\mathrm{mm}$, ft, USNM, NNE." Spell out numbers "one through "nine" in expository text, but use digits in all other cases if possible. Use of the metric system of measurement is preferable; where use of the English system is unavoidable. supply metric equivalents in parentheses. Use the decimal system for precise measurements and relationships, common fractions for approximations. Use day/month/year sequence for dates: "9 April 1976." For months in tabular listings or data sections. use three-letter abbreviations with no periods: ' Jan. Mar, Jun," etc. Omit space between initials of a personal name: "J.B. Jones.

Arrange and paginate sequentially every sheet of manuscript in the following order: (1) title page. (2) abstract. (3) contents, (4) foreword and/or preface, (5) text, (6) appendixes. (7) notes section, (8) glossary, (9) kibliography, (10) legends, (11) tables. Index copy may be submitted at page proof stage, but plans for an index should be indicated when manuscript is submitted. 

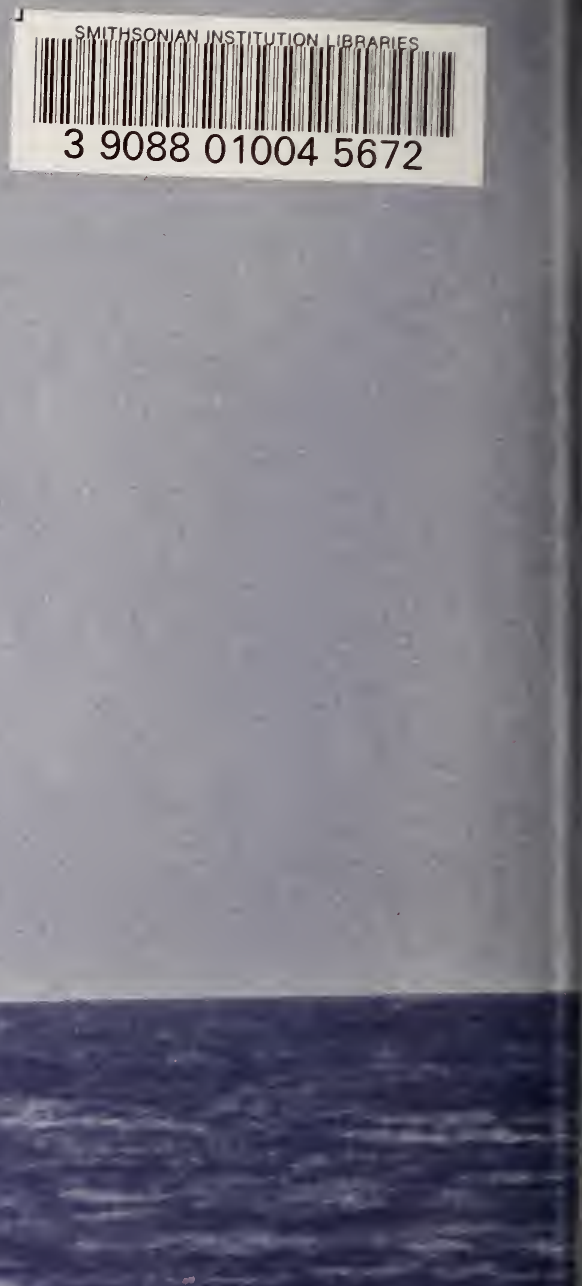

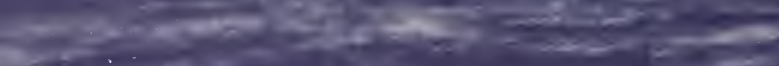

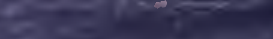

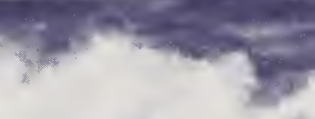

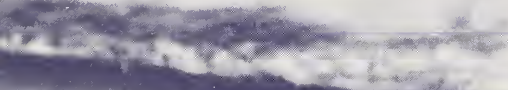

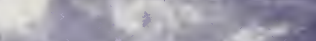

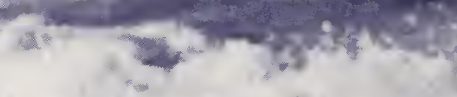

and 